l.

NUREG/CR-4691

SAND86-1562

Vol. 1

\title{
MELCOR Accident Consequence Code System (MACCS)
}

User's Guide

Prepared by D. I. Chanin, J. L. Sprung, L. T. Ritchie, H-N Jow

Sandia National Laboratories

Prepared for

U.S. Nuclear Regulatory Commission

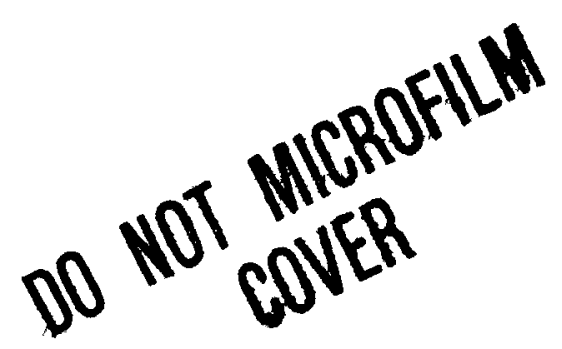




\section{AVAILABILITY NOTICE}

\section{Availability of Relerence Materials Cited in NRC Publications}

Most documents clted in NRC publications will be avallable from one of the following sources:

1. The NRC Publlc Document Room, 2120 L Street, NW, Lower Level, Washington, DC 20555

2. The Superintendent of Documents, U.S. Government Printing Office, P.O. Box 37082, Washington, DC 20013-7082

3. The National Technical Information Service, Springfield, VA 22161

Although the listing that follows represents the majorlty of documents clted in NRC publications, it is not Intended to be exhaustive.

Referenced documents avallable for Inspection and copying for a fee from the NRC Public Document Room Include NRC correspondence and internal NRC memoranda; NRC Office of inspection and Enforcement bulletins, circulars, information notlces, Inspection and Investlgation notlces; Licensee Event Reports; vendor reports and correspondence; Commission papers; and appllcant and licensee documents and correspondence.

The following documents in the NUREG series are avallable for purchase from the GPO Sales Program: formal NRC staff and contractor reports, NAC-sponsored conference proceedings, and NRC booklets and brochures. Also avallable are Regulatory Guldes, NRC regulations in the Code of Federal Regulations, and Nuclear Regulatory Commission issuances.

Documents avallable from the National Technical Information Servlce include NUREG series reports and technical reports prepared by other federal agencles and reports prepared by the Atomic Energy Commission, forerunner agency to the Nuclear Regulatory Commission.

Documents avallable from public and speclal technical libraries include all open literature items, such as books, Journal and perlodlcal articles, and transactions. Federal Register notices, federal and state legislation, and congressional reports can usually be obtalned from these libraries.

Documents such as theses, dlssertations, forelon reports and translations, and non-NRC conference proceedings are avallable for purchase from the organization sponsoring the publication clted.

Single coples of NRC draft reports are avallable free, to the extent of supply, upon written request to the Office of Information Resources Management, Distribution Section, U.S. Nuclear Regulatory Commission, Washington, DC 20555.

Coples of industry codes and standards used in a substantive manner in the NRC regulatory process are maintalned at the NRC Library, 7920 Norfolk Avenue, Bethesda, Maryland, and are avallable there for reference use by the publlc. Codes and standards are usually copyrighted and may be purchased from the originating organization or, If they are Amerlcan National Standards, from the American National Standards Institute, 1430 Broadway. New York, NY 10018.

\section{DISCLAIMER NOTICE}

This report was prepared as an account of work sponsored by an agency of the United States Govemment. Neither the United States Government nor any agency thereof, or any of their employees, makes any warranty, expresed or implied, or assumes any legal liability of responsibility for any third panty's use, or the results of such use, of any information, apparatus, product or process disclosed in this report, or represents that its use by such third party would not infringe privately owned rights. 


\section{DISCLAIMER}

This report was prepared as an account of work sponsored by an agency of the United States Government. Neither the United States Government nor any agency Thereof, nor any of their employees, makes any warranty, express or implied, or assumes any legal liability or responsibility for the accuracy, completeness, or usefulness of any information, apparatus, product, or process disclosed, or represents that its use would not infringe privately owned rights. Reference herein to any specific commercial product, process, or service by trade name, trademark, manufacturer, or otherwise does not necessarily constitute or imply its endorsement, recommendation, or favoring by the United States Government or any agency thereof. The views and opinions of authors expressed herein do not necessarily state or reflect those of the United States Government or any agency thereof. 


\section{DISCLAIMER}

Portions of this document may be illegible in electronic image products. Images are produced from the best available original document. 


\title{
MELCOR Accident Consequence Code System (MACCS)
}

\author{
User's Guide
}

Manuscript Completed: December 1989

Date Published: February 1990

Prepared by

D. I. Chanin, * J. L. Sprung, L. T.Ritchie, H-N Jow

\section{DISCLAIMER}

Sandia National Laboratories

Albuquerque, NM 87185

-Technadyne Engineering Consultants, Inc. Albuquerque, NM

Prepared for

Division of Systems Research

Office of Nuclear Regulatory Research

U.S. Nuclear Regulatory Commission

Washington, DC 20555

NRC FIN A1853
This report was prepared as an account of work sponsored by an agency of the United States Government. Neither the United States Government nor any agency thereof, nor any of their employees, makes any warranty, express or implied, or assumes any legal liability or responsibility for the accuracy, completeness, or usefulness of any information, apparatus, product, of process disclosed, or represents that its use would not infringe privately owned rights. Refermanufacturer, or otherwise does not product, process, or service by trade name, trademark, manufacturer, or otherwise does not necessarily constitute or imply its endorsement, recomand opinions of authors the United States Government or any agency thereof. The views United States Government or any agency thereof.

\section{MASTER}

BISI WIBUTION OF THIS OOCUMENT IS UNLIMREE:

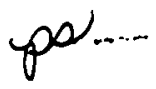




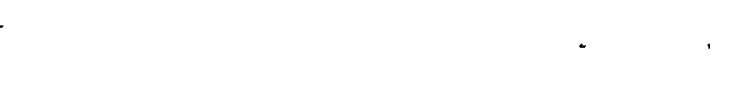




\section{ABSTRACT}

This report describes the MACCS computer code. The purpose of this code is to simulate the impact of severe accidents at nuclear power plants on the surrounding environment. MACCS has been developed for the U.S. Nuclear Regulatory Commission to replace the previous CRAC2 code, and it incorporates many improvements in modeling flexibility in comparison to CRAC2.

The principal phenomena considered in MACCS are atmospheric transport, mitigative actions based on dose projection, dose accumulation by a number of pathways including food and water ingestion, early and latent health effects, and economic costs.

The MACCS code can be used for a variety of applications. These include (1) probabilistic risk assessment (PRA) of nuclear power plants and other nuclear facilities, (2) sensitivity studies to gain a better understanding of the parameters important to PRA, and (3) cost-benefit analysis.

This report is composed of three volumes. Volume $I$, the User's Guide, describes the input data requirements of the MACCS code and provides directions for its use as illustrated by three sample problems. Volume II, the Model Description, describes the underlying models that are implemented in the code, and Volume III, the Programmer's Reference Manual, describes the code's structure and database management. 
CONTENTS

Page

USER'S OVERVIEW

Introduction

MACCS Structure

User Input Processing

Sample Problems

1

References for User's Overview

$\begin{array}{lll}1.0 & \text { ATMOS USER INPUT FILE } & 12\end{array}$

1.1 Introduction to ATMOS 12

1.2 Run Identification (RI) Data 12

1.3 Geometry (GE) Data 13

1.4 Nuclide (IS) Data 14

1.5 Wet Deposition (WD) Data 17

1.6 Dry Deposition (DD) Data 18

1.7 Dispersion Parameter (DP) Data 19

1.8 Plume Meander (PM) Data 21

1.9 Plume Rise (PR) Data 23

1.10 Wake Effects (WE) Data 24

1.11 Release Description (RD) Data 25

1.12 Output Control (OC) Data 32

1.13 Meteorological Sampling (M1) Specification $\quad 34$

1.14 Boundary Weather (M2) Data 36

1.15 Fixed Start Time (M3) Data 37

1.16 Meteorological Bin Sampling (M4) Data 38

1.17 User Supplied Weather Sequence (M5) Data 43

References for Chapter 1

$\begin{array}{lll}2.0 & \text { EARLY USER INPUT FILE } & 45\end{array}$

2.1 Introduction to EARLY 45

2.2 Miscellaneous (MI) Data 46

2.3 Population Distribution (PD) Data 49

2.4 Organ Definition (OD) Data 51

2.5 Shielding and Exposure (SE) Data 53

2.6 Evacuation Zone (EZ) Data 55

2.7 Shelter and Relocation (SR) Data 58

2.8 Early Fatality (EF) Data 63

2.9 Early Injury (EI) Data 67

2.10 Latent Cancer (LC) Data 69

2.11 Generation of Consequence Distributions 73

2.12 User Requested Cases of a Given Health Effect (T1) 75

2.13 User Requested Early Fatality Radius (T2) 77

2.14 User Requested Population Exceeding a Dose
Threshold (T3)

2.15 User Requested Average Individual Risk (T4) 79 
CONTENTS

(Continued)

Page

2.16 User Requested Population Dose (T5) 81

2.17 User Requested Centerline Dose vs. Distance (T6) 82

2.18 User Requested Centerline Risk vs. Distance (T7) 84

2.19 User Requested Population Weighted Risk (T8) 86

$\begin{array}{ll}\text { References for Chapter } 2 & 87\end{array}$

$\begin{array}{lll}3.0 & \text { CHRONC USER INPUT FILE } & \mathbf{8 8}\end{array}$

3.1 Introduction to CHRONC 88

3.2 Problem Identification Data 88

3.3 Emergency Response Cost Data 89

3.4 Long-Term Protective Action Data 89

3.5 Decontamination Plan Data 91

3.6 Interdiction Plan Cost Data 95

3.7 Groundshine Weathering Data 96

3.8 Resuspension Weathering Data 97

3.9 Regional Characteristics Data 98

3.10 Ingestion Transfer Factors Data 101

3.11 Crop Share and Growing Season Data 119

3.12 Food Pathway Protective Action Guide Data 121

3.13 Diagnostic Trace Options 124

3.14 User Requested Population Dose Results 125

3.15 User Requested Economic Costs Results 128

3.16 User Requested Action Distance Results 130

3.17 User Requested Impacted Area/Population Results 131

References for Chapter $3 \quad 133$

\section{APPENDICES}

A. GLOSSARY OF VARIABLES IN THE USER INPUT FILES A-1

A.1 Alphabetized Glossary A-1

B. AUXILIARY INPUT FILES B-1

B.1 Meteorological Data File Format B-1

B. 2 Dose Conversion Factors File Format B-4

B. 3 Site Data File Format B-6

C. PROCEDURE FILES TO RUN MACCS C-1

C.1 MACCS Procedure File c-1

D. SAMPLE PROBLEM INPUT AND OUTPUT FILES D-1

D. 1 Dose Conversion Factors File - DOSDATA D-2

D.2 Site Data File for the Sample Problems - SURSIT D-19

$\begin{array}{ll}\text { D. } 3 \text { List Output File for Sample Problem A } & \text { D-24 }\end{array}$ 
This report provides the documentation of the MACCS computer code, which performs probabilistic calculations of potential offsite consequences of the atmospheric releases of radioactive material in reactor accidents. Sandia National Laboratories (SNL) developed the code for the U.S. Nuclear Regulatory Commission (NRC). The report consists of three volumes -- Volume I being the User's Guide; Volume II, the Model Description; and Volume III, the Programmer's Reference Manual.

With the publication of this report, the MACCS code is released for use within the NRC and for the benefit of other interested users. The MACCS code supersedes the earlier NRC consequence codes, namely, CRAC and CRAC2. The code, its formatted data files, and two pre-processor programs, namely, DOSFAC and MAXGC, which generate certain types of data for the code, are available on magnetic tape from the National Energy Software Center, Argonne National Laboratory, 9700 South Cass Avenue, Argonne, Illinois 60439.

The MACCS code has evolved through several draft versions. The current version (i.e., Version 1.5), simply called MACCS, has been substantially improved and subjected to rigorous quality assurance and verification processes. Idaho National Engineering Laboratory (INEL) performed line-by-line checking of the individual code modules to (a) assess the internal and interfacing consistencies and (b) verify that the FORTRAN statements correctly represent the algorithms, statistical techniques, input data requirements, and output capabilities. INEL's efforts were to ensure that the intended models were implemented into a consistent and essentially error-free computer code as specified by state-of-the-art coding standards for large scientific computer programs. Mr. Ulf Tveten, Institute of Energy Technology, Kjeller, Norway, under a subcontract from SNL, performed a comprehensive review of the chronic exposure pathway modeling in MACCS and compared it with those in the latest versions of the consequence codes that are being used, or planned to be completed in the near future, in several. member countries of the Organization for Economic Cooperation and Development (OECD). INEL, Mr. Tveten, and SNL were interactively involved in the processes of quality assurance, verification, review, identification of errors and implementation of their correction, and model updating. These processes were largely completed before the MACCS code was used for consequence analysis for the second draft of NUREG-1150. INEL's quality assurance and verification report will be published as NUREG/CR-5376. Mr. Tveten's chronic exposure pathway review report will be published as NUREG/CR-5377.

An NRC effort is under way for comparing MACCS with similar codes of earlier vintage using the benchmark problems of the International Consequence Code Comparison Study. This study was sponsored by the OECD, Nuclear Energy Agency (NEA), Committee on the Safety of Nuclear Installations (CSNI), and was completed in 1983. The staff findings will be published as NUREG-1364. Further, it is also planned that MACCS will participate in the forthcoming NEA/CSNI-sponsored consequence code comparison study scheduled to be completed in 1992. Several other new generation consequence codes from the OECD member countries will also participate in the study. The NRC staff will be assisted by Brookhaven National Laboratory in performing the required analysis using MACCS for the study. 
Some of the major new features of MACCS are: (a) improved approximation of the Gaussian crosswind concentration profile, (b) improved health effect models, (c) improved weather sampling, (d) treatment of multiphase release with capability for treatment of change in the wind direction at the reactor between the release phases, (e) detailed chronic exposure pathway modeling, (f) inclusion of inhalation of resuspended radionuclides as an early exposure pathway, (g) provision for more complex emergency response and long-term protective measures, and (h) code flexibility, so that virtually all model parameters can now be provided by the user via input.

The item ( $h$ ) above is a very useful feature of MACCS that will facilitate the analysis of consequence uncertainties due to uncertainties in the model parameters. However, the user now has to prepare much more data, involving multiple disciplines, for input. This introduces the potential for an inexperienced user to produce distorted results because of improper or inconsistent data.

MACCS continues to use a straight line Gaussian plume dispersion and transport model like its predecessors, CRAC and CRAC2. Although this model is very convenient for probabilistic calculations of consequences using a large number of weather samples, care should be exercised in the MACCS applications to any deterministic, or real-time, situations because of such limitations of the model.

Additional improvements in MACCS will be undertaken in the near future. These include incorporation of latent cancer effect models for high-LET radiation (discussed in the BEIR IV report) and any changes that may be dictated by the recently revised assessment of latent cancer risks of radiation (discussed in the BEIR $V$ report). Research for improvements in these areas is under way. In the longer term, additional areas for improvement will be identified by comparing MACCS with other full-scope consequence codes, such as CONDOR (United Kingdom), UFOMOD (Federal Republic of Germany), and COSYMA (Commission of the European Communities).

The MACCS code represents a significant advancement in the development of severe accident analysis methods. Comments based on use of the code would be greatly appreciated and should be forwarded to the undersigned.

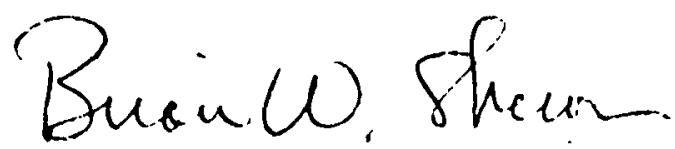

Brian W. Sheron, Director Division of Systems Research Office of Nuclear Regulatory Research 


\section{ACKNOWLEDGMENTS}

The authors would like to thank Sarbes Acharya of the U.S. Nuclear Regulatory Commission and Chuck Dobbe of the Idaho National Engineering Laboratory for their valuable contributions to this report. 


\section{USER'S OVERVIEW}

\section{Introduction}

Sandia National Laboratories has developed a new severe accident riskassessment code, MACCS (MELCOR [We84] Accident Consequence Code System), for the U.S. Nuclear Regulatory Commission as a replacement for the CRAC2 [Ri83] code. The MACCS code models the off-site consequences of radioactive releases; that is, it considers atmospheric transport and deposition, mitigative actions, dosimetry, health effects, and economic costs. The MACCS code is available on magnetic tape from the National Energy Software Center, Argonne National Laboratory, 9700 South Cass Avenue, Argonne, Illinois, 60439.

Our objectives in developing MACCS were (1) to develop a code structure that facilitates the performance of sensitivity and uncertainty analyses, (2) to provide flexibility for performing site-specific consequence analyses, (3) to provide a modular structure that permits incorporation of future modeling improvements, and (4) to provide a portable program that can be used on most large computer systems. The coding conforms to the FORTRAN 77 ANSI standard [AN78].

To facilitate performing uncertainty and sensitivity analyses, the value of almost every parameter used in the MACCS code may be changed by the user through input. Please refer to Volume 2 of this document [Jo89] for a description of the model parameters which cannot be modified through user input. Because of this flexibility, the user is asked to provide significantly more input data than in previous consequence models. Therefore, we have made every effort to develop an input format that is self-documenting in order to make the code easy to use.

Unless specified otherwise, all times used in the code are referenced to the time of accident initiation. This point in time is normally referred to as "scram." Throughout MACCS, SI units have been used to the greatest possible extent. Table 1 contains conversion factors between commonly used SI and English units.

Table 1

Conversion Factors for SI Units

\begin{tabular}{|c|c|c|c|}
\hline Quantity & SI Unit & English Unit & Conversion \\
\hline $\begin{array}{l}\text { absorbed dose } \\
\text { dose equivalent } \\
\text { radioactivity } \\
\text { distance } \\
\text { area } \\
\text { area } \\
\text { power } \\
\text { speed }\end{array}$ & $\begin{array}{l}\text { Gray (Gy) } \\
\text { sievert (Sv) } \\
\text { becquerel (Bq) } \\
\text { meter (m) } \\
\text { sq kilometer } \\
\text { hectare (ha) } \\
\text { Watt }(\mathrm{W}) \\
\text { meter } / \mathrm{sec}(\mathrm{m} / \mathrm{s})\end{array}$ & $\begin{array}{l}\text { rad } \\
\text { rem } \\
\text { curie (Ci) } \\
\text { mile (mi) } \\
\text { sq mile } \\
\text { acre } \\
\text { Btu/hour } \\
\text { miles/hour (mph) }\end{array}$ & $\begin{aligned} 1 \mathrm{~Gy} & =100 \mathrm{rad} \\
1 \mathrm{~Sv} & =100 \mathrm{rem} \\
1 \mathrm{~Bq} & =2.7 \mathrm{E}-11 \mathrm{Ci} \\
1 \mathrm{~m} & =6.2 \mathrm{E}-3 \mathrm{mi} \\
1 \mathrm{sq} \mathrm{km} & =0.386 \mathrm{sq} \mathrm{mi} \\
1 \mathrm{ha} & =2.5 \mathrm{acres} \\
1 \mathrm{~W} & =3.4 \mathrm{Btu} / \mathrm{hour} \\
1 \mathrm{~m} / \mathrm{s} & =2.2 \mathrm{mph}\end{aligned}$ \\
\hline
\end{tabular}


This report describes the input data required by the MACCS program. The remainder of this overview section presents an overview to the structure of MACCS and a description of MACCS user input processing. Chapters 1 , 2 , and 3 provide descriptions of the data requirements for each of the main modules in MACCS (ATMOS, EARLY and CHRONC). A glossary of all the input variables defined in Chapters 1 to 3 is presented in Appendix $A$. The formats of the auxiliary input files (weather data, dose conversion factors, and site descriptive data) are described in Appendix $B$. A description of the procedure file that controls MACCS is supplied in Appendix C. Finally, listings of some of the input and output files for the sample problems are provided in Appendix $D$.

It is hoped that this document will allow a knowledgeable consequence analyst to exercise MACCS. This report is not intended to provide a description of the models used within MACCS or to describe the method of their implementation. A description of the MACCS models is available as Volume 2 of this document [Jo89]. A Programmer's Reference Manual is available as Volume 3 [Ro89].

\section{MACCS Structure}

This section is intended to provide sufficient information so that the reader may understand the general sequence of the MACCS calculations.

The internal documentation of MACCS is intended to provide a detailed picture of the code structure. The program itself begins with a set of comment cards which present the hierarchical control structure of MACCS in graphical form.

Every subroutine or function program unit in MACCS begins with a stylized block of information that states: (1) the purpose of the program unit, (2) the name(s) of the program unit which reference it, (3) a glossary of the variables it utilizes, (4) a glossary of the program units it references, (5) the name and date of its authorship, and (6) a history of its modification.

As a further aid, the program units of MACCS are arranged in the order in which they are first referenced.

A MACCS calculation consists of three phases: (1) input processing and validation, (2) phenomenological modeling, and (3) output processing.

The calculations begin with the processing of all input to the code. Extensive error checking is used so that any input errors are located and diagnosed before attempting to perform the modeling phase of the calculations. If any errors are detected, the program will try to validate as much of the subsequent input as possible in order to facilitate the debugging process but execution of the program will be terminated before attempting to perform the next phase. 
The second phase of the calculations is where all the phenomenological modeling occurs. The sequence in which the phenomena are evaluated closely follows the temporal order of events in the real world which would occur in the event of a reactor accident. The phenomenological models are for the most part based on empirical data and the solutions they entail are usually analytical in nature and computationally straightforward.

The modeling phase of MACCS is subdivided into three parts: ATMOS, EARLY, and CHRONC. ATMOS treats the atmospheric transport and dispersion of material and its deposition from the air. EARLY models the effect of the accident on the surrounding area during an emergency action period which can have a duration of up to one week. CHRONC considers the Impact in the period subsequent to the emergency action period and up to $t=i n f i n i t y$. The following list describes the sequence of phenomenological modeling in ATMOS, EARLY, and CHRONC.

\section{ATMOS :}

define the weather conditions

initialize the dimensions of the plume

calculate radioactive decay occurring before release

determine whether there will be any plume rise

calculate the arrival time of the plume

calculate the amount of material remaining after wet deposition

calculate the dispersion of the plume

calculate radioactive decay during transport

determine the amount of plume rise of a buoyant plume

calculate a prospective $x / Q$ that ignores deposition

calculate the amount of material remaining after dry deposition

calculate the amount of material lost from dry and wet deposition

together

calculate average air and ground concentrations

deplete the inventory of the plume due to deposition

update the particle size distribution for depletion

EARLY:

calculate the histogram approximating the Gaussian distribution

calculate the cloudshine correction factor

calculate the doses in the relocation zone (non-shelterees, non-

evacuees)

calculate the doses to shelterees and evacuees while they are

stationary

calculate the doses to evacuees while they are moving

calculate the health effect risks

produce the EARLY results requested by the user

CHRONC :

calculate the ground concentrations on the spatial grid

determine the efficiency of the first year growing season food pathway 
calculate how long people are gone from home for relocation or evacuation determine what actions are needed during the intermediate phase period determine what doses are incurred during the intermediate phase period find a set of actions to meet exposure criteria in the long-term period determine the effectiveness and cost of decontamination/interdiction determine if the selected actions are cost-effective accumulate the resulting doses and costs from all pathways and actions produce the CHRONC results requested by the user

The data needed to define the models described above are specified through three user input files with the names: ATMOS, EARLY, and CHRONC. The requirements for preparation of these files are described in Chapters 1,2 , and 3 of this document.

In some cases, the user may not need to exercise all of the code's features. For instance, if all that is needed is a calculation of air and ground concentrations as a function of distance, only the ATMOS module need be exercised and EARLY and CHRONC can be skipped. As another example, if a sensitivity study on emergency response assumptions is being performed, there may be no need to run the CHRONC module.

The skipping of modules is controlled by the specification of a control variable in the previous module's user input file. A control variable in the ATMOS User Input File (ENDAT1) can be used to signify that both EARLY and CHRONC are to be skipped. By setting a control variable on the EARLY User Input File (ENDAT2), CHRONC alone can be skipped. If any module is inactivated in this way, there is no need to prepare its corresponding user input file.

of the three phenomenological modules in MACCS, the ATMOS module is the only one which must always be exercised. Depending on the value of userspecified parameters in the ATMOS User Input File, results can be generated for either single weather trials or multiple weather trials.

The OUTPUT module generates complementary cumulative distribution functions (CCDFs) [NR82] of the results generated by EARLY and CHRONC when the user requests that those results be produced as described in Section 2.11. This is done for both single and multiple weather trial runs. The results that can be produced are defined in Sections 2.12 to 2.19 , and Sections 3.13 to 3.17 . There is currently no provision for the production of any CCDF results from ATMOS such as air or ground concentrations, or contaminated area.

A CCDF is generated internally for all of the user requested consequence measures. For each of these CCDFs, the code always produces a one-line summary describing various aspects of the distribution function that is written to the List Output File. 
For any subset of the results, the user can, in addition, cause the code to print out the entire CCDF table. This feature of printing out the CCDF table is under user control and is described in each of the sections where the user requests the production of the individual consequence measures.

The results from the OUTPUT module are presented individually for each emergency response strategy, and also as a weighted sum of the combined results. Consequences calculated by both EARLY and CHRONC (such as cancer cases) are summed together in the overall weighted sum. In addition, the weighting fractions associated with the individual emergency response scenarios of EARLY (up to three are allowed) are automatically combined according to the values of "fraction of the people" or "fraction of the time" specified on the EARLY User Input File (WTFRAC).

There is no provision for specifying each accident's expected rate of occurrence (accident frequency). All consequence measures calculated by MACCS are conditional on the occurrence of a particular accident. For directions on the interpretation of the consequence values presented by the OUTPUT module, please refer to Section 2.11 of this document.

MACCS can handle multiple source terms in a single run. When more than one source term is specified, the results for each are presented on the output listing in the order of their appearance on the ATMOS User Input File. The OUTPUT module will print a description of all the results for each source term before going on to the next source term. The code is currently dimensioned to handle up to 60 source terms at a time.

When the user has requested that multiple weather trials be performed according to weather category sampling (Section 1.16), the user has the capability of having the OUTPUT module present a table of relative contribution from each weather category sampling bin (Section 2.2). This enables the user to see what type of weather conditions contribute the most to the mean consequence value of each result on the output listing. These tables can only be produced if weather category sampling is being used (METCOD-2). This feature is especially useful now that the user has some control over the bins defining weather category since it can provide a means of "fine-tuning" the bin definitions. Section 2.11 provides additional information on the interpretation of the relative contribution tables.

Parameters defining both the rain intensity and rain distance bin definitions are specified on the ATMOS User Input File as described in Section 1.16. In contrast to the categorization of rain events, which is under the user's control as defined in Section 1.16, the initial condition bins for stability-class and wind-speed remain hard-wired into the code with no provision for easy modification by the user. It is hoped that a future version of MACCS will give the user complete flexibility in defining the weather category bins. 
The user input files for MACCS are processed by a free-field input processor, INPRE, that was developed to emulate the function of the INPPRE/CRACKR package which was originally developed for MELCOR. The replacement for the INPPRE/CRACKR package was developed for MACCS in order to facilitate the portability and ease of maintenance of the MACCS code. The INPRE package is portable without any modification to any computer system with an ANSI standard FORTRAN compiler [AN78].

The format of the input files was designed to maximize their readability. The input processor allows comments to be freely interspersed with the data which allows the input files to be essentially self-documenting.

Because of the requirements of the INPRE free-field input processor, certain restrictions on the format of the input data files are necessary. The input data files consist of a sequence of card images, with each card allowed a length of up to 100 characters. The sequence of cards is delimited by a special terminator record. A terminator record is a card with a period (.) in column one. Each card in a file is either a comment card or a data card. Comment cards are denoted by an asterisk (*) in column one. Data cards are required to begin in column one with an eleven character record identifier consisting of eight or nine alphanumeric identification characters followed by two or three numeric characters which are used for sequencing. An example of a record identifier is RIATNAM1001.

The input processor begins by sorting all the data cards in the file according to the collating sequence of their record identifiers. If more than one data card has the same record identifier, the last card encountered is used and all earlier cards with the same identifier are ignored. Cards may appear in any order. The ordering of data cards is only important for cards sharing the same record identifier.

Multiple source terms for ATMOS and multiple emergency response strategies for EARLY can be specified by the addition of "change cards" positioned at the end of the ATMOS and EARLY user input files. Each set of change cards is separated from the rest by a period (.) in Column 1. ATMOS allows up to 59 sets of source term change cards and EARLY allows up to two sets of emergency response change cards. Each change card set must specify a new text field to describe the source term or scenario and at least one previously defined input value must be redefined in each set of change cards. If the user tries to override a previously defined input variable that is not in the data blocks for source term (Section 1.11 ) or emergency response (Sections 2.6 and 2.7 ), those cards will be ignored by the program.

Every card appearing in the source term and emergency response change card sets must have been previously supplied in the base case input for that file. That is, when the change cards are being processed, there is no capability of adding new cards to the input file database. The change cards can only be used to replace previously defined data items in the 
base case input. The change card processor simply replaces a previously defined data card with the new card which has the same record identifier. If a set of change cards contains data cards with record identifiers which have not been defined in the base case input, it is possible that spurious results will be generated. All of the MACCS User Input Files must end with a period in column one.

Data required by MACCS can be of four different types: logical, character, integer, or real. Logical values are represented as either .TRUE. or .FALSE. as defined in the ANSI FORTRAN 77 standard [AN78]. Character values can be any ASCII string delimited by apostrophes ('). If the string has no embedded blanks, the apostrophes are optional provided that the string is not interpreted to be a data item of another type. Integer values can be preceded with a minus sign or an optional plus sign but they cannot have a decimal point. Real values can be written either as an optionally signed number with a decimal point (FORTRAN $F$ format) or else in exponential notation (FORTRAN $E$ format) as defined in the ANSI standard.

The determination of the type of each item encountered is performed as follows: the code first determines if the item is of type logical, if it isn't, it checks to see if it is of type integer, if it isn't, it checks to see if it is of type real. Any item that is neither logical, integer nor real is considered to be of type character.

More than one item can be placed on a card. Multiple items on a card are delimited either by a comma or a blank. The presence of additional blanks between items has no effect on how the items are interpreted. Multiple commas between items are not allowed.

The input processor will not convert the type of the values that it encounters from real to integer or vice versa. If an integer value is required by one of the program modules, then an integer value must be supplied by the user. If a decimal point appears in what should be an integer value, an error flag will be set and execution will be terminated upon completion of the input processing phase. Likewise, the absence of a decimal point in what should be a real value will be interpreted as an input error and cause the error flag to be set.

In addition to checking that each data item encountered is of the correct type, the input processor determines the validity of the numeric input parameters (both integer and real) by checking whether they fall within the specified range. The allowable range of each datum is listed in this report. Character string values have a length between specified limits; these limits are also listed here. If a value is encountered that falls outside the required range, the error flag will be set and a diagnostic message issued to the output file. This message will list the minimum and maximum allowable values for the item in order to facilitate the debugging process. 
If any input errors are detected, the code will usually attempt to process as much as possible of the subsequent input. In some instances though, the occurrence of an input error will cause an immediate termination of the program's execution.

A data element consists of either a single value (scalar) or a set of values which are all of the same type (array). Within a user input file for a particular program module, the data that it requires has been broken up into groups of functionally related quantities. These data groups are described in separate sections of the following chapters.

The record identifier used for scalar values is always of the same form. The first two characters are a mnemonic for the data group to which it belongs. For example, the mnemonic for the Run Identification Data is RI. The next six characters of the record identifier are the name of the FORTRAN variable within the program that is used to store the value. The record identifier is always 001 . Only the first item found on the card for a scalar quantity will be processed. Anything to the right of the data item is ignored by the input processor. This allows the use of descriptive comments alongside of the data item. This feature should only be used for scalar values.

Arrays are found in the input files in two different forms. The first method is similar to the approach used for scalars. A starting record identifier is constructed the same as above: a two letter mnemonic, followed by the six letter variable name, followed by 001 . More than one value can appear on the data card separated by blanks or a comma. You may place as many values on the card as will fit into the 100 columns. Successive cards with ascending sequence numbers are processed until all the required data items have been supplied. The record identifiers of the succeeding cards differ from the starting record identifier only in their last three digits.

The second method used for arrays is more structured. Several arrays of the same length whose values are related appear as columns across the page. The arrays can be of different type but they all share the same record identifier. The name of a program variable is not used to construct the record identifier since more than one variable receives its value from this "data block." Instead of reading array values from left to right in rows, the arrays are read from top to bottom in columns.

All of the input parameters used by the program modules will be described in this report. Unless specified otherwise, all of these parameters are required to be supplied. Each input parameter is described in a stylized block of text which presents the following information: (1) the FORTRAN variable name used in the code, (2) the type of the data item (integer, real, logical or character), (3) an indication of whether only a single value is required (Scalar), or multiple values are required (array), (4) minimum and maximum allowable values (or lengths), (5) a statement describing the variable, and (6) an example of the variable's usage. 
The next section of this document describes the Sample Problems which are being supplied with the MACCS release tape.

Sample Problems

Three sample problems are provided to illustrate the use of MACCS. These will be referred to as Problems A, B, and C. Due to the length of the output listings it is impractical to include them all in this document's printed text but they are listed on microfiche and enclosed in the rear jacket of this report. On the magnetic tape used for distributing MACCS, all of the input and output files for the Sample Problems are available in machine-readable form.

Throughout the body of this document, data cards similar to those supplied in the Sample Problem input files are used to illustrate the appearance of the data in those files. The parameter values found on these data cards are for the most part identical to the values used in the MACCS calculations for the second draft of NUREG-1150 [NR89]. It is the responsibility of each MACCS user to ensure the appropriateness of all data in the MACCS input files which they prepare.

Problem A shows how MACCS can be used for probabilistic risk assessment (PRA) studies to address a variety of needs including regulatory studies and conventional parametric variation sensitivity studies. It illustrates how MACCS can automatically loop on source terms and emergency response assumptions in a single run of the code. The weather category bin sampling method is used in this problem to estimate the distribution of consequences which could result from an accident if the time of the accident's occurrence is unknown. Automatic looping is illustrated as follows: the ATMOS User Input File for Problem A causes results to be calculated for two hypothetical source terms, and the EARLY User Input File specifies two different emergency response scenarios ( 95 percent evacuation and 5 percent non-evacuation as was done for the first draft of NUREG-1150 [NR87]. An additional sheltering case is presented for the purpose of illustration. There is no provision for looping on the CHRONC module and only a single set of assumptions is ever used to determine the modeling in the long-term chronic exposure phase of the calculations.

The list output produced by Sample Problem $A$ is presented its entirety in Appendix D.3. Since all of the input processed by MACCS is echoed to the list output file, this file illustrates both input and output of the code.

Problem B illustrates how MACCS can be used to examine one of the weather sequences which was selected in the weather sampling used for Problem A. This type of examination is usually done when some type of unusual result is noticed on the output listing and the user wishes to determine if the calculations are being properly performed. The output listing of Problem A shows that the peak value of economic cost from the 
first source term was $\$ 2.71 E 11$ and it was obtained from the calculation of weather trial number 2. By going back to the first part of the listing, we can see that this trial began at day $=157$ and hour $=10$ on the Surry weather data file.

For Problem B, the ATMOS User Input File of Problem A has been modified to run only the first source term and to calculate results for only the single weather sequence which led to the peak economic cost as described above. We have turned on the diagnostic print options in all three of the User Input Files by changing the values of the following input parameters from zero to one: IDEBUG in ATMOS, IPRINT in EARLY, and KSWDSC in CHRONC. A large amount of printed output will result.

Just by themselves, the numbers printed on the debug output listing may be hard to interpret, but they could be helpful if it is necessary to examine intermediate steps in the calculations.

In Problem $C$, the code is set up to run the source term of Problem $B$ with constant meteorology; D-stability, $5 \mathrm{~m} / \mathrm{s}$ wind speed, no rain. A uniform population distribution of 50 people/square-km is used for the calculations and therefore there is no need for a Site Data File. Since wind direction is not defined for constant meteorology, the value of IPLUME is changed from 2 to 1 . In this run, the diagnostic print statements of Problem $B$ have been turned off and only the standard presentation of results from the OUTPUT module appears on the output listing.

Once MACCS is installed on a computer system, the user can verify that all is well by running the sample problems and comparing the resulting output files to those on the distribution magnetic tape which were generated on a VAX/VMS system. Since MACCS is intended to be portable to any computer, the numeric output values by and large should be identical or very close to identical. The only significant deviation might lie in the probabilities of non-zero and peak value consequences since the precision of arithmetic and the handling of underflow will vary on different computer systems.

If the user is working with a VAX/VMS system, the MACCS command file included on the installation tape may be used to execute the three sample problems as follows after the input files files have been loaded and the program compiled and linked. The sample problems can be executed by typing the following three command lines.

\section{A- QMACCS IN1A IN2A IN3A METSUR SURSIT TEMPA \\ B- QMACCS IN1B IN2B IN3B METSUR SURSIT TEMPB \\ C- QMACCS IN1C IN2C IN3C " " " TEMPC}

The output from these sample problems will be found in the three files: TEMPA.OUT, TEMPB.OUT, and TEMPC.OUT. Sample Problem $C$ does not require a meteorological data file (METSUR) or a Site Data File (SURSIT), since it uses constant weather conditions and a uniform population density. 
References

AN78 "American National Standard Programming Language FORTRAN," ANSI X3.9-1978, available from American National Standards Institute, 1430 Broadway, New York, NY 10018, copyrighted.

Jo89 Jow, H.N., et al., MELCOR Accident Consequence Code System (MACCS) Volume 2: Model Description, NUREG/CR-4691, SAND86-1562, Sandia National Laboratories, Albuquerque, NM, 1990.

NR82 U.S. Nuclear Regulatory Commission, PRA Procedures Guide, NUREG/CR2300, Vol. 2, 1982, available from National Technical Information Service, Springfield, Virginia, 22161.

NR87 U.S. Nuclear Regulatory Commission, Reactor Risk Reference Document, USNRC Draft Report NUREG-1150, February 1987.

NR89 U.S. Nuclear Regulatory Commission, Severe Accident Risks: An Assessment for Five U.S. Nuclear Power Plants, Second Draft NUREG-1150, June 1989.

Ri83 Ritchie, L. T., Calculations of Reactor Accident Consequences Version 2 CRAC2: Computer Code, User's Guide, NUREG/CR-2326, SAND81-1994, Sandia National Laboratories, Albuquerque, NM, 1983.

Ro89 Ro11stin, J.A., et al., MELCOR Accident Consequence Code System (MACCS) Volume 3: Programmer's Reference Manual, NUREG/CR-4691, SAND86-1562, Sandia National Laboratories, Albuquerque, NM, 1990.

We84 Weigand, G. G., Thermal-Hydraulic Process Modeling in Risk Analysis: An Assessment of the Relevant Systems, Structures, and Phenomena,

- NUREG/CR-3986, SAND84-1219, Sandia National Laboratories, Albuquerque, NM, 1984. 


\subsection{ATMOS USER INPUT FILE}

\subsection{Introduction to ATMOS}

The ATMOS program calculates the dispersion and deposition of material released to the atmosphere as a function of downwind distance. It utilizes a Gaussian plume model with Pasquill-Gifford dispersion parameters [NR82]. The phenomena that ATMOS treats are (1) building wake effects, (2) buoyant plume rise, (3) plume dispersion during transport, (4) wet and dry deposition, and (5) radioactive decay.

At the midpoint of each spatial interval along the transport path, air and ground concentrations for all the radionuclides are calculated as well as miscellaneous information about plume size, height, and transport timing. This data is stored in common blocks which are used later by the EARLY and CHRONC modules of MACCS.

Transport and deposition in ATMOS are treated with a one-dimensional model. Concentration values are calculated only for the plume centerline. There is no calculation in ATMOS of off-centerline concentrations. The adjustment for off-axis location is handled in the the EARLY and CHRONC modules.

Several different options for specifying weather conditions are available to the user. These include two weather sampling options: (1) category bin sampling and (2) strictly random sampling; as well as three different methods of specifying a single weather trial: (1) constant weather conditions, (2) fixed start time in the weather file, and (3) user supplied 120 hour weather sequence.

It is up to the user to specify the various parameters needed for these calculations. There are no default values. All of this information is supplied through the user input file to ATMOS and all of the input parameters are described in this chapter.

\subsection{Run Identification (RI) Data}

In order to identify the computer run that is being performed, the user is required to supply a text field that will be printed on all of the list output produced for this run by MACCS. All of the MACCS programs obtain the current date and time from the computer operating system so it is not necessary to include information of that type. In addition to this text field, a text field describing the source term is supplied separately in the Release Description Data Block which defines the source term.

Variable Name - ATNAM1

Variable Type - Character, Scalar

Allowed Range - $1<-$ length $\Leftrightarrow 80$

Purpose - Identifies this MACCS calculation. This identification information will be printed at the top of all pages of the OUTPUT listing. 
Example Usage:

*

* GENERAL DESCRIPTIVE TITLE DESCRIBING THE ATMOSPHERIC ASSUMPTION FOR

* THESE RUNS

*

RIATNAM1001 'IN1A.INP, SURRY, SAMPLE PROBLEM A, ATMOS INPUT'

Note to user: The asterisk in column one denotes a comment.

\subsection{Geometry (GE) Data}

A polar grid coordinate system is used by MACCS to represent the region surrounding the reactor. The reactor itself is always located at the centerpoint of the coordinate system $(r-0)$. The data in this section defines the grid spacing between spatial elements in the radial direction. All of the consequence calculations performed by MACCS are stored on the basis of the radial spacing defined here. For example, air and ground concentrations are calculated to be representative of the entire length of the spatial element (not just its centerpoint).

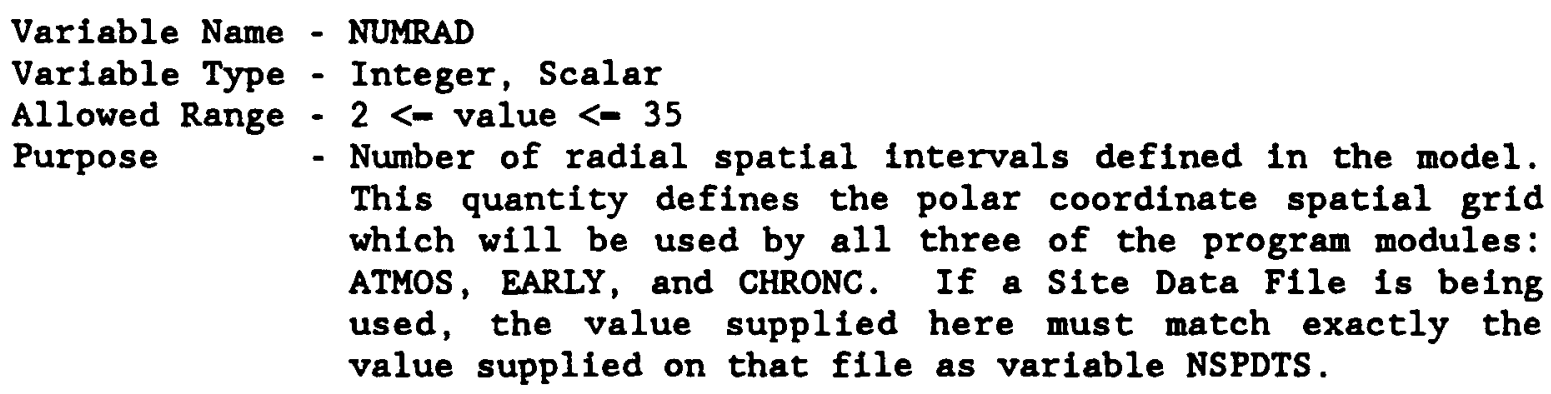

Example Usage:

*

* NUMber of radial spatial elements

*

GENUMRAD001 26

Variable Name - SPAEND

Variable Type - Real, Array

Allowed Range - $0.001<-$ value <- 9999. (kilometers)

Purpose

- Distance in kilometers to the endpoints of the spatial intervals. If a site Data File is being used, the values supplied here must be within 10 percent of the corresponding parameter values supplied on that file for the array SPDSTS.

Note: The spacing between adjacent spatial intervals should be at least 0.1 kilometers. 
Example Usage:

*

* SPATIAL ENDPOINT DISTANCES IN KILOMETERS

$\begin{array}{lrrrrr}\text { GESPAENDO01 } & 0.16 & 0.52 & 1.21 & 1.61 & 2.13 \\ \text { GESPAEND002 } & 3.22 & 4.02 & 4.83 & 5.63 & 8.05 \\ \text { GESPAEND003 } & 11.27 & 16.09 & 20.92 & 25.75 & 32.19 \\ \text { GESPAEND004 } & 40.23 & 48.28 & 64.37 & 80.47 & 112.65 \\ \text { GESPAEND005 } & 160.93 & 241.14 & 321.87 & 563.27 & 804.67\end{array}$

Note to user: Elements in the array must be separated by blanks or a comma. The number of items per card is left to the user's discretion. The sequence numbers of the record identifiers must be in ascending order.

\subsection{Nuclide (IS) Data}

This section defines the physical characteristics of the nuclides that are to be modeled. These nuclides are divided into groups that share similar physical and chemical characteristics. Information on radioactive decay (half-life and decay schemes) is also provided here.

Variable Name - NUMISO

Variable Type - Integer, Scalar

Allowed Range - 1 <- value <- 150

Purpose - Number of nuclides defined in the model. Several other input parameters use this value to determine the number of values which are required to be supplied. The Dose Conversion Data File must have dose conversion data for - each of the nuclides defined here.

Example Usage:

*

* NUMBER OF NUCLIDES

*

ISNUMISO001 60

Variable Name - MAXGRP

Variable Type - Integer, Scalar

Allowed Range - 1 <- value <- 10

Purpose

- Number of chemical element groups defined in the model. Each nuclide will be assigned to a chemical element group, e.g., noble gases. The wet and dry deposition characteristics of a nuclide, as well as its release fraction, depend on the number of the element group (IGROUP) to which it is assigned. 


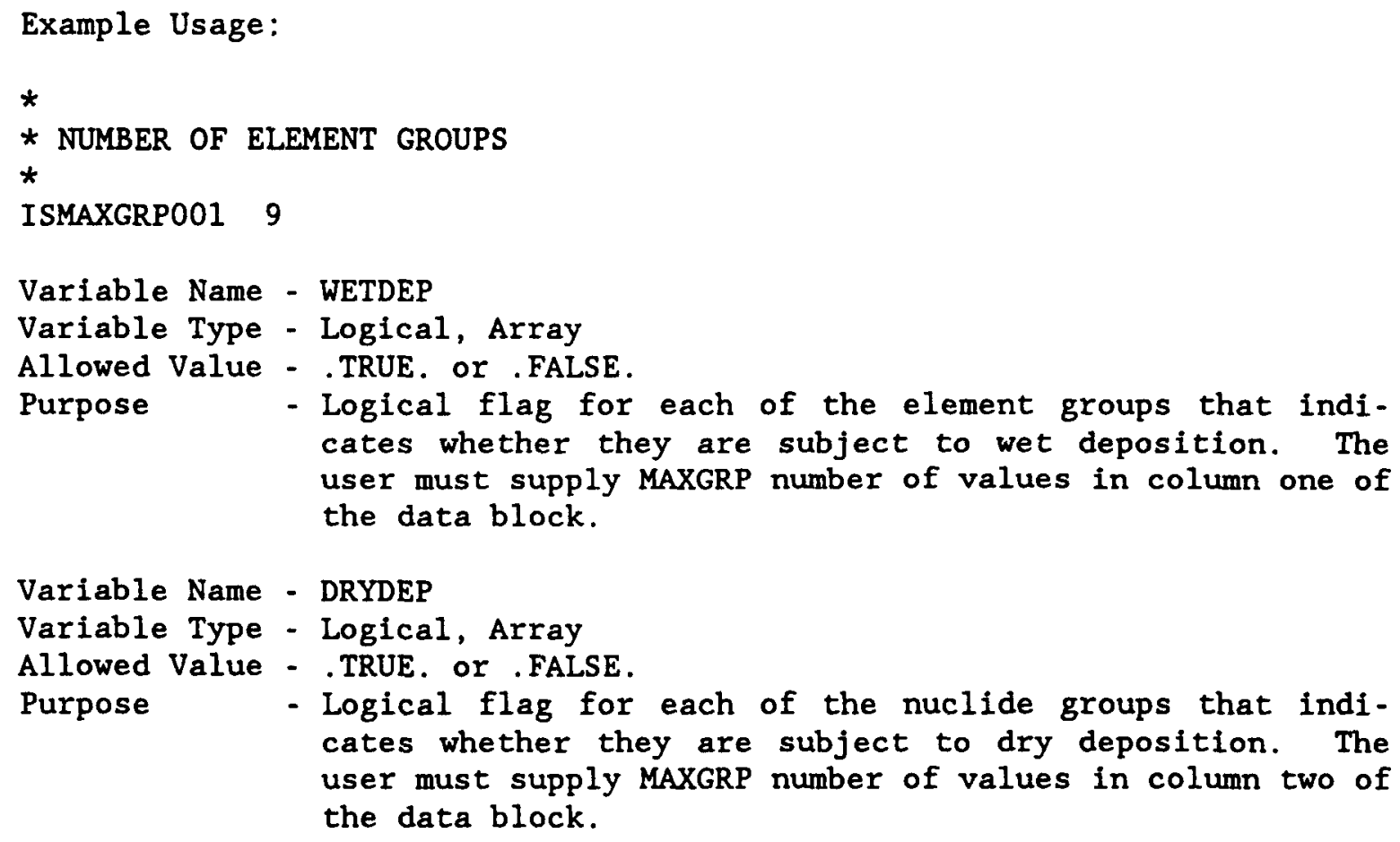
cates whether they are subject to wet deposition. The user must supply MAXGRP number of values in column one of the data block.

Variable Name - DRYDEP

Variable Type - Logical, Array

Allowed Value - .TRUE. or .FALSE.

Purpose - Logical flag for each of the nuclide groups that indicates whether they are subject to dry deposition. The user must supply MAXGRP number of values in column two of the data block.

Example Usage of WETDEP, and DRYDEP:

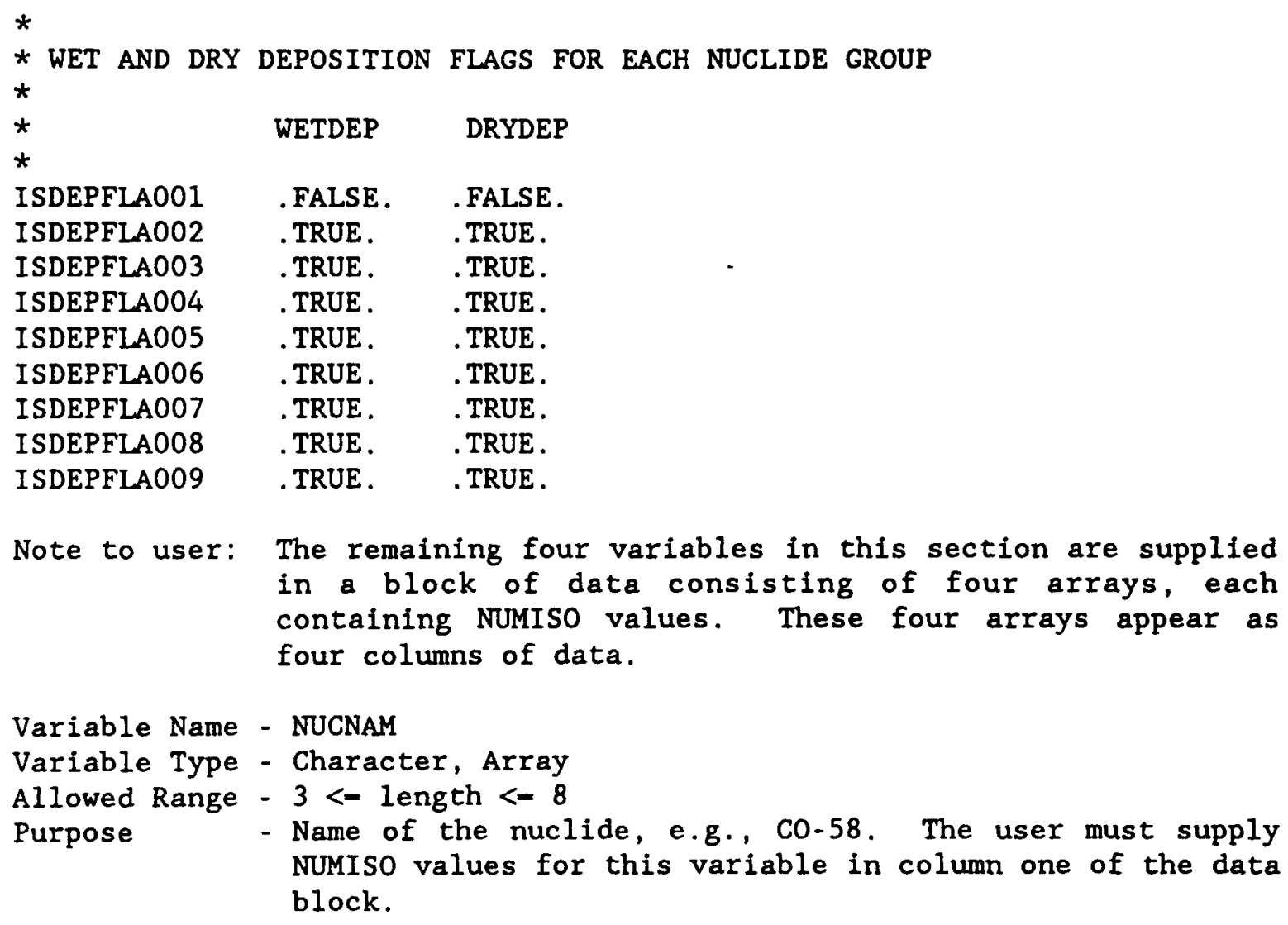




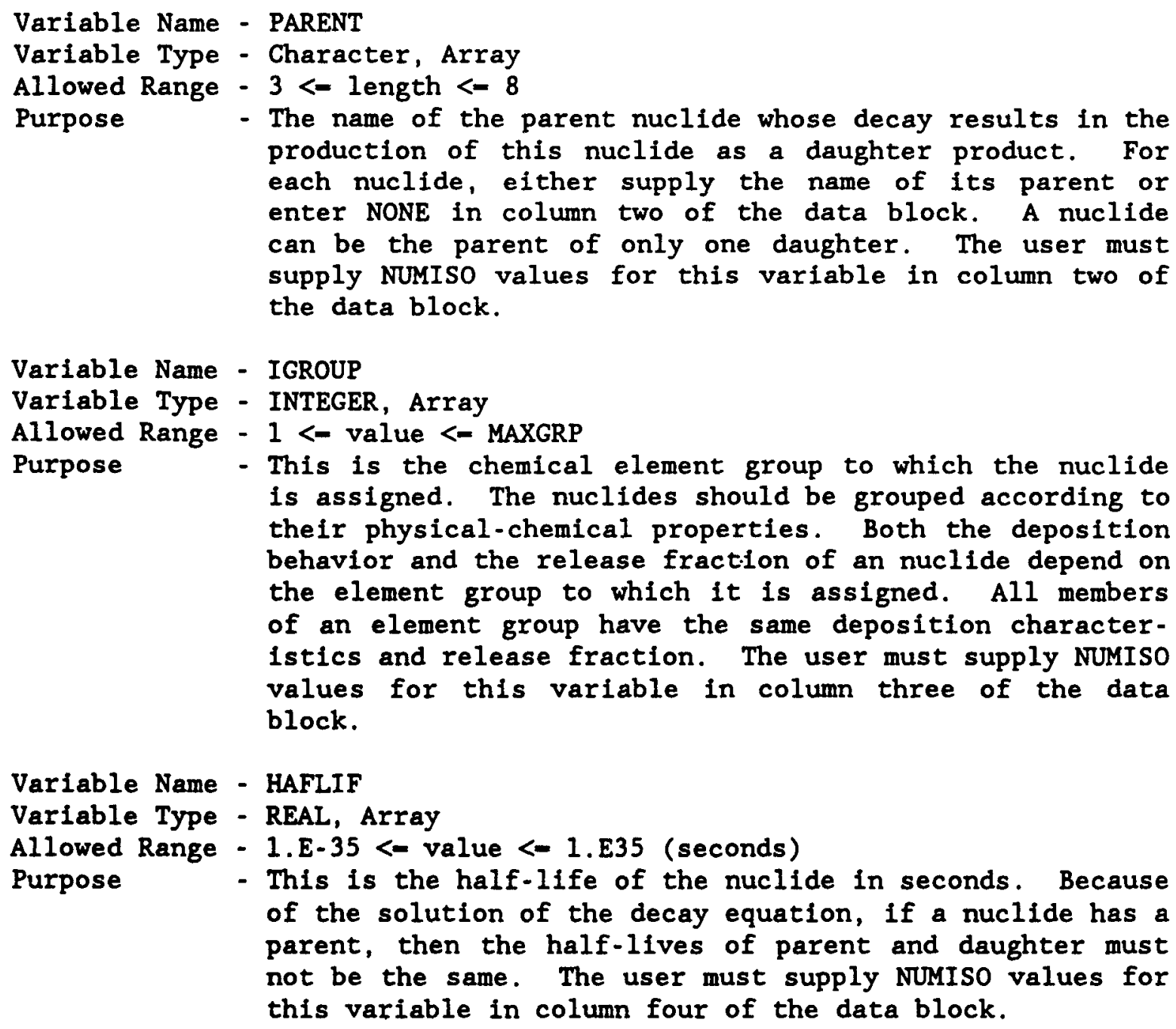

Example Usage of NUCNAM, PARENT, IGROUP, HAFLIF:

* NUCLIDE GROUP DATA FOR 9 NUCLIDE GROUPS

*

*

NUCNAM PARENT IGROUP HAFLIF

ISOTPGRP001

ISOTPGRPO02

ISOTPGRPO03

ISOTPGRP004

ISOTPGRPO05

ISOTPGRP006

ISOTPGRPO07

ISOTPGRP008

ISOTPGRPOO9

ISOTPGRP010

ISOTPGRP011

ISOTPGRP012

ISOTPGRPO13

$\begin{array}{lrll}C O-58 & \text { NONE } & 6 & 6.160 E+06 \\ \text { CO-60 } & \text { NONE } & 6 & 1.660 E+08 \\ \text { KR }-85 & \text { NONE } & 1 & 3.386 E+08 \\ \text { KR }-85 M & \text { NONE } & 1 & 1.613 E+04 \\ \text { KR }-87 & \text { NONE } & 1 & 4.560 E+03 \\ \text { KR }-88 & \text { NONE } & 1 & 1.008 E+04 \\ \text { RB }-86 & \text { NONE } & 3 & 1.611 E+06 \\ \text { SR }-89 & \text { NONE } & 5 & 4.493 E+06 \\ \text { SR }-90 & \text { NONE } & 5 & 8.865 E+08 \\ \text { SR }-91 & \text { NONE } & 5 & 3.413 E+04 \\ Y-90 & \text { SR-90 } & 7 & 2.307 E+05 \\ Y-91 & \text { SR-91 } & 7 & 5.080 E+06 \\ Z R-95 & \text { NONE } & 7 & 5.659 E+06\end{array}$




\begin{tabular}{|c|c|c|c|c|c|}
\hline ISOTPGRP014 & ZR-97 & NONE & 7 & $6.048 E+04$ & \\
\hline ISOTPGRP015 & NB -95 & ZR-95 & 7 & $3.033 E+06$ & \\
\hline ISOTPGRP016 & MO-99 & NONE & 6 & $2.377 E+05$ & \\
\hline ISOTPGRP017 & TC-99M & MO-99 & 6 & $2.167 E+04$ & \\
\hline ISOTPGRP018 & RU-103 & NONE & 6 & $3.421 E+06$ & \\
\hline ISOTPGRP019 & RU-105 & NONE & 6 & $1.598 E+04$ & \\
\hline ISOTPGRP020 & RU-106 & NONE & 6 & $3.188 \mathrm{E}+07$ & \\
\hline ISOTPGRP021 & RH-105 & $R U-105$ & 6 & $1.278 \mathrm{E}+05$ & \\
\hline ISOTPGRP022 & SB - 127 & NONE & 4 & $3.283 E+05$ & \\
\hline ISOTPGRP023 & $S B-129$ & NONE & 4 & $1.562 \mathrm{E}+04$ & \\
\hline ISOTPGRP024 & TE- 127 & SB - 127 & 4 & 3. $366 E+04$ & \\
\hline ISOTPGRP025 & $\mathrm{TE}-127 \mathrm{M}$ & NONE & 4 & $9.418 \mathrm{E}+06$ & \\
\hline ISOTPGRP026 & TE- 129 & SB-129 & 4 & $4.200 E+03$ & \\
\hline ISOTPGRP027 & TE-129M & NONE & 4 & $2.886 E+06$ & \\
\hline ISOTPGRP028 & TE-131M & NONE & 4 & $1.080 \mathrm{E}+05$ & \\
\hline ISOTPGRP029 & TE- 132 & NONE & 4 & $2.808 E+05$ & \\
\hline ISOTPGRP030 & $I-131$ & $\mathrm{TE}-131 \mathrm{M}$ & 2 & $6.947 E+05$ & \\
\hline ISOTPGRP031 & I - 132 & TE- 132 & 2 & $8.226 E+03$ & \\
\hline ISOTPGRP032 & I - 133 & NONE & 2 & $7.488 E+04$ & \\
\hline ISOTPGRP033 & I - 134 & NONE & 2 & $3.156 \mathrm{E}+03$ & \\
\hline ISOTPGRP034 & $I-135$ & NONE & 2 & $2.371 \mathrm{E}+04$ & \\
\hline ISOTPGRP035 & $X E-133$ & I - 133 & 1 & $4.571 E+05$ & \\
\hline ISOTPGRP036 & $X E-135$ & $I-135$ & 1 & $3.301 E+04$ & \\
\hline ISOTPGRP037 & CS -134 & NONE & 3 & $6.501 E+07$ & \\
\hline ISOTPGRP038 & CS -136 & NONE & 3 & $1.123 E+06$ & \\
\hline ISOTPGRP039 & CS -137 & NONE & 3 & $9.495 E+08$ & \\
\hline ISOTPGRP040 & $B A-140$ & NONE & 9 & $1.105 E+06$ & \\
\hline ISOTPGRP041 & $L A-140$ & $B A-140$ & 7 & $1.448 \mathrm{E}+05$ & \\
\hline ISOTPGRP042 & CE- 141 & $L A-141$ & 8 & $2.811 E+06$ & PARENT ADDED \\
\hline ISOTPGRP0 43 & CE -143 & NONE & 8 & $1.188 \mathrm{E}+05$ & \\
\hline ISOTPGRP044 & CE- 144 & NONE & 8 & $2.457 E+07$ & \\
\hline ISOTPGRP045 & PR-143 & $C E-143$ & 7 & $1.173 E+06$ & \\
\hline ISOTPGRP046 & ND -147 & NONE & 7 & $9.495 E+05$ & \\
\hline ISOTPGRP047 & $N P-239$ & NONE & 8 & $2.030 E+05$ & \\
\hline ISOTPGRP048 & PU-238 & $C M-242$ & 8 & $2.809 E+09$ & \\
\hline ISOTPGRP049 & PU- 239 & $N P-239$ & 8 & $7.700 E+11$ & \\
\hline ISOTPGRP050 & PU- 240 & $C M-244$ & 8 & $2.133 E+11$ & \\
\hline ISOTPGRP051 & PU- 241 & NONE & 8 & $4.608 E+08$ & \\
\hline ISOTPGRP052 & $A M-241$ & PU-241 & 7 & 1. $366 \mathrm{E}+10$ & \\
\hline ISOTPGRP053 & CM- 242 & NONE & 7 & $1.408 \mathrm{E}+07$ & \\
\hline ISOTPGRP054 & CM- 244 & NONE & 7 & $5.712 \mathrm{E}+08$ & \\
\hline ISOTPGRP055 & SR-92 & NONE & 5 & $9.756 \mathrm{E}+03$ & NEW \\
\hline ISOTPGRP056 & $Y-92$ & SR-92 & 7 & $1.274 \mathrm{E}+04$ & NEW \\
\hline ISOTPGRP057 & $Y-93$ & NONE & 7 & $3.636 \mathrm{E}+04$ & NEW \\
\hline ISOTPGRP058 & BA-139 & NONE & 9 & $4.986 E+03$ & NEW \\
\hline ISOTPGRP059 & $L A-141$ & NONE & 7 & $1.418 \mathrm{E}+04$ & NEW \\
\hline ISOTPGRP060 & LA- 142 & NONE & 7 & $5.724 E+03$ & NEW \\
\hline
\end{tabular}

\subsection{Wet Deposition (WD) Data}

The washout model predicts how much material is deposited on the ground by rainfall. Washout is a function of both rain duration and rain 
intensity. The fraction remaining after wet deposition is: EXP (-CWASH1 * rain duration * rain intensity ** CWASH2), where rain duration is in seconds and rain intensity is in millimeters per hour [Br81].

Variable Name - CWASHI

Variable Type - Real, Scalar

Allowed Range - 0 . <- value <- 1.

Purpose - The linear term of the washout function.

Example Usage:

*

* WASHOUT COEFFICIENT NUMBER ONE, LINEAR FACTOR

*

WDCWASH1001 9.5E-5 (HELTON AFTER JONES, 1986)

Variable Name - CWASH2

Variable Type - Real, Scalar

Allowed Range - 0 . <- value <- 1 .

Purpose - The exponential term of the washout function.

Example Usage:

*

* WASHOUT COEFFICIENT NUMBER TWO, EXPONENTIAL FACTOR

$\star$

WDCWASH2001 0.8 (HELTON AFTER JONES, 1986)

\subsection{Dry Deposition (DD) Data}

Dry deposition is modeled using the source depletion method [NR82]. The concentration of material at any single point on the ground is the product of the integrated ground-level air concentration times the deposition velocity. The material in each element group can be distributed among several particle size groups, with each element group having a different distribution of material among the particle size groups. The particle size distribution of each element group is specified in the Release Description Data. All of the plume segments will have the same distribution of particle sizes at the time of their release, but this distribution will change with time as the plumes travel downwind.

Variable Name - NPSGRP

Variable Type - Integer, Scalar

Allowed Range - 1 <- value <- 10

Purpose

- The number of particle size groups that will be used for the dry deposition model. A deposition velocity must be specified for each representative particle size group.

Example Usage:

$\star$ 


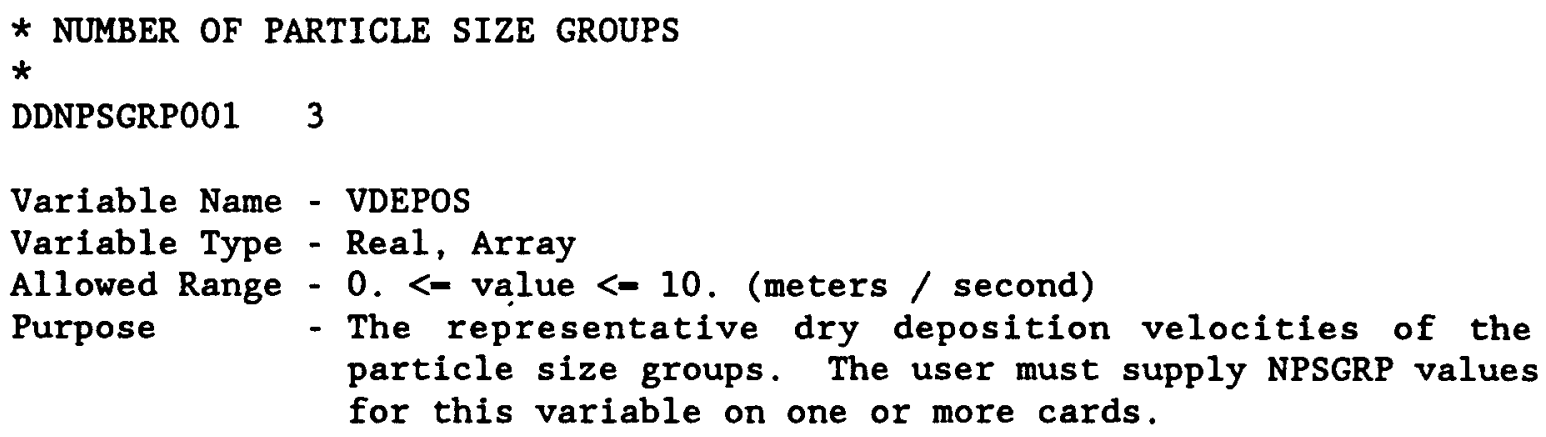

Example Usage:

*

* DEPOSITION VELOCITY OF EACH PARTICLE SIZE GROUP (M/S)

DDVDEPOS001 $0.001 \quad 0.01 \quad 0.02$

\subsection{Dispersion Parameter (DP) Data}

The Gaussian plume model of atmospheric dispersion uses spatially dependent dispersion parameters, sigma-y and sigma-z. Sigma-y and sigma$z$ are functions of the form:

$$
\begin{aligned}
& \text { sigma-y }=\text { CYSIGA (X) } \star * \text { CYSIGB, and } \\
& \text { sigma-z }=\text { CZSIGA (X) } \star \star \text { CZSIGB, }
\end{aligned}
$$

where sigma-y, sigma-z, and $X$ (the downwind distance from the source), are all in meters.

Variable Name - CYSIGA

Variable Type - Real, Array

Allowed Range - 1.E-35 <- value <- 10.

Purpose

- The linear term of the expression for sigma-y. The user must supply six values of CYSIGA, one for each of the six Pasquill-Gifford stability classes (classes A through F).

Example Usage:

*

* LINEAR TERM OF THE EXPRESSION FOR SIGMA-Y, 6 STABILITY CLASSES

*

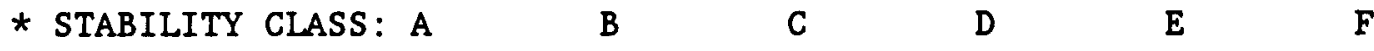

$\begin{array}{lllllll}* & & & & & & \\ \text { DPCYSIGA001 } & 0.3658 & 0.2751 & 0.2089 & 0.1474 & 0.1046 & 0.0722\end{array}$

Variable Name - CYSIGB

Variable Type - Real, Array

Allowed Range - 1.E-35<- value <- 10.

Purpose

- The exponential term of the expression for sigma-y. The user must supply six values of CYSIGB, one for each of the six Pasquill-Gifford stability classes (classes A through F). 
Example Usage:

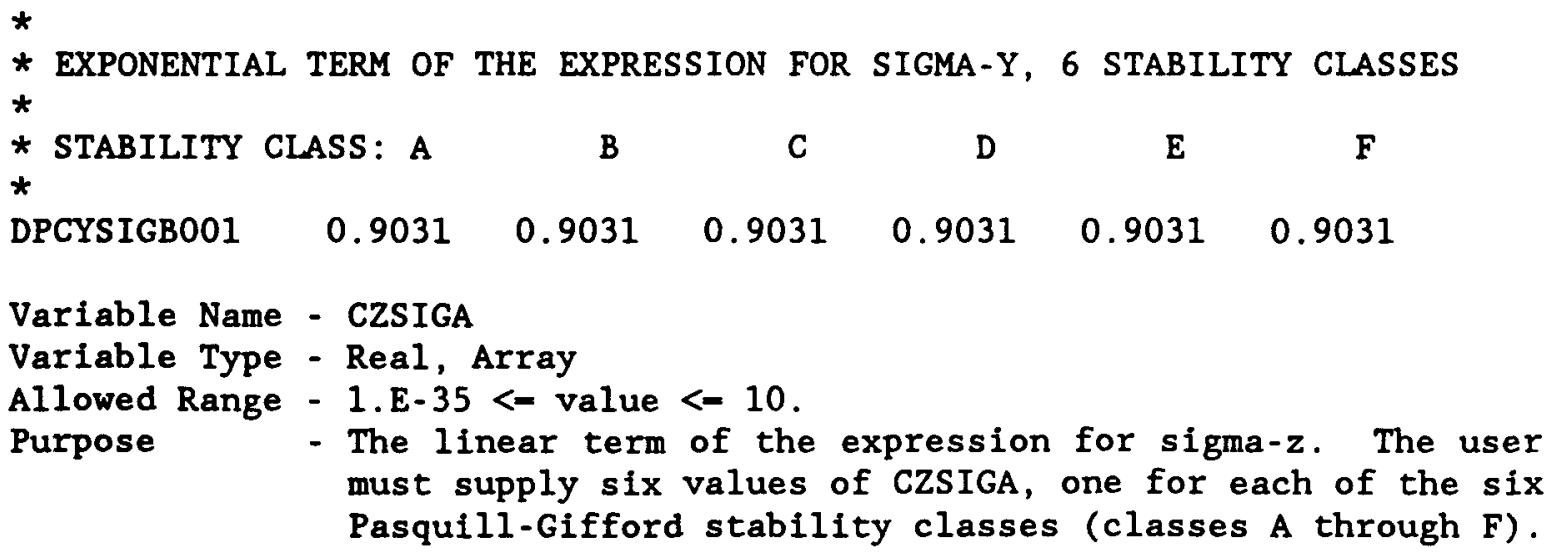

Example Usage:

*

* LINEAR TERM OF THE EXPRESSION FOR SIGMA-Z, 6 STABILITY CLASSES

*

$\begin{array}{llllllll}* & \text { STABILITY CLASS: A } & \text { B } & \text { C } & \text { D } & \text { E } & \text { F }\end{array}$

*

$\begin{array}{lllllll}\text { DPCZSIGA001 } & 2.5 \mathrm{E}-4 & 1.9 \mathrm{E}-3 & 0.2 & 0.3 & 0.4 & 0.2\end{array}$

Variable Name - CZSIGB

Variable Type - Real, Array

Allowed Range - 1.E-35 <- value <- 10.

Purpose - The exponential term of the expression for sigma-z. The user must supply six values of CZSIGB, one for each of the six Pasquill-Gifford stability classes (classes A through F).

Example Usage:

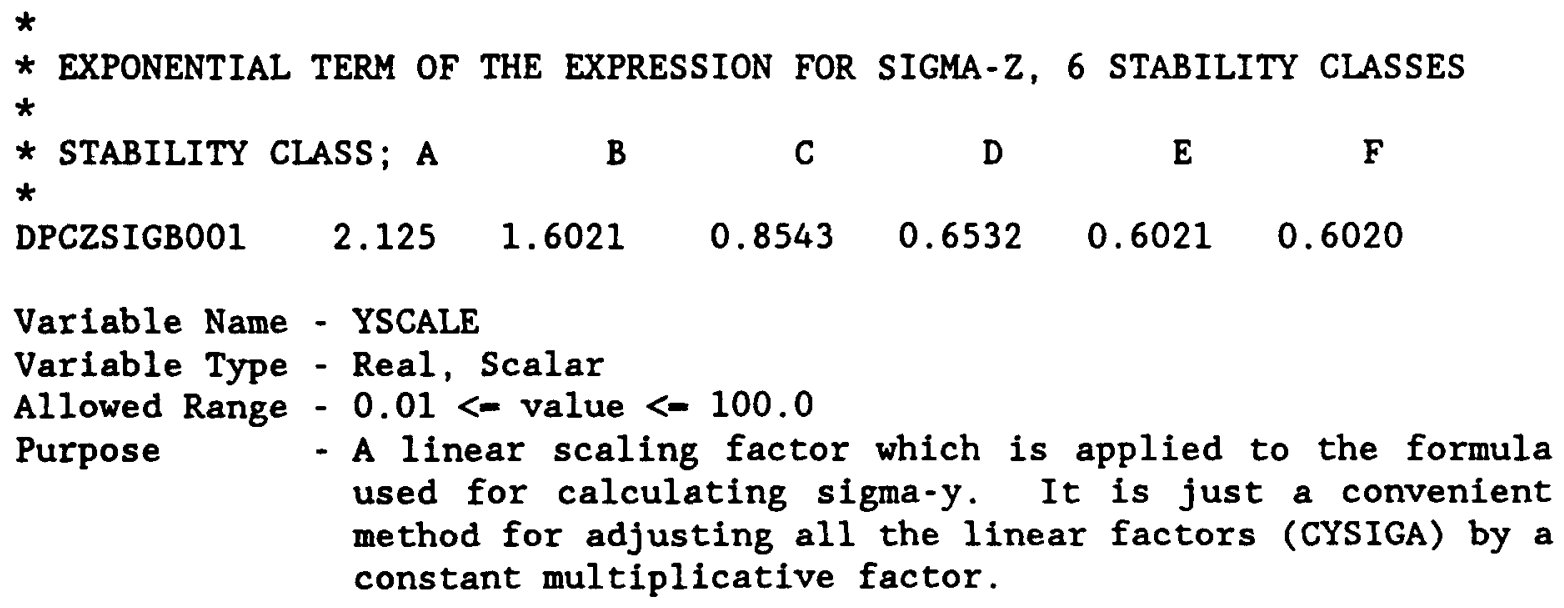




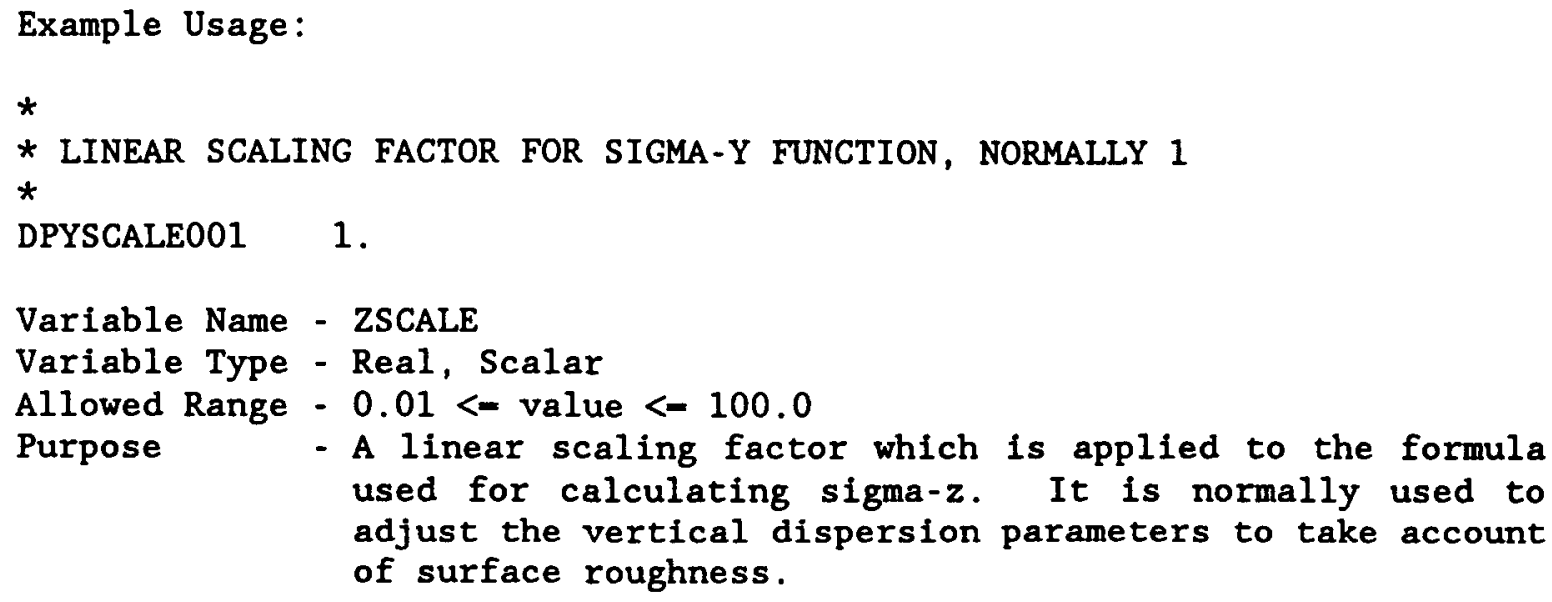
used for calculating sigma-z. It is normally used to adjust the vertical dispersion parameters to take account of surface roughness.

Example Usage:

*

* LINEAR scaling factor for sigma-Z function, * NORMALLY USED FOR SURFACE ROUGHNESS LENGTH CORRECTION.

* $(\mathrm{Z1} / \mathrm{Z0}) * * 0.2$, FROM CRAC2 WE HAVE (10 CM / $3 \mathrm{CM}) * * 0.2=1.27$

*

DPZSCALE001 1.27

\subsection{Plume Meander (PM) Data}

In order to account for the effect of plume meander during transport of the plume, an expansion factor, EXPFAC, is calculated which serves to widen the plumes in the cross-wind direction. It acts as a linear factor on sigma-y during the calculation of $x / Q$, but it does not affect the rate of growth of sigma-y. A two part function is used. The expansion factors used for different plume segments are independent of each other. If the release duration of the plume segment is less than or equal to BRKPNT, then the following formula will be used,

$$
\text { EXPFAC }=\text { (plume-segment-release-duration / TIMBAS) } \star \star \text { XPFAC1. }
$$

If the plume segment duration exceeds BRKPNT, then a different factor is used for the exponent of the function,

$$
\text { EXPFAC - (plume-segment-release-duration / TIMBAS) } \star \star \text { XPFAC2. }
$$

In both expressions, the duration of the plume segment is limited to 10 hours. A warning is printed on the output listing if the user specifies a release duration exceeding 10 hours.

Variable Name - TIMBAS

Variable Type - Real, Scalar

Allowed Range - 60. <- value <- 36000. (seconds)

Purpose - The time base associated with the parameterization of the plume meander adjustment factor (seconds). 


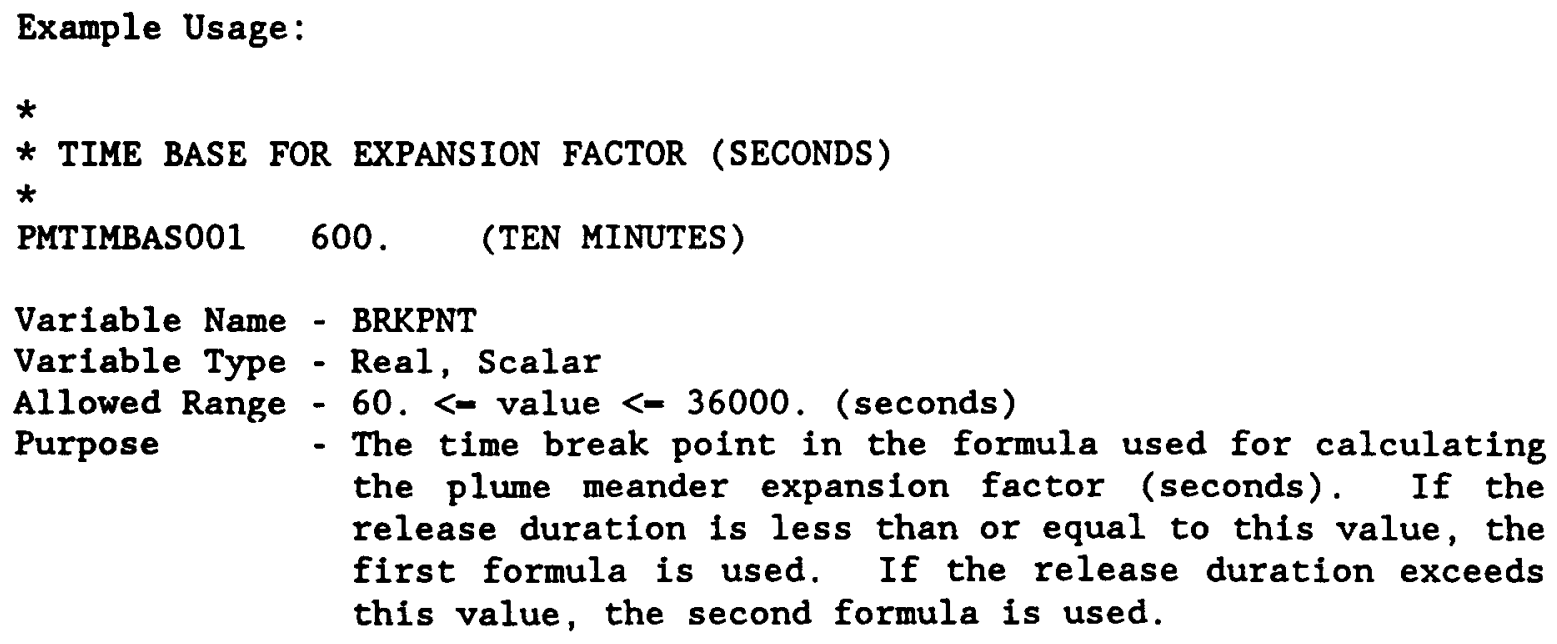
meander expansion factor for releases having release durations that are less than or equal to BRKPNT.

Example Usage:

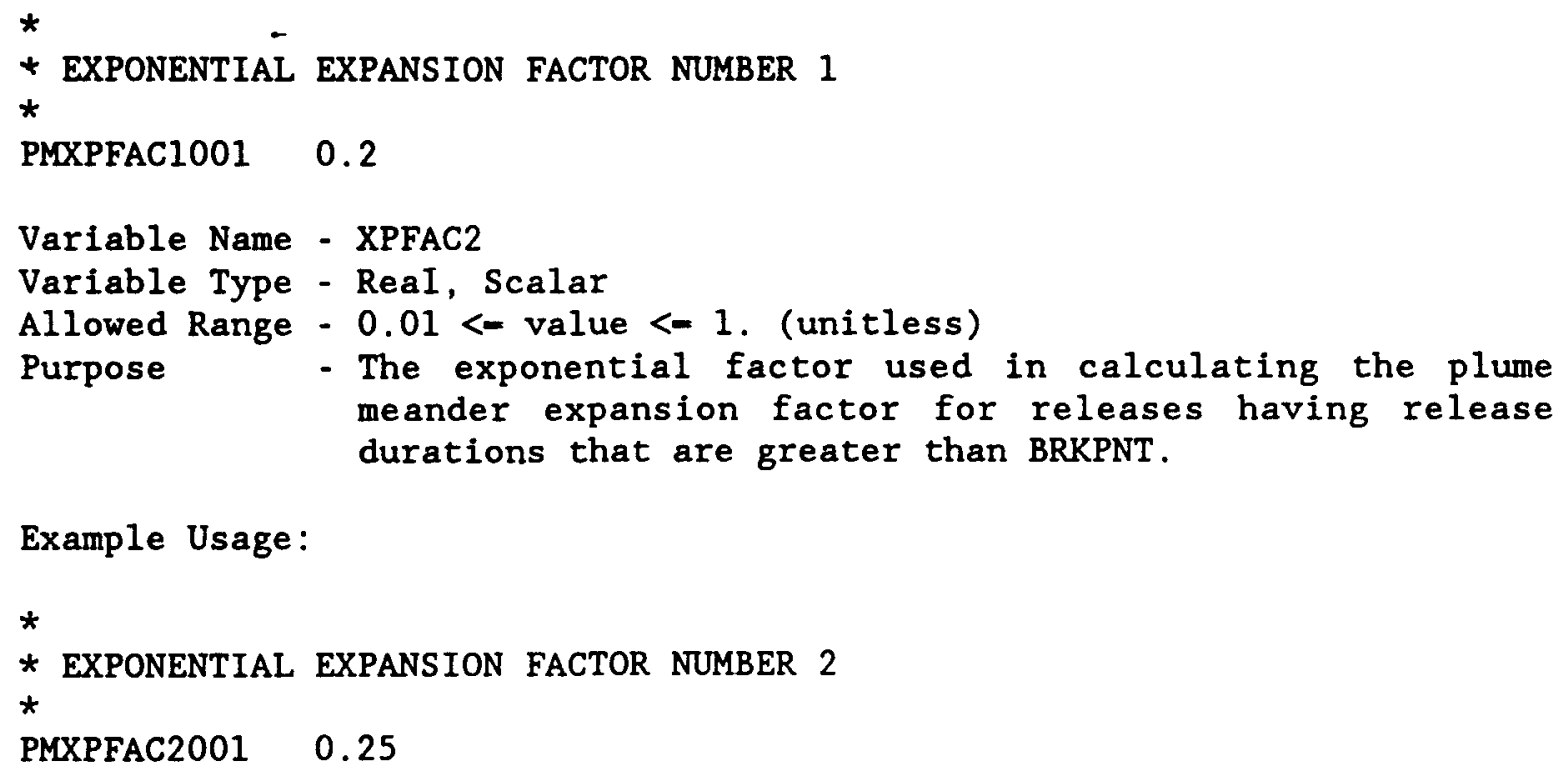




\subsection{Plume Rise (PR) Data}

A plume rise model based on the recommendations of Gary Briggs is incorporated into MACCS. There are three basic components of the MACCS plume rise model: (1) entrainment of buoyant plumes in a building wake, (2) plume rise under unstable and neutral conditions (classes $A$ to D), and (3) plume rise under stable conditions (classes $E$ to $F$ ). These component models are described in the MACCS Model Description [Jo89]. The individual numeric coefficients utilized by these models are hardwired into the code with no provision for their convenient modification by the user.

While it is not possible for the user to vary the individual coefficients utilized by the three components of the plume rise model, it is possible to modify their end results by the specification of linear scaling factors that are described in this section.

Variable Name - SCLCRW

Variable Type - Real, Scalar

Allowed Range - 0.01 <- value <- 100.

Purpose

- Linear scaling factor on the critical wind speed used in determining if buoyant plumes will be trapped in the turbulent wake of the reactor building complex. Parameter values less than 1 . make plume rise less likely to occur because plume liftoff occurs only if the ambient wind speed at the time of release is less than the calculated critical wind speed.

If there is a need to ignore the effect of building wake entrainment, this can be achieved by setting SCLCRW to its maximum allowable value.

Example Usage:

*

* SCALING FACTOR FOR THE CRITICAL WIND SPEED FOR ENTRAINMENT OF A BUOYANT

* PLUME

* (USED BY FUNCTION CAUGHT)

*

PRSCLCRWO01 1.

Variable Name - SCLADP

Variable Type - Real, Scalar

Allowed Range - 0.01 <- value <- 100.0 (unitless)

Purpose

- Linear scaling factor on the plume rise formula used for determining the amount of plume rise that will occur when unstable or neutral atmospheric conditions occur (classes A through D). 


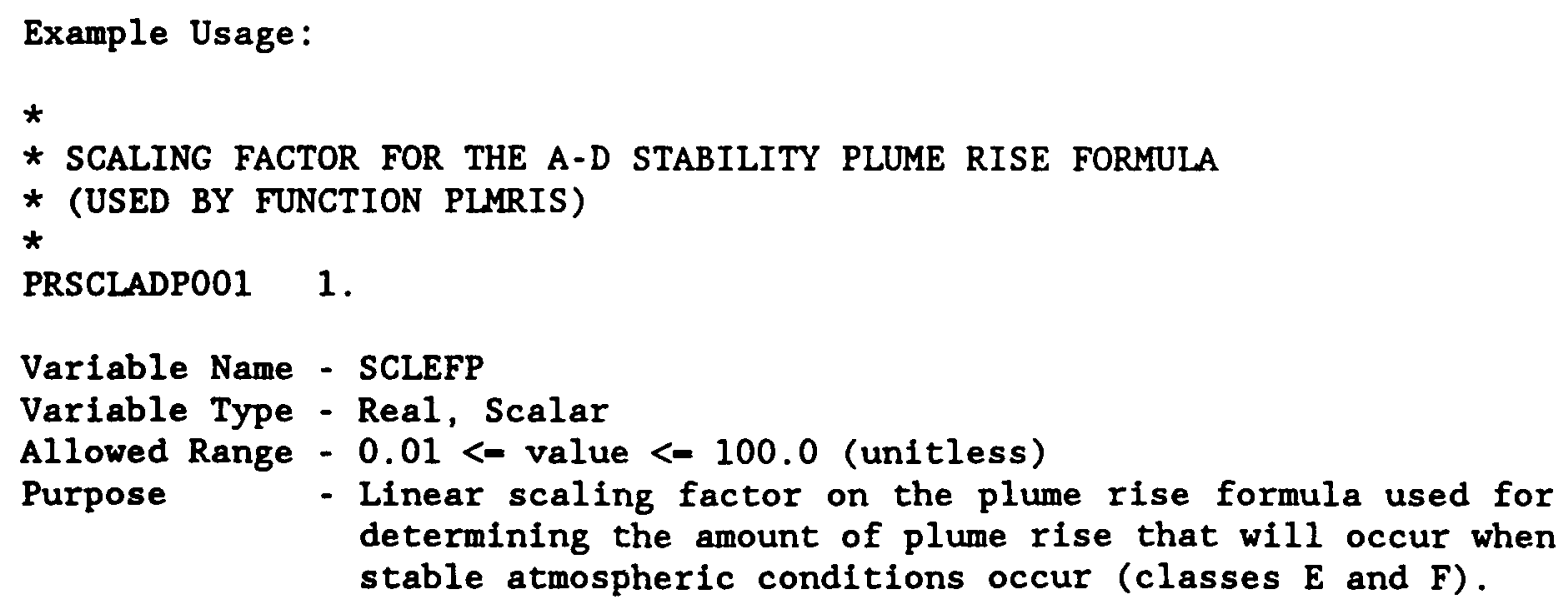

Example Usage:

*

* SCALING FACTOR FOR THE E-F STABILITY PLUME RISE FORMULA

* (USED BY FUNCTION PLMRIS)

*

PRSCLEFP001 1.

\subsection{Wake Effects (WE) Data}

The initial size of the plume is determined by the width and height of the reactor building wake. Sigma-y is initialized to width/4.3, and sigma-z is initialized to height/2.15. The height of the building wake is also used to determine if the plume is entrained in the turbulent region surrounding the building. Consequently, in addition to determining the initial plume size, the wake-height is utilized in the determination of whether or not buoyant plume rise will occur.

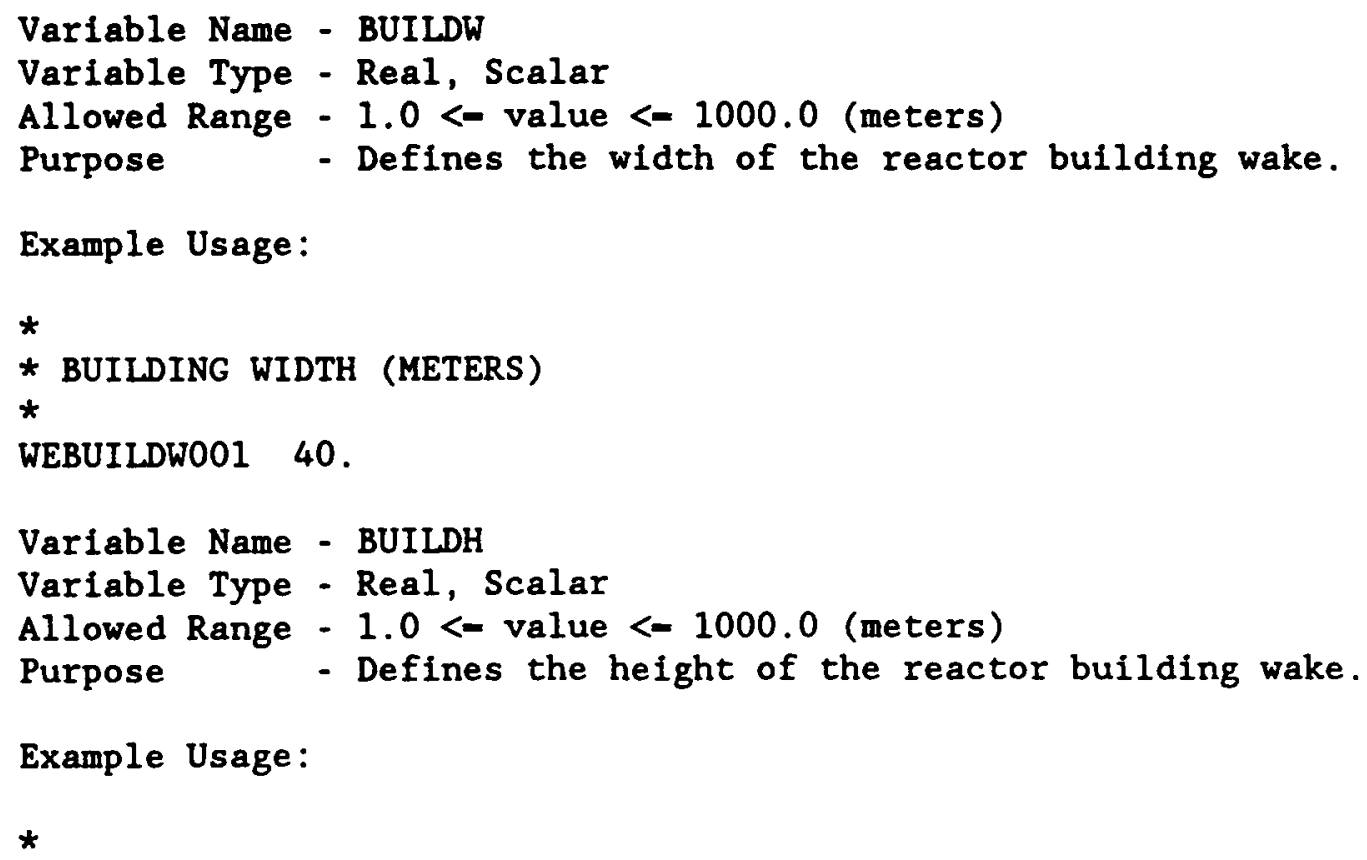


* BUILDING HEIGHT (METERS)

*

WEBUILDH001 50 .

\subsection{Release Description (RD) Data}

ATMOS can handle multiple plume segments in order to treat a release which has a composition that varies with time. The plume segments that comprise a release can be separated by a time gap, or can directly follow the preceding segment, but they are not allowed to overlap. The plume segments can be released at different heights, have different heat contents, and have different durations, but they will all share the same particle size distribution.

MACCS incorporates the capability for calculating the consequences from up to 60 different source terms in a single run of the code. This is accomplished by appending "change cards" to the ATMOS User Input File. The first source term is defined in the main body of the ATMOS User Input File. Up to 59 additional source terms can be defined through change card sets positioned at the end of the file.

The delimiter used to separate the change card sets is a period (.) in column one. The end of the file is also signified by a period in column one. All of the MACCS User Input Files must end with a period in column one. The sample ATMOS user input file listed in Appendix D.1 is an example illustrating the use of change cards for a Probabilistic Risk Assessment (PRA) application of the code.

The purpose of the change card processing in ATMOS is solely to allow modification of the previously specified Release Description Data. If data items from another data block appear in the change cards, they will be ignored. Each set of change cards must include a new value of ATNAM2, a text field describing the source term. Also, each set of change cards must specify a change in at least one of the numeric input variables described in this data block.

Variable Name - ATNAM2

Variable Type - Character, Scalar

Allowed Range - 1 <- length <- 80

Purpose - Identifies the name of the source term being studied. This name will be printed on all pages of the OUTPUT listing. A unique name must be specifled for each source term.

Example Usage:

$\star$

* SPECIFIC DESCRIPTIVE TEXT DESCRIBING THIS PARTICULAR SOURCE TERM

*

RDATNAM2001 'HYPOTHETICAL SOURCE TERM NUMBER 1' 


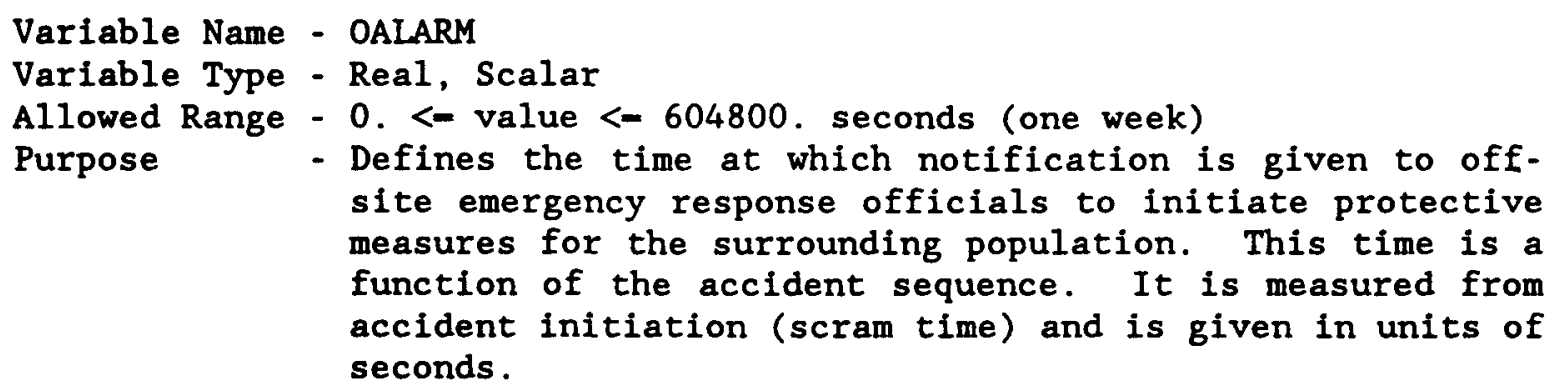

Example Usage:

*

* TIME AFTER aCcident INITIATION AT WHICH THE OFFSITE ALARM IS RUNG

* EVACUATION WILL BEGIN EDELAY SECONDS AFTERWARDS, SEE THE EARLY INPUT

* FILE

*

RDOALARMO01 17280.

Variable Name - NUMREL

Variable Type - Integer, Scalar

Allowed Range - 1 <- value <- 4

Purpose - Defines the number of plumes that will be released.

Note: If the multiple source term feature is being used, it is not possible to provide a larger value for NUMREL in the change cards than the value which was defined previously. That is, the values of NUMREL specified on change cards cannot be larger than the value of NUMREL specified in the base case data.

Example Usage:

*

* NUMBER OF PLUME SEgMENTS THAT ARE RELEASED

*

RDNUMRELO01 2

Variable Name - MAXRIS

Variable Type - Integer, Scalar

Allowed Range - 1 <- value <- NUMREL

Purpose - Specifies which plume segment is to be considered risk dominant. The selection of the risk dominant plume is usually based on its potential for causing early fatalities. Release of the risk dominant plume always begins at the selected meteorological start time of the weather sequence.

Example Usage:

*

* SELECTION OF RISK DOMINANT PLUME SEGMENT

$\star$ 


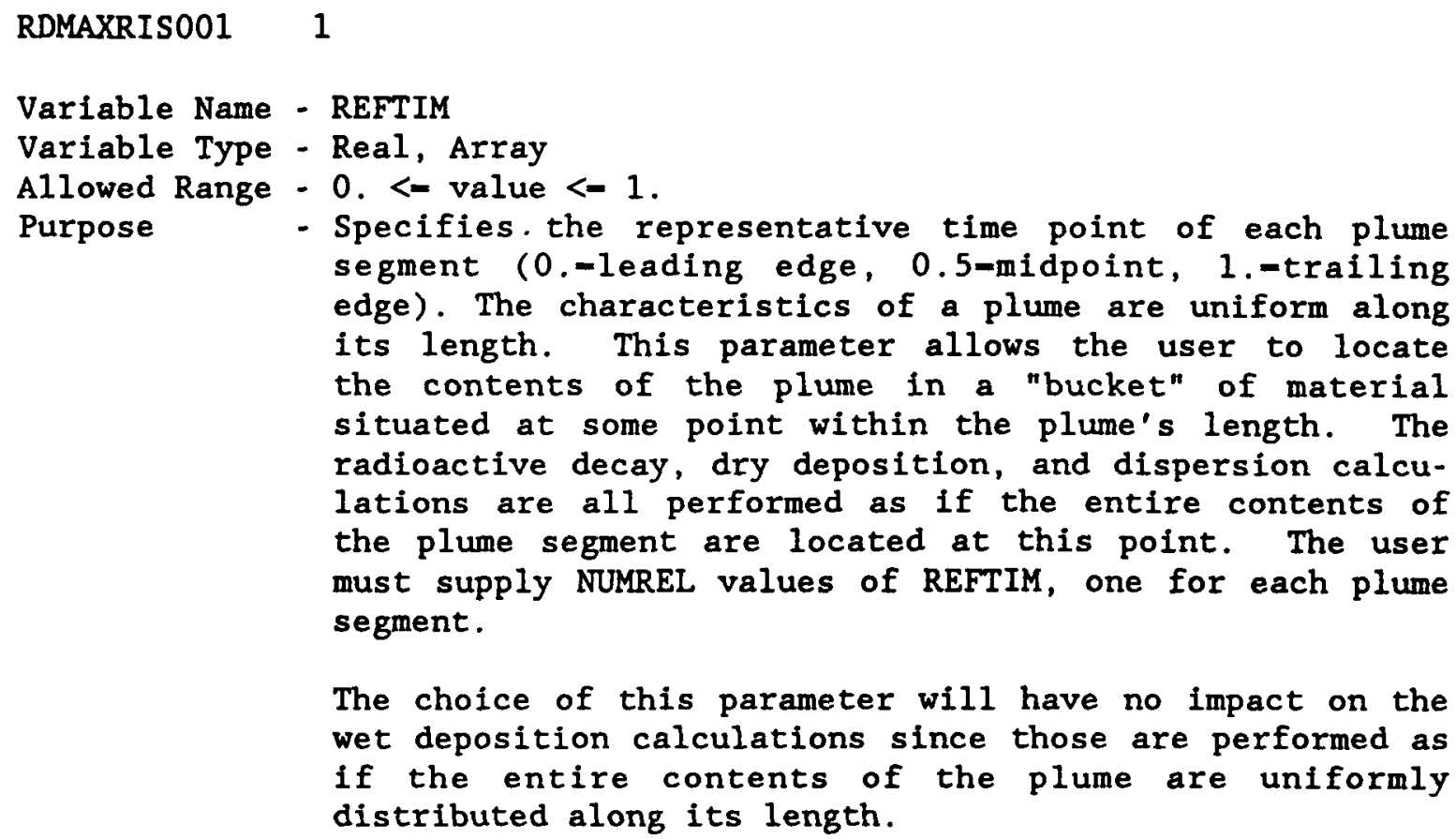

The choice of this parameter will have no impact on the wet deposition calculations since those are performed as if the entire contents of the plume are uniformly distributed along its length.

Example Usage:

*

* REPRESENTATIVE TIME POINT FOR DISPERSION AND RADIOACTIVE DECAY

*

RDREFTIMO01 0.0 .5 (CORRESPONDING TO HEAD AND MIDPOINT WEATHER)

Variable Name - PLHEAT

Variable Type - Rea1, Array

Allowed Range - $0.0<$ value <- 1. E10 (Watts)

Purpose - Specifies the rate of release of sensible heat in each plume segment. This quantity should be calculated as the amount of sensible heat in the plume segment divided by the duration of the plume segment. The value specified here is used to determine the amount of bouyant plume rise which will occur. The user must supply NUMREL values of PLHEAT, one for each plume segment.

Example Usage:

*

* HEAT CONTENT OF THE RELEASE SEGMENTS (WATTS)

* a VAlue specified fOR EACH OF THE RELEASE SEgments

*

RDPLHEAT001 $\quad 6.37 E+06 \quad 3.43 E+06$

Variable Name - PLHITE

Variable Type - Real, Array

Allowed Range - $0.0<=$ value $<-1000.0$ (meters) 
Purpose - Specifies the height above ground level at which each plume segment is released. The user must supply NUMREL values of PLHITE, one for each plume segment.

Example Usage:

$\star$

* HEIGHT OF THE PLUME SEgMENTS AT RELEASE (METERS)

* a VALUE SPECIFIED FOR EACH OF THE RELEASE SEGMENTS

*

RDPLHITEOO1 30.30.

Variable Name - PLUDUR

Variable Type - Real, Array

Allowed Range - $60.0<-$ value <- 86400.0 (seconds) ONE DAY

Purpose - Specifies the duration in seconds of each plume segment. The user must supply NUMREL values of PLUDUR, one for each plume segment.

Example Usage:

*

* DURATION OF THE PLUME SEgMENTS (SECONDS)

* a VALUE SPECIFIED FOR EACH OF THE RELEASE SEgMENTS

*

RDPLUDUR001 9000. 12588.

Variable Name - PDELAY

Variable Type - Real, Array

Allowed Range - 0 . <- value <- 345600.0 (seconds) (4 DAYS)

Purpose - Specifies the start time of each plume segment in seconds from the time of accident initiation, e.g., reactor scram. The user must supply NUMREL values for PDELAY, one for each plume segment.

Example Usage:

*

* TIME OF RELEASE FOR EACH PLUME (SECS FROM SCRAM)

* A VALUE SPECIFIED FOR EACH OF THE RELEASE SEGMENTS

*

RDPDELAY001 17280.26280.

Variable Name - PSDIST

Variable Type - Real, Array

Allowed Range - 0 . <- value <- 1 .

Purpose

- Defines the fraction of the released material allocated to each of the particle size (deposition velocity) bins. All of the plume segments must use the same distribution of material among these bins. The user must specify one data card for each of the chemical element groups. The number of chemical element groups was defined as variable 
MAXGRP in Section 1.4. On each of these cards, the user must supply an allocation fraction to be associated with each of the particle size groups. The number of particle size groups was defined as the input variable NPSGRP in Section 1.6 .

Example Usage:

*

* PARTICLE SIZE DISTRIBUTION OF EACH ELEMENT GROUP

*

* $\quad 0.001 \quad 0.010 .02$ DEPOSITION VELOCITY OF EACH GROUP (M/S)

*

$\begin{array}{llll}\text { RDPSDIST001 } & 0.1 & 0.8 & 0.1 \\ \text { RDPSDIST002 } & 0.1 & 0.8 & 0.1 \\ \text { RDPSDIST003 } & 0.1 & 0.8 & 0.1 \\ \text { RDPSDIST004 } & 0.1 & 0.8 & 0.1 \\ \text { RDPSDIST005 } & 0.1 & 0.8 & 0.1 \\ \text { RDPSDIST006 } & 0.1 & 0.8 & 0.1 \\ \text { RDPSDIST007 } & 0.1 & 0.8 & 0.1 \\ \text { RDPSDIST008 } & 0.1 & 0.8 & 0.1 \\ \text { RDPSDIST009 } & 0.1 & 0.8 & 0.1\end{array}$

Variable Name - CORINV

Variable Type - Real, Array

Allowed Range - 0 . <- value <- 1. E35 (becquerels)

Purpose

- Defines the inventory of each nuclide present in the reactor at the time of accident initiation (scram). The inventory is given in units of becquerels (disintegrations/second). All of the nuclides which have been previously defined on the list of nuclides (NUCNAM array in Section 1.4) must be listed here. The nuclide names and their respective inventories may appear in any order.

Example Usage:

* 3578 MWTH BWR CORE INVENTORY, 80 0/0 CAPACITY, 3/3 SHUTDOWN INVENTORY

*

*

*

*

RDCORINV001

RDCORINVO02

RDCORINV003

RDCORINV004

RDCORINV005

RDCORINV006

RDCORINV007

RDCORINV008

RDCORINVO09

SUPPLIED BY D.E. BENNETT, $6 / 17 / 86$

NUCNAM

CORINV (BECQUERELS)

RDCORINV010

$\begin{array}{ll}\text { CO-58 } & 2.024 \mathrm{E}+16 \\ \text { CO-60 } & 2.423 \mathrm{E}+16 \\ \mathrm{KR}-85 & 3.317 \mathrm{E}+16 \\ \mathrm{KR}-85 \mathrm{M} & 1.206 \mathrm{E}+18 \\ \mathrm{KR}-87 & 2.193 \mathrm{E}+18 \\ \mathrm{KR}-88 & 2.960 \mathrm{E}+18 \\ \text { RB }-86 & 1.856 \mathrm{E}+15 \\ \text { SR }-89 & 3.673 \mathrm{E}+18 \\ \text { SR }-90 & 2.599 \mathrm{E}+17 \\ \text { SR-91 } & 4.771 \mathrm{E}+18\end{array}$




\begin{tabular}{|c|c|c|}
\hline RDCORINV011 & SR-92 & $4.984 E+18$ \\
\hline RDCORINV012 & $Y-90$ & $2.783 E+17$ \\
\hline RDCORINV013 & $Y-91$ & $4.482 E+18$ \\
\hline RDCORINV014 & $Y-92$ & $5.004 E+18$ \\
\hline RDCORINV015 & $Y-93$ & $5.690 \mathrm{E}+18$ \\
\hline RDCORINV016 & ZR-95 & $5.899 E+18$ \\
\hline RDCORINV017 & ZR-97 & $6.073 E+18$ \\
\hline RDCORINV018 & NB -95 & $5.581 E+18$ \\
\hline RDCORINV019 & MO-99 & $6.436 \mathrm{E}+18$ \\
\hline RDCORINV020 & TC-99M & $5.554 E+18$ \\
\hline RDCORINV021 & RU-103 & $4.877 E+18$ \\
\hline RDCORINV022 & RU-.105 & $3.254 \mathrm{E}+18$ \\
\hline RDCORINVO23 & RU-106 & $1.327 \mathrm{E}+18$ \\
\hline RDCORINV024 & RH-105 & $2.429 \mathrm{E}+18$ \\
\hline RDCORINV025 & SB - 127 & $3.077 E+17$ \\
\hline RDCORINV026 & SB - 129 & $1.068 \mathrm{E}+18$ \\
\hline RDCORINV027 & TE - 127 & $2.979 E+17$ \\
\hline RDCORINV028 & $\mathrm{TE}-127 \mathrm{M}$ & $4.010 \mathrm{E}+16$ \\
\hline RDCORINV029 & TE - 129 & $1.002 \mathrm{E}+18$ \\
\hline RDCORINV030 & TE-129M & $2.634 \mathrm{E}+17$ \\
\hline RDCORINV031 & TE-131M & $5.058 \mathrm{E}+17$ \\
\hline RDCORINV032 & TE-132 & $4.944 E+18$ \\
\hline RDCORINV033 & I - 131 & $3.417 E+18$ \\
\hline RDCORINV034 & I - 132 & $5.020 E+18$ \\
\hline RDCORINV035 & I - 133 & $7.172 \mathrm{E}+18$ \\
\hline RDCORINV036 & $I-134$ & $7.850 E+18$ \\
\hline RDCORINV037 & $I-135$ & $6.751 E+18$ \\
\hline RDCORINV038 & $X E-133$ & $7.182 E+18$ \\
\hline RDCORINV039 & $X E-135$ & $1.707 E+18$ \\
\hline RDCORINV040 & CS - 134 & $5.596 \mathrm{E}+17$ \\
\hline RDCORINV041 & CS -136 & $1.501 \mathrm{E}+17$ \\
\hline RDCORINV0 42 & CS -137 & $3.350 E+17$ \\
\hline RDCORINV043 & BA -139 & $6.612 E+18$ \\
\hline RDCORINV044 & $B A-140$ & $6.522 \mathrm{E}+18$ \\
\hline RDCORINV045 & LA -140 & $6.655 E+18$ \\
\hline RDCORINV046 & $L A-141$ & $6.145 E+18$ \\
\hline RDCORINV047 & LA-142 & $5.912 E+18$ \\
\hline RDCORINV048 & CE-141 & $5.922 E+18$ \\
\hline RDCORINV049 & $\mathrm{CE}-143$ & $5.765 \mathrm{E}+18$ \\
\hline RDCORINV050 & CE -144 & $3.841 E+18$ \\
\hline RDCORINV051 & PR -143 & $5.643 E+18$ \\
\hline RDCORINV052 & ND -147 & $2.522 E+18$ \\
\hline RDCORINV053 & NP - 239 & $7.516 \mathrm{E}+19$ \\
\hline RDCORINV054 & PU- 238 & $5.226 E+15$ \\
\hline RDCORINV055 & PU - 239 & $1.325 E+15$ \\
\hline RDCORINV056 & $P U-240$ & $1.659 E+15$ \\
\hline RDCORINV057 & PU - 241 & $2.856 E+17$ \\
\hline RDCORINV058 & $A M-241$ & $2.903 E+14$ \\
\hline RDCORINV059 & $C M-242$ & $7.667 E+16$ \\
\hline RDCORINV060 & CM - 244 & $4.137 E+15$ \\
\hline
\end{tabular}




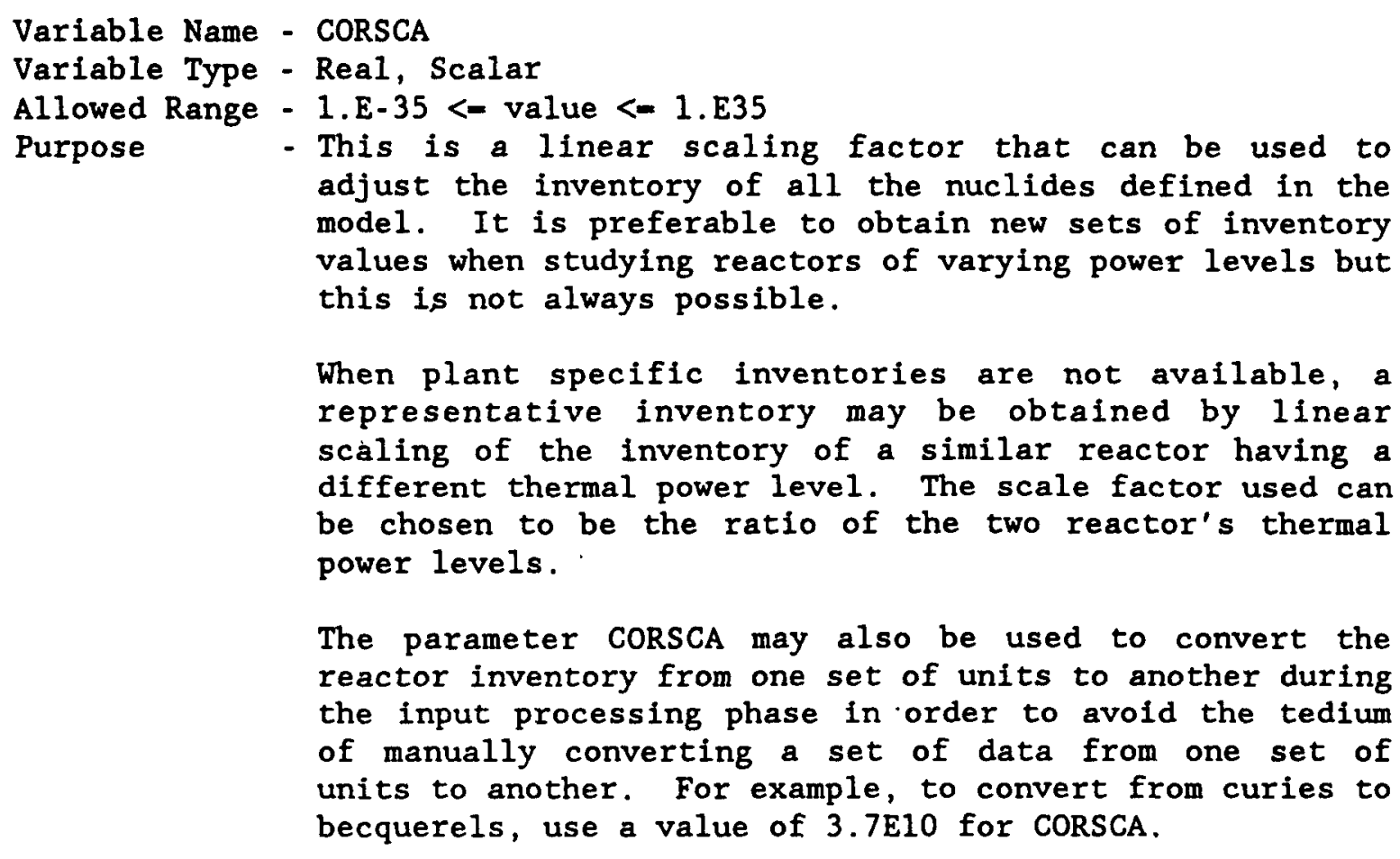

Example Usage:

*

* SCALING FACTOR TO ADJUST THE CORE INVENTORY

*

RDCORSCA001 $0.715 *$ SURRY

Variable Name - RELFRC

Variable Type - Real, Array

Allowed Range - 0 . <- value <- 1.

Purpose - Defines the release fractions for each of the plume segments. One card is supplied for each plume and it contains as many values as there are element groups. All components of an element group are released from the reactor in the same fraction.

Release fractions are applied to the core inventory at the time of scram to calculate the amount of radioactivity for each nuclide allocated to each plume segment. During the delay period before the plume's release, radioactive decay and buildup of daughter products is calculated by adjustments to the release inventory of each individual plume segment. This means that any buildup of daughter products is released in the same fraction as its parent.

For example, consider the case of radioiodine which decays to radioxenon during holdup inside the containment after accident initiation (scram). Let us assume that 
the Noble gases (xenon) have a release fraction of one and the iodine group has a release fraction of one-half. The interpretation of these release fractions is as follows. After adjusting for radioactive decay up to the time of release, all of the xenon in the reactor at the time of scram will be released (release-fraction-1). Because of the assumption that daughters are released according to the same fraction as their parents, the daughter product buildup of xenon which resulted from the decay of radiolodine will be subject to a release fraction of one-half (the release fraction of the iodine).

This approach for treatment of the in-plant processes prior to release is consistent with the methodology of the Source Term Code Package (STCP) and MELCOR. It may not be appropriate for other source term generation programs.

Example Usage:

*

* RELEASE FRACTIONS FOR NUCLIDE GROUPS IN RELEASE

*

* GENERIC SOURCE TERM A

*

$\begin{array}{llllllllll} & \mathrm{XE} / \mathrm{KR} & \mathrm{I} & \mathrm{CS} & \mathrm{TE} & \mathrm{SR} & \mathrm{RU} & \mathrm{LA} & \mathrm{CE} & \mathrm{BA}\end{array}$

$\dot{x}$

*

RDRELFRC001 7.0E-01 4.7E-01 4.7E-01 3.4E-01 3.E-01 5.E-03 3.E-02 4.5E-02 2.7E-01

RDRELFRC002 2.9E-01 1.0E-02 9.0E-03 5.3E-03 2.E-03 4.E-04 1.E-04 9.6E-04 2.3E-03

\subsection{Output-Control (OC) Data}

The user has the option of looking at tables of dispersion data for all of the trials that are performed. This information includes air and ground concentrations, sigma-y and sigma-z values, and arrival and departure times for each plume segment at each spatial interval. This data is written to the standard output file (TAPE6).

Variable Name - ENDAT1

Variable Type - Logical, Scalar

Allowed Value - .TRUE. or .FALSE.

Purpose - Control flag which allows the user to execute only the ATMOS module. A value of .TRUE. tells the code that EARLY and CHRONC will not be run. When this is done, the User Input Files for EARLY and CHRONC and a site Data File need not be supplied. If the user wishes to skip execution of only the CHRONC module, that can be accomplished through the input variable ENDAT2 on the EARLY User Input File. 


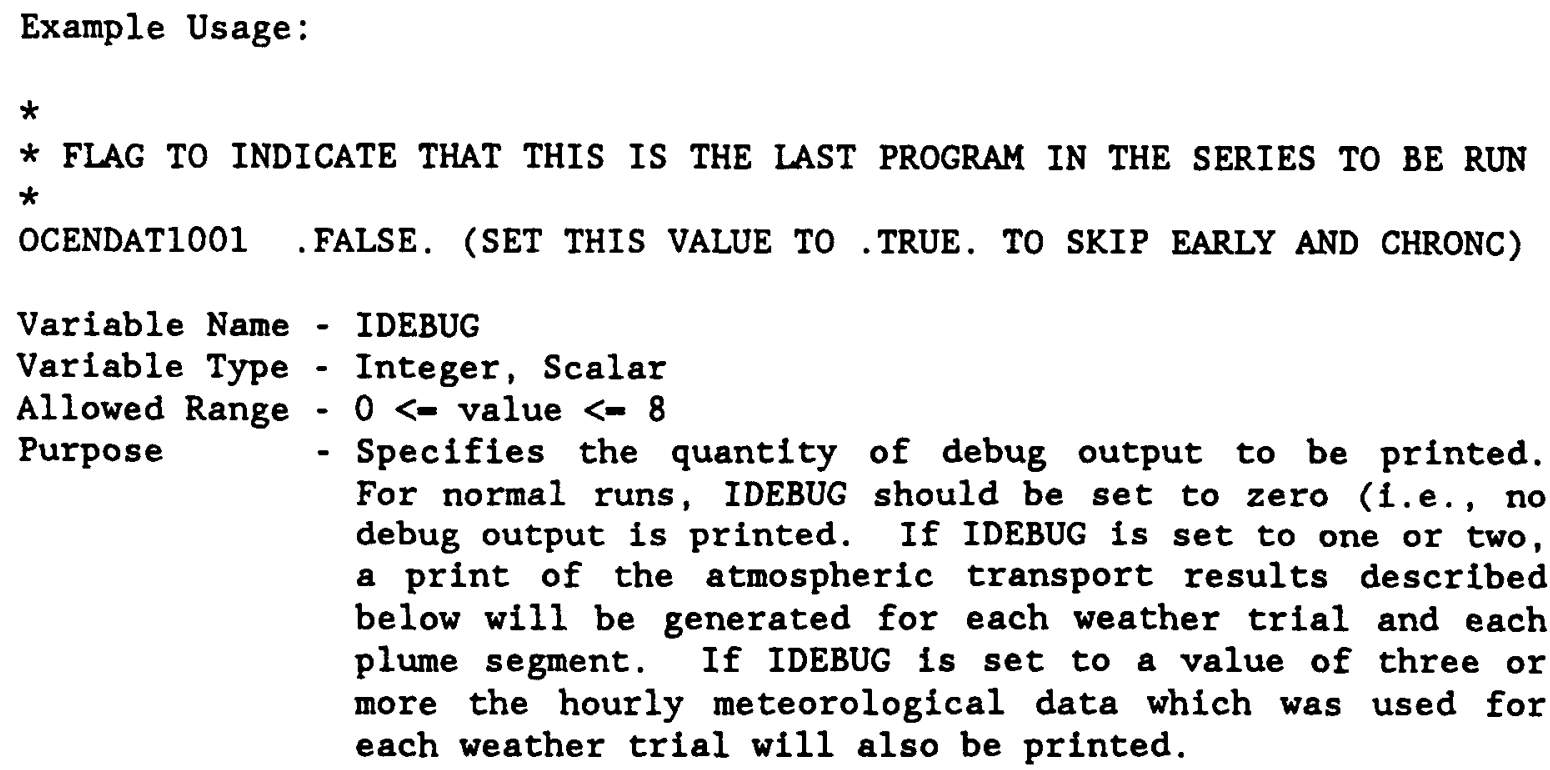

The debug output for atmospheric transport prints the results described below.

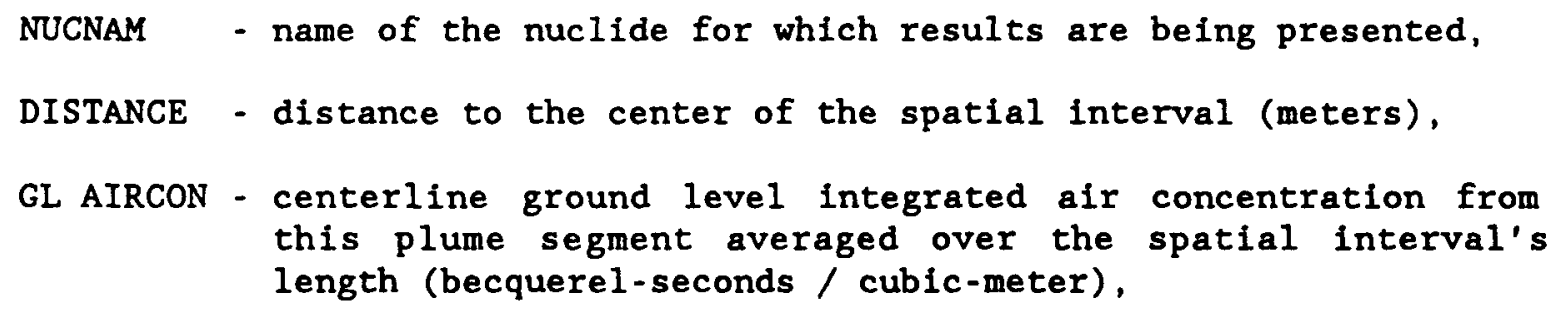

GL X/Q - centerline ground level chi over $Q(x / Q)$, ratio of air concentration, chi, to source strength $Q$, in SI units, averaged over the interval's length,

WETREM - fraction of material remaining in the plume segment after wet deposition over the spatial interval's length,

DRYREM - fraction of material remaining in the plume segment after dry deposition over the spatial interval's length,

REMINV - adjusted source strength of the plume upon entering each spatial interval after adjustment for losses in the previous intervals due to radioactive decay and wet and dry deposition (becquerels),

PLSIGY - horizontal dispersion parameter sigma-y averaged over the spatial interval's length (meters), 

PLSIGZ - vertical dispersion parameter sigma-z averaged over the spatial interval's length (meters),
WEATHER - indices to the first and last hours of the weather sequence used for determining atmospheric conditions during transport across each spatial interval,
HTFCTR - ratio of the centerline ground level air concentration ( $z=0$ ) to the plume centerline air concentration $(z-H)$,
AVGHIT - average height (H) of the plume as it traversed the spatial interval (meters),
TIMCEN - time after accident initiation at which the leading edge of the plume arrived at the center of the spatial interval (seconds),

TIMOVH - duration for which the plume was overhead at the centerpoint of the spatial interval (seconds).

Example Usage:

$\star$

OCIDEBUG001 1 (REQUEST A TRACE OF ATMOSPHERIC DISPERSION)

Variable Name - NUCOUT

Variable Type - Character, Scalar

Allowed Range - $3<-$ length $<-8$

Purpose

- Specifies which nuclide will appear on the dispersion listing if one is produced. The dispersion listing is only produced if IDEBUG is greater than zero. The specified nuclide name must appear on the previously defined Iist of nuclides, NUCNAM, defined in Section 1.4.

This item is only required if IDEBUG is greater than zero.

Example Usage:

*

* NAME OF THE NUCLIDE TO BE LISTED ON THE DISPERSION LISTINGS

*

OCNUCOUT001 CS-137

\subsection{Meteorological Sampling (M1) Specification}

There are five options available to the user for specifying the weather data that will be used by ATMOS. The code can either be used to run a single weather sequence or multiple weather sequences.

If a single weather sequence is desired, there are three ways to specify the weather. The user can either (1) specify data for 120 hours of 
weather on the ATMOS User Input File, (2) specify a starting day and hour in the weather data file for the weather sequence, or (3) specify constant weather conditions. For the specified starting day and hour option, the program will obtain 120 hours of weather data from the weather file beginning at the specified date and time. A file of hourly weather data covering a period of one year $(8,760$ hours) is required if the fixed start time or either of the weather sampling options are to be used. The format of this file is described in Appendix C.1.

The two methods of weather sampling are: (1) a modified version of the weather bin sampling method used by CRAC2 [Ri84], and (2) a stratified purely random sampling approach.

The weather bin sampling method sorts weather sequences into categories and assigns a probability to each category according to their initial conditions (wind speed and stability class) and the occurrence of rain (intensity and distance). Because the rain bins depend on rain intensity as well as the downwind distance at which rain occurs, the user is required to supply parameters defining the rain weather bins as part of the ATMOS User Input File. The definitions of the other weather bins, those defined by initial stability class and wind speed, are hard-wired in the code. The definitions of those initial condition weather bins are not the same as used in CRAC2. In addition to changing the breakpoints used to categorize wind speed, the wind speed slowdown bins of CRAC2 have been removed. A description of the MACCS weather sampling algorithm can be found in the MACCS Model Description [Jo89].

The stratified random sampling method allows the user to sample weather from each day of the year after division of each day into one, two, three or four equal time periods. Each weather sequence selected is considered to have the same probability of occurrence, that is,

$$
\text { - } \quad P=\frac{1 \ldots}{\text { total-selected-samples }}
$$

Because of the flexiblity that ATMOS affords in the specification of the geometric grid, it is necessary to guard against the possibility of running out of weather data. It is possible that 120 hours of weather may not suffice to carry all the plume segments out to the last spatial interval. Also, the user may wish to cause the occurrence of rain in the outermost spatial intervals in order to prevent radioactive material from escaping consideration. For these reasons, the user must specify a set of boundary weather conditions.

Depending on the option selected by the user, different data items will be required by ATMOS.

Variable Name - METCOD

Variable Type - Integer, Scalar

Allowed Range - $1<$ value <- 5 
Purpose

- Meteorological sampling option code:

1 - fixed start time in the weather file (day,hour),

2 - weather bin sampling,

3 - 120 hours of weather supplied by the user,

4 - constant weather conditions (use boundary weather),

5 - stratified random sampling from each day of the year.

Example Usage:

*

* meteorological sampling option code:

*

MIMETCOD001 2

\subsection{Boundary Weather (M2) Data}

Boundary Weather Data are required for all possible values of METCOD. This data block specifies the weather conditions that will be used if 120 hours of recorded weather data do not transport the last plume through the limiting spatial interval for measured weather, LIMSPA. The boundary weather data are also used for predicting the behavior of the plume at all spatial intervals beyond LIMSPA.

For the case of constant weather, METCOD-4, the boundary weather data in this section determines the constant weather conditions that will be used. The boundary weather is used throughout the atmospheric calculations and the value of LIMSPA is ignored by the program in this case.

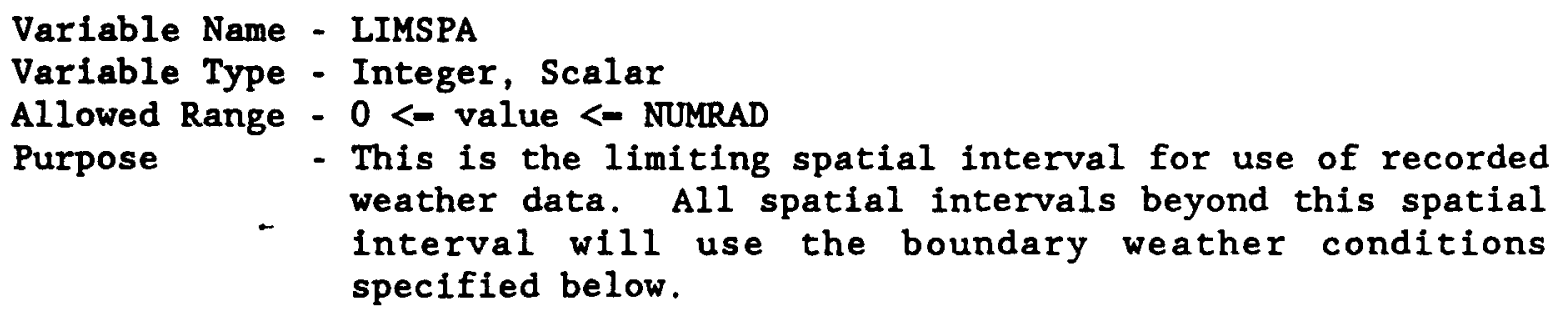
weather data. All spatial intervals beyond this spatial interval will use the boundary weather conditions specified below.

If a value of zero is specified, then the boundary weather conditions will be used right from the start. If METCOD-4, the value of LIMSPA is ignored.

Example Usage:

*

* LAST SPATIAL INTERVAL FOR MEASURED WEATHER

*

M2LIMSPA001 25

Variable Name - BNDMXH

Variable Type - Real, Scalar

Allowed Range - 1.E2 <- value <- 1.E4 (meters)

Purpose - This is the mixing layer height that will be used for the boundary weather conditions. 


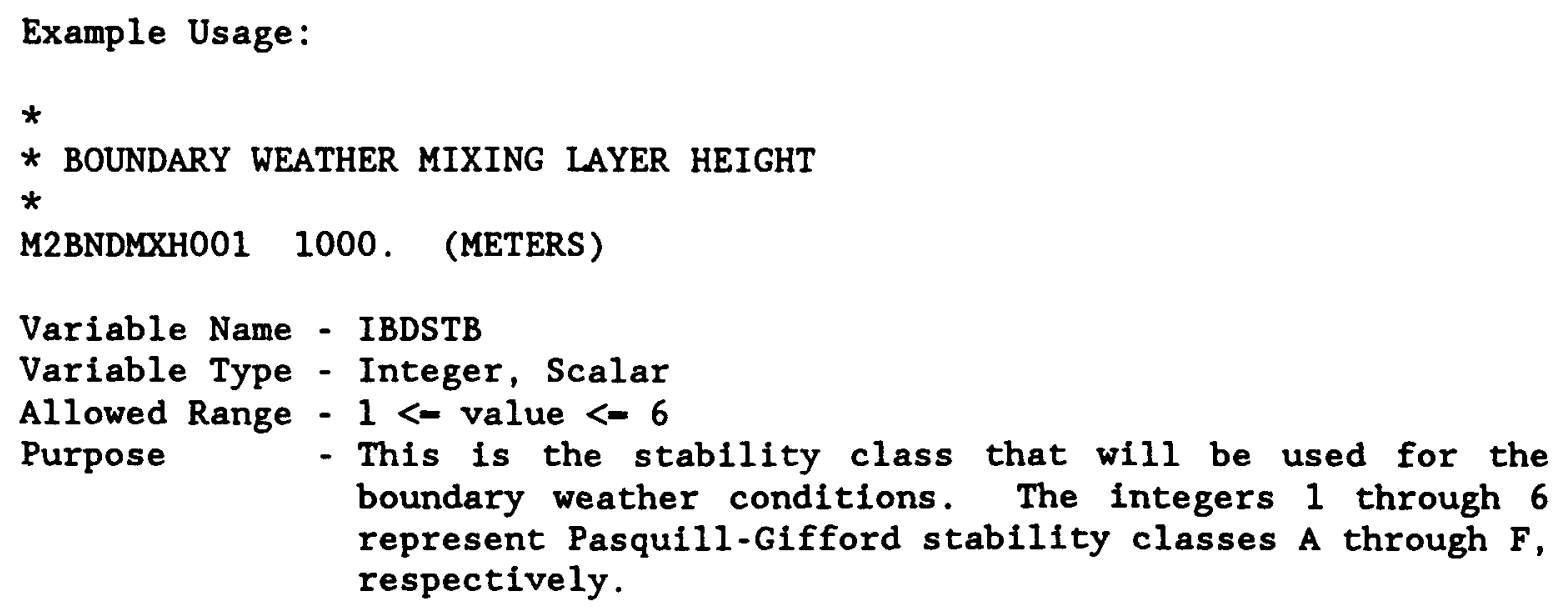

*

* Boundary wEATHER WIND SPEED

*

M2BNDWND001 $0.5 \quad(M / S)$

\subsection{Fixed Start Time (M3) Data}

The data in this section must be supplied for all values of METCOD except METCOD-2 (meteorological bin sampling), and METCOD-5 (stratified random 
sampling). The data is needed for all of these option choices because the food pathway calculations of the CHRONC module depend on the accident's day of occurrence.

If the user has chosen METCOD=1, the values of ISTRDY and ISTRHR specify the starting day and hour in the weather file of the single weather trial that will be performed.

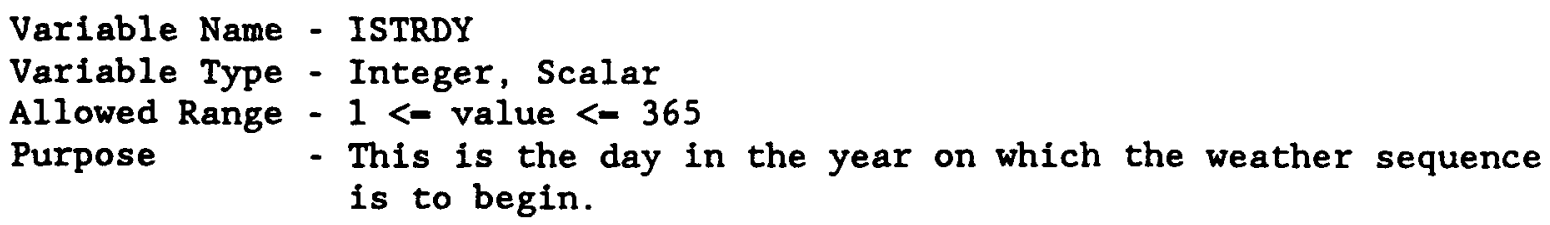

Example Usage:

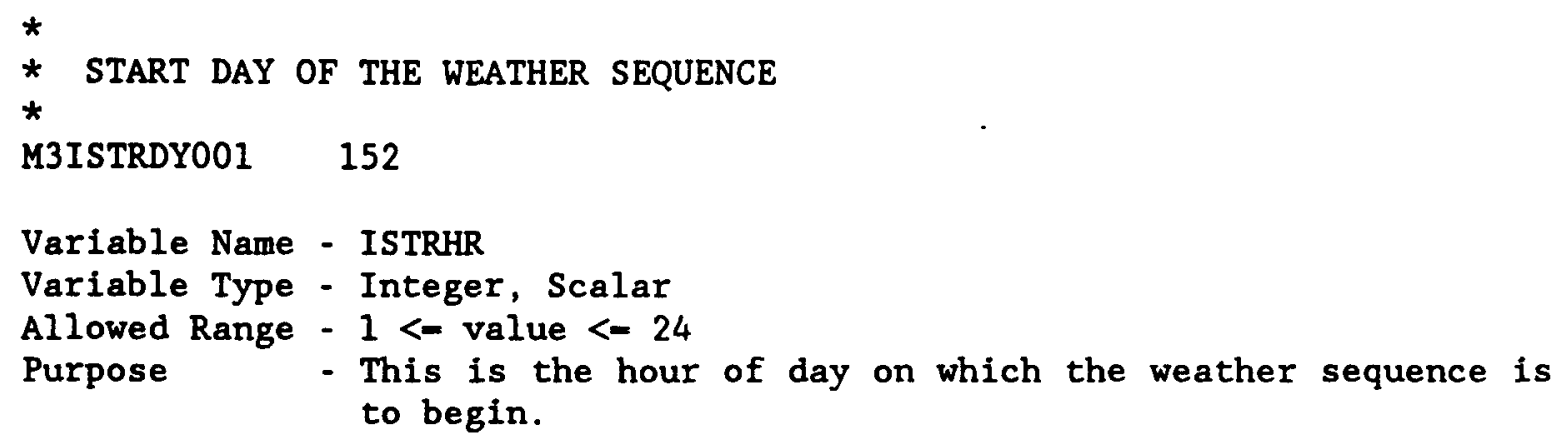

\subsection{Meteorological Bin Sampling (M4) Data}

The data in this section must be supplied if the user chooses METCOD-2 . This sampling method requires that the meteorological data be sorted into a set of weather bins. The bins are defined to represent rain conditions in different distance intervals downwind from the accident site together with sixteen bins for initial conditions (stability class and wind speed).

Definition of the rain intensities and distance intervals that define the rain bins is the responsibility of the user. The user must specify either two or three rain intensities which are used as breakpoints in the categorization of rain rate (NRINTN). A rain intensity of zero is not allowed. For example, if the user specifies two rain intensity breakpoints of $1 \mathrm{~mm} / \mathrm{hr}$ and $4 \mathrm{~mm} / \mathrm{hr}$, the following three rain intensity bins, where $x$ is the rain intensity, will result: 
1. $0 \mathrm{~mm} / \mathrm{hr}<\mathrm{x}<-1 \mathrm{~mm} / \mathrm{hr}$,

2. $1 \mathrm{~mm} / \mathrm{hr}<\mathrm{x}<-4 \mathrm{~mm} / \mathrm{hr}$,

3. $4 \mathrm{~mm} / \mathrm{hr}<\mathrm{x}$.

The concept of rain distance intervals used in MACCS is similar to that used by CRAC2 [Ri84] but MACCS requires that the user specify the rain distances to be used in the weather categorization. Let us suppose that the user specifies $2,4,8$, and $16 \mathrm{~km}$ as four distance intervals (NRNINT). Then these values define the following four rain distance intervals:

1. $0 \mathrm{~km}<-$ distance of first rain occurrence $<-2 \mathrm{~km}$,

2. $2 \mathrm{~km}<$ distance of first rain occurrence $<-4 \mathrm{~km}$,

3. $4 \mathrm{~km}<$ distance of first rain occurrence $<=8 \mathrm{~km}$,

4. $8 \mathrm{~km}<$ distance of first rain occurrence $<-16 \mathrm{~km}$.

The total number of weather bins, $N$, is determined by the expression $\mathrm{N}=\mathrm{NRNINT*}(\mathrm{NRINTN}+1)+16$, where NRNINT is the number of distance intervals and NRINTN is the number of rain intensity breakpoints. Thus, the total number of defined bins can range from twenty-eight to forty depending on the values supplied by the user.

The sixteen initial condition weather bins are hard-wired into the code as follows:

$\begin{array}{rcl}\text { METBIN } & \text { STABILITY } & \text { WIND-SPEED (u) } \\ 1 & \text { A/B } & 0 \mathrm{~m} / \mathrm{s}<\mathrm{u}<-3 \mathrm{~m} / \mathrm{s} \\ 2 & \text { A/B } & 3 \mathrm{~m} / \mathrm{s}<\mathrm{u} \\ & \text { C/D } & 0 \mathrm{~m} / \mathrm{s}<\mathrm{u}<-1 \mathrm{~m} / \mathrm{s} \\ 3 & \text { C/D } & 1 \mathrm{~m} / \mathrm{s}<\mathrm{u}<-2 \mathrm{~m} / \mathrm{s} \\ 4 & \text { C/D } & 2 \mathrm{~m} / \mathrm{s}<\mathrm{u}<=3 \mathrm{~m} / \mathrm{s} \\ 5 & \text { C/D } & 3 \mathrm{~m} / \mathrm{s}<\mathrm{u}<-5 \mathrm{~m} / \mathrm{s} \\ 6 & \text { C/D } & 5 \mathrm{~m} / \mathrm{s}<\mathrm{u}<-7 \mathrm{~m} / \mathrm{s} \\ 7 & \text { C/D } & 7 \mathrm{~m} / \mathrm{s}<\mathrm{u} \\ 8 & \text { E } & 0 \mathrm{~m} / \mathrm{s}<\mathrm{u}<-1 \mathrm{~m} / \mathrm{s} \\ & \text { E } & 1 \mathrm{~m} / \mathrm{s}<\mathrm{u}<-2 \mathrm{~m} / \mathrm{s} \\ 9 & \text { E } & 2 \mathrm{~m} / \mathrm{s}<\mathrm{u}<=3 \mathrm{~m} / \mathrm{s} \\ 10 & \text { E } & 3 \mathrm{~m} / \mathrm{s}<\mathrm{u} \\ 11 & & \\ 12 & \text { F } & 0 \mathrm{~m} / \mathrm{s}<\mathrm{u}<-1 \mathrm{~m} / \mathrm{s} \\ & \text { F } & 1 \mathrm{~m} / \mathrm{s}<\mathrm{u}<-2 \mathrm{~m} / \mathrm{s} \\ 13 & \text { F } & 2 \mathrm{~m} / \mathrm{s}<\mathrm{u}<-3 \mathrm{~m} / \mathrm{s} \\ 14 & \text { F } & 3 \mathrm{~m} / \mathrm{s}<\mathrm{u}\end{array}$

Note that the definition of the initial condition weather categories are not the same as those used by CRAC2. Also, MACCS does not consider wind speed slowdowns in the weather categorization. 
The user controls how many weather sequences are chosen from each weather bin by the choice of a value for NSMPLS, defined later in this section. This can be done in two different ways: either (1) request that the same number of weather sequences are to be chosen from each bin and specify the number of sequences, NSMPLS, to be selected $(1<-$ NSMPLS $<=10)$, or else (2) specify a non-uniform sampling from the categories (NSMPLS-0) as defined below.

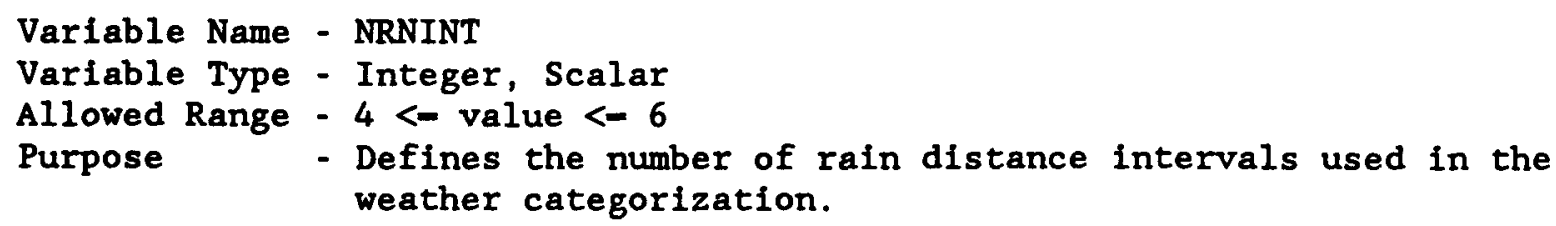
must lie within 10 percent of the spatial interval endpoint distances (variable SPAEND in Section 1.3), i.e., $0.9 * \operatorname{SPAEND}(i)<-\operatorname{RNDSTS}(j)<-1.1 * \operatorname{SPAEND}(i)$, for each $j$ and some value of 1 . The user must supply NRNINT unique values in ascending order.

Example Usage:

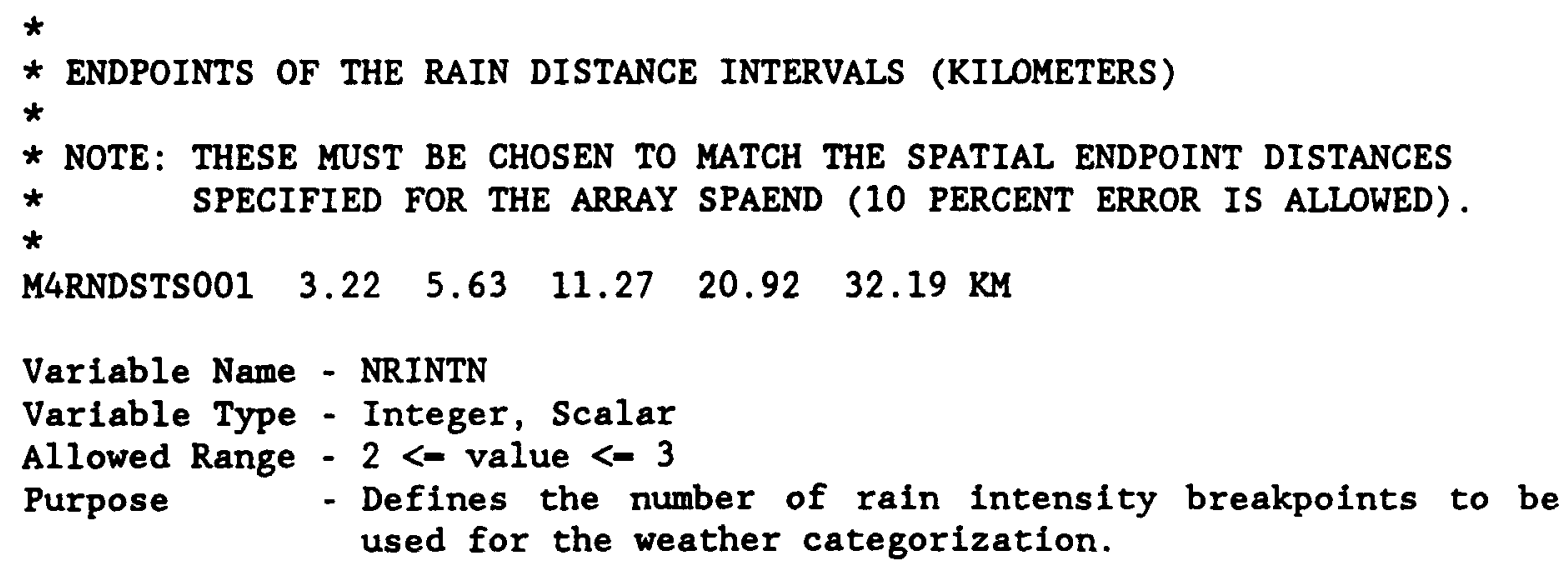


Example Usage:

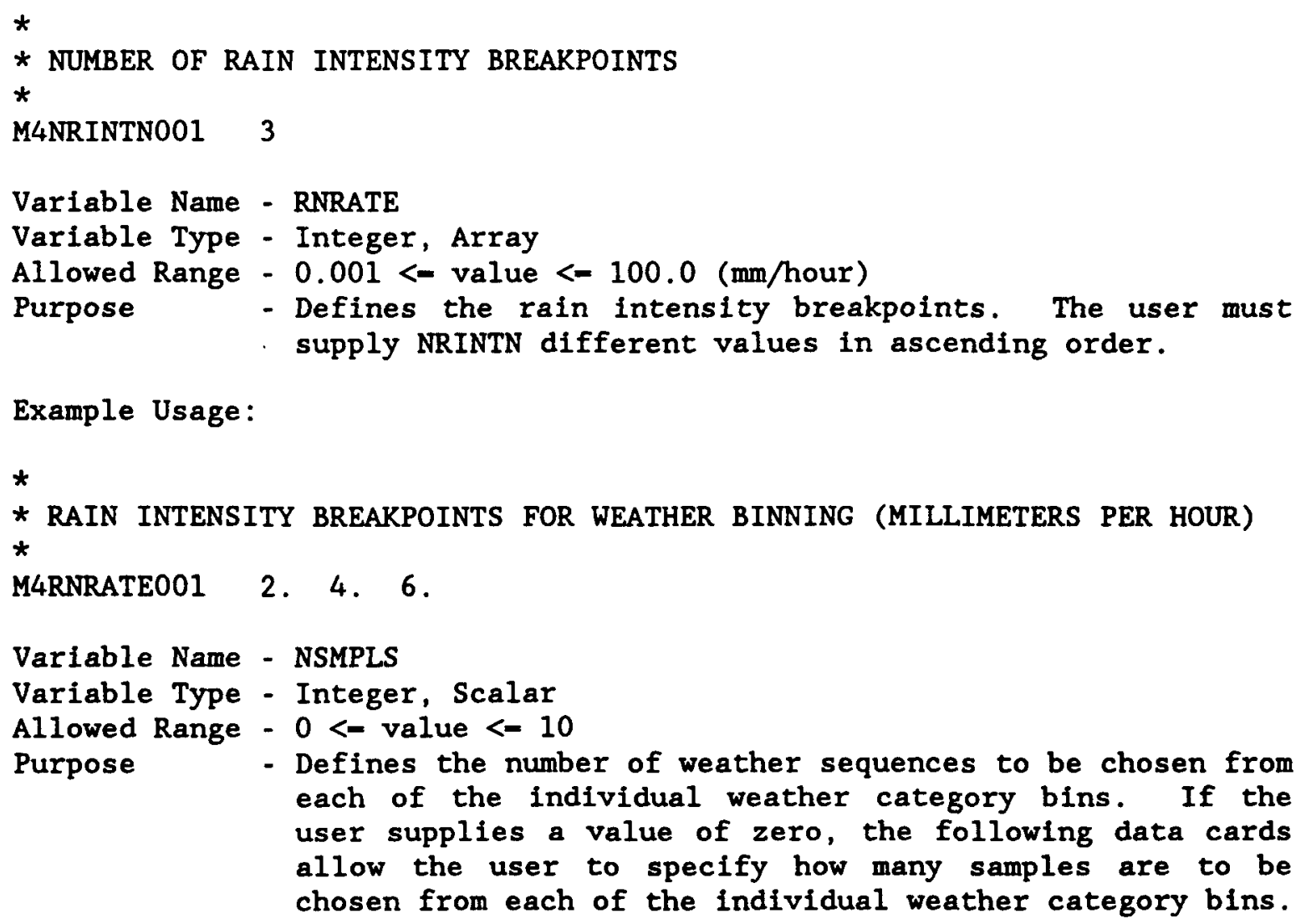

The random number generator of MACCS is included in the FORTRAN source code and therefore runs made on different types of computers should select identical sets of weather sequences. 


\section{Example Usage:}

*

* INITIAI SEED FOR RANDOM NUMBER GENERATOR

$\star$

M4IRSEED001 79

Note: the following cards in this section are only needed if NSMPLS $=0$.

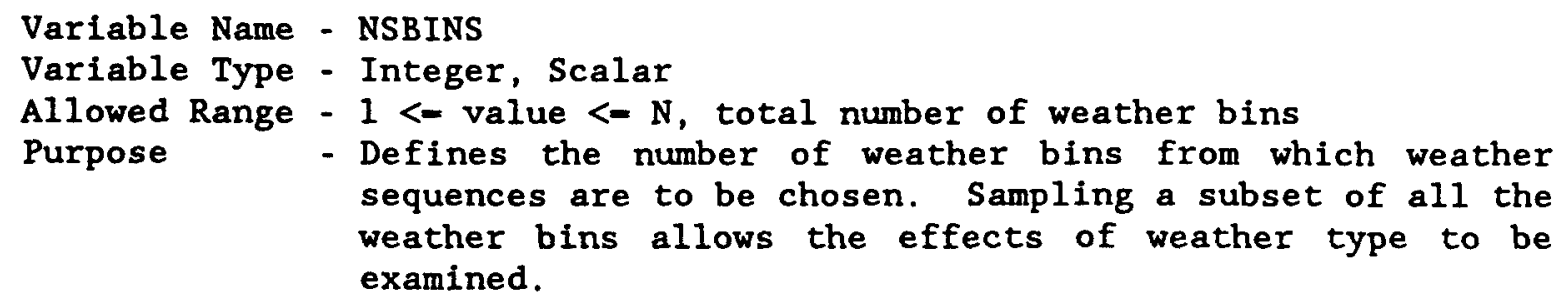
would like to be selected from the specified weather bin. If the requested number of sequences cannot be found, the code will select all of the sequences in the specified bin. The user must supply NSBINS values in column two of the data block.

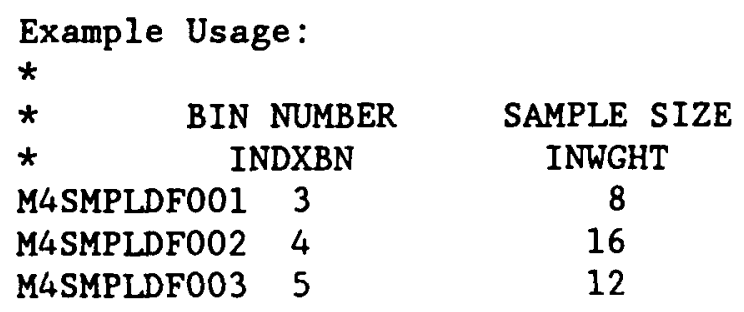


$\begin{array}{lll}\text { M4SMPLDF004 } & 6 & 4 \\ \text { M4SMPLDF005 } & 7 & 4 \\ \text { M4SMPLDF006 } & 8 & 4\end{array}$

\subsection{User Supplied Weather Sequence (M5) Data}

The data in this section must be supplied if the user chooses METCOD-3 . There must be one data card for each hour of weather in the sequence. The five arrays in this section are supplied in a block of data as columns.

Variable Name - HRMXHT

Variable Type - Real, Array

Allowed Range - 1.E2 <- length <- $1 . E 4$ (meters)

Purpose - These are the mixing layer heights that will be used for the single trial. They are given in units of meters. The user must supply 120 values of ISTAB in column one of the data block.

Note: The atmospheric dispersion model currently being used cannot accommodate a mixing layer height that varies with time during a weather sequence. The single value of mixing height which will be used in the atmospheric model is the largest value in the following set of values: the 120 values supplied here, and the boundary weather mixing layer height, BNDMXH.

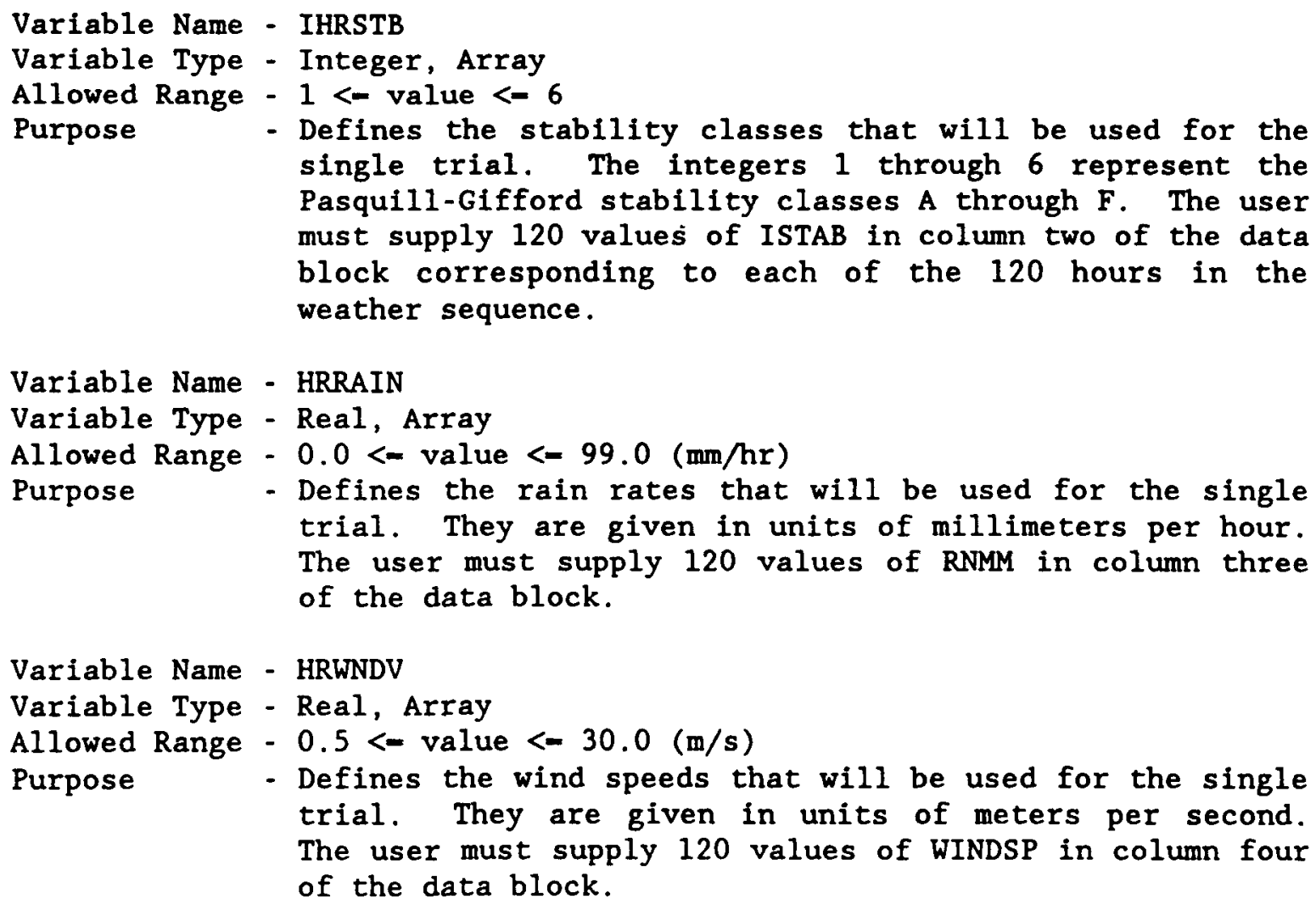




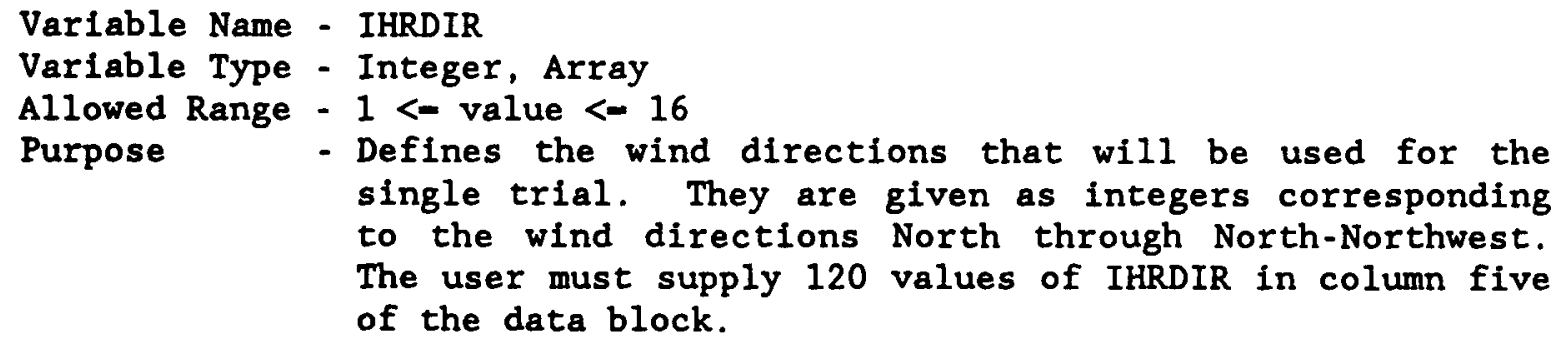

\section{References}

Br81 Brenk, H.D., and K.J. Vogt, "The Calculation of Wet Deposition from Radioactive Plumes," Nuclear Safety, Vol. 22, No. 3 (1981).

Jo89 Jow, H.N., et al., MELCOR ACcident Consequence Code System (MACCS) Volume 2: Model Description, NUREG/CR-4691, SAND86-1562, Sandia National Laboratories, Albuquerque, MM, 1990.

NR82 U.S. Nuclear Regulatory Commission, PRA Procedures Guide, NUREG/CR2300, Vol. 2, 1982, available for purchase from National Technical Information Service, Springfield, Virginia, 22161.

Ri84 Ritchie, L.T., CRAC2 Mode1 Description, NUREG/CR-2326, SAND81-1994, Sandia National Laboratories, Albuquerque, NM, 1983. 


\subsection{EARLY USER INPUT FILE}

\subsection{Introduction to EARLY}

The EARLY module models the time period immediately following a reactor accident. This period is commonly referred to as the emergency phase. It may extend up to one week after the arrival of the first plume at any downwind spatial interval. The subsequent intermediate and long-term periods are treated by CHRONC. The user may specify scenarios that make use of evacuation, sheltering, and dose-dependent relocation. Results can be calculated for combinations of scenarios weighted either by time fractions (frequencies of occurrence) or population fractions (fraction of the population engaging in the specified behavior).

The EARLY module has the capability for combining results from up to three different emergency response scenarios. This is accomplished by appending "change cards" to the EARLY User Input File. The first emergency response scenario is defined in the main body of the EARLY User Input File. Up to two additional emergency response scenarios can be defined through change card sets positioned at the end of the file.

The delimiter used to separate the change card sets is a period (.) in column one. The end of the file is also signified by a period in column one. All of the MACCS User Input Files must end with a period in column one. The sample EARLY User Input File listed in Appendix D.2 is an example illustrating the use of change cards for a PRA application of the code.

The purpose of the change card processing in EARLY is solely to allow modification of the previously specified emergency response scenario data. Any cards appearing in the change card sets must have been previously defined in either the Evacuation Zone Data (Section 2.6) or the Shelter and Relocation Data (Section 2.7). If data items from another data block appear in the change cards, they will be 1 gnored. Each set of change cards must include a new value of EANAM2, a text field describing the emergency response scenario. Also, each set of change cards must produce a change in at least one of the numeric input variables described in the Evacuation Zone Data or in the Shelter and Relocation Data.

EARLY calculates the doses and health effects that result from exposure during the emergency response period for up to three different emergency response scenarios and these are written in binary form to units 31 to 33. The results calculated by CHRONC are written to unit 34. All of these files have the same format.

For results which are calculated by both EARLY and CHRONC, such as population dose and cancer cases, the OUTPUT module automatically adds the value of the consequence calculated by CHRONC to the value of the same consequence measure produced by EARLY in order to generate the "overall combined" results. If more than one EARLY emergency response scenario is being run, these results are combined according to the weighting fractions supplied by the user on the EARLY User Input File, 
and the weighted sum is then combined with the CHRONC result to produce the overall result.

Whenever results are combined by the code, the listing produced by the OUTPUT module will present the overall combination of results as well as each of the individual components from which it is constructed.

It is up to the user to specify the various parameters needed for these calculations. There are no default values. In addition to specifying the characteristics of the model, the user has complete control over the output produced by EARLY and must explicitly specify which results are to be produced. All of this information is supplied through the User Input File for EARLY and all of the input parameters are described in this chapter.

The User Input File for EARLY consists of ten data blocks used to define the simulation and an additional eight data blocks used to specify the output desired by the user. These eighteen data blocks are described below.

\subsection{Miscellaneous (MI) Data}

The user must supply information to identify the run, define the histogram approximating the crosswind Gaussian distribution, and also supply information describing the handling of the wind-rose. It is possible to specify a single wind-rose that will override the wind-roses that were calculated in ATMOS for each of the weather category sampling bins. The user must also specify how to treat changes in wind direction. There are three options to choose from and these are described below.

Variable Name - EANAMI

Variable Type - Character, Scalar

Allowed Range - $1<-$ length <- 80

Purpose - Identifies a name describing the EARLY calculations. This is printed on all pages of the OUTPUT listing. A name describing the particular emergency response assumption will be requested in addition to this name.

Example Usage:

* * GENERAL DESCRIPTIVE INFORMATION FOR THE "EARLY" INPUT FILE * MIEANAM1001 'IN2A.INP, SURRY, SAMPLE PROBLEM A, EARLY INPUT'

Variable Name - ENDAT2

Variable Type - Logical, Scalar

Allowed Value - .TRUE. or .FALSE.

Purpose - Control flag which allows the user to execute only the ATMOS and EARLY modules while skipping execution of the CHRONC module. If CHRONC is to be skipped, there is no 
need for the user to specify a CHRONC User Input File. A value of .TRUE. causes MACCS to skip the CHRONC module. A value of .FALSE. causes the CHRONC module to be executed.

Example Usage:

*

* Flag to indicate that this is the last pRogram IN THE SERIES to be RUN *

MIENDAT2001 .FALSE. (SET THIS VALUE TO .TRUE. TO SKIP CHRONC)

Variable Name - IPLUME

Variable Type - Integer, Scalar

Allowed Range - 1 <- value <- 3

Purpose

- Dispersion model option code:

1 - Straight line dispersion model:

All plume segments travel in the same direction. Each set of modeling results is rotated around the sixteen compass directions (population sectors) to yield sixteen sets of results for each weather trial.

2 - Wind-shift plume dispersion model with rotation: Each plume segment in the release travels in the direction that the wind is blowing at the time that its representative time point (REFTIM in Section 1.11) leaves the reactor. Each set of modeling results is rotated around the sixteen compass directions (population sectors) to yield sixteen sets of results for each weather trial.

3 - Wind-shift dispersion model without rotation:

Each plume segment in the release travels in the direction that the wind is blowing at the time that its representative time point (REFTIM in section 1.11) leaves the reactor. No rotation of the wind shift pattern is performed. Each weather trial yields one set of results.

Example Usage:

*

* Dispersion model option CODE: 1- StRaight LINE,

* 2- WIND-SHIFT WITH ROTATION,

* 3- WIND-SHIFT WITHOUT ROTATION.

MIIPLUMEO01 2

Variable Name - NUMFIN

Variable Type - Integer, Scalar

Allowed Range - 3, 5, or 7

Purpose - Number of fine grid subdivisions used by the model. A step function is used to approximate the Gaussian 
distribution of the plume in the crosswind direction. Each of the 22.5 degree sectors is subdivided into NUMFIN fine grid elements, with doses and risks being uniform in each of the fine grid elements.

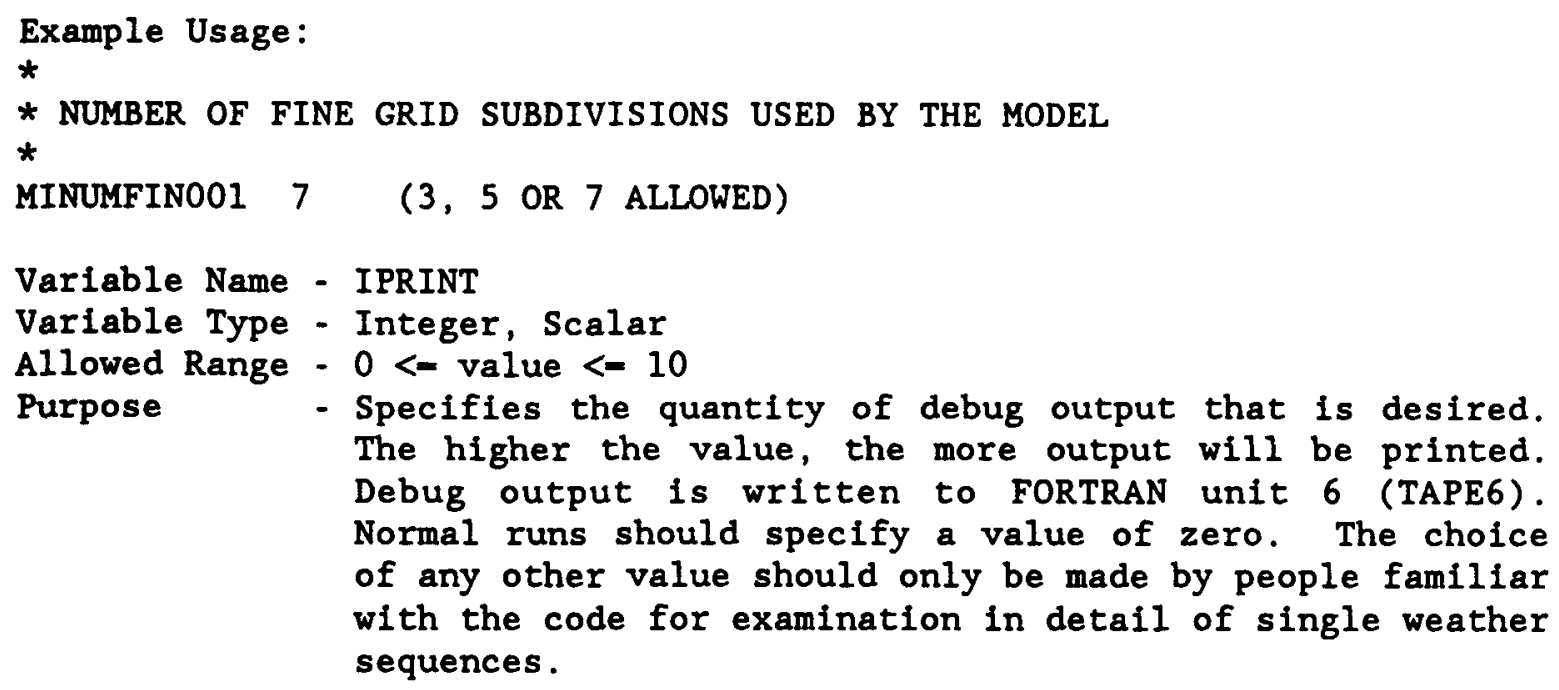

For various values of IPRINT, the code will print out intermediate results on the list output file. These are described below.

IPRINT 21 : skin dose conversion factors, centerline doses for all organs (if IPLUME-1), Gaussian histogram and cloudshine correction factors, return code values (RETCOD).

IPRINT $\geq 2$ : final groundshine dose rate for each organ, each plume segment.

IPRINT 24: total acute dose for organs. 2 and 3 , early fatality, early injury, and cancer risk values for each spatial element.

IPRINT $\geq 8$ : acute dose to organs 2 and 3 after completion of subroutine RELZON, acute dose to organs 2 and 3 after completion of subroutine ESTAT.

Example Usage:

*

* LEVEL OF DEBUG OUTPUT REQUIRED, NORMAL RUNS SHOULD SPECIFY ZERO

*

MIIPRINT001 0

Variable Name - RISCAT

Variable Type - Logical, Scalar

Allowed Value - .TRUE. or .FALSE.

Purpose - If the option of weather category bin sampling was chosen by the user in the ATMOS User Input File (METCOD=2), the display of results produced by the OUTPUT module can show 
the relative contribution of each of the weather category bins to the mean consequence value.

Example Usage:

*

* FLAG INDICATING IF RISK CONTRIBUTION IS TO BROKEN DOWN BY WEATHER

* CATEgORY bIN ON THE OUTPUT LISTING

*

MIRISCATO01 .FALSE.

Variable Name - OVRRID

Variable Type - Logical, Scalar

Allowed Value - .TRUE. or .FALSE.

Purpose - Specifies whether the wind-rose probabilities are to be supplied by the user. If the weather sampling option was chosen in ATMOS (METCOD-2), wind-roses for each weather sampling bin have been passed down from ATMOS. Those wind-roses will be used if OVRRID - .FALSE. If no windrose is available to EARLY, a uniform wind-rose will be used, that is, $\mathrm{P}=0.0625$ in each direction.

Example Usage:

$\star$

* FLAG INDICATING IF WIND-ROSES FROM ATMOS ARE TO BE OVERRIDDEN

*

MIOVRRIDOO1 .FALSE. (USE THE WIND ROSE CALCULATED FOR EACH WEATHER BIN)

Note to user: The following data is only required if (OVRRID-.TRUE.).

Variable Name - WINROS

Variable Type - Real, Array

Allowed Range - 0 . <- value <- 1 .

Purpose - These are the probabilities of the wind blowing from the site into each of the sixteen compass sectors (rotating clockwise from $N$ to $N N W$ ).

The sum of these values must be between 0.95 and 1.05 . The user must supply 16 values in rows on one or more data cards.

Example Usage:

*

* SITING STUDY WINDROSE FOR THE PEACH BOTTOM SITE

*

MIWINROS001 8.521E-02 6.360E-02 4.605E-02 5.189E-02 6.869E-02 9.493E-02

MIWINROS002 1.145E-01 1.090E-01 6.019E-02 4.326E-02 3.148E-02 3.238E-02

MIWINROS003 3.383E-02 4.625E-02 5.446E-02 6.424E-02

\subsection{Population Distribution (PD) Data}

The user must supply information to define the polar coordinate population distribution surrounding the site. This information can be supplied 
from the Site Data File or a uniform population distribution can be specified by the user. The format of the Site Data File is described in Appendix B.3.

Note: Whatever values are supplied here will be used by both the EARLY and the CHRONC modules in defining the characteristics of the region surrounding the site. There is no possibility of having EARLY and CHRONC use different population distributions.

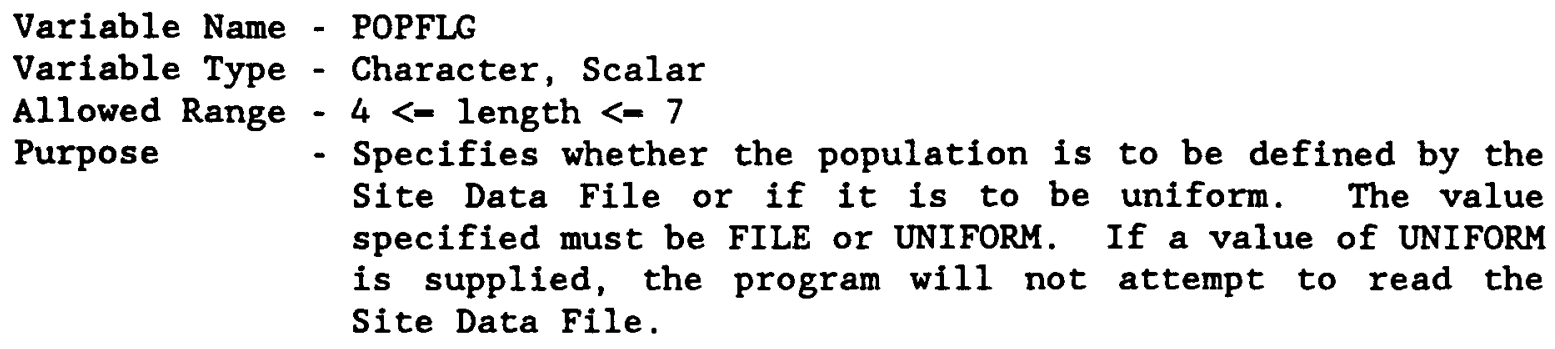

Example Usage:

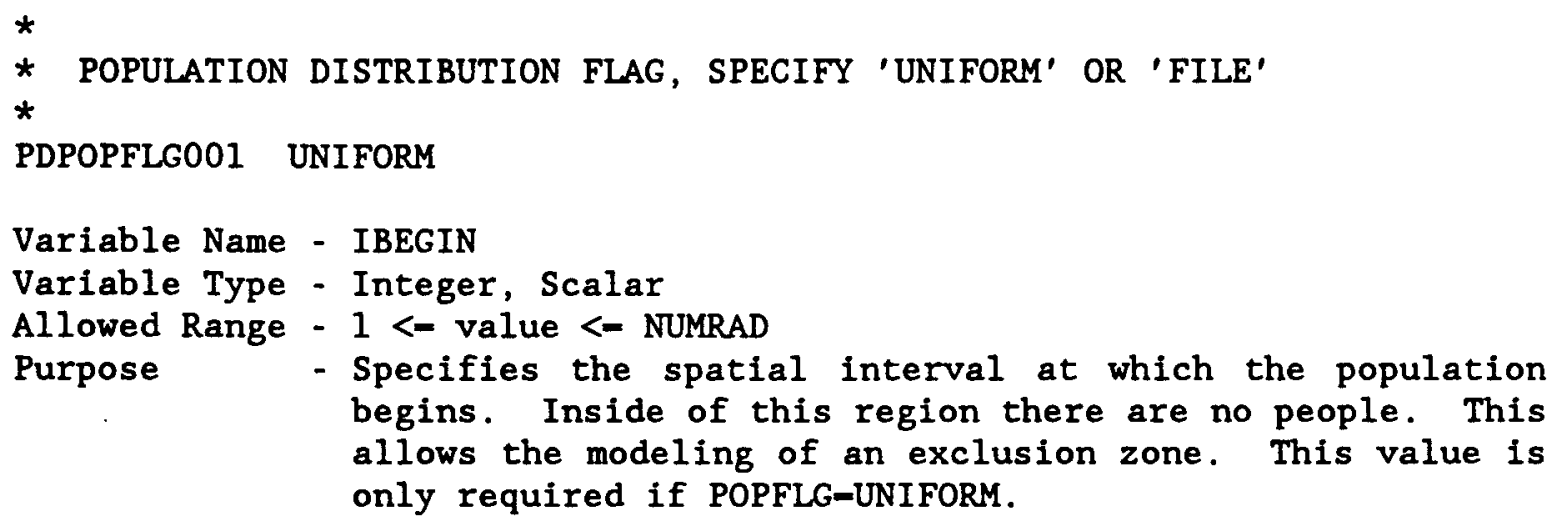
begins. Inside of this region there are no people. This allows the modeling of an exclusion zone. This value is only required if POPFLG-UNIFORM.

Example Usage:

*

* First spatial interval is an eXClusion zone

*

PDIBEGINO01 2

Variable Name - POPDEN

Variable Type - Real, Scalar

Allowed Range - $0.0<-$ value <- 1.E6 (people / square-kilometer)

Purpose - Specifies the uniform population density of the region. This value is only required if POPFLG-UNIFORM.

Example Usage:

* ASSUME 100 PEOPLE PER SQ. KILOMETER

PDPOPDENO01 100 . 


\subsection{Organ Definition (OD) Data}

The MACCS code will only calculate doses for the organs defined in this section. The shelter and relocation model (variable CRIORG), and the health effects models (prompt fatalities, early injuries, and latent cancers) must only reference organs which are on the list of organs. The same is true of the dose related output options (results of type 3,5 , and 6). MACCS will check to ensure that appropriate dosimetry data are available on the Dose Conversion Factors Data File for all of these organs.

MACCS can calculate two kinds of doses: lifetime dose and effective acute dose.

Lifetime dose is utilized for determining the need for mitigative actions and for calculating the cancer induction and population dose results. It represents the 0 to 50 year dose commitment to an ICRP reference man [IC79].

Effective acute doses are used for calculating the acute health effects in the EARLY module. The acute health effects are "early fatalities" and "early injuries." A full description of the meaning of effective acute dose can be found in Section 2.8, Early Fatality Data.

The list of organs for which dosimetry data are available is presented below.

\begin{tabular}{|c|c|c|c|}
\hline \multicolumn{4}{|c|}{ EFFECTIVE } \\
\hline & ACUTE & LIFETIME & \\
\hline 'SKIN' & YES & NO & \\
\hline 'LUNGS' & YES & YES & \\
\hline 'RED MARR' & YES & YES & (red bone marrow) \\
\hline ' SMALL IN' & YES & YES & (small intestine) \\
\hline ' LOWER LI' & YES & YES & (lower large intestine) \\
\hline ' STOMACH' & YES & YES & \\
\hline ' THYROID' & NO & YES & \\
\hline ' THYROIDH' & YES & YES & $\begin{array}{l}\text { (pseudo-thyroid for calculating health } \\
\text { effects) }\end{array}$ \\
\hline ' EDEWBODY' & No & YES & (effective whole body dose equivalent) \\
\hline 'BONE SUR' & No & YES & (bone surface) \\
\hline 'BREAST' & No & YES & \\
\hline 'OVARIES' & No & YES & \\
\hline 'TESTES' & No & YES & \\
\hline
\end{tabular}

If the Dose Conversion Factors Data File supplied with MACCS is to be used for the calculations, the organ names must be spelled exactly as above. If some other dose factor file is used, its organ names must be identical to those input to EARLY in the ORGNAM list defined below.

Apostrophes are mandatory if the organ name has any embedded blanks. Any reference to an organ that cannot be found on the organ list (ORGNAM) will cause the error flag to be set and execution will be inhibited. 


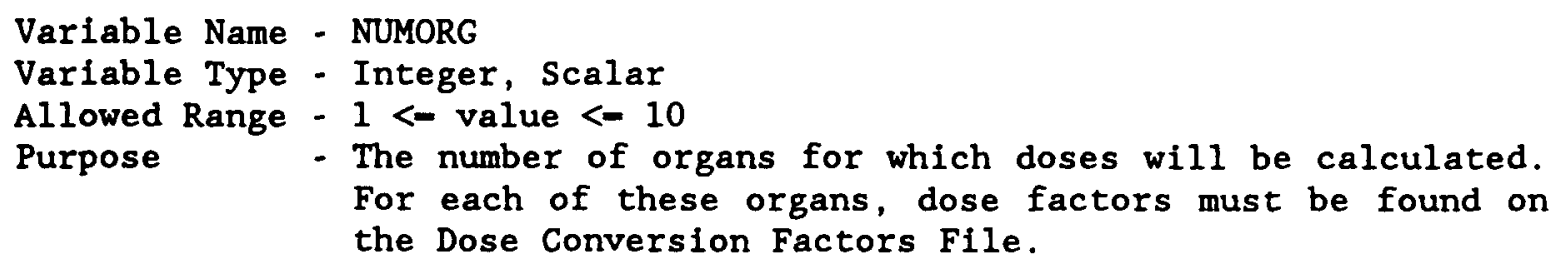

Example Usage:

*

* NUMBER OF ORGANS DEFINED FOR HEALTH EFFECTS

*

ODNUMORGO01 9

Variable Name - ORGNAM

Variable Type - Character, Array

Allowed Range - $2<-$ length $<-10$

Purpose - The names of the NORGAN organs defined in the model. For each of these organs, dose conversion factors must must be found on the Dose Conversion Factors File. The skin organ receives special handling. If skin doses are to be calculated, SKIN must be the first organ name on the 1ist. If skin doses are not required, an organ called NULL must occupy the first position. Do not use NULL anywhere but in the first position on the list.

Note: The CHRONC module normally only calculates doses to the cancer target organs defined in Section 2.10. If the user would like to calculate the long-term dose to an organ which is not defined to be a cancer target organ, such as the effective whole body dose equivalent (EDEWBODY), that organ name must appear as the second organ on the list of organs being defined here.

If the user specifies the long-term critical organ, CRTOCR, defined in Section 3.4, to be an organ which is not a cancer target organ, a fatal error will result unless that organ appears as the number two organ on the list of organs below.

Example Usage:

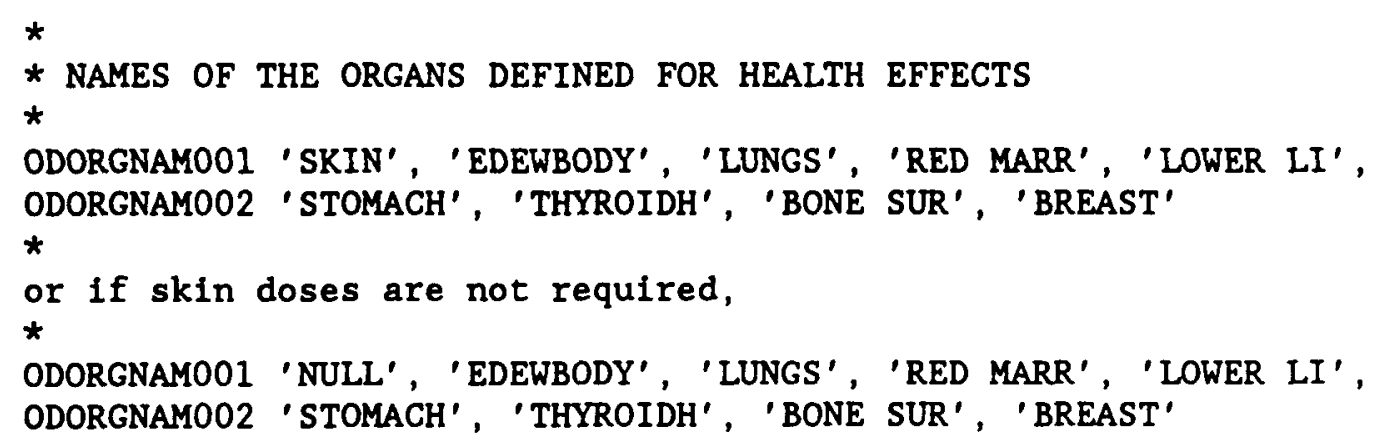




\subsection{Shielding and Exposure (SE) Data}

This section defines the shielding factors for exposure to cloudshine, groundshine, inhalation and deposition to skin for three types of activities (normal activity, evacuation, and sheltering). A breathing rate is also specified for each type of activity. In addition, the resuspension parameters to be used for the emergency phase time period (EARLY), the resuspension coefficient and resuspension half-life are also defined.

Some of the parameter values defined in this section are used in both the EARLY and the CHRONC modules; these are: (1) the normal activity groundshine and (2) inhalation protection factors as well as the (3) normal activity breathing rate. The CHRONC module does not calculate the effects resulting from direct exposure to the radioactive cloud, therefore it makes no use of the (1) cloudshine and (2) deposition to skin shielding factors. The long-term resuspension parameters are defined on the basis of data supplied in the CHRONC User Input File and the emergency phase resuspension parameters defined here are not used in the CHRONC module.

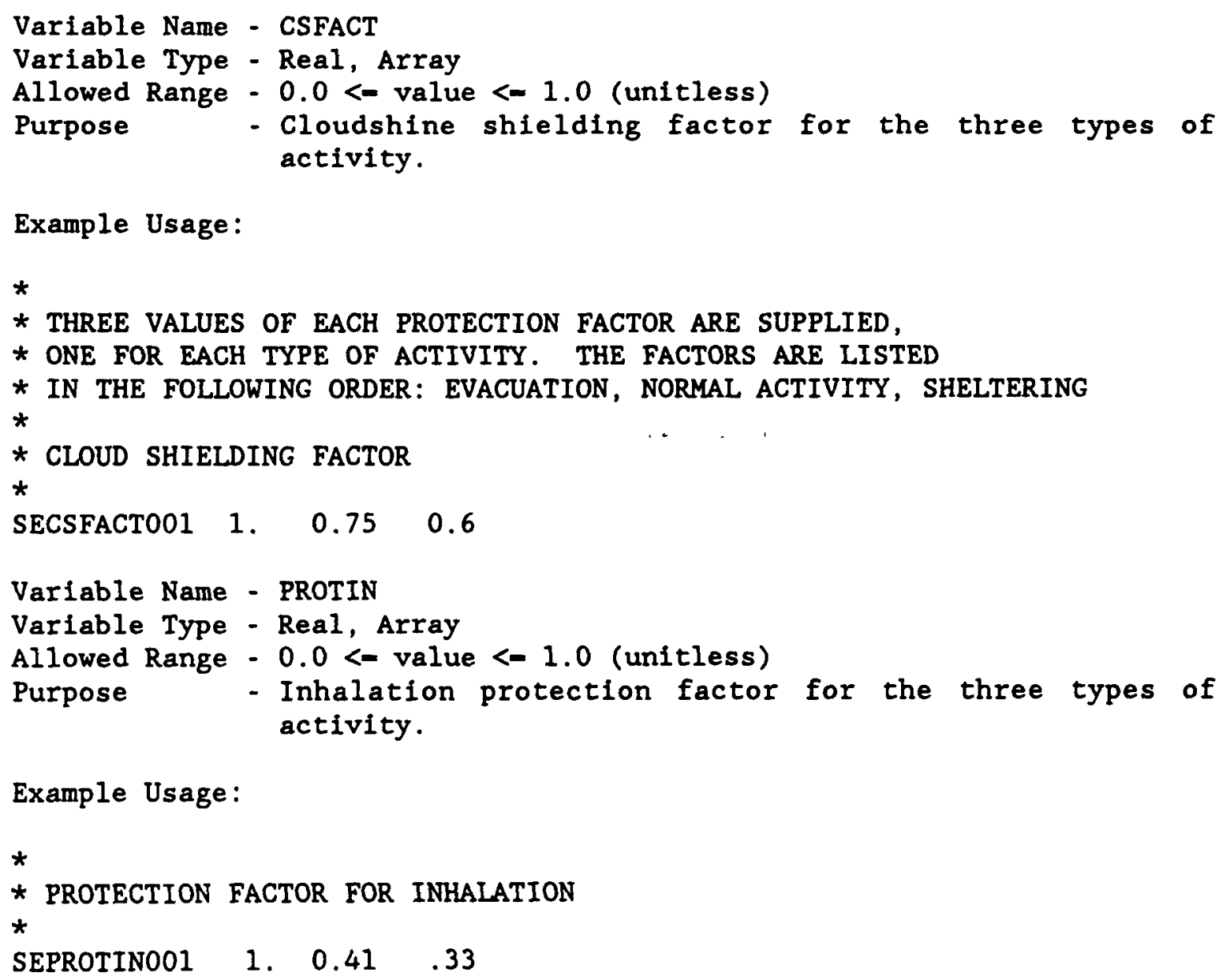




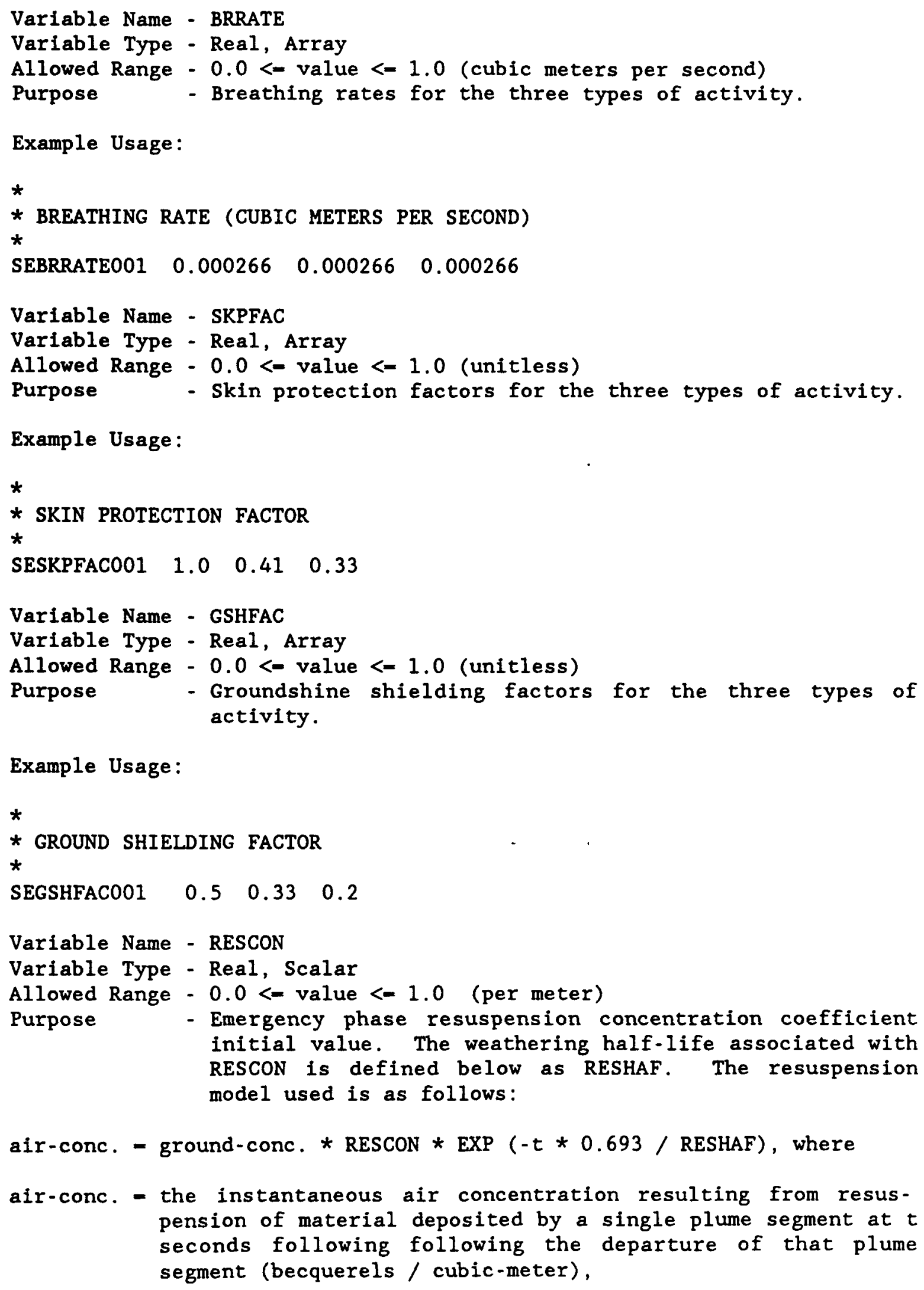
initial value. The weathering half-life associated with RESCON is defined below as RESHAF. The resuspension model used is as follows: 


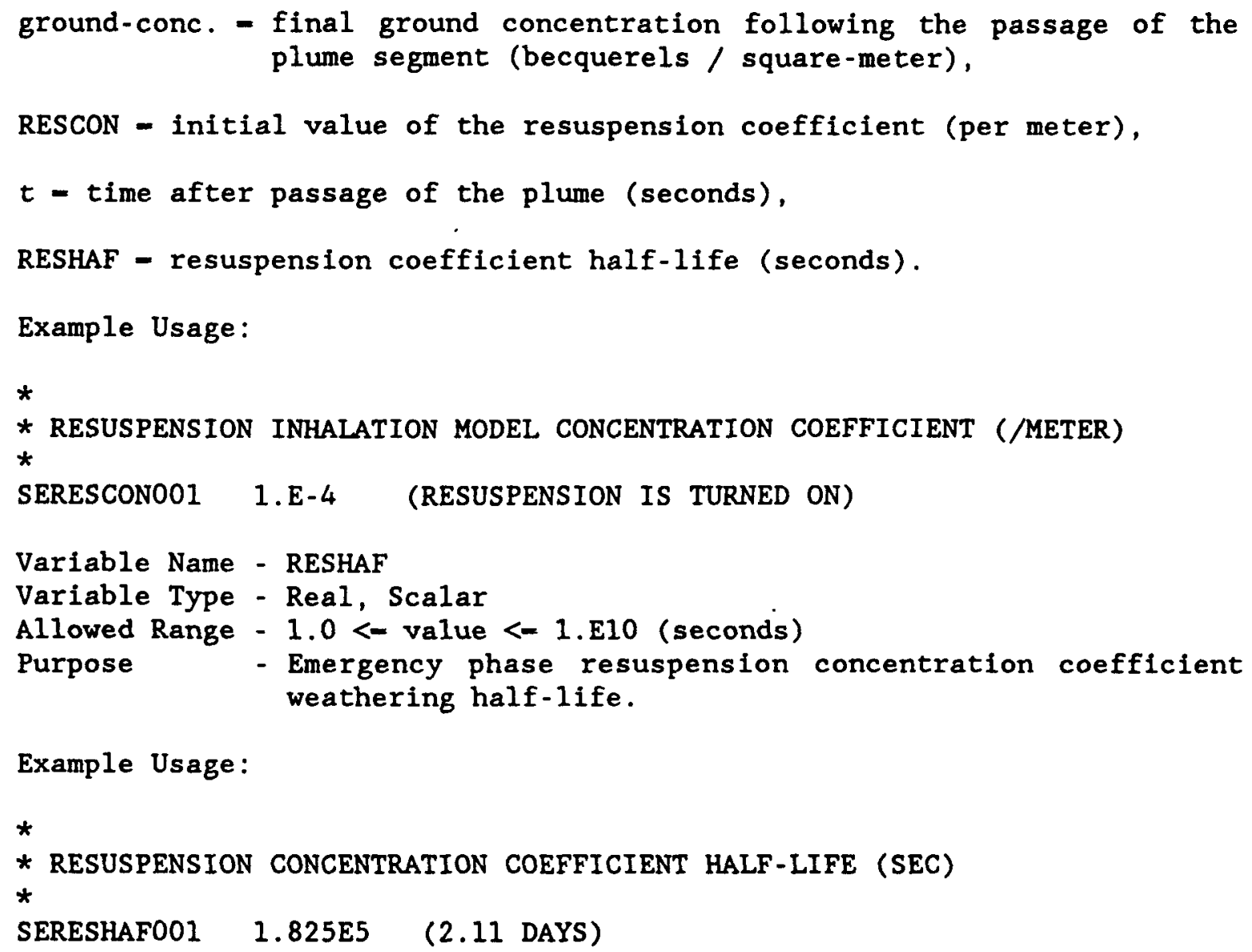

\subsection{Evacuation Zone (EZ) Data}

In the current implementation, MACCS is limited to an evacuation model where the only movement allowed is radially outward.

All individuals between the first and last evacuating intervals (INIEVA and [ASEVA(3)] are assumed to evacuate. Evacuating individuals travel radially outward at a constant speed and are subject to radiation exposure until they leave the LASMOV spatial interval whereupon they are assumed to avoid all further exposure.

Within the evacuation zone, up to three regions with different evacuation delay times may be defined. The three times associated with these regions are specified in EDELAY. Outer boundaries of the three evacuation rings are specified in LASEVA. Some of these values may be zero, indicating that the region is null.

Variable Name - EANAM2

Variable Type - Character, Scalar

Allowed Range - $1 \ll$ length $<=80$

Purpose - Identifies the name of the emergency response scenario being studied. This name will be printed on all pages of of the OUTPUT listing. A unique name must be specified for each emergency response. 


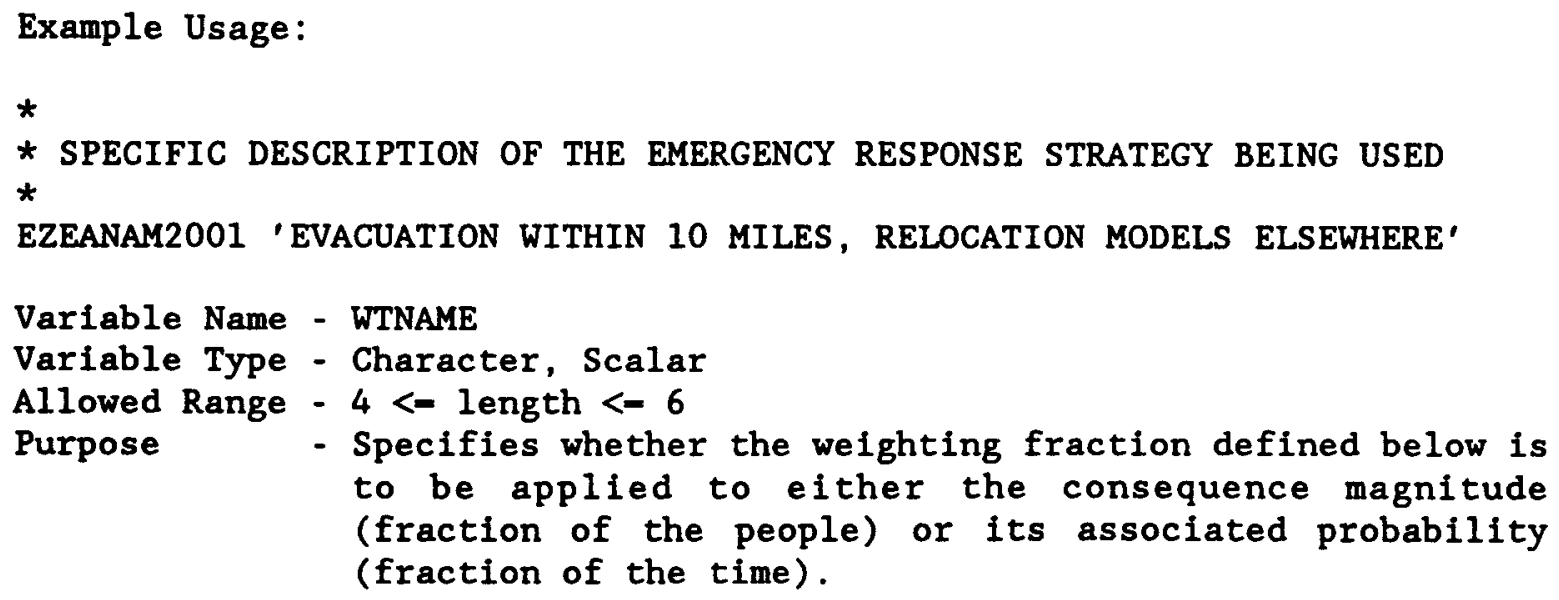

If the user specifies a value of zero, there will be no evacuation and no further data in this section are required. 


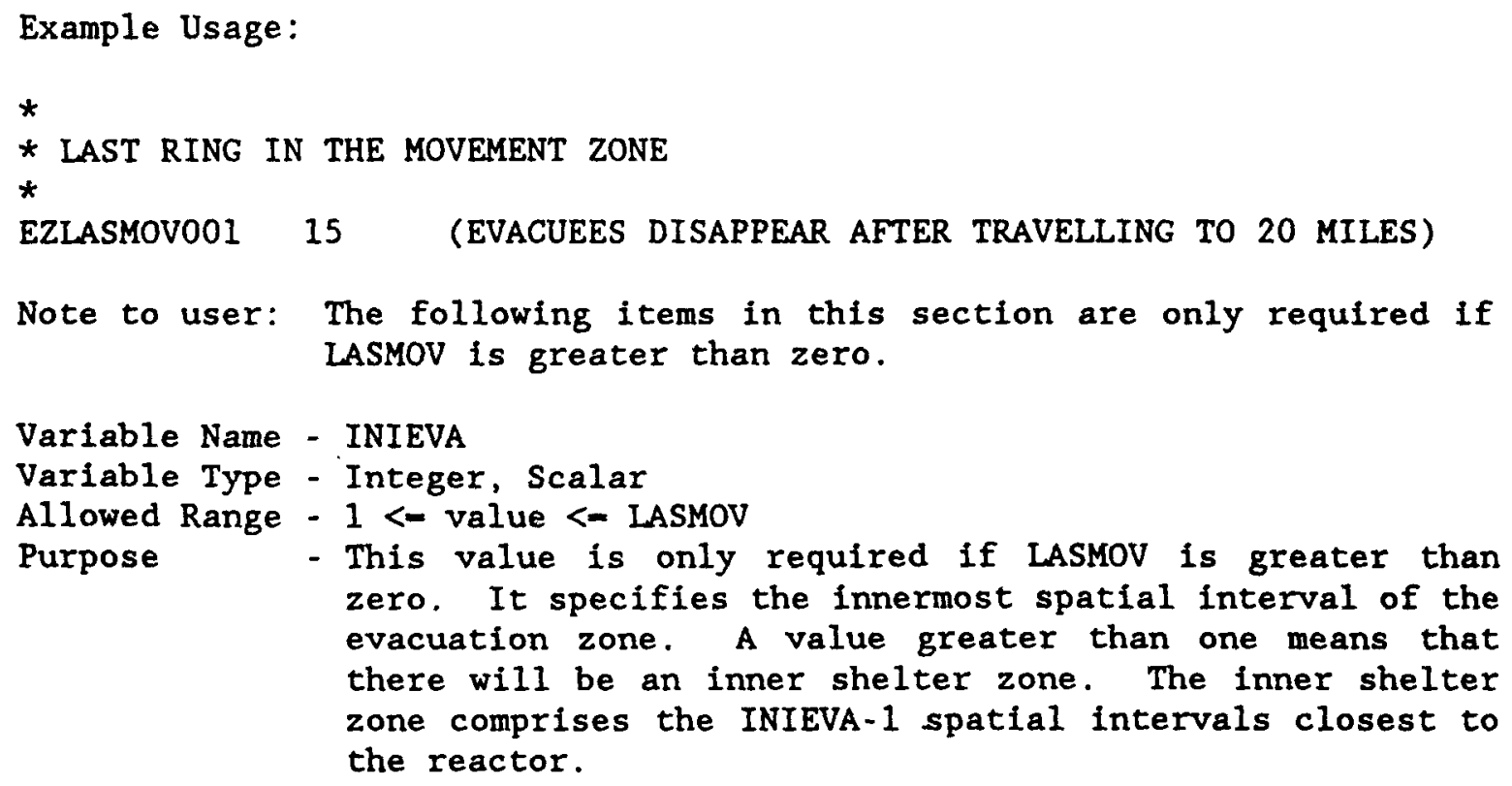

Example Usage:

$\star$

* First spatial interval in the evacuation zone

*

EZINIEVAOO1 1 (NO INNER SHELTER ZONE)

Variable Name - LASEVA

Variable Type - Integer, Array

Allowed Range - $0<-$ value <- LASMOV

Purpose - The outer spatial intervals marking off the three evacuation delay zones from nearest to farthest. These allow a phased evacuation. The user must supply three values. A value of zero for any of the rings rings causes that zone to be null. The non-zero values must not be less than INIEVA and they must be in ascending order.

Example Usage:

*

* OUTER bounds of THE 3 EVACUATION zONES (O MEANS the zONE IS NOT

* DEFINED)

*

EZLASEVA001 00012 (EVACUATION FROM A SINGLE ZONE OUT TO 10 MILES)

Variable Name - EDELAY

Variable Type - Real, Array

Allowed Range - 0 . <- value $<-86400.0$ (seconds) (one day)

Purpose - Defines the time necessary for evacuees to get moving. Before evacuating, evacuees can receive doses from the plume segments while continuing normal activity. The 
user must supply evacuation delay times for each of the three evacuation rings even if some of them are null. Evacuees remain in place until EDELAY seconds have elapsed from the off-site alarm time, OALARM, (see Section 1.11) and then they begin their evacuation.

Note: The reference point for evacuation (alarm time) is different from the reference time for relocation actions (time after first plume arrival).

Example Usage:

*

* evacuation delay times for the 3 eVAC zones

*

EZEDELAY001 0. 0. 7200. (SURRY)

Variable Name - ESPEED

Variable Type - Real, Scalar

Allowed Range - $0.1<-$ value <- 1.E6 (meters / second)

Purpose - The speed at which all evacuees travel radially outward.

Example Usage:

*

* Radial evacuation SPEed (METERs / SECOND)

*

EZESPEED001 $1.8 \quad$ (SURRY)

\subsection{Shelter and Relocation (SR) Data}

The user can specify the existence of two shelter zones; one inside of the evacuation zone, the other outside of it. Both of these zones are optional. The inner shelter zone is defined if a value greater than one was specified for INIEVA. The outer shelter zone is defined if LASHE2 is assigned a non-zero value. Even though these zones are optional, the code requires that all of the parameters described in this section be supplied.

Variable Name - TTOSH1

Variable Type - Real, Scalar

Allowed Range - $0.0<$ value $<-86400.0$ (seconds) (one day)

Purpose - Defines the time to take shelter in the inner shelter zone. People here take shelter TTOSH1 seconds after the offsite alarm time, OALARM, (see Section 1.11). Normal activity shielding factors are used up to the time at which individuals take shelter. Upon taking shelter, the shelter shielding factors are used.

Note: The reference point for sheltering (alarm) is different from reference time for relocation actions (first plume arrival). 


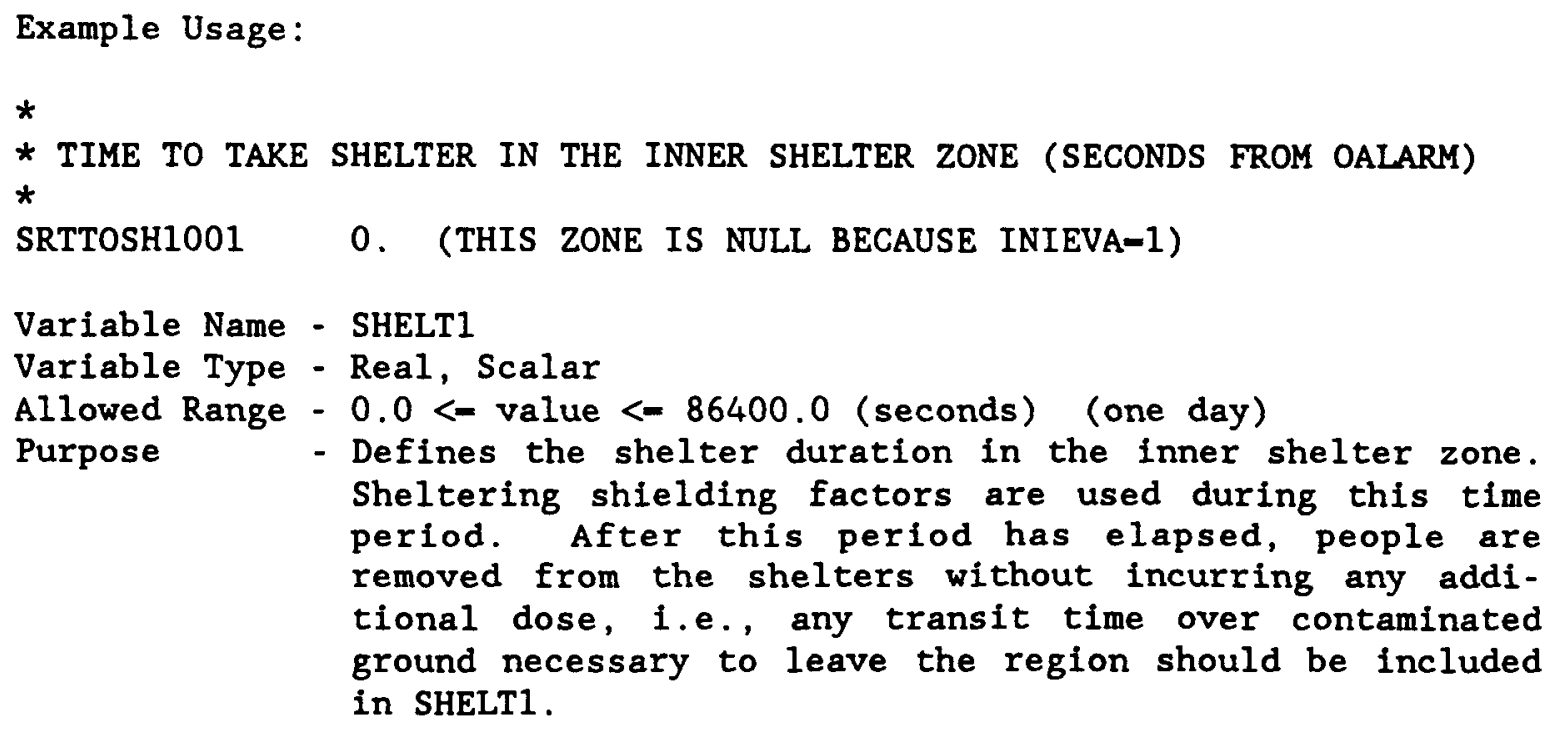
Sheltering shielding factors are used during this time period. After this period has elapsed, people are removed from the shelters without incurring any additional dose, i.e., any transit time over contaminated ground necessary to leave the region should be included in SHELT1.

Example Usage:

*

* SHELTER TIME IN THE INNER SHELTER ZONE (SECONDS)

*

SRSHELT1001 0. (THIS ZONE IS NULL BECAUSE INIEVA-1)

Variable Name - LASHE2

Variable Type - Integer, Scalar

Allowed Range - 0 <- value <- NUMRAD

Purpose - Defines the outermost ring of the outer shelter zone. The relocation zone begins at the next interval. If there is an evacuation (LASMOV $>0$ ), and a non-zero value is specified for LASHE2, then IASHE2 must be greater than the outermost evacuating interval.

Example Usage:

*

* LAST RING OF THE OUTER SHELTER ZONE

*

SRLASHE2001 0 (THIS ZONE IS NULL)

Variable Name - TTOSH2

Variable Type - Real, Scalar

Allowed Range - $0.0<-$ value <- 86400.0 (seconds) (one day)

Purpose - Defines the time to take shelter in the outer shelter zone. People here take shelter TTOSH2 seconds after the offsite alarm time, OALARM, (see Section 1.11). Normal activity shielding factors are used up to the time at which individuals take shelter. Upon taking shelter, the shelter shielding factors are used. 


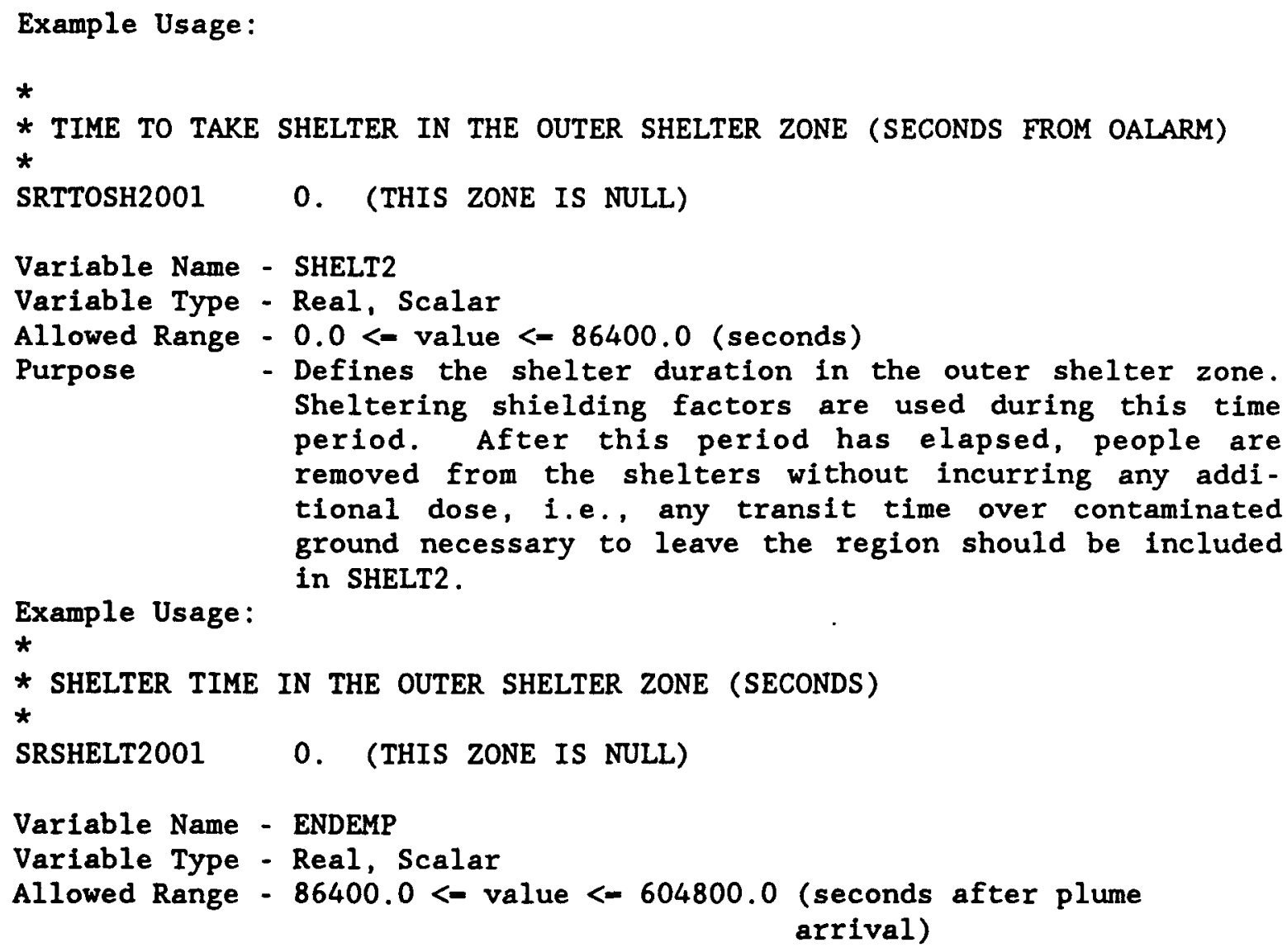

Purpose - Defines the duration of the emergency phase period. EARLY only calculates doses that would be received during the emergency phase time period. Doses at each spatial interval are cut off at ENDEMP seconds after the arrival of the first plume segment to reach the interval. This cutoff applies to all individuals, no matter where they are located. Any subsequent doses will be calculated by CHRONC.

ENDEMP is also the duration for which evacuees and shelterees are kept away from their homes if there was any contamination in the coarse grid element in which they reside.

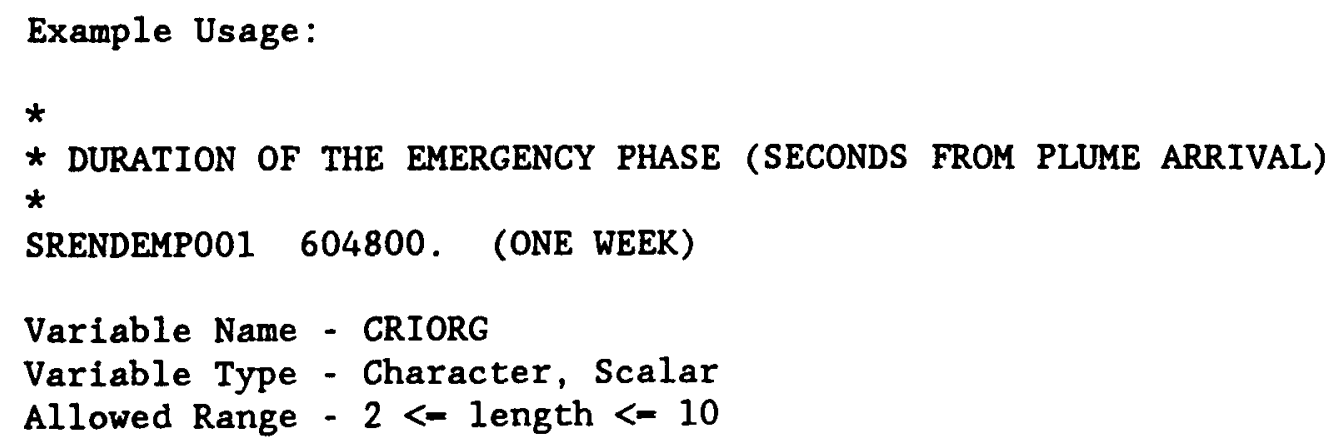


Purpose

- Defines the critical organ for relocation decisions during the emergency phase period considered by EARLY. In order to determine whether people can remain in the relocation zone, the total committed dose to the critical organ of an individual who remained in place for the entire emergency phase is calculated. The critical organ must be found on the list of organs, ORGNAM, defined in Section 2.4 .

Example Usage:

*

* CRItical organ for RELOCATion

*

SRCRIORGO01 'EDEWBODY'

Variable Name - TIMHOT

Variable Type - Real, Scalar

Allowed Range - 0.0 <alue <- ENDEMP (seconds after plume arrival)

Purpose - Defines the hot spot relocation action time. Hot spot relocation can only occur for individuals residing outside of the emergency response zone. That is, doses to people awaiting evacuation or protected in shelters will not be affected by the hot spot relocation model.

If the total dose commitment to any individual in a coarse grid element in the relocation region would exceed DOSHOT sieverts to the critical organ, CRIORG, for someone remaining there for the entire emergency phase (ENDEMP), people in that element are relocated at TIMHOT seconds after the arrival of the first plume at that distance.

For the purpose of evaluating the need for hot spot and normal relocation, the total dose commitment is the dose which would be received by an individual who remained in place for the entire emergency phase period while engaging in "normal" activity. The pathways used for calculating the total dose commitment are: cloudshine, groundshine, direct inhalation; and resuspension inhalation.

Any individuals relocated due to hot spots are removed from the problem for the duration of the emergency phase and receive no additional dose during the emergency phase.

Example Usage:

*

* HOT SPOT RELOCATION TIME (SECONDS FROM PLUME ARRIVAL)

$\star$ 


\begin{abstract}
Variable Name - TIMNRM
Variable Type - Real, Scalar

Allowed Range - TIMHOT <- value <- ENDEMP (seconds after plume arrival)

Purpose - Defines the normal relocation action time. Normal relocation can only occur for individuals residing outside of the emergency response zone. That is, doses to people awaiting evacuation or protected in shelters will not be affected by the normal relocation model.

If the total dose commitment to any individual in a coarse grid element in the relocation region would exceed DOSNRM sieverts to the critical organ, CRIORG, for someone remaining there for the entire emergency phase (ENDEMP), people in that element are relocated at TIMNRM seconds after the arrival of the first plume at that distance. Any individuals subject to normal relocation are removed from contaminated areas for the duration of the emergency phase and receive no additional dose during the emergency phase.

For the purpose of evaluating the need for hot spot and normal relocation, the total dose commitment is the dose which would be received by an individual who remained in place for the entire emergency phase period while engaging in "normal" activity. The pathways used for calculating the total dose commitment are: cloudshine, groundshine, direct inhalation, and resuspension inhalation.
\end{abstract}

Example Usage:

*

* NORMAL RELOCATION TIME (SECONDS FROM PLUME ARRIVAL)

*

SRTIMNRMO01 86400. (ONE DAY)

\author{
Variable Name - DOSHOT \\ Variable Type - Real, Scalar \\ Allowed Range - $0.0<-$ value <- 100.0 (sieverts) \\ Purpose - Defines the hot spot relocation dose threshold. If the \\ total dose commitment to individuals outside of the \\ evacuation and sheltering zones who remained stationary \\ for the entire emergency phase period would exceed \\ DOSHOT, those people would be relocated (removed) at the \\ hot-spot relocation time (TIMHOT).
}




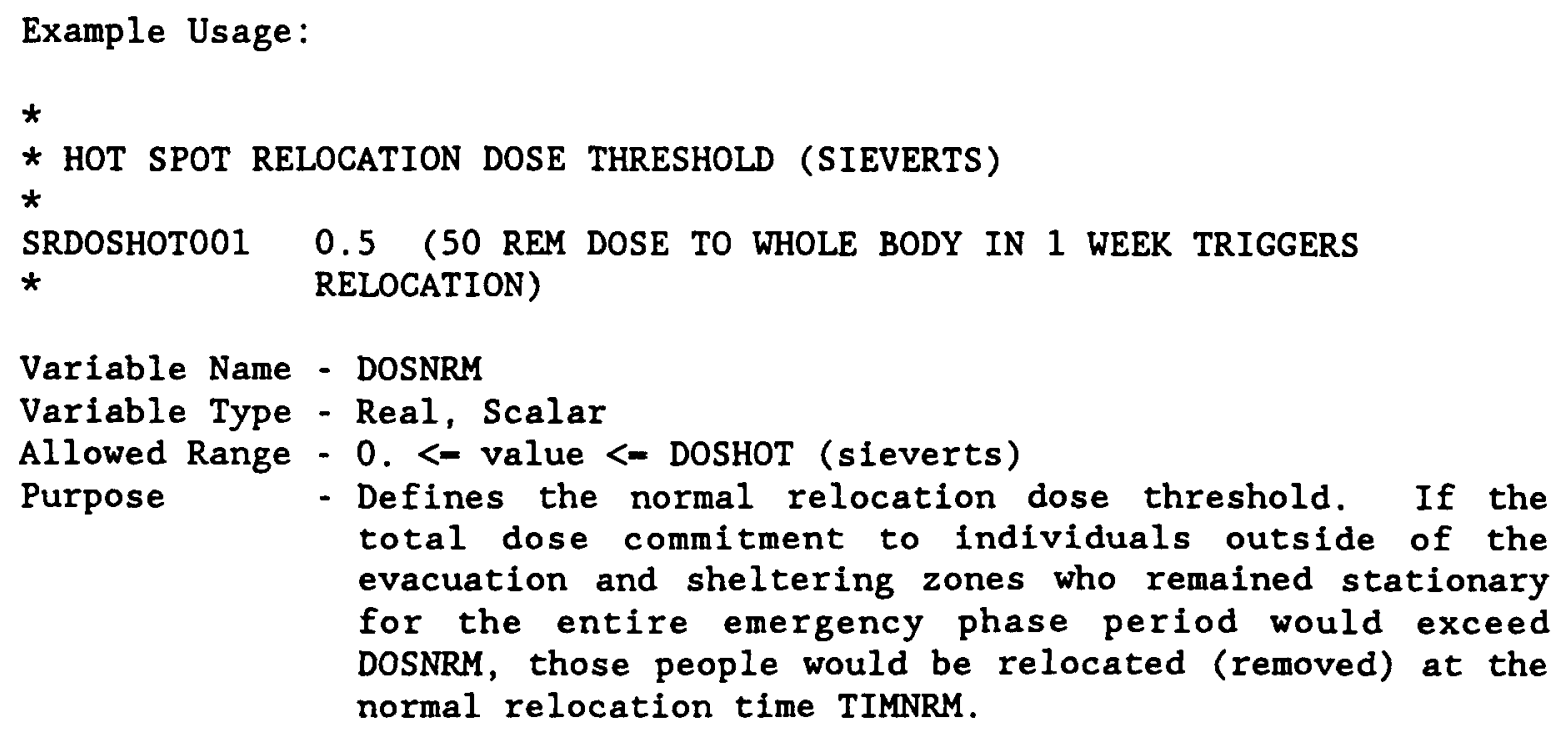

\subsection{Early Fatality (EF) Data}

The individual risk of prompt fatality is modeled in MACCS using a two parameter Weibull function here called a hazard function [Ev85]. The hazard function is used to sum the cumulative risk from a number of potential types of damage as follows:

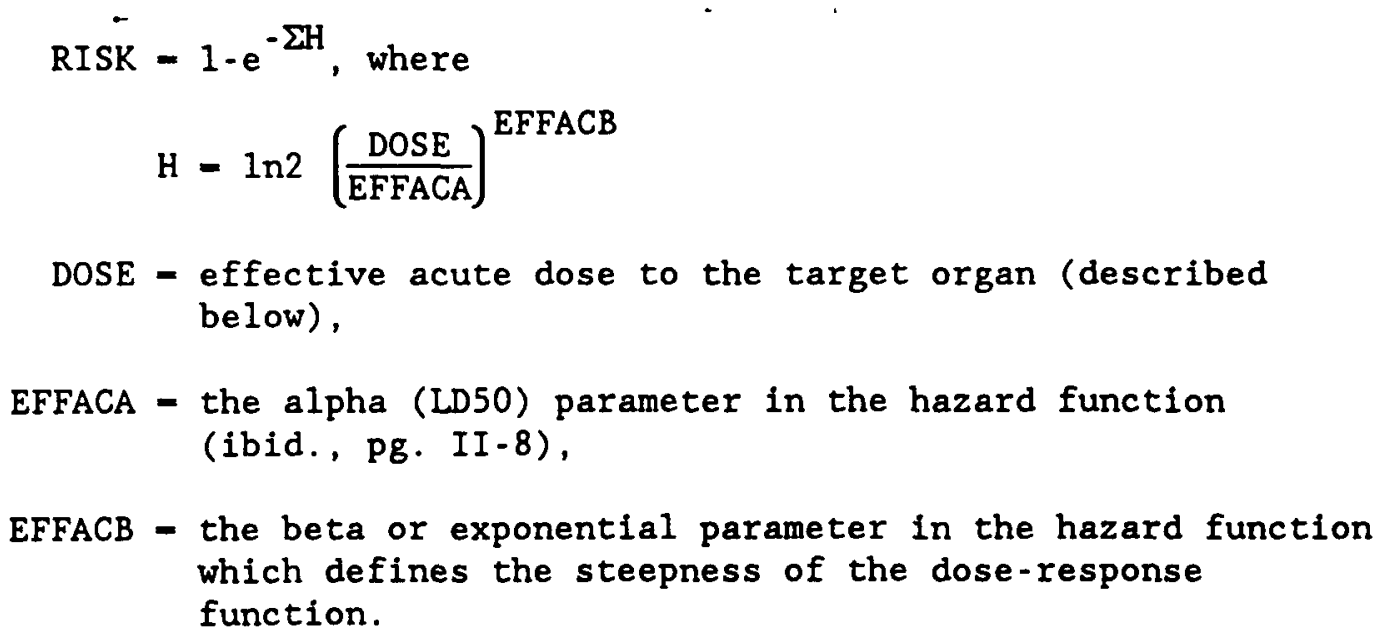

A dose threshold is incorporated into the early fatality model in the following manner. If the dose to any of the target organs is below the user specified threshold (see EFFTHR below), then the hazard (H) from that organ is set to zero. 
When radioactive material is inhaled and retained in the respiratory system, an individual may continue to receive a radiation dose for long periods of time after the material was inhaled. Depending on particle size and chemical form, clearance mechanisms may remove the material from the body or transport it away from the respiratory system to other organs of the body. For this reason, it does not make sense to refer to radiation dose without specifying the time period of interest. The concept of lifetime dose commitment is widely used [IC79]. As applied in MACCS, it refers to the dose received over the fifty year period following inhalation by a standard reference man who is 30 years old. This lifetime dose is used to determine the need for mitigative actions and for calculating cancers and population dose.

Implementation of the Harvard Report [Ev85] early health effects models requires a calculational method which takes account of dose protraction. MACCS treats protraction by using for all early health effects a series of $L D_{50}$ or $D_{50}$ values that apply to a sequential set of time periods of fixed length.

For the calculation of early fatalities and injuries in MACCS, a new measure of dose was defined in order to reduce the computational demands of the calculations. Throughout this document it will be referred to as "effective acute" dose.

The effective acute dose, $D_{0}$, is that dose which if delivered entirely in one day would induce the same health effects as the actual dose that was delivered over many days. Thus,

$$
H=\ln 2\left(\frac{D_{e}}{\alpha_{1}}\right)^{\beta}=\ln 2\left(\begin{array}{ll}
\Sigma & D_{t} \\
t & \alpha_{t}
\end{array}\right)^{\beta}
$$

Where $D_{0}=C F_{0}$ and $D_{t}=C F_{t}$ in which $C$ is the amount of material inhaled from the plume, $F_{\text {. }}$ is the effective acute dose conversion factor, and $F_{t}$ is the dose conversion factor for the actual dose $D_{t}$ delivered in time period $t$. Substitution now yields

$$
F_{e}=\sum_{t}^{\frac{\alpha_{1}}{\alpha_{t}}} F_{t}
$$

Effective acute dose conversion factors are only supplied for the organs used for calculating the early health effects. The other organs on the dose conversion file have been given effective acute dose conversion factors of -1 , which prevents their inadvertent use since any resulting doses would be negative. The dose reduction factors, i.e.,

$$
\frac{a_{1}}{a_{t}}
$$

currently being used for effective acute doses are as follows: 
EFFECTIVE ACUTE DOSE REDUCTION FACTORS (unitless)

$\begin{array}{llll} & \text { TIME PERIOD AFTER EXPOSURE (DAYS) } \\ & \underline{1-7} \quad \underline{7-14} \quad \underline{14-30} \quad \underline{30-200} \underline{200-365}\end{array}$

$\begin{array}{lrrrlll}\text { RED MARR } & 1 . & .5 & .5 & .25 & & \\ \text { LUNGS } & 1 . & .0625 & .0625 & .027 & .027 & .0109 \\ \text { THYROID } & 1 . & .2 & .2 & .2 & & \\ \text { STOMACH } & 1 . & .37 & & & \\ \text { LOWER LI } & 1 . & .43 & & & \\ \text { SMALI IN } & 1 . & .43 & & & \end{array}$

The generation of dose conversion factors is currently done by a preprocessor program which reads a database supplied by Oak Ridge National Laboratory. This pre-processor aggregates the data into a condensed format containing only the numbers needed by MACCS.

For bone marrow death (with supportive treatment), the Harvard Report shows LD50 values of 4.5 Gy for the 0 to 1 day time period, 9 Gy for the 1 to 14 day time period, and $18 \mathrm{~Gy}$ for the 14 to 30 day time period. Instead of calculating three different red marrow doses and applying the three different values of LD50 to calculate risk, we calculate a single red marrow dose using the effective acute dose conversion factor.

So, according to the above table, effective acute red marrow dose is 100 percent of the first day's dose, 50 percent of the next 13 day's dose, and 25 percent of the next 16 day's dose. This acute dose is then used in the risk equation with the LD50 for the 0 to 1 day time period in order to obtain the hazard of bone marrow syndrome.

The dose protraction effect described here is only applied to the internal exposure resulting from inhalation. Dose from the direct exposure pathways in EARLY (groundshine-and cloudshine) are summed together with no reduction factor being applied even if the exposure lasted more than one day. The current implementation of the early health effects models does not distinguish between cloudshine, groundshine, and the 0 to 1 day inhalation dose. They are equally effective in causing damage.

The error introduced by attributing all the direct exposure dose to the first day is small and is in the direction of overestimating risk. In most cases, emergency plans should ensure that no individuals are permitted to remain on contaminated ground for much longer than a day if dose levels were high enough to pose a risk of early health effects.

Resuspension inhalation is another area where the importance of the pathway is overestimated somewhat. The inhalation dose from resuspended material is treated identically to the inhalation dose from direct inhalation of the plume. That is, it is treated as though all the resuspended material was inhaled at the beginning of the exposure period (timezero). 
Information in this section is only used to control the calculation of individual risk. Results to be processed by OUTPUT, e.g., total cases of early fatality, average individual risk of early fatality, and centerline risk vs. distance of early fatality, are described in later sections of this document. In order for the code to produce early fatality results, the early fatality model must be defined in this section.

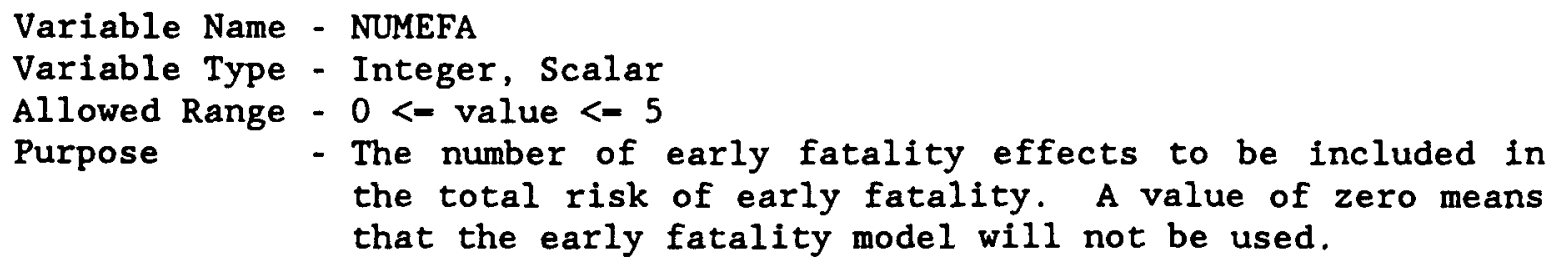

Example Usage:

*

* NUMBER OF EARLY FATALITY EFFECTS

*

EFNUMEFA001 3

Note to user: The remaining parameters in this section are only required if NUMEFA is greater than zero.

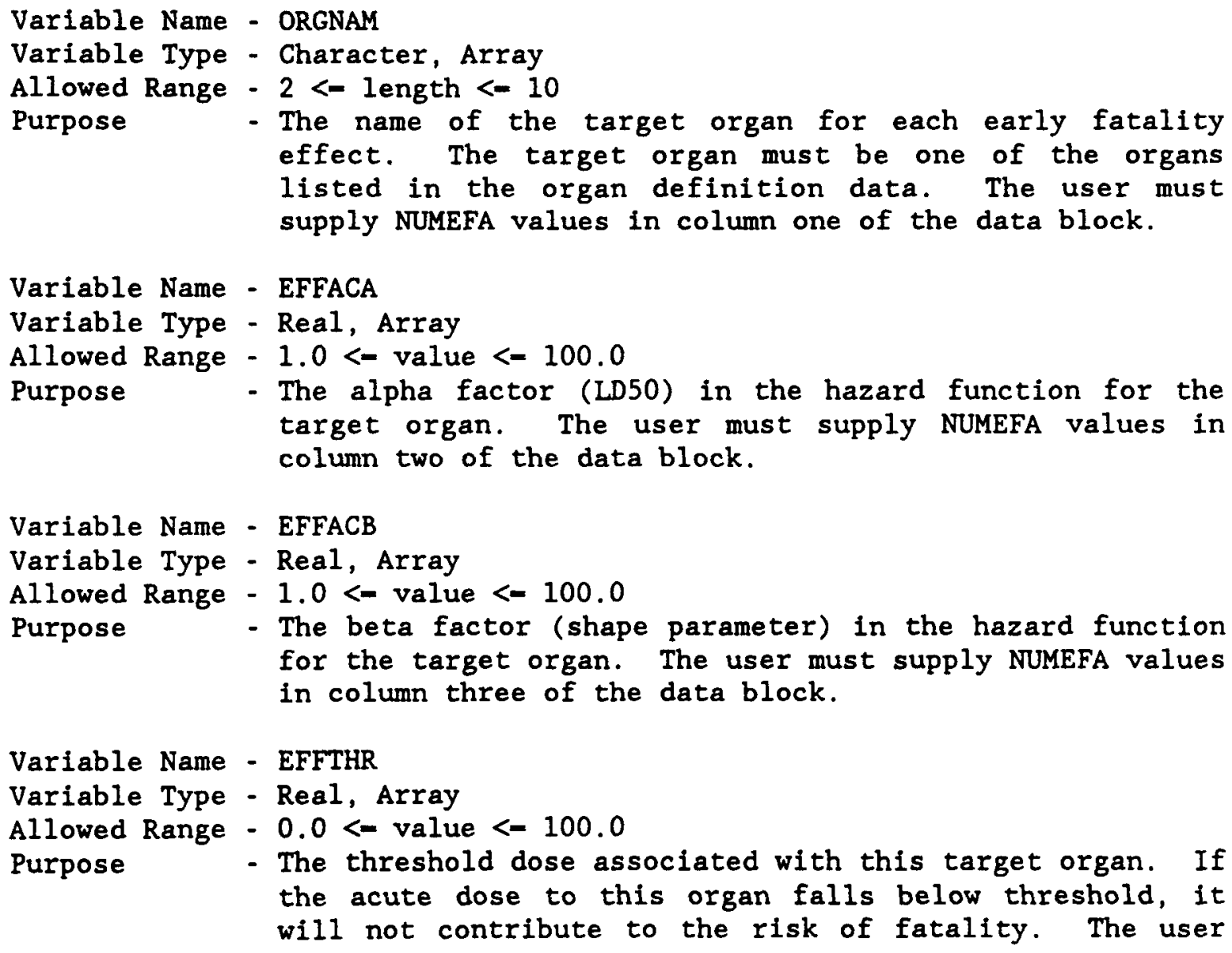


must supply NUMEFA values in column four of the data block.

Example Usage of EFFACA and EFFACB:

$\begin{array}{llrrc}* & & & \\ * & \text { ORGNAM } & \text { EFFACA } & \text { EFFACB } & \text { EFFTHR } \\ \text { * } & & & & \\ \text { EFATAGRP001 'RED MARR' } & 3.8 & 5.0 & 1.5 \\ \text { EFATAGRP002 'LUNGS' } & 10.0 & 7.0 & 5.0 \\ \text { EFATAGRP003 'LOWER LI' } & 15.0 & 10.0 & 8.0\end{array}$

\subsection{Early Injury (EI) Data}

The individual risk of each type of early injury is modeled in MACCS using an approach analagous to that used for early fatality risk [Ev85]. The early injury risk model differs from the early fatality model in that instead of summing the damage from more than one organ, only a single organ is used. The early injury risk function is as follows:

$$
\begin{aligned}
\text { RISK }= & 1-e^{-H}, \text { where } \\
& H-\ln 2\left(\frac{\text { DOSE }}{\text { EIFACA }}\right)^{\text {EIFACB }} \\
\text { DOSE = } & \text { effective acute dose (described in Section 2.8) to the } \\
& \text { target organ, } \\
\text { EIFACA }= & \text { the alpha }\left(D_{50}\right) \text { parameter in the hazard function } \\
& \text { (ibid., Pg. II-8), } \\
\text { EIFACB }= & \text { the beta or exponential parameter in the hazard function } \\
& \text { which defines the steepness of the dose-response function. }
\end{aligned}
$$

A dose threshold is incorporated into the early fatality model in the following manner. If the dose to any of the target organs is below the user specified threshold (see EITHRE below), then the risk of this type of early injury is set to zero.

In addition to the values described above, the user must specify the fraction of the population that is susceptible to the injury, EISUSC. Information in this section is only used to control the calculation of individual risk. Results to be processed by OUTPUT, e.g., total cases of a given injury, average individual risk of a given injury, and centerline risk vs. distance of a given injury, are described in later sections of this document. In order for the code to produce early injury results, the injuries must be defined in this section.

Variable Name - NUMEIN

Variable Type - Integer, Scalar

Allowed Range - $0<=$ value $<=10$ 
Purpose - The number of different types of early injuries that will be calculated. A value of zero means that no early injuries will be calculated.

Example Usage:

*

* NUMBER OF EARLY INJURY EFFECTS

*

EINUMEINO01 7

Note to user: The remaining parameters in this section are only required if NUMEIN is greater than zero. The six arrays are arranged as rows of data in a single data block.

Variable Name - EINAME

Variable Type - Character, Array

Allowed Range - 1 <- length <- 16

Purpose - The name of each type of early injury. The user may specify any name. Apostrophes are mandatory if there are any embedded blanks. The user must supply NUMEIN values in column one of the data block.

Variable Name - ORGNAM

Variable Type - Character, Array

Allowed Range - $2<-$ length $<=10$

Purpose

- The name of the target organ for each type of early injury. The target organ must be one of the organs listed in the organ definition data. The user must supply NUMEIN values in column two of the data block.

Variable Name - EISUSC

Variable Type - Real, Array

Allowed Range - $0.0<-$ value <- 1.0

Purpose - The fraction of the population that is susceptible to the early injury. The user must supply NUMEIN values in column three of the data block.

Variable Name - EITHRE

Variable Type - Real, array

Allowed Range - $0.0<-$ value $<-1000.0$

Purpose - The threshold dose below which the risk of the injury is zero. The user must supply NUMEIN values in column four of the data block.

Variable Name - EIFACA

Variable Type - Real, Array

Allowed Range - $1.0<-$ value <- 1000.0

Purpose - The alpha factor (D50) in the hazard function for the injury. The user must supply NUMEIN values in column five of the data block. 


\begin{tabular}{|c|c|c|c|c|c|c|}
\hline $\begin{array}{l}\text { Variable Nan } \\
\text { Variable Ty } \\
\text { Allowed Rang } \\
\text { Purpose }\end{array}$ & $\begin{aligned} & \text { ne } \text { EIFACB } \\
& \text { pe - Real, Array } \\
& \text { ge - } 1.0<=\text { length } \\
& \text { - The beta (sha } \\
& \text { injury. The } \\
& \text { of the data b. }\end{aligned}$ & $\begin{array}{l}<=100.0 \\
\text { ape) factor } \\
\text { user must s } \\
\text { lock. }\end{array}$ & $\begin{array}{l}\text { in th } \\
\text { supply } N\end{array}$ & $\begin{array}{l}\text { he hazal } \\
\text { NUMEIN }\end{array}$ & $\begin{array}{l}\text { ard func } \\
\text { values }\end{array}$ & $\begin{array}{l}\text { etion fo } \\
\text { in colur }\end{array}$ \\
\hline Example Usag & ge of EINAME, EISUS & SC, EITHRE, & EIFACA & $A$ and $E I$ & SFFACB : & \\
\hline * & & & & & & \\
\hline * & EINAME & ORGNAM & EISUSC & EITHRE & EIFACA & EIFACB \\
\hline EINJUGRP001 & 'PRODROMAL VOMIT' & ' STOMACH' & 1. & .5 & 2. & 3. \\
\hline EINJUGRP002 & ' DIARRHEA' & ' STOMACH' & 1. & 1. & 3. & 2.5 \\
\hline EINJUGRP003 & 'PNEUMONITIS' & 'LUNGS' & 1 . & 5. & 10. & 7. \\
\hline EINJUGRI & ' SKIN ERYTHEMA' & 'SKIN' & 1. & 3. & 6. & 5. \\
\hline EINJUGI & 'TRANSEPIDERMAL' & 'SKIN' & 1. & 10. & 20. & \\
\hline & 'THYROIDITIS' & ' THYROIDH' & 1 . & 40. & 240 . & \\
\hline EINJUG & 'HYPOTHYROIDISM' & 'THYROIDH' & 1. & 2. & 60. & 1.3 \\
\hline
\end{tabular}

\subsection{Latent Cancer (LC) Data}

This section defines the cancer induction models which are used by both the EARLY and the CHRONC modules.

Cancer risk is modeled differently in the EARLY and CHRONC modules. In the EARLY module, which treats the period immediately following the accident, individual doses can be quite high near the reactor. This calls for the use of two cancer risk models in EARLY: the first for low and medium dose levels (linear-quadratic), and the second for high dose levels where an upper-bound limit to the cancer dose-response function is utilized.

During the long-term phase modeled by CHRONC, it is assumed that exposure of the population will be limited to low levels by mitigative actions and therefore the quadratic term of the linear-quadratic cancer risk equation is ignored. Cancer risk is calculated by CHRONC using only the linear term of the linear-quadratic dose response function which is used by EARLY.

In the period modeled by EARLY, a linear-quadratic dose-response relationship is used if the dose to the target organ is less than a userspecified limit, ACTHRE, (ibid., p. I-23).

The dose response function used in this case is of the form:

$$
\text { risk - DOSE * CFRISK * (DOSEFA + DOSE * DOSEFB }) \text { * ACSUSC, }
$$

where DOSE is the 0 to 50 year lifetime dose commitment to the target organ, and CFRISK, DOSEFA, DOSEFB, and ACSUSC are input parameters described below. 
If dose to the target organ is greater than the dose limit ACTHRE, the upper bound linear dose response relationship is used:

$$
\text { risk }=\text { DOSE * CFRISK * (DOSEFA + ACTHRE * DOSEFB }) * \text { ACSUSC. }
$$

In the long-term phase modeled by CHRONC, cancer risk from all pathways (groundshine, resuspension inhalation, and ingestion) are modeled with the linear dose response function given below:

$$
\text { risk }=\text { DOSE * CFRISK * DOSEFA * ACSUSC, }
$$

where the meaning of DOSE and CFRISK depend on whether individual risk (groundshine and resuspension) or collective risk (ingestion and decontamination worker's doses) is being calculated.

These three cancer fatality equations are easily depicted as follows:

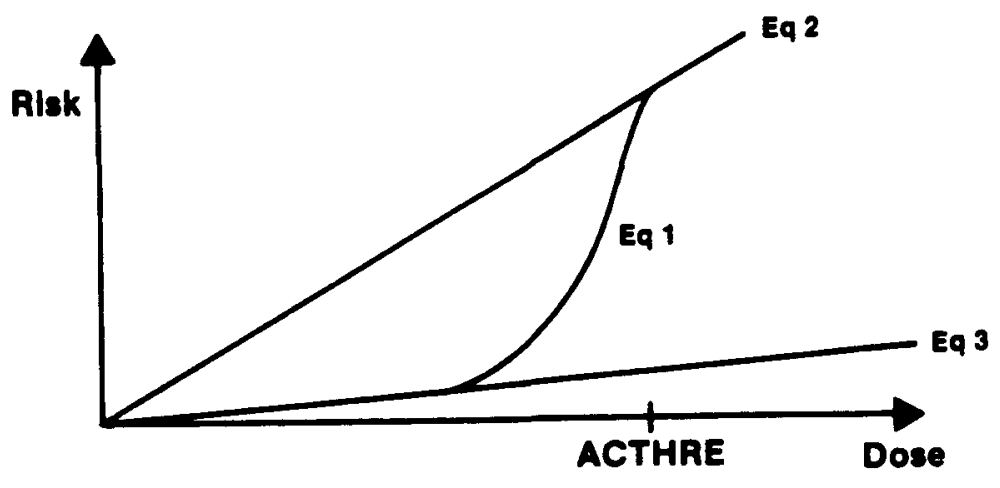

The long-term phase model does not calculate an individual's level of dose resulting from ingestion of contaminated food and water or performing decontamination work in the contaminated area. The dose from these pathways is attributed to society as a whole. This necessitates the use of a linear dose response function for calculating the resulting cancers from those societal pathways.

Risk of latent cancer injury is calculated separately for each cancer syndrome. The risk factors for cancer injury are calculated using the same equations as are used to calculate cancer fatalities except that CIRISK is used instead of CFRISK. The user must specify the fraction of the population that is susceptible to each type of cancer in ACSUSC.

Information in this section is only used to control the calculation of individual risk. Results to be processed by OUTPUT, e.g., total cases of a given cancer, average individual risk of a given cancer, and centerline risk of a given cancer versus distance, are described in later sections of this document. In order for the code to produce cancer results, the cancers must be defined in this section.

Variable Name - NUMACA

Variable Type - Integer, Scalar

Allowed Range - $0<-$ value $<=10$ 
Purpose $\quad$ The number of different types of latent cancer effects
that will be calculated. A value of zero means that
latent cancer effects will not be calculated.

Example Usage:

*

* NUMBER of CANCER EFFEĆtS

*

LCNUMACAOOI 7

Note: The remaining parameters in this section are only required if NUMACA is greater than zero.

Variable Name - ACTHRE

Variable Type - Real, Scalar

Allowed Range - $0.0<-$ value <- 10.0 (sieverts)

Purpose - The lower dose limit for the linear dose response relationship. For doses less than ACTHRE, the linearquadratic relationship is used.

Note: The parameter ACTHRE is used only in the EARLY module. It is not used in the CHRONC module.

Example Usage:

*

* DOSE THRESHOLd FOR LINEAR dOSE RESPONSE (SV)

*

LCACTHRE001 1.5

Note: The next seven variables are supplied in a block of data as columns. An example of their usage will follow.

Variable Name - ACNAME

Variable Type - Character, Array

Allowed Range - $1<-$ length <- 10

Purpose - The name of each type of latent cancer effect. The user may specify any name. Apostrophes are mandatory if there are any embedded blanks. The user must supply NUMACA values in column one of the data block.

Variable Name - ORGNAM

Variable Type - Character, Array

Allowed Range - $2<-$ length <- 10

Purpose - The name of the target organ for each type of latent cancer effect. The target organ must be one of the organs listed in the organ definition data. The user must supply NUMACA values in column two of the data block. 


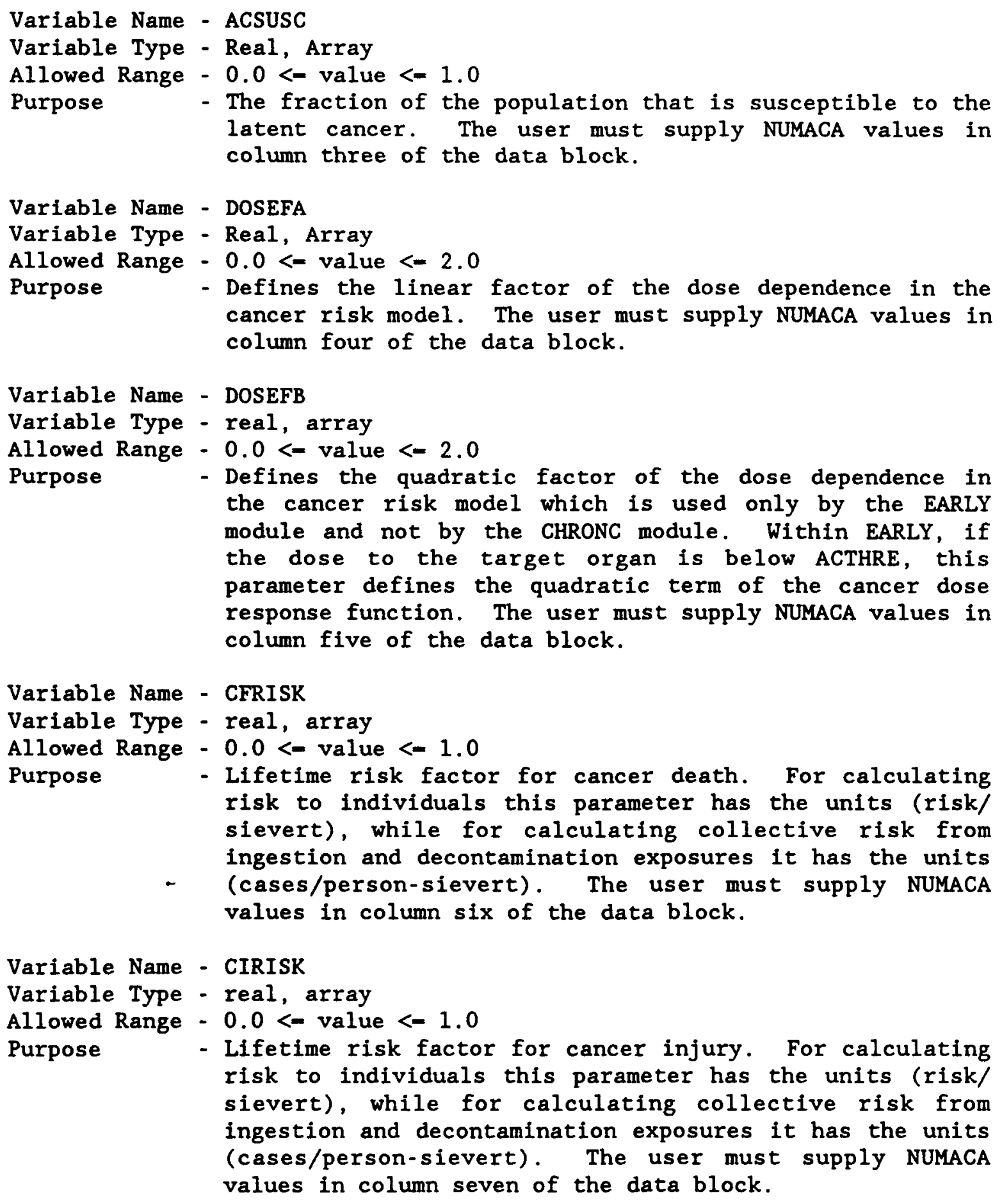
the cancer risk model which is used only by the EARLY module and not by the CHRONC module. Within EARLY, if the dose to the target organ is below ACTHRE, this parameter defines the quadratic term of the cancer dose response function. The user must supply NUMACA values in column five of the data block.

Variable Name - CFRISK

Variable Type - real, array

Allowed Range - $0.0<-$ value $<-1.0$

Purpose - Lifetime risk factor for cancer death. For calculating risk to individuals this parameter has the units (risk) sievert), while for calculating collective risk from ingestion and decontamination exposures it has the units - (cases/person-sievert). The user must supply NUMACA values in column six of the data block.

Variable Name - CIRISK

Variable Type - real, array

Allowed Range - $0.0<-$ value $<-1.0$

Purpose - Lifetime risk factor for cancer injury. For calculating risk to individuals this parameter has the units (risk) sievert), while for calculating collective risk from ingestion and decontamination exposures it has the units (cases/person-sievert). The user must supply NUMACA values in column seven of the data block.

Example Usage of ACNAME, ACSUSC, DOSEFA, DOSEFB, CFRISK and CIRISK:

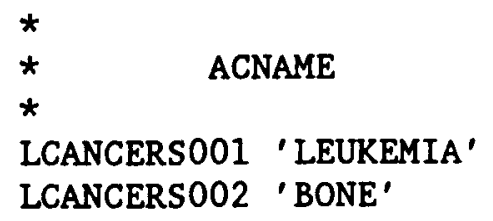

LCANCERSO01 'LEUKEMIA'
LCANCERSO02 'BONE'

\begin{tabular}{lrrrrr}
\multicolumn{2}{c}{ ORGNAM ACSUSC } & DOSEFA & DOSEFB & CFRISK & CIRISK \\
'RED MARR' & 1. & .39 & .61 & $3.7 \mathrm{E}-3$ & $3.7 \mathrm{E}-3$ \\
'BONE SUR' & 1. & .39 & .61 & $1.5 \mathrm{E}-4$ & $1.5 \mathrm{E}-4$
\end{tabular}




$\begin{array}{ll}\text { LCANCERSO03 ' } & \text { BREAST' } \\ \text { LCANCERS004 'LUNG' } \\ \text { LCANCERSO05 'THYROID' } \\ \text { LCANCERSO06 'GI' } \\ \text { LCANCERS007 'OTHER' }\end{array}$

\subsection{Generation of Consequence Distributions}

Under the control of parameters supplied by the user on the EARLY and CHRONC User Input Files, the EARLY and CHRONC modules can calculate a variety of different consequence measures to portray the impact of a reactor accident on the surrounding region.

The user has total control over the results that will be produced. By choosing appropriate values in the user input files, the user can ensure that the code does not perform unnecessary calculations.

This affords a great deal of flexibility but it also requires that the user anticipate which results will be of interest. If any are omitted, it is necessary to correct the user input and rerun the program.

In this regard, please remember that a result can only be produced if the model needed for its calculation has been previously defined in the appropriate section. If any results pertaining to health effects are requested, risk factors for that model must have been supplied in the sections entitled Early Fatality (EF), Early Injury (EI), and Latent Cancer (LC).

EARLY can produce eight different types of results. These are described in the next eight sections of this document. Some of these eight types of results can also be calculated by CHRONC, but some cannot. For instance, both EARLY and CHRONC calculate cancer cases and population dose, but EARLY alone calculates early fatalities, and CHRONC alone calculates economic costs.

If the user requests EARLY to produce a result that can also be produced by CHRONC, the code will ensure that it will be produced by both EARLY and CHRONC. Whenever a result can be produced by both modules, this will be called out in the following eight sections.

Neither EARLY nor CHRONC generate Complementary Cumulative Distribution Functions (CCDFs) of the results that they calculate. As EARLY and CHRONC generate the requested consequence measures, those numbers are written to binary files on units 31 to 34 for later processing into CCDFs.

The generation of CCDFs is performed by the OUTPUT module of MACCS. It reads the binary files of consequence measures and automatically combines the results in a predetermined way. The user has no direct control over the OUTPUT module other than through the EARLY and CHRONC data blocks which control the generation of consequence measures. 
The CCDF is an estimate of the distribution of consequence magnitudes. The variability of consequence values in MACCS CCDFs is due solely to the uncertainty of the weather conditions existing at the time of the accident.

If a consequence measure was calculated by both EARLY and CHRONC, the output module will present those results and their CCDFs separately for EARLY and CHRONC. Also, the output module will sum the results of EARLY and CHRONC and provide the CCDFs of their sums. The contribution of up to three sets of results generated by EARLY can be combined together according to the weighting fractions described in Section 2.6. These weighting fractions can apply to either consequence magnitude (fraction of the people), or the consequence probability (fraction of the time). The weighted sum of all consequence measures, calculated by summation of results from separate runs of EARLY, are presented at the beginning of each section of the listing produced by OUTPUT. Following the overall weighted sum, the results from each of its individual components are presented.

The following material describes the format of the listing produced by the OUTPUT module. It is recommended that the reader of this section refer to Appendix D.3 which presents a listing of the output from Sample Problem A. This output file, named LISTA, is included on microfiche with this report and is also included on the magnetic tape used for distribution of MACCS.

At the top of each page is printed the date and time of the run and a page number. The numbering of pages begins with the first page produced by the OUTPUT module.

The leftmost 38 columns of the page provide the name of the consequence measure. These names are split into two parts: the general and the specific. For example we have

"HEALTH EFFECTS CASES"

as the general name and

"ERL FAT/TOTAL 0-1609 KM"

as the specific part of the name. The consequence measure being presented on this line is the number of early fatality cases expected to occur in the region beginning at the reactor $(0 \mathrm{~km}$ radius on our polar grid) and extending outward in a concentric ring to the spatial interval with an outer boundary at $1609 \mathrm{~km}$.

Going across the page from left to right, there are ten columns of numeric data which provide a summary of the CCDF generated by the OUTPUT module. These are described as follows:

PROB NON-ZERO - Conditional probability of having a non-zero consequence estimate, conditional on the occurrence of the release under consideration. 


\section{MEAN - The average (expected) consequence over all weather}

trials. This is calculated by taking the sum of all the products [(consequence value) X (associated probability of that value)] for each weather trial.

50TH QUANTILE - The median of the estimated distribution function.

90TH QUANTILE - Based on the estimated distribution function, there is a 10 percent chance this consequence magnitude will be exceeded.

95TH QUANTILE - Based on the estimated distribution function, there is a 5 percent chance this consequence magnitude will be exceeded.

99TH QUANTILE - Based on the estimated distribution function, there is a 1 percent chance this consequence magnitude will be exceeded.

99.9TH QUANTILE - Based on the estimated distribution function, there is a 0.1 percent chance this consequence magnitude will be exceeded.

PEAK CONS - The largest consequence magnitude obtained from the full set of weather trials which were examined.

PEAK PROB - The probability associated with the largest consequence magnitude.

PEAK TRIAL - In the series of weather samples, the sequence number of the weather trial which gave rise to the largest consequence magnitude. By going back to the ATMOS output listing, the user can determine the start time (day and hour) of this weather sequence.

\subsection{User Requested Cases of a Given Health Effect (T1)}

EARLY can calculate the number of health effect cases which will occur within a range of distances. The risk models for these health effects must have been previously defined in the proper section.

Note: Any cancer results requested here are automatically produced by CHRONC so that the results can be combined by OUTPUT. In the results calculated by CHRONC, the cancer values include direct exposure cancers in the population residing in the region as well as the indirect exposure cancers resulting from both ( 1 ) consumption of food and water produced in the region and (2) exposure of decontamination workers working in the region.

Variable Name - NUM1

Variable Type - Integer, Scalar

Allowed Range - $0<=$ value $<=40$ 


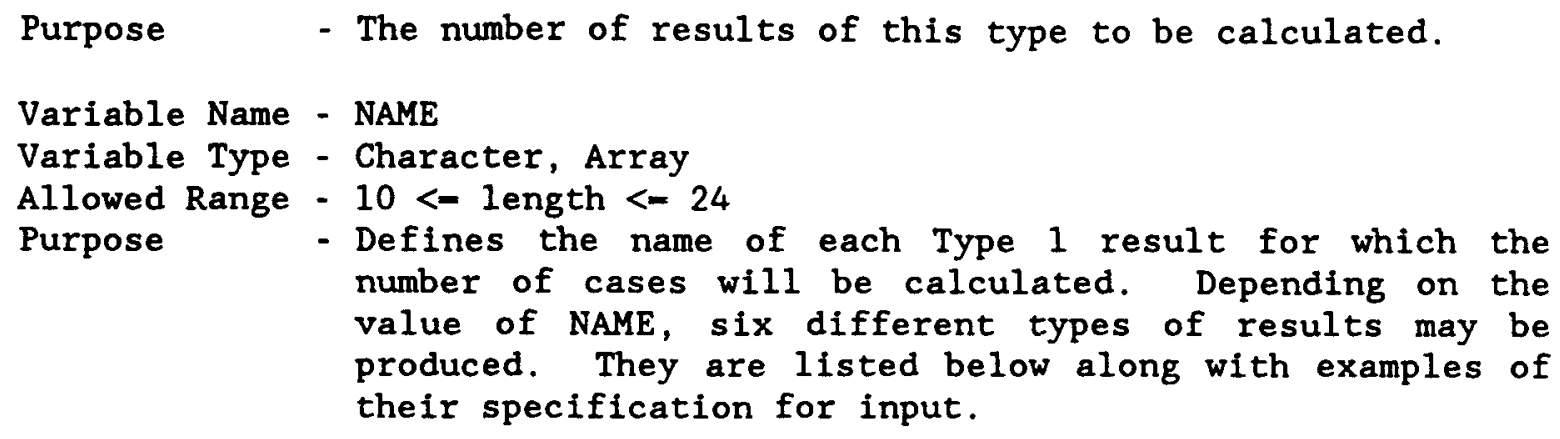

Note: In order to obtain the CCDF tables of a consequence measure requested in this section, append the character string 'CCDF' to the line requesting that result as the fourth item on the data card. The CCDF tables will be printed on the list output File (unit 6).

Example Usage:

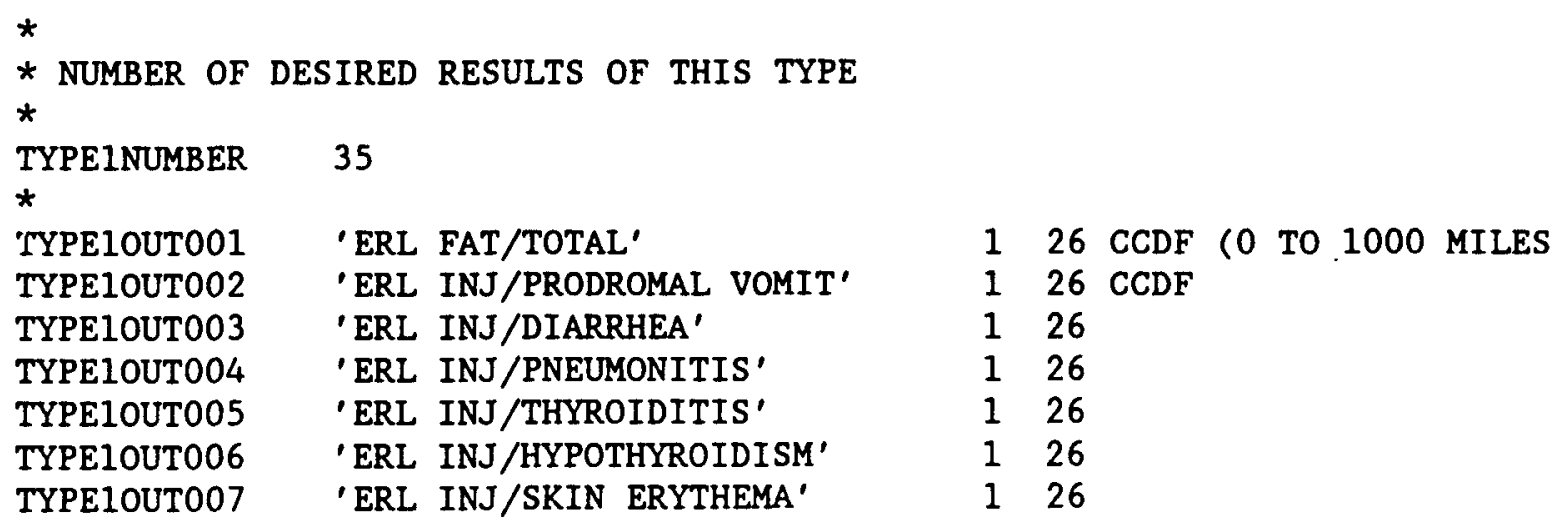




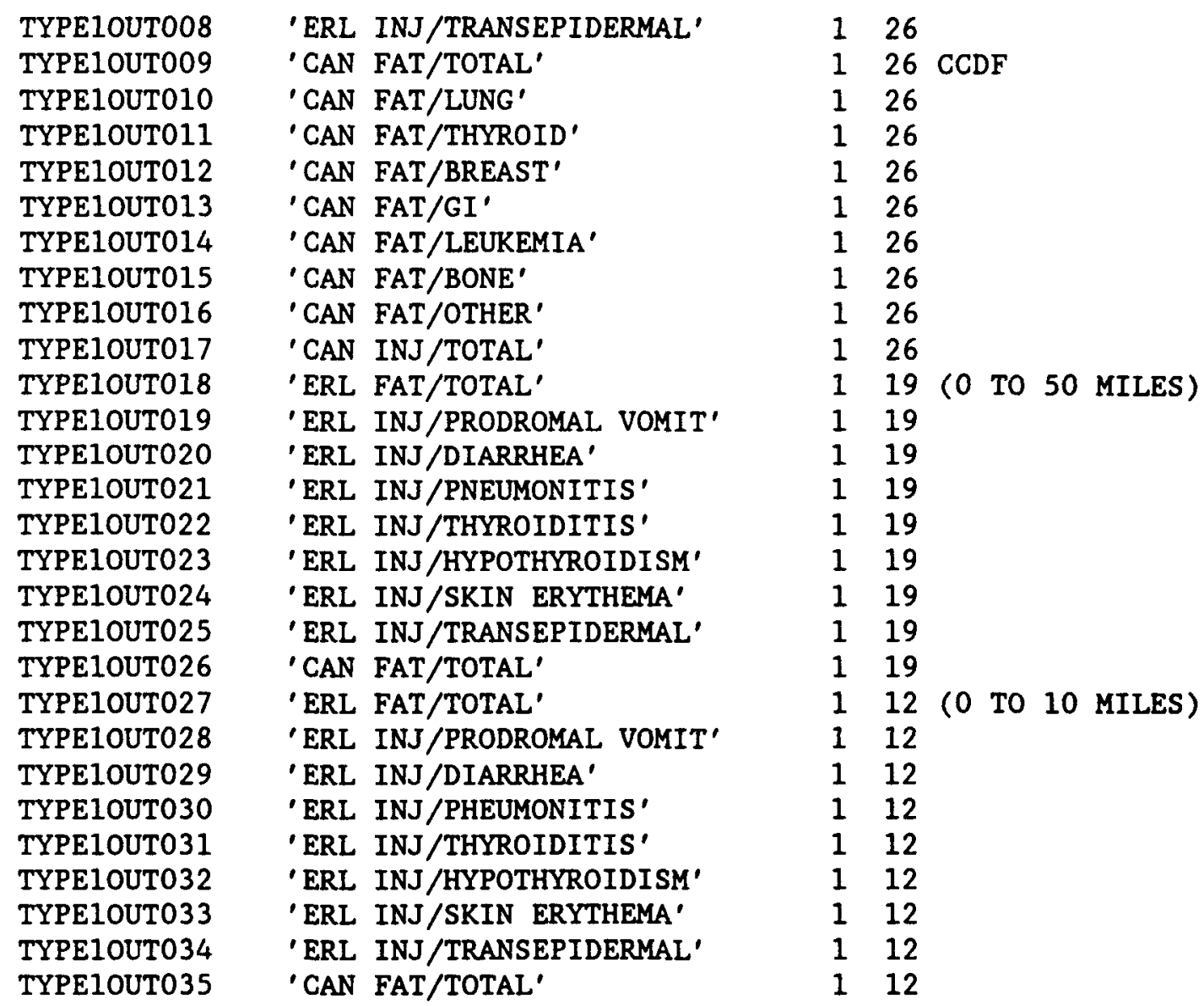

\subsection{User Requested Early Fatality Radius (T2)}

It may be of interest to know the greatest distance at which a specified level of early fatality risk is exceeded. By turning on this result, the user can obtain information about the size of the region in which early fatalities are predicted to occur.

Variable Name - NUM2

Variable Type - Integer, Scalar

Allowed Range - $0<-$ value <- 10

Purpose - The number of results of this type to be calculated.

Variable Name - RISTHR

Variable Type - Real, Scalar

Allowed Range - $0.0<-$ value <- 1.0

Purpose - Defines the risk threshold used for calculating the fatality radius (reported in kilometers). The user must supply NUM2 values in column one of the data block. This is the only array in the data block.

Note: In order to obtain the CCDF tables of a consequence measure requested in this section, append the character string 'CCDF' to the line requesting that result as the second item on the data 
card. The CCDF tables will be printed on the List Output File (unit 6).

Example Usage:

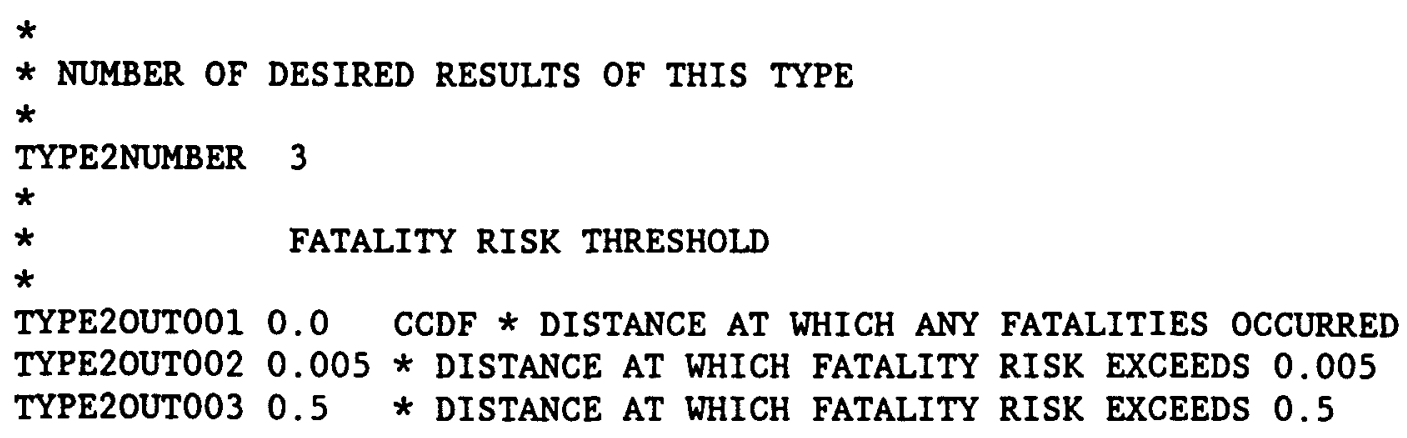

\subsection{User Requested Population Exceeding a Dose Threshold (T3)}

Within the EARLY module alone, it may be of interest to know how many people received doses exceeding certain levels. This information can be obtained by requesting the production of the result described below. It is important to remember that this consequence measure is calculated solely on the basis of the dose calculations performed in the EARLY module. There is no provision for examining an analogous result for the CHRONC module, or for determining the number of people whose total dose from both EARLY and CHRONC exceeded a certain level.

The user can specify two type of doses to be used for the comparison: acute and lifetime. "Acute" dose is used by the code for calculating early fatality and early injury health effects (see Section 2.8). For each organ, the "acute" dose is calculated with set of dose reduction factors defined in that section. The lifetime dose is used for the calculation of cancer fatality and cancer injury health effects (see Section 2.10). It represents the 0 to 50 year lifetime dose commitment to a reference man which results from exposure during the emergency phase (EARLY) time period.

Variable Name - NUM3

Variable Type - Integer, Scalar

Allowed Range - $0<-$ value <- 10

Purpose - The number of results of this type to be calculated.

Variable Name - NAME

Variable Type - Character, Scalar

Allowed Range - $2<-$ length <- 10

Purpose - Defines the name of the organ to which the dose threshold applies. This organ must be found on the list of organs, ORGNAM. The user must supply NUM3 values in column one of the data block. 


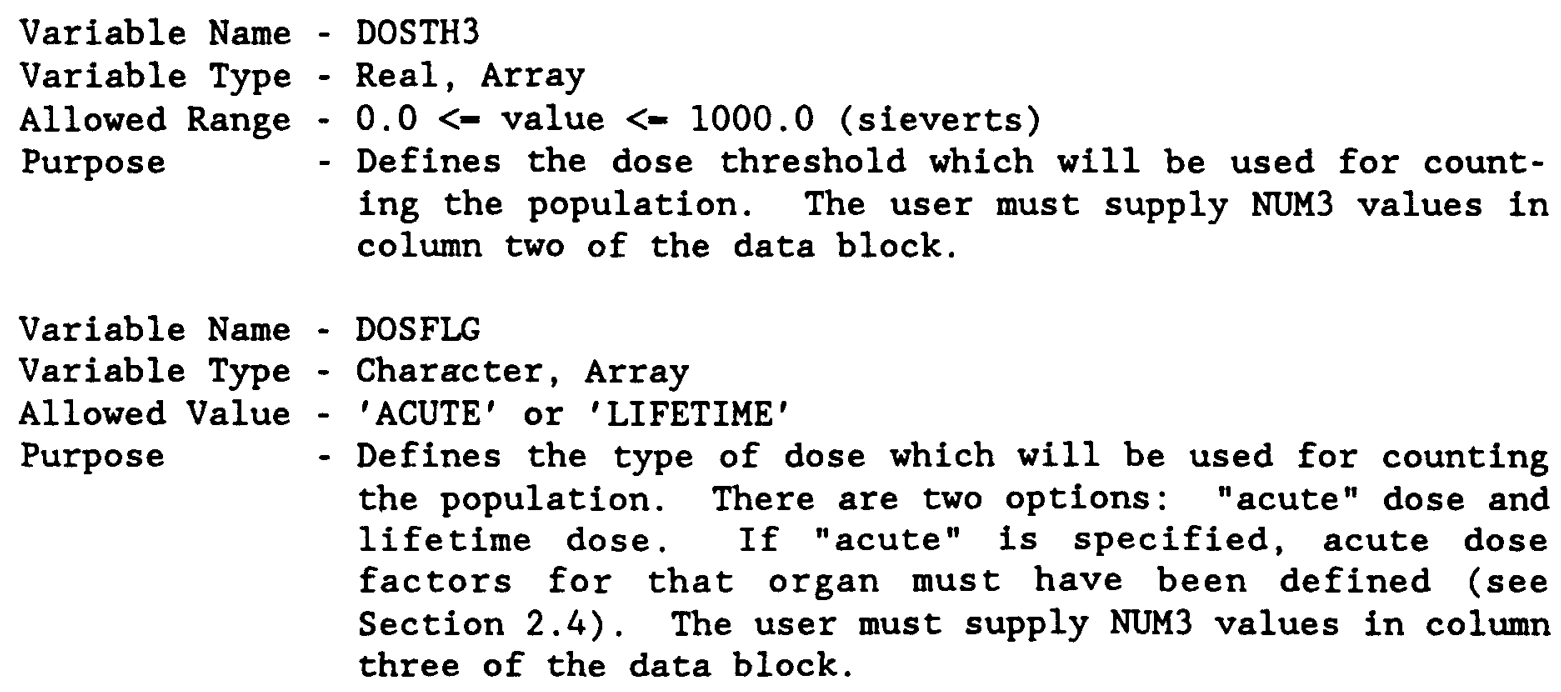

Note: In order to obtain the CCDF tables of a consequence measure requested in this section, append the character string 'CCDF' to the line requesting that result as the fourth item on the data card. The CCDF tables will be printed on the List Output File (unit 6).

Example Usage:

*

* NUMBER OF DESIRED RESULTS OF THIS TYPE

*

TYPE3NUMBER 3

* oRgan Name dose threshold (SV) dose flag

*

$\begin{array}{llll}\text { TYPE3OUT001 } & \text { 'RED MARR' } & 1.5 & \text { ACUTE } \\ \text { TYPE3OUT002 } & \text { 'LUNGS' } & 5.0 & \text { ACUTE } \\ \text { TYPE3OUT003 } & \text { 'EDEWBODY' } & 0.05 . & \text { LIFETIME }\end{array}$

\subsection{User Requested Average Individual Risk (T4)}

Average individual risk is obtained by taking the sum of the risk values in all sectors at a given distance and dividing it by the number of sectors.

Note: Any cancer results requested here are automatically produced by CHRONC so that the results can be combined by OUTPUT. When this result is produced by CHRONC, it is only a measure of the risk from direct exposure to people at that distance. Direct exposure includes the cloudshine, groundshine, and inhalation dose to the people living around the reactor.

The risk presented in this result does not include doses from ingestion of food and water by the region's population or doses to decontamination workers working in the region. 


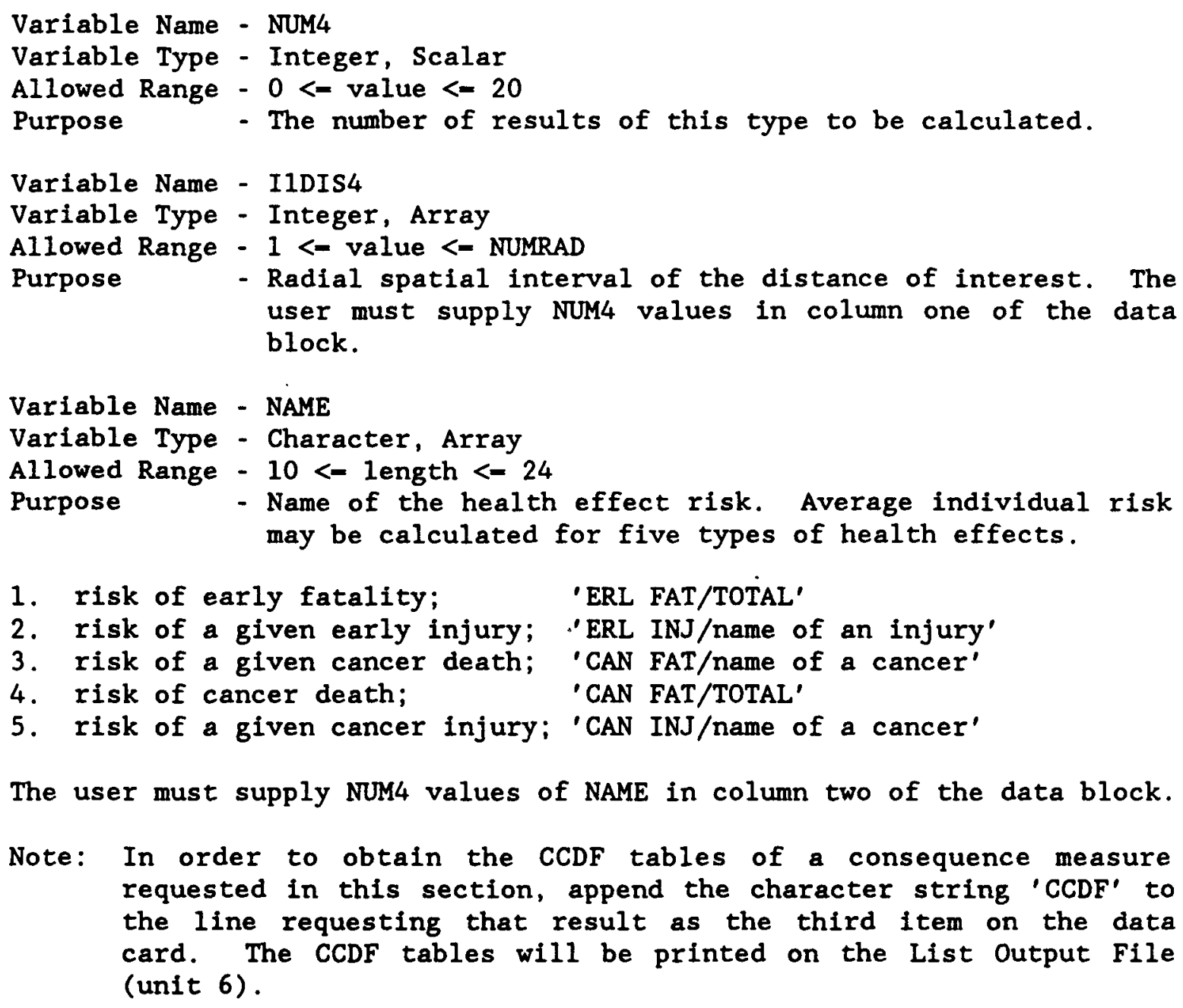

The user must supply NUM4 values of NAME in column two of the data block.

Note: In order to obtain the CCDF tables of a consequence measure requested in this section, append the character string 'CCDF' to the line requesting that result as the third item on the data card. The CCDF tables will be printed on the List Output File (unit 6).

Example Usage:

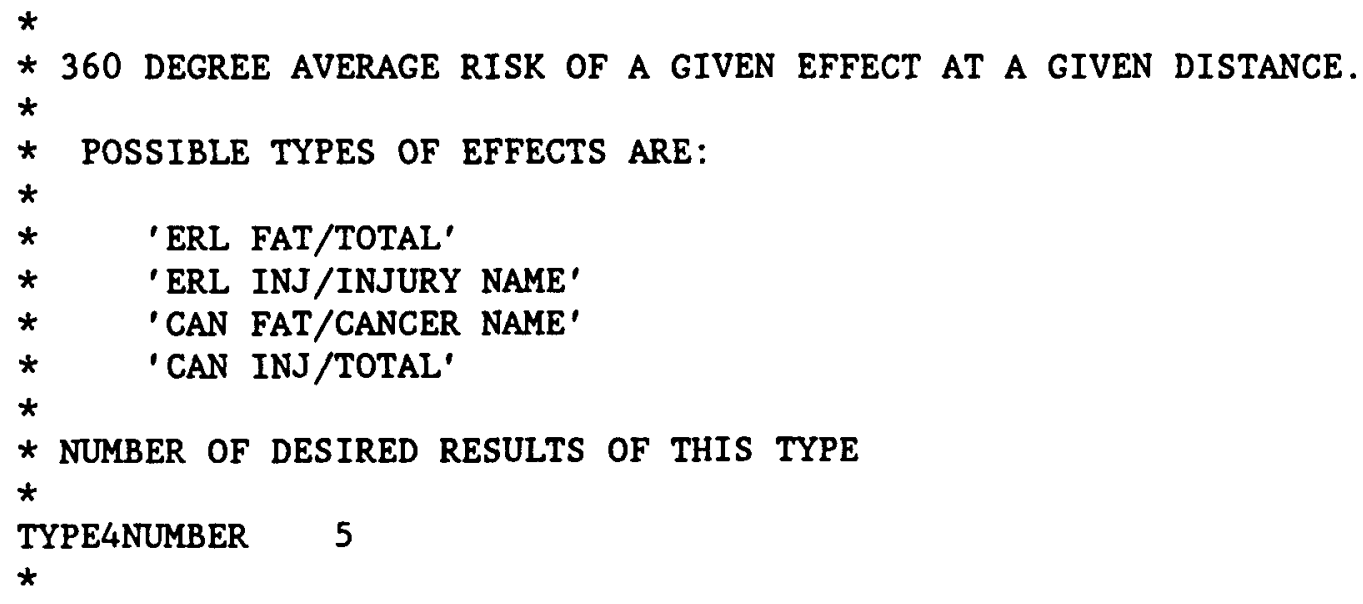




$\begin{array}{lcl}* & \text { RADIAL INDEX } & \text { TYPE OF EFFECT } \\ * & & \text { 'ERL FAT/TOTAL' } \\ \text { TYPE4OUT001 } & 1 & \text { 'ERL FAT/TOTAL' } \\ \text { TYPE40UT002 } & 2 & \text { 'ERL FAT/TOTAL' } \\ \text { TYPE40UT003 } & 3 & \text { 'ERL FAT/TOTAL' } \\ \text { TYPE40UT004 } & 4 & \text { 'ERL FAT/TOTAL' } \\ \text { TYPE40UT005 } & 5 & \end{array}$

\subsection{User Requested Population Dose (T5)}

The total long-term population dose to a given organ resulting from the contamination of a specified region can be calculated. The user must supply the name of the target organ as well as the inner and outer spatial intervals of the region of interest. If only the EARLY module is being run, this result reflects only the pathways considered by EARLY. If both EARLY and CHRONC are being run, the population dose from all pathways is included in the calculation. The CHRONC pathways include both (1) food and water ingestion doses resulting from material deposited in the region and (2) doses to decontamination workers working in the region. On the output listing, population dose is presented in units of person-sieverts.

Variable Name - NUM5

Variable Type - Integer, Scalar

Allowed Range - $0<-$ value <- 10

Purpose - The number of results of this type to be calculated.

Variable Name - NAME

Variable Type - Character, Scalar

Allowed Range - 2 <- length <- 10

Purpose

- Defines the name of the organ to which the population dose applies. This organ must be found on the list of organs, ORGNAM. The user must supply NUM5 values in column one of the data block.

Variable Name - I1DIS5

Variable Type - Integer, Array

Allowed Range - 1 <- value <- NUMRAD

Purpose - Defines the inner spatial interval of the region of interest for this result. The user must supply NUM5 values in column two of the data block.

Variable Name - I2DIS5

Variable Type - Integer, Array

Allowed Range - I1DIS5 <- value <- NUMRAD

Purpose - Defines the outer spatial interval of the region of interest for this result. The user must supply NUM5 values in column three of the data block.

Note: In order to obtain the CCDF tables of a consequence measure requested in this section, append the character string 'CCDF' to the line requesting that result as the fourth item on the data card. The CCDF tables will be printed on the List Output File (unit 6). 


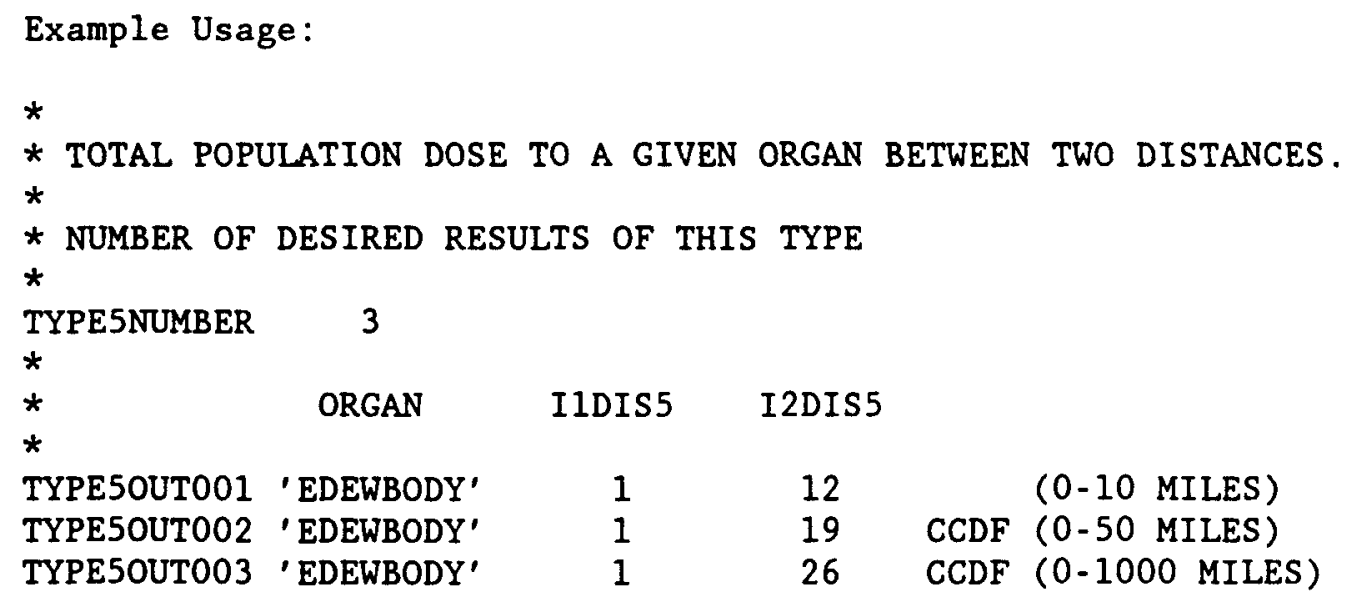

\subsection{User Requested Centerline Dose vs. Distance (T6)}

If the straight-line plume model was chosen (IPLUME=1), the code can keep track of centerline dose between a range of distances for the various pathways. The centerline dose at each distance is treated as a separate result and OUTPUT will generate a set of results for each of the radial spatial intervals within the specified range.

Depending on the exposure pathways specified, this result will be calculated by EARLY and/or CHRONC (see below). If both EARLY and CHRONC are being run, then CHRONC will automatically produce all of the results for the pathways which it considers. If pathways 'GRD', 'RES LIF', or 'TOT LIF' are chosen, those results are automatically produced by CHRONC so that the results can be combined by OUTPUT. The other pathway values are only produced by EARLY.

For a description of effective acute dose, please refer to Section 2.8.

An alternative method for examining centerline dose from EARLY alone is to set the output control variable, IPRINT, to a value greater than zero. If this is done, a listing of dose vs. distance for all the organs will be printed on the list output. Since this is written for each weather trial, it is recommended that this be done only for single weather trial runs.

Variable Name - NUM6

Variable Type - Integer, Scalar

Allowed Range - $0<-$ value <- 10

Purpose - The number of results of this type to be calculated.

Note: unless IPLUME-1, you must specify a value of zero. PATHNM DESCRIPTION

1. CLD cloudshine dose (EARLY only),

2. GRD groundshine dose (EARLY and CHRONC), 
3. INH ACU effective acute dose from inhalation of the plume as it passed overhead (EARLY only),

4. INH IIF lifetime dose commitment from inhalation of the plume as it passed overhead (EARLY only),

5. TOT ACU total effective acute dose (EARLY only) being the sum of CLD, GRD, INH ACU, and RES ACU,

6. TOT LIF total lifetime dose commitment from all direct exposure pathways (EARLY and CHRONC) being the sum of CLD, GRD, INH LIF, and RES LIF,

7. RES LIF lifetime dose commitment from inhalation of resuspended material after plume passage (EARLY and CHRONC),

8. RES ACU effective acute dose from inhalation of resuspended material after plume passage (EARLY only).

Note: The pathway name for 'SKIN' will ignored by the code since there is only one exposure pathway for skin (direct dry deposition to the skin). The user must supply a value for the pathway name in all cases. For the case of skin as the target organ, it does not matter which of the eight pathways listed above is supplied.

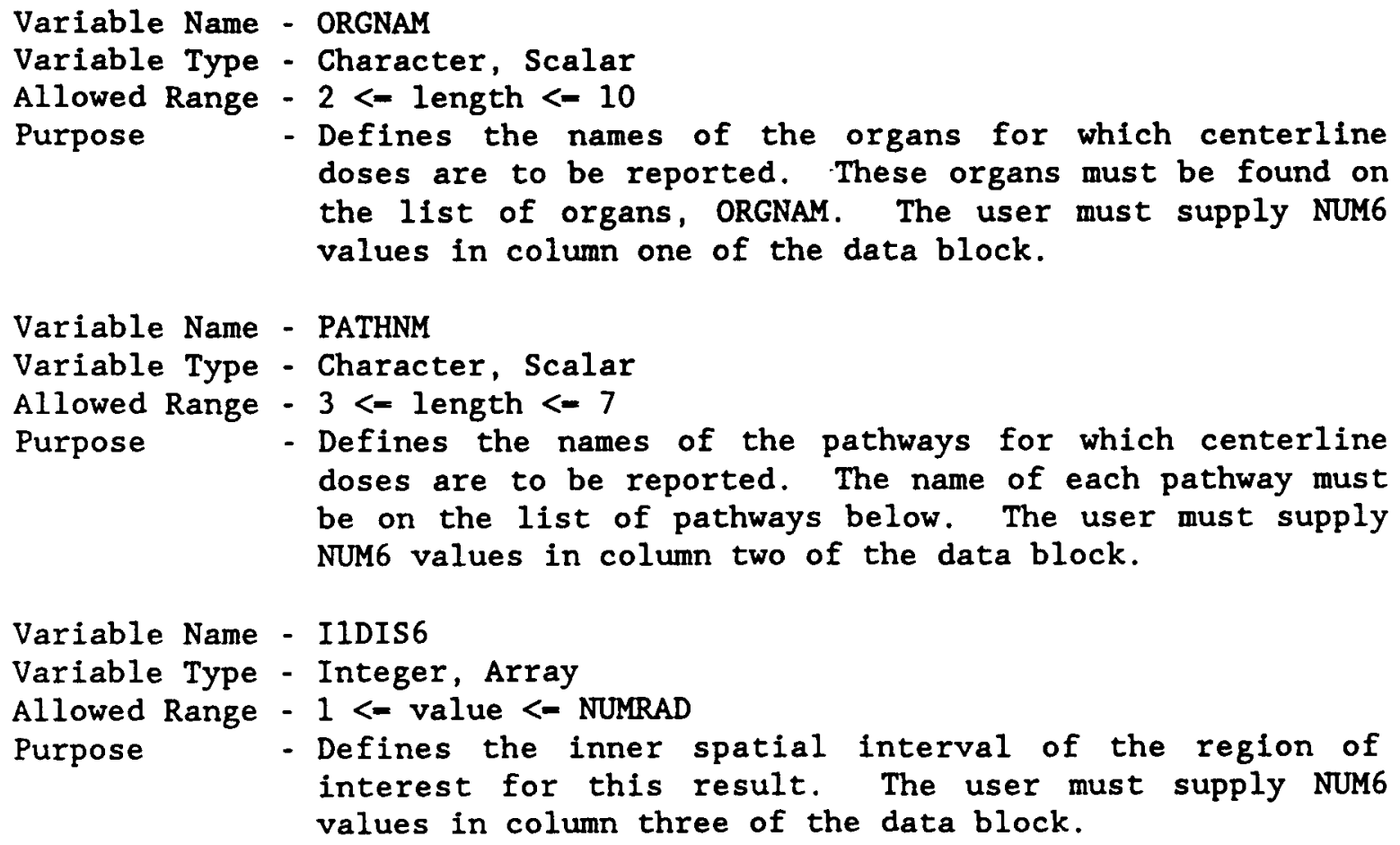




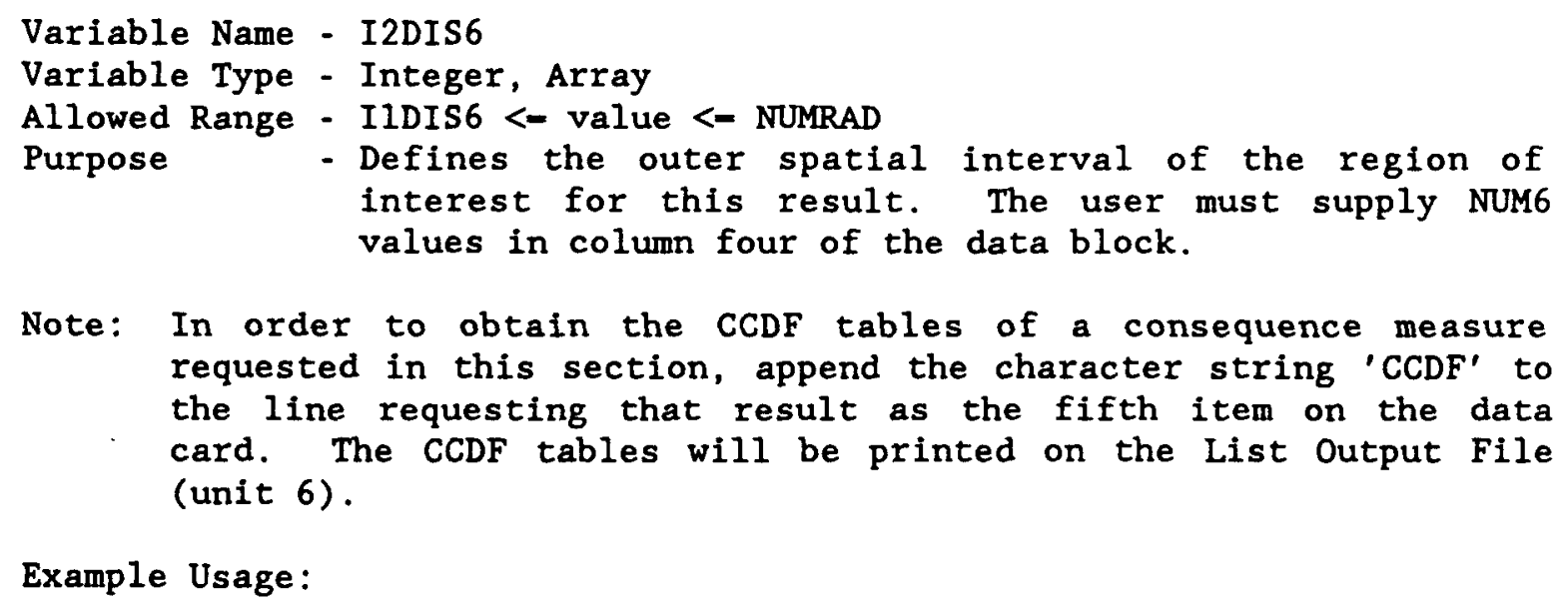

Note: In order to obtain the CCDF tables of a consequence measure requested in this section, append the character string 'CCDF' to the line requesting that result as the fifth item on the data card. The CCDF tables will be printed on the List Output File (unit 6).

Example Usage:

\subsection{User Requested Centerline Risk vs. Distance (T7)}

If the straight-line plume model was chosen (IPLUME-1), the code can keep track of centerline risk between two specified spatial intervals for the various types of health effects. Centerline risk is calculated for hypothetical individuals located directly under the path of the plume who are exposed to the Gaussian peak of the air and ground concentrations. The centerline risk at each distance in the region is treated as a separate result and OUTPUT will generate a distribution of the consequence measure for each of the spatial intervals within the specified range.

Note: Any cancer results requested here are automatically produced by CHRONC so that the results can be combined by OUTPUT. When this result is produced by CHRONC, it is only a measure of the risk from direct exposure to people at those distances. Direct exposure includes the cloudshine, groundshine, and inhalation dose to the resident population in the specified region.

The risk presented in this result does not include societal doses from ingestion of any food and water contaminated as a result of the accident or doses to decontamination workers working in the contaminated area. 


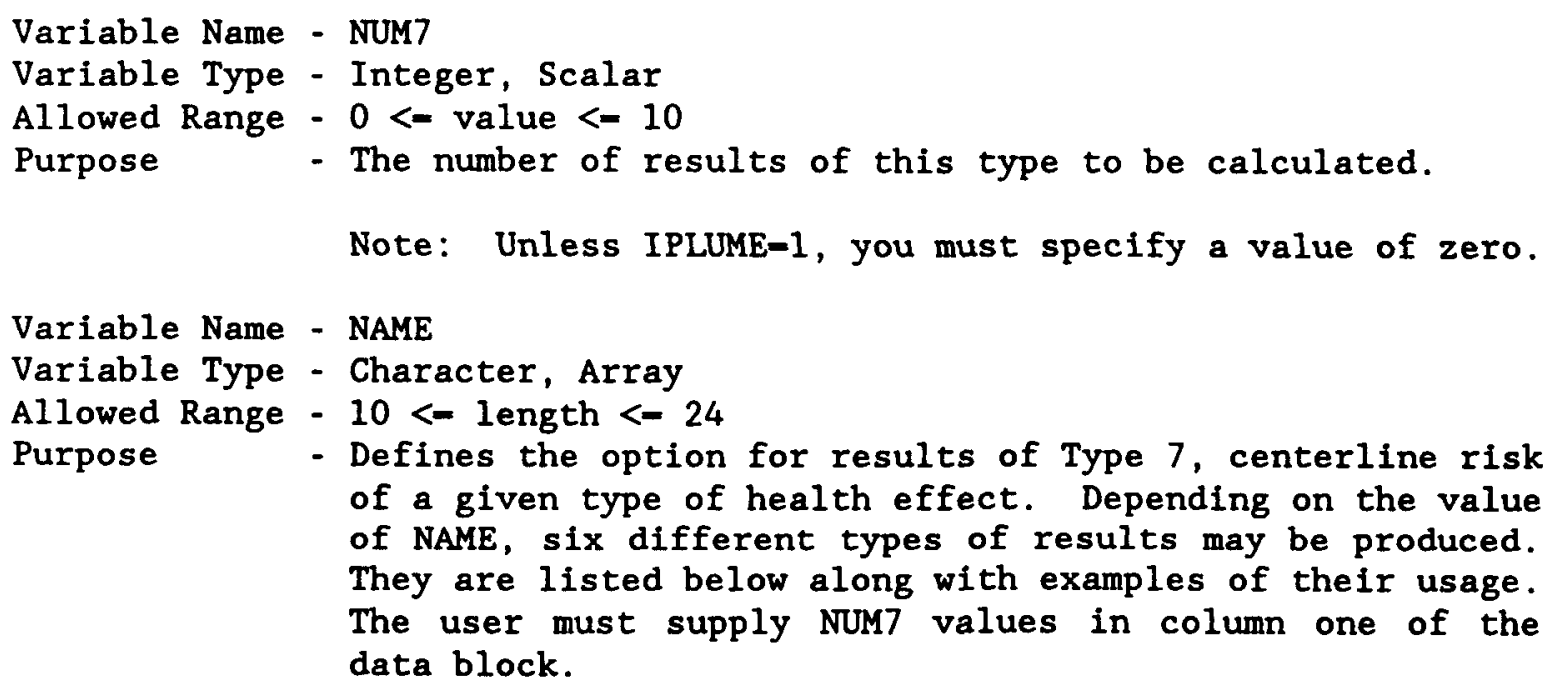

Note: In order to obtain the CCDF tables of a consequence measure requested in this section, append the character string 'CCDF' to the line requesting that result as the fourth item on the data card. The CCDF tables will be printed on the List Output File (unit 6).

Example Usage:

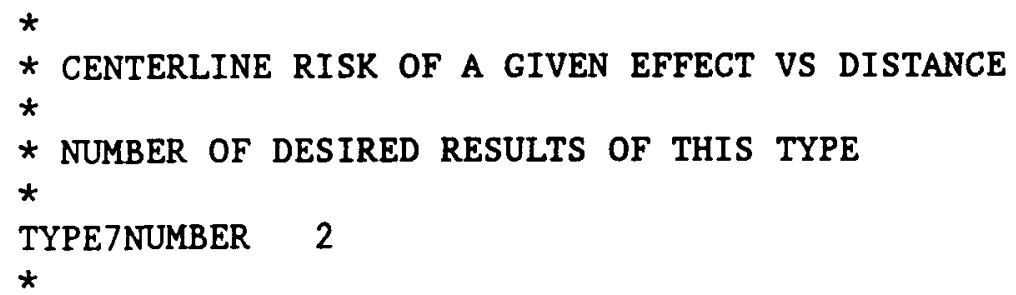




$\begin{array}{llccl}* & \text { NAME } & \text { IIDIS7 } & \text { I2DIS7 } & \\ * & & & & \\ \text { TYPE7OUT001 } & \text { 'ERL FAT/TOTAL' } & 1 & 19 & (0-50 \text { MILES }) \\ \text { TYPE7OUT002 } & \text { 'CAN FAT/TOTAL' } & 1 & 26 & (0-1000 \text { MILES })\end{array}$

\subsection{User Requested Population Weighted Risk (T8)}

Population weighted health effect risk is obtained by calculating the cases of a health effect in a certain region and then dividing by the total population in the region. It takes account of both the population distribution and the wind-rose.

Note: Any cancer results requested here are automatically produced by CHRONC so that the results can be combined by OUTPUT. When this result is produced by CHRONC, it is only a measure of the risk from direct exposure to the resident population in the specified region. The direct exposure pathways are: cloudshine, groundshine, direct inhalation, and resuspension inhalation.

The exposure period considered by the CHRONC module has a duration of one million years and therefore it predicts the cancers expected to occur over that period of time. Population weighted risk in CHRONC is calculated by determining the expected cancer cases over a million years and dividing that by the current population in the region.

The risk presented in this result does not include the societal pathways of (1) ingestion of contaminated food and water or (2) doses to decontamination workers working in the area.

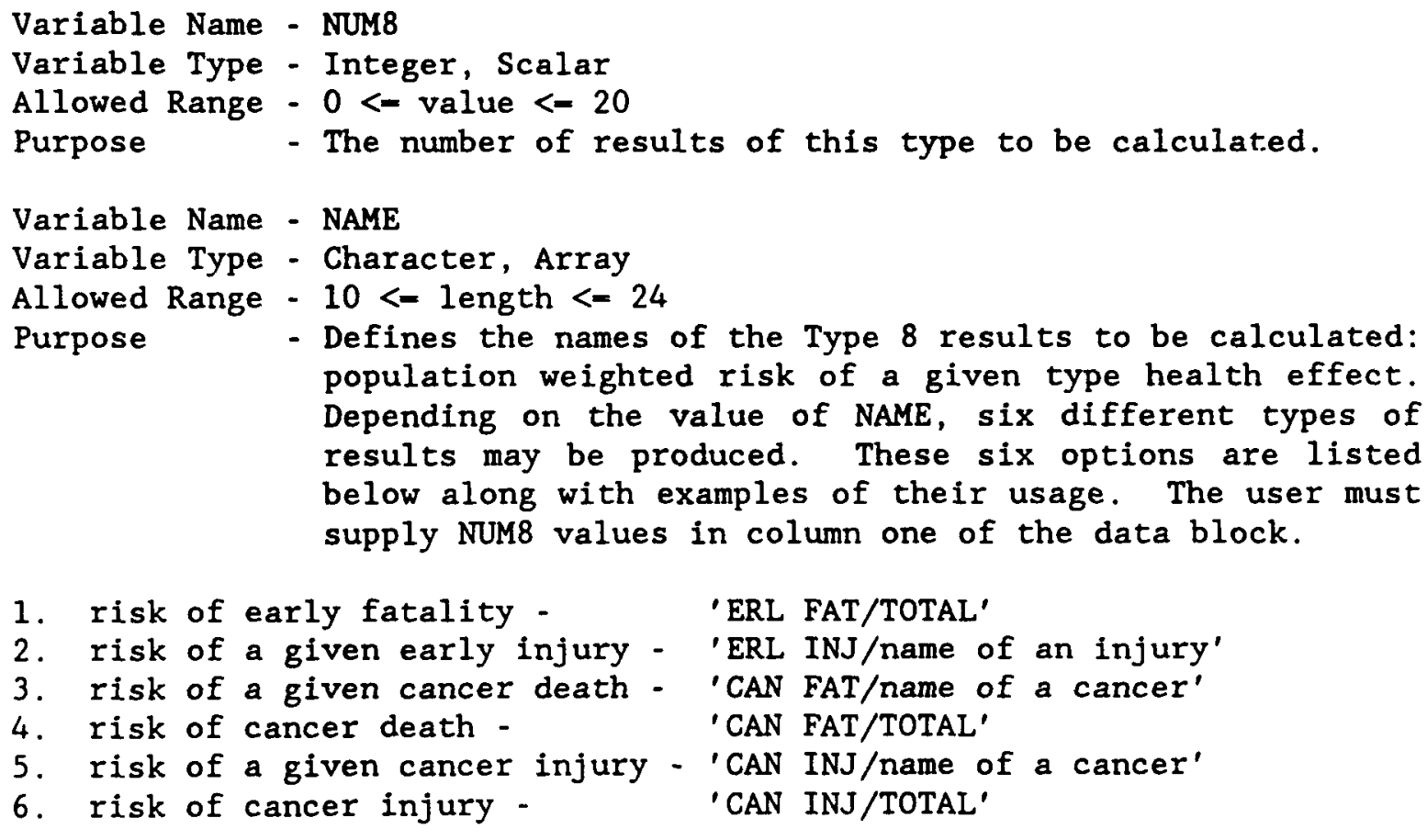
population weighted risk of a given type health effect. Depending on the value of NAME, six different types of results may be produced. These six options are listed below along with examples of their usage. The user must supply NUM8 values in column one of the data block. 


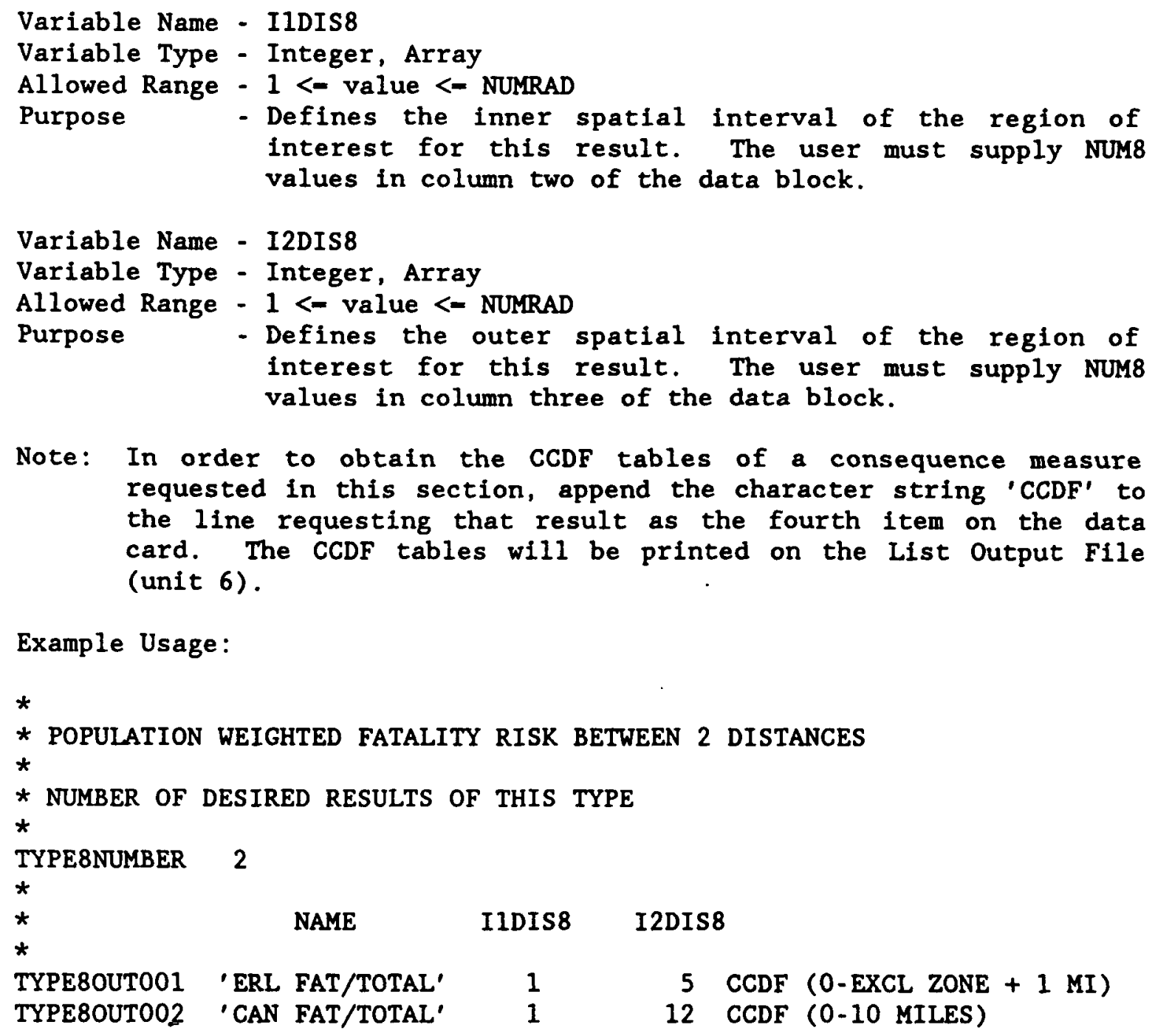

Note: In order to obtain the CCDF tables of a consequence measure requested in this section, append the character string 'CCDF' to the line requesting that result as the fourth item on the data card. The CCDF tables will be printed on the list Output File (unit 6).

Example Usage:

*

* POPUlation WEIGHTEd FATALITY RISK BETWEEN 2 DISTANCES

*

* NUMBER OF DESIRED RESULTS OF THIS TYPE

*

TYPE8NUMBER 2

*

* NAME IIDIS8 I2DIS8

*

TYPE8OUT001 'ERL FAT/TOTAL' 1 5 CCDF (0-EXCL ZONE + $1 \mathrm{MI})$

TYPE8OUT002 'CAN FAT/TOTAL' 1 12 CCDF (0-10 MILES)

\section{$\underline{\text { References }}$}

Ev85 Evans, J.S., D.W. Moeller and D.W. Cooper, Health Effects Model for Nuclear Power Plant Consequence Analysis, Sandia National Laboratories, Albuquerque, NM, 87185, NUREG/CR-4214, SAND85-7815, July 1986.

IC79 ICRP, Limits for Intake of Radionuclides by Workers, ICRP Publication 30, (Oxford: Pergamon Press, 1979). 


\subsection{CHRONC USER INPUT FILE}

\subsection{Introduction to CHRONC}

The CHRONC module simulates the events which occur following the emergency phase time period modeled by EARLY. Various long-term protective actions may be taken during this time period to limit radiation doses to acceptable levels. The parameters defining these protective actions are under user-control and all of them are described in this chapter.

CHRONC calculates the individual health effects that result from both (1) direct exposure to contaminated ground and from inhalation of resuspended materials as well as (2) indirect health effects caused by the consumption of contaminated food and water by individuals who could reside both on and off of the computational grid. Both the direct and indirect exposure pathways are integrated over an essentially infinite period following the accident. That is, population dose and the resulting health effects are calculated for an exposure period which extends beyond the lifetime of those individuals alive at the time of the accident.

CHRONC also calculates the economic costs of the long-term protective actions as well as the cost of the emergency response actions which were modeled in the EARLY module of MACCS.

It is up to the user to specify the various parameters needed for these calculations. There are no default values for the parameters described in this section. In addition to specifying the characteristics of the model, the user has complete control over the output produced by CHRONC and must explicitly specify which results are to be produced. All of this information is supplied through the User Input File to CHRONC and all of the input parameters are described in this chapter.

The User Input File for the CHRONC module consists of eleven data blocks which define the simulation and an additional five data blocks used to specify the output desired by the user. These sixteen data blocks are described below. The other input data requirements and the output requirements of the CHRONC module are also discussed below.

\subsection{Problem Identification Data}

The problem identification data block is used to identify the CHRONC input data file and the calculations which are being performed.

Variable Name - CHNAME

Variable Type - Character, Scalar

Allowed Range - 1 <- length <- 80

Purpose - Describes this particular CHRONC input data file. Choose a text string that briefly describes the input data file; the assumptions and model choices of this particular application of the CHRONC module. 
This text will be printed on all of the MACCS list output as an aid in identification of the results. It is not necessary to include the date of the run since that information is automatically included.

Example Usage:

CHCHNAME001 'SURRY CHRONC INPUT FOR FINAL NUREG-1150 CALCULATIONS'

\subsection{Emergency Response Cost Data}

The emergency response cost data block is used to define the compensation costs for people who are subject to the emergency actions of evacuation, sheltering, or relocation. Evacuation is solely under the control of models defined in the EARLY module. Relocation of individuals can occur either during the EARLY emergency phase or during the CHRONC intermediate phase period. In the presentation of economic cost results (Section $3.15)$, the cost of evacuation and the cost of relocation are summed together and there is no possibility of examining them individually.

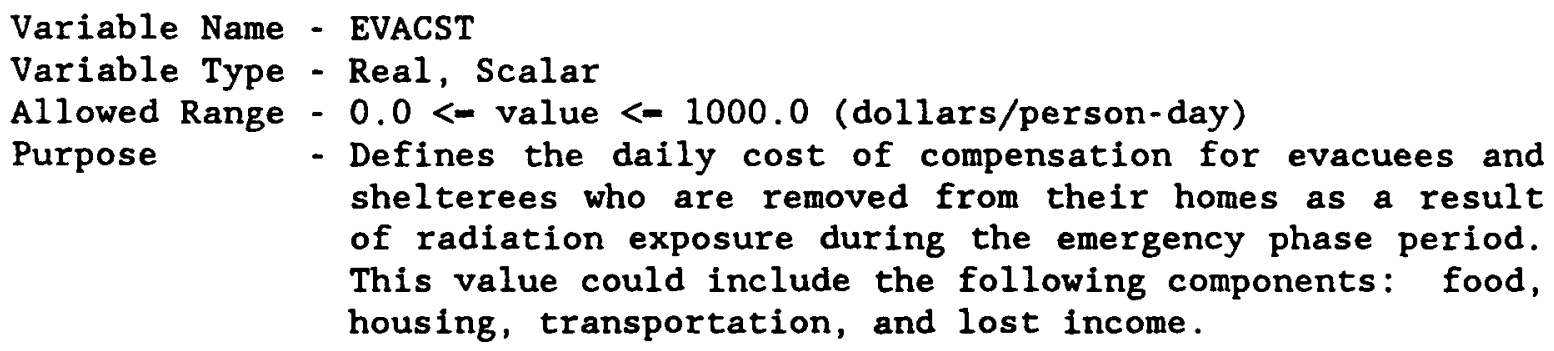

Example Usage:

CHEVACSTO01 27.00

Variable Name - RELCST

Variable Type - Real, Scalar

Allowed Range - 0.0 <- value <- 1000.0 (dollars/person-day)

Purpose - Defines the daily cost of compensation for individuals relocated due to either hot-spot or normal reiocation during the emergency phase period, or due to intermediate phase relocation modeled by CHRONC. This value could include the following components: food, housing, trans. portion, and lost income.

Example Usage:

CHRELCST001 27.00

\subsection{Long-Term Protective Action Data}

The long-term protective action data block defines the intermediate and long-term action time periods as well as the maximum doses which people are allowed to receive during these periods. The maximum allowable doses defined here are used to determine the need for relocation, decontamination, interdiction or condemnation. 


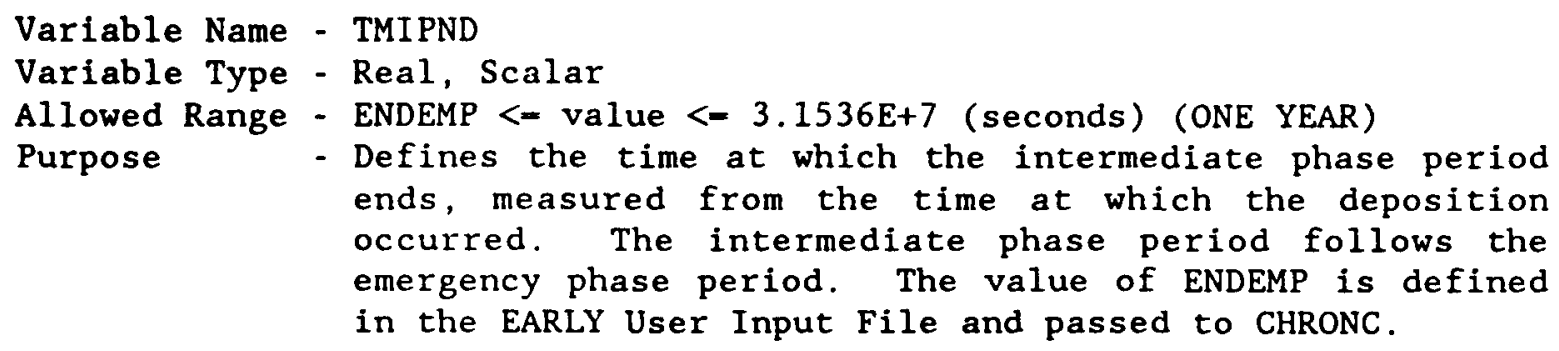

Example Usage:

CHTMIPND001 604800. (7 DAYS, NO INTERMEDIATE PHASE)

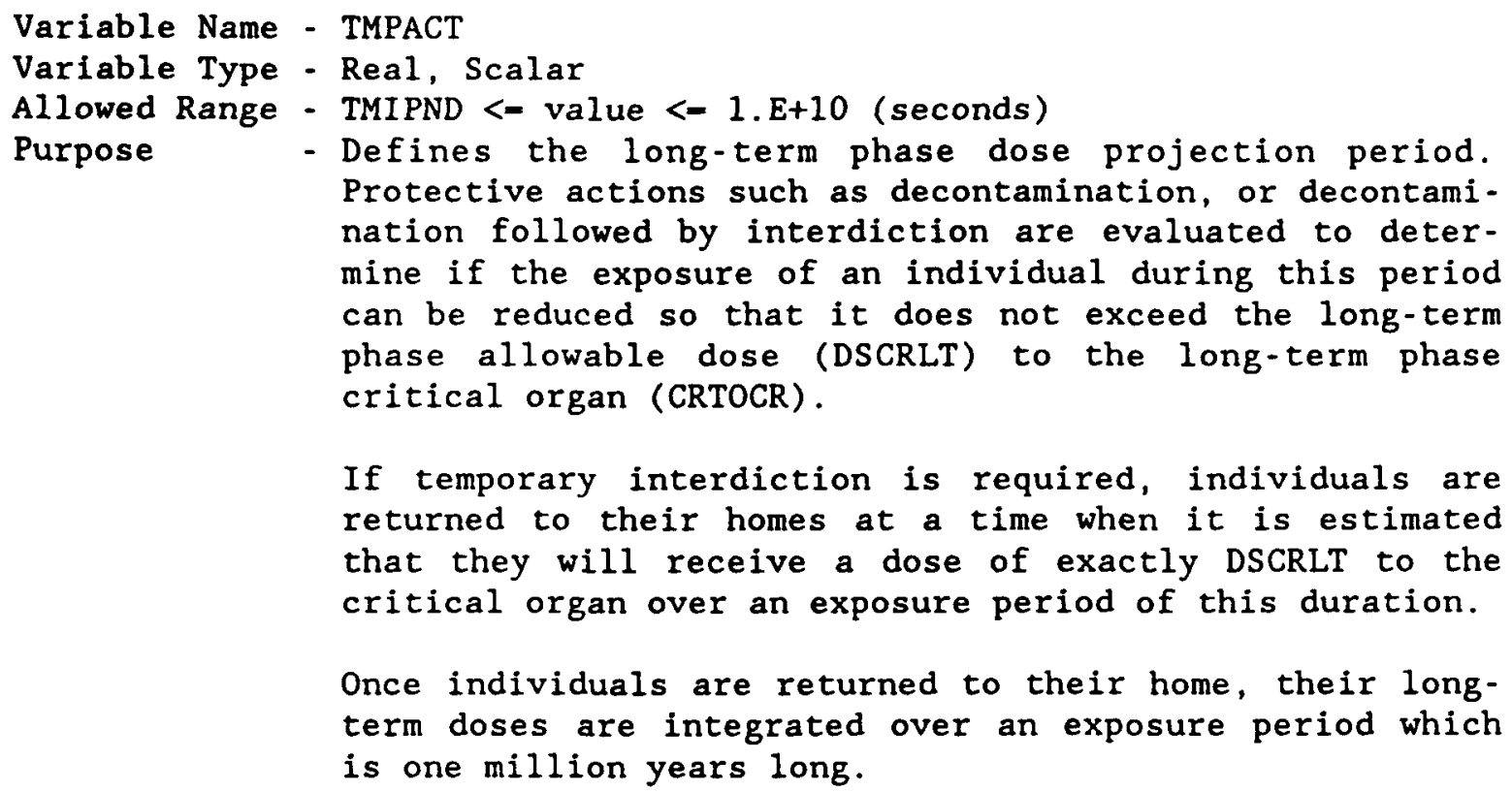

If temporary interdiction is required, individuals are returned to their homes at a time when it is estimated that they will receive a dose of exactly DSCRLT to the critical organ over an exposure period of this duration.

Once individuals are returned to their home, their longterm doses are integrated over an exposure period which is one million years long.

Example Usage:

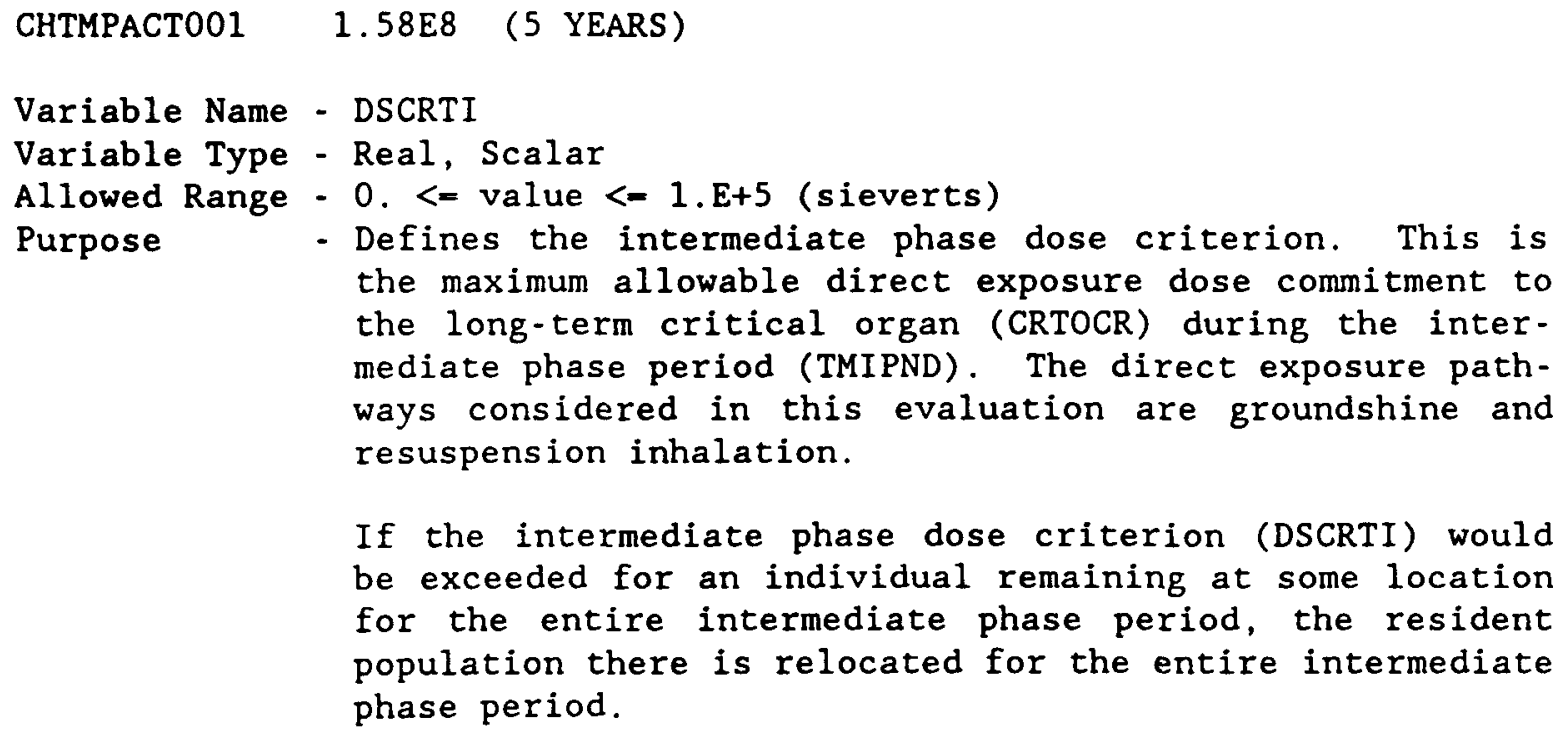
the maximum allowable direct exposure dose commitment to the long-term critical organ (CRTOCR) during the intermediate phase period (TMIPND). The direct exposure pathways considered in this evaluation are groundshine and resuspension inhalation.

If the intermediate phase dose criterion (DSCRTI) would be exceeded for an individual remaining at some location for the entire intermediate phase period, the resident population there is relocated for the entire intermediate phase period. 
Example Usage:

CHDSCRTIO01 1.0E5 (NO INTERMEDIATE PHASE RELOCATION)

Variable Name - DSCRLT

Variable Type - Real, Scalar

Allowed Range - 1.E-20<- value <- 1.E+5 (sieverts)

Purpose

- Defines the long-term phase dose criterion. This is the maximum allowable direct exposure dose commitment to the long-term critical organ (CRTOCR) during the long-term phase action period (TMPACT).

If this dose criterion would be exceeded in any spatial element during the long-term action period, mitigative actions such as decontamination or decontamination followed by temporary interdiction would be employed in order to limit the dose to the critical organ so that the allowable dose level is not exceeded in that spatial grid element. The long-term critical organ is defined below in this data block.

If it is not possible to reduce doses to this level in a cost-effective manner, the property is condemned and the resident population is permanently relocated.

Example Usage:

CHDSCRLT001 0.04 (2 REM IN FIRST YEAR, 0.5 REM PER YEAR FOR YRS 2-5)

Variable Name - CRTOCR

Variable Type - Character, Scalar

Allowed Range - $2<=$ length <- 10

Purpose - - Defines the long-term phase critical organ. If the total direct exposure dose commitment to this organ in a grid element would exceed the dose criteria in either the intermediate phase period (TMIPND) or the long-term phase period (TMPACT), protective actions would be taken in order to limit that dose to acceptable levels.

Example Usage:

CHCRTOCROO1 'EDEWBODY'

\subsection{Decontamination Plan Data}

The decontamination plan data block defines the decontamination actions which may be taken during the long-term period in order to reduce doses to acceptable levels. These data define the decontamination strategies which are possible, their effectiveness and their cost. Each decontamination level represents an alternative decontamination strategy that would reduce the projected long-term groundshine and resuspension doses by a factor called the dose reduction factor. Up to three levels of decontamination can be defined. 
The object of decontamination is to reduce projected doses below the long-term dose criterion in a cost-effective manner. If the maximum decontamination level is insufficient to restore an area to immediate habitability, a period of temporary interdiction following that maximum decontamination level is considered in order to allow for dose reduction due to radioactive decay and weathering. If the property cannot be made habitable within thirty years or if the cost of reclaiming the habitability of the property exceeds the cost of condemning it, the property will be condemned and permanently withdrawn from use.

Decontamination cost is divided into two categories and these two types of cost are calculated separately. Area dependent decontamination cost represents the cost of farmland decontamination in a grid element. Area dependent decontamination is a function of the area of the grid element devoted to agriculture (see FRCFRM, defined in Section 3.9). Population dependent decontamination represents the cost of non-farmland decontamination. Population dependent decontamination cost is a function of the population residing in the grid element. The strategy of decontamination within a grid element is independent of the type of area being decontaminated.

For a given decontamination level, the same decontamination time and decontamination effectiveness apply to both farmland and non-farmland, but the two costs are unique and are maintained independently for each type of decontamination. Due to the requirement that the recovery of property must be cost-effective, it is possible, in a given element, that decontamination of non-farmland is performed, but farmland is instead condemned.

Decontamination of a grid element serves to reduce the contamination level in that element by the dose reduction factor associated with the decontamination effort which is being applied. Everything else being equal, a decontamination factor of ten will cause the integrated dose over any exposure period to be reduced by a factor of ten.

During the decontamination period, which is assumed to begin at the end of the intermediate phase time period (beginning of the long-term phase period) population from areas that will be decontaminated is assumed to be relocated to uncontaminated areas, and the associated cost from loss of use is calculated in the same manner as temporary interdiction.

While engaged in cleanup efforts, decontamination workers are assumed to wear respiratory protection devices. Therefore, they accumulate only groundshine doses. These doses and the cancer fatalities that they induce contribute to the aggregated doses and cases of cancer fatalities tabulated in the MACCS output. 
Decontamination reduces direct exposure doses (both groundshine and resuspension) caused by contamination of land and buildings. Many decontamination processes (e.g., plowing, fire-hosing) reduce groundshine and resuspension doses by mixing surface contamination down into the ground. Since these processes may not move contamination out of the root zone, MACCS assumes that farmland decontamination reduces direct exposure doses to farmers without reducing uptake of radioactivity by root systems. Thus, decontamination of farmland does not reduce the ingestion doses produced by consumption of crops contaminated by root uptake.

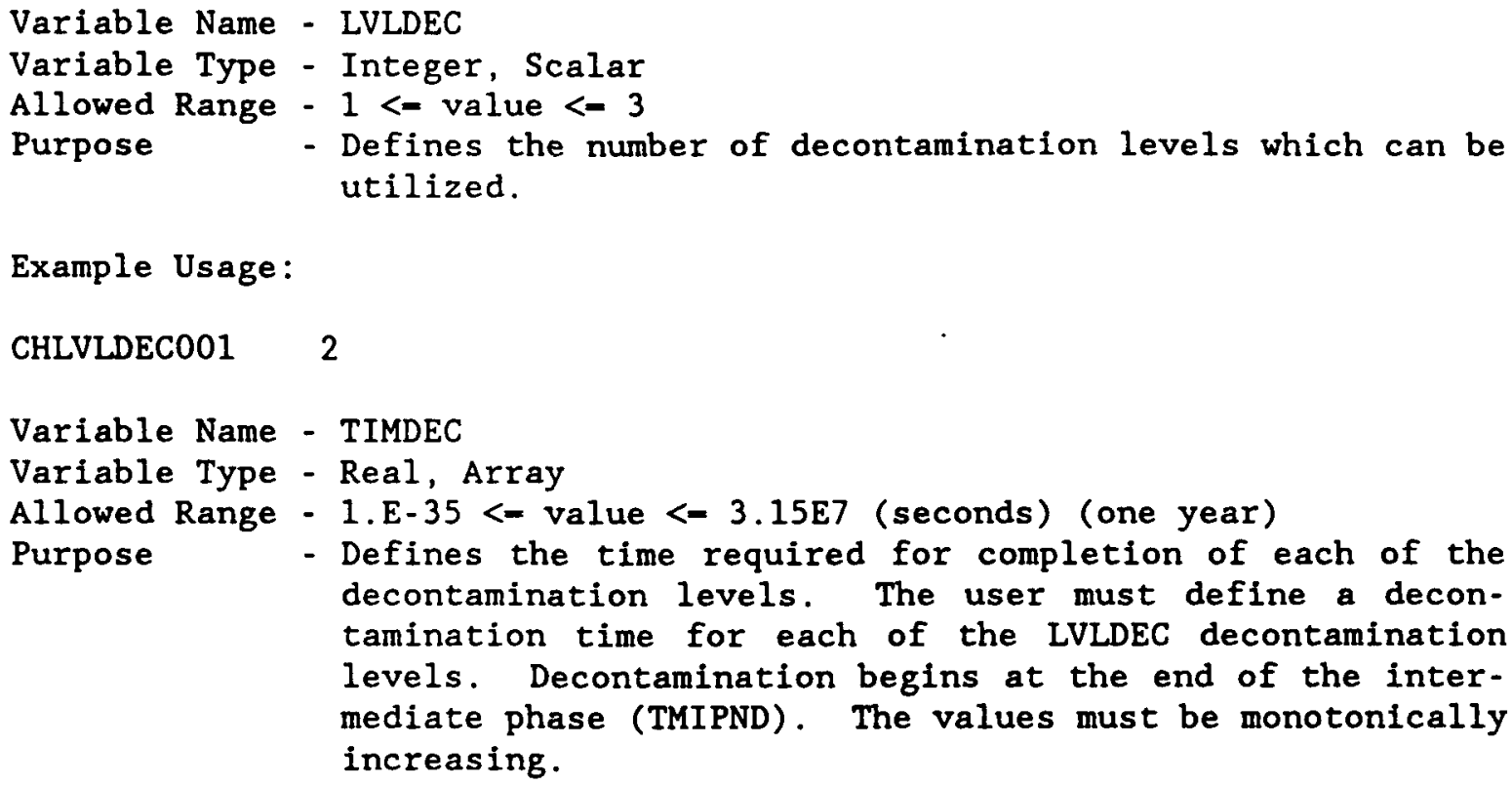

Example Usage:

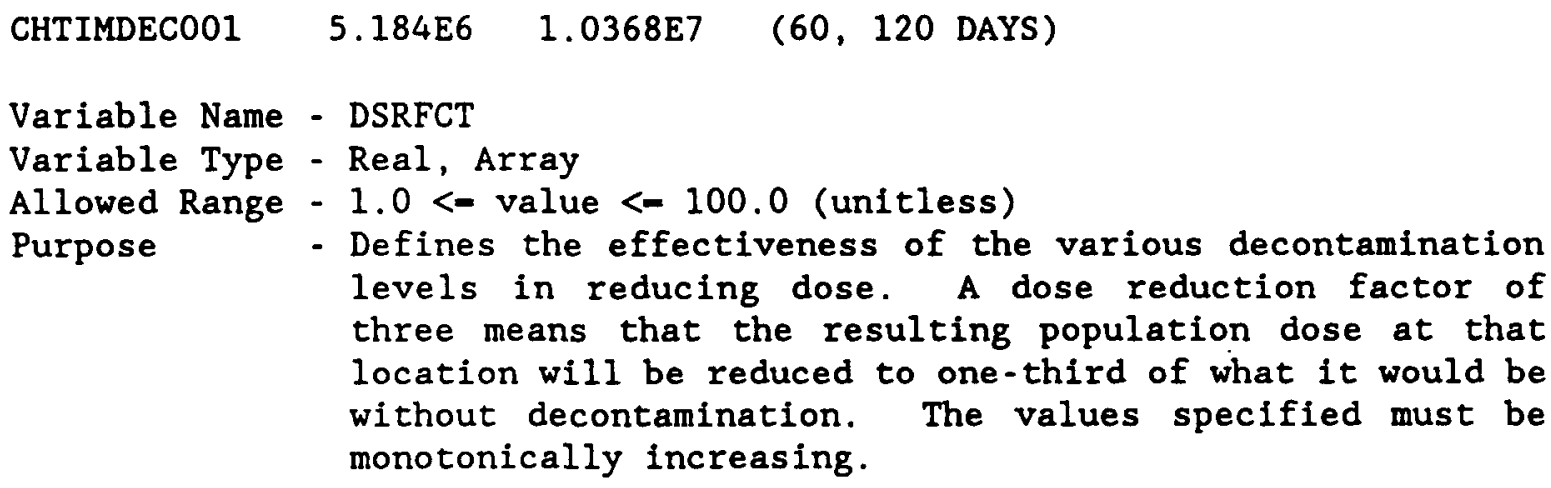

Example Usage:

CHDSRFCT001 3. 15.

Variable Name - CDFRM

Variable Type - Real, Array

Allowed Range $-0.0<=$ value $<-1 . E+5$ (dollars/hectare) 


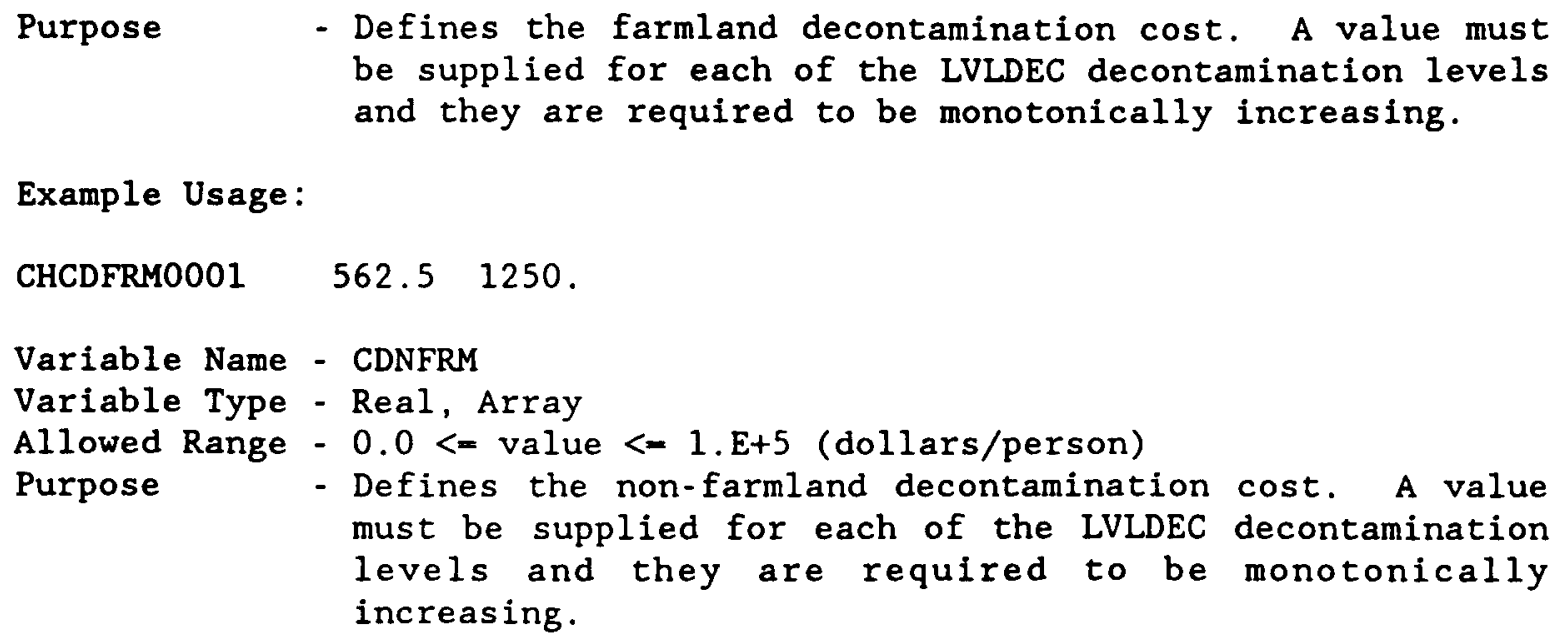
be supplied for each of the LVLDEC decontamination levels and they are required to be monotonically increasing.

Example Usage:

CHCDFRMO001 562.51250.

Variable Name - CDNFRM

Variable Type - Real, Array

Allowed Range - $0.0<=$ value $<=1 . E+5$ (dollars/person)

Purpose - Defines the non-farmland decontamination cost. A value must be supplied for each of the LVLDEC decontamination levels and they are required to be monotonically increasing.

Example Usage:

CHCDNFRM001 3000. 8000 .

Note to user: The remaining parameters in this section are used for the sole purpose of calculating the dose received by decontamination workers.

Variable Name - FRFDL

Variable Type - Real, Array

Allowed Range - $0.0<=$ value $<=1.0$ (unitless)

Purpose - Defines the fraction of the farmland decontamination cost that is due to labor. A value must be supplied for each of the LVLDEC decontamination levels.

Example Usage:

$\begin{array}{lll}\text { CHFRFDL0001 } & .3 & .35\end{array}$

Variable Name - FRNFDL

Variable Type - Real, Array

Allowed Range - $0.0<=$ value $<=1.0$ (unitless)

Purpose - Defines the fraction of the non-farmland decontamination cost that is due to labor. A value must be supplied for each of the LVLDEC decontamination levels.

Example Usage:

CHFRNFDLO01 $\quad .7 \quad .5$

Variable Name - TFWKF

Variable Type - Real, Array

Allowed Range - $0.0<$ value < 1.0 (unitless)

Purpose - Defines the fraction of the decontamination period

(TIMDEC) that a farmland decontamination worker spends in 
the contaminated area. A value must be supplied for each of the LVLDEC decontamination levels.

Example Usage:

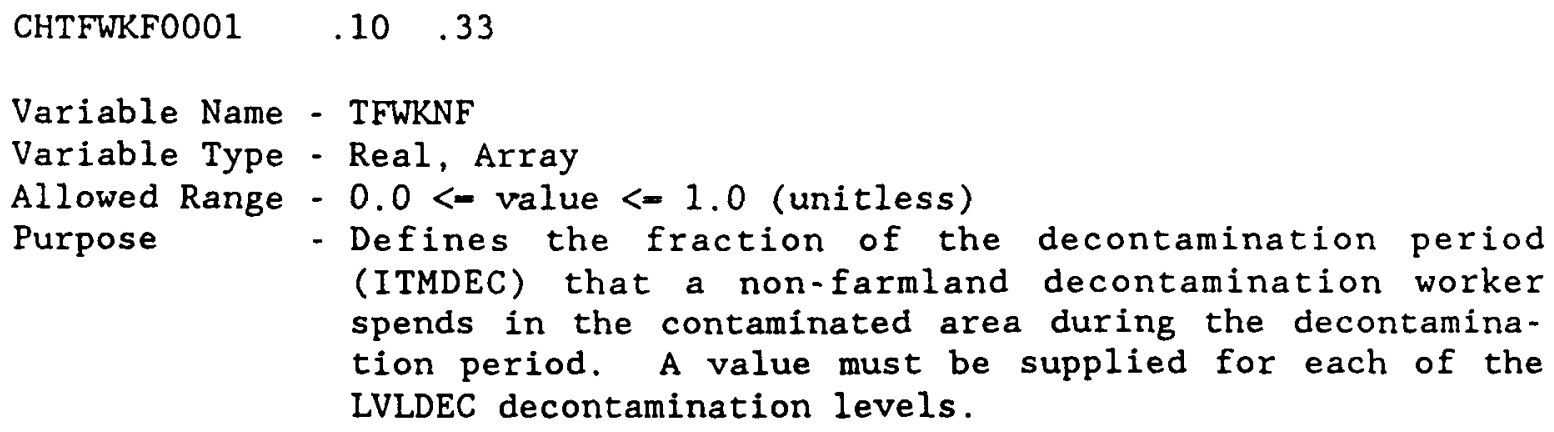

\subsection{Interdiction Plan Cost Data}

The Interdiction Plan Cost Data block defines the parameters needed for the calculation of the cost of interdiction. The data supplied here are combined with data in the Site Data File and the Regional Characteristics Data in the course of the calculations.

The model used in MACCS for assessing the cost of interdiction is based on the model described in Appendix VI of the Reactor Safety Study [NR75] as selected by Rick Burke [Bu84]. It is currently applied to calculate the economic cost of loss of use during both decontamination and temporary interdiction periods.

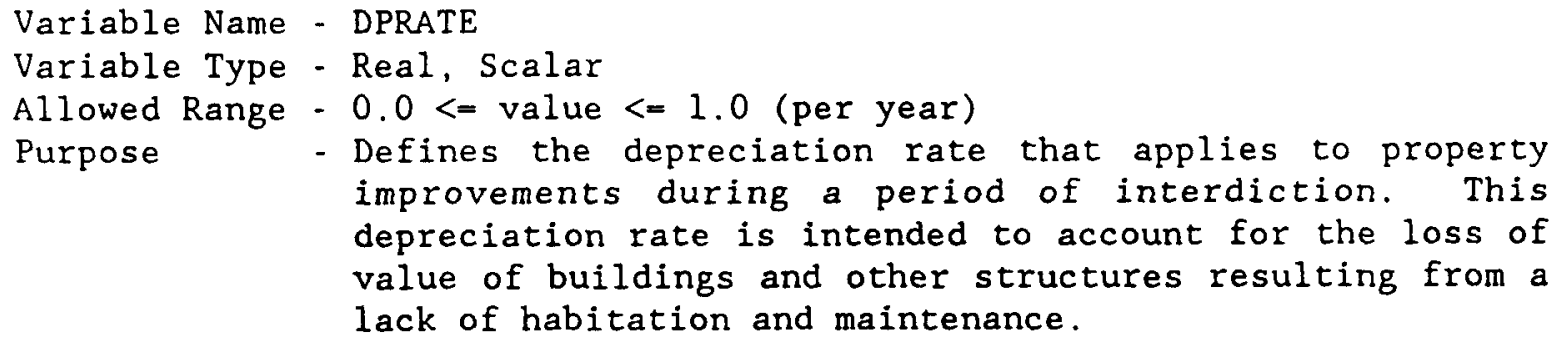




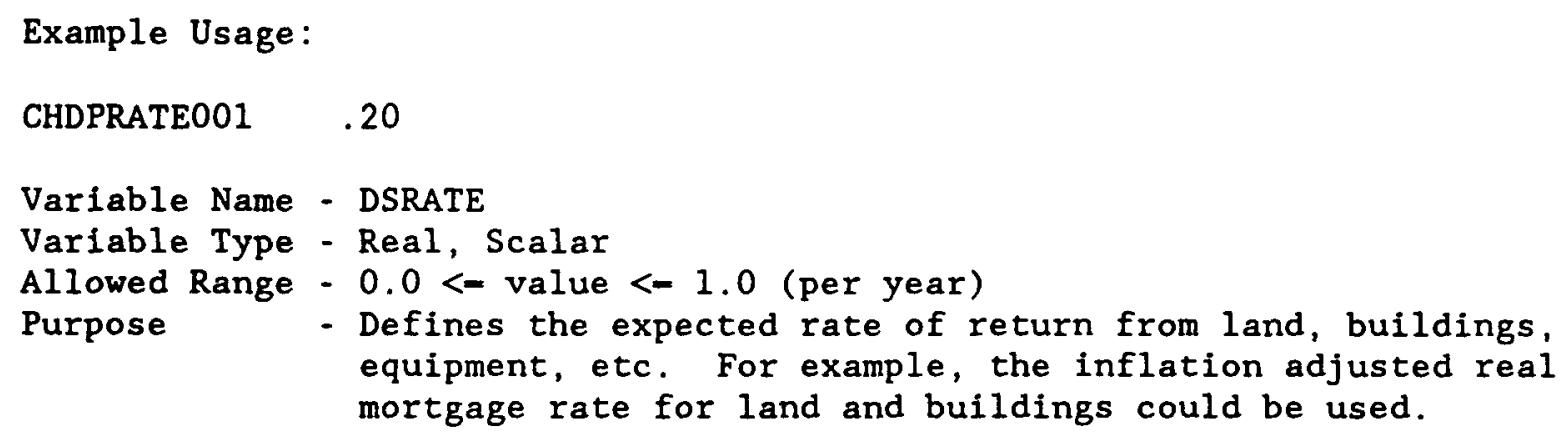

Example Usage:

CHDSRATE001 . .12

Variable Name - POPCST

Variable Type - Real, Scalar

Allowed Range - 1.E-35<- value <- 1.E+6 (dollars/person)

Purpose - Defines the per-capita removal cost for temporary or permanent relocation of population and businesses in a region rendered uninhabitable during the long-term phase time period. This cost is assessed if any of the following actions are required: decontamination alone, decontamination followed by interdiction, or condemnation. This value should be derived in a way that takes account of both personal and corporate income losses for a transitional period as well as moving expenses.

Example Usage:

CHPOPCST001 5000 .

\subsection{Groundshine Weathering Data}

The groundshine weathering definition data block defines the groundshine weathering equation, Gale's equation [Ga64]. The groundshine weathering relationship is defined as

$$
\begin{aligned}
\mathrm{GW}(t)= & \operatorname{GWCOEF}(1) * \exp [-\ln (2) * t / \operatorname{TGWHLF}(1)]+\ldots+ \\
& \operatorname{GWCOEF}(n) * \exp [-\ln (2) * t / \operatorname{TGWHLF}(n)]
\end{aligned}
$$

where $G W(t)$ represents the groundshine weathering at time $t$, given the weathering coefficients, GWCOEF, and the weathering half-lives, TGWHLF. The user must specify the number of terms, $n$, in the relationship and values for the weathering coefficients and the weathering half-lives.

Variable Name - NGWTRM

Variable Type - Integer, Scalar

Allowed Range - $1<=$ value $<-2$

Purpose - Defines the number of terms in the groundshine weathering relationship. 


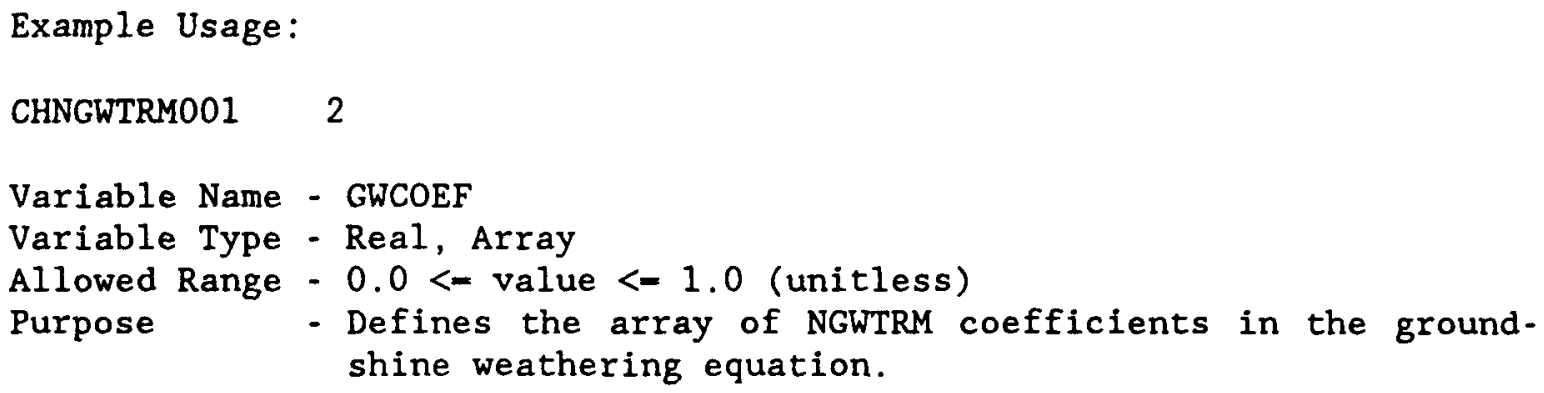

Example Usage:

CHGWCOEFOO1 $0.5 \quad 0.5 \quad$ (JON HELTON)

Variable Name - TGWHLF

Variable Type - Real, Array

Allowed Range - 1.E-35 <- value <- $1 . E+12$ (seconds)

Purpose - Defines the array of NGWTRM half-lives in the groundshine weathering equation.

Example Usage:

CHTGWHLFOO1 1.6E7 2.8E9 (JON HELTON)

\subsection{Resuspension Weathering Data}

The resuspension weathering definition data block defines the resuspension weathering equation. The resuspension weathering relationship is defined as

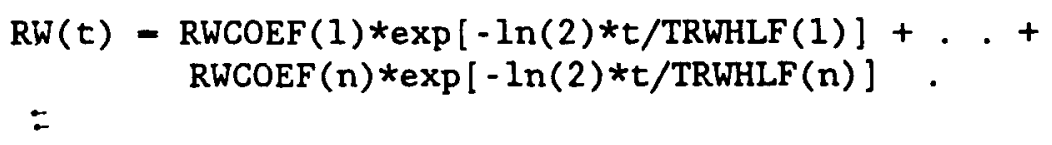

where $R W(t)$ represents the resuspension weathering at time $t$, given the weathering coefficients, RWCOEF, and the weathering half-lives, TRWHLF. The user must specify the number of terms, $n$, in the relationship and the values for the weathering coefficients and weathering half-lives.

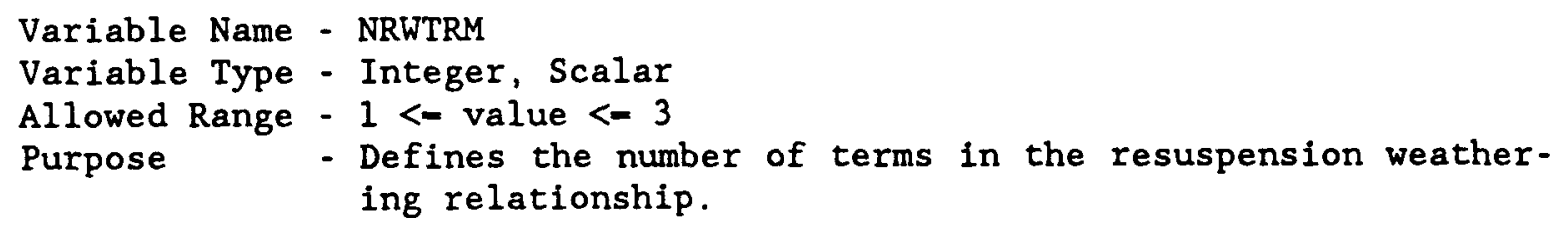

Example Usage:

CHNRWTRMOO1 3

Variable Name - RWCOEF

Variable Type - Real, Array

Allowed Range - $0.0<=$ value <- 1.0 (per meter)

Purpose - Defines the array of NRWTRM coefficients in the resuspension weathering equation. 


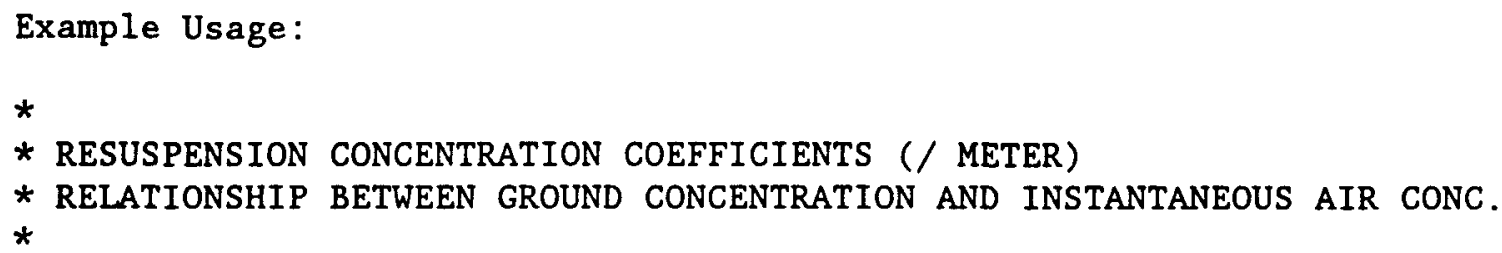

\subsection{Regional Characteristics Data}

The Regional Characteristics data block defines the aggregate economic and agricultural characteristics of the area surrounding the accident site.

If mitigative actions are necessary to restrict exposure to allowable levels, this data is used to evaluate the cost-effectiveness of those actions. For instance, if the average property values supplied here indicate that reclaiming a piece of land will cost more than the cost of condemning it, the land will be condemned instead of reclaimed.

Once a set of actions at a certain distance is decided upon, these actions may be "rotated" around the circle if the user has chosen the option of IPLUME-1 or IPLUME-2 as defined in Section 2.2. Now, in some of these wind directions, there could be an extremely valuable property. It is implicit in our calculational method that the cost-effectiveness test can not take account of this non-homogeneity of the valuation field because of the need for performing a rotation of the chosen actions around the circle.

The choice of actions is always determined by the aggregate characteristics of the region, but the calculation of the resulting consequence measures can be done in two different ways depending on the value of the input variable POPFLG which is defined in Section 2.3.

When a uniform population distribution is being used (POPFLG=UNIFORM), all of data in this section is used for the calculation of the CHRONC consequence measures. However, if the user requests that a Site Data 
File be utilized (POPFLG-FILE), the first four parameters defined in this section are not actually used in the calculations though they are all required to be supplied. When a site Data File is being utilized, the values corresponding to these four parameters are obtained from the Site Data File for each individual spatial element on the computational grid.

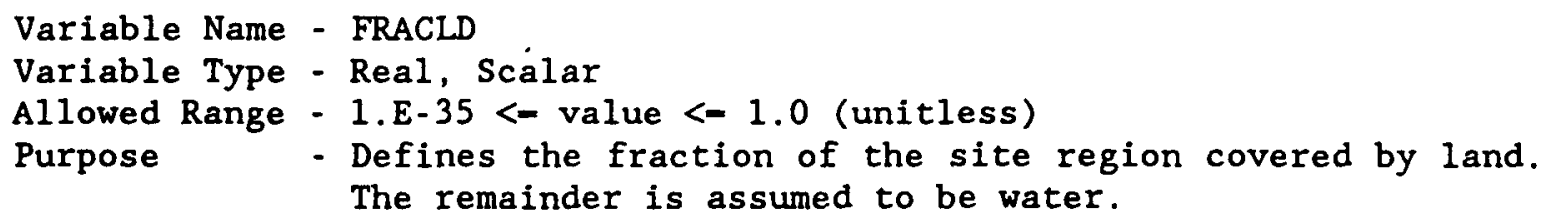

Note: If a site Data File is being used (POPFLG-FILE), the value of this input parameter is not used by the code, nevertheless a valid parameter value must be supplied.

Example Usage:

CHFRACLDO01 0.95

Variable Name - FRCFRM

Variable Type - Real, Scalar

Allowed Range - 1.E-35<- value <- 1.0 (unitless)

Purpose - Defines the average fraction of land in the region devoted to farm production. The land area under cultivation is as follows:

cultivated-area - total-area * FRACLD * FRCFRM

Note: If a Site Data File is being used (POPFLG-FILE), the value of this input parameter is not used by the code, nevertheless a valid parameter value must be supplied.

Example Usage:

CHFRCFRMO01 0.382 VIRGINIA

Variable Name - FRMPRD

Variable Type - Real; Scalar

Allowed Range - $0.0<-$ value <- 1.E+5 (dollars/hectare)

Purpose - Defines the value of the average annual farm production (gross sales) in the region.

Note: If a Site Data File is being used (POPFLG-FILE), the value of this input parameter is not used by the code, nevertheless a valid parameter value must be supplied. 


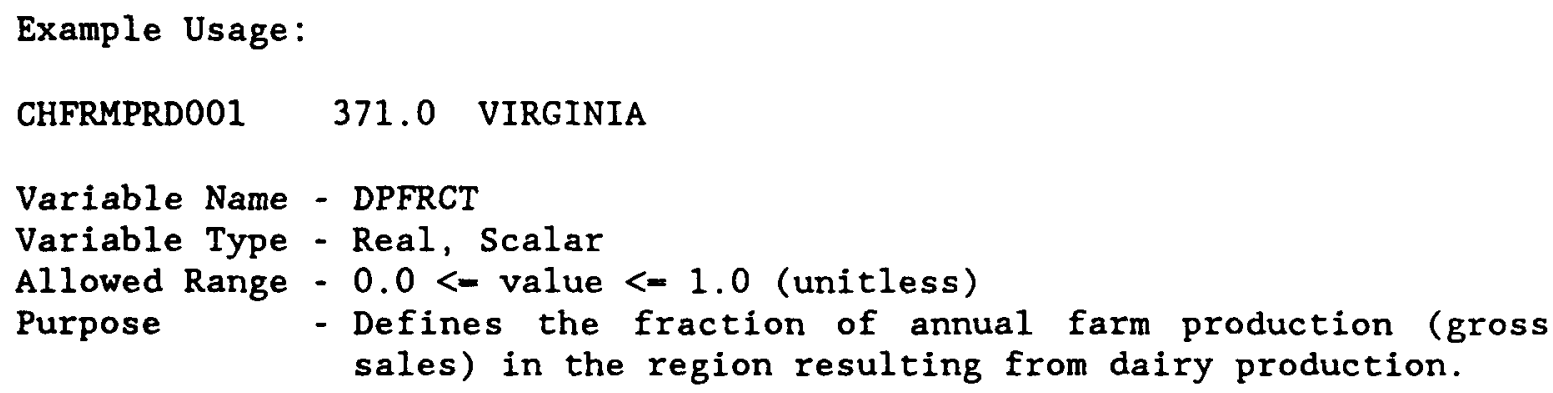

Note: If a Site Data File is being used (POPFLG-FILE), the value of this input parameter is not used by the code, nevertheless a valid parameter value must be supplied.

Example Usage:

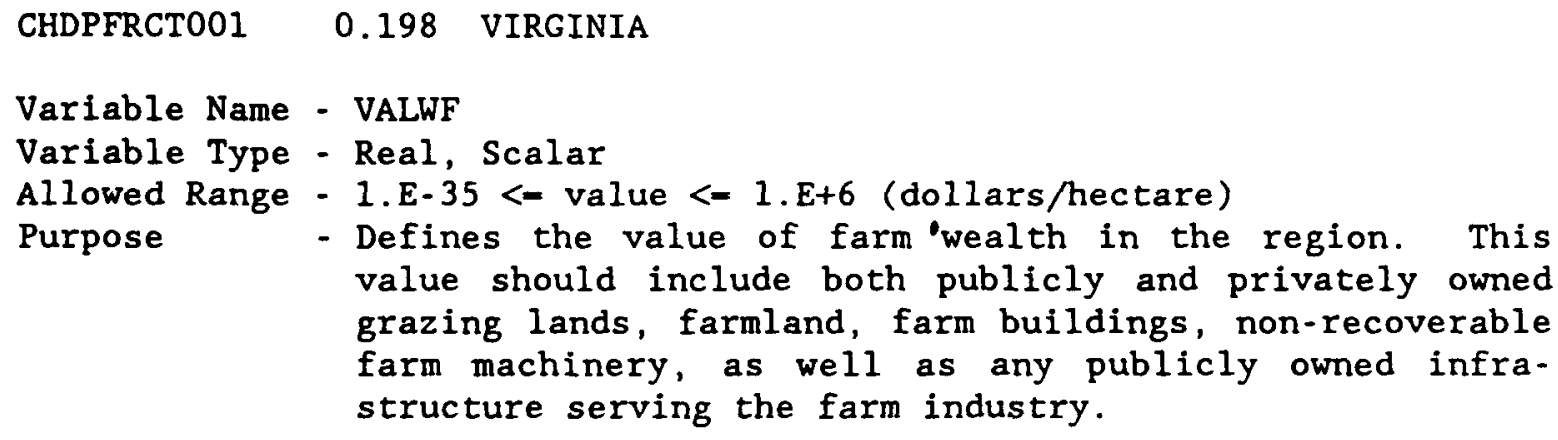
value should include both publicly and privately owned grazing lands, farmland, farm buildings, non-recoverable farm machinery, as well as any publicly owned infrastructure serving the farm industry.

Example Usage:

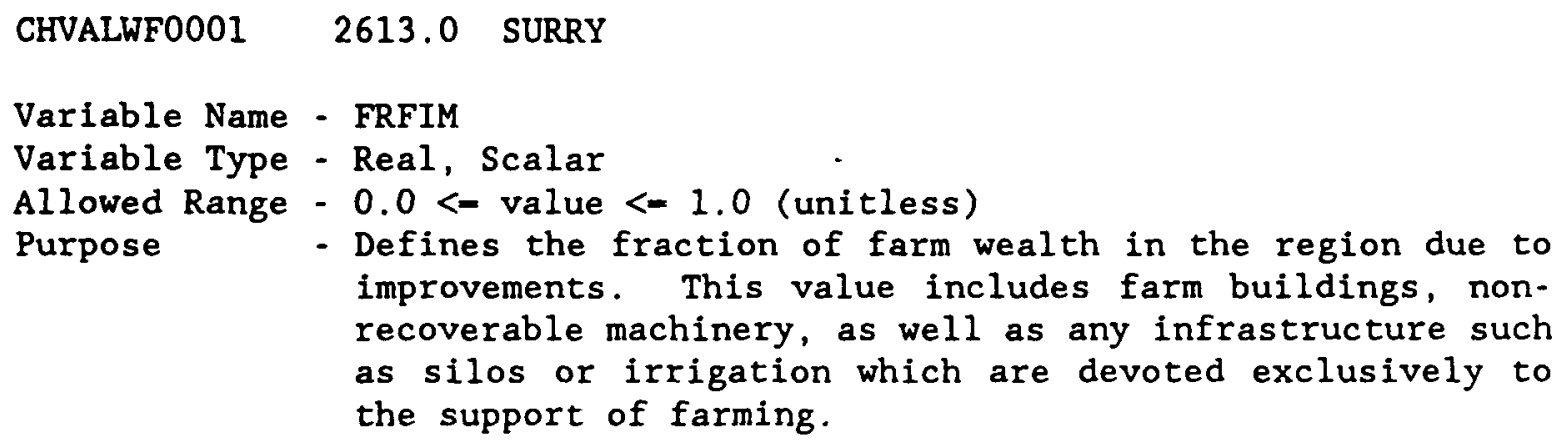

Example Usage:

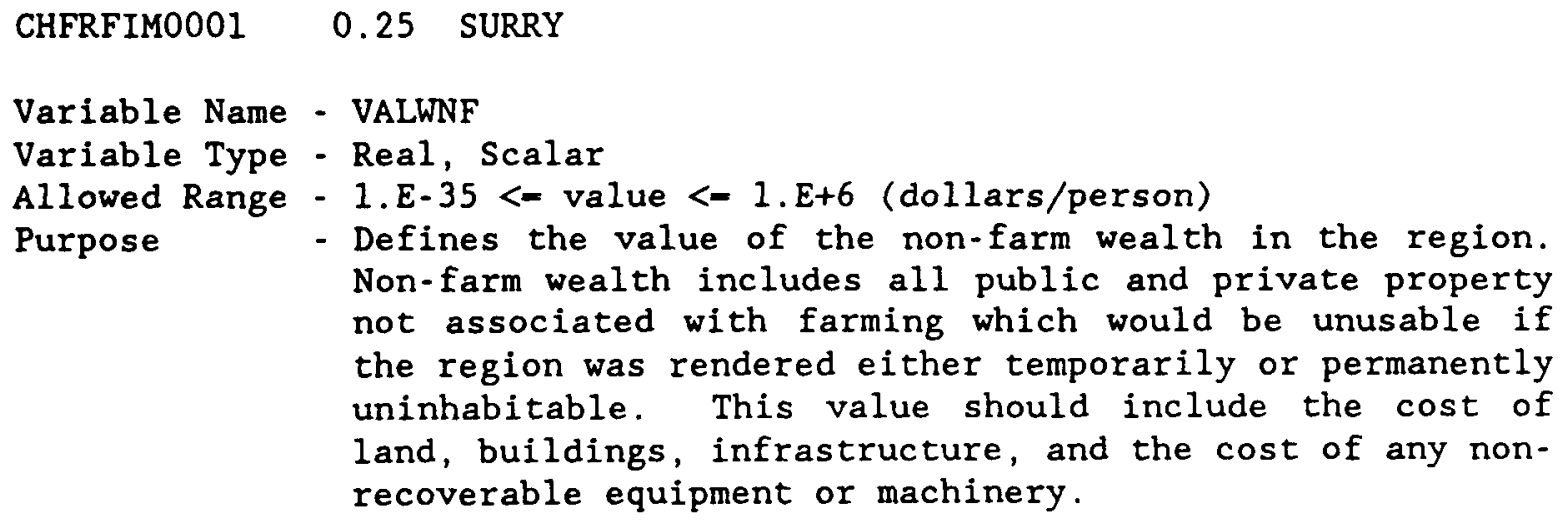


Example Usage:

CHVALWNF001 84000. VIRGINIA

Variable Name - FRNFIM

Variable Type - Real, scalar

Allowed Range - $0.0<-$ value <- 1.0 (unitless)

Purpose - Defines the fraction of non-farm wealth in the region due to improvements. This value includes buildings, infrastructure such as roads and utilities as well as any nonrecoverable equipment or machinery.

Example Usage:

CHFRNFIM001 $\quad 0.8$

\subsection{Ingestion Transfer Factors Data}

When radioactive material is deposited on land some fraction of this material may make its way through the food chain and ultimately be consumed by man in the form of contaminated food or drinking water. The ingestion pathway is modeled in MACCS as a series of transfer processes which the material must traverse between the time of deposition and the consumption of the contaminated food products by humans and which decrease the amount of material passed on to the next step.

To calculate the population dose resulting from the accumulated contamination of an area, it is necessary to know the efficiency of the entire food chain taken as a whole in transferring material from the ground to man. MACCS calculates the overall efficiency of the two ingestion pathways (food and water) by multiplication of all of the individual transfer factors which are described below.

Variable Name - NFICRP

Variable Type - Integer, Scalar

Allowed Range - $1<-$ value <- 10

Purpose - Defines the number of crop categories which will be used by the food pathway model.

Variable Name - NAMCRP

Variable Type - Character, Array

Allowed Range - $6<=$ length <- 20

Purpose - Defines the name of a crop category used in the food pathway model. The user must supply NFICRP names in column one of the data block.

Note: Within the code, there is a distinction between two types of crops: those harvested at the end of the growing season, and those harvested continuously over the entire growing season. The first seven letters of the crop names supplied here are used to distinguish between these two types of crops. 
If a crop's name begins with 'PASTURE', it is harvested continuously; if it doesn't, it is harvested at the end of the growing season.

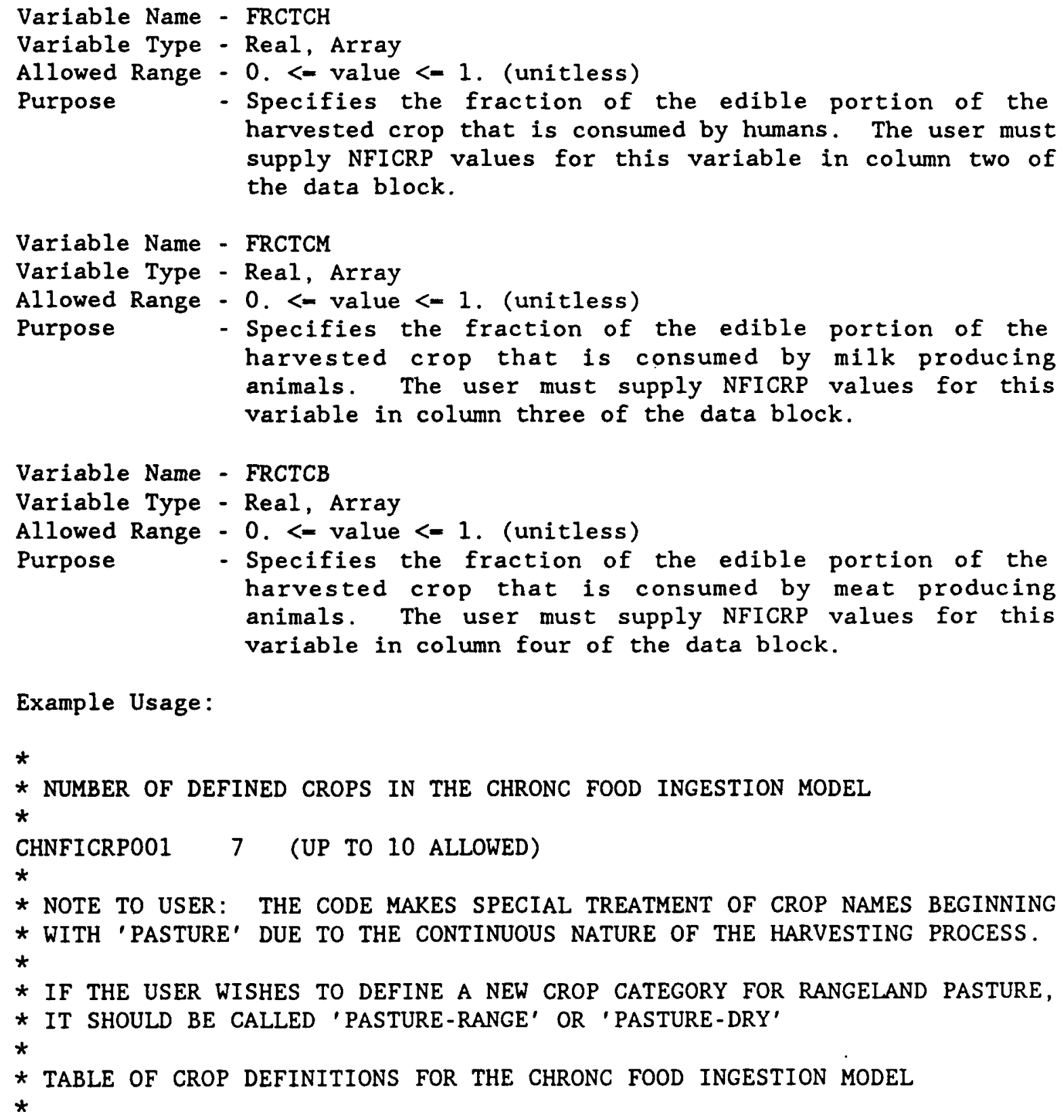
harvested crop that is consumed by humans. The user must supply NFICRP values for this variable in column two of the data block.

Variable Name - FRCTCM

Variable Type - Real, Array harvested crop that is consumed by milk producing animals. The user must supply NFICRP values for this variable in column three of the data block.

Variable Name - FRCTCB

Variable Type - Real, Array

Allowed Range - 0 . <- value <- 1. (unitless)

Purpose - Specifies the fraction of the edible portion of the harvested crop that is consumed by meat producing animals. The user must supply NFICRP values for this variable in column four of the data block.

Example Usage:

*

* NUMBER Of DEFINEd CROPS IN THE CHRONC FOOD INGESTION MODEL

*

CHNFICRP001 7 (UP TO 10 ALLOWED)

*

* NOTE TO USER: THE CODE MAKES SPECIAL TREATMENT OF CROP NAMES BEgINNING

* WITH 'PASTURE' DUE TO THE CONTINUOUS NATURE OF THE HaRVESTING PROCESS.

*

* IF THE USER WISHES TO DEFINE A NEW CROP CATEgORY FOR RANGELAND PASTURE, * IT SHOULD BE CALLED 'PASTURE-RANGE' OR 'PASTURE-DRY'

*

* TABLE OF CROP DEFINITIONS FOR THE CHRONC FOOD INGESTION MODEL

* 


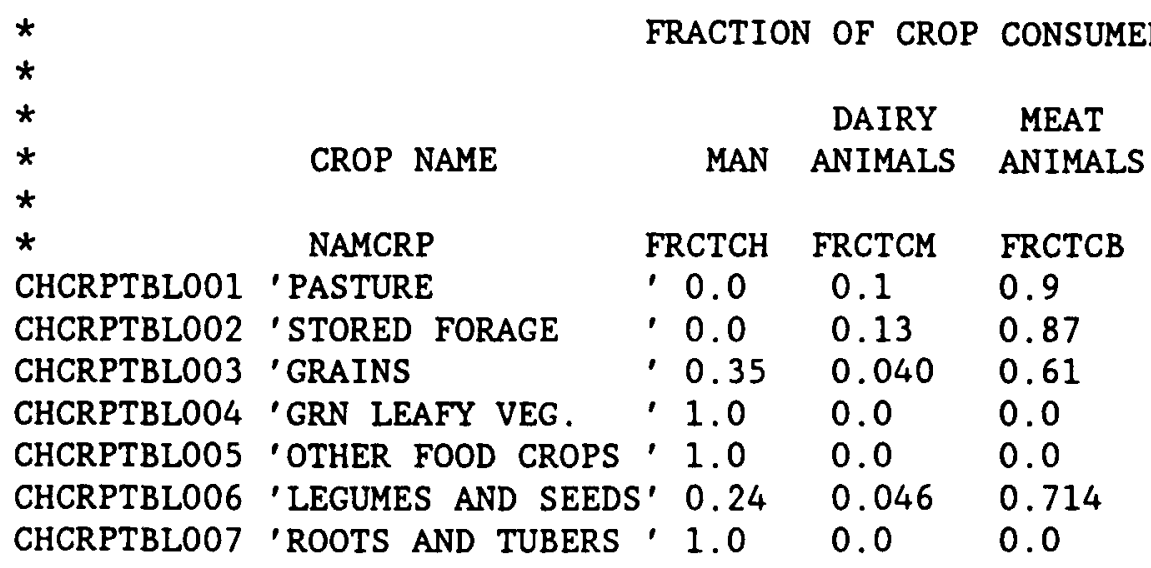

Note: The data in the following section describing the water ingestion factors is required to be supplied on the CHRONC User Input File but it is only used in the calculations if the user has chosen the option of a uniform population density surrounding the site (in Section 2.3, POPFLG=' UNIFORM').

When radioactive material is deposited on the ground, it is expected that some fraction of this material will make its way into drinking water consumed by humans [He85]. MACCS models this uptake into drinking water as two separate paths: (1) deposition of material directly onto fresh water bodies, and (2) deposition of material onto land with subsequent washoff into fresh water bodies.

The water ingestion model assumes that the area surrounding the site is divided into two categories: water and land. The radioactive material deposited on a spatial element is apportioned between water and land according to the fraction of the region "covered by land" (see variable FRACLD in the previous section).

For coastal sites, where both fresh water and ocean need to be treated, it is recommended that the user supply a Site Data File where the limitation of having only one kind of watershed can be overcome. A Site Data File can define up to four types of watersheds. One of those watersheds, e.g., ocean, can be defined to have a zero uptake fraction.

The user is required to supply three numeric parameters in the following section, which define the behavior of the single watershed which is utilized if a uniform population density has been specified.

of the material deposited directly onto water or transferred from land to water, the fraction represented by WINGF determines how much of that material will eventually be consumed by humans. There is no adjustment of WINGF within the code to account for radioactive decay.

of the material that has been deposited on land, some fraction makes its way through runoff into the fresh water supply over a relatively short period after deposition. This fraction is specified by the value of WSHRTI. 
The remainder of the material deposited on land is assumed to be washed off to the fresh water supply at a constant fractional rate over the time from $t=0$ to t-infinity. The rate at which this subsequent washoff occurs is specified by the value of the rate constant WSHRTA.

The code uses the values of the two variables, WSHRTI and WSHRTA, and evaluates the integral of the washoff fraction in a way which takes account of radioactive decay for the material deposited on land surfaces. The model is described in [He85]. The evaluation of this integral produces a numeric value, $F$, which represents the fraction of material falling on land that will be eventually transferred to surface water bodies that supply drinking water.

With this value of $F$, we can now calculate the uptake fraction for material deposited on solid ground. It is simply WINGF * F.

The input variable WINGF is the ratio between the total amount of a radionuclide consumed via the drinking water pathway (i.e., by the entire population of the region surrounding the reactor) and the amount of that radionuclide entering potable surface-water bodies. Typically, WINGF would be derived by the MACCS user from a model for radionuclide movement in the surface-water system in the surrounding region.

The models used to determine WINGF can vary in complexity from the very simple (e.g., a single uniformly mixed cell) to the very complex (e.g., three dimensional fluid transport with temporal and spatial variability). The value of WINGF supplied with the Sample Problem, 5.E-6, has been derived in a very simple manner.

Variable Name - NUMWPI

Variable Type - Integer, Scalar

Allowed Range - 1 <- value <- 10

Purpose - Defines the number of nuclides to be considered by the drinking water pathway. The drinking water nuclides must be a subset of the food ingestion nuclides (defined by NFIISO and NAMIPI in the following text).

Variable Name - NAMWPI

Variable Type - Character, Array

Allowed Range - $2<-$ length <- 10

Purpose

- Defines the name of a nuclide used in the drinking water pathway. The user must supply NUMWPI nuclide names in column one of the data block.

Note: For the purpose of calculating water ingestion doses, there is no provision for modeling the buildup of any radioactive daughter products which result from decay. There is no provision for accounting for the water ingestion dose resulting from radioactive daughter products of the water pathway nuclides defined in NAMWPI. 


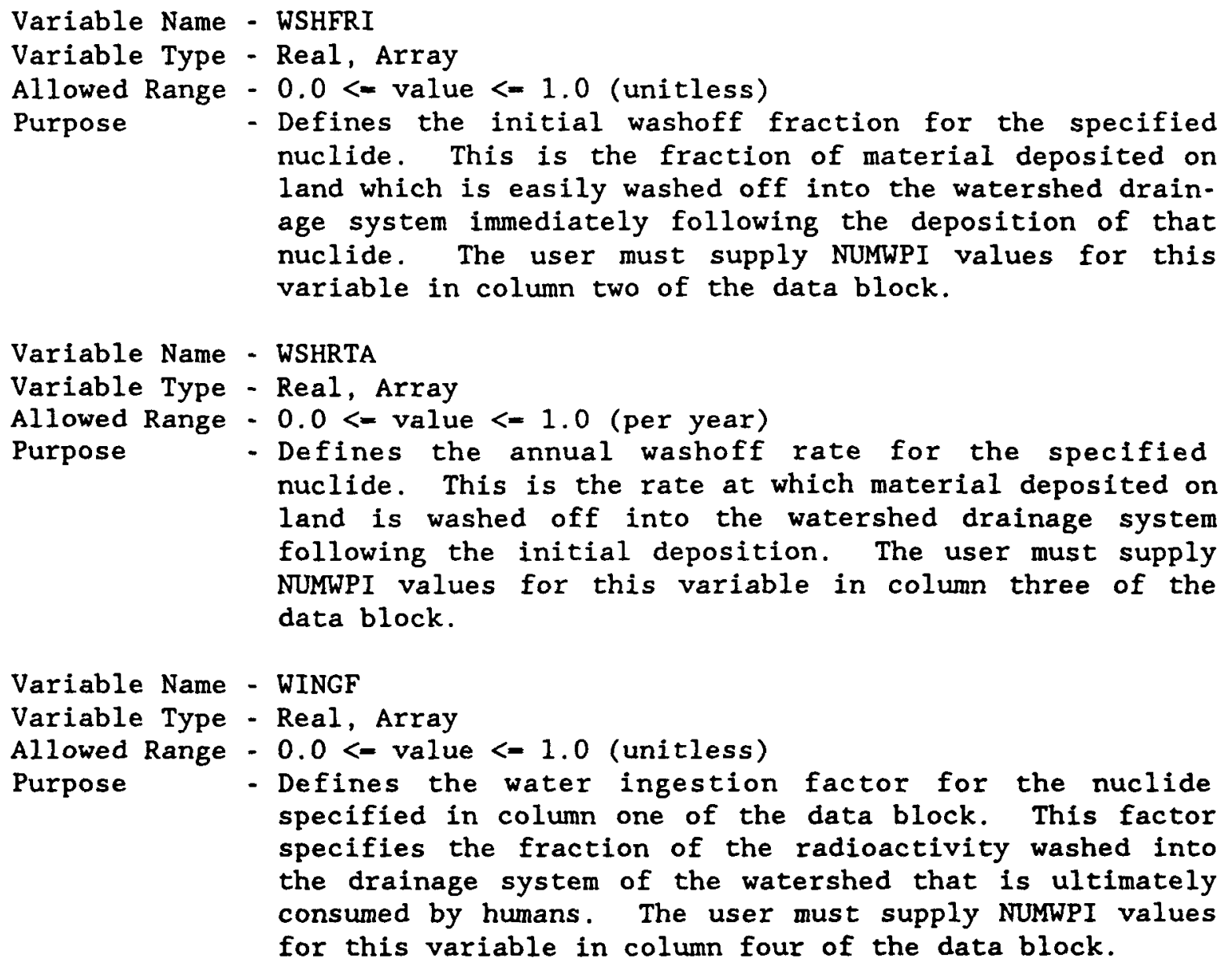
nuclide. This is the fraction of material deposited on land which is easily washed off into the watershed drainage system immediately following the deposition of that nuclide. The user must supply NUMWPI values for this variable in column two of the data block.

Variable Name - WSHRTA

Variable Type - Real, Array

Allowed Range - $0.0<-$ value <- 1.0 (per year)

Purpose - Defines the annual washoff rate for the specified nuclide. This is the rate at which material deposited on land is washed off into the watershed drainage system following the initial deposition. The user must supply NUMWPI values for this variable in column three of the data block.

Variable Name - WINGF

Variable Type - Real, Array

Allowed Range - $0.0<-$ value <- 1.0 (unitless)

Purpose - Defines the water ingestion factor for the nuclide specified in column one of the data block. This factor specifies the fraction of the radioactivity washed into the drainage system of the watershed that is ultimately consumed by humans. The user must supply NUMWPI values for this variable in column four of the data block.

Note: If a site Data File is being used (POPFLG-FILE), the value of WINGF is not used by the code, nevertheless a valid parameter value must be supplied.

Example Usage:

*

* NUMBER OF NUCLIDES IN THE CHRONC DRINKING WATER PATHWAY MODEL

$\star$

CHNUMWPI001 4

k

* TABLE OF NUCLIDES, WASHOFF FRACTIONS, AND INGESTION FACTORS FOR THE

* CHRONIC DRINKING WATER PATHWAY MODEL. THE SET OF DRINKING WATER

* PATHWAY NUCLIDES MUST BE A SUBSET OF THE SET OF NUCLIDES SPECIFIED

* FOR THE FOOD PATHWAY MODEL.

* 


\begin{tabular}{|c|c|c|c|c|}
\hline * & & $\begin{array}{l}\text { INITIAL } \\
\text { WASHOFF }\end{array}$ & $\begin{array}{l}\text { ANNUAL } \\
\text { WASHOFF }\end{array}$ & $\begin{array}{l}\text { INGESTION FACTOR } \\
\text { ((BO INGESTED) }\end{array}$ \\
\hline * & $\begin{array}{l}\text { WATER } \\
\text { NICITF }\end{array}$ & WASHUFF & WASHOFF & [(BQ INGESTED)] \\
\hline * & NUCLIDE & FRACIIUN & RATE & (BQ IN WAIEK)] \\
\hline * & NAMWPI & WSHFRI & WSHRTA & WINGF \\
\hline CHWTRISOOOI & SR-89 & 0.01 & 0.004 & $5.0 E-6$ \\
\hline CHWTRISO002 & SR - 90 & 0.01 & 0.004 & $5.0 \mathrm{E}-6$ \\
\hline CHWTRISO003 & CS -134 & 0.005 & 0.001 & $5.0 \mathrm{E}-6$ \\
\hline CHWTRISO004 & CS -137 & 0.005 & 0.001 & $5.0 E-6$ \\
\hline
\end{tabular}

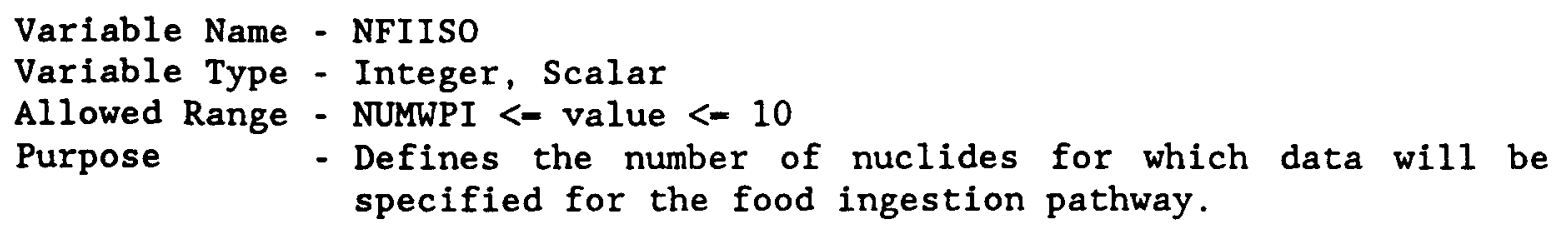
specified for the food ingestion pathway.

Note: There is no provision for accounting for the food ingestion dose resulting from radioactive daughter products of the food pathway nuclides defined in NAMIPI.

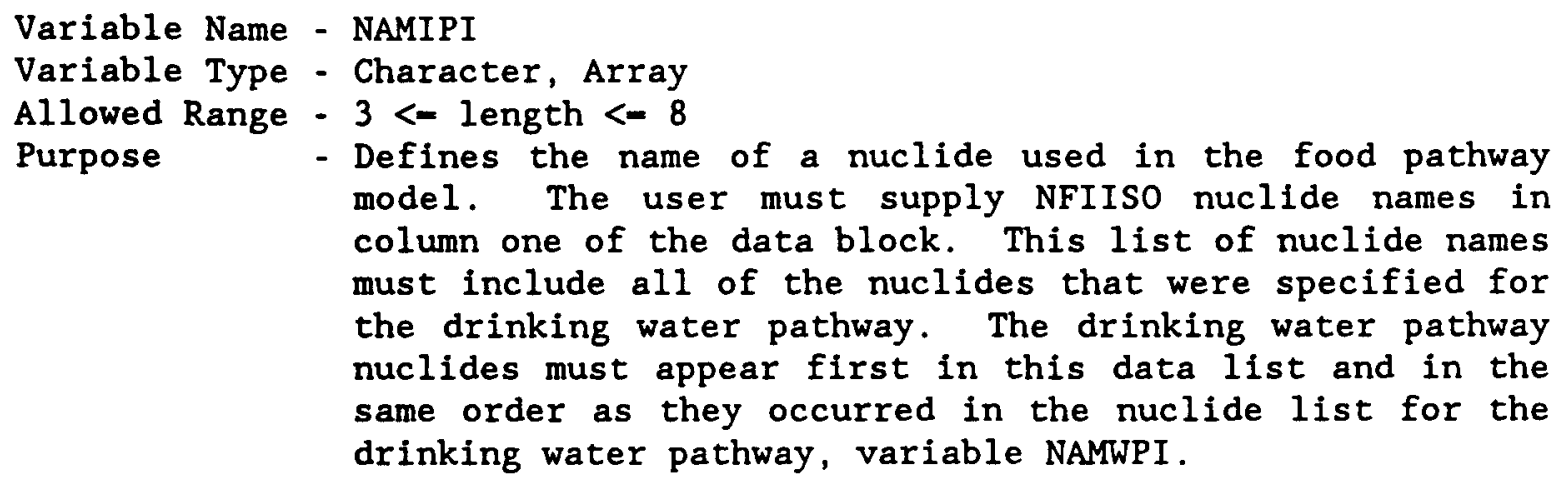
model. The user must supply NFIIso nuclide names in column one of the data block. This list of nuclide names must include all of the nuclides that were specified for the drinking water pathway. The drinking water pathway nuclides must appear first in this data list and in the same order as they occurred in the nuclide list for the drinking water pathway, variable NAMWPI.

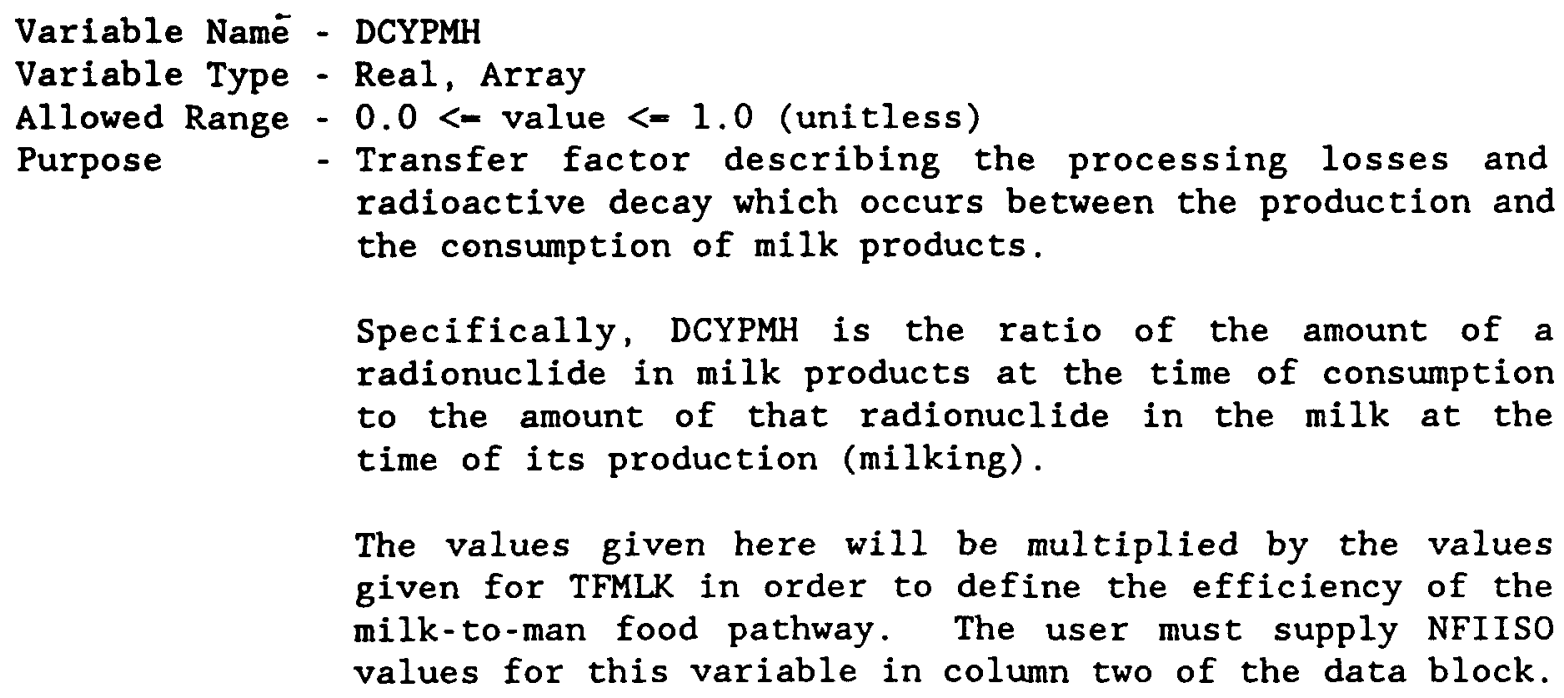

- Transfer factor describing the processing losses and radioactive decay which occurs between the production and the consumption of milk products.

Specifically, DCYPMH is the ratio of the amount of a radionuclide in milk products at the time of consumption to the amount of that radionuclide in the milk at the time of its production (milking).

The values given here will be multiplied by the values given for TFMLK in order to define the efficiency of the milk-to-man food pathway. The user must supply NFIISO values for this variable in column two of the data block. 


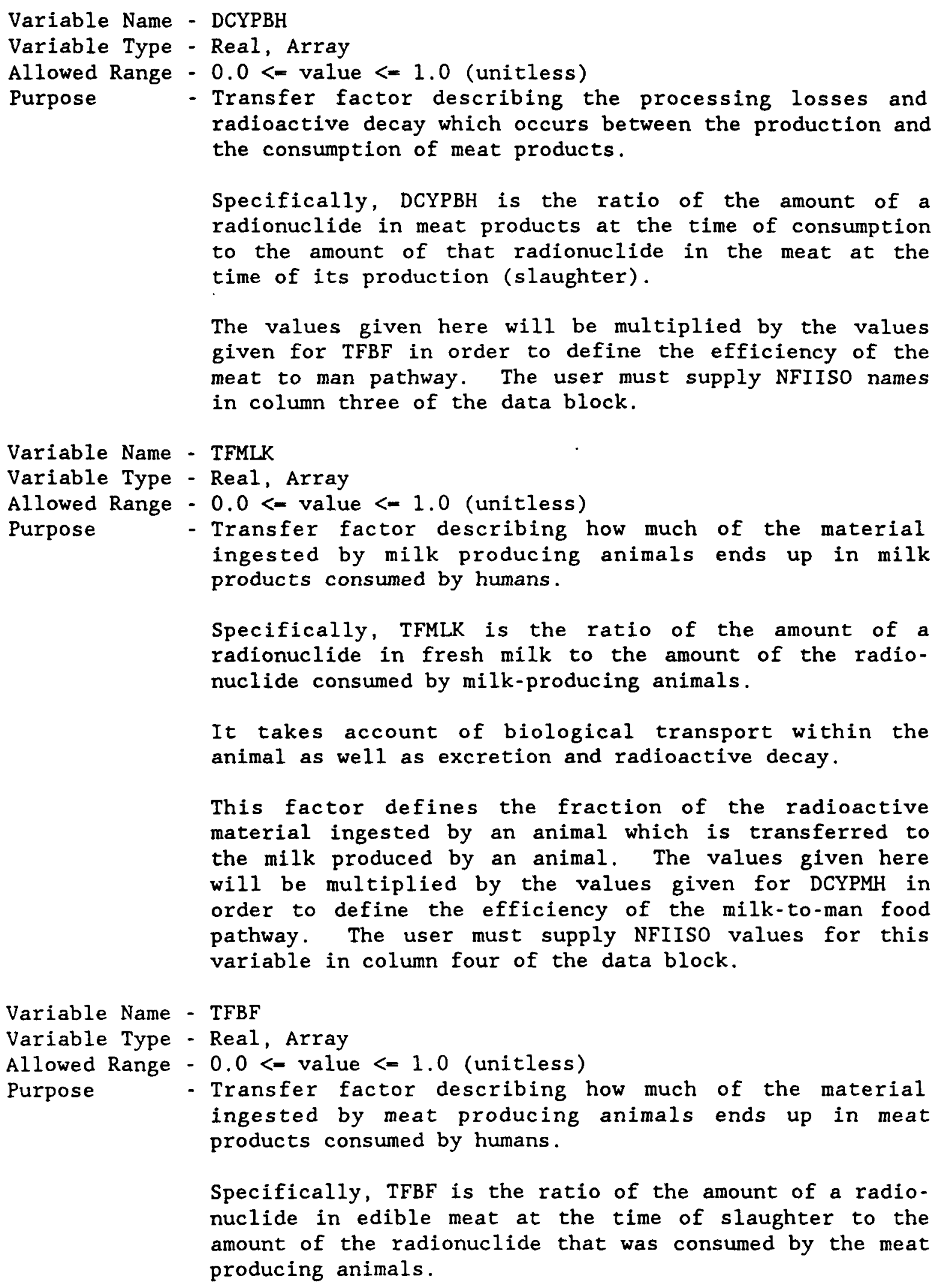


It takes account of biological transport within the animals as well as excretion and radioactive decay.

This factor defines the fraction of the radioactive material ingested by the animal which is transferred to the meat products produced from the animal. The values given here will be multiplied by the values given for DCYBH to define the efficiency of the meat to man food pathway. The user must supply NFIISO values for this variable in column five of the data block.

Example Usage:

*

* NUMBER OF NUCLIDES IN THE CHRONC FOOD INGESTION MODEL

*

CHNFIISO001 6 (UP TO 10 ALLOWED, DAUGHTER BUILDUP IS NOT TREATED)

*

* TABLE OF NUCLIDE DEFINITIONS IN THE CHRONC INGESTION PATHWAY MODEL

*

* NUClides THAT WERE DEFINED IN THE WATER PATHWAY DATA ABOVE MUST BE A

* SUBSET OF THE CHRONC INGESTION PATHWAY NUCLIDES. THE WATER

* PATHWAY NUCLIDES MUST BE LISTED FIRST IN THIS DATA BLOCK AND IN

* THE SAME ORDER AS THEY WERE LISTED IN THE WATER PATHWAY DATA

* BLOCK

*

$\star$

$\star$

*

$\star$

$\star$

*

CHISODEFOOI

CHISODEF002

CHISODEF003

CHISODEF004

CHISODEF005

CHISODEF006

\begin{tabular}{|c|c|c|c|c|}
\hline \multirow{3}{*}{$\begin{array}{l}\text { INGESTION } \\
\text { NUCLIDE }\end{array}$} & \multicolumn{2}{|c|}{ RETENTION FACTORS } & \multicolumn{2}{|c|}{ TRANSFER FACTORS } \\
\hline & PROCESS I & NG AND DECAY & (BQ & ESTED) ] \\
\hline & MILK/MAN & MEAT/MAN & MILK & MEAT \\
\hline NAMIPI & DCYPMH & DCYPBH & TFMLK & TFBF \\
\hline SR-89 & 0.66 & 0.77 & 0.022 & 0.00022 \\
\hline SR-90 & 1.0 & 1.0 & 0.022 & 0.00022 \\
\hline CS -134 & 1.0 & 1.0 & 0.11 & 0.023 \\
\hline CS -137 & 1.0 & 1.0 & 0.11 & 0.024 \\
\hline$I-131$ & 0.28 & 0.18 & 0.13 & 0.0024 \\
\hline$I-133$ & 0.002 & 0.0 & 0.062 & 0.0011 \\
\hline
\end{tabular}

Variable Name - TCROOT

Variable Type - Real, Array

Allowed Range - $0.0<-$ value $<=1.0$ (unitless)

Purpose

- Defines the transfer factor for the long-term transfer of radionuclides from soil to edible crops.

Specifically, TCROOT is the ratio of the amount of a radionuclide ultimately taken up from soil into edible portions of a crop to the amount of that radionuclide that was deposited onto the soil. 
The long-term transfer of nuclides from soil to plants occurs principally by uptake (sorption) by plant root systems. Transfer to plant surfaces by rainsplash and by deposition of materials resuspended from surface soil can also contribute to this pathway. In addition, the ingestion of contaminated soil by animals grazing on pastureland may also contribute to the overall dose.

The values of TCROOT supplied in the data block should be calculated by integrating the overall uptake rate over the period from timezero to infinity. In other words, TCROOT incorporates the total uptake over all time after the initial deposition.

As nuclides are removed from the soil compartment not only by root uptake but also by radioactive decay, percolation, and irreversible chemical binding, all of these processes should be incorporated into the derivation of TCROOT .

The annual rate at which the material is made unavailable due to these processes is specified by the input variable QROOT which is defined at the end of this section.

Values of TCROOT must be specified for each crop category for all nuclides treated by the food pathway model (i.e., for all possible nuclide/crop combinations).

The required data are entered in a block as a twodimensional array. The first column of the block repeats the list of food pathway nuclides. This list must be ordered exactly the same as the original definition of the variable NAMIPI.

All the other columns of the data block present values of TCROOT for one crop category and all food nuclides. The order of the columns in going from left to right is assumed to be the same as is specified by NAMCRP in Section 3.11 .

Example Usage:

* TRANSFER FACTOR FROM SOIL TO PLANT BY ROOT-UPTAKE (AND BY SOIL

* INGESTION FOR GRAZING ON PASTURE) INTEGRATED OVER ALL TIME [(BQ TRANS -

* FERRED)/(BQ DEPOSITED)] 


\begin{tabular}{|c|c|c|c|c|c|c|c|c|}
\hline * & & & STORED & & $\begin{array}{l}\text { GRN } \\
\text { LEAFY }\end{array}$ & $\begin{array}{l}\text { OTHER } \\
\text { FOOD }\end{array}$ & $\begin{array}{l}\text { LEGUMES } \\
\text { AND }\end{array}$ & $\begin{array}{l}\text { ROOTS } \\
\text { AND }\end{array}$ \\
\hline t & NUCLIDE & PASTURE & FORAGE & GRAINS & VEG & CROPS & SEEDS & TUBE \\
\hline & NAMISO & TCROOT & TCROOT & TCROOT & TCROOT & TCROOT & TCROOT & \\
\hline ITCROOT001 & & & $1.3 E$ & $4.3 E-5$ & $1.7 \mathrm{E}$ & & $3.7 E-4$ & \\
\hline ITCROOT002 & SR- & $2.6 \mathrm{E}-2$ & $9.0 \mathrm{E}-2$ & $3.3 \mathrm{E}$ & 1. $3 E-2$ & $E-4$ & $2.8 E-2$ & 8.41 \\
\hline HTCROOT003 & CS - 134 & 1. $3 E-3$ & $7.1 \mathrm{E}-4$ & $3.5 E-5$ & $1.4 \mathrm{E}-5$ & 1. $1 E-4$ & $9.3 E-5$ & $5.6 \mathrm{E}$ \\
\hline ITCROOT004 & CS -137 & $6.9 E-3$ & 1. $5 \mathrm{E}-3$ & $7.6 E-5$ & $3.0 E-5$ & 2. $3 E-4$ & $2.0 \mathrm{E}-4$ & $1.2 \mathrm{E}$ \\
\hline ITCROOT005 & & $1.6 \mathrm{E}-4$ & 0.0 & 0.0 & 0.0 & 0.0 & 0.0 & \\
\hline ITCROOT006 & I -133 & $1.7 \mathrm{E}-6$ & 0.0 & 0.0 & 0.0 & 0.0 & 0.0 & \\
\hline
\end{tabular}

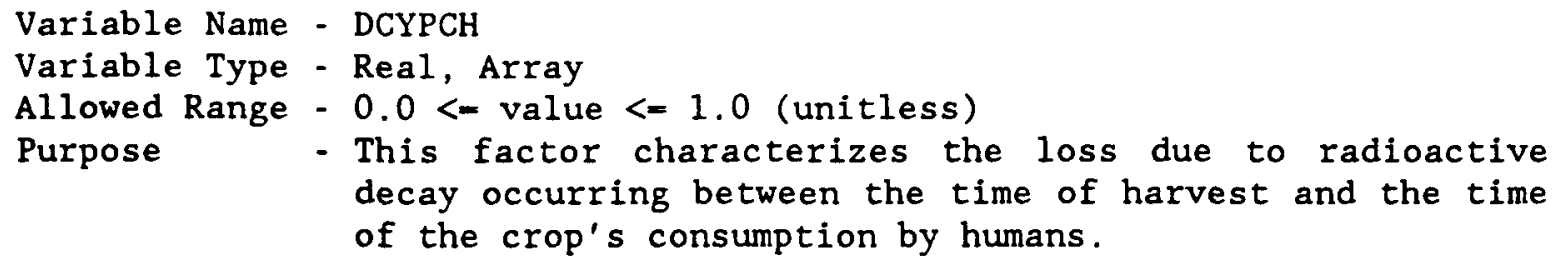

- This factor characterizes the loss due to radioactive decay occurring between the time of harvest and the time of the crop's consumption by humans.

Specifically, DCYPCH is the ratio between the amount of a radionuclide present in a crop at the time of its consumption and the amount of that radionuclide in the crop at the time of harvest after taking account of losses due to radioactive decay.

The data supplied here are used in calculating ingestion dose arising from both direct deposition onto growing crops and the long-term uptake processes of subsequent growing seasons.

This factor is applied only to crops that are directly consumed by humans (e.g., grains, vegetables, legumes, etc.). Any values supplied for crops not consumed by humans will have no impact on the calculations.

The values give here are multiplied by the corresponding values of FPLSCH, which characterizes processing losses, to obtain the overall transfer factor for this part of the food chain.

Values of DCYPCH must be specified for each crop category for all nuclides treated by the food pathway model (i.e., for all possible nuclide/crop combinations).

The required data are entered in a block as a two-dimensional array. The first column of the block repeats the list of food pathway nuclides. This list must be ordered exactly the same as the original definition of the variable NAMIPI. 
All the other columns of the data block present values of DCYPCH for one crop category and all food nuclides. The order of the columns in going from left to right is assumed to be the same as is specified by NAMCRP in Section 3.11.

\begin{tabular}{|c|c|c|c|c|c|c|c|c|}
\hline \multicolumn{9}{|c|}{$\begin{array}{l}\text { * RADIOACTIVE DECAY RETENTION FACTORS (I.E., } 1 \text { - F WHERE F - FRACTION OF } \\
\text { * RADIOACTIVITY LOST BY DECAY) FOR NUCLIDES IN CROPS FROM TIME OF HARVEST } \\
\text { * TO TIME OF CONSUMPTION BY HUMANS (FRACTION RETAINED) } \\
\text { * }\end{array}$} \\
\hline * & & & & & GRN & OTHER & LEGUMES & ROOTS \\
\hline * & & & STORED & & LEAFY & FOOD & AND & AND \\
\hline $\begin{array}{l}\star \\
\star\end{array}$ & NUCLIDE & PASTURE & FORAGE & GRAINS & VEG & CROPS & SEEDS & TUBERS \\
\hline$*$ & NAMISO & DCYPCH & DCYPCH & DCYPCH & DCYPCH & DCYPCH & DCYPCH & DCYPCH \\
\hline СHDCYPCHOO1 & SR- 89 & 0.0 & 0.0 & 0.18 & 0.67 & 0.21 & 0.18 & 0.18 \\
\hline CHDCYPCHOO2 & SR-90 & 0.0 & 0.0 & 0.99 & 1.0 & 0.99 & 0.99 & 0.99 \\
\hline CHDCYPCHOO3 & $\mathrm{CS}-134$ & 0.0 & 0.0 & 0.84 & 0.96 & 0.85 & 0.84 & 0.84 \\
\hline CHDCYPCHOO4 & CS -137 & 0.0 & 0.0 & 0.99 & 1.0 & 0.99 & 0.99 & 0.99 \\
\hline CHDCYPCHOO5 & $I-131$ & 0.0 & 0.0 & 0.0099 & 0.21 & 0.024 & 0.0099 & 0.0099 \\
\hline СHDCYРCH0O6 & I -133 & 0.0 & 0.0 & 0.0 & 0.0 & 0.0 & 0.0 & 0.0 \\
\hline
\end{tabular}

\begin{abstract}
Variable Name - DCYPCM
Variable Type - Real, Array

Allowed Range - $0.0<$ value $<=1.0$ (unitless)

Purpose

- This factor characterizes the loss due to radioactive decay occurring between the time of harvest and the time of the crop's consumption by meat-producing animals.

Specifically, DCYPCM is the ratio of the amount of a radionuclide in the crop at the time of its consumption to the amount of that radionuclide in the crop at the time of harvest.
\end{abstract}

The data supplied here are used in calculating ingestion dose arising from both direct deposition onto growing crops and the long-term uptake processes of subsequent growing seasons.

This factor is applied only to crops that are directly consumed by milk-producing animals (e.g., pasture and forage). Any values supplied for crops not consumed by milk producing animals will have no impact on the calculations.

For pasture crops, harvest and consumption are simultaneous and so DCYPCM should be set to 1.0 for the pasture crop categories. 
Values of DCYPCM must be specified for each crop category for all nuclides treated by the food pathway model (i.e., for all possible nuclide/crop combinations).

The required data are entered in a block as a two-dimensional array. The first column of the block repeats the list of food pathway nuclides. This list must be ordered exactly the same as the original definition of the variable NAMIPI.

All the other columns of the data block present values of DCYPCM for one crop category and all food nuclides. The order of the columns in going from left to right is assumed to be the same as is specified by NAMCRP in Section 3.11.

Example Usage:

* RETENTION FACTORS FOR NUCLIDES IN CROPS FROM TIME OF HARVEST TO TIME OF

* CONSUMPTION BY MILK-PRODUCING ANIMALS (FRACTION RETAINED). FACTORS

* REFLECTS LOSSES DUE TO RADIOACTIVE DECAY.

*

$\star$

*

*

*

*

CHDCYPCMO01

CHDCYPCMOO2

СНDCYPCM003

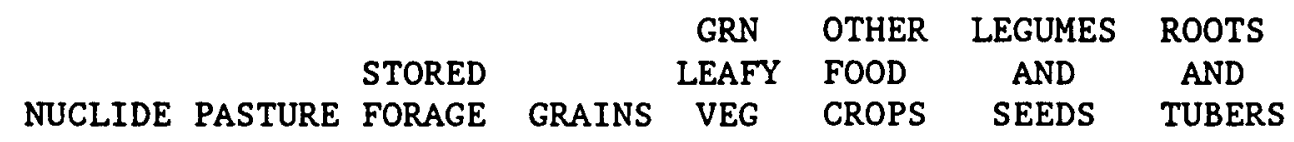

CHDCYPCM004 CS -137

NAMISO

DCYPCM DCYPCM

DCYPCM DCYPCM DCYPCM

DCYPCM DCYPCM

SR-89

1.0

0.37

0.20

0.0

0.0

0.20

0.0

1.0

0.99

0.99

0.0

0.0

0.99

0.0

CHDCYPCM005 I-131

1.0

0.99

0.85

0.0

$0.0 \quad 0.85$

0.0

CHDCYPCM006 I-133

1.0

0.063

0.99

0.0

$0.0 \quad 0.99$

0.0

1.0

0.0068

0.0034

0.0

$0.0 \quad 0.032$

0.0

0.0

0.0034

0.0

\begin{abstract}
Variable Name - DCYPCB
Variable Type - Real, Array

Allowed Range - $0.0<-$ value <- 1.0 (unitless)

Purpose - This factor specifies the retention of material after radioactive decay occurs between the time of harvest and the time of consumption by meat producing animals.

Specifically, DCYPCB is the ratio of the amount of a radionuclide in the crop at the time of its consumption to the amount of that radionuclide in the crop at the time of harvest.

This factor is applied only to crops that are directly consumed by meat-producing animals (e.g., pasture and forage). Any values supplied for crops not consumed by meat producing animals will have no impact on the calculations.
\end{abstract}


Values of DCYPCB must be specified for each crop category for all nuclides treated by the food pathway model (i.e., for all possible nuclide/crop combinations).

The required data are entered in a block as a two-dimensional array. The first column of the block repeats the list of food pathway nuclides. This list must be ordered exactly the same as the original definition of the variable NAMIPI.

All the other columns of the data block present values of DCYPCB for one crop category and all food nuclides. The order of the columns in going from left to right is assumed to be the same as is specified by NAMCRP in Section 3.11 .

Example Usage:

* RETENTION FACTORS FOR NUCLIDES IN CROPS FROM TIME OF HARVEST TO TIME OF

* CONSUMPTION BY MEAT-PRODUCING ANIMALS (FRACTION RETAINED). FACTOR

* REFLECTS LOSSES DUE TO RADIOACTIVE DECAY.

*

$\star$

$*$

*

*

$*$

\title{
CHDC
}

STORED

GRN OTHER LEGUMES ROOTS

STORED LEAFY FOOD AND AND

NUCLIDE PASTURE FORAGE GRAINS VEG CROPS SEEDS TUBERS

NAMISO DCYPCB DCYPCB DCYPCB DCYPCB DCYPCB DCYPCB DCYPCB

CHDCYPCBOO2 SR-90

1.0

0.37

0.20

1.0

0.99

0.99

0.0

0.0

0.20

0.0

CHDCYPCBO03 CS -134

1.0

0.92

0.85

0.0

0.0

0.99

0.0

0.85

0.0

CHDCYPCB004 CS-137

1.0

0.99

0.99

0.0

0.0

0.99

0.0

0.032

1.0

0.0068

$0.032 \quad 0.0$

0.0

0.0034

0.0

0.0

CHDCYPCBO06 I- 133

$0.0034 \quad 0.0$

(1)

\begin{abstract}
Variable Name - FPLSCH
Variable Type - Real, Array

Allowed Range - $0.0<=$ value $<=1.0$ (unitless)

Purpose

- This factor specifies the fraction of material retained in the harvested crop after accounting for the losses resulting from its preparation for consumption by humans.

Specifically, FPLSCH is the ratio of the amount of a radionuclide in the crop after it has been processed for human consumption to the amount of that radionuclide in the crop before processing.

The retention factor reflects the fraction of radioactive material in the edible portion of the plant which is retained after washing, peeling, or cooking.
\end{abstract}


The values supplied here apply only to crops which are directly consumed by humans. Any values specified for crops such as pasture which are supplied here will have no impact on the calculations.

The values given here are multiplied with the corresponding values of DCYPCH in order to obtain the overall transfer factor for this part of the food chain.

Values of FPLSCH must be specified for each crop category for all nuclides treated by the food pathway model (i.e., for all possible nuclide/crop combinations).

The required data are entered in a block as a twodimensional array. The first column of the block repeats the list of food pathway nuclides. This list must be ordered exactly the same as the original definition of the variable NAMIPI.

AlI the other columns of the data block present values of FPLSCH for one crop category and all food nuclides. The order of the columns in going from left to right is assumed to be the same as is specified by NAMCRP in Section 3.11.

Example Usage:

* CROP PROCESSING AND PREPARATION RETENTION FACTORS FOR NUCLIDES IN FOOD

* CROPS CONSUMED BY HUMANS (FRACTION RETAINED). FACTORS REFLECT LOSS OF

* NUCLIDES FROM FOODS DUE TO PROCESSING (E.G., WASHING OF FRUIT, PEELING

* OF POTATOES, LOSSES DURING CANNING) AND FOOD PREPARATION (COOKING) FROM

* THE TIME OF PROCESSING OF THE HARVESTED CROP TO THE TIME OF CONSUMPTION

* BY HUMANS. FACTORS DO NOT REFLECT LOSSES DUE TO RADIOACTIVE DECAY.

*

*

*

*

*

*

CHFPLSCHOO1 SR-89

CHFPLSCHOO2 SR-90

GRN OTHER LEGUMES ROOTS

STORED LEAFY FOOD AND AND

NUCLIDE PASTURE FORAGE GRAINS VEG CROPS SEEDS TUBERS

CHFPLSCH003 CS -134

FPLSCH

FPLSCH

FPLSCH FPLSCH FPLSCH FPLSCH FPLSCH

CHFPLSCH004 CS -137

0.0

0.0

0.25

0.5

0.71

0.8

0.8

CHFPLSCHOO5 I-131

0.0

0.0

0.25

0.5

0.71

0.8

0.8

0.0

0.0

0.25

0.5

0.71

0.8

0.8

0.0

0.0

0.25

0.5

0.71

0.8

0.8

CHFPLSCHOO6 I-133

0.0

0.0

0.33

0.5

0.71

0.8

0.8

0.33

0.5

0.71

0.8

0.8

When an accident occurs during the growing season, part of the radioactive material deposited on farmland will be retained on plant surfaces and the remainder will fall on the ground. Between the time of deposition and the time of harvest, radioactive material can be lost from plant surfaces due to weathering, radioactive decay, translocation to interior portions of the plant, and the harvesting process. 
The fraction of radioactive material which is removed from the air due to dry and wet deposition that ends up in edible portions of the harvested plant is here called the growing crop retention factor. Specifically, this factor is defined to be the ratio between the amount of a radionuclide present in the crop at harvest and the total amount of material initially deposited onto the land used for producing that crop.

For all crops except pasture, harvesting occurs at the end of the growing season. The harvesting of pasture differs from the other crops in that it is a continuous process. In MACCS it is assumed that grazing takes exactly one growing season to harvest the year's entire production of the pasture crop. The numerical integration used in the pasture dose calculations is therefore different from that used for the other crop categories. The type of integration performed by the code is determined by the name given to the crop categories. Crop names beginning with "PASTURE" are treated differently from the rest.

For crops where the edible portion of the plant is exposed to the environment, weathering losses over the period from deposition to harvesting will decrease the amount of retained as a function of time. That is, the longer the time between deposition and harvesting, the lower the resultant dose.

Crops such as grains and legumes with the edible portions internal to the plant may show the opposite behavior with material being absorbed into the plant over time. Since this is a slow process and data on the translocation rate is hard to obtain, the user should try to define an average retention factor appropriate for deposition onto crops that is independent of time in the growing season and not use the weathering model for these types of crops.

The situation is further complicated by the fact that the available data suggests that total retention for grains is greatest when the deposition occurs near the middle of the growing season rather than at the end of it.

Both types of crops are modeled with a weathering equation that can have up to three exponential terms, each with a different weathering rate. For the types of crops not subject to weathering losses (i.e., grains, legumes) a very long half-life can be specified for the weathering rate as a way of replacing the weathering function with a constant transfer fraction.

In MACCS the removal of radioactivity from plant surfaces by weathering is treated as a sum of terms that have the following form:

$$
\text { CTCOEF } \cdot e^{-\lambda t}
$$

where CTCOEF represents the fraction of material, deposited on a cultivated field that is removed by weathering with a decay rate of $\lambda$. 
CTCOEF equals the product of two quantities: the interception fraction and the availability fraction. The interception fraction is the fraction of material deposited onto a field that is intercepted by crop surfaces and the availability fraction is the fraction of material deposited onto crop surfaces that is weathered away with the half-life CTHALF.

Because translocation from the plant surface to interior portions influences the retention of radioactivity for grains, legumes, and root crops, the weathering model just described is not appropriate for these crop categories but as discussed in the next paragraph, the model can be modified through user-defined input parameters to, in effect, yield a transfer function which is constant with respect to time.

For the sample data presented in this section, the efficiency of grains, legumes, and root crops in transferring radioactivity was not derived in the same way as the data was derived for the other types of crops. For the grain, legume, and "other" categories, an empirical transfer factor derived from fallout studies was used, where the empirical factor represents the combined effects of interception, weathering, translocation to seeds, and root-uptake.

Since long-term uptake is treated separately from the growing-season pathway, this may cause the long-term uptake dose from grains and legumes to be double counted in the first growing season. However, as root uptake in a single season is typically small relative to the contamination resulting from direct deposition, the potential impact of such a double-counting is unlikely to be significant.

For convenience, this empirical factor is input as a value of CTCOEF. Since this empirical value includes the effects of weathering, the exponential part of the weathering decay expression associated with this empirical value for CTCOEF is reduced to a value close to unity by setting CTHALF equal to one million years ( $3.15 E 13$ seconds).

For all food pathway nuclides, values of CTCOEF and CTHALF are required for each term in the weathering expression for each crop category. Unnecessary weathering terms are eliminated by setting CTCOEF equal to zero and CTHALF equal to unity for those terms.

Variable Name - NTTRM

Variable Type - Integer, Scalar

Allowed Range - 1 <= value <= 3

Purpose - Defines the number of terms used in the growing crop retention model. 


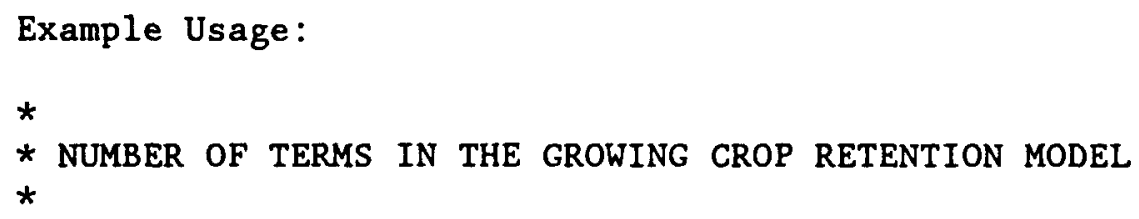

For each term of the weathering equation (and there can be up to three of them) values of CTCOEF must be specified for each crop category for all nuclides treated by the food pathway model.

The required data are entered in up to three data blocks for each of the possible terms of the weathering equation with each of the data blocks being arranged in the same manner as the variables described earlier (FPLSCH, DCYPCH, etc.).

The values of CTCOEF entered in different data blocks are distinguished from each other by the ninth character in the record identifier. For variable CTCOEF, the record identifier has the form CHCTCOEFNMM where $\mathrm{N}$ is one for the first weathering term, two for the second weathering term, and three for the third weathering term, and MM varies from 01 for the first food pathway nuclide to NN for the last food pathway nuclide.

The data format for CTCOEF is identical to that used for the subsequent variable CTHALF. There should be a oneto-one correspondence between the data supplied for these two variables.

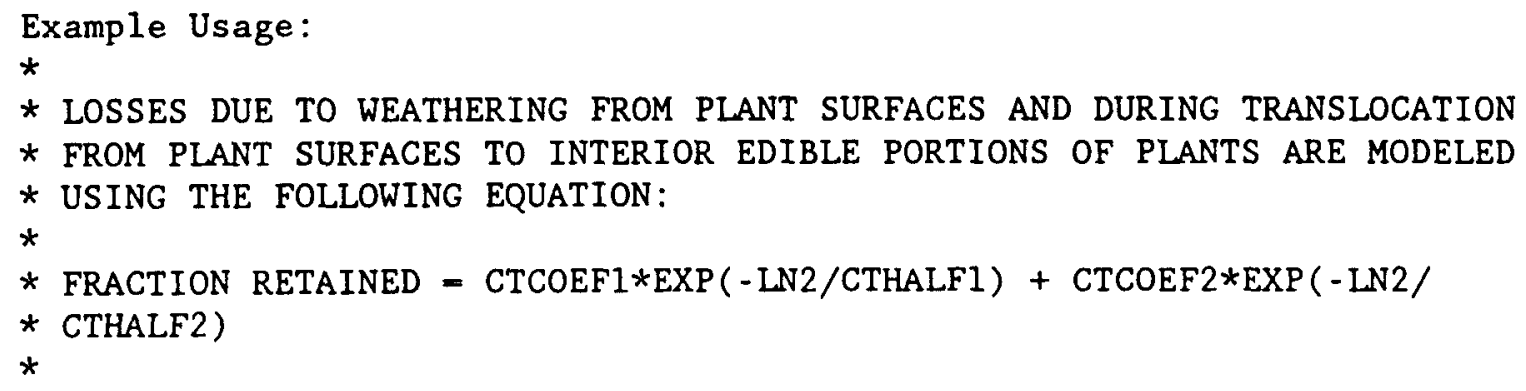


* FOR PASTURE, STORED FORAGE, GREEN LEAFY VEGETABLES, AND OTHER FOOD

* CROPS, THIS EQUATION IS USED AS A TWO TERM WEATHERING EQUATION. FOR

* GRAINS, LEGUMES AND SEEDS, AND ROOTS AND TUBERS WHERE RADIOACTIVITY IS

* CONSUMED ONLY IF TRANSLOCATED TO EDIBLE PORTIONS OF THE PLANT, THIS

* EQUATION IS REDUCED TO A TRANSLOCATION TRANSFER FACTOR BY SETTING

* CTCOEF2 TO ZERO, CTHALF2 TO ONE SECOND, AND CTHALF1 TO ABOUT ONE

* MILlion yEARS (1E13 SECONDS). WHEN USED TO MODEL TRANSLOCATION, THE

* VALUE OF THE TRANSLOCATION TRANSFER FACTOR IS DEVELOPED FROM FALLOUT

* Data and is inPUt as the value of ctcoefl.

*

* TWO TIME PERIODS ARE USED FOR WEATHERING, THE FIRST IS 14 DAYS LONG

* (1.21E6 SECONDS) AND THE SECOND IS 50 DAYS LONG (4.32E6 SECONDS).

*

* DIRECT DEPOSITION TRANSFER COEFFICIENTS BY CHRONIC INGESTION MODEL

* NUCLIDE [(BQ TRANSFERRED)/(BQ DEPOSITED)]

*

$\star$

*

$\star$

* TERM1

CHCTCOEF101

CHCTCOEF 102

CHCTCOEF103

CHCTCOEF104

CHCTCOEF105

CHCTCOEF106

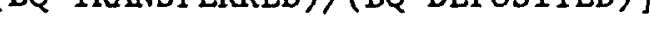

STORED

GRN OTHER LEGUMES ROOTS

NUCLIDE PASTURE FORAGE GRAINS VEG CROPS SEEDS TUBERS

*

* TERM 2

CHCTCOEF201

CHCTCOEF202

CHCTCOEF203

CHCTCOEF204

CHCTCOEF205

SR -89

0.3

0.2

0.01

0.24

0.2

0.005

0.0006

SR -90

0.3

0.2

0.01

0.24

0.2

0.005

0.0006

CS-134

0.3

0.2

0.05

0.24

0.2

0.01

0.025

CS-137

0.3

0.2

0.05

0.24

0.2

0.01

0.025

I -133

0.3

0.2

0.0

0.24

0.0

0.0

0.0

0.0

CHCTCOEF206

SR- 89

0.076

0.05

0.0

0.06

0.05

0.0

0.0

SR -90

0.076

0.05

0.0

0.06

0.05

0.0

0.0

CS -134

0.076

0.05

0.0

0.06

0.05

0.0

0.0

CS-137

0.076

0.05

0.0

0.06

0.05

0.0

0.0

I -131

0.076

0.05

0.0

0.06

0.05

0.0

0.0

$0.0 \quad 0.06$

0.05

0.0

0.0

Variable Name - CTHALF

Variable Type - Real, Array

Allowed Range - $1 .<$ value <- 1.E14 (seconds)

Purpose

- Half-life values for the various terms of the weathering model. The values supplied for CTHALF in this data block must be properly paired with the corresponding values of CTCOEF defined in the previous data block.

For each term of the weathering equation (there can be up to three) values of CTHALF must be specified for each crop category for all nuclides treated by the food pathway model.

The required data are entered in up to three data blocks for each of the possible terms of the weathering equation with each of the data blocks being arranged in the same manner as the variables described earlier (FPLSCH, DCYPCH, etc.). 
The values of CTHALF entered in different data blocks are distinguished from each other by the ninth character in the record identifier. For variable CTHALF, the record identifier identifier has the form CHCTHALFNMM where $\mathrm{N}$ is one for the first weathering term, two for the second weathering term, and three for the third weathering term, and MM varies from 01 for the first food pathway nuclide to NN for the last food pathway nuclide.

The data format for CTHALF is identical to that used for the preceding variable CTCOEF. There should be a one-toone correspondence between the data supplied for these two variables.

\begin{tabular}{|c|c|c|c|c|c|c|c|c|}
\hline $\begin{array}{l}\text { * CROP TRANS } \\
\text { * }\end{array}$ & SFER HALF & - LIVES BY & CHRONC & INGESTIC & ON MODEI & L NUCLIDI & E (SECON & \\
\hline * & & & & & GRN & OTHER & LEGUMES & ROOTS \\
\hline * & & & STORED & & LEAFY & FOOD & AND & AND \\
\hline * TERMI & NUCLIDE & PASTURE & FORAGE & GRAINS & VEG & CROPS & SEEDS & TUBERS \\
\hline CHCTHALF101 & SR -89 & $1.21 \mathrm{E} 6$ & $1.21 \mathrm{E} 6$ & $1 E 13$ & $1.21 \mathrm{E} 6$ & $1.21 \mathrm{E} 6$ & $1 E 13$ & $1 E 13$ \\
\hline CHCTHALF 102 & SR - 90 & $1.21 \mathrm{E} 6$ & $1.21 \mathrm{E} 6$ & $1 E 13$ & $1.21 E 6$ & $1.21 \mathrm{E} 6$ & $1 \mathrm{E} 13$ & $1 E 13$ \\
\hline CHCTHALF 103 & CS -134 & $1.21 \mathrm{E} 6$ & $1.21 \mathrm{E} 6$ & $1 \mathrm{E} 13$ & $1.21 \mathrm{E} 6$ & $1.21 \mathrm{E} 6$ & $1 \mathrm{E} 13$ & $1 E 13$ \\
\hline CHCTHALF 104 & CS -137 & $1.21 \mathrm{E} 6$ & $1.21 \mathrm{E} 6$ & $1 \mathrm{E} 13$ & $1.21 \mathrm{E} 6$ & $1.21 \mathrm{E} 6$ & $1 E 13$ & $1 E 13$ \\
\hline CHCTHALF105 & I - 131 & $1.21 \mathrm{E} 6$ & $1.21 \mathrm{E} 6$ & 1.0 & $1.21 \mathrm{E} 6$ & $1.21 \mathrm{E} 6$ & 1.0 & 1.0 \\
\hline $\begin{array}{l}\text { CHCTHALF106 } \\
\star\end{array}$ & I - 133 & 1. $21 \mathrm{E} 6$ & $1.21 \mathrm{E} 6$ & 1.0 & $1.21 \mathrm{E} 6$ & $1.21 \mathrm{E} 6$ & 1.0 & 1.0 \\
\hline * TERM2 & & & & & & & & \\
\hline CHCTHALF201 & SR - 89 & 4. $32 \mathrm{E} 6$ & $4.32 \mathrm{E} 6$ & 1.0 & $4.32 \mathrm{E} 6$ & $4.32 \mathrm{E} 6$ & 1.0 & 1.0 \\
\hline CHCTHALF202 & SR-90 & $4.32 \mathrm{E} 6$ & 4. $32 E 6$ & 1.0 & 4. $32 E 6$ & 4. $32 E 6$ & 1.0 & 1.0 \\
\hline CHCTHALF203 & CS -134 & 4. $32 \mathrm{E} 6$ & $4.32 E 6$ & 1.0 & $4.32 \mathrm{E} 6$ & $4.32 \mathrm{E} 6$ & 1.0 & 1.0 \\
\hline CHCTHALF204 & CS -137 & $4.32 \mathrm{E} 6$ & $4.32 E 6$ & $1: 0$ & $4: 32 E 6$ & $4.32 \mathrm{E} 6$ & 1.0 & 1.0 \\
\hline CHCTHALF205 & $I-131$ & $4.32 \mathrm{E} 6$ & 4. $32 \mathrm{E} 6$ & 1.0 & $4.32 \mathrm{E} 6$ & $4.32 \mathrm{E} 6$ & 1.0 & 1.0 \\
\hline CHCTHALF206 & I - 133 & $4.32 \mathrm{E} 6$ & 4. $32 \mathrm{E} 6$ & 1.0 & $4.32 \mathrm{E} 6$ & $4.32 \mathrm{E} 6$ & 1.0 & 1.0 \\
\hline
\end{tabular}

\subsection{Crop Share and Growing Season Data}

In the previous section, the user has defined the characteristics of a number of different crop categories with regard to their efficiency in transferring radioactive material to man through the food chain. If MACCS is being used without a Site Data File (see Section 2.3), the data in this section is used to supply the information necessary to calculate the doses resulting from the food ingestion pathway.

Note: In the current implementation, the data in this section is always required to be supplied. But if the user is supplying a Site Data File (POPFLG - 'FILE' in Section 2.3), then the data supplied here will have no impact on the calculations because it will be superseded by the corresponding data on that file. 


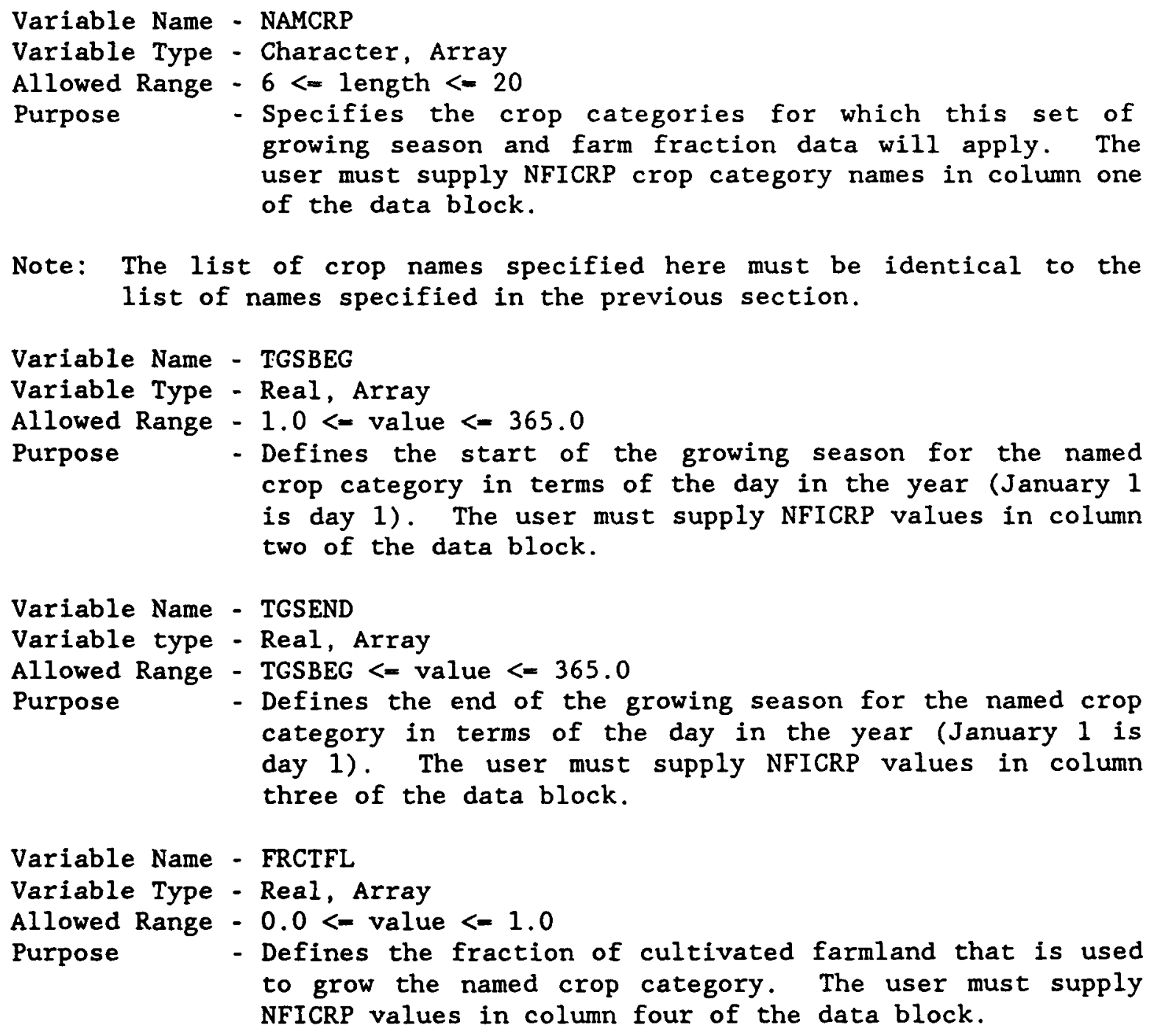

Note: The list of crop names specified here must be identical to the list of names specified in the previous section.

\begin{tabular}{|c|c|c|c|c|}
\hline$\star$ & & GROI & JING & \\
\hline * & & SEASON & (DAYS) & FARMLAND \\
\hline * & CROP NAME & START & END & SHARE \\
\hline & & & & \\
\hline * & NAMCRP & TGSBEG & TGSEND & FRCTFL \\
\hline CHCRPRGNO01 & ' PASTURE & , 90. & 270 & 0.41 \\
\hline CHCRPRGNOO2 & ' STORED FORAGE & - 150 . & 240 . & 0.13 \\
\hline CHCRPRGNO03 & 'GRAINS & - 150. & 240 . & 0.21 \\
\hline CHCRPRGNOO4 & 'GRN LEAFY VEGETABLES & $S^{\prime} 150$. & 240. & 0.002 \\
\hline CHCRPRGNOO5 & 'OTHER FOOD CROPS & . 150 & 240 . & 0.004 \\
\hline CHCRPRGN006 & ' LEGUMES AND SEEDS & - 150. & 240 . & 0.15 \\
\hline CHCRPRGN007 & 'ROOTS AND TUBERS & - 150. & 240 . & 0.003 \\
\hline
\end{tabular}




\subsection{Food Pathway Protective Action Guide Data}

In the event of an accident at a nuclear power plant, it is likely that an assessment of the accident's impact on agricultural production in the surrounding region will be performed. Based on a projection of dose to an individual consuming locally produced food products, the local authorities will determine if these food products are safe to eat. If the food production is judged to be unsafe, two kinds of actions can be taken: disposal of already growing crops, and restriction of agricultural production in subsequent growing seasons.

The stringency (degree of protection) for both types of mitigative actions are specified by the user through input parameters described in this section. All of these parameters are specified in terms of allowable ground concentration and will be referred to as "action guides."

For an accident that occurs during the growing season, there are two types of actions which can occur: (1) disposal of milk and its products, and (2) disposal of crops other than milk (non-milk crops). All agricultural production is divided between these two categories. The action guides used for determining if these two actions are necessary are the input variables PSCMLK and PSCOTH which are defined below.

For high levels of contamination, it may be necessary to restrict agricultural production for a number of years subsequent to the accident. In order to evaluate if this is necessary, a single set of values for allowable ground concentration (variable GCMAXR) is used. During the longterm interdiction period, either all crops (both milk and non-milk) can be produced, or no crops can be produced.

For accidents which occur outside of the growing season, MACCS will, in all cases, evaluate only the long-term action guide (GCMAXR). Outside of the growing season, the growing season action guides PSCMLK and PSCOTH are never evaluated since crop disposal can only occur during the growing season.

For accidents which occur during the growing season however, both types of criteria (growing-season and long-term) may be evaluated by the code. It is up to the user to determine whether these two types of evaluation are performed either (1) independently of each other (uncoupled), or (2) in such a way that the exceedance of one type of criteria will automatically force the exceedance of the other criteria (coupled). The choice of these two options is defined by the user-specified input parameter, COUPLD, defined below.

When the two types of criteria are chosen to be evaluated independently, there are no interactions between the growing-season pathway and the long-term pathway. The resultant doses and economic costs from these two components of the food model are calculated in a completely independent manner. 
If the user chooses the option of a coupled evaluation, the following rules define the interactions between two types of actions. Whenever the long-term criteria (GCMAXR) are exceeded, the disposal of any growing crops (both milk and non-milk) will automatically be triggered. Alternatively, whenever both milk and non-milk crop disposal are called for as a result of exceeding both the PSCMLK and PSCOTH criteria, the code will automatically impose at least one year of long-term farmland interdiction.

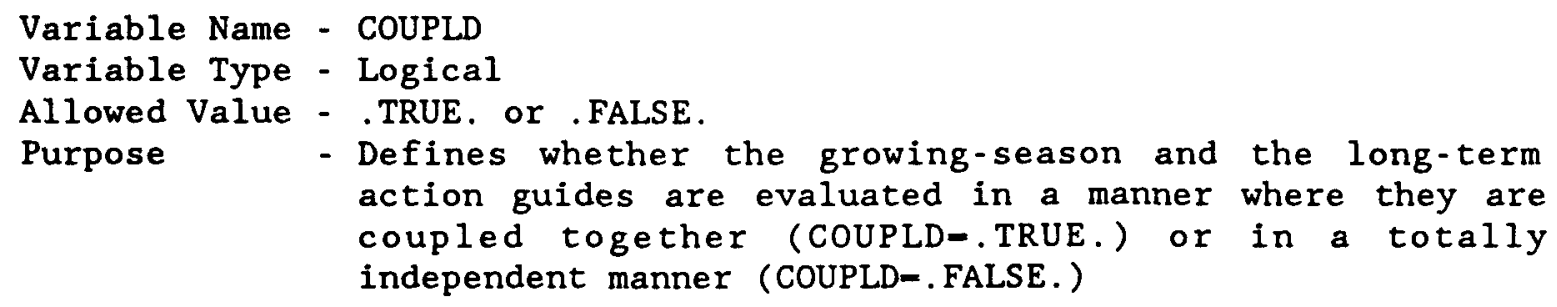

Example Usage:

CHCOUPLDO01 .FALSE. (THE TWO TYPES OF TESTS ARE INDEPENDENT)

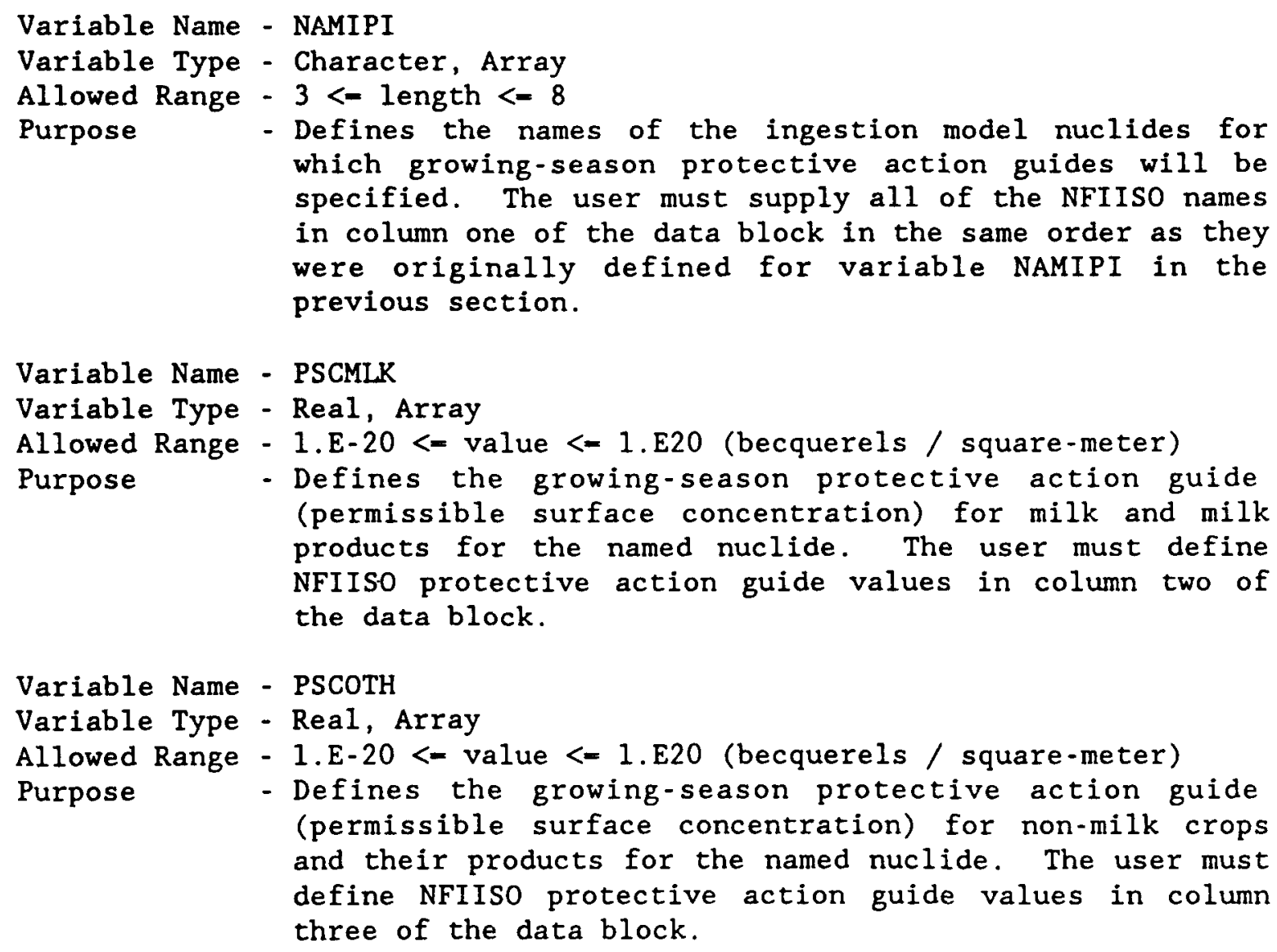
which growing-season protective action guides will be specified. The user must supply all of the NFIISO names in column one of the data block in the same order as they were originally defined for variable NAMIPI in the previous section. 


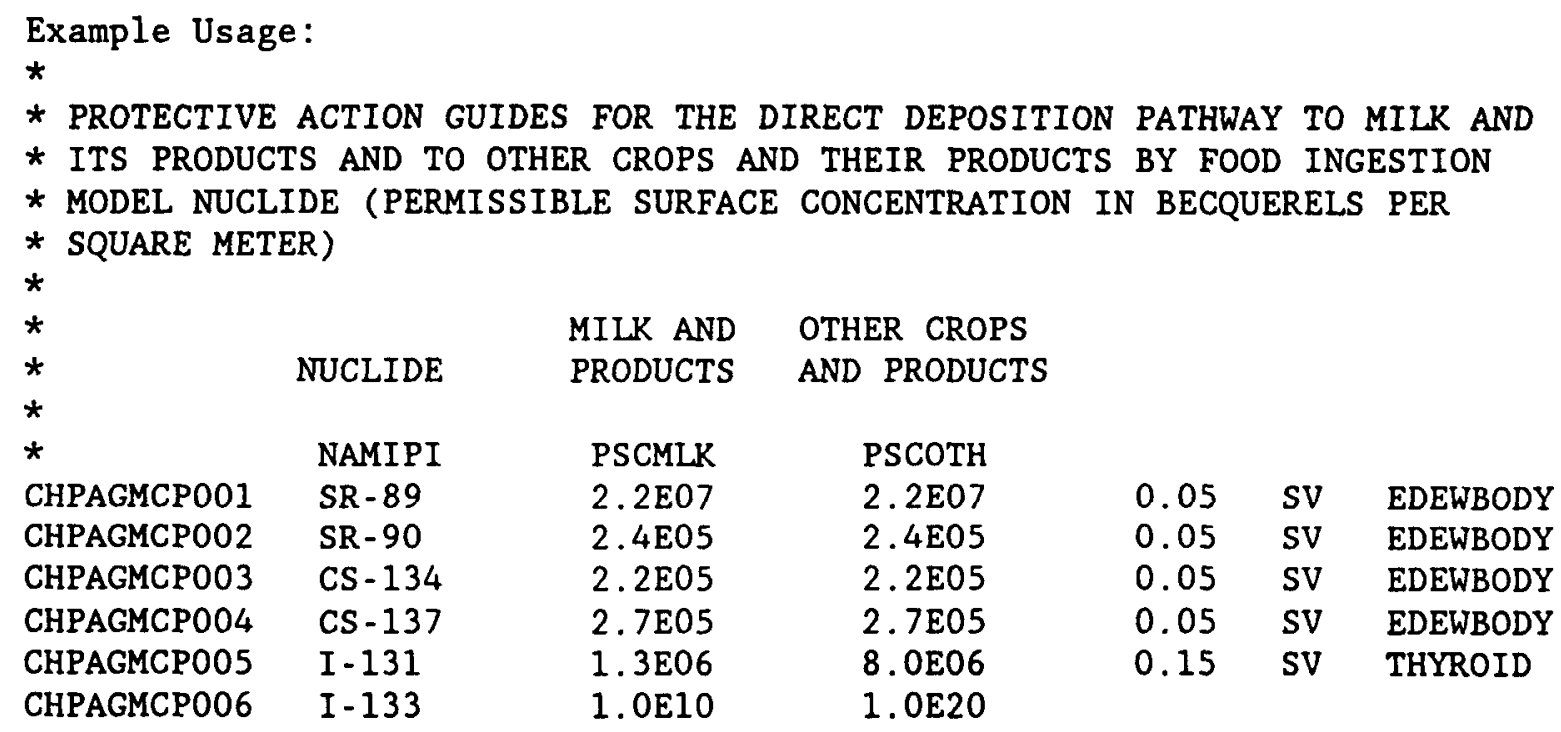

The long-term uptake fractions for root-uptake and soil ingestion by animals have been previously defined by variable TCROOT. These uptake fractions are integrated over all time, that is, from $t=0$ to $t=i n f i n i t y$. MACCS allows the user to define a model for the temporary interdiction of the long-term uptake pathway if certain ground contamination levels are exceeded at the time of the accident. If this model is activated, and temporary interdiction of long-term uptake is needed, the period of temporary interdiction is the shortest number of whole years which allow the long-term criteria to be met. The longest allowed period of longterm interdiction is eight years. If eight years of weathering and radioactive decay are insufficient, the farmland is condemned and permanently removed from production.

The data defining this interdiction model is defined below. It consists of two parts: the criteria to be met for each ingestion nuclide, and a rate constant for the decrease of the nuclide's availability over the temporary interdiction period. Values for these parameters must be supplied in the same order as was originally used to define the list of nuclides used in the ingestion model, the initial definition of NAMIPI.

Variable Name - NAMIPI

Variable Type - Character, Array

Allowed Range - $3<-$ length $<-8$

Purpose - Defines the name of the food ingestion nuclide whose permissible surface concentration (protective action guide) for long-term production and weathering depletion rate are being defined in the adjacent two columns. The user must name the NFIISO food ingestion nuclides in column one of the data block in the same order as they were originally defined. 



action guide) for long-term crop production for the named nuclide. The user must supply NFIISO values for this parameter in column two of the data block.

Variable Name - QROOT

Variable Type - Real, Array

Allowed Range - 0 . <- value <- 1.E35 (per year)

Purpose - Defines the rate constant for the decrease in availability of the named nuclide. The following processes should be considered in the specification of the value for this parameter:

1. radioactive decay,

2. irreversible chemical binding to the soil,

3. percolation downwards into the soil,

4. uptake into plants or ingestion by animals.

The value assigned to QROOT is used to determine how effective temporary interdiction of the long-term agricultural pathway will be in reducing ingestion doses. For example, if the depletion rate is .5 per year, a year of temporary interdiction will cause the integrated dose to be $\operatorname{EXP}(-.5)$ of what it would be if there were no interdiction.

The user must define NFIISO values for this parameter in column three of the data block.

Example Usage:

*

$\star$

*

$\star$

*

*

CHPAGLTSO01

CHPAGLTSO02

CHPAGLTSOO3

CHPAGLTS004

CHPAGLTSO0 5

CHPAGLTS006

$\begin{array}{lcc}\text { NUCLIDE } & \begin{array}{c}\text { PERMISSIBLE } \\ \text { SURFACE } \\ \text { CONCENTRATION }\end{array} & \begin{array}{c}\text { ANNUAL } \\ \text { DEPLETION } \\ \text { RATE }\end{array} \\ \text { NAMIPI } & \text { GCMAXR } & \text { QROOT } \\ \text { SR-89 } & 1.8 E 08 & 4.9 \\ \text { SR-90 } & 3.7 \text { E04 } & 0.065 \\ \text { CS }-134 & 4.1 \text { E06 } & 0.59 \\ \text { CS }-137 & 1.8 \text { E06 } & 0.28 \\ \text { I-131 } & 1.0 \text { 20 } & 32.0 \\ \text { I-133 } & 1.0 \text { E20 } & 290.0\end{array}$

0.005 SV EDEWBODY

INTEGRATED FROM

$\mathrm{T}=0$ TO $\mathrm{T}=$-INFINITY

QROOT

0.065

0.28

290.0

\subsection{Diagnostic Trace Options}

The diagnostic options data block is defined to allow a user to select as special output eight different diagnostic results generated during the use of the CHRONC module. These selected diagnostic results are written to the List Output File, FORTRAN unit 6. 


\begin{abstract}
Variable Name - KSWTCH
Variable Type - Integer, Array

Allowed Range - $0<=$ value $<=10$

Purpose - Defines the set of special diagnostics to be printed by CHRONC. This variable is used to enable the generation and printing of intermediate calculations on the output listing. The user should understand that the amount of output generated by these options can be very large. The definition of the output option associated with each element of the array KSWTCH follows:

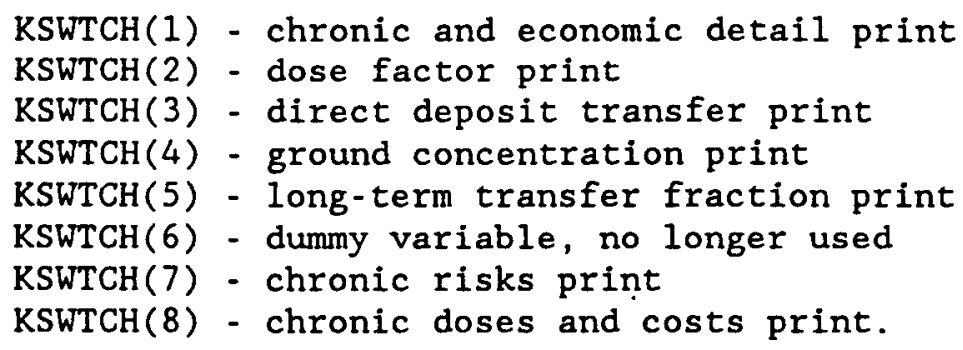

It is recommended that the user set the value of each of these options to zero for normal calculations. Specification of any value greater than zero will generate a large amount of printed output. Therefore, the trace output option should only be used for single weather trial runs. The most useful of these options is KSWTCH (8). An investigation into the CHRONC intermediate results should begin with setting KSWTCH ( 8 ) to one.
\end{abstract}

Example Usage:

*

* DETAILED PRINT OPTION CONTROL SWITCHES

* (KCEPNT, KDFPNT, KDTPNT, KGCPNT, KLTPNT, KWTPNT, KSWRSK, KSWDSC)

*

$\begin{array}{lllllllll}\text { CHKSWTCHOOI } & 0 & 0 & 0 & 0 & 0 & 0 & 0 & 0\end{array}$

\title{
3.14 User Requested Population Dose Results
}

The CHRONC module calculates the long-term population dose broken down by pathway for each of the cancer target organs defined on the EARLY User Input File (see Section 2.10). The option to examine this breakdown of long-term population doses for selected cancer organs is controlled by the user as defined in this section. The breakdown of long-term population dose results has no corresponding result in the EARLY module. The EARLY module produces only one consequence measure relating to population dose, the TYPE 5 result, total population dose.

No long-term population dose results for a defined cancer organ are produced on the output listing unless the user specifically requests them. Each request for the breakdown of the long-term population dose to an organ produces the block of twelve dose results identified below (each 
result identification consists of the capitalized result name followed by a description of the result). All of the dose results are computed in person-sieverts.

1. Total Long-Term Pathways Dose - total long-term population dose from groundshine and resuspension, from the consumption of contaminated food, from the ingestion of contaminated surface water, and from decontamination work,

2. Long-Term Direct Exposure Pathways - total long-term population dose to resident population from groundshine and resuspension,

3. Total Ingestion Pathways Dose - total long-term population dose from the consumption of contaminated dairy products, contaminated nondairy products and contaminated water,

4. Long-Term Groundshine Dose - total long-term population dose received by resident population from groundshine,

5. Long-Term Resuspension Dose - total long-term population dose received by resident population from resuspension,

6. Pop. Dependent Decontamination Dose - total long-term population dose received from groundshine by workers performing "population dependent" (non-farm) decontamination (decontamination workers receive no inhalation dose),

7. Area Dependent Decontamination Dose - total long-term population dose received from groundshine by workers performing "area dependent" (farmland) decontamination (decontamination workers receive no inhalation dose),

8. Milk Growing-Season Dose - total long-term population dose resulting from consumption of milk and dairy products contaminated as a result of deposition onto crops during the growing season,

9. Crop Growing-Season Dose - total long-term population dose resulting from consumption of non-milk food products contaminated as a result of deposition onto crops during the growing season,

10. Milk Long-Term Dose - total long-term population dose resulting from consumption of milk and dairy products contaminated by long-term uptake in the period following the accident,

11. Crop Long-Term Dose - total long-term population dose resulting from consumption of non-dairy crops and their products contaminated by long-term uptake in the period following the accident,

12. Water Ingestion Dose - total long-term population dose from ingestion of contaminated surface water.

Variable Name - NXUM9

Variable Type - Integer, Scalar 


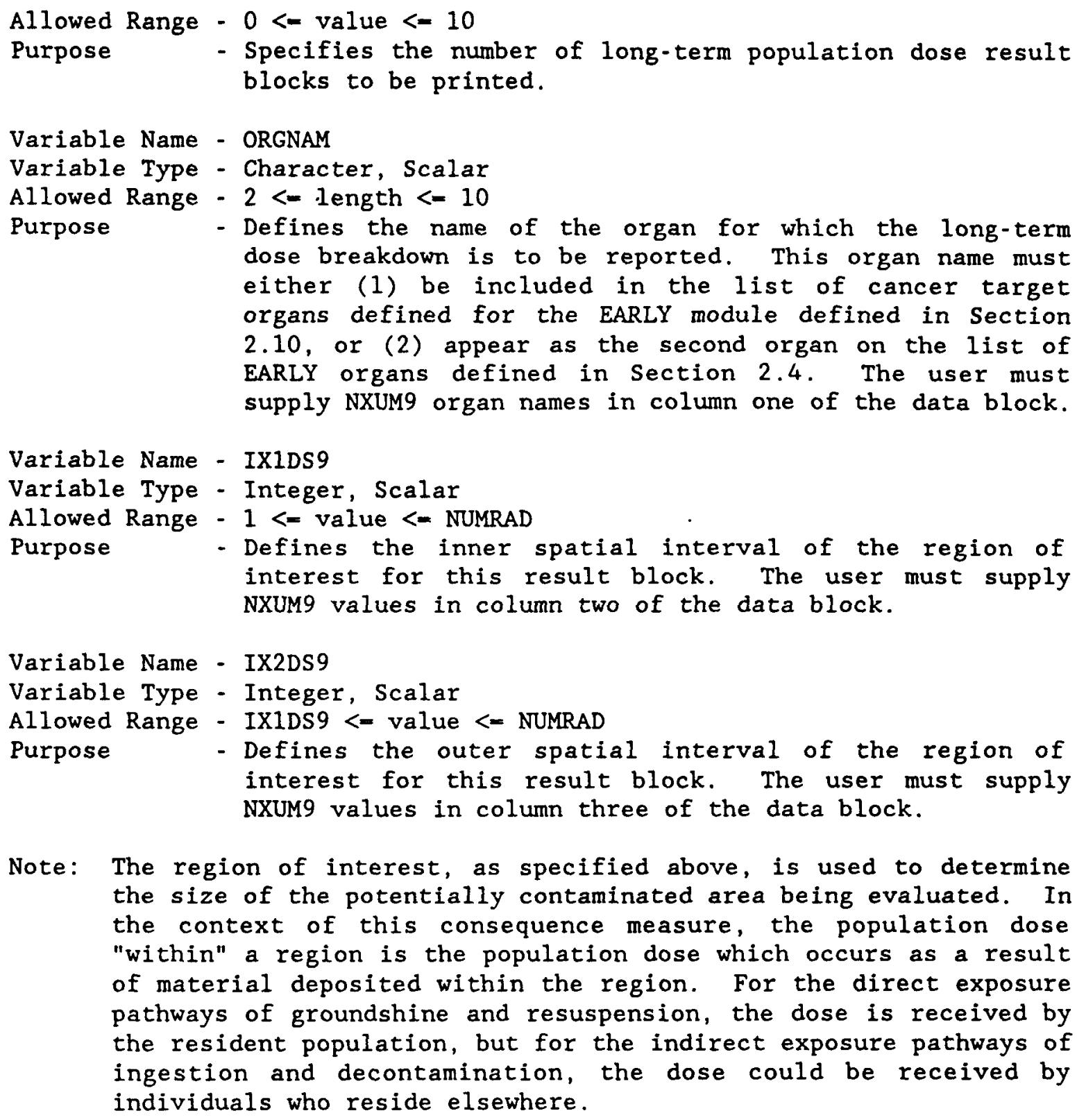

Note: The region of interest, as specified above, is used to determine the size of the potentially contaminated area being evaluated. In the context of this consequence measure, the population dose "within" a region is the population dose which occurs as a result of material deposited within the region. For the direct exposure pathways of groundshine and resuspension, the dose is received by the resident population, but for the indirect exposure pathways of ingestion and decontamination, the dose could be received by individuals who reside elsewhere.

Additional Note: In order to obtain the CCDF tables of a consequence measure requested in this section, append the character string ' $C C D F$ ' to the line requesting that result as the fourth item on the data card. The CCDF tables will be printed on the List Output File (unit 6).

Example Usage:

$\star$

* DEFINE THE TYPE 9 RESULTS

*

* LONG-TERM POPUlation DOSE IN A GIVEN REgION BROKEN DOWN BY

* THE 12 PATHWAYS

*

* NUMBER OF RESULTS OF THIS TYPE THAT ARE BEING REQUESTED 


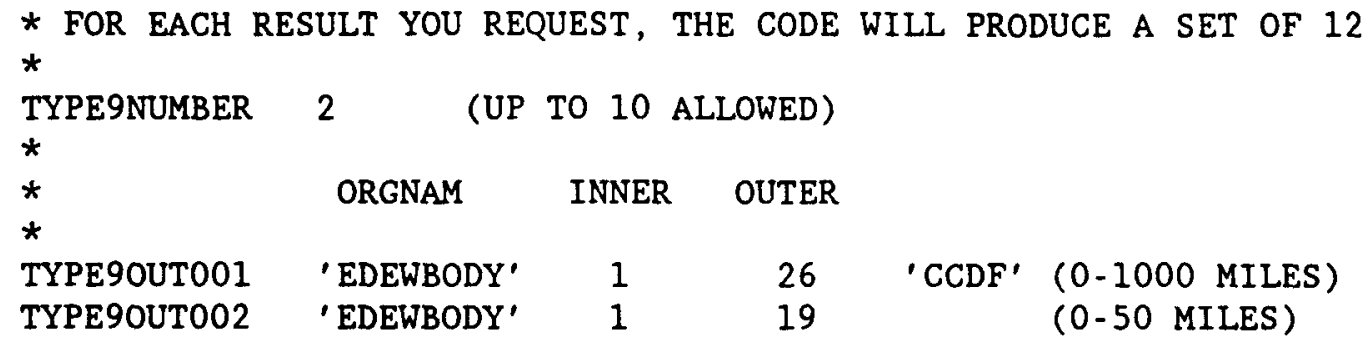

\subsection{User Requested Economic Costs Results}

The CHRONC module calculates the economic costs of all the long-term protective actions as well as the cost of the emergency response actions which were treated in the EARLY module. The option to print these economic results is controlled by the user.

No economic costs are printed unless the user specifically requests them. Each request for economic results produces the block of twelve economic results described below. Each result is identified by the result name used on the output file together with a description of the result (each result identification consists of the capitalized result name followed by a description of the result). All of the economic costs are computed in dollars.

Total Economic Cost - the sum of Pop. and Area Dependent Costs,

Pop. Dependent Costs - the sum of Pop. Dependent Decontamination, Inter diction, and Condemnation Costs,

Area Dependent Costs - the sum of Area Dependent Decontamination, Interdiction, Condemnation Costs as well as Milk and Crop Disposal Costs,

Pop. Dependent Decontamination Cost - non-farm property decontamination cost,

Area Dependent Decontamination Cost - farm property decontamination cost, Pop. Dependent Interdiction Cost - depreciation and deterioration of nonfarm property during the period it cannot be used during both decontamination and interdiction plus the cost of population removal (POPCST in Section 3.6),

Area Dependent Interdiction Cost - depreciation and deterioration of farm property during the period it cannot be used during both decontamination and interdiction,

Pop. Dependent Condemnation Cost - compensation paid for permanent loss of non-farm property plus the cost of 
population removal (POPCST in Section $3.6)$, Area Dependent Condemnation Cost - compensation paid for permanent loss
of farm property,

Evacuation and Relocation Cost - per diem costs to compensate people for being away from home due to evacuation and relocation during the emergency phase (EARLY) and also for relocation during the intermediate phase (CHRONC),

Note: When more than one emergency response scenario is being evaluated by the EARLY module, the presentation of evacuation and relocation cost is calculated on the basis of the scenario which was defined last.

Milk Disposal Cost - compensation for lost milk sales during a quarter of a year. This cost is incurred if the accident occurs during the growing season and any of the following conditions are found:

1. the growing-season milk action guide (PSCMLK) is exceeded, or

2. any decontamination actions are required, or

3. if COUPLD=.TRUE. AND any long-term interdiction is required,

Crop Disposal Cost - compensation for lost non-milk crop sales during a full year. This cost is incurred if the accident occurs during the growing season and any of the following conditions are found:

1. the growing-season non-milk action guide (PSCOTH) is exceeded, or

2. any decontamination actions are required, or

3. if COUPLD=.TRUE. AND any long-term interdiction is required.

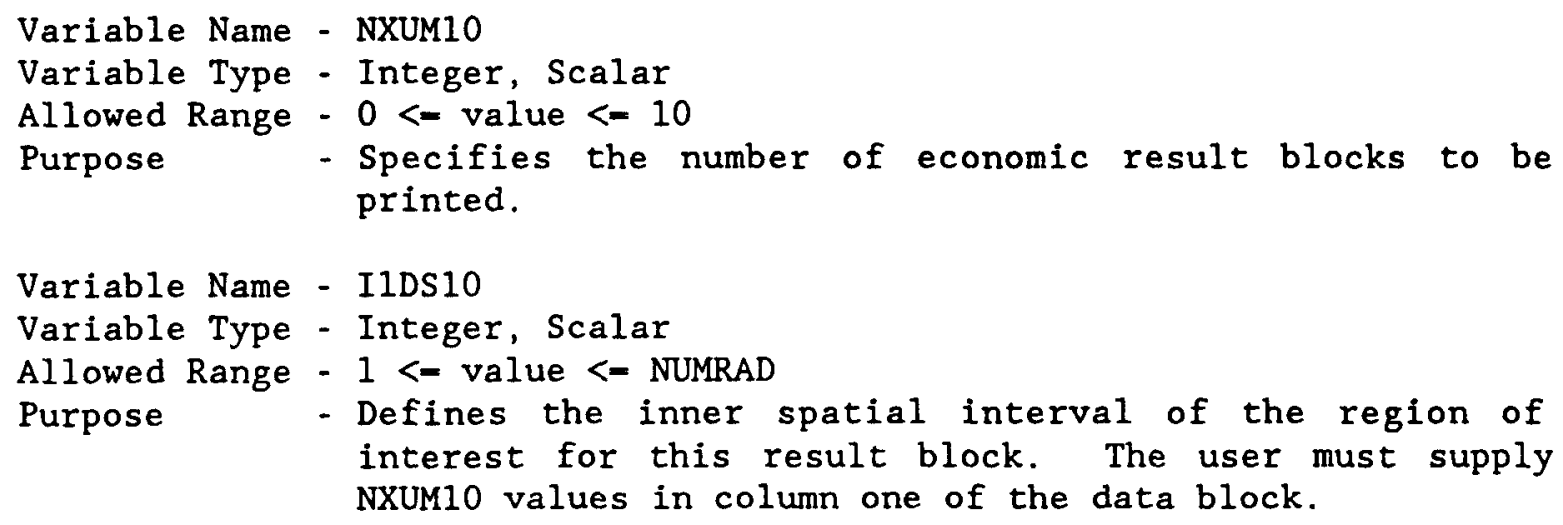




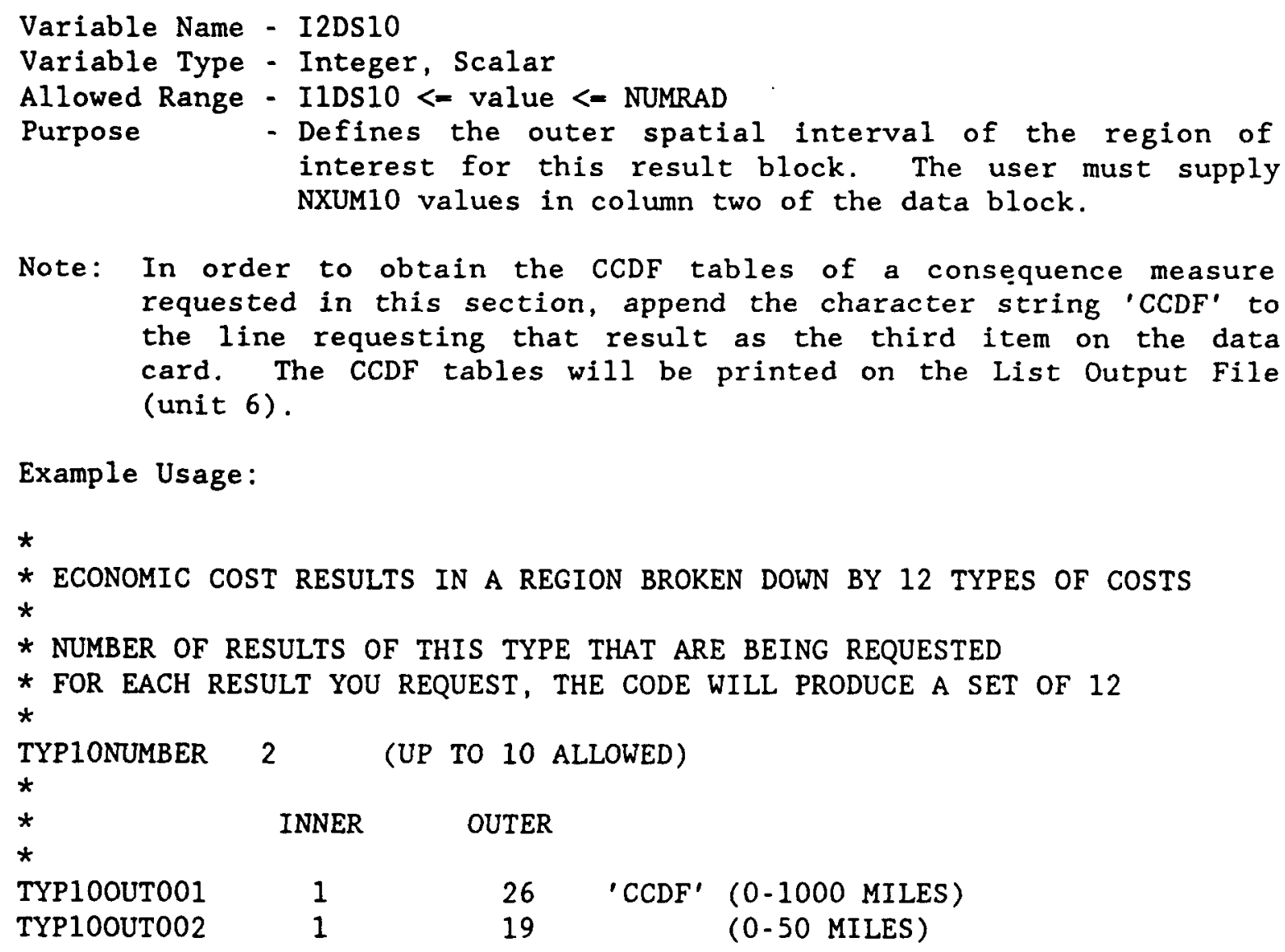

Note: In order to obtain the CCDF tables of a consequence measure requested in this section, append the character string 'CCDF' to the line requesting that result as the third item on the data card. The CCDF tables will be printed on the List Output File (unit 6).

Example Usage:

*

* ECONOMIC COST RESULTS IN A REgION BROKEN DOWN BY 12 TYPES OF COSTS

*

* NUMBER OF RESULTS OF THIS TYPE THAT ARE BEING REQUESTED

* FOR EACH RESULT YOU REQUEST, THE CODE WILL PRODUCE A SET OF 12

*

TYP1ONUMBER 2 (UP TO 10 ALLOWED)

*

* INNER OUTER

*

TYP100UT001 $1126 \quad$ 'CCDF' (0-1000 MILES)

$\begin{array}{llll}\text { TYP10OUT002 } & 1 & 19 & (0-50 \text { MILES })\end{array}$

\subsection{User Requested Action Distance Results}

The long-term protective actions that result from the calculations of the CHRONC module depend on the data supplied by the user. Associated with the long-term actions of decontamination, interdiction and crop disposal are the maximum distances to which these actions are implemented. The user must specify whether or not these maximum action distance results are to be printed.

The option to print or not print these long-term action distances is controlled by a flag specified by the user. The flag value. TRUE. will produce the eight maximum action distance results that are described below. Each result is identified by the result name used on the output file together with a description of the result (each result identification consists of the capitalized result name followed by a description of the result). All of the distances are reported in kilometers $(\mathrm{km})$.

Area Dependent Decontamination Dist. - maximum distance at which farmland decontamination is required,

Pop. Dependent Decontamination Dist. - maximum distance at which nonfarmland decontamination is required, 


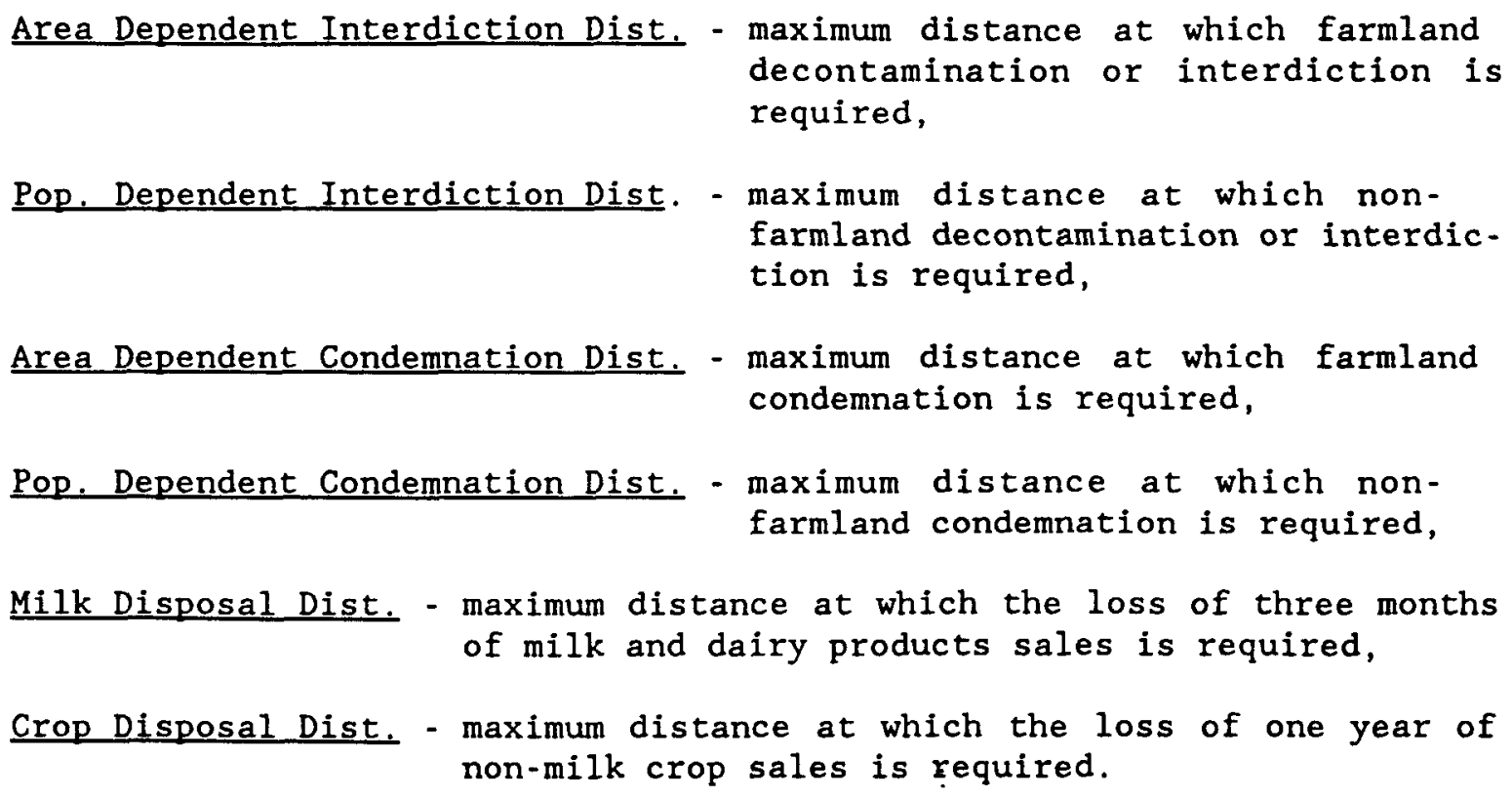

The flag value. FALSE. will eliminate the maximum action distance results from the output.

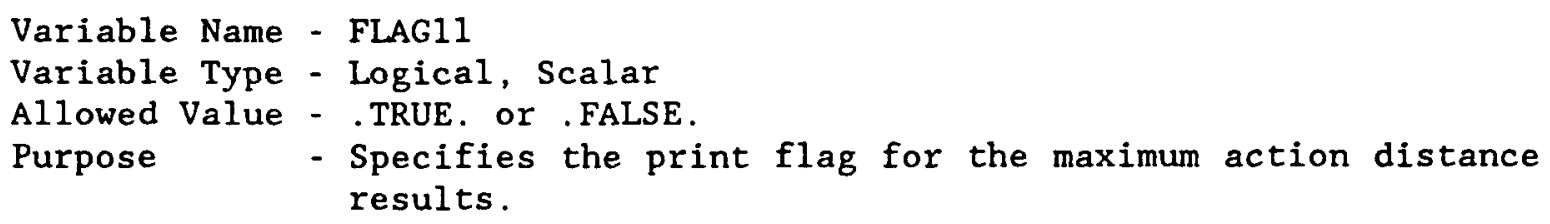

Note: In order to obtain the CCDF tables of a consequence measure requested in this section, append the character string 'CCDF' to the line requesting that result as the second item on the data card. The CCDF tables will be printed on the List Output File (unit 6).

Example Usage:

*

* DEFine a flag that CONTROLS THE PRODUCTION OF THE ACTION Distance

* RESULTS

*

* SPECIFying a VALUE OF. TRUe. TURNS ON ALL 8 OF THE aCtion Distance

* RESUlts, a VALUE of . False. Will eliminate THE aCtion Distance Results

* FROM THE OUTPUT.

*

TYP11FLAG11 . TRUE. CCDF * WILL BE PRODUCED FOR EACH OF THE SIX RESULTS

\subsection{User Requested Impacted Area/Population Results}

The long-term protective actions that result from the calculations of the CHRONC module depend on the data supplied by the user. Associated with the long-term actions of decontamination, interdiction, condemnation, and 
crop disposal are the areas and populations which are impacted by these actions. The option to print these impacted area/population results is controlled by the user.

No impacted area/population results are printed unless the user specifically requests them. Each request for impacted area/population results produces the block of eight results identified below (each result identification consists of the capitalized result name followed by a description of the result). All area results are reported in hectares and all population results are reported as number of individuals.

Decontamination Area (Hectares) - area within which farmland decontamination was required,

Decontamination Pop. (Individuals) - population of areas that required decontamination of non-farm property,

Interdiction Area (Hectares) - farmland area which required either decontamination or interdiction,

Interdiction Pop. (Individuals) - population of areas that required either decontamination or interdiction of non-farm property,

Condemnation Area (Hectares) - area within which farmland condemnation was required,

Condemnation Pop. (Individuals) - population of areas that required condemnation of non-farm property,

Milk Disposal Area (Hectares) - affected area requiring the loss of milk and dairy products sales for three months,

Crop Disposal Area (Hectares) - affected area requiring the loss of nonmilk crop sales for a year.

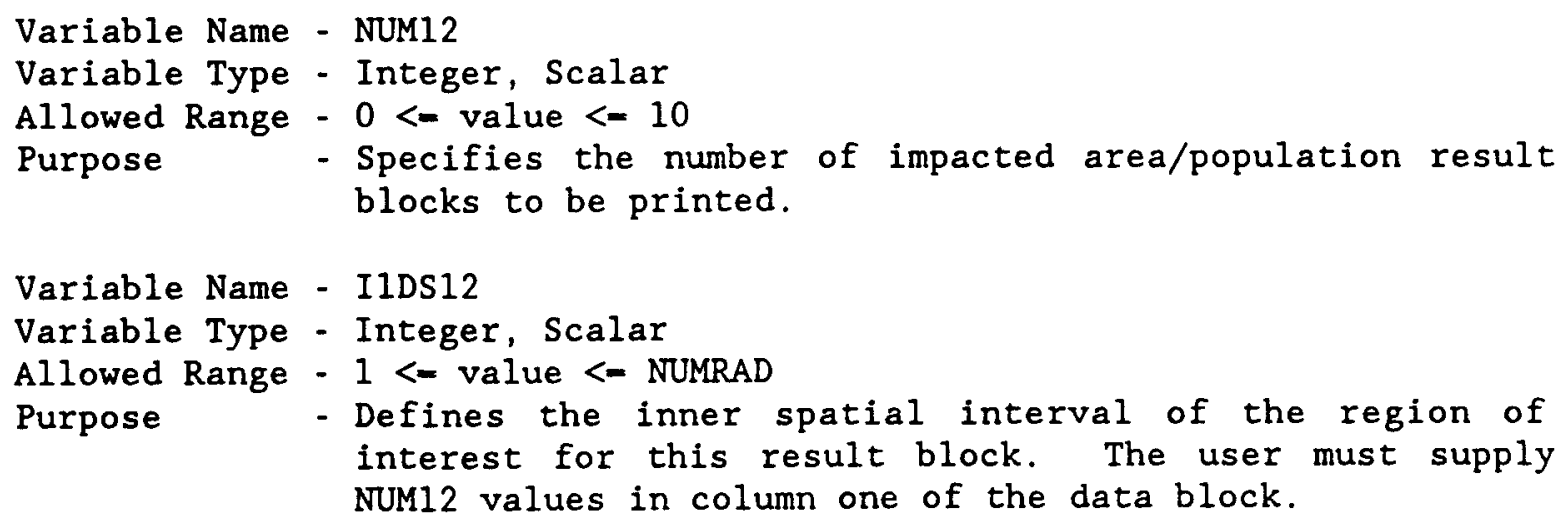




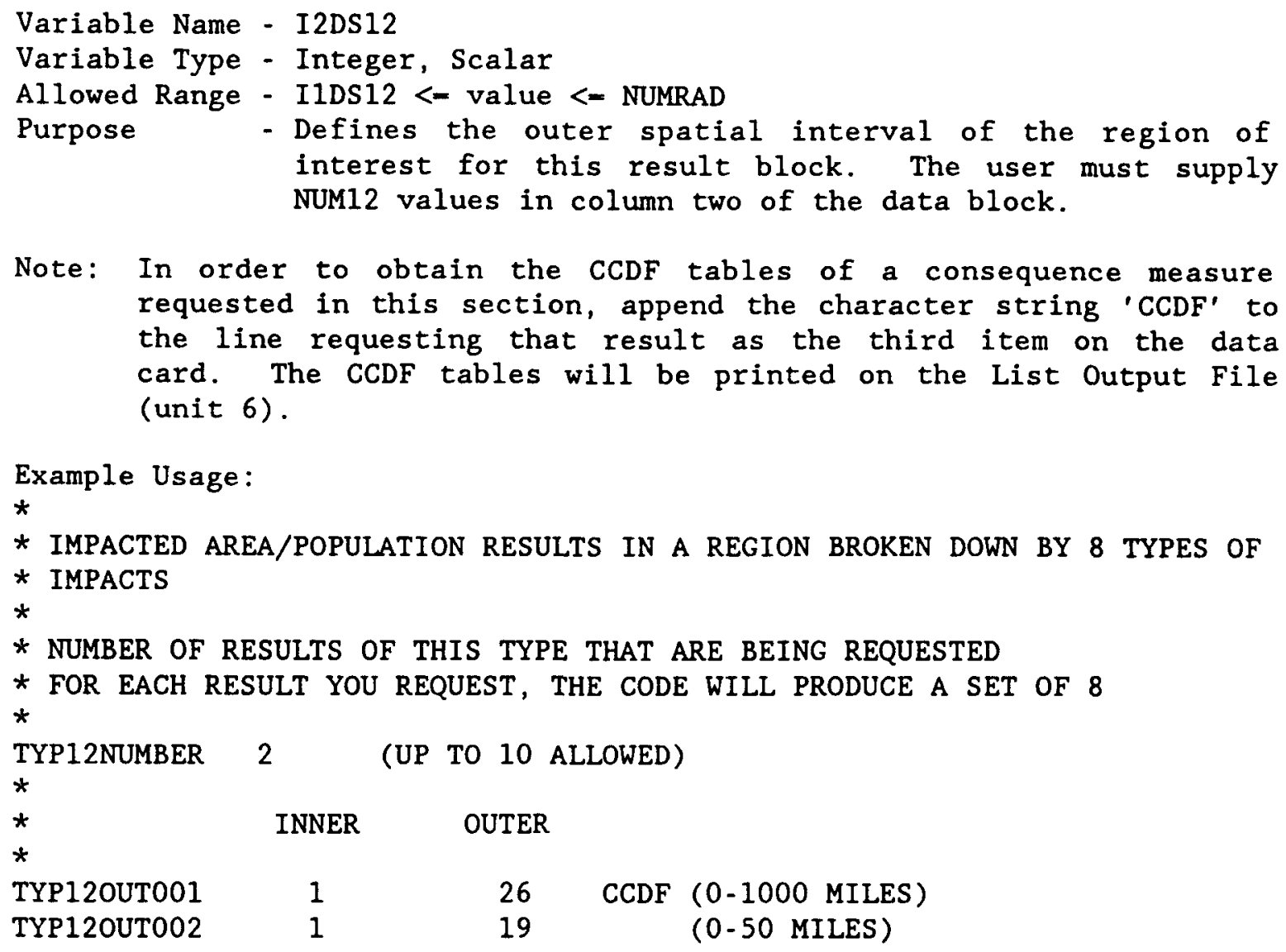

\section{References}

Bu84 Burke, R.P., D.C. Aldrich, and N.C. Rasmussen, Economic Risks of Nuclear Power Reactor Accidents, NUREG/CR-3673, SAND84-0178, Sandia National Laboratories, Albuquerque, NM, April 1984.

Ga64 Gale, H.J., D.L.O. Miller and E.M.R. Fisher, "Weathering of Caesium137 in Soil," Nature, Vol. 201, pp. 257-261, 1964.

He85 Helton, J.C., A.B. Muller and A. Bayer, "Contamination of Surface Water Bodies After Reactor Accidents by Erosion of Atmospherically Deposited Radionclides," Health Physics, Vol. 48, No. 6 (1985).

NR75 U.S. Nuclear Regulatory Commission, Reactor Safety Study: An Assessment of Accident Risks in U.S. Commercial Nuclear Power Plants, Washington, DC, WASH-1400, 1975. 


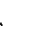




\section{A.1 Alphabetized Glossary}

This appendix is a glossary of the input names for the MACCS code. The name of the User Input File in which each variable belongs is noted. The location in the User's Guide where the variable is defined is given by section number. Each variable name is also briefly described.

\begin{tabular}{|c|c|c|c|}
\hline $\begin{array}{l}\text { VARIABLE } \\
\text { NAME } \\
\end{array}$ & $\begin{array}{l}\text { INPUT } \\
\text { FILE }\end{array}$ & SECT. & DESCRIPTION \\
\hline ACNAME & EARLY & 2.10 & Latent Cancer Effect Table \\
\hline ACSUSC & EARLY & 2.10 & Population Susceptible to Cancer \\
\hline ACTHRE & EARLY & 2.10 & Linear Response Dose Threshold \\
\hline ATNAM1 & ATMOS & 1.2 & Text Describing the ATMOS Assumptions \\
\hline ATNAM2 & ATMOS & 1.11 & Text Describing the Source Term \\
\hline BNDMXH & ATMOS & 1.14 & Boundary Weather Mixing Layer Height \\
\hline BNDRAN & ATMOS & 1.14 & Boundary Weather Rain Rate \\
\hline BNDWND & ATMOS & 1.14 & Boundary Weather Wind Speed \\
\hline BRKPNT & ATMOS & 1.8 & Break Point Time for Plume Meander \\
\hline BRRATE & EARLY & 2.5 & Breathing Rate Table \\
\hline BUILDH & ATMOS & 1.10 & Reactor Building Height \\
\hline BUILDW & ATMOS & 1.10 & Reactor Building width \\
\hline CDFRM & CHRONC & 3.5 & Farmland Decontamination Cost Table \\
\hline CDNFRM & CHRONC & 3.5 & Non-Farm Decontamination Cost Table \\
\hline CFRISK & EARLY & 2.10 & Lifetime Cancer Fatality Risk Factors \\
\hline CHNAME & CHRONC & 3.2 & CHRONC Problem Identification \\
\hline CIRISK & EARLY & 2.10 & Lifetime Cancer Injury Risk Factors \\
\hline CORINV & ATMOS & 1.11 & Table of Core Inventory by Nuclide \\
\hline CORSCA & ATMOS & 1.11 & Linear Scaling Factor on Core Inventory \\
\hline COUPLD & CHRONC & 3.12 & Coupling Flag for Food Actions \\
\hline CRIORG & EARLY & 2.7 & Critical Organ for Emergency Phase \\
\hline CRTOCR & CHRONC & 3.4 & Critical Organ for Long-Term Mitigation \\
\hline CSFACT & EARLY & 2.5 & Cloudshine Shielding Factor Table \\
\hline CTCOEF & CHRONC & 3.10 & Growing-Season Transfer Coefficient \\
\hline CTHALF & CHRONC & 3.10 & Growing-Season Weathering Half-Life \\
\hline CWASH1 & ATMOS & 1.5 & Linear Coefficient for Washout \\
\hline CWASH2 & ATMOS & 1.5 & Exponential Term for Washout \\
\hline CYSIGA & ATMOS & 1.7 & Linear Coefficient for Sigma-y \\
\hline CYSIGB & ATMOS & 1.7 & Exponential Term for Sigma-y \\
\hline CZSIGA & ATMOS & 1.7 & Linear Coefficient for Sigma-z \\
\hline CZSIGB & ATMOS & 1.7 & Exponential Term for Sigma-z \\
\hline DCYPBH & CHRONC & 3.10 & Decay/Processing Retention Meat-to-Humans \\
\hline DCYPCB & CHRONC & 3.10 & Decay/Processing Retention Crops-to-Meat \\
\hline DCYPCH & CHRONC & 3.10 & Decay/Processing Retention Crops-to-Humans \\
\hline DCYPCM & CHRONC & 3.10 & Decay/Processing Retention Crops-to-Milk \\
\hline DCYPMH & CHRONC & 3.10 & Decay/Processing Retention Milk-to-Humans \\
\hline DLBCST & CHRONC & 3.5 & Decontamination Worker Labor Cost \\
\hline DOSEFA & EARLY & 2.10 & Cancer Dose Response Alpha Factors \\
\hline
\end{tabular}




\begin{tabular}{|c|c|c|c|}
\hline $\begin{array}{l}\text { VARIABLE } \\
\text { NAME } \\
\end{array}$ & $\begin{array}{l}\text { INPUT } \\
\text { FILE } \\
\end{array}$ & $\underline{\text { SECT }}$ & DESCRIPTION \\
\hline DOSEFB & EARLY & 2.10 & Cancer Dose Response Beta Factors \\
\hline DOSFLG & EARLY & 2.14 & Acute/Lifetime Flag for Result 3 \\
\hline DOSHOT & EARLY & 2.7 & Normal Relocation Dose Threshold \\
\hline DOSNRM & EARLY & 2.7 & Hot Spot Relocation Dose Threshold \\
\hline DOSTH3 & EARLY & 2.14 & Dose Threshold for Result 3 \\
\hline DPFRCT & CHRONC & 3.9 & Farm Production Dairy Fraction \\
\hline DPRATE & CHRONC & 3.6 & Property Depreciation Rate \\
\hline DRYDEP & ATMOS & 1.4 & Dry Deposition Flag Table \\
\hline DSCRLT & CHRONC & 3.4 & Long-Term Phase Dose Criterion \\
\hline DSCRTI & CHRONC & 3.4 & Intermediate Phase Dose Criterion \\
\hline DSRATE & CHRONC & 3.6 & Societal Discount Rate for Property \\
\hline DSRFCT & CHRONC & 3.5 & Table of Decontamination Factors \\
\hline EANAM1 & EARLY & 2.2 & Text Describing the EARIY Assumptions \\
\hline EANAM2 & EARLY & 2.6 & Text Describing the Emergency Response \\
\hline EDELAY & EARLY & 2.6 & Evacuation Delay Times for 3 Rings \\
\hline EFFACA & EARLY & 2.8 & Hazard Function Fatal Alpha Factors \\
\hline EFFACB & EARLY & 2.8 & Hazard Function Fatal Beta Factors \\
\hline EIFACA & EARLY & 2.9 & Hazard Function Injury Alpha Factors \\
\hline EIFACB & EARLY & 2.9 & Hazard Function Injury Beta Factors \\
\hline EINAME & EARLY & 2.9 & Table of Early Injury Effect Names \\
\hline EISUSC & EARLY & 2.9 & Susceptible Population Fraction Table \\
\hline EITHRE & EARLY & 2.9 & Early Injury Dose Threshold Table \\
\hline ENDAT1 & ATMOS & 1.2 & Flag Indicating Only Run Atmos \\
\hline ENDAT2 & EARLY & 2.2 & Flag Indicating Only Run Atmos and Early \\
\hline ENDEMP & EARLY & 2.7 & Duration of the Early Phase \\
\hline ESPEED & EARLY & 2.6 & Radial Evacuation Speed \\
\hline EVACST & CHRONC & 3.3 & Evacuation Cost Per Person-Day \\
\hline FLAG11 & CHRONC & 3.16 & Flag for Maximum Distance Results \\
\hline FPLSCH & CHRONC & 3.10 & Food Processing Losses Crops-to-Humans \\
\hline FRACLD & CHRONC & 3.9 & Fraction of Site Area That is Land \\
\hline FRCFRM & CHRONC & 3.9 & Fraction of Site Land Used for Farming \\
\hline FRCTCB & CHRONC & 3.10 & Fraction of Crop Consumed by Meat Animals \\
\hline FRCTCH & CHRONC & 3.10 & Fraction of Crop Consumed by Humans \\
\hline FRCTCM & CHRONC & 3.10 & Fraction of Crop Consumed by Milk Animals \\
\hline FRCTFL & CHRONC & 3.11 & Fraction of Farmland Used for Each Crop \\
\hline FRFDL & CHRONC & 3.5 & Farm Labor Cost Fraction Table \\
\hline FRFIM & CHRONC & 3.9 & Farm Wealth Improvements Fraction \\
\hline FRMPRD & CHRONC & 3.9 & Average Annual Farm Production Value \\
\hline FRNFDL & CHRONC & 3.5 & Non-Farm Labor Cost Fraction Table \\
\hline FRNFIM & CHRONC & 3.9 & Non-Farm Wealth Improvements Fraction \\
\hline GCMAXR & CHRONC & 3.12 & Long-Term Uptake to Milk or Crops Pags \\
\hline GSHFAC & EARLY & 2.5 & Groundshine Shielding Factor Table \\
\hline GWCOEF & CHRONC & 3.7 & Groundshine Weathering Coefficients \\
\hline HAFLIF & ATMOS & 1.4 & Table of Nuclide Half-Lives \\
\hline HRMXHT & ATMOS & 1.17 & Table of Mixing Layer Heights \\
\hline HRRAIN & ATMOS & 1.17 & Table of Rain Rates \\
\hline HRWNDV & ATMOS & 1.17 & Table of Wind Speeds \\
\hline IIDIS1 & EARLY & 2.12 & Inner Spatial Interval for Result 1 \\
\hline I1DIS4 & EARLY & 2.15 & Spatial Interval for Result 4 \\
\hline
\end{tabular}




\begin{tabular}{|c|c|c|c|}
\hline $\begin{array}{c}\text { VARIABLE } \\
\text { NAME } \\
\end{array}$ & $\begin{array}{l}\text { INPUT } \\
\text { FILE }\end{array}$ & SECT, & DESCRIPTION \\
\hline I1DIS5 & EARLY & 2.16 & Inner Spatial Interval for Result 5 \\
\hline IIDIS6 & EARIY & 2.17 & Inner Spatial Interval for Result 6 \\
\hline I1DIS7 & EARLY & 2.18 & Inner Spatial Interval for Result 7 \\
\hline I1DIS8 & EARLY & 2.19 & Inner Spatial Interval for Result 8 \\
\hline I1DS10 & CHRONC & 3.15 & Inner Spatial Interval for Result 10 \\
\hline IIDS12 & CHRONC & 3.17 & Inner Spatial Interval for Result 12 \\
\hline I2DIS1 & EARLY & 2.12 & Outer Spatial Interval for Result 1 \\
\hline I2DIS5 & EARLY & 2.16 & Outer Spatial Interval for Result 5 \\
\hline I2DIS6 & EARLY & 2.17 & Outer Spatial Interval for Result 6 \\
\hline I2DIS7 & EARLY & 2.18 & Outer Spatial Interval for Result 7 \\
\hline I2DIS8 & EARLY & 2.19 & Outer Spatial Interval for Result 8 \\
\hline I2DS10 & CHRONC & 3.15 & Outer Spatial Interval for Result 10 \\
\hline I2DS12 & CHRONC & 3.17 & Outer Spatial Interval for Result 12 \\
\hline IBDSTB & ATMOS & 1.14 & Boundary Weather Stability Class \\
\hline IBEGIN & EARLY & 2.3 & Interval at Which Population Begins \\
\hline IDEBUG & ATMOS & 1.12 & Debug Switch for Dispersion Print \\
\hline IGROUP & ATMOS & 1.4 & Table of the Nuclide Groups \\
\hline IHRDIR & ATMOS & 1.17 & Table of Wind Directions \\
\hline IHRSTB & ATMOS & 1.17 & Table of Stability Classes \\
\hline INIEVA & EARLY & 2.6 & First Evacuation Interval \\
\hline INDXBN & ATMOS & 1.16 & Index to Bin Number Used for Met Sample \\
\hline INWGHT & ATMOS & 1.16 & Number of Samples to Take From the Bin \\
\hline IPLUME & ATMOS & 2.2 & Plume Model Dispersion Code \\
\hline IPRINT & EARLY & 2.2 & Quantity of Early Debug Output \\
\hline IRSEED & ATMOS & 1.16 & Seed for Random Number Generator \\
\hline ISTRDY & ATMOS & 1.15 & Starting Day of the Accident \\
\hline ISTRHR & ATMOS & 1.15 & Starting Hour of the Accident \\
\hline KSWTCH & CHRONC & 3.13 & Diagnostic Output Option Switch Table \\
\hline LASEVA & EARLY & 2.6 & Table of Last Evacuating Intervals \\
\hline LASHE2 & EARLY & 2.7 & Last Ring of Outer Shelter Zone \\
\hline LASMOV & EARLY & 2.6 & Last Evacuation Movement Interval \\
\hline LIMSPA & ATMOS & 1.14 & Last Spatial Interval for Recorded Weather \\
\hline LVLDEC & CHRONC & 3.5 & Number of Decontamination Levels \\
\hline MAXGRP & ATMOS & 1.4 & Number of Nuclide Groups \\
\hline MAXRIS & ATMOS & 1.11 & Selection of Risk Dominant Plume \\
\hline METCOD & ATMOS & 1.13 & Meteorological Sampling Option Code \\
\hline NAMCRP & CHRONC & 3.10 & Names of the Crop Categories \\
\hline NAMCRP & CHRONC & 3.11 & Names of the Crop Categories \\
\hline NAME & EARLY & 2.12 & Result Type 1 Health Effect Name \\
\hline$n$ & EARLY & 2.14 & Result Type 3 Organ Name \\
\hline$"$ & EARLY & 2.15 & Result Type 4 Health Effect Name \\
\hline$"$ & EARLY & 2.16 & Result Type 5 Organ Name \\
\hline$"$ & EARLY & 2.18 & Result Type 7 Health Effect Name \\
\hline$n$ & EARLY & 2.19 & Result Type 8 Health Effect Name \\
\hline NAMIPI & CHRONC & 3.10 & Names of Food Ingestion Nuclides \\
\hline NAMIPI & CHRONC & 3.12 & Names of Food Ingestion Nuclides \\
\hline NAMWPI & CHRONC & 3.10 & Names of Water Ingestion Nuclides \\
\hline NFICRP & CHRONC & 3.10 & Number of Crop Categories \\
\hline NFIISO & CHRONC & 3.10 & Number of Nuclides for Food Ingestion \\
\hline
\end{tabular}




\begin{tabular}{|c|c|c|c|}
\hline $\begin{array}{l}\text { VARIABLE } \\
\text { NAME } \\
\end{array}$ & $\begin{array}{l}\text { INPUT } \\
\text { FILE } \\
\end{array}$ & SECT. & DESCRIPTION \\
\hline NGWTRM & CHRONC & 3.7 & Terms in Groundshine Weathering Equation \\
\hline NPSGRP & ATMOS & 1.6 & Number of Particle Size Groups \\
\hline NRINTN & ATMOS & 1.16 & Number of Rain Intensity Breakpoints \\
\hline NRNINT & ATMOS & 1.16 & Number of Rain Distance Intervals \\
\hline NRWTRM & CHRONC & 3.8 & Terms in Resuspension Weathering Equation \\
\hline NSBINS & ATMOS & 1.16 & Number of Weather Bins to Sample \\
\hline NSMPLS & ATMOS & 1.16 & Number of Weather Samples Per Bin \\
\hline NTTRM & CHRONC & 3.10 & Number of Terms Growing-Season to Food \\
\hline NUCNAM & ATMOS & 1.4 & Table of the Nuclide Names \\
\hline NucouT & ATMOS & 1.12 & Nuclide Used in Dispersion Print \\
\hline NUM1 & EARLY & 2.12 & Number of Health Effect Results \\
\hline NUM2 & EARLY & 2.13 & Number of Early Fatality Radius Results \\
\hline NUM3 & EARLY & 2.14 & Number of Early Dose Threshold Results \\
\hline NUM4 & EARLY & 2.15 & Number of Avg Individual Risk Results \\
\hline NUM5 & EARLY & 2.16 & Number of Population Dose Results \\
\hline NUM6 & EARLY & 2.17 & Number of Centeriine Dose/Distance Results \\
\hline NUM7 & EARLY & 2.18 & Number of Centerline Risk/Distance Results \\
\hline NUM8 & EARLY & 2.19 & Number of Population Weighted Risk Results \\
\hline NUM12 & CHRONC & 3.17 & Number of Affected Area Results \\
\hline NUMACA & EARLY & 2.10 & Number of Acute Exposure Cancers \\
\hline NUMEFA & EARLY & 2.8 & Number of Early Fatality Effects \\
\hline NUMEIN & EARLY & 2.9 & Number of Early Injury Effects \\
\hline NUMFIN & EARLY & 2.2 & Number of Fine Grid Subdivisions \\
\hline NUMISO & ATMOS & 1.4 & Number of Defined Nuclides \\
\hline NUMORG & EARLY & 2.4 & Number of Defined Organs \\
\hline NUMRAD & ATMOS & 1.3 & Number of Spatial Intervals \\
\hline NUMREL & ATMOS & 1.11 & Number of Released Plume Segments \\
\hline NUMWPI & CHRONC & 3.10 & Number of Nuclides for Water Ingestion \\
\hline NXUM10 & CHRONC & 3.15 & Number of Economic Cost Results \\
\hline OALARM & ATMOS & 1.11 & Off-Site Alarm Time \\
\hline ORGNAM & EARLY & 2.4 & Table of Defined Organ Names \\
\hline " & EARLY & 2.8 & Early Fatality Target Organ Table \\
\hline " & EARIY & 2.9 & Early Injury Target Organ Table \\
\hline " & EARLY & 2.10 & Latent Cancer Target Organ Table \\
\hline " & EARLY & 2.17 & Organ Name of the Centerline Doses \\
\hline OVRRID & EARLY & 2.2 & Wind-Rose Probability Override \\
\hline PARENT & ATMOS & 1.4 & Table of Nuclide Parent Names \\
\hline PDELAY & ATMOS & 1.11 & Table of Plume Release Times \\
\hline PATHNM & EARLY & 2.17 & Pathway Code for Result 6 \\
\hline PLHEAT & ATMOS & 1.11 & Table of Plume Heat Contents \\
\hline PLHITE & ATMOS & 1.11 & Table of Plume Release Heights \\
\hline PLUDUR & ATMOS & 1.11 & Table of Plume Segment Durations \\
\hline POPCST & CHRONC & 3.6 & Population Removal Cost \\
\hline POPDEN & EARLY & 2.3 & Average Regional Population Density \\
\hline POPFLG & EARLY & 2.3 & Population Distribution Flag \\
\hline PROTIN & EARLY & 2.5 & Inhalation Protection Factor Table \\
\hline PSCMLK & CHRONC & 3.12 & Growing-Season to Milk P.A.G. \\
\hline PSCOTH & CHRONC & 3.12 & Growing-Season to Crops P.A.G. \\
\hline PSDIST & ATMOS & 1.11 & Particle Size Distribution by Group \\
\hline
\end{tabular}




\begin{tabular}{|c|c|c|c|}
\hline $\begin{array}{l}\text { VARIABLE } \\
\text { NAME } \\
\end{array}$ & $\begin{array}{l}\text { INPUT } \\
\text { FILE } \\
\end{array}$ & SECT. & DESCRIPTION \\
\hline QROOT & CHRONC & 3.12 & Long-Term Uptake Effectiveness Decay Rate \\
\hline REFTIM & ATMOS & 1.11 & Plume Reference Time Point \\
\hline RELCST & CHRONC & 3.3 & Relocation Cost Per Person-Day \\
\hline RELFRC & ATMOS & 1.11 & Table of Release Fractions by Group \\
\hline RESCON & EARLY & 2.5 & Resuspension Concentration Coefficient \\
\hline RESHAF & EARLY & 2.5 & Resuspension Concentration Half-Iife \\
\hline RISCAT & EARLY & 2.2 & Risk by Weather Category Flag \\
\hline RISTHR & EARLY & 2.13 & Risk Threshold for Fatality Radius \\
\hline RNDSTS & ATMOS & 1.16 & Rain Distances Used for Binning \\
\hline RNRATE & ATMOS & 1.16 & Rain Intensities Used for Binning \\
\hline RWCOEF & CHRONC & 3.8 & Resuspension Weathering Coefficients \\
\hline SCLADP & ATMOS & 1.9 & Scaling Factor for A-D Plume Rise \\
\hline SCLCRW & ATMOS & 1.9 & Scaling Factor for Critical Wind Speed \\
\hline SCLEFP & ATMOS & 1.9 & Scaling Factor for E-F Plume Rise \\
\hline SHELT1 & EARLY & 2.7 & Shelter Duration for Inner Zone \\
\hline SHELT2 & EARLY & 2.7 & Shelter Duration for Outer Zone \\
\hline SKPFAC & EARLY & 2.5 & Skin Protection Factor Table \\
\hline SPAEND & ATMOS & 1.3 & Endpoints of Spatial Intervals \\
\hline TCROOT & CHRONC & 3.10 & Transfer Factor Long-Term Uptake \\
\hline TFBF & CHRONC & 3.10 & Transfer Factor Animal-to-Meat \\
\hline TFMILK & CHRONC & 3.10 & Transfer Factor Animal-to-Milk \\
\hline TFWKF & CHRONC & 3.5 & Table of Farm Worker's Work Fraction \\
\hline TFWKNF & CHRONC & 3.5 & Table Non-Farm Workers Work Fraction \\
\hline TGSBEG & CHRONC & 3.11 & Growing Season Start Time for Each Crop \\
\hline TGSEND & CHRONC & 3.11 & Growing Season End Time for Each Crop \\
\hline TGWHLF & CHRONC & 3.7 & Groundshine Weathering Half-Lives \\
\hline TIMBAS & ATMOS & 1.8 & Time Base for Plume Meander \\
\hline TIMDEC & CHRONC & 3.5 & Table of Decontamination Times \\
\hline TIMHOT & EARLY & 2.7 & Hot Spot Relocation Time \\
\hline TIMNRM & EARLY & 2.7 & Normal Relocation Time \\
\hline TMIPND & CHRONC & 3.4 & Time Intermediate Phase Ends \\
\hline TMPACT & CHRONC & 3.4 & Time Action Period Ends \\
\hline TRWHLF & CHRONC & 3.8 & Resuspension Weathering Half-Lives \\
\hline TTOSH1 & EARLY & 2.7 & Take Shelter Time for Inner Zone \\
\hline TTOSH2 & EARLY & 2.7 & Take Shelter Time for Outer Zone \\
\hline VALWF & CHRONC & 3.9 & Value of Farm Wealth \\
\hline VALWNF & CHRONC & 3.9 & Value of Non-Farm Wealth \\
\hline VDEPOS & ATMOS & 1.6 & Table of Dry Deposition Velocities \\
\hline WETDEP & ATMOS & 1.4 & Wet Deposition Flag Table \\
\hline WINGF & CHRONC & 3.10 & Water Transfer Fraction to Humans \\
\hline WINROS & EARLY & 2.2 & Table of Wind-Rose Probabilities \\
\hline WSHFRI & CHRONC & 3.10 & Initial Water Washoff Fraction \\
\hline WSHRTA & CHRONC & 3.10 & Annual Water Washoff Fraction \\
\hline WTFRAC & EARLY & 2.6 & Weighting Fraction for Combined Results \\
\hline WTNAME & EARLY & 2.6 & Type of Weighting (People, Time) \\
\hline XPFAC1 & ATMOS & 1.8 & Base Time Meander Expansion Factor \\
\hline XPFAC2 & ATMOS & 1.8 & Break Point Time Expansion Factor \\
\hline YSCALE & ATMOS & 1.7 & Scale Factor for Horizontal Dispersion \\
\hline ZSCALE & ATMOS & 1.7 & Scale Factor for Vertical Dispersion \\
\hline
\end{tabular}




\section{.}


APPENDIX B

\section{AUXILIARY INPUT FILES}

\section{B.1 Meteorological Data File Format}

For METCOD values of $1,,^{\prime} 2$ or 5 (see Section 1.13), a meteorological data file must be provided by the user. The file consists of one year of hourly recordings (8760) of the wind direction, wind speed, atmospheric stability and accumulated precipitation. Generally, the data are taken from either the reactor site or from a nearby weather station.

The meteorological data file used in MACCS is a formatted file of eyereadable text. The format is similar to that used in the CRAC2 consequence model. If an additional title card is added at the beginning of the file, CRAC2 meteorological data files may be used with MACCS. There are a total of 8763 records in the meteorological data file. The first two records contain identification information. Up to 80 characters may be used on each line. This header information is printed on the output listing. Records 3 through 8762 contain hourly meteorological observations, one hour per line. The format of the information is as follows:

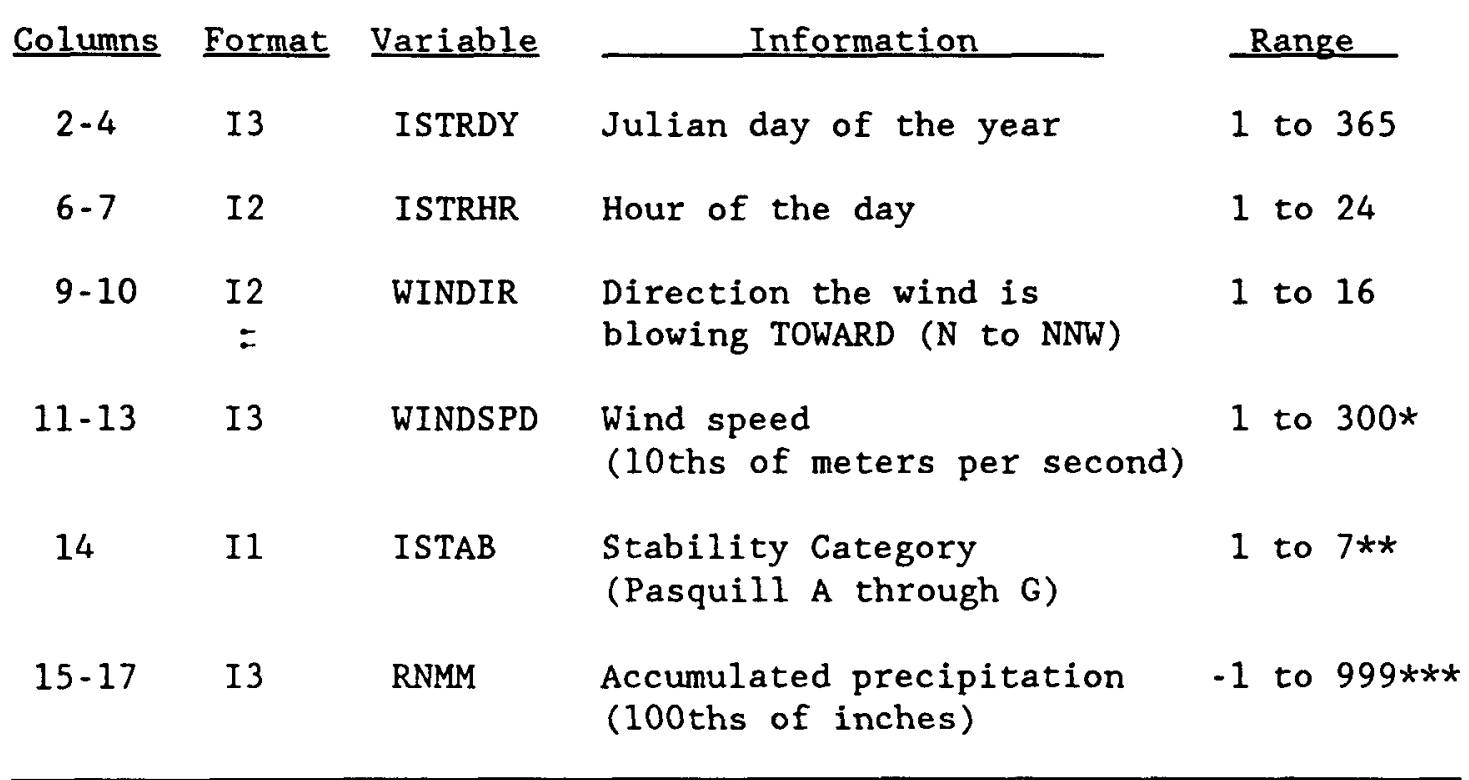

*Values between 1 and 4 are automatically changed to $5(0.5 \mathrm{~m} / \mathrm{s})$. $* *$ A value of 7 is automatically changed to 6 by the code. $* * *$ Some meteorological data files use -1 to indicate a trace of precipitation during the hour. MACCS assumes these values to be zero. 
The 8763rd record contains a table of eight values of mixing layer height. Two values of mixing height are supplied for each of the four seasons of the year. The first of these two values corresponds to the morning mixing height and the second to the afternoon height. In the current implementation, the larger of these two values and the value of the boundary weather mixing height is used by the code.

In its present form, that atmospheric model implemented in MACcs does not allow a change in mixing layer to occur during transport of the plume. Mixing layer height is assumed to be constant and therefore only a single value is used by the code. Only seasonal variability can be considered for this parameter. The start day of each weather sequence determines the season in which that sequence lies.

Note: The following data is supplied in units of hundreds of meters.

\begin{tabular}{|c|c|c|c|c|}
\hline Columns & Format & Variable & Information & Range \\
\hline $1-10$ & F10.0 & $\operatorname{HEIGHT}(1,1)$ & Morning-winter mixing height & to 999 . \\
\hline $11-2$ & F10 & HEIGH & Morning-spring mixing height & 0 . to 999 . \\
\hline $21-30$ & F10.0 & $\operatorname{HEIGHT}(3,1)$ & Morning-summer mixing height & to 999 . \\
\hline $31-40$ & F10.0 & $\operatorname{HEIGHT}(4,1)$ & Morning-autumn mixing height & 0. to 999 . \\
\hline & F10.0 & $\operatorname{HEIGHT}(1,2)$ & Afternoon-winter mixing height & 0 . to 999 . \\
\hline & F10.0 & $\operatorname{HEIGHT}(2,2)$ & Afternoon-spring mixing height & 0 . to 999 . \\
\hline $61-70$ & F10.0 & $\operatorname{HEIGHT}(3,2)$ & Afternoon-summer mixing height & to 999 . \\
\hline $71-80$ & $F 10.0$ & $\operatorname{HEIGHT}(4,2)$ & Afternoon-autumn mixing height & 0. to 999 . \\
\hline
\end{tabular}

An example of the meteorological data file is given in below. The figure shows the first and last ten records of the file. For example, the weather conditions for day one, hour one (record number three) are: wind direction - blowing toward the $S E$, wind speed of $2.6 \mathrm{~m} / \mathrm{sec}$, stability 4 (category D), and accumulated precipitation of 0.07 inches $(1.8 \mathrm{~mm}$ ) in the hour. The winter, spring, summer and autumn mixing heights for neutral and unstable conditions (record 8763) are all 1200 meters.

\section{Portion of the Meteorological Data File Used In MACCS}

The first and last ten records are shown.

U.S. NATIONAL WEATHER SERVICE METEOROLOGICAL DATA FILE FOR NEW YORK, NY. USED FOR THE INDIAN POINT REACTOR SITE. D. CHANIN, SANDIA 5058465447

$\begin{array}{rrrr}1 & 1 & 70264 & 7 \\ 1 & 2 & 60624 & 7 \\ 1 & 3 & 80414 & 2 \\ 1 & 4 & 80364 & -1 \\ 1 & 5 & 90314 & 0 \\ 1 & 6 & 110464 & 0 \\ 1 & 7 & 110264 & -1\end{array}$


$18 \begin{array}{llll}110414 & -1\end{array}$

.

$\begin{array}{llll}365 & 16 & 120154 \quad 4\end{array}$

$\begin{array}{llll}365 & 17 & 110154 & 4\end{array}$

$\begin{array}{llll}365 & 18 & 130214 & 4\end{array}$

$\begin{array}{llll}365 & 19 & 130154 & 3\end{array}$

$\begin{array}{llll}365 & 20 & 130104 & 2\end{array}$

$\begin{array}{llll}365 & 21 & 130154 & 2\end{array}$

$\begin{array}{llll}365 & 22 & 140104 & 2\end{array}$

$\begin{array}{llll}365 & 23 & 130104 & 2\end{array}$

$\begin{array}{llll}365 & 24 & 140104 & 2\end{array}$
12 .

12.

12.

12.

B -3 


\section{B.2 Dose Conversion Factors File Format}

A dosimetry data file is required for both the EARLY and CHRONC modules. Since the dosimetry data file is generally the same for all calculations, the file is attached directly by the procedure files used to run MACCS; the user does not need to specify the name of the dosimetry file. The dosimetry data in the file are dose factors for: cloudshine ( $\mathrm{Sv}-\mathrm{m} * * 3 / \mathrm{Bq}-$ sec), groundshine integrated for 8 hours ( $\mathrm{Sv}-\mathrm{m} * * 2 / \mathrm{Bq}$ ), groundshine integrated for 7 days $(\mathrm{Sv}-\mathrm{m} * * 2 / \mathrm{Bq}$ ), groundshine dose rate (Sv-m**2/Bqsec), internal doses from inhalation used with the early health effects model $(\mathrm{Sv} / \mathrm{Bq})$, internal doses from inhalation for lifetime exposure $(\mathrm{Sv} / \mathrm{Bq})$, and internal doses from ingestion ( $\mathrm{Sv} / \mathrm{Bq})$. If an organ is not considered in the early health effects model, no internal dose for early exposure from inhalation is given; the values are set to -1.0 . The integrated dose factors for groundshine include any contribution from daughter nuclides.

The current dosimetry file contains data for 60 radionuclides and 12 organs. A description of the method used to calculate the dosimetry data is provided in Appendix D of the MACCS Model Description (Volume 2 of this document).

The dosimetry data file used in MACCS is a formatted file. The first two records contain identification information. Up to 80 characters may be used on each line. This header information is printed on the output listing. The next line gives the number of organs included in the file. The format for this line is I10. The organ names are specified next, one name per line. The spelling must be the same as that used in all other program data files. Next the number of nuclides included in the file is given. The format is again I10. The names of the nuclides are given on the following lines, one name per line. The spelling of the nuclide names must again be the same as in all other program modules. Following the list of nuclides, there are two lines in the dosimetry data file that are not read but aid user understanding of the file. On the next line is the name of the first nuclide followed on the next lines by the organ names and the associated dosimetry data. The format of the dosimetry data is as follows:

\begin{tabular}{|c|c|c|c|}
\hline Column & Variable & Format & Identification \\
\hline $1-10$ & FILORG & A10 & Name of the organ \\
\hline $11-20$ & CDCF & F10.0 & Cloudshine dose conversion factor \\
\hline $21-30$ & $\operatorname{IGDCF}(I, J, 1)$ & F10.0 & $\begin{array}{l}\text { 8-hour integrated groundshine dose } \\
\text { factor }\end{array}$ \\
\hline $31-40$ & $\operatorname{IGDCF}(I, J, 2)$ & F10.0 & $\begin{array}{l}\text { 7-day integrated groundshine dose } \\
\text { factor }\end{array}$ \\
\hline
\end{tabular}




\begin{tabular}{|c|c|c|c|}
\hline Column & Variable & Format & Identification \\
\hline $41-50$ & GRDCF & F10.0 & Groundshine dose-rate factor \\
\hline $51-60$ & $\operatorname{IDCF}(I, J, 1)$ & F10.0 & $\begin{array}{l}\text { Inhalation dose factor used only with } \\
\text { the early health effects model }\end{array}$ \\
\hline $61-70$ & $\operatorname{IDCF}(I, J, 2)$ & F10.0 & $\begin{array}{l}\text { Inhalation dose factor for lifetime } \\
\text { exposure }\end{array}$ \\
\hline $71-80$ & DFING & F10.0 & Ingestion dose factor \\
\hline
\end{tabular}

A complete listing of the current MACCS Dose Conversion Factors Data File is included in this document as Appendix D.1. 


\section{B.3 Site Data File Format}

In the Site Data File, the user specifies the population distribution and land use information for the region surrounding the site. Contained in the Site Data File are the geometry data used for the site (spatial intervals), population distribution, fraction of the area that is land, watershed data for the liquid pathways model, information on agricultural land use and growing seasons, and regional economic information. An example of a Site Data File is provided in Appendix D.2.

The decision on whether to use a Site Data File is determined by a flag set in the EARLY User Input File (see Section 2.3). If a Site Data File is not being used, the EARLY User Input File determines the population density to be used by both the EARLY and CHRONC modules. It is not possible for the user to supply differing population data for the two modules.

The Site Data File used in MACCS is a formatted file. The data must appear exactly as described in this Appendix and in exactly the same order. In contrast to the User Input Files for MACCS, which are processed by a free-format input processor, the Site Data File is processed with fixed format FORTRAN READ statements.

The use of fixed format READ statements requires that the user exercise special attention to line up the data items in their proper fields. Any numeric items specified in exponential format (e.g., 1.E-6) must be right-justified in the field.

In contrast to the User Input Files, where every value is validated by the code to ensure that it lies within a range of allowable values, the validation performed on the Site Data is only partially complete. Some of the input parameters on this file are rigorously checked to ensure that they fall within the allowed range, while other values are not checked at all. It is recommended that the user exercise scrupulous care in constructing a Site Data File. It is very important that all items appear in their proper fields and that all numeric values lie within the range of acceptable values. Failure to conform to these requirements may lead to the generation of spurious results.

The first two records of the site Data File contain identification information. Up to 80 characters may be used on each line. This header information is printed on the output listing.

Following the descriptive text fields, there are six data cards which specify the amount of data which is being supplied on the file. The values defined on these data cards must be consistent with the MACCS model definition data defined by the ATMOS, EARLY, and CHRONC User Input Files. In other words, the value of the Site Data File input variable and the corresponding comparison variable must be identical. If any 
inconsistencies are detected, execution of the program will be terminated.

An example of the data cards described up to this point is included for the purpose of illustration.

MACCS SITE DATA FILE FOR SURRY (JLS, 11/10/88)

SECPOP POP DISTRIBUTION FROM 1980 CENSUS DATA ALTERED USING 0-10 MI NRC

26 SPATIAL INTERVALS

16 WIND DIRECTIONS

7 CROP CATEGORIES

4 WATER PATHWAY ISOTOPES

2 WATERSHEDS

59 ECONOMIC REGIONS

The format of the data is as follows:

\begin{tabular}{cccccccc}
\hline Line & Columns & Format & $\begin{array}{c}\text { Input } \\
\text { Variable }\end{array}$ & Identification & $\begin{array}{c}\text { Comparison } \\
\text { Variable }\end{array}$ & $\begin{array}{c}\text { Allowed } \\
\text { Range }\end{array}$ \\
4 & $1-4$ & I4 & NSPDTS & $\begin{array}{l}\text { Number of spatial } \\
\text { intervals }\end{array}$ & NUMRAD & $2-35$ \\
5 & $1-4$ & I4 & NWNDIR & $\begin{array}{l}\text { Number of wind } \\
\text { direction sectors }\end{array}$ & NUMCOR & $16-16$ \\
6 & $1-4$ & I4 & NCPGZN & $\begin{array}{l}\text { Number of crops } \\
\text { to be considered }\end{array}$ & NFICRP & $1-10$ \\
7 & $1-4$ & I4 & NWTRSH & $\begin{array}{l}\text { Number of watershed } \\
\text { categories }\end{array}$ & NUMWPA & $1-4$ \\
8 & $1-4$ & I4 & NECRGN & $\begin{array}{l}\text { Number of water } \\
\text { pathway nuclides } \\
\text { regions }\end{array}$ & NUMWPI & $1-10$ \\
\hline
\end{tabular}

Eight blocks of site data follow the introductory block described above. Each of these data blocks is introduced by a separator line that identifies the block of data to follow. The first line of each data block must be the separator for that block. Since each separator is read with a format of $1 \mathrm{X}, \mathrm{A} 22$, the first character of the separator line is ignored and the following 22 characters must match the identification field for that specific data block. 


\section{Geometry Data Block}

Geometry information is used to specify the population and land use data. The geometry data consist of the distance in kilometers to the endpoints of the spatial intervals. This grid definition must agree with the grid used in the ATMOS User Input File (see Section 1.3). A relative error of 10 percent in the endpoint distances is allowed. For larger discrepancies in the geometry data, the error flag will be set and execution will terminate upon completion of the Site Data File input processing.

The first line of the geometry information contains the 22 character separator beginning with "SPATIAL DISTANCES" in column 2. Next, the endpoint distances in kilometers are specified, eight values per line, using the format described below. As many lines as are needed to define the spatial distances are used. The formatting of this information is illustrated below by presentation of the spatial distance data for a single wind direction sector.

Note: The spacing between adjacent spatial intervals should be at least 0.1 kilometer.

\begin{tabular}{rrrrrrrr} 
SPATIAL & DISTANCES & \multicolumn{7}{c}{ } & & & & \\
0.16 & 0.52 & 1.21 & 1.61 & 2.13 & 3.22 & 4.02 & 4.83 \\
5.63 & 8.05 & 11.27 & 16.09 & 20.92 & 25.75 & 32.19 & 40.23 \\
48.28 & 64.37 & 80.47 & 112.65 & 160.93 & 241.14 & 321.87 & 563.27 \\
804.67 & 1609.34 & & & & & &
\end{tabular}

The format used to process the data is as follows:

\begin{tabular}{ccccccc}
\hline Columns & Format & $\begin{array}{c}\text { Input } \\
\text { Variable }\end{array}$ & Information & $\begin{array}{c}\text { Comparison } \\
\text { Variable }\end{array}$ & $\begin{array}{c}\text { Allowed } \\
\text { Range }\end{array}$ \\
\hline 1.80 & $8 E 10.2$ & SPDSTS & $\begin{array}{c}\text { Spatial interval } \\
\text { endpoints (km) }\end{array}$ & SPAEND & $0.001-9999.0$ \\
\hline
\end{tabular}

\section{Population Data Block}

The population data for each element in the spatial grid is defined here. The first line of the data block contains the 22 character separator beginning with "POPULATION" in column 2. Next, the number of people in each element is given for the first sector (The first sector is assumed to be centered on North). The population data are specified, eight values per line, using the format described below. As many lines as needed to cover all the spatial elements in the sector are used. Proceeding in a clockwise rotation, the population data for the second (NNE) and subsequent sectors follow. Data for all 16 wind directions (sectors) must be provided. Data for each sector begins on a new line. The formatting of this information is illustrated below by presentation 
of the population data for a single wind direction sector for 26 radial distance elements.

POPULATION

$\begin{array}{rrrrrrrr}0 . & 0 . & 0 . & 0 . & 0 & 0 . & 4 . & 5 . \\ 6 . & 25 . & 3341 . & 7107 . & 2173 . & 0 . & 1305 . & 474 . \\ 2252 . & 2945 . & 5403 . & 20169 . & 112004 . & 3431358 . & 1355700 . & 2742710 . \\ 2487346 . & 104331 . & & & & & & \end{array}$

The format used to process the data is as follows:

\begin{tabular}{lllll}
\hline Columns & Format & Variable & Identification & Allowed Range \\
$1-80$ & $8 E 10.0$ & POPDAT & Population data & $0.0-1 . E 9$ \\
\hline
\end{tabular}

\section{Land Fraction Block}

The fraction of each spatial element that is land (as opposed to lakes, oceans, etc.) must be defined. The first line of the data block contains the 22 character separator beginning with "LAND FRACTION" in column 2. Next, the fraction of area that is land in each radial spatial interval of the first sector is given. All values must be between zero and one. A value of zero means the element has no land, a value of one means the element is all land. The land fraction data are specified, 16 values per line, with the format described below. As many lines as needed to define all the spatial intervals in the sector are used. The land fraction data for the second and subsequent sectors follow in a clockwise rotation. Data for all 16 wind direction sectors must be provided. The data for each sector begins on a new line. The formatting of this information is illustrated below by presentation of the land fraction data for a single wind direction sector.

LAND FRACTION

$\begin{array}{llllllllllllll}1.00 & 1.00 & 0.00 & 0.00 & 0.00 & 0.00 & 0.00 & 0.00 & 0.00 & 0.80 & 1.00 & 1.00 & 0.95\end{array}$

$0.75 \quad 0.70 \quad 0.85$

The format used to process the data is as follows:

\begin{tabular}{llllc}
\hline Columns & Format & Variable & Identification & Allowed Range \\
\cline { 4 - 5 } $1-80$ & $16 \mathrm{~F} 5.2$ & FRCLND & Land fraction & $0.0-1.0$ \\
\hline
\end{tabular}

\section{Region Identification Block}

This data block identifies each of the spatial intervals with an economic region. A region can be a state, a county, or any division desired. A 
description of the economic data used for each region is given later in this Appendix. The first line in the datablock contains the 22 character separator beginning with "REGION INDEX" in column 2. The next line contains two-digit integers associating a region index with each of the spatial elements in the first sector. The data are specified with the format defined below. All of the region indices for one sector will fit on one line. The region indices for the second and subsequent sectors are on the following lines, a new line for each sector in a clockwise rotation. A total of 16 lines are required. For example, a region index of 09 means that economic data for region number nine will be used for the spatial element. (See the section of this Appendix on Regional Economic Data.) The formatting of this information is illustrated below by a presentation of the region identification data for a single wind direction sector.

REGION INDEX

444450505050505050444444444444444444444418187283054

The format used to process the data is as follows:

\begin{tabular}{lcccc}
\hline Columns & Format & Variable & Identification & Allowed Range \\
$1-80$ & 4012 & INDREG & Region index & 1 - NECRGN \\
\hline
\end{tabular}

\section{Watershed Identification Block}

Each of the spatial intervals in the grid must be associated with one of the watershed classes. The definition of the efficiency of these watershed classes is described later in this appendix in the "WATERSHED DEFINITION" block. The watershed identification data block begins with the 22 character separator beginning with "WATERSHED INDEX" in column 2. The next line contains two-digit integers associating a watershed type with each of the spatial elements in the first sector. The data are specified with the format defined below. All of the watershed type indices for one sector will fit on a single line. The watershed indices for the second and subsequent sectors are on the following lines, a new line for each sector in a clockwise rotation. A total of 16 lines are required. For example, a watershed index of 1 means that the water ingestion factor for watershed type 1 will be used for all material deposited on that spatial element. A watershed index of 2 means that the water ingestion factor for watershed type 2 will be used for all material deposited on that spatial element. The formatting of this information is illustrated below by the presentation of the watershed data for a single wind direction sector.

WATERSHED INDEX

$\begin{array}{llllllllllllllllllllllllll}1 & 1 & 2 & 2 & 2 & 2 & 2 & 2 & 2 & 1 & 1 & 1 & 1 & 1 & 1 & 1 & 1 & 1 & 1 & 1 & 1 & 1 & 1 & 1 & 3 & 3\end{array}$ 
The format used to process the data is as follows:

\begin{tabular}{|c|c|c|c|c|}
\hline Columns & Format & Variable & Identification & Allowed Range \\
\hline $1-80$ & $40 I 2$ & INDWTR & Watershed index & 1 - NWTRSH \\
\hline
\end{tabular}

\section{Agricultural Data Block}

The length of the growing season and the average fraction of the farmland area at the site devoted to each crop type must be specified. These fractions, FRCLP, need not sum to exactly one, but their sum should not exceed a value of one. If these values sum to a value less than one, that sum indicates the fraction of farmland in production in an average year (some fraction of farmland may be fallow). Data must be given for each of the NCPGZN crop categories. The data block begins with the separator "CROP SEASON AND SHARE" in column 2. The formatting of this information is illustrated below by the presentation of the agricultural data for the seven crop categories used in the example Site Data File.

$\begin{array}{llrl}\text { CROP SEASON AND SHARE } & & \\ 1 & \text { PASTURE } & 90.270 . & 0.41 \\ 2 & \text { STORED FORAGE } & 150.240 . & 0.13 \\ 3 & \text { GRAINS } & 150.240 . & 0.21 \\ 4 & \text { GRN LEAFY VEGETABLES } & 150.240 . & 0.002 \\ 5 & \text { OTHER FOOD CROPS } & 150.240 . & 0.004 \\ 6 & \text { LEGUMES AND SEEDS } & 150.240 . & 0.15 \\ 7 & \text { ROOTS AND TUBERS } & 150.240 . & 0.003\end{array}$

The format used to process the data is as follows:

\begin{tabular}{|c|c|c|c|c|}
\hline Columns & Format & Variable & Identification & Allowed Range \\
\hline $1-4$ & I4 & I & Crop index & 1 - NCPGZN \\
\hline $6-25$ & A20 & CROP & Crop name & N.A. \\
\hline $26-30$ & F5. 0 & GBEG & $\begin{array}{l}\text { Day of the year the growing } \\
\text { season begins }\end{array}$ & 1.0 - GEND \\
\hline $31-35$ & F5. 0 & GEND & $\begin{array}{l}\text { Day of the year the growing } \\
\text { season ends }\end{array}$ & GBEG -365.0 \\
\hline $36-45$ & F10.0 & FRCLCP & $\begin{array}{l}\text { Fraction of the site- } \\
\text { averaged farmland devoted } \\
\text { to this crop }\end{array}$ & $0.0-1.0$ \\
\hline
\end{tabular}




\section{Watershed Data Block}

The data block begins with the 22 character separator beginning with "WATERSHED DEFINITION" in column 2. For each of the NUMWPI (NWPISO) nuclides considered in the liquid pathways model, an initial washoff fraction and an annual washoff rate (fraction/year) must be specified together with an ingestion factor (becquerel-ingested/becquerel-in-water) for each of the NUMWPA (NWTRSH) watershed classes. The formatting of this information is illustrated below by the presentation of the watershed data for the four water ingestion nuclides used on the example Site Data File.

$\begin{array}{rlccc}\text { WATERSHED DEFINITION } & \text { RIVER } & \text { OCEAN } & \text { LAKE } \\ 1 & \text { SR }-89 & 5.0 \mathrm{E}-6 & 0.0 & 2.0 \mathrm{E}-7 \\ 2 \mathrm{SR}-90 & 5.0 \mathrm{E}-6 & 0.0 & 2.0 \mathrm{E}-7 \\ 3 \mathrm{CS}-134 & 5.0 \mathrm{E}-6 & 0.0 & 2.0 \mathrm{E}-6 \\ 4 \mathrm{CS}-137 & 5.0 \mathrm{E}-6 & 0.0 & 4.0 \mathrm{E}-6\end{array}$

The format used to process the data is as follows:

\begin{tabular}{cclllc}
\hline Columns & Format & Variable & \multicolumn{1}{c}{ Identification } & Allowed Range \\
$1-4$ & I4 & I & Nuclide index & 1 - NWPISO \\
$6-13$ & A8 & NMISO & Nuclide name & N.A. \\
$16-25$ & E10.1 & DUMMY & No longer used & N.A. \\
$26-35$ & E10.1 & DUMMY & No longer used & N.A. \\
$36-45$ & E10.1 & WTRINF(1) & $\begin{array}{l}\text { Ingestion factor for } \\
\text { watershed class 1 }\end{array}$ & $0.0-1.0$ \\
$46-55$ & E10.1 & WTRINF(2) & $\begin{array}{l}\text { Ingestion factor for } \\
\text { watershed class 2 }\end{array}$ & $0.0-1.0$ \\
$56-65$ & E10.1 & WTRINF(3) & $\begin{array}{l}\text { Ingestion factor for } \\
\text { watershed class } 3\end{array}$ & $0.0-1.0$ \\
$66-75$ & E10.1 & WTRINF(4) & $\begin{array}{l}\text { Ingestion factor for } \\
\text { watershed class 4 }\end{array}$ & $0.0-1.0$ \\
\hline
\end{tabular}

Regional Economic Data Block

Economic data must be specified for each of the NECRGN economic regions. The data block begins with the separator "REGIONAL ECONOMIC DATA" in column 2. An economic region can be a county, a state or any other userspecified region. The data provided in this block are similar to those contained in the CRAC2 "Economic" subgroup but converted to metric units. 
The index of the region is used with the "REGION INDEX" data block (described earlier in this Appendix) to associate each element of the spatial grid with an economic region. The formatting of this information is illustrated below by the presentation of the economic data for the first economic region on the example Site Data File, Alabama.

REGIONAL ECONOMIC DATA

1 ALA $\quad 354^{\circ} .040 \quad 459 . \quad 1824.62000$.

The format used to process this data is as follows:

\begin{tabular}{|c|c|c|c|c|}
\hline Columns & Format & Variable & Identification & Allowed Range \\
\hline $1-4$ & 14 & I & Region index number & 1 - NECRGN \\
\hline $6-15$ & A10 & NMRGN & Region identification & N.A. \\
\hline $21-25$ & F5. 3 & FRMFRC & $\begin{array}{l}\text { Fraction of land devoted } \\
\text { to farming in the region }\end{array}$ & $0.0-1.0$ \\
\hline $26-30$ & F5. 3 & DPF & $\begin{array}{l}\text { Fraction of farm sales } \\
\text { resulting from dairy } \\
\text { production in the region }\end{array}$ & $0.0-1.0$ \\
\hline $31-40$ & F10.1 & ASFP & $\begin{array}{l}\text { Annual average farm sales } \\
\text { for the region ( } \$ / \text { hectare) }\end{array}$ & $0.0-1 . E 9$ \\
\hline $41-50$ & F10.1 & VFRM & $\begin{array}{l}\text { Average farmland value } \\
\text { for the region ( } \$ / \text { hectare) }\end{array}$ & $0.0-1 . \mathrm{E} 9$ \\
\hline $51-60$ & F10.1 & VNFRM & $\begin{array}{l}\text { Average non-farm value } \\
\text { for the region ( } \$ / \text { person) }\end{array}$ & $0.0-1 . \mathrm{E} 9$ \\
\hline
\end{tabular}

A complete listing of a sample Site Data File is supplied in this document as Appendix D.2. 
APPENDIX C

PROCEDURE FILES TO RUN MACCS

\section{C.1 MACCS Procedure File}

MACCS has been installed on a VAX/VMS computer system at Sandia National Laboratories in Albuquerque and on a Cray UNICOS system at the Idaho National Engineering Laboratories. Instructions are provided here for using the VAX/VMS versions of MACCS.

\section{VAX/NMS Command File to run MACCS}

On VAX machines, MACCS is executed under the control of command file MACCS.COM. The argument list for MACCS.COM consists of six file names that define the data files which can be used by MACCS for both input and output. A parameter with a default value can be skipped by specifying the null string " ".

Example Usage of MACCS:

QMACCS IN1A IN2A IN3A METSUR SURSIT LISTA

(list output is written to LISTA.OUT)

QMACCS IN1A IN2A IN3A METSUR SURSIT LISTA

Parameter List for execution of MACCS;

1. ATMOS User Input File: IN1A

This parameter is always required. It must be a valid pathname to a previously created file to which the user has read access.

2. EARLY User Input File: IN2A

This parameter can be omitted if the user is only exercising the ATMOS module (ENDAT1 set to .TRUE. on the ATMOS input). If this parameter is specified, it must be a valid pathname to a previously created file to which the user has read access.

3. CHRONC User Input File: IN3A

This parameter can be omitted if the user is skipping the CHRONC module (if ENDAT1 was set to .TRUE. on the ATMOS input or ENDAT2 was set to . TRUE. on the EARLY input). If this parameter is specified, it must be a valid pathname to a previously created file to which the user has read access.

4. Meteorological Data File: METSUR

Name of a weather data file with a year of hourly recordings. This file is described in Appendix B.1 of this document. The name supplied here must be a valid pathname to a previously created file to which the user has read access. The parameter may be omitted if 
a single weather sequence is defined on the ATMOS User Input File (METCOD $=3$ or 4 ).

5. Site Data File: SURSIT

Name of a Site Data File for the surrounding region. This file is described in Appendix B.3 of this document. The name supplied here must be a valid pathname to a previously created file to which the user has read access. The parameter may be omitted if a uniform population density is defined on the EARLY User Input File (POPFLG .TRUE.).

6. List Output File: IISTA

Name of the file on which to write the list output of the run. If this argument is omitted, the results will be written to the system default for FORTRAN unit number 6 . If a name is specified here, it must be a valid pathname to a file which the user can write upon. 
In order to illustrate their appearance, two input files and one output file are included in this appendix. The two input files are the Dose Conversion Factors File and the Sample Problem Site Data File. The formats of those input files are described in Appendices B.2 and B.3. The output file presented here is that produced by Sample Problem A which illustrates how MACCS can be used for PRA applications. Sample Problem A is described on page 9 of this document. 


\section{D.1 Dose Conversion Factors File - DOSDATA}

MACCS DOSE CONVERSION FILE: CHANGED BY D. CHANIN 1-NOV-89, 15:18:00 APPLY THE THYROID REDUCTION FACTORS TO ONLY I-131

12 ORGANS DEFINED IN THIS FILE:

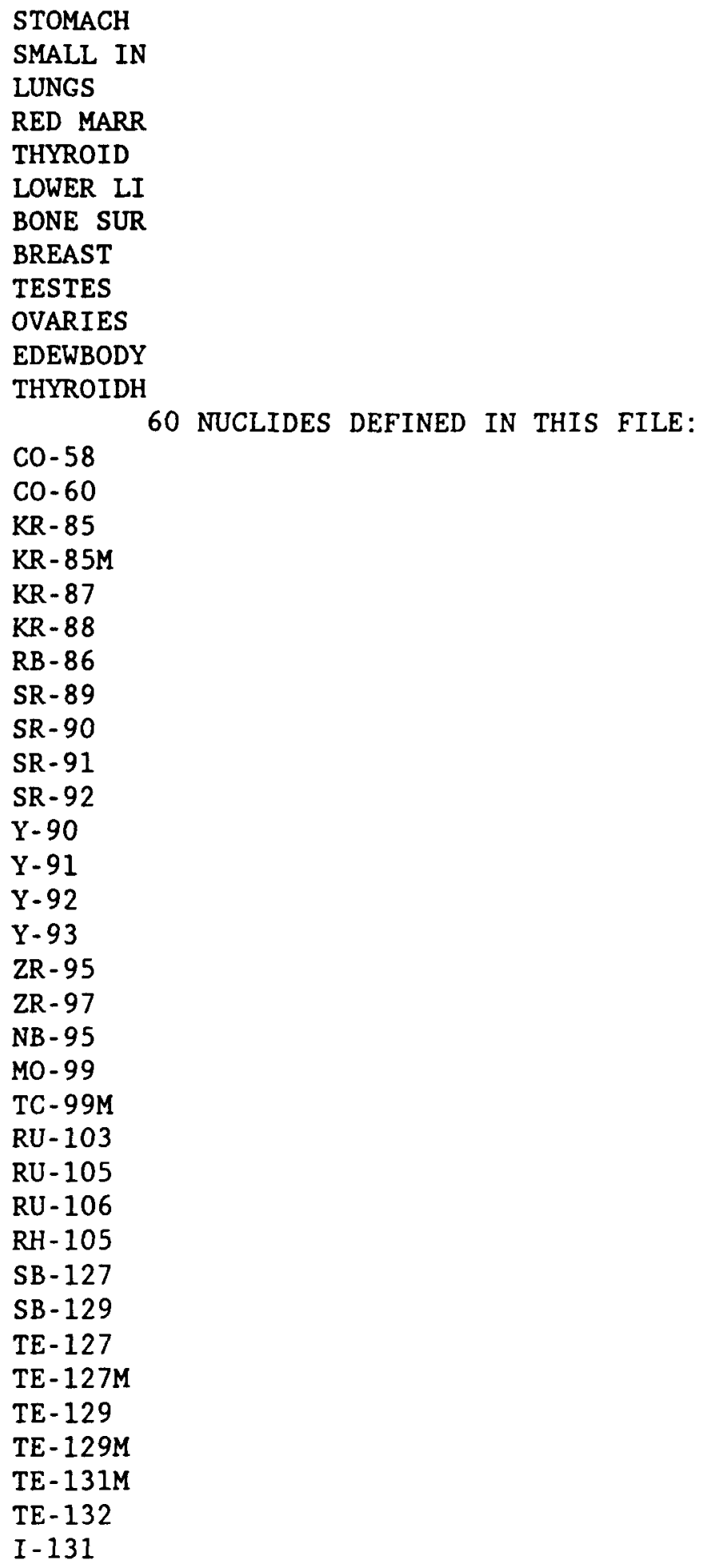




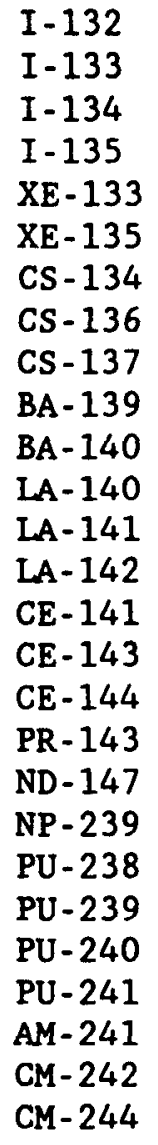

CLOUDSHINE GROUND GROUND GROUND INHALED INHALED INGESTION SHINE 8HR SHINE 7DAY SHINE RATE ACUTE CHRONIC

Co- 58

STOMACH $3.520 \mathrm{E}-14 \quad 1.979 \mathrm{E}-11 \quad 4.023 \mathrm{E}-10 \quad 6.881 \mathrm{E}-16 \quad 1.558 \mathrm{E}-10 \quad 1.394 \mathrm{E}-09 \quad 3.853 \mathrm{E}-10$

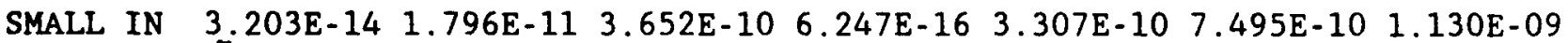

LUNGS $\quad 3.805 \mathrm{E}-14 \quad 2.143 \mathrm{E}-11 \quad 4.356 \mathrm{E}-10 \quad 7.452 \mathrm{E}-16 \quad 7.599 \mathrm{E}-10 \quad 1.601 \mathrm{E}-08 \quad 8.510 \mathrm{E}-11$

RED MARR 3.869E-14 2.179E-11 4.430E-10 7.579E-16 $1.577 \mathrm{E}-10 \quad 9.228 \mathrm{E}-10 \quad 2.601 \mathrm{E}-10$

THYROID 4.788E-14 2.690E-11 $5.469 \mathrm{E}-10 \quad 9.354 \mathrm{E}-16-1.000 \mathrm{E}+00 \quad 8.704 \mathrm{E}-10 \quad 6.308 \mathrm{E}-11$

LOWER LI $3.456 \mathrm{E}-14$ 1.942E-11 $3.948 \mathrm{E}-10 \quad 6.754 \mathrm{E}-16 \quad 9.144 \mathrm{E}-10 \quad 1.989 \mathrm{E}-09 \quad 3.962 \mathrm{E}-09$

BONE SUR 4.249E-14 2.389E-11 4.857E-10 8.308E-16-1.000E+00 6.926E-10 $1.252 \mathrm{E}-10$

BREAST $\quad 4.566 E-142.571 E-11 \quad 5.228 E-10 \quad 8.942 E-16-1.000 E+00 \quad 9.367 E-10 \quad 1.788 E-10$

TESTES $\quad 5.074 E-14 \quad 2.845 E-11 \quad 5.784 E-10 \quad 9.894 E-16-1.000 E+00 \quad 1.060 E-10 \quad 1.614 E-10$

OVARIES $\quad 3.456 \mathrm{E}-14 \quad 1.942 \mathrm{E}-11 \quad 3.948 \mathrm{E}-10 \quad 6.754 \mathrm{E}-16-1.000 \mathrm{E}+00 \quad 6.166 \mathrm{E}-10 \quad 1.041 \mathrm{E}-09$

EDEWBODY 4.398E-14 2.459E-11 5.000E-10 8.553E-16-1.000E+00 3.088E-09 8.206E-10

THYROIDH $4.788 E-14 \quad 2.690 E-11 \quad 5.469 E-10 \quad 9.354 E-16 \quad 6.142 E-11 \quad 8.704 E-10 \quad 6.308 E-11$

CO-60

STOMACH $\quad 9.132 \mathrm{E}-14 \quad 4.602 \mathrm{E}-11 \quad 9.655 \mathrm{E}-10 \quad 1.598 \mathrm{E}-15 \quad 3.840 \mathrm{E}-10 \quad 2.726 \mathrm{E}-08 \quad 1.611 \mathrm{E}-09$

SMALL IN $8.530 \mathrm{E}-14 \quad 4.301 \mathrm{E}-11 \quad 9.022 \mathrm{E}-10 \quad 1.494 \mathrm{E}-15 \quad 8.077 \mathrm{E}-10 \quad 7.046 \mathrm{E}-09 \quad 3.591 \mathrm{E}-09$

LUNGS $\quad 9.862 \mathrm{E}-14 \quad 4.986 \mathrm{E}-11 \quad 1.046 \mathrm{E}-09 \quad 1.731 \mathrm{E}-15 \quad 3.511 \mathrm{E}-09 \quad 3.448 \mathrm{E}-07 \quad 8.768 \mathrm{E}-10$

RED MARR 9.957E-14 5.032E-11 $1.055 \mathrm{E}-09$ 1.747E-15 3.986E-10 $1.718 \mathrm{E}-08$ 1.311E-09

THYROID $\quad 1.230 E-13 \quad 6.219 E-11 \quad 1.305 E-09 \quad 2.159 E-15-1.000 E+00 \quad 1.615 E-08 \quad 7.843 E-10$

LOWER LI 9.069E-14 4.575E-11 9.597E-10 1.589E-15 2.386E-09 7.916E-09 1.113E-08

BONE SUR 1.056E-13 5.333E-11 $1.119 \mathrm{E}-09$ 1.852E-15-1.000E+00 $1.353 \mathrm{E}-08 \quad 9.415 \mathrm{E}-10$

BREAST 1.164E-13 5.872E-11 $1.232 \mathrm{E}-09 \quad 2.039 \mathrm{E}-15-1.000 \mathrm{E}+00 \quad 1.843 \mathrm{E}-08 \quad 1.100 \mathrm{E}-09$

$\begin{array}{llllllll}\text { TESTES } & 1.297 \mathrm{E}-13 & 6.548 \mathrm{E}-11 & 1.373 \mathrm{E}-09 & 2.274 \mathrm{E}-15-1.000 \mathrm{E}+00 & 1.697 \mathrm{E}-09 & 1.075 \mathrm{E}-09\end{array}$ 
OVARIES $\quad 8.879 \mathrm{E}-14 \quad 4.484 \mathrm{E}-11 \quad 9.406 \mathrm{E}-10 \quad 1.557 \mathrm{E}-15-1.000 \mathrm{E}+00 \quad 4.753 \mathrm{E}-09 \quad 3.187 \mathrm{E}-09$ EDEWBODY 1.125E-13 5.666E-11 1.189E-09 1.968E-15-1.000E+00 5.948E-08 2.839E-09 THYROIDH 1.230E-13 6.219E-11 $1.305 \mathrm{E}-09 \quad 2.159 \mathrm{E}-15 \quad 1.465 \mathrm{E}-10 \quad 1.615 \mathrm{E}-08 \quad 7.843 \mathrm{E}-10$ $K R-85$

$\begin{array}{lllllllll}\text { STOMACH } & 7.674 \mathrm{E}-17 & 0.000 \mathrm{E}+00 & 0.000 \mathrm{E}+00 & 0.000 \mathrm{E}+00 & 6.757 \mathrm{E}-14 & 7.007 \mathrm{E}-14 & 0.000 \mathrm{E}+00\end{array}$ SMALL IN $6.881 \mathrm{E}-17 \quad 0.000 \mathrm{E}+00 \quad 0.000 \mathrm{E}+00 \quad 0.000 \mathrm{E}+00 \quad 6.781 \mathrm{E}-14 \quad 7.007 \mathrm{E}-14 \quad 0.000 \mathrm{E}+00$ LUNGS RED MARR THYROID LOWER LI BONE SUR BREAST TESTES OVARIES EDEWBODY THYROIDH KR - 85M STOMACH SMALL IN LUNGS RED MARR THYROID LOWER LI BONE SUR BREAST TESTES OVARIES $8.340 E-17 \quad 0.000 E+00 \quad 0.000 E+00$ $0.000 \mathrm{E}+00 \quad 4.671 \mathrm{E}-13 \quad 4.708 \mathrm{E}-13 \quad 0.000 \mathrm{E}+00$ $\begin{array}{llllllll}8.562 \mathrm{E}-17 & 0.000 \mathrm{E}+00 & 0.000 \mathrm{E}+00 & 0.000 \mathrm{E}+00 & 6.808 \mathrm{E}-14 & 7.007 \mathrm{E}-14 & 0.000 \mathrm{E}+00\end{array}$ $\begin{array}{llllll}1.037 \mathrm{E}-16 & 0.000 \mathrm{E}+00 & 0.000 \mathrm{E}+00 & 0.000 \mathrm{E}+00-1.000 \mathrm{E}+00 & 7.007 \mathrm{E}-14 & 0.000 \mathrm{E}+00\end{array}$ $\begin{array}{lllllllll}7.484 \mathrm{E}-17 & 0.000 \mathrm{E}+00 & 0.000 \mathrm{E}+00 & 0.000 \mathrm{E}+00 & 6.781 \mathrm{E}-14 & 7.007 \mathrm{E}-14 & 0.000 \mathrm{E}+00\end{array}$ $\begin{array}{llllllll}9.767 \mathrm{E}-17 & 0.000 \mathrm{E}+00 & 0.000 \mathrm{E}+00 & 0.000 \mathrm{E}+00-1.000 \mathrm{E}+00 & 7.007 \mathrm{E}-14 & 0.000 \mathrm{E}+00\end{array}$ $\begin{array}{lllllll}1.037 \mathrm{E}-16 & 0.000 \mathrm{E}+00 & 0.000 \mathrm{E}+00 & 0.000 \mathrm{E}+00-1.000 \mathrm{E}+00 & 7.007 \mathrm{E}-14 & 0.000 \mathrm{E}+00\end{array}$ $\begin{array}{lllllll}1.123 \mathrm{E}-16 & 0.000 \mathrm{E}+00 & 0.000 \mathrm{E}+00 & 0.000 \mathrm{E}+00-1.000 \mathrm{E}+00 & 7.007 \mathrm{E}-14 & 0.000 \mathrm{E}+00\end{array}$ $\begin{array}{lllllll}7.484 \mathrm{E}-17 & 0.000 \mathrm{E}+00 & 0.000 \mathrm{E}+00 & 0.000 \mathrm{E}+00-1.000 \mathrm{E}+00 & 7.007 \mathrm{E}-14 & 0.000 \mathrm{E}+00\end{array}$ $\begin{array}{llllllll}2.315 \mathrm{E}-16 & 0.000 \mathrm{E}+00 & 0.000 \mathrm{E}+00 & 0.000 \mathrm{E}+00-1.000 \mathrm{E}+00 & 1.180 \mathrm{E}-13 & 0.000 \mathrm{E}+00\end{array}$

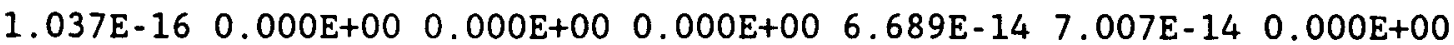

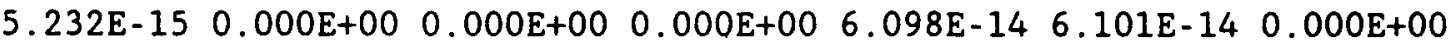
$\begin{array}{llllllll}4.566 \mathrm{E}-15 & 0.000 \mathrm{E}+00 & 0.000 \mathrm{E}+00 & 0.000 \mathrm{E}+00 & 6.101 \mathrm{E}-14 & 6.104 \mathrm{E}-14 & 0.000 \mathrm{E}+00\end{array}$ $\begin{array}{llllllll}5.771 E-15 & 0.000 E+00 & 0.000 E+00 & 0.000 E+00 & 4.804 E-13 & 4.804 E-13 & 0.000 E+00\end{array}$ $\begin{array}{llllllll}5.549 \mathrm{E}-15 & 0.000 \mathrm{E}+00 & 0.000 \mathrm{E}+00 & 0.000 \mathrm{E}+00 & 6.369 \mathrm{E}-14 & 6.372 \mathrm{E}-14 & 0.000 \mathrm{E}+00\end{array}$ $\begin{array}{lllllll}7.737 \mathrm{E}-15 & 0.000 \mathrm{E}+00 & 0.000 \mathrm{E}+00 & 0.000 \mathrm{E}+00-1.000 \mathrm{E}+00 & 5.532 \mathrm{E}-14 & 0.000 \mathrm{E}+00\end{array}$ $\begin{array}{llllllll}5.105 \mathrm{E}-15 & 0.000 \mathrm{E}+00 & 0.000 \mathrm{E}+00 & 0.000 \mathrm{E}+00 & 5.912 \mathrm{E}-14 & 5.915 \mathrm{E}-14 & 0.000 \mathrm{E}+00\end{array}$ $\begin{array}{lllllll}8.467 \mathrm{E}-15 & 0.000 \mathrm{E}+00 & 0.000 \mathrm{E}+00 & 0.000 \mathrm{E}+00-1.000 \mathrm{E}+00 & 6.103 \mathrm{E}-14 & 0.000 \mathrm{E}+00\end{array}$

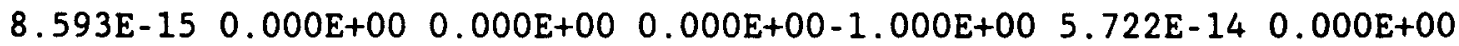
$\begin{array}{llllll}7.959 \mathrm{E}-15 & 0.000 \mathrm{E}+00 & 0.000 \mathrm{E}+00 & 0.000 \mathrm{E}+00-1.000 \mathrm{E}+00 & 5.514 \mathrm{E}-14 & 0.000 \mathrm{E}+00\end{array}$ EDEWBODY 7.222E-15 $0.000 \mathrm{E}+00 \quad 0.000 \mathrm{E}+00 \quad 0.000 \mathrm{E}+00-1.000 \mathrm{E}+00 \quad 1.110 \mathrm{E}-13 \quad 0.000 \mathrm{E}+00$ THYROIDH $\quad 7.737 \mathrm{E}-15 \quad 0.000 \mathrm{E}+00 \quad 0.000 \mathrm{E}+00 \quad 0.000 \mathrm{E}+00 \quad 5.529 \mathrm{E}-14 \quad 5.532 \mathrm{E}-14 \quad 0.000 \mathrm{E}+00$ $\mathrm{KR}-87$ STOMACH $\begin{array}{lllllll}3.161 E-14 & 0.000 E+00 & 0.000 E+00 & 0.000 E+00 & 2.218 E-13 & 2.218 E-13 & 0.000 E+00\end{array}$ $\begin{array}{lllllllllllllll}\text { SMALL IN } 2.946 \mathrm{E}-14 & 0.000 \mathrm{E}+00 & 0.000 \mathrm{E}+00 & 0.000 \mathrm{E}+00 & 2.210 \mathrm{E}-13 & 2.210 \mathrm{E}-13 & 0.000 \mathrm{E}+00\end{array}$ LUNGS RED MARR THYROID $\begin{array}{llllllll}3.393 \mathrm{E}-14 & 0.000 \mathrm{E}+00 & 0.000 \mathrm{E}+00 & 0.000 \mathrm{E}+00 & 2.386 \mathrm{E}-12 & 2.386 \mathrm{E}-12 & 0.000 \mathrm{E}+00\end{array}$ $\begin{array}{llllllll}3.456 \mathrm{E}-14 & 0.000 \mathrm{E}+00 & 0.000 \mathrm{E}+00 & 0.000 \mathrm{E}+00 & 2.179 \mathrm{E}-13 & 2.179 \mathrm{E}-13 & 0.000 \mathrm{E}+00\end{array}$ LOWER LI BONE SUR BREAST TESTES OVARIES EDEWBODY THYROIDH KR -88 $\begin{array}{llllllllll}\text { STOMACH } & 1.064 E-13 & 0.000 E+00 & 0.000 E+00 & 0.000 E+00 & 3.745 E-13 & 3.745 E-13 & 0.000 E+00\end{array}$ SMALL IN $1.001 \mathrm{E}-13 \quad 0.000 \mathrm{E}+00 \quad 0.000 \mathrm{E}+00 \quad 0.000 \mathrm{E}+00 \quad 3.780 \mathrm{E}-13 \quad 3.780 \mathrm{E}-13 \quad 0.000 \mathrm{E}+00$ LUNGS RED MARR THYROID $\begin{array}{lllllll}4.091 \mathrm{E}-14 & 0.000 \mathrm{E}+00 & 0.000 \mathrm{E}+00 & 0.000 \mathrm{E}+00-1.000 \mathrm{E}+00 & 2.149 \mathrm{E}-13 & 0.000 \mathrm{E}+00\end{array}$ $\begin{array}{llllllll}3.133 \mathrm{E}-14 & 0.000 \mathrm{E}+00 & 0.000 \mathrm{E}+00 & 0.000 \mathrm{E}+00 & 2.250 \mathrm{E}-13 & 2.250 \mathrm{E}-13 & 0.000 \mathrm{E}+00\end{array}$ $\begin{array}{lllllll}3.678 \mathrm{E}-14 & 0.000 \mathrm{E}+00 & 0.000 \mathrm{E}+00 & 0.000 \mathrm{E}+00-1.000 \mathrm{E}+00 & 2.139 \mathrm{E}-13 & 0.000 \mathrm{E}+00\end{array}$

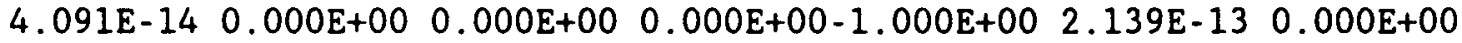

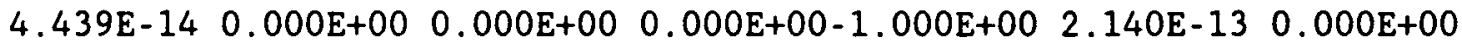
$\begin{array}{lllllll}2.933 \mathrm{E}-14 & 0.000 \mathrm{E}+00 & 0.000 \mathrm{E}+00 & 0.000 \mathrm{E}+00-1.000 \mathrm{E}+00 & 2.220 \mathrm{E}-13 & 0.000 \mathrm{E}+00\end{array}$ $\begin{array}{lllllll}3.958 \mathrm{E}-14 & 0.000 \mathrm{E}+00 & 0.000 \mathrm{E}+00 & 0.000 \mathrm{E}+00-1.000 \mathrm{E}+00 & 4.816 \mathrm{E}-13 & 0.000 \mathrm{E}+00\end{array}$ $\begin{array}{lllllll}4.091 E-14 & 0.000 E+00 & 0.000 E+00 & 0.000 E+00 & 2.149 E-13 & 2.149 E-13 & 0.000 E+00\end{array}$ LOWER LI BONE SUR BREAST TESTES $\begin{array}{lllllll}1.140 \mathrm{E}-13 & 0.000 \mathrm{E}+00 & 0.000 \mathrm{E}+00 & 0.000 \mathrm{E}+00 & 4.218 \mathrm{E}-12 & 4.218 \mathrm{E}-12 & 0.000 \mathrm{E}+00\end{array}$ $\begin{array}{llllllll}1.156 E-13 & 0.000 E+00 & 0.000 E+00 & 0.000 E+00 & 3.666 E-13 & 3.666 E-13 & 0.000 E+00\end{array}$ $\begin{array}{lllllll}1.367 \mathrm{E}-13 & 0.000 \mathrm{E}+00 & 0.000 \mathrm{E}+00 & 0.000 \mathrm{E}+00-1.000 \mathrm{E}+00 & 3.538 \mathrm{E}-13 & 0.000 \mathrm{E}+00\end{array}$ $\begin{array}{llllllll}1.059 E-13 & 0.000 E+00 & 0.000 E+00 & 0.000 E+00 & 3.971 E-13 & 3.972 E-13 & 0.000 E+00\end{array}$ $\begin{array}{lllllll}1.205 \mathrm{E}-13 & 0.000 \mathrm{E}+00 & 0.000 \mathrm{E}+00 & 0.000 \mathrm{E}+00-1.000 \mathrm{E}+00 & 3.508 \mathrm{E}-13 & 0.000 \mathrm{E}+00\end{array}$ $\begin{array}{lllllll}1.357 \mathrm{E}-13 & 0.000 \mathrm{E}+00 & 0.000 \mathrm{E}+00 & 0.000 \mathrm{E}+00-1.000 \mathrm{E}+00 & 3.556 \mathrm{E}-13 & 0.000 \mathrm{E}+00\end{array}$

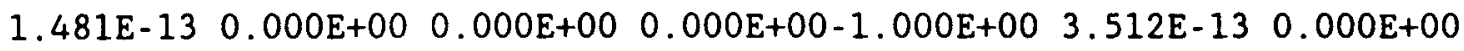


OVARIES

EDEWBODY

THYROIDH

RB- 86

STOMACH

SMALL IN

LUNGS

RED MARR

THYROID

LOWER LI

BONE SUR

BREAST

TESTES

OVARIES

EDEWBODY

THYROIDH

SR - 89

STOMACH

SMALL IN

LUNGS

RED MARR

THYROID

LOWER LI

BONE SUR

BREAST

TESTES

OVARIES

EDEWBODY

THYROIDH

SR - 90

STOMACH

SMALL IN

LUNGS

RED MARR

THYROID

LOWER LI

BONE SUR

BREAST

TESTES

OVARIES

EDEWBODY

THYROIDH

SR - 91

STOMACH

SMALL IN

LUNGS

RED MARR

THYROID

LOWER LI

BONE SUR

BREAST

TESTES
$9.881 \mathrm{E}-14 \quad 0.000 \mathrm{E}+00 \quad 0.000 \mathrm{E}+00 \quad 0.000 \mathrm{E}+00-1.000 \mathrm{E}+00 \quad 3.890 \mathrm{E}-13 \quad 0.000 \mathrm{E}+00$ $\begin{array}{lllllll}9.850 \mathrm{E}-14 & 0.000 \mathrm{E}+00 & 0.000 \mathrm{E}+00 & 0.000 \mathrm{E}+00-1.000 \mathrm{E}+00 & 8.454 \mathrm{E}-13 & 0.000 \mathrm{E}+00\end{array}$ $\begin{array}{llllllll}1.367 \mathrm{E}-13 & 0.000 \mathrm{E}+00 & 0.000 \mathrm{E}+00 & 0.000 \mathrm{E}+00 & 3.537 \mathrm{E}-13 & 3.538 \mathrm{E}-13 & 0.000 \mathrm{E}+00\end{array}$

$\begin{array}{lllllll}3.488 \mathrm{E}-15 & 1.806 \mathrm{E}-12 & 3.361 \mathrm{E}-11 & 6.310 \mathrm{E}-17 & 3.020 \mathrm{E}-10 & 1.453 \mathrm{E}-09 & 2.912 \mathrm{E}-09\end{array}$ $\begin{array}{lllllll}3.203 E-15 & 1.670 E-12 & 3.108 E-11 & 5.835 E-17 & 2.042 E-10 & 1.347 E-09 & 2.163 E-09\end{array}$ 3.742E-15 1.951E-12 3.631E-11 6.818E-17 1.539E-09 3.298E-09 2.137E-09 $\begin{array}{llllllll}3.805 E-15 & 1.979 E-12 & 3.682 E-11 & 6.913 E-17 & 8.078 E-10 & 2.362 E-09 & 3.789 E-09\end{array}$ 4.725E-15 2.460E-12 4.577E-11 8.593E-17-1.000E+00 1.333E-09 2.137E-09 $\begin{array}{lllllll}3.425 \mathrm{E}-15 & 1.788 \mathrm{E}-12 & 3.327 \mathrm{E}-11 & 6.247 \mathrm{E}-17 & 2.041 \mathrm{E}-10 & 1.349 \mathrm{E}-09 & 2.167 \mathrm{E}-09\end{array}$ 4.059E-15 2.115E-12 3.935E-11 7.388E-17-1.000E+00 4.260E-09 6.841E-09

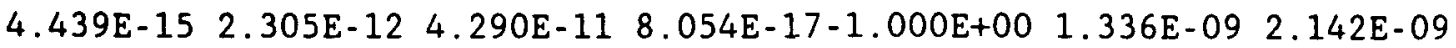
4.947E-15 2.578E-12 4.797E-11 9.006E-17-1.000E+00 1.346E-09 2.160E-09 $\begin{array}{llllll}3.393 E-15 & 1.770 E-12 & 3.293 E-11 & 6.183 E-17-1.000 E+00 ~ & 1.332 E-09 & 2.137 E-09\end{array}$ 4.722E-15 3.909E-12 7.274E-11 $1.366 \mathrm{E}-16-1.000 \mathrm{E}+00 \quad 1.801 \mathrm{E}-09 \quad 2.545 \mathrm{E}-09$ $\begin{array}{lllllll}4.725 \mathrm{E}-15 & 2.460 \mathrm{E}-12 & 4.577 \mathrm{E}-11 & 8.593 \mathrm{E}-17 & 2.497 \mathrm{E}-10 & 1.333 \mathrm{E}-09 & 2.137 \mathrm{E}-09\end{array}$

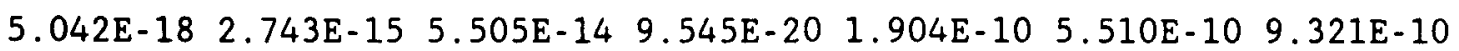
$\begin{array}{lllllll}4.598 E-18 & 2.497 E-15 & 5.011 E-14 & 8.689 E-20 & 2.759 E-10 & 6.284 E-10 & 1.433 E-09\end{array}$ $\begin{array}{lllllll}5.422 \mathrm{E}-18 & 2.952 \mathrm{E}-15 & 5.925 \mathrm{E}-14 & 1.027 \mathrm{E}-19 & 1.302 \mathrm{E}-09 & 2.186 \mathrm{E}-09 & 2.584 \mathrm{E}-10\end{array}$ $\begin{array}{lllllll}5.518 \mathrm{E}-18 & 2.998 \mathrm{E}-15 & 6.017 \mathrm{E}-14 & 1.043 \mathrm{E}-19 & 9.360 \mathrm{E}-10 & 5.651 \mathrm{E}-09 & 3.261 \mathrm{E}-09\end{array}$ $\begin{array}{lllllll}6.849 \mathrm{E}-18 & 3.727 \mathrm{E}-15 & 7.480 \mathrm{E}-14 & 1.297 \mathrm{E}-19-1.000 \mathrm{E}+00 & 4.475 \mathrm{E}-10 & 2.584 \mathrm{E}-10\end{array}$

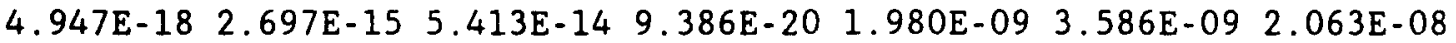
$5.961 \mathrm{E}-18 \quad 3.235 \mathrm{E}-15 \quad 6.492 \mathrm{E}-14$ 1.126E-19-1.000E+00 $8.355 \mathrm{E}-09 \quad 4.820 \mathrm{E}-09$ $6.437 \mathrm{E}-18 \quad 3.508 \mathrm{E}-15 \quad 7.041 \mathrm{E}-14 \quad 1.221 \mathrm{E}-19-1.000 \mathrm{E}+00 \quad 4.475 \mathrm{E}-10 \quad 2.584 \mathrm{E}-10$ $7.198 \mathrm{E}-18 \quad 3.909 \mathrm{E}-15 \quad 7.846 \mathrm{E}-14 \quad 1.360 \mathrm{E}-19-1.000 \mathrm{E}+00 \quad 4.475 \mathrm{E}-10 \quad 2.584 \mathrm{E}-10$ $4.947 \mathrm{E}-18 \quad 2.688 \mathrm{E}-15 \quad 5.395 \mathrm{E}-14 \quad 9.354 \mathrm{E}-20-1.000 \mathrm{E}+00 \quad 4.476 \mathrm{E}-10 \quad 2.584 \mathrm{E}-10$ $3.796 \mathrm{E}-16 \quad 1.457 \mathrm{E}-12 \quad 2.924 \mathrm{E}-11 \quad 5.069 \mathrm{E}-17-1.000 \mathrm{E}+00 \quad 1.787 \mathrm{E}-09 \quad 2.503 \mathrm{E}-09$ $\begin{array}{lllllll}6.849 \mathrm{E}-18 & 3.727 \mathrm{E}-15 & 7.480 \mathrm{E}-14 & 1.297 \mathrm{E}-19 & 8.878 \mathrm{E}-11 & 4.475 \mathrm{E}-10 & 2.584 \mathrm{E}-10\end{array}$ $\begin{array}{lllllll}0.000 E+00 & 0.000 E+00 & 0.000 E+00 & 0.000 E+00 & 1.219 E-10 & 2.365 E-09 & 1.570 E-09\end{array}$ $\begin{array}{lllllll}0.000 E+00 & 0.000 E+00 & 0.000 E+00 & 0.000 E+00 & 1.686 E-10 & 2.402 E-09 & 1.802 E-09\end{array}$ $\begin{array}{lllllll}0.000 E+00 & 0.000 E+00 & 0.000 E+00 & 0.000 E+00 & 6.993 E-10 & 3.422 E-09 & 1.333 E-09\end{array}$ $\begin{array}{llllllll}0.000 E+00 & 0.000 E+00 & 0.000 E+00 & 0.000 E+00 & 1.725 E-09 & 3.051 E-07 & 1.752 E-07\end{array}$

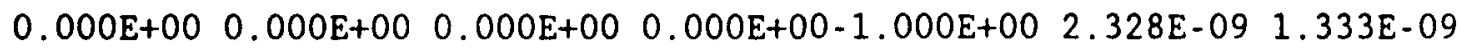

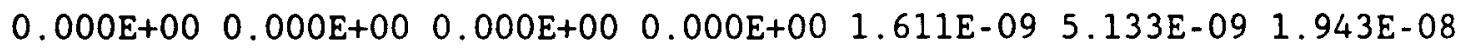
$\begin{array}{lllllll}0.000 \mathrm{E}+00 & 0.000 \mathrm{E}+00 & 0.000 \mathrm{E}+00 & 0.000 \mathrm{E}+00-1.000 \mathrm{E}+00 & 6.758 \mathrm{E}-07 & 3.881 \mathrm{E}-07\end{array}$ $\begin{array}{lllllll}0.000 \mathrm{E}+00 & 0.000 \mathrm{E}+00 & 0.000 \mathrm{E}+00 & 0.000 \mathrm{E}+00-1.000 \mathrm{E}+00 & 2.328 \mathrm{E}-09 & 1.333 \mathrm{E}-09\end{array}$ $\begin{array}{llllllll}0.000 \mathrm{E}+00 & 0.000 \mathrm{E}+00 & 0.000 \mathrm{E}+00 & 0.000 \mathrm{E}+00-1.000 \mathrm{E}+00 & 2.328 \mathrm{E}-09 & 1.333 \mathrm{E}-09\end{array}$

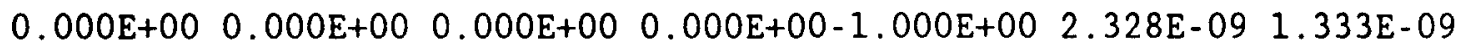
$9.537 \mathrm{E}-17 \quad 2.464 \mathrm{E}-13 \quad 3.113 \mathrm{E}-11 \quad 5.000 \mathrm{E}-18-1.000 \mathrm{E}+00 \quad 5.919 \mathrm{E}-08 \quad 3.518 \mathrm{E}-08$

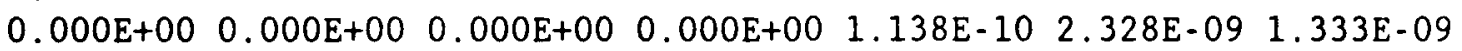

$\begin{array}{lllllll}3.581 E-14 & 1.456 E-11 & 3.424 E-11 & 6.938 E-16 & 1.653 E-10 & 1.731 E-10 & 8.625 E-10\end{array}$ $\begin{array}{lllllll}3.254 E-14 & 1.324 E-11 & 3.112 E-11 & 6.305 E-16 & 2.333 E-10 & 2.419 E-10 & 1.353 E-09\end{array}$ $\begin{array}{llllllll}3.873 E-14 & 1.575 E-11 & 3.702 E-11 & 7.501 E-16 & 8.672 E-10 & 9.243 E-10 & 3.289 E-11\end{array}$ $3.936 \mathrm{E}-14 \quad 1.599 \mathrm{E}-11 \quad 3.760 \mathrm{E}-11 \quad 7.620 \mathrm{E}-16 \quad 7.944 \mathrm{E}-11 \quad 1.446 \mathrm{E}-10 \quad 1.233 \mathrm{E}-10$ $4.869 \mathrm{E}-14 \quad 1.971 \mathrm{E}-11 \quad 4.633 \mathrm{E}-11 \quad 9.388 \mathrm{E}-16-1.000 \mathrm{E}+00 \quad 4.434 \mathrm{E}-11 \quad 2.641 \mathrm{E}-11$ $\begin{array}{llllllll}3.516 \mathrm{E}-14 & 1.425 \mathrm{E}-11 & 3.350 \mathrm{E}-11 & 6.787 \mathrm{E}-16 & 5.220 \mathrm{E}-10 & 6.234 \mathrm{E}-10 & 3.929 \mathrm{E}-09\end{array}$ $4.322 \mathrm{E}-14 \quad 1.756 \mathrm{E}-11 \quad 4.133 \mathrm{E}-11 \quad 8.375 \mathrm{E}-16-1.000 \mathrm{E}+00 \quad 1.424 \mathrm{E}-10 \quad 9.812 \mathrm{E}-11$ $4.662 \mathrm{E}-14 \quad 1.895 \mathrm{E}-11 \quad 4.458 \mathrm{E}-11 \quad 9.034 \mathrm{E}-16-1.000 \mathrm{E}+00 \quad 4.814 \mathrm{E}-11 \quad 5.224 \mathrm{E}-11$ $5.136 \mathrm{E}-142.089 \mathrm{E}-11 \quad 4.914 \mathrm{E}-11 \quad 9.957 \mathrm{E}-16-1.000 \mathrm{E}+00 \quad 4.335 \mathrm{E}-11 \quad 4.141 \mathrm{E}-11$ 
OVARIES EDEWBODY THYROIDH SR-92

STOMACH

SMALL IN LUNGS

RED MARR THYROID

LOWER LI BONE SUR BREAST TESTES OVARIES EDEWBODY THYROIDH Y -90

STOMACH

SMALL IN LUNGS

RED MARR THYROID

LOWER LI BONE SUR BREAST TESTES OVARIES EDEWBODY THYROIDH Y - 91

STOMACH SMALL IN LUNGS

RED MARR THYROID LOWER LI BONE SUR BREAST TESTES OVARIES EDEWBODY THYROIDH Y-92 STOMACH SMALL IN IUNGS RED MARR THYROID LOWER LI BONE SUR BREAST TESTES
$3.507 \mathrm{E}-14 \quad 1.425 \mathrm{E}-11 \quad 3.350 \mathrm{E}-11 \quad 6.787 \mathrm{E}-16-1.000 \mathrm{E}+00 \quad 6.763 \mathrm{E}-11 \quad 2.120 \mathrm{E}-10$ $3.160 \mathrm{E}-14 \quad 1.404 \mathrm{E}-11 \quad 3.184 \mathrm{E}-11 \quad 6.435 \mathrm{E}-16-1.000 \mathrm{E}+00 \quad 2.615 \mathrm{E}-10 \quad 6.790 \mathrm{E}-10$ $4.869 \mathrm{E}-14 \quad 1.971 \mathrm{E}-11 \quad 4.633 \mathrm{E}-11 \quad 9.388 \mathrm{E}-16 \quad 3.583 \mathrm{E}-11 \quad 4.434 \mathrm{E}-11 \quad 2.641 \mathrm{E}-11$

$4.883 \mathrm{E}-14 \quad 1.157 \mathrm{E}-11 \quad 1.422 \mathrm{E}-11 \quad 8.403 \mathrm{E}-16 \quad 1.104 \mathrm{E}-10 \quad 1.108 \mathrm{E}-10 \quad 5.294 \mathrm{E}-10$

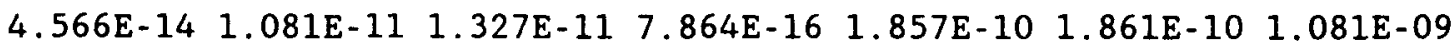
$5.264 \mathrm{E}-14$ 1.253E-11 $1.539 \mathrm{E}-11 \quad 9.101 \mathrm{E}-16 \quad 7.104 \mathrm{E}-10 \quad 7.173 \mathrm{E}-10 \quad 1.948 \mathrm{E}-11$ $5.327 \mathrm{E}-14 \quad 1.266 \mathrm{E}-11 \quad 1.556 \mathrm{E}-11 \quad 9.196 \mathrm{E}-16 \quad 4.114 \mathrm{E}-11 \quad 4.213 \mathrm{E}-11 \quad 4.225 \mathrm{E}-11$ $6.532 \mathrm{E}-14$ 1.556E-11 $1.913 \mathrm{E}-11 \quad 1.129 \mathrm{E}-15-1.000 \mathrm{E}+00 \quad 2.288 \mathrm{E}-11 \quad 1.418 \mathrm{E}-11$ $\begin{array}{lllllll}4.852 \mathrm{E}-14 & 1.152 \mathrm{E}-11 & 1.415 \mathrm{E}-11 & 8.371 \mathrm{E}-16 & 3.252 \mathrm{E}-10 & 3.369 \mathrm{E}-10 & 2.179 \mathrm{E}-09\end{array}$ $\begin{array}{lllllll}5.644 \mathrm{E}-14 & 1.343 \mathrm{E}-11 & 1.653 \mathrm{E}-11 & 9.735 \mathrm{E}-16-1.000 \mathrm{E}+00 & 5.591 \mathrm{E}-11 & 3.874 \mathrm{E}-11\end{array}$ 6.247E-14 1.481E-11 1.821E-11 $1.075 \mathrm{E}-15-1.000 \mathrm{E}+00 \quad 2.549 \mathrm{E}-11 \quad 2.743 \mathrm{E}-11$ $6.913 \mathrm{E}-14 \quad 1.643 \mathrm{E}-11 \quad 2.020 \mathrm{E}-11 \quad 1.192 \mathrm{E}-15-1.000 \mathrm{E}+00 \quad 2.176 \mathrm{E}-11 \quad 1.903 \mathrm{E}-11$ 4.725E-14 1.123E-11 $1.381 \mathrm{E}-11 \quad 8.149 \mathrm{E}-16-1.000 \mathrm{E}+00 \quad 3.134 \mathrm{E}-11 \quad 8.079 \mathrm{E}-11$ $6.019 \mathrm{E}-14 \quad 1.536 \mathrm{E}-11 \quad 1.953 \mathrm{E}-11 \quad 1.043 \mathrm{E}-15-1.000 \mathrm{E}+00 \quad 1.741 \mathrm{E}-10 \quad 4.451 \mathrm{E}-10$ $\begin{array}{lllllll}6.532 E-14 & 1.556 \mathrm{E}-11 & 1.913 \mathrm{E}-11 & 1.129 \mathrm{E}-15 & 2.241 \mathrm{E}-11 & 2.288 \mathrm{E}-11 & 1.418 \mathrm{E}-11\end{array}$

$\begin{array}{lllllll}0.000 E+00 & 0.000 E+00 & 0.000 E+00 & 0.000 E+00 & 3.745 E-10 & 4.263 E-10 & 1.060 E-09\end{array}$ $\begin{array}{lllllll}0.000 \mathrm{E}+00 & 0.000 \mathrm{E}+00 & 0.000 \mathrm{E}+00 & 0.000 \mathrm{E}+00 & 8.713 \mathrm{E}-10 & 1.022 \mathrm{E}-09 & 2.557 \mathrm{E}-09\end{array}$ $\begin{array}{lllllll}0.000 \mathrm{E}+00 & 0.000 \mathrm{E}+00 & 0.000 \mathrm{E}+00 & 0.000 \mathrm{E}+00 & 3.311 \mathrm{E}-09 & 9.309 \mathrm{E}-09 & 1.264 \mathrm{E}-14\end{array}$ $\begin{array}{llllllll}0.000 \mathrm{E}+00 & 0.000 \mathrm{E}+00 & 0.000 \mathrm{E}+00 & 0.000 \mathrm{E}+00 & 8.668 \mathrm{E}-12 & 1.507 \mathrm{E}-11 & 3.664 \mathrm{E}-13\end{array}$ $\begin{array}{lllllll}0.000 \mathrm{E}+00 & 0.000 \mathrm{E}+00 & 0.000 \mathrm{E}+00 & 0.000 \mathrm{E}+00-1.000 \mathrm{E}+00 & 5.183 \mathrm{E}-13 & 1.264 \mathrm{E}-14\end{array}$ $\begin{array}{lllllll}0.000 \mathrm{E}+00 & 0.000 \mathrm{E}+00 & 0.000 \mathrm{E}+00 & 0.000 \mathrm{E}+00 & 6.544 \mathrm{E}-09 & 1.262 \mathrm{E}-08 & 3.145 \mathrm{E}-08\end{array}$

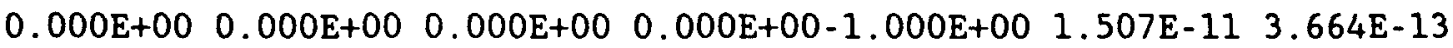
$\begin{array}{llllll}0.000 \mathrm{E}+00 & 0.000 \mathrm{E}+00 & 0.000 \mathrm{E}+00 & 0.000 \mathrm{E}+00-1.000 \mathrm{E}+00 & 5.184 \mathrm{E}-13 & 1.268 \mathrm{E}-14\end{array}$

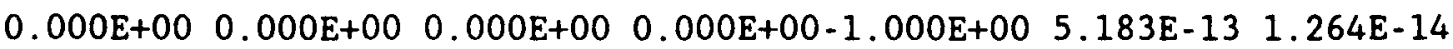
$\begin{array}{lllllll}0.000 \mathrm{E}+00 & 0.000 \mathrm{E}+00 & 0.000 \mathrm{E}+00 & 0.000 \mathrm{E}+00-1.000 \mathrm{E}+00 & 5.191 \mathrm{E}-13 & 1.438 \mathrm{E}-14\end{array}$

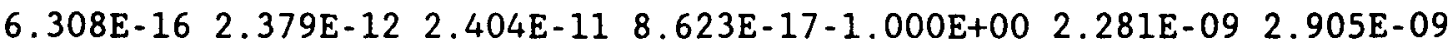
$\begin{array}{lllllll}0.000 \mathrm{E}+00 & 0.000 \mathrm{E}+00 & 0.000 \mathrm{E}+00 & 0.000 \mathrm{E}+00 & 3.153 \mathrm{E}-13 & 5.183 \mathrm{E}-13 & 1.264 \mathrm{E}-14\end{array}$

$\begin{array}{lllllll}1.316 \mathrm{E}-16 & 6.690 \mathrm{E}-14 & 1.351 \mathrm{E}-12 & 2.328 \mathrm{E}-18 & 2.740 \mathrm{E}-10 & 3.431 \mathrm{E}-10 & 6.914 \mathrm{E}-10\end{array}$ $\begin{array}{lllllll}1.224 E-16 & 6.234 E-14 & 1.259 E-12 & 2.169 E-18 & 6.656 E-10 & 8.421 E-10 & 1.737 E-09\end{array}$ $\begin{array}{lllllll}1.424 \mathrm{E}-16 & 7.246 \mathrm{E}-14 & 1.463 \mathrm{E}-12 & 2.521 \mathrm{E}-18 & 5.123 \mathrm{E}-09 & 9.836 \mathrm{E}-08 & 2.017 \mathrm{E}-13\end{array}$ $\begin{array}{lllllll}1.436 \mathrm{E}-16 & 7.310 \mathrm{E}-14 & 1.476 \mathrm{E}-12 & 2.543 \mathrm{E}-18 & 2.798 \mathrm{E}-11 & 3.174 \mathrm{E}-10 & 6.562 \mathrm{E}-12\end{array}$ $\begin{array}{llllll}1.782 \mathrm{E}-16 & 9.060 \mathrm{E}-14 & 1.830 \mathrm{E}-12 & 3.152 \mathrm{E}-18-1.000 \mathrm{E}+00 & 8.479 \mathrm{E}-12 & 1.288 \mathrm{E}-13\end{array}$ $\begin{array}{lllllll}1.306 E-16 & 6.644 E-14 & 1.342 E-12 & 2.312 E-18 & 6.908 E-09 & 1.455 E-08 & 3.027 E-08\end{array}$ $\begin{array}{llllll}1.528 \mathrm{E}-16 & 7.765 \mathrm{E}-14 & 1.568 \mathrm{E}-12 & 2.702 \mathrm{E}-18-1.000 \mathrm{E}+00 & 3.166 \mathrm{E}-10 & 6.108 \mathrm{E}-12\end{array}$ $\begin{array}{llllll}1.677 \mathrm{E}-16 & 8.540 \mathrm{E}-14 & 1.725 \mathrm{E}-12 & 2.971 \mathrm{E}-18-1.000 \mathrm{E}+00 & 8.908 \mathrm{E}-12 & 5.545 \mathrm{E}-13\end{array}$ $\begin{array}{lllll}1.874 \mathrm{E}-16 & 9.570 \mathrm{E}-14 & 1.933 \mathrm{E}-12 & 3.330 \mathrm{E}-18-1.000 \mathrm{E}+00 & 6.404 \mathrm{E}-12 \quad 4.137 \mathrm{E}-13\end{array}$

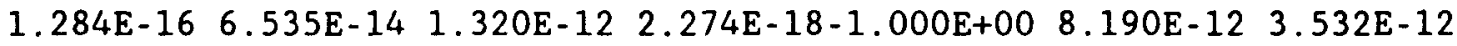
5.509E-16 1.594E-12 3.218E-11 5.544E-17-1.000E+00 1.312E-08 2.571E-09 $\begin{array}{lllllll}1.782 E-16 & 9.060 E-14 & 1.830 E-12 & 3.152 E-18 & 6.243 E-13 & 8.479 E-12 & 1.288 E-13\end{array}$

$9.228 \mathrm{E}-15 \quad 2.473 \mathrm{E}-12 \quad 3.125 \mathrm{E}-12 \quad 1.700 \mathrm{E}-16 \quad 1.703 \mathrm{E}-10 \quad 1.705 \mathrm{E}-10 \quad 1.420 \mathrm{E}-09$ $\begin{array}{lllllll}8.498 E-15 & 2.274 E-12 & 2.875 E-12 & 1.563 E-16 & 2.382 E-10 & 2.390 E-10 & 2.000 E-09\end{array}$ $\begin{array}{lllllll}9.989 E-15 & 2.671 E-12 & 3.376 E-12 & 1.836 E-16 & 1.242 E-09 & 1.249 E-09 & 1.393 E-12\end{array}$ $\begin{array}{lllllll}1.012 \mathrm{E}-14 & 2.708 \mathrm{E}-12 & 3.423 \mathrm{E}-12 & 1.861 \mathrm{E}-16 & 2.061 \mathrm{E}-12 & 2.079 \mathrm{E}-12 & 4.930 \mathrm{E}-12\end{array}$ $\begin{array}{lllllll}1.249 \mathrm{E}-14 & 3.344 \mathrm{E}-12 & 4.227 \mathrm{E}-12 & 2.299 \mathrm{E}-16-1.000 \mathrm{E}+00 & 1.054 \mathrm{E}-12 & 1.776 \mathrm{E}-13\end{array}$

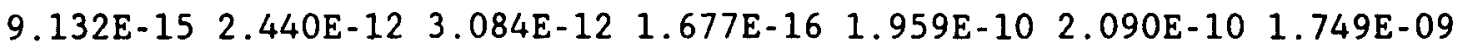

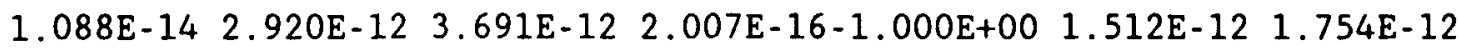
$1.186 \mathrm{E}-143.178 \mathrm{E}-12 \quad 4.017 \mathrm{E}-12 \quad 2.185 \mathrm{E}-16-1.000 \mathrm{E}+00 \quad 1.503 \mathrm{E}-12 \quad 3.564 \mathrm{E}-12$

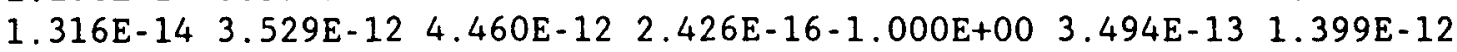


OVARIES $\quad 9.006 \mathrm{E}-15 \quad 2.413 \mathrm{E}-12 \quad 3.049 \mathrm{E}-12 \quad 1.658 \mathrm{E}-16-1.000 \mathrm{E}+00 \quad 2.628 \mathrm{E}-12 \quad 1.971 \mathrm{E}-11$ EDEWBODY $1.238 \mathrm{E}-14 \quad 5.018 \mathrm{E}-12 \quad 6.342 \mathrm{E}-12 \quad 3.449 \mathrm{E}-16-1.000 \mathrm{E}+00 \quad 2.125 \mathrm{E}-10 \quad 5.153 \mathrm{E}-10$ THYROIDH $\quad 1.249 \mathrm{E}-14 \quad 3.344 \mathrm{E}-12 \quad 4.227 \mathrm{E}-12 \quad 2.299 \mathrm{E}-16 \quad 1.049 \mathrm{E}-12 \quad 1.054 \mathrm{E}-12 \quad 1.776 \mathrm{E}-13$ Y-93

$\begin{array}{lllllllll}\text { STOMACH } & 3.361 E-15 & 1.321 E-12 & 3.127 E-12 & 5.961 E-17 & 2.810 E-10 & 2.896 E-10 & 1.280 E-09\end{array}$ SMALL IN $3.127 \mathrm{E}-15$ 1.223E-12 $2.895 \mathrm{E}-12 \quad 5.518 \mathrm{E}-17 \quad 5.455 \mathrm{E}-10 \quad 5.711 \mathrm{E}-10 \quad 2.524 \mathrm{E}-09$ LUNGS RED MARR THYROID LOWER LI BONE SUR BREAST TESTES OVARIES EDEWBODY THYROIDH ZR-95 STOMACH SMALL IN LUNGS RED MARR THYROID LOWER LI BONE SUR BREAST TESTES OVARIES EDEWBODY THYROIDH ZR - 97

STOMACH SMALL IN LUNGS

RED MARR THYROID LOWER LI BONE SUR BREAST TESTES OVARIES EDEWBODY THYROIDH NB -95 STOMACH $\begin{array}{lllllll}3.647 \mathrm{E}-15 & 1.427 \mathrm{E}-12 & 3.377 \mathrm{E}-12 & 6.437 \mathrm{E}-17 & 2.176 \mathrm{E}-09 & 2.524 \mathrm{E}-09 & 8.661 \mathrm{E}-13\end{array}$ $\begin{array}{lllllll}3.710 \mathrm{E}-15 & 1.448 \mathrm{E}-12 & 3.427 \mathrm{E}-12 & 6.532 \mathrm{E}-17 & 3.495 \mathrm{E}-12 & 4.018 \mathrm{E}-12 & 4.936 \mathrm{E}-12\end{array}$ 4.503E-15 1.771E-12 4.192E-12 7.991E-17-1.000E+00 9.250E-13 1.259E-13

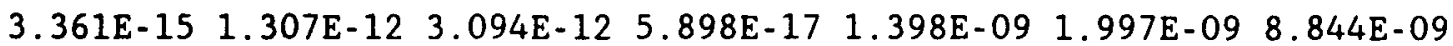
4.059E-15 1.602E-12 3.793E-12 7.230E-17-1.000E+00 3.134E-12 $1.726 \mathrm{E}-12$ 4.439E-15 1.757E-12 4.159E-12 7.927E-17-1.000E+00 1.742E-12 3.121E-12 4.788E-15 1.883E-12 4.458E-12 $\quad 8.498 \mathrm{E}-17-1.000 \mathrm{E}+00 \quad 6.650 \mathrm{E}-13 \quad 1.777 \mathrm{E}-12$ $\begin{array}{llllll}3.203 E-15 & 1.251 E-12 & 2.961 E-12 & 5.644 E-17-1.000 E+00 ~ & 5.307 E-12 & 2.194 E-11\end{array}$ 4.977E-15 4.053E-12 9.594E-12 1.829E-16-1.000E+00 5.829E-10 1.232E-09 $\begin{array}{lllllll}4.503 E-15 & 1.771 E-12 & 4.192 E-12 & 7.991 E-17 & 8.313 E-13 & 9.250 E-13 & 1.259 E-13\end{array}$

$\begin{array}{lllllll}2.660 E-14 & 1.509 E-11 & 3.258 E-10 & 5.232 E-16 & 1.541 E-10 & 1.085 E-09 & 3.568 E-10\end{array}$ $2.407 \mathrm{E}-14 \quad 1.363 \mathrm{E}-11 \quad 2.943 \mathrm{E}-10 \quad 4.725 \mathrm{E}-16 \quad 3.296 \mathrm{E}-10 \quad 9.802 \mathrm{E}-10 \quad 1.119 \mathrm{E}-09$ $\begin{array}{lllllll}2.876 \mathrm{E}-14 & 1.628 \mathrm{E}-11 & 3.516 \mathrm{E}-10 & 5.644 \mathrm{E}-16 & 1.137 \mathrm{E}-09 & 1.843 \mathrm{E}-08 & 2.342 \mathrm{E}-11\end{array}$ $2.924 \mathrm{E}-14 \quad 1.656 \mathrm{E}-11 \quad 3.575 \mathrm{E}-10 \quad 5.740 \mathrm{E}-16 \quad 2.845 \mathrm{E}-10 \quad 3.207 \mathrm{E}-09 \quad 2.135 \mathrm{E}-10$ $3.615 \mathrm{E}-142.049 \mathrm{E}-11 \quad 4.425 \mathrm{E}-10 \quad 7.103 \mathrm{E}-16-1.000 \mathrm{E}+00 \quad 7.790 \mathrm{E}-10 \quad 8.238 \mathrm{E}-12$ $2.607 \mathrm{E}-14 \quad 1.473 \mathrm{E}-11 \quad 3.181 \mathrm{E}-10 \quad 5.105 \mathrm{E}-16 \quad 1.573 \mathrm{E}-09 \quad 4.164 \mathrm{E}-09 \quad 7.778 \mathrm{E}-09$

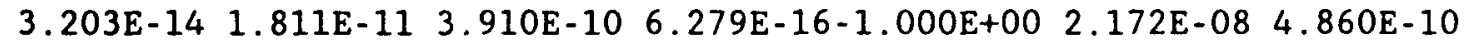
$3.456 \mathrm{E}-14 \quad 1.948 \mathrm{E}-11 \quad 4.207 \mathrm{E}-10 \quad 6.754 \mathrm{E}-16-1.000 \mathrm{E}+00 \quad 9.280 \mathrm{E}-10 \quad 1.047 \mathrm{E}-10$

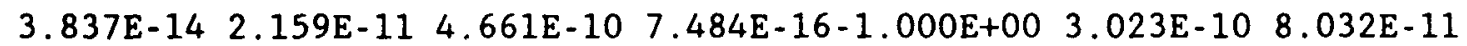
$2.616 \mathrm{E}-14 \quad 1.482 \mathrm{E}-11 \quad 3.199 \mathrm{E}-10 \quad 5.137 \mathrm{E}-16-1.000 \mathrm{E}+00 \quad 8.361 \mathrm{E}-10 \quad 8.153 \mathrm{E}-10$ $3.299 \mathrm{E}-14 \quad 1.873 \mathrm{E}-11 \quad 4.043 \mathrm{E}-10 \quad 6.493 \mathrm{E}-16-1.000 \mathrm{E}+00 \quad 4.327 \mathrm{E}-09 \quad 1.027 \mathrm{E}-09$ $3.615 \mathrm{E}-142.049 \mathrm{E}-11 \quad 4.425 \mathrm{E}-10 \quad 7.103 \mathrm{E}-16 \quad 6.141 \mathrm{E}-11 \quad 7.790 \mathrm{E}-10 \quad 8.238 \mathrm{E}-12$ $\begin{array}{lllllll}5.527 \mathrm{E}-14 & 2.431 \mathrm{E}-11 & 9.498 \mathrm{E}-11 & 1.083 \mathrm{E}-15 & 3.772 \mathrm{E}-10 & 4.175 \mathrm{E}-10 & 1.213 \mathrm{E}-09\end{array}$

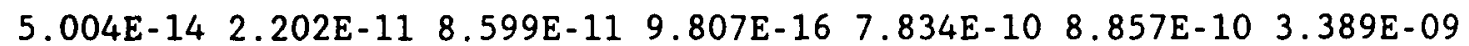
$\begin{array}{lllllll}5.975 E-14 & 2.626 \mathrm{E}-11 & 1.026 \mathrm{E}-10 & 1.170 \mathrm{E}-15 & 2.789 \mathrm{E}-09 & 3.957 \mathrm{E}-09 & 1.759 \mathrm{E}-11\end{array}$ $6.084 \mathrm{E}-142.673 \mathrm{E}-11 \quad 1.044 \mathrm{E}-10 \quad 1.191 \mathrm{E}-15 \quad 1.079 \mathrm{E}-10 \quad 1.396 \mathrm{E}-10 \quad 1.297 \mathrm{E}-10$ $\begin{array}{llllll}7.481 E-14 & 3.301 E-11 & 1.290 E-10 & 1.471 E-15-1.000 E+00 & 3.744 E-11 & 2.662 E-12\end{array}$ $\begin{array}{lllllll}5.419 E-14 & 2.387 E-11 & 9.324 E-11 & 1.063 E-15 & 2.609 E-09 & 4.271 E-09 & 1.795 E-08\end{array}$ $6.691 \mathrm{E}-142.949 \mathrm{E}-111.153 \mathrm{E}-10 \quad 1.315 \mathrm{E}-15-1.000 \mathrm{E}+00 \quad 1.217 \mathrm{E}-10 \quad 4.538 \mathrm{E}-11$ $7.210 \mathrm{E}-14 \quad 3.172 \mathrm{E}-11 \quad 1.240 \mathrm{E}-10 \quad 1.414 \mathrm{E}-15-1.000 \mathrm{E}+00 \quad 5.790 \mathrm{E}-11 \quad 8.094 \mathrm{E}-11$ $\begin{array}{lllllll}7.937 \mathrm{E}-14 & 3.496 \mathrm{E}-11 & 1.366 \mathrm{E}-10 & 1.558 \mathrm{E}-15-1.000 \mathrm{E}+00 & 2.986 \mathrm{E}-11 & 5.200 \mathrm{E}-11\end{array}$ $\begin{array}{llllll}5.415 \mathrm{E}-14 & 2.380 \mathrm{E}-11 & 9.302 \mathrm{E}-11 & 1.061 \mathrm{E}-15-1.000 \mathrm{E}+00 & 1.696 \mathrm{E}-10 & 6.219 \mathrm{E}-10\end{array}$ 8.623E-15 5.200E-12 1.857E-11 2.118E-16-1.000E+00 1.067E-09 2.288E-09 $\begin{array}{lllllll}7.481 E-14 & 3.301 \mathrm{E}-11 & 1.290 \mathrm{E}-10 & 1.471 \mathrm{E}-15 & 2.788 \mathrm{E}-11 & 3.744 \mathrm{E}-11 & 2.662 \mathrm{E}-12\end{array}$ SMALL IN LUNGS RED MARR THYROID LOWER LI BONE SUR BREAST TESTES

$\begin{array}{llllllll}2.778 E-14 & 1.557 \mathrm{E}-11 & 3.063 \mathrm{E}-10 & 5.422 \mathrm{E}-16 & 1.287 \mathrm{E}-10 & 6.362 \mathrm{E}-10 & 2.796 \mathrm{E}-10\end{array}$ $2.515 \mathrm{E}-14 \quad 1.411 \mathrm{E}-11 \quad 2.776 \mathrm{E}-10 \quad 4.915 \mathrm{E}-16 \quad 2.838 \mathrm{E}-10 \quad 5.201 \mathrm{E}-10 \quad 9.116 \mathrm{E}-10$ $\begin{array}{lllllll}3.000 E-14 & 1.684 \mathrm{E}-11 & 3.314 \mathrm{E}-10 & 5.866 \mathrm{E}-16 & 5.701 \mathrm{E}-10 & 8.319 \mathrm{E}-09 & 2.743 \mathrm{E}-11\end{array}$ $3.051 E-14 \quad 1.711 E-11 \quad 3.367 E-10 \quad 5.961 E-16 \quad 1.212 E-10 \quad 4.425 E-10 \quad 1.993 E-10$ $3.773 \mathrm{E}-14 \quad 2.121 \mathrm{E}-11 \quad 4.173 \mathrm{E}-10 \quad 7.388 \mathrm{E}-16-1.000 \mathrm{E}+00 \quad 3.582 \mathrm{E}-10 \quad 1.184 \mathrm{E}-11$ $2.724 \mathrm{E}-14 \quad 1.529 \mathrm{E}-11 \quad 3.009 \mathrm{E}-10 \quad 5.327 \mathrm{E}-16 \quad 9.234 \mathrm{E}-10 \quad 1.928 \mathrm{E}-09 \quad 4.009 \mathrm{E}-09$ $3.330 \mathrm{E}-14 \quad 1.866 \mathrm{E}-11 \quad 3.672 \mathrm{E}-10 \quad 6.501 \mathrm{E}-16-1.000 \mathrm{E}+00 \quad 5.161 \mathrm{E}-10 \quad 2.965 \mathrm{E}-10$ $3.583 \mathrm{E}-14 \quad 2.012 \mathrm{E}-11 \quad 3.959 \mathrm{E}-10 \quad 7.008 \mathrm{E}-16-1.000 \mathrm{E}+00 \quad 4.062 \mathrm{E}-10 \quad 1.074 \mathrm{E}-10$ $3.995 \mathrm{E}-142.230 \mathrm{E}-11 \quad 4.388 \mathrm{E}-10 \quad 7.769 \mathrm{E}-16-1.000 \mathrm{E}+00 \quad 6.507 \mathrm{E}-11 \quad 9.664 \mathrm{E}-11$ 
OVARIES

EDEWBODY

THYROIDH

MO-99

STOMACH

SMALL IN

LUNGS

RED MARR

THYROID

LOWER LI

BONE SUR

BREAST

TESTES

OVARIES

EDEWBODY

THYROIDH

TC-99M

STOMACH

SMALL IN

LUNGS

RED MARR

THYROID

LOWER LI

BONE SUR

BREAST

TESTES

OVARIES

EDEWBODY

THYROIDH

RU-103

STOMACH

SMALL IN

LUNGS

RED MARR

THYROID

LOWER LI

BONE SUR

BREAST

TESTES

OVARIES

EDEWBODY

THYROIDH

RU-105

STOMACH

SMALL IN

LUNGS

RED MARR

THYROID

LOWER LI

BONE SUR

BREAST

TESTES
$2.730 \mathrm{E}-14 \quad 1.529 \mathrm{E}-11 \quad 3.009 \mathrm{E}-10 \quad 5.327 \mathrm{E}-16-1.000 \mathrm{E}+00 \quad 4.310 \mathrm{E}-10 \quad 8.030 \mathrm{E}-10$ $3.449 \mathrm{E}-14 \quad 1.937 \mathrm{E}-11 \quad 3.812 \mathrm{E}-10 \quad 6.748 \mathrm{E}-16-1.000 \mathrm{E}+00 \quad 1.652 \mathrm{E}-09 \quad 7.029 \mathrm{E}-10$ $3.773 \mathrm{E}-14 \quad 2.121 \mathrm{E}-11 \quad 4.173 \mathrm{E}-10 \quad 7.388 \mathrm{E}-16 \quad 4.278 \mathrm{E}-11 \quad 3.582 \mathrm{E}-10 \quad 1.184 \mathrm{E}-11$

$\begin{array}{lllllll}5.518 \mathrm{E}-15 & 3.820 \mathrm{E}-12 & 5.368 \mathrm{E}-11 & 1.100 \mathrm{E}-16 & 1.894 \mathrm{E}-10 & 2.325 \mathrm{E}-10 & 5.135 \mathrm{E}-10\end{array}$ $4.978 \mathrm{E}-15 \quad 3.408 \mathrm{E}-12 \quad 4.751 \mathrm{E}-11 \quad 9.894 \mathrm{E}-17 \quad 4.221 \mathrm{E}-10 \quad 5.189 \mathrm{E}-10 \quad 1.249 \mathrm{E}-09$ 5.993E-15 4.163E-12 5.884E-11 $1.192 \mathrm{E}-16 \quad 1.482 \mathrm{E}-09 \quad 4.292 \mathrm{E}-09$ 1.506E-11 6.057E-15 4.111E-12 5.687E-11 $1.202 \mathrm{E}-16 \quad 3.096 \mathrm{E}-11 \quad 5.074 \mathrm{E}-11 \quad 7.972 \mathrm{E}-11$ $7.579 \mathrm{E}-15 \quad 5.376 \mathrm{E}-12 \quad 7.737 \mathrm{E}-11 \quad 1.513 \mathrm{E}-16-1.000 \mathrm{E}+00 \quad 1.516 \mathrm{E}-11 \quad 1.034 \mathrm{E}-11$ $\begin{array}{lllllll}5.422 E-15 & 3.740 E-12 & 5.251 E-11 & 1.078 E-16 & 2.860 E-09 & 5.516 E-09 & 1.367 E-08\end{array}$ $6.913 \mathrm{E}-15 \quad 5.189 \mathrm{E}-12 \quad 7.849 \mathrm{E}-11 \quad 1.386 \mathrm{E}-16-1.000 \mathrm{E}+00 \quad 4.290 \mathrm{E}-11 \quad 6.669 \mathrm{E}-11$ $\begin{array}{llllll}7.388 \mathrm{E}-15 & 5.483 \mathrm{E}-12 & 8.176 \mathrm{E}-11 & 1.487 \mathrm{E}-16-1.000 \mathrm{E}+00 & 2.748 \mathrm{E}-11 & 3.422 \mathrm{E}-11\end{array}$ $7.991 \mathrm{E}-15 \quad 5.622 \mathrm{E}-12 \quad 8.026 \mathrm{E}-11 \quad 1.595 \mathrm{E}-16-1.000 \mathrm{E}+00 \quad 1.220 \mathrm{E}-112.719 \mathrm{E}-11$ $5.359 \mathrm{E}-15 \quad 3.624 \mathrm{E}-12 \quad 4.983 \mathrm{E}-11 \quad 1.065 \mathrm{E}-16-1.000 \mathrm{E}+00 \quad 9.503 \mathrm{E}-112.174 \mathrm{E}-10$ $7.164 \mathrm{E}-15 \quad 5.694 \mathrm{E}-12 \quad 7.867 \mathrm{E}-11 \quad 1.667 \mathrm{E}-16-1.000 \mathrm{E}+00 \quad 1.075 \mathrm{E}-09 \quad 1.362 \mathrm{E}-09$ $7.579 \mathrm{E}-15 \quad 5.376 \mathrm{E}-12 \quad 7.737 \mathrm{E}-11 \quad 1.513 \mathrm{E}-16 \quad 5.884 \mathrm{E}-12 \quad 1.516 \mathrm{E}-11 \quad 1.034 \mathrm{E}-11$

4.154E-15 1.731E-12 2.875E-12 9.196E-17 $1.437 \mathrm{E}-11 \quad 1.519 \mathrm{E}-11 \quad 7.169 \mathrm{E}-11$

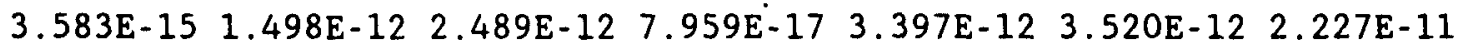
$\begin{array}{lllllll}4.598 \mathrm{E}-15 & 1.928 \mathrm{E}-12 & 3.203 \mathrm{E}-12 & 1.024 \mathrm{E}-16 & 2.918 \mathrm{E}-11 & 3.061 \mathrm{E}-11 & 3.151 \mathrm{E}-12\end{array}$ $\begin{array}{lllllll}4.217 E-15 & 1.755 E-12 & 2.915 E-12 & 9.323 E-17 & 2.296 E-12 & 2.389 E-12 & 6.273 E-12\end{array}$ $\begin{array}{llllll}6.342 \mathrm{E}-15 & 2.656 \mathrm{E}-12 & 4.412 \mathrm{E}-12 & 1.411 \mathrm{E}-16-1.000 \mathrm{E}+00 & 2.088 \mathrm{E}-11 & 8.449 \mathrm{E}-11\end{array}$

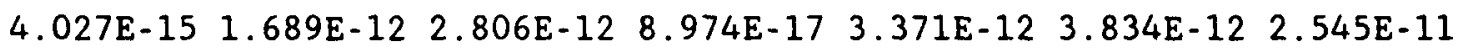

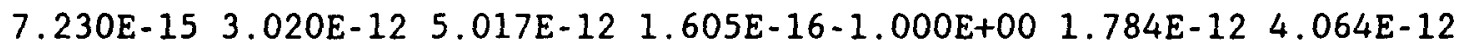
$7.230 \mathrm{E}-15 \quad 3.050 \mathrm{E}-12 \quad 5.067 \mathrm{E}-12 \quad 1.620 \mathrm{E}-16-1.000 \mathrm{E}+00 \quad 1.520 \mathrm{E}-12 \quad 3.563 \mathrm{E}-12$

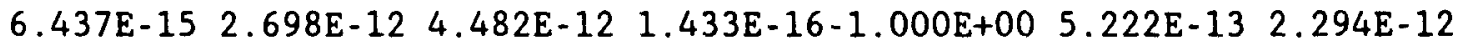
$3.615 \mathrm{E}-15 \quad 1.510 \mathrm{E}-12 \quad 2.509 \mathrm{E}-12 \quad 8.023 \mathrm{E}-17-1.000 \mathrm{E}+00 \quad 1.703 \mathrm{E}-12 \quad 9.738 \mathrm{E}-12$ $5.729 \mathrm{E}-15 \quad 2.418 \mathrm{E}-12 \quad 4.017 \mathrm{E}-12 \quad 1.285 \mathrm{E}-16-1.000 \mathrm{E}+00 \quad 7.451 \mathrm{E}-12 \quad 1.673 \mathrm{E}-11$ $6.342 \mathrm{E}-15 \quad 2.656 \mathrm{E}-12 \quad 4.412 \mathrm{E}-12 \quad 1.411 \mathrm{E}-16 \quad 1.968 \mathrm{E}-11 \quad 2.088 \mathrm{E}-11 \quad 8.449 \mathrm{E}-11$

$\begin{array}{lllllll}1.662 \mathrm{E}-14 & 9.838 \mathrm{E}-12 & 1.950 \mathrm{E}-10 & 3.426 \mathrm{E}-16 & 1.212 \mathrm{E}-10 & 5.047 \mathrm{E}-10 & 3.143 \mathrm{E}-10\end{array}$ $\begin{array}{lllllll}1.487 \mathrm{E}-14 & 8.816 \mathrm{E}-12 & 1.747 \mathrm{E}-10 & 3.070 \mathrm{E}-16 & 2.683 \mathrm{E}-10 & 4.713 \mathrm{E}-10 & 8.509 \mathrm{E}-10\end{array}$ $\begin{array}{lllllll}1.808 E-14 & 1.075 E-11 & 2.131 E-10 & 3.745 E-16 & 9.950 E-10 & 1.560 E-08 & 7.350 E-11\end{array}$ $\begin{array}{lllllll}1.849 \mathrm{E}-14 & 1.093 \mathrm{E}-11 & 2.166 \mathrm{E}-10 & 3.806 \mathrm{E}-16 & 8.176 \mathrm{E}-11 & 3.183 \mathrm{E}-10 & 1.666 \mathrm{E}-10\end{array}$ $2.246 \mathrm{E}-14 \quad 1.331 \mathrm{E}-11 \quad 2.638 \mathrm{E}-10 \quad 4.635 \mathrm{E}-16-1.000 \mathrm{E}+00 \quad 2.564 \mathrm{E}-10 \quad 6.289 \mathrm{E}-11$ $\begin{array}{lllllll}1.624 \mathrm{E}-14 & 9.656 \mathrm{E}-12 & 1.914 \mathrm{E}-10 & 3.363 \mathrm{E}-16 & 1.489 \mathrm{E}-09 & 3.135 \mathrm{E}-09 & 6.534 \mathrm{E}-09\end{array}$ $2.119 \mathrm{E}-14 \quad 1.258 \mathrm{E}-11 \quad 2.493 \mathrm{E}-10 \quad 4.381 \mathrm{E}-16-1.000 \mathrm{E}+00 \quad 2.371 \mathrm{E}-10 \quad 9.661 \mathrm{E}-11$ $2.257 \mathrm{E}-141.346 \mathrm{E}-11 \quad 2.671 \mathrm{E}-10 \quad 4.694 \mathrm{E}-16-1.000 \mathrm{E}+00 \quad 3.103 \mathrm{E}-10 \quad 1.200 \mathrm{E}-10$ $2.427 \mathrm{E}-14 \quad 1.442 \mathrm{E}-11 \quad 2.859 \mathrm{E}-10 \quad 5.023 \mathrm{E}-16-1.000 \mathrm{E}+00 \quad 6.971 \mathrm{E}-11 \quad 1.220 \mathrm{E}-10$ $1.614 \mathrm{E}-14 \quad 9.565 \mathrm{E}-12 \quad 1.896 \mathrm{E}-10 \quad 3.331 \mathrm{E}-16-1.000 \mathrm{E}+00 \quad 3.070 \mathrm{E}-10 \quad 5.712 \mathrm{E}-10$ $2.107 \mathrm{E}-14 \quad 1.249 \mathrm{E}-11 \quad 2.476 \mathrm{E}-10 \quad 4.351 \mathrm{E}-16-1.000 \mathrm{E}+00 \quad 2.485 \mathrm{E}-09 \quad 8.278 \mathrm{E}-10$ $\begin{array}{lllllll}2.246 E-14 & 1.331 E-11 & 2.638 E-10 & 4.635 E-16 & 2.856 E-11 & 2.564 E-10 & 6.289 E-11\end{array}$

$2.784 \mathrm{E}-14 \quad 9.261 \mathrm{E}-12 \quad 1.410 \mathrm{E}-11 \quad 5.581 \mathrm{E}-16 \quad 7.924 \mathrm{E}-11 \quad 8.095 \mathrm{E}-11 \quad 4.980 \mathrm{E}-10$ $2.511 E-14 \quad 8.313 E-12 \quad 1.265 E-11 \quad 5.010 E-16 \quad 1.205 E-10 \quad 1.248 E-10 \quad 7.898 E-10$ $\begin{array}{lllllll}3.019 \mathrm{E}-14 & 9.999 \mathrm{E}-12 & 1.525 \mathrm{E}-11 & 6.025 \mathrm{E}-16 & 4.975 \mathrm{E}-10 & 5.725 \mathrm{E}-10 & 6.210 \mathrm{E}-12\end{array}$ $\begin{array}{lllllll}3.076 \mathrm{E}-14 & 1.021 \mathrm{E}-11 & 1.558 \mathrm{E}-11 & 6.152 \mathrm{E}-16 & 7.221 \mathrm{E}-12 & 7.688 \mathrm{E}-12 & 2.340 \mathrm{E}-11\end{array}$ $3.805 E-14 \quad 1.258 E-11 \quad 1.919 E-11 \quad 7.579 E-16-1.000 E+00 \quad 4.145 E-12 \quad 1.813 E-12$ $2.727 \mathrm{E}-14 \quad 9.051 \mathrm{E}-12 \quad 1.379 \mathrm{E}-11 \quad 5.454 \mathrm{E}-16 \quad 2.058 \mathrm{E}-10 \quad 3.048 \mathrm{E}-10 \quad 1.339 \mathrm{E}-09$ $3.456 \mathrm{E}-14 \quad 1.154 \mathrm{E}-11 \quad 1.775 \mathrm{E}-11 \quad 6.944 \mathrm{E}-16-1.000 \mathrm{E}+00 \quad 4.616 \mathrm{E}-12 \quad 8.893 \mathrm{E}-12$ $3.710 \mathrm{E}-14 \quad 1.232 \mathrm{E}-11 \quad 1.893 \mathrm{E}-11 \quad 7.420 \mathrm{E}-16-1.000 \mathrm{E}+00 \quad 6.609 \mathrm{E}-12 \quad 1.591 \mathrm{E}-11$ $4.027 \mathrm{E}-14 \quad 1.337 \mathrm{E}-112.040 \mathrm{E}-11 \quad 8.054 \mathrm{E}-16-1.000 \mathrm{E}+00 \quad 1.523 \mathrm{E}-12 \quad 7.596 \mathrm{E}-12$ 
OVARIES

EDEWBODY

THYROIDH

RU-106

STOMACH

SMALL IN

LUNGS

RED MARR

THYROID

LOWER LI

BONE SUR

BREAST

TESTES

OVARIES

EDEWBODY

THYROIDH

RH - 105

STOMACH

SMALI IN

LUNGS

RED MARR

THYROID

LOWER LI

BONE SUR

BREAST

TESTES

OVARIES

EDEWBODY

THYROIDH

SB - 127

STOMACH

SMALL IN

LUNGS

RED MARR

THYROID

LOWER II

BONE SUR

BREAST

TESTES

OVARIES

EDEWBODY

THYROIDH

SB - 129

STOMACH

SMALL IN

LUNGS

RED MARR

THYROID

LOWER LI

BONE SUR

BREAST

TESTES
$2.714 \mathrm{E}-14 \quad 8.994 \mathrm{E}-12 \quad 1.365 \mathrm{E}-11 \quad 5.422 \mathrm{E}-16-1.000 \mathrm{E}+00 \quad 1.590 \mathrm{E}-11 \quad 9.669 \mathrm{E}-11$

$3.519 \mathrm{E}-14 \quad 1.210 \mathrm{E}-11 \quad 1.848 \mathrm{E}-11 \quad 7.292 \mathrm{E}-16-1.000 \mathrm{E}+00 \quad 1.235 \mathrm{E}-10 \quad 2.868 \mathrm{E}-10$

$3.805 \mathrm{E}-14 \quad 1.258 \mathrm{E}-11 \quad 1.919 \mathrm{E}-11 \quad 7.579 \mathrm{E}-16 \quad 3.876 \mathrm{E}-12 \quad 4.145 \mathrm{E}-12 \quad 1.813 \mathrm{E}-12$

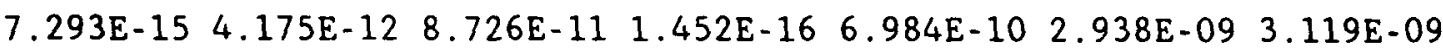
6.564E-15 3.765E-12 7.869E-11 $1.310 \mathrm{E}-16 \quad 1.589 \mathrm{E}-09 \quad 3.397 \mathrm{E}-09 \quad 5.548 \mathrm{E}-09$

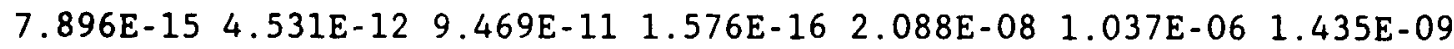
8.054E-15 4.622E-12 9.660E-11 $1.608 \mathrm{E}-16 \quad 8.744 \mathrm{E}-11 \quad 1.770 \mathrm{E}-09 \quad 1.483 \mathrm{E}-09$ $9.862 \mathrm{E}-15 \quad 5.652 \mathrm{E}-12 \quad 1.181 \mathrm{E}-10 \quad 1.966 \mathrm{E}-16-1.000 \mathrm{E}+00 \quad 1.733 \mathrm{E}-09 \quad 1.432 \mathrm{E}-09$

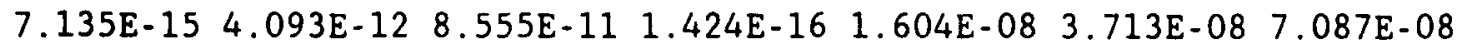
$9.037 \mathrm{E}-15 \quad 5.187 \mathrm{E}-12 \quad 1.084 \mathrm{E}-10 \quad 1.804 \mathrm{E}-16-1.000 \mathrm{E}+00 \quad 1.620 \mathrm{E}-09 \quad 1.445 \mathrm{E}-09$ 9.672E-15 5.552E-12 1.160E-10 1.931E-16-1.000E+00 1.798E-09 1.457E-09 $1.056 \mathrm{E}-14 \quad 6.053 \mathrm{E}-12 \quad 1.265 \mathrm{E}-10 \quad 2.106 \mathrm{E}-16-1.000 \mathrm{E}+00 \quad 1.154 \mathrm{E}-09 \quad 1.474 \mathrm{E}-09$ 7.103E-15 4.075E-12 8.517E-11 1.417E-16-1.000E+00 1.308E-09 1.656E-09 $1.010 \mathrm{E}-14 \quad 9.017 \mathrm{E}-12 \quad 1.885 \mathrm{E}-10 \quad 3.137 \mathrm{E}-16-1.000 \mathrm{E}+00 \quad 1.289 \mathrm{E}-07 \quad 7.419 \mathrm{E}-09$ $9.862 \mathrm{E}-15 \quad 5.652 \mathrm{E}-12 \quad 1.181 \mathrm{E}-10 \quad 1.966 \mathrm{E}-16 \quad 4.022 \mathrm{E}-11 \quad 1.733 \mathrm{E}-09 \quad 1.432 \mathrm{E}-09$

$2.600 \mathrm{E}-15 \quad 1.488 \mathrm{E}-12 \quad 9.870 \mathrm{E}-12 \quad 5.581 \mathrm{E}-17 \quad 6.353 \mathrm{E}-11 \quad 7.262 \mathrm{E}-11 \quad 1.946 \mathrm{E}-10$ $2.315 \mathrm{E}-15 \quad 1.327 \mathrm{E}-12 \quad 8.804 \mathrm{E}-12 \quad 4.978 \mathrm{E}-17 \quad 1.353 \mathrm{E}-10 \quad 1.578 \mathrm{E}-10 \quad 4.427 \mathrm{E}-10$ $2.841 E-15 \quad 1.631 E-12 \quad 1.082 E-11 \quad 6.120 E-17 \quad 4.797 E-10 \quad 9.509 E-10 \quad 3.847 E-12$ $2.936 \mathrm{E}-15 \quad 1.682 \mathrm{E}-12 \quad 1.116 \mathrm{E}-11 \quad 6.310 \mathrm{E}-17 \quad 5.385 \mathrm{E}-12 \quad 7.746 \mathrm{E}-12 \quad 1.463 \mathrm{E}-11$

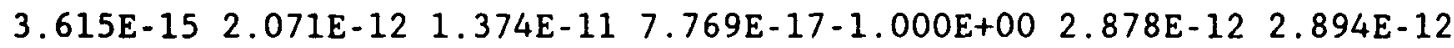
$2.556 \mathrm{E}-15 \quad 1.462 \mathrm{E}-12 \quad 9.701 \mathrm{E}-12 \quad 5.486 \mathrm{E}-17 \quad 7.389 \mathrm{E}-10 \quad 1.347 \mathrm{E}-09 \quad 3.781 \mathrm{E}-09$ $3.647 \mathrm{E}-15 \quad 2.088 \mathrm{E}-12 \quad 1.385 \mathrm{E}-11 \quad 7.832 \mathrm{E}-17-1.000 \mathrm{E}+00 \quad 4.441 \mathrm{E}-12 \quad 6.731 \mathrm{E}-12$ $3.805 \mathrm{E}-15 \quad 2.189 \mathrm{E}-12 \quad 1.452 \mathrm{E}-11 \quad 8.213 \mathrm{E}-17-1.000 \mathrm{E}+00 \quad 5.592 \mathrm{E}-12 \quad 8.959 \mathrm{E}-12$ $3.837 \mathrm{E}-15 \quad 2.206 \mathrm{E}-12 \quad 1.464 \mathrm{E}-11 \quad 8.276 \mathrm{E}-17-1.000 \mathrm{E}+00 \quad 2.827 \mathrm{E}-12 \quad 7.209 \mathrm{E}-12$ $\begin{array}{llllll}2.416 \mathrm{E}-15 & 1.386 \mathrm{E}-12 & 9.197 \mathrm{E}-12 & 5.200 \mathrm{E}-17-1.000 \mathrm{E}+00 & 2.105 \mathrm{E}-11 & 5.798 \mathrm{E}-11\end{array}$ $\begin{array}{llllll}3.449 \mathrm{E}-15 & 2.012 \mathrm{E}-12 & 1.335 \mathrm{E}-11 & 7.546 \mathrm{E}-17-1.000 \mathrm{E}+00 & 2.573 \mathrm{E}-10 & 3.980 \mathrm{E}-10\end{array}$

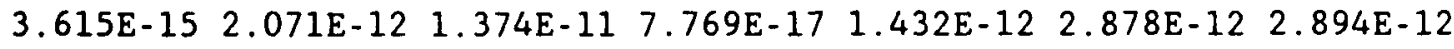

$2.337 \mathrm{E}-14 \quad 1.314 \mathrm{E}-11 \quad 1.623 \mathrm{E}-10 \quad 4.693 \mathrm{E}-16 \quad 2.278 \mathrm{E}-10 \quad 3.267 \mathrm{E}-10 \quad 5.597 \mathrm{E}-10$ $2.106 \mathrm{E}-14 \quad 1.181 \mathrm{E}-11 \quad 1.459 \mathrm{E}-10 \quad 4.217 \mathrm{E}-16 \quad 5.199 \mathrm{E}-10 \quad 7.090 \mathrm{E}-10 \quad 1.630 \mathrm{E}-09$ 2.534E-14 1.420E-11 1.755E-10 5.074E-16 $1.712 \mathrm{E}-09 \quad 6.915 \mathrm{E}-09 \quad 1.574 \mathrm{E}-11$ $2.584 \mathrm{E}-14 \quad 1.456 \mathrm{E}-111.799 \mathrm{E}-10 \quad 5.200 \mathrm{E}-16 \quad 9.334 \mathrm{E}-11 \quad 1.547 \mathrm{E}-10 \quad 1.317 \mathrm{E}-10$ $\begin{array}{llllll}3.171 \mathrm{E}-14 & 1.784 \mathrm{E}-11 & 2.205 \mathrm{E}-10 & 6.374 \mathrm{E}-16-1.000 \mathrm{E}+00 & 6.144 \mathrm{E}-11 & 4.642 \mathrm{E}-12\end{array}$ 2.289E-14 1.287E-11 $1.590 \mathrm{E}-10 \quad 4.598 \mathrm{E}-16 \quad 3.663 \mathrm{E}-09 \quad 7.432 \mathrm{E}-09 \quad 1.959 \mathrm{E}-08$ $2.908 \mathrm{E}-14 \quad 1.634 \mathrm{E}-11 \quad 2.019 \mathrm{E}-10 \quad 5.835 \mathrm{E}-16-1.000 \mathrm{E}+00 \quad 1.331 \mathrm{E}-10 \quad 5.225 \mathrm{E}-11$ $\begin{array}{llllll}3.111 E-14 & 1.749 E-11 & 2.161 E-10 & 6.247 E-16-1.000 E+00 & 9.102 E-11 & 7.574 E-11\end{array}$ $\begin{array}{lllllll}3.393 \mathrm{E}-14 & 1.909 \mathrm{E}-11 & 2.358 \mathrm{E}-10 & 6.818 \mathrm{E}-16-1.000 \mathrm{E}+00 & 4.588 \mathrm{E}-11 & 5.894 \mathrm{E}-11\end{array}$ $\begin{array}{llllll}2.280 E-14 & 1.278 E-11 & 1.579 E-10 & 4.566 E-16-1.000 E+00 & 2.521 E-10 & 6.148 E-10\end{array}$ $2.951 \mathrm{E}-14 \quad 1.702 \mathrm{E}-11 \quad 2.093 \mathrm{E}-10 \quad 6.088 \mathrm{E}-16-1.000 \mathrm{E}+00 \quad 1.633 \mathrm{E}-09 \quad 1.948 \mathrm{E}-09$ $\begin{array}{lllllll}3.171 E-14 & 1.784 E-11 & 2.205 E-10 & 6.374 E-16 & 2.443 E-11 & 6.244 E-11 & 4.642 E-12\end{array}$

$\begin{array}{llllllll}5.264 E-14 & 1.651 E-11 & 2.316 E-11 & 9.830 E-16 & 1.226 E-10 & 1.235 E-10 & 7.245 E-10\end{array}$ $\begin{array}{lllllll}4.820 \mathrm{E}-14 & 1.512 \mathrm{E}-11 & 2.120 \mathrm{E}-11 & 9.006 \mathrm{E}-16 & 1.888 \mathrm{E}-10 & 1.912 \mathrm{E}-10 & 1.473 \mathrm{E}-09\end{array}$ $\begin{array}{lllllll}5.676 E-14 & 1.785 E-11 & 2.503 E-11 & 1.062 E-15 & 8.357 E-10 & 8.954 E-10 & 9.379 E-12\end{array}$ $\begin{array}{lllllll}5.771 E-14 & 1.811 \mathrm{E}-11 & 2.540 \mathrm{E}-11 & 1.078 \mathrm{E}-15 & 1.608 \mathrm{E}-11 & 1.654 \mathrm{E}-11 & 3.661 \mathrm{E}-11\end{array}$ $\begin{array}{lllllll}7.103 \mathrm{E}-14 & 2.233 \mathrm{E}-11 & 3.132 \mathrm{E}-11 & 1.329 \mathrm{E}-15-1.000 \mathrm{E}+00 & 9.720 \mathrm{E}-12 & 1.464 \mathrm{E}-12\end{array}$ $\begin{array}{lllllll}5.169 \mathrm{E}-14 & 1.624 \mathrm{E}-11 & 2.278 \mathrm{E}-11 & 9.672 \mathrm{E}-16 & 2.014 \mathrm{E}-10 & 2.321 \mathrm{E}-10 & 1.929 \mathrm{E}-09\end{array}$ $6.247 \mathrm{E}-14 \quad 1.970 \mathrm{E}-11 \quad 2.764 \mathrm{E}-11 \quad 1.170 \mathrm{E}-15-1.000 \mathrm{E}+00 \quad 1.481 \mathrm{E}-11 \quad 1.332 \mathrm{E}-11$ $6.786 \mathrm{E}-142.143 \mathrm{E}-11 \quad 3.008 \mathrm{E}-11 \quad 1.272 \mathrm{E}-15-1.000 \mathrm{E}+00 \quad 1.281 \mathrm{E}-112.560 \mathrm{E}-11$ $7.515 \mathrm{E}-142.363 \mathrm{E}-11 \quad 3.315 \mathrm{E}-11 \quad 1.405 \mathrm{E}-15-1.000 \mathrm{E}+00 \quad 5.415 \mathrm{E}-12 \quad 1.105 \mathrm{E}-11$ 
OVARIES

EDEWBODY

THYROIDH

TE-127

STOMACH

SMALL IN

LUNGS

RED MARR

THYROID

LOWER LI

BONE SUR

BREAST

TESTES

OVARIES

EDEWBODY

THYROIDH

TE-127M

STOMACH

SMALL IN

LUNGS

RED MARR

THYROID

LOWER LI

BONE SUR

BREAST

TESTES

OVARIES

EDEWBODY

THYROIDH

TE-129

STOMACH

SMALL IN

LUNGS

RED MARR

THYROID

LOWER LI

BONE SUR

BREAST

TESTES

OVARIES

EDEWBODY

THYROIDH

TE-129M

STOMACH

SMALL IN

LUNGS

RED MARR

THYROID

LOWER LI

BONE SUR

BREAST

TESTES $\begin{array}{llllll}5.137 \mathrm{E}-14 & 1.608 \mathrm{E}-11 & 2.255 \mathrm{E}-11 & 9.576 \mathrm{E}-16-1.000 \mathrm{E}+00 & 2.148 \mathrm{E}-11 & 1.514 \mathrm{E}-10\end{array}$ $\begin{array}{llllll}6.528 \mathrm{E}-14 & 2.129 \mathrm{E}-11 & 2.998 \mathrm{E}-11 & 1.238 \mathrm{E}-15-1.000 \mathrm{E}+00 & 1.740 \mathrm{E}-10 & 4.830 \mathrm{E}-10\end{array}$

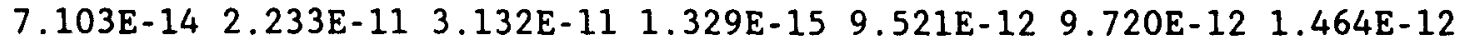

$\begin{array}{lllllll}1.639 \mathrm{E}-16 & 7.509 \mathrm{E}-14 & 1.679 \mathrm{E}-13 & 3.456 \mathrm{E}-18 & 4.321 \mathrm{E}-11 & 4.465 \mathrm{E}-11 & 2.425 \mathrm{E}-10\end{array}$

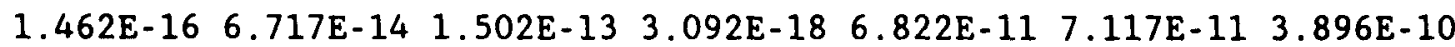
$\begin{array}{lllllll}1.788 \mathrm{E}-16 & 8.198 \mathrm{E}-14 & 1.833 \mathrm{E}-13 & 3.773 \mathrm{E}-18 & 3.727 \mathrm{E}-10 & 4.258 \mathrm{E}-10 & 2.885 \mathrm{E}-12\end{array}$

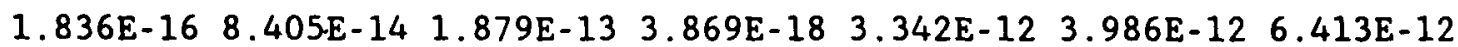
$2.245 \mathrm{E}-16 \quad 1.033 \mathrm{E}-13 \quad 2.310 \mathrm{E}-13 \quad 4.756 \mathrm{E}-18-1.000 \mathrm{E}+00 \quad 1.844 \mathrm{E}-12 \quad 2.855 \mathrm{E}-12$ $\begin{array}{lllllll}1.605 \mathrm{E}-16 & 7.371 \mathrm{E}-14 & 1.648 \mathrm{E}-13 & 3.393 \mathrm{E}-18 & 1.640 \mathrm{E}-10 & 2.275 \mathrm{E}-10 & 1.265 \mathrm{E}-09\end{array}$ $2.191 \mathrm{E}-16 \quad 1.006 \mathrm{E}-13 \quad 2.248 \mathrm{E}-13 \quad 4.630 \mathrm{E}-18-1.000 \mathrm{E}+00 \quad 4.063 \mathrm{E}-12 \quad 6.424 \mathrm{E}-12$ $2.312 \mathrm{E}-16 \quad 1.061 \mathrm{E}-13 \quad 2.372 \mathrm{E}-13 \quad 4.883 \mathrm{E}-18-1.000 \mathrm{E}+00 \quad 1.878 \mathrm{E}-12 \quad 2.998 \mathrm{E}-12$ $2.410 \mathrm{E}-16 \quad 1.109 \mathrm{E}-13 \quad 2.479 \mathrm{E}-13 \quad 5.105 \mathrm{E}-18-1.000 \mathrm{E}+00 \quad 1.828 \mathrm{E}-12 \quad 2.940 \mathrm{E}-12$ $\begin{array}{lllll}1.560 \mathrm{E}-16 & 7.165 \mathrm{E}-14 & 1.602 \mathrm{E}-13 & 3.298 \mathrm{E}-18-1.000 \mathrm{E}+00 & 2.029 \mathrm{E}-12 \quad 4.040 \mathrm{E}-12\end{array}$ $\begin{array}{llllll}0.000 \mathrm{E}+00 & 0.000 \mathrm{E}+00 & 0.000 \mathrm{E}+00 & 0.000 \mathrm{E}+00-1.000 \mathrm{E}+00 & 8.597 \mathrm{E}-11 & 1.874 \mathrm{E}-10\end{array}$ $\begin{array}{lllllll}2.245 \mathrm{E}-16 & 1.033 \mathrm{E}-13 & 2.310 \mathrm{E}-13 & 4.756 \mathrm{E}-18 & 1.590 \mathrm{E}-12 & 1.844 \mathrm{E}-12 & 2.855 \mathrm{E}-12\end{array}$

$\begin{array}{lllllll}4.566 \mathrm{E}-17 & 7.804 \mathrm{E}-14 & 2.953 \mathrm{E}-12 & 1.884 \mathrm{E}-18 & 8.892 \mathrm{E}-11 & 2.302 \mathrm{E}-10 & 2.115 \mathrm{E}-10\end{array}$ $3.165 \mathrm{E}-17 \quad 5.837 \mathrm{E}-14 \quad 2.406 \mathrm{E}-12 \quad 1.287 \mathrm{E}-18 \quad 1.832 \mathrm{E}-10 \quad 3.690 \mathrm{E}-10 \quad 4.338 \mathrm{E}-10$ 6.247E-17 1.013E-13 $3.555 \mathrm{E}-12 \quad 2.616 \mathrm{E}-18 \quad 1.799 \mathrm{E}-09 \quad 3.337 \mathrm{E}-08 \quad 9.597 \mathrm{E}-11$ 2.632E-17 5.643E-14 2.669E-12 1.034E-18 2.769E-10 5.309E-09 5.373E-09 $1.237 \mathrm{E}-16 \quad 1.852 \mathrm{E}-13 \quad 5.663 \mathrm{E}-12 \quad 5.296 \mathrm{E}-18-1.000 \mathrm{E}+00 \quad 9.635 \mathrm{E}-11$ 9.411E-11 $\begin{array}{lllllll}4.059 \mathrm{E}-17 & 7.121 \mathrm{E}-14 & 2.788 \mathrm{E}-12 & 1.662 \mathrm{E}-18 & 2.309 \mathrm{E}-09 & 5.719 \mathrm{E}-09 & 1.109 \mathrm{E}-08\end{array}$ $1.069 \mathrm{E}-16 \quad 1.615 \mathrm{E}-13 \quad 5.126 \mathrm{E}-12 \quad 4.503 \mathrm{E}-18-1.000 \mathrm{E}+00 \quad 2.055 \mathrm{E}-08 \quad 2.086 \mathrm{E}-08$ $3.038 \mathrm{E}-16 \quad 4.141 \mathrm{E}-13 \quad 1.042 \mathrm{E}-11 \quad 1.322 \mathrm{E}-17-1.000 \mathrm{E}+00 \quad 1.097 \mathrm{E}-10 \quad 9.733 \mathrm{E}-11$

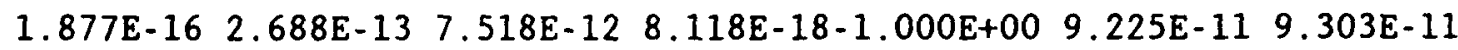
$4.503 \mathrm{E}-17 \quad 7.676 \mathrm{E}-14 \quad 2.865 \mathrm{E}-12 \quad 1.877 \mathrm{E}-18-1.000 \mathrm{E}+00 \quad 1.100 \mathrm{E}-10 \quad 1.248 \mathrm{E}-10$ $1.412 \mathrm{E}-16$ 1.758E-13 3.615E-12 6.111E-18-1.000E+00 5.801E-09 2.224E-09 $1.237 E-16 \quad 1.852 E-13 \quad 5.663 E-12 \quad 5.296 E-18 \quad 1.337 E-11 \quad 9.635 E-11 \quad 9.411 E-11$

$\begin{array}{llllllll}1.842 E-15 & 2.273 E-13 & 2.293 E-13 & 3.805 E-17 & 1.553 E-11 & 1.553 E-11 & 3.970 E-10\end{array}$ $\begin{array}{lllllll}1.652 E-15 & 2.027 E-13 & 2.044 E-13 & 3.393 E-17 & 1.083 E-11 & 1.083 E-11 & 2.730 E-10\end{array}$

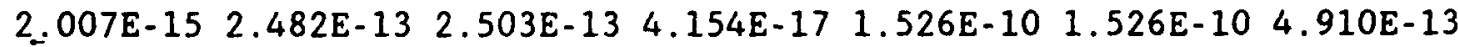
$2.042 E-15 \quad 2.501 E-13 \quad 2.522 E-13 \quad 4.186 E-17 \quad 6.131 E-13 \quad 6.131 E-13 \quad 7.610 E-13$ $2.527 E-15 \quad 3.145 E-13 \quad 3.172 E-13 \quad 5.264 E-17-1.000 E+00 \quad 5.088 E-13 \quad 3.350 E-13$ $\begin{array}{lllllll}1.804 E-15 & 2.217 E-13 & 2.235 E-13 & 3.710 E-17 & 1.838 E-12 & 1.839 E-12 & 3.700 E-11\end{array}$ $2.375 E-152.956 E-13 \quad 2.981 E-13 \quad 4.947 E-17-1.000 E+00 \quad 7.058 E-13 \quad 5.990 E-13$ $2.610 E-15 \quad 3.334 E-13 \quad 3.363 E-13 \quad 5.581 E-17-1.000 E+00 \quad 5.381 E-13 \quad 6.050 E-13$ $2.737 E-15 \quad 3.429 E-13 \quad 3.458 E-13 \quad 5.740 E-17-1.000 E+00 \quad 4.522 E-13 \quad 3.750 E-13$ $\begin{array}{llllll}1.782 E-15 & 2.198 E-13 & 2.216 E-13 & 3.678 E-17-1.000 E+00 ~ & 5.052 E-13 & 1.580 E-12\end{array}$ $2.685 E-15 \quad 5.525 E-13 \quad 5.572 E-13 \quad 9.248 E-17-1.000 E+00 \quad 2.085 E-11 \quad 5.430 E-11$ $2.527 E-15 \quad 3.145 E-13 \quad 3.172 E-13 \quad 5.264 E-17 \quad 5.088 E-13 \quad 5.088 E-13 \quad 3.350 E-13$

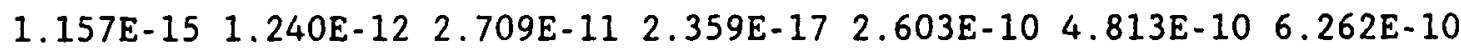

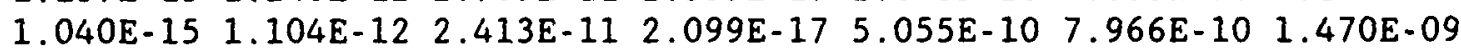

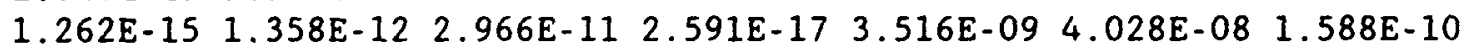
$\begin{array}{lllllll}1.259 E-15 & 1.344 E-12 & 2.940 E-11 & 2.524 E-17 & 4.854 E-10 & 3.038 E-09 & 3.432 E-09\end{array}$ $1.617 \mathrm{E}-15 \quad 1.753 \mathrm{E}-12 \quad 3.820 \mathrm{E}-11 \quad 3.393 \mathrm{E}-17-1.000 \mathrm{E}+00 \quad 1.555 \mathrm{E}-10 \quad 1.566 \mathrm{E}-10$

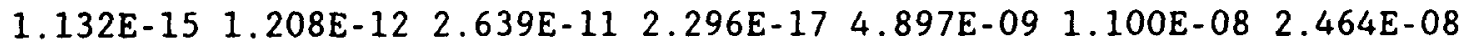

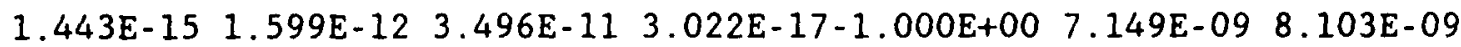
$1.687 E-15 \quad 1.936 E-12 \quad 4.203 E-11 \quad 3.869 E-17-1.000 E+00 \quad 1.684 E-10 \quad 1.657 E-10$ $1.754 E-15 \quad 1.932 E-12 \quad 4.207 E-11 \quad 3.773 E-17-1.000 E+00 \quad 1.390 E-10 \quad 1.604 E-10$ 
$\begin{array}{lllllll}\text { OVARIES } \quad 1.138 \mathrm{E}-15 & 1.209 \mathrm{E}-12 & 2.640 \mathrm{E}-11 & 2.318 \mathrm{E}-17-1.000 \mathrm{E}+00 & 1.783 \mathrm{E}-10 & 2.405 \mathrm{E}-10\end{array}$ EDEWBODY 1.644E-15 2.813E-12 6.190E-11 5.035E-17-1.000E+00 6.466E-09 2.885E-09 THYROIDH $\quad 1.617 \mathrm{E}-15 \quad 1.753 \mathrm{E}-12 \quad 3.820 \mathrm{E}-11 \quad 3.393 \mathrm{E}-17 \quad 3.040 \mathrm{E}-11 \quad 1.555 \mathrm{E}-10 \quad 1.566 \mathrm{E}-10$ TE-131M

$\begin{array}{lllllllll}\text { STOMACH } & 5.525 \mathrm{E}-14 & 2.755 \mathrm{E}-11 & 1.751 \mathrm{E}-10 & 1.048 \mathrm{E}-15 & 2.208 \mathrm{E}-10 & 2.758 \mathrm{E}-10 & 6.627 \mathrm{E}-10\end{array}$ SMALL IN $5.016 \mathrm{E}-14 \quad 2.504 \mathrm{E}-11 \quad 1.589 \mathrm{E}-10 \quad 9.528 \mathrm{E}-16 \quad 3.920 \mathrm{E}-10 \quad 4.928 \mathrm{E}-10 \quad 1.600 \mathrm{E}-09$ LUNGS RED MARR THYROID LOWER LI BONE SUR BREAST TESTES OVARIES EDEWBODY THYROIDH TE- 132 STOMACH SMALL IN LUNGS RED MARR THYROID LOWER LI BONE SUR BREAST TESTES OVARIES EDEWBODY THYROIDH I-131 STOMACH 5.966E-14 2.979E-11 1.895E-10 1.133E-15 1.203E-09 2.231E-09 6.208E-11 $\begin{array}{lllllll}6.028 \mathrm{E}-14 & 3.011 \mathrm{E}-11 & 1.918 \mathrm{E}-10 & 1.145 \mathrm{E}-15 & 9.441 \mathrm{E}-11 & 1.386 \mathrm{E}-10 & 2.393 \mathrm{E}-10\end{array}$ $7.492 \mathrm{E}-14 \quad 3.757 \mathrm{E}-11 \quad 2.390 \mathrm{E}-10 \quad 1.430 \mathrm{E}-15-1.000 \mathrm{E}+00 \quad 3.280 \mathrm{E}-08 \quad 3.908 \mathrm{E}-08$ $\begin{array}{lllllll}5.424 \mathrm{E}-14 & 2.710 \mathrm{E}-11 & 1.722 \mathrm{E}-10 & 1.031 \mathrm{E}-15 & 1.333 \mathrm{E}-09 & 2.367 \mathrm{E}-09 & 8.102 \mathrm{E}-09\end{array}$ $6.713 \mathrm{E}-14 \quad 3.366 \mathrm{E}-11 \quad 2.159 \mathrm{E}-10 \quad 1.281 \mathrm{E}-15-1.000 \mathrm{E}+00 \quad 2.608 \mathrm{E}-10 \quad 3.698 \mathrm{E}-10$

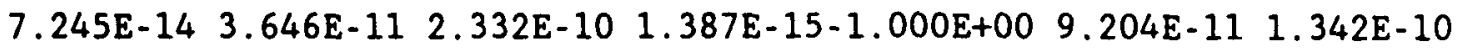
$7.928 \mathrm{E}-14 \quad 3.970 \mathrm{E}-11 \quad 2.529 \mathrm{E}-10 \quad 1.511 \mathrm{E}-15-1.000 \mathrm{E}+00 \quad 4.542 \mathrm{E}-11 \quad 9.816 \mathrm{E}-11$ $5.350 \mathrm{E}-142.671 \mathrm{E}-11 \quad 1.695 \mathrm{E}-10 \quad 1.016 \mathrm{E}-15-1.000 \mathrm{E}+00 \quad 2.335 \mathrm{E}-10 \quad 7.370 \mathrm{E}-10$

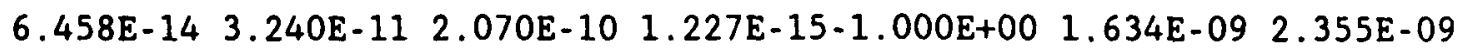

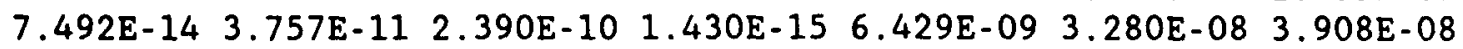

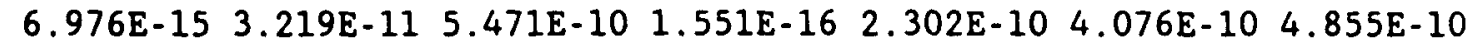
6.152E-15 2.912E-11 4.957E-10 $1.364 \mathrm{E}-16 \quad 3.127 \mathrm{E}-10 \quad 5.081 \mathrm{E}-10 \quad 7.662 \mathrm{E}-10$ $\begin{array}{lllllll}7.706 \mathrm{E}-15 & 3.493 \mathrm{E}-11 & 5.930 \mathrm{E}-10 & 1.722 \mathrm{E}-16 & 5.309 \mathrm{E}-10 & 1.641 \mathrm{E}-09 & 2.937 \mathrm{E}-10\end{array}$ $7.642 \mathrm{E}-15 \quad 3.531 \mathrm{E}-11 \quad 6.006 \mathrm{E}-10 \quad 1.681 \mathrm{E}-16 \quad 2.500 \mathrm{E}-10 \quad 3.951 \mathrm{E}-10 \quad 4.064 \mathrm{E}-10$ $1.021 \mathrm{E}-14 \quad 4.414 \mathrm{E}-11 \quad 7.469 \mathrm{E}-10 \quad 2.308 \mathrm{E}-16-1.000 \mathrm{E}+00 \quad 5.284 \mathrm{E}-08 \quad 4.695 \mathrm{E}-08$ $\begin{array}{lllllll}6.849 E-15 & 3.154 E-11 & 5.362 E-10 & 1.519 E-16 & 8.207 E-10 & 1.574 E-09 & 3.764 E-09\end{array}$ $1.075 E-14 \quad 4.024 E-11 \quad 6.752 E-10 \quad 2.419 E-16-1.000 E+00 \quad 7.249 E-10 \quad 8.537 E-10$ $1.154 \mathrm{E}-14 \quad 4.369 \mathrm{E}-11 \quad 7.321 \mathrm{E}-10 \quad 2.686 \mathrm{E}-16-1.000 \mathrm{E}+00 \quad 3.312 \mathrm{E}-10 \quad 3.126 \mathrm{E}-10$ $1.078 \mathrm{E}-14 \quad 4.683 \mathrm{E}-11 \quad 7.922 \mathrm{E}-10 \quad 2.461 \mathrm{E}-16-1.000 \mathrm{E}+00 \quad 3.374 \mathrm{E}-10 \quad 3.256 \mathrm{E}-10$ 6.247E-15 3.114E-11 5.313E-10 1.395E-16-1.000E+00 3.866E-10 5.058E-10 $9.502 E-15 \quad 4.131 E-11 \quad 6.992 E-10 \quad 2.153 E-16-1.000 E+00 \quad 2.229 E-09 \quad 2.132 E-09$

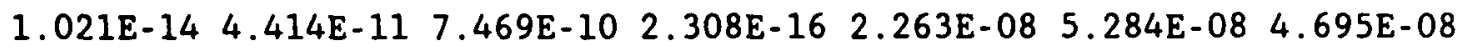

SMALL IN LUNGS RED MARR THYROID LOWER LI BONE SUR BREAST TESTES OVARIES EDEWBODY THYROIDH $I-132$

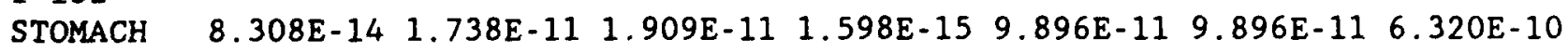
SMALI IN $7.547 \mathrm{E}-14 \quad 1.579 \mathrm{E}-11 \quad 1.735 \mathrm{E}-11 \quad 1.452 \mathrm{E}-15 \quad 1.245 \mathrm{E}-11 \quad 1.245 \mathrm{E}-11 \quad 3.160 \mathrm{E}-11$ LUNGS RED MARR THYROID LOWER LI BONE SUR BREAST TESTES $\begin{array}{lllllll}8.974 E-14 & 1.879 E-11 & 2.065 E-11 & 1.728 E-15 & 2.702 E-10 & 2.703 E-10 & 2.640 E-11\end{array}$ $9.132 \mathrm{E}-14 \quad 1.910 \mathrm{E}-11 \quad 2.099 \mathrm{E}-11 \quad 1.757 \mathrm{E}-15 \quad 1.401 \mathrm{E}-11 \quad 1.401 \mathrm{E}-11 \quad 2.450 \mathrm{E}-11$ $\begin{array}{lllllll}1.126 E-13 & 2.352 E-11 & 2.584 E-11 & 2.163 E-15-1.000 E+00 ~ & 1.726 E-09 & 3.842 E-09\end{array}$ 8.149E-14 $1.703 \mathrm{E}-11 \quad 1.871 \mathrm{E}-11 \quad 1.566 \mathrm{E}-15 \quad 1.126 \mathrm{E}-11 \quad 1.127 \mathrm{E}-11 \quad 2.750 \mathrm{E}-11$ $9.989 E-142.090 E-11 \quad 2.296 E-11 \quad 1.922 E-15-1.000 E+00 \quad 1.242 E-112.190 E-11$

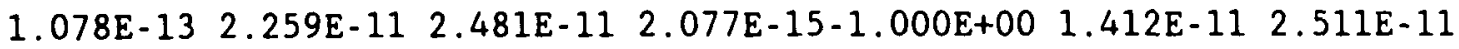

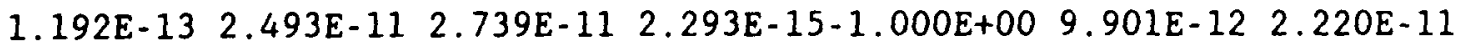


OVARIES

EDEWBODY

THYROIDH

I - 133

STOMACH

SMALL IN

LUNGS

RED MARR

THYROID

LOWER LI

BONE SUR

BREAST

TESTES

OVARIES

EDEWBODY

THYROIDH

I - 134

STOMACH

SMALL IN

LUNGS

RED MARR

THYROID

LOWER LI

BONE SUR

BREAST

TESTES

OVARIES

EDEWBODY

THYROIDH

I - 135

STOMACH

SMALL IN

LUNGS

RED MARR

THYROID

LOWER LI

BONE SUR

BREAST

TESTES

OVARIES

EDEWBODY

THYROIDH

XE - 133

STOMACH

SMALL IN

LUNGS

RED MARR

THYROID

LOWER LI

BONE SUR

BREAST

TESTES

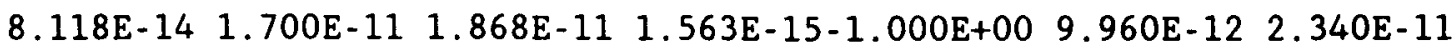
$\begin{array}{llllll}1.032 \mathrm{E}-13 & 2.203 \mathrm{E}-11 & 2.420 \mathrm{E}-11 & 2.025 \mathrm{E}-15-1.000 \mathrm{E}+00 & 1.026 \mathrm{E}-10 & 1.814 \mathrm{E}-10\end{array}$ $\begin{array}{lllllll}1.126 \mathrm{E}-13 & 2.352 \mathrm{E}-11 & 2.584 \mathrm{E}-11 & 2.163 \mathrm{E}-15 & 1.721 \mathrm{E}-09 & 1.726 \mathrm{E}-09 & 3.842 \mathrm{E}-09\end{array}$

$2.121 \mathrm{E}-14 \quad 1.076 \mathrm{E}-11 \quad 4.720 \mathrm{E}-11 \quad 4.249 \mathrm{E}-16 \quad 1.023 \mathrm{E}-10 \quad 1.046 \mathrm{E}-10 \quad 5.533 \mathrm{E}-10$ $\begin{array}{lllllll}1.915 E-14 & 9.713 E-12 & 4.252 E-11 & 3.837 E-16 & 2.006 E-11 & 2.150 E-11 & 4.041 E-11\end{array}$ $2.305 \mathrm{E}-14$ 1.172E-11 5.158E-11 4.630E-16 7.085E-10 8.162E-10 4.518E-11 $2.350 \mathrm{E}-14 \quad 1.196 \mathrm{E}-11 \quad 5.196 \mathrm{E}-11 \quad 4.725 \mathrm{E}-16 \quad 2.454 \mathrm{E}-11 \quad 2.717 \mathrm{E}-11 \quad 4.313 \mathrm{E}-11$ $2.873 E-14 \quad 1.462 E-11 \quad 6.491 E-11 \quad 5.771 E-16-1.000 E+00 \quad 4.855 E-08 \quad 9.102 E-08$ $2.077 \mathrm{E}-14$ 1.052E-11 $4.605 \mathrm{E}-11 \quad 4.154 \mathrm{E}-16 \quad 1.910 \mathrm{E}-112.049 \mathrm{E}-11 \quad 3.883 \mathrm{E}-11$ $2.645 \mathrm{E}-14 \quad 1.350 \mathrm{E}-11 \quad 6.041 \mathrm{E}-11 \quad 5.327 \mathrm{E}-16-1.000 \mathrm{E}+00 \quad 2.518 \mathrm{E}-11 \quad 4.080 \mathrm{E}-11$ $2.829 \mathrm{E}-14 \quad 1.439 \mathrm{E}-11 \quad 6.509 \mathrm{E}-11 \quad 5.676 \mathrm{E}-16-1.000 \mathrm{E}+00 \quad 2.931 \mathrm{E}-11 \quad 4.688 \mathrm{E}-11$ $3.079 \mathrm{E}-14 \quad 1.566 \mathrm{E}-11 \quad 6.965 \mathrm{E}-11 \quad 6.183 \mathrm{E}-16-1.000 \mathrm{E}+00 \quad 1.947 \mathrm{E}-11 \quad 3.632 \mathrm{E}-11$ $2.071 \mathrm{E}-14$ 1.052E-11 4.602E-11 4.154E-16-1.000E+00 1.934E-11 3.573E-11 $2.685 \mathrm{E}-14 \quad 1.427 \mathrm{E}-11 \quad 6.312 \mathrm{E}-11 \quad 5.637 \mathrm{E}-16-1.000 \mathrm{E}+00 \quad 1.583 \mathrm{E}-09 \quad 2.800 \mathrm{E}-09$ $2.873 \mathrm{E}-14 \quad 1.462 \mathrm{E}-11 \quad 6.491 \mathrm{E}-11 \quad 5.771 \mathrm{E}-16 \quad 2.389 \mathrm{E}-08 \quad 4.855 \mathrm{E}-08 \quad 9.102 \mathrm{E}-08$

$9.672 \mathrm{E}-14 \quad 8.230 \mathrm{E}-12 \quad 8.245 \mathrm{E}-12 \quad 1.811 \mathrm{E}-15 \quad 7.081 \mathrm{E}-11 \quad 7.081 \mathrm{E}-11 \quad 5.480 \mathrm{E}-10$ 8.847E-14 7.524E-12 7.537E-12 1.655E-15 5.521E-12 $5.521 \mathrm{E}-12 \quad 1.600 \mathrm{E}-11$

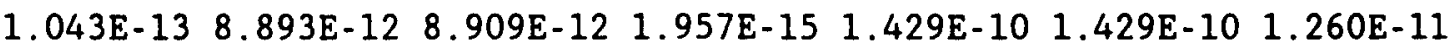

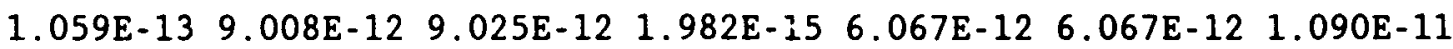

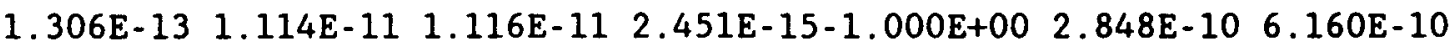
$9.513 \mathrm{E}-14 \quad 8.100 \mathrm{E}-12 \quad 8.115 \mathrm{E}-12 \quad 1.782 \mathrm{E}-15 \quad 4.800 \mathrm{E}-12 \quad 4.800 \mathrm{E}-12 \quad 1.290 \mathrm{E}-11$ $1.148 \mathrm{E}-13 \quad 9.787 \mathrm{E}-12 \quad 9.804 \mathrm{E}-12 \quad 2.153 \mathrm{E}-15-1.000 \mathrm{E}+00 \quad 5.296 \mathrm{E}-12 \quad 9.290 \mathrm{E}-12$

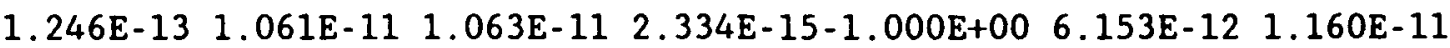

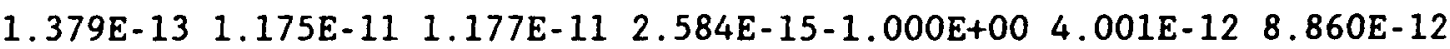
$9.418 \mathrm{E}-14 \quad 8.028 \mathrm{E}-12 \quad 8.043 \mathrm{E}-12 \quad 1.766 \mathrm{E}-15-1.000 \mathrm{E}+00 \quad 4.235 \mathrm{E}-12 \quad 1.100 \mathrm{E}-11$ $\begin{array}{llllll}1.204 \mathrm{E}-13 & 1.042 \mathrm{E}-11 & 1.044 \mathrm{E}-11 & 2.292 \mathrm{E}-15-1.000 \mathrm{E}+00 & 3.548 \mathrm{E}-11 & 6.630 \mathrm{E}-11\end{array}$ $\begin{array}{lllllll}1.306 \mathrm{E}-13 & 1.114 \mathrm{E}-11 & 1.116 \mathrm{E}-11 & 2.451 \mathrm{E}-15 & 2.848 \mathrm{E}-10 & 2.848 \mathrm{E}-10 & 6.160 \mathrm{E}-10\end{array}$

6.093E-14 2.170E-11 4.265E-11 $1.065 E-15 \quad 9.819 E-11 \quad 9.855 E-11 \quad 5.403 E-10$ 5.689E-14 2.020E-11 3.951E-11 9.932E-16 $1.878 \mathrm{E}-11 \quad 1.899 \mathrm{E}-11 \quad 4.108 \mathrm{E}-11$ $\begin{array}{lllllll}6.589 \mathrm{E}-14 & 2.354 \mathrm{E}-11 & 4.632 \mathrm{E}-11 & 1.154 \mathrm{E}-15 & 4.291 \mathrm{E}-10 & 4.396 \mathrm{E}-10 & 3.737 \mathrm{E}-11\end{array}$ $\begin{array}{llllllll}6.658 E-14 & 2.377 E-11 & 4.682 E-11 & 1.165 E-15 & 2.194 E-11 & 2.231 E-11 & 3.638 E-11\end{array}$ 8.172E-14 2.917E-11 5.765E-11 1.427E-15-1.000E+00 8.435E-09 1.778E-08 $\begin{array}{llllllll}6.056 \mathrm{E}-14 & 2.154 \mathrm{E}-11 & 4.231 \mathrm{E}-11 & 1.057 \mathrm{E}-15 & 1.765 \mathrm{E}-11 & 1.784 \mathrm{E}-11 & 3.937 \mathrm{E}-11\end{array}$ $7.106 \mathrm{E}-14 \quad 2.561 \mathrm{E}-11 \quad 5.157 \mathrm{E}-11 \quad 1.242 \mathrm{E}-15-1.000 \mathrm{E}+00 \quad 1.997 \mathrm{E}-11 \quad 3.342 \mathrm{E}-11$ $\begin{array}{llllll}7.823 E-14 & 2.817 E-11 & 5.639 E-11 & 1.370 E-15-1.000 E+00 & 2.334 E-11 & 3.844 E-11\end{array}$ 8.672E-14 3.094E-11 6.113E-11 1.514E-15-1.000E+00 1.521E-11 3.213E-11 $5.897 \mathrm{E}-14 \quad 2.091 \mathrm{E}-11 \quad 4.088 \mathrm{E}-11 \quad 1.029 \mathrm{E}-15-1.000 \mathrm{E}+00 \quad 1.699 \mathrm{E}-11 \quad 3.616 \mathrm{E}-11$ $7.257 \mathrm{E}-14 \quad 2.643 \mathrm{E}-11 \quad 5.277 \mathrm{E}-11 \quad 1.285 \mathrm{E}-15-1.000 \mathrm{E}+00 \quad 3.308 \mathrm{E}-10 \quad 6.041 \mathrm{E}-10$ 8.172E-14 2.917E-11 5.765E-11 1.427E-15 7.172E-09 8.435E-09 1.778E-08

$\begin{array}{lllllll}9.450 \mathrm{E}-16 & 0.000 \mathrm{E}+00 & 0.000 \mathrm{E}+00 & 0.000 \mathrm{E}+00 & 1.312 \mathrm{E}-13 & 1.451 \mathrm{E}-13 & 0.000 \mathrm{E}+00\end{array}$ $\begin{array}{lllllll}7.991 E-16 & 0.000 E+00 & 0.000 E+00 & 0.000 E+00 & 1.368 E-13 & 1.498 E-13 & 0.000 E+00\end{array}$ $\begin{array}{lllllll}1.113 \mathrm{E}-15 & 0.000 \mathrm{E}+00 & 0.000 \mathrm{E}+00 & 0.000 \mathrm{E}+00 & 3.508 \mathrm{E}-13 & 3.716 \mathrm{E}-13 & 0.000 \mathrm{E}+00\end{array}$ $\begin{array}{lllllll}7.293 E-16 & 0.000 E+00 & 0.000 E+00 & 0.000 E+00 & 1.558 E-13 & 1.686 E-13 & 0.000 E+00\end{array}$

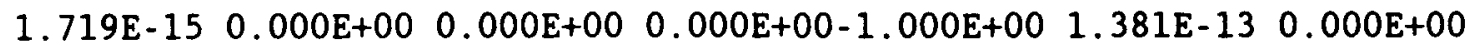
$\begin{array}{lllllll}8.689 E-16 & 0.000 E+00 & 0.000 E+00 & 0.000 E+00 & 1.357 E-13 & 1.486 E-13 & 0.000 E+00\end{array}$ $\begin{array}{lllllll}1.976 \mathrm{E}-15 & 0.000 \mathrm{E}+00 & 0.000 \mathrm{E}+00 & 0.000 \mathrm{E}+00-1.000 \mathrm{E}+00 & 1.616 \mathrm{E}-13 & 0.000 \mathrm{E}+00\end{array}$

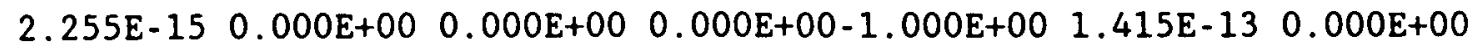

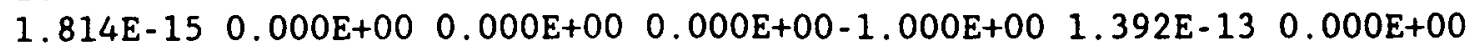


OVARIES $\quad 8.340 \mathrm{E}-16 \quad 0.000 \mathrm{E}+00 \quad 0.000 \mathrm{E}+00 \quad 0.000 \mathrm{E}+00-1.000 \mathrm{E}+00 \quad 1.464 \mathrm{E}-13 \quad 0.000 \mathrm{E}+00$ $\begin{array}{lllllll}\text { EDEWBODY } 1.551 E-15 & 0.000 E+00 & 0.000 E+00 & 0.000 E+00-1.000 E+00 & 1.762 E-13 & 0.000 E+00\end{array}$ $\begin{array}{llllllll}\text { THYROIDH } \quad 1.719 \mathrm{E}-15 & 0.000 \mathrm{E}+00 & 0.000 \mathrm{E}+00 & 0.000 \mathrm{E}+00 & 1.212 \mathrm{E}-13 & 1.381 \mathrm{E}-13 & 0.000 \mathrm{E}+00\end{array}$ $\mathrm{XE}-135$

$\begin{array}{lllllllll}\text { STOMACH } & 8.213 \mathrm{E}-15 & 0.000 \mathrm{E}+00 & 0.000 \mathrm{E}+00 & 0.000 \mathrm{E}+00 & 2.546 \mathrm{E}-13 & 2.575 \mathrm{E}-13 & 0.000 \mathrm{E}+00\end{array}$ $\begin{array}{lllllll}\text { SMALL IN } 7.293 \mathrm{E}-15 & 0.000 \mathrm{E}+00 & 0.000 \mathrm{E}+00 & 0.000 \mathrm{E}+00 & 2.560 \mathrm{E}-13 & 2.586 \mathrm{E}-13 & 0.000 \mathrm{E}+00\end{array}$ LUNGS RED MARR THYROID LOWER LI BONE SUR BREAST TESTES OVARIES EDEWBODY THYROIDH CS -134 STOMACH $\begin{array}{lllllll}9.006 \mathrm{E}-15 & 0.000 \mathrm{E}+00 & 0.000 \mathrm{E}+00 & 0.000 \mathrm{E}+00 & 7.645 \mathrm{E}-13 & 7.685 \mathrm{E}-13 & 0.000 \mathrm{E}+00\end{array}$ $\begin{array}{lllllll}9.228 \mathrm{E}-15 & 0.000 \mathrm{E}+00 & 0.000 \mathrm{E}+00 & 0.000 \mathrm{E}+00 & 2.532 \mathrm{E}-13 & 2.554 \mathrm{E}-13 & 0.000 \mathrm{E}+00\end{array}$ $\begin{array}{lllllll}1.167 \mathrm{E}-14 & 0.000 \mathrm{E}+00 & 0.000 \mathrm{E}+00 & 0.000 \mathrm{E}+00-1.000 \mathrm{E}+00 & 2.341 \mathrm{E}-13 & 0.000 \mathrm{E}+00\end{array}$ $\begin{array}{lllllll}8.118 \mathrm{E}-15 & 0.000 \mathrm{E}+00 & 0.000 \mathrm{E}+00 & 0.000 \mathrm{E}+00 & 2.479 \mathrm{E}-13 & 2.504 \mathrm{E}-13 & 0.000 \mathrm{E}+00\end{array}$

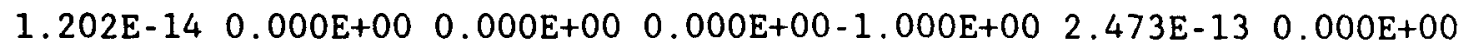

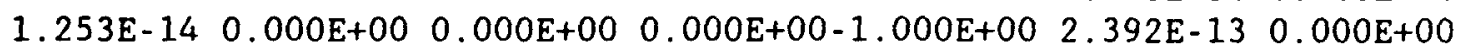
$\begin{array}{llllll}1.227 \mathrm{E}-14 & 0.000 \mathrm{E}+00 & 0.000 \mathrm{E}+00 & 0.000 \mathrm{E}+00-1.000 \mathrm{E}+00 & 2.321 \mathrm{E}-13 & 0.000 \mathrm{E}+00\end{array}$

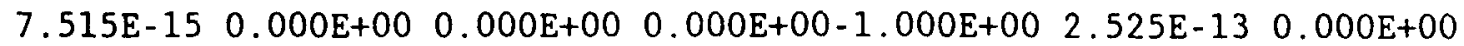

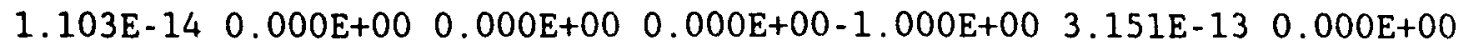
$\begin{array}{lllllll}1.167 E-14 & 0.000 E+00 & 0.000 E+00 & 0.000 E+00 & 2.308 E-13 & 2.341 E-13 & 0.000 E+00\end{array}$

SMALL IN LUNGS RED MARR THYROID LOWER LI BONE SUR BREAST TESTES OVARIES EDEWBODY THYROIDH CS - 136 STOMACH $\begin{array}{lllllll}5.581 E-14 & 3.159 E-11 & 6.615 E-10 & 1.097 E-15 & 3.462 E-10 & 1.251 E-08 & 2.009 E-08\end{array}$ $\begin{array}{lllllll}5.042 \mathrm{E}-14 & 2.858 \mathrm{E}-11 & 5.984 \mathrm{E}-10 & 9.925 \mathrm{E}-16 & 3.382 \mathrm{E}-10 & 1.372 \mathrm{E}-08 & 2.178 \mathrm{E}-08\end{array}$ $\begin{array}{lllllll}6.025 E-14 & 3.424 E-11 & 7.169 E-10 & 1.189 E-15 & 8.600 E-10 & 1.173 E-08 & 1.746 E-08\end{array}$ $\begin{array}{lllllll}6.152 \mathrm{E}-14 & 3.488 \mathrm{E}-11 & 7.303 \mathrm{E}-10 & 1.211 \mathrm{E}-15 & 9.057 \mathrm{E}-10 & 1.178 \mathrm{E}-08 & 1.868 \mathrm{E}-08\end{array}$ $7.579 \mathrm{E}-14 \quad 4.292 \mathrm{E}-11 \quad 8.986 \mathrm{E}-10 \quad 1.490 \mathrm{E}-15-1.000 \mathrm{E}+00 \quad 1.113 \mathrm{E}-08 \quad 1.764 \mathrm{E}-08$ $\begin{array}{lllllll}5.454 E-14 & 3.095 E-11 & 6.481 E-10 & 1.075 E-15 & 3.349 E-10 & 1.372 E-08 & 2.177 E-08\end{array}$ $6.786 \mathrm{E}-14 \quad 3.844 \mathrm{E}-11 \quad 8.049 \mathrm{E}-10 \quad 1.335 \mathrm{E}-15-1.000 \mathrm{E}+00 \quad 1.091 \mathrm{E}-08 \quad 1.731 \mathrm{E}-08$ $7.293 \mathrm{E}-14 \quad 4.136 \mathrm{E}-11 \quad 8.661 \mathrm{E}-10 \quad 1.436 \mathrm{E}-15-1.000 \mathrm{E}+00 \quad 1.082 \mathrm{E}-08 \quad 1.714 \mathrm{E}-08$

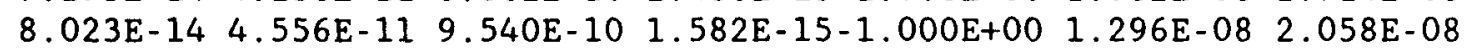
$5.454 \mathrm{E}-14 \quad 3.105 \mathrm{E}-11 \quad 6.500 \mathrm{E}-10 \quad 1.078 \mathrm{E}-15-1.000 \mathrm{E}+00 \quad 1.133 \mathrm{E}-08 \quad 1.798 \mathrm{E}-08$ $6.968 \mathrm{E}-14 \quad 3.966 \mathrm{E}-11 \quad 8.304 \mathrm{E}-10 \quad 1.377 \mathrm{E}-15-1.000 \mathrm{E}+00 \quad 1.253 \mathrm{E}-08 \quad 1.975 \mathrm{E}-08$ $\begin{array}{lllllll}7.579 E-14 & 4.292 \mathrm{E}-11 & 8.986 \mathrm{E}-10 & 1.490 \mathrm{E}-15 & 4.736 \mathrm{E}-10 & 1.113 \mathrm{E}-08 & 1.764 \mathrm{E}-08\end{array}$

SMALL IN LUNGS RED MARR THYROID

$7.864 \mathrm{E}-14 \quad 4.255 \mathrm{E}-11 \quad 7.539 \mathrm{E}-10 \quad 1.490 \mathrm{E}-15 \quad 3.756 \mathrm{E}-10 \quad 1.965 \mathrm{E}-09 \quad 3.384 \mathrm{E}-09$ $\begin{array}{lllllll}7.166 E-14 & 3.884 E-11 & 6.882 E-10 & 1.360 E-15 & 3.663 E-10 & 2.114 E-09 & 3.408 E-09\end{array}$ $\begin{array}{llllllll}8.498 E-14 & 4.608 E-11 & 8.165 E-10 & 1.614 E-15 & 6.540 E-10 & 2.311 E-09 & 2.617 E-09\end{array}$ $\begin{array}{lllllll}8.593 E-14 & 4.653 E-11 & 8.245 E-10 & 1.630 E-15 & 7.018 E-10 & 1.855 E-09 & 2.952 E-09\end{array}$ LOWER LI BONE SUR BREAST TESTES OVARIES EDEWBODY THYROIDH CS -137 $\begin{array}{llllllll}\text { STOMACH } & 2.010 E-14 & 1.149 E-11 & 2.430 E-10 & 4.020 E-16 & 2.251 E-10 & 8.606 E-09 & 1.390 E-08\end{array}$ SMALL IN $1.812 \mathrm{E}-14 \quad 1.037 \mathrm{E}-11 \quad 2.194 \mathrm{E}-10 \quad 3.630 \mathrm{E}-16 \quad 1.956 \mathrm{E}-10 \quad 9.043 \mathrm{E}-09 \quad 1.436 \mathrm{E}-08$ LUNGS RED MARR THYROID LOWER LI BONE SUR BREAST TESTES

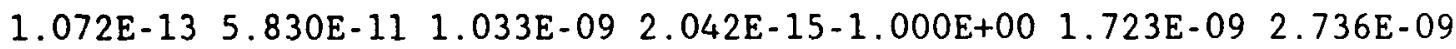
$\begin{array}{llllll}7.737 E-14 & 4.191 E-11 & 7.427 E-10 & 1.468 E-15 & 3.617 E-10 & 2.108 E-09 \\ 3 & 3.404 E-09\end{array}$ $9.481 \mathrm{E}-145.178 \mathrm{E}-11 \quad 9.176 \mathrm{E}-10 \quad 1.814 \mathrm{E}-15-1.000 \mathrm{E}+00 \quad 1.691 \mathrm{E}-09 \quad 2.697 \mathrm{E}-09$ $\begin{array}{llllll}1.027 \mathrm{E}-13 & 5.604 \mathrm{E}-11 & 9.930 \mathrm{E}-10 & 1.963 \mathrm{E}-15-1.000 \mathrm{E}+00 & 1.658 \mathrm{E}-09 & 2.636 \mathrm{E}-09\end{array}$

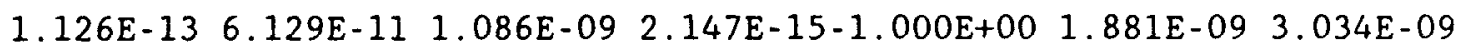

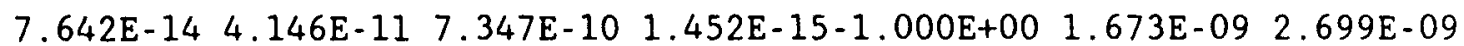
$9.792 \mathrm{E}-14 \quad 5.320 \mathrm{E}-11 \quad 9.427 \mathrm{E}-10 \quad 1.863 \mathrm{E}-15-1.000 \mathrm{E}+00 \quad 1.990 \mathrm{E}-09 \quad 3.062 \mathrm{E}-09$

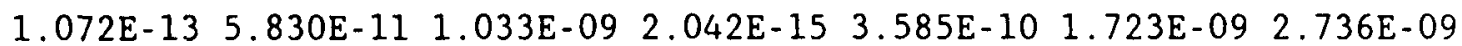
$2.178 \mathrm{E}-14 \quad 1.243 \mathrm{E}-11 \quad 2.630 \mathrm{E}-10 \quad 4.350 \mathrm{E}-16 \quad 8.287 \mathrm{E}-10 \quad 8.799 \mathrm{E}-09 \quad 1.266 \mathrm{E}-08$ $2.217 \mathrm{E}-14 \quad 1.260 \mathrm{E}-11 \quad 2.666 \mathrm{E}-10 \quad 4.410 \mathrm{E}-16 \quad 5.625 \mathrm{E}-10 \quad 8.295 \mathrm{E}-09 \quad 1.316 \mathrm{E}-08$ $2.730 \mathrm{E}-14$ 1.560E-11 $3.301 \mathrm{E}-10 \quad 5.460 \mathrm{E}-16-1.000 \mathrm{E}+00 \quad 7.919 \mathrm{E}-09 \quad 1.257 \mathrm{E}-08$ $\begin{array}{lllllll}1.968 E-14 & 1.123 E-11 & 2.376 E-10 & 3.930 E-16 & 1.953 E-10 & 9.063 E-09 & 1.439 E-08\end{array}$ $2.460 \mathrm{E}-14 \quad 1.406 \mathrm{E}-11 \quad 2.974 \mathrm{E}-10 \quad 4.920 \mathrm{E}-16-1.000 \mathrm{E}+00 \quad 7.933 \mathrm{E}-09 \quad 1.258 \mathrm{E}-08$ $2.643 \mathrm{E}-141.509 \mathrm{E}-11 \quad 3.192 \mathrm{E}-10 \quad 5.280 \mathrm{E}-16-1.000 \mathrm{E}+00 \quad 7.823 \mathrm{E}-09 \quad 1.240 \mathrm{E}-08$ $2.904 \mathrm{E}-14$ 1.655E-11 3.500E-10 5.790E-16-1.000E+00 8.758E-09 1.390E-08 
OVARIES

EDEWBODY

THYROIDH

BA- 139

STOMACH

SMALL IN

LUNGS

RED MARR

THYROID

LOWER LI

BONE SUR

BREAST

TESTES

OVARIES

EDEWBODY

THYROIDH

BA - 140

STOMACH

SMALL IN

LUNGS

RED MARR

THYROID

LOWER LI

BONE SUR

BREAST

TESTES

OVARIES

EDEWBODY

THYROIDH

LA - 140

STOMACH

SMALL IN

LUNGS

RED MARR

THYROID

LOWER LI

BONE SUR

BREAST

TESTES

OVARIES

EDEWBODY

THYROIDH

LA - 141

STOMACH

SMALL IN

LUNGS

RED MARR

THYROID

LOWER LI

BONE SUR

BREAST

TESTES $\begin{array}{lllll}1.974 \mathrm{E}-14 & 1.123 \mathrm{E}-11 & 2.376 \mathrm{E}-10 & 3.930 \mathrm{E}-16-1.000 \mathrm{E}+00 & 8.107 \mathrm{E}-09 \\ 1.286 \mathrm{E}-08\end{array}$

$2.526 \mathrm{E}-141.459 \mathrm{E}-11 \quad 3.085 \mathrm{E}-10 \quad 5.104 \mathrm{E}-16-1.000 \mathrm{E}+00 \quad 8.628 \mathrm{E}-09 \quad 1.355 \mathrm{E}-08$ $2.730 \mathrm{E}-14$ 1.560E-11 3.301E-10 5.460E-16 2.960E-10 7.919E-09 1.257E-08

$\begin{array}{llllllll}1.157 E-15 & 1.742 E-13 & 1.775 E-13 & 2.467 E-17 & 9.184 E-11 & 9.184 E-11 & 6.880 E-10\end{array}$ $\begin{array}{llllllll}1.024 \mathrm{E}-15 & 1.539 \mathrm{E}-13 & 1.567 \mathrm{E}-13 & 2.178 \mathrm{E}-17 & 7.425 \mathrm{E}-11 & 7.425 \mathrm{E}-11 & 5.560 \mathrm{E}-10\end{array}$ $\begin{array}{lllllll}1.278 E-15 & 1.928 \mathrm{E}-13 & 1.964 \mathrm{E}-13 & 2.730 \mathrm{E}-17 & 2.529 \mathrm{E}-10 & 2.529 \mathrm{E}-10 & 4.450 \mathrm{E}-13\end{array}$

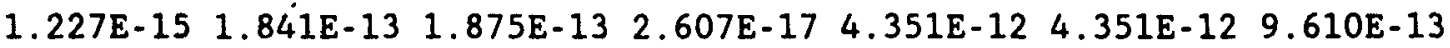

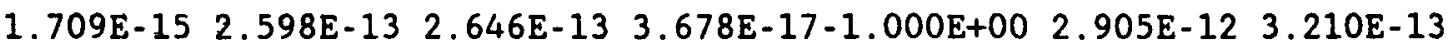
$1.138 \mathrm{E}-15 \quad 1.711 \mathrm{E}-13 \quad 1.743 \mathrm{E}-13 \quad 2.423 \mathrm{E}-17 \quad 1.573 \mathrm{E}-11 \quad 1.573 \mathrm{E}-11 \quad 1.000 \mathrm{E}-10$ $\begin{array}{llllll}1.826 \mathrm{E}-15 & 2.777 \mathrm{E}-13 & 2.829 \mathrm{E}-13 & 3.932 \mathrm{E}-17-1.000 \mathrm{E}+00 & 4.354 \mathrm{E}-12 & 6.400 \mathrm{E}-13\end{array}$ $\begin{array}{lllllll}1.887 \mathrm{E}-15 & 2.911 \mathrm{E}-13 & 2.966 \mathrm{E}-13 & 4.122 \mathrm{E}-17-1.000 \mathrm{E}+00 & 2.947 \mathrm{E}-12 & 5.700 \mathrm{E}-13\end{array}$ $\begin{array}{llllll}1.763 E-15 & 2.687 \mathrm{E}-13 & 2.737 \mathrm{E}-13 & 3.805 \mathrm{E}-17-1.000 \mathrm{E}+00 & 2.870 \mathrm{E}-12 & 3.620 \mathrm{E}-13\end{array}$

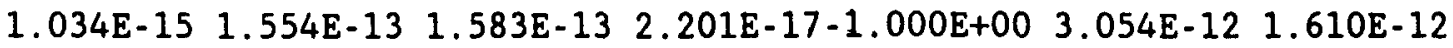
$2.164 \mathrm{E}-15$ 8.125E-13 8.276E-13 $1.150 \mathrm{E}-16-1.000 \mathrm{E}+00 \quad 4.686 \mathrm{E}-11 \quad 1.080 \mathrm{E}-10$

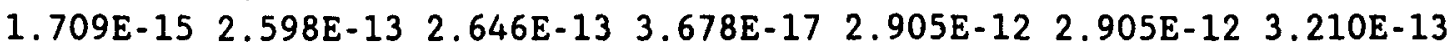

$6.405 E-15 \quad 6.623 E-12 \quad 5.941 E-10 \quad 1.332 E-16 \quad 1.416 E-10 \quad 3.561 E-10 \quad 5.836 E-10$ $5.708 \mathrm{E}-15 \quad 6.025 \mathrm{E}-12 \quad 5.512 \mathrm{E}-10 \quad 1.189 \mathrm{E}-16 \quad 2.874 \mathrm{E}-10 \quad 5.526 \mathrm{E}-10 \quad 1.691 \mathrm{E}-09$ $6.976 \mathrm{E}-15 \quad 7.209 \mathrm{E}-12 \quad 6.438 \mathrm{E}-10 \quad 1.455 \mathrm{E}-16 \quad 9.303 \mathrm{E}-10 \quad 1.678 \mathrm{E}-09 \quad 6.920 \mathrm{E}-11$ $7.071 \mathrm{E}-15 \quad 7.296 \mathrm{E}-12 \quad 6.525 \mathrm{E}-10 \quad 1.471 \mathrm{E}-16 \quad 4.739 \mathrm{E}-10 \quad 1.221 \mathrm{E}-09 \quad 4.219 \mathrm{E}-10$ 8.752E-15 9.019E-12 7.951E-10 1.842E-16-1.000E+00 2.721E-10 5.583E-11 6.247E-15 6.515E-12 5.891E-10 1.300E-16 2.334E-09 4.364E-09 2.630E-08 8.340E-15 8.317E-12 7.069E-10 1.754E-16-1.000E+00 2.317E-09 5.331E-10 $8.942 \mathrm{E}-15 \quad 9.129 \mathrm{E}-12 \quad 7.788 \mathrm{E}-10 \quad 1.918 \mathrm{E}-16-1.000 \mathrm{E}+00 \quad 3.023 \mathrm{E}-10 \quad 1.609 \mathrm{E}-10$ $9.418 \mathrm{E}-15 \quad 9.696 \mathrm{E}-12 \quad 8.495 \mathrm{E}-10 \quad 1.991 \mathrm{E}-16-1.000 \mathrm{E}+00 \quad 2.908 \mathrm{E}-10 \quad 1.467 \mathrm{E}-10$ $6.152 \mathrm{E}-15 \quad 6.374 \mathrm{E}-12 \quad 5.705 \mathrm{E}-10 \quad 1.284 \mathrm{E}-16-1.000 \mathrm{E}+00 \quad 4.480 \mathrm{E}-10 \quad 9.877 \mathrm{E}-10$ 8.333E-15 8.930E-12 7.628E-10 1.875E-16-1.000E+00 1.020E-09 2.545E-09

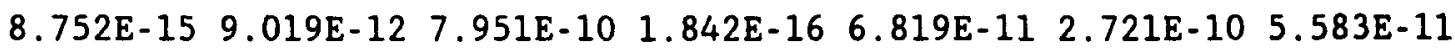

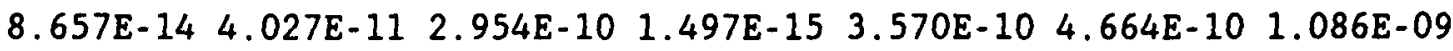

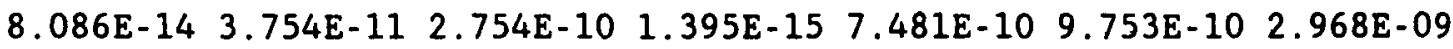
$9.354 \mathrm{E}-14 \quad 4.359 \mathrm{E}-11 \quad 3.198 \mathrm{E}-10 \quad 1.620 \mathrm{E}-15$ 1.958E-09 $4.213 \mathrm{E}-09 \quad 4.000 \mathrm{E}-11$ $9.481 \mathrm{E}-14 \quad 4.419 \mathrm{E}-11 \quad 3.242 \mathrm{E}-10 \quad 1.643 \mathrm{E}-15 \quad 1.440 \mathrm{E}-10 \quad 2.124 \mathrm{E}-10 \quad 2.816 \mathrm{E}-10$

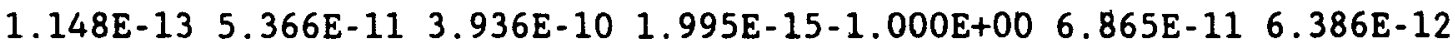
$\begin{array}{lllllll}8.593 E-14 & 4.001 E-11 & 2.935 E-10 & 1.487 E-15 & 2.946 E-09 & 5.498 E-09 & 1.750 E-08\end{array}$ $1.008 \mathrm{E}-13 \quad 4.726 \mathrm{E}-11 \quad 3.467 \mathrm{E}-10 \quad 1.757 \mathrm{E}-15-1.000 \mathrm{E}+00 \quad 1.406 \mathrm{E}-10 \quad 9.757 \mathrm{E}-11$ $1.116 \mathrm{E}-13 \quad 5.212 \mathrm{E}-11 \quad 3.824 \mathrm{E}-10 \quad 1.937 \mathrm{E}-15-1.000 \mathrm{E}+00 \quad 1.453 \mathrm{E}-10 \quad 1.800 \mathrm{E}-10$ $1.227 \mathrm{E}-13 \quad 5.724 \mathrm{E}-11 \quad 4.199 \mathrm{E}-10 \quad 2.128 \mathrm{E}-15-1.000 \mathrm{E}+00 \quad 5.875 \mathrm{E}-11 \quad 1.217 \mathrm{E}-10$ 8.276E-14 3.864E-11 2.835E-10 1.436E-15-1.000E+00 4.534E-10 1.334E-09 $1.071 \mathrm{E}-13 \quad 5.107 \mathrm{E}-113.746 \mathrm{E}-10$ 1.898E-15-1.000E $300.1 .315 \mathrm{E}-09 \quad 2.290 \mathrm{E}-09$ $\begin{array}{lllllll}1.148 \mathrm{E}-13 & 5.366 \mathrm{E}-11 & 3.936 \mathrm{E}-10 & 1.995 \mathrm{E}-15 & 3.768 \mathrm{E}-11 & 6.865 \mathrm{E}-11 & 6.386 \mathrm{E}-12\end{array}$

$\begin{array}{lllllll}1.566 \mathrm{E}-15 & 4.158 \mathrm{E}-13 & 6.939 \mathrm{E}-13 & 2.667 \mathrm{E}-17 & 9.818 \mathrm{E}-11 & 9.900 \mathrm{E}-11 & 9.310 \mathrm{E}-10\end{array}$ $\begin{array}{lllllll}1.475 E-15 & 3.913 E-13 & 6.421 E-13 & 2.511 E-17 & 1.430 E-10 & 1.445 E-10 & 1.370 E-09\end{array}$ $\begin{array}{lllllll}1.700 \mathrm{E}-15 & 4.510 \mathrm{E}-13 & 7.574 \mathrm{E}-13 & 2.892 \mathrm{E}-17 & 8.303 \mathrm{E}-10 & 8.904 \mathrm{E}-10 & 2.715 \mathrm{E}-13\end{array}$ $\begin{array}{lllllll}1.712 E-15 & 4.545 \mathrm{E}-13 & 7.461 \mathrm{E}-13 & 2.917 \mathrm{E}-17 & 5.104 \mathrm{E}-12 & 6.845 \mathrm{E}-12 & 1.073 \mathrm{E}-12\end{array}$ $\begin{array}{lllllll}2.099 E-15 & 5.594 \mathrm{E}-13 & 9.643 \mathrm{E}-13 & 3.583 \mathrm{E}-17-1.000 \mathrm{E}+00 & 2.447 \mathrm{E}-12 & 5.284 \mathrm{E}-14\end{array}$ $\begin{array}{lllllll}1.563 \mathrm{E}-15 & 4.152 \mathrm{E}-13 & 6.889 \mathrm{E}-13 & 2.664 \mathrm{E}-17 & 1.440 \mathrm{E}-10 & 1.672 \mathrm{E}-10 & 1.448 \mathrm{E}-09\end{array}$ $\begin{array}{lllllll}1.804 \mathrm{E}-15 & 4.812 \mathrm{E}-13 & 8.877 \mathrm{E}-13 & 3.073 \mathrm{E}-17-1.000 \mathrm{E}+00 & 2.502 \mathrm{E}-11 & 6.220 \mathrm{E}-13\end{array}$ $\begin{array}{llllll}2.001 E-15 & 5.310 \mathrm{E}-13 & 9.646 \mathrm{E}-13 & 3.393 \mathrm{E}-17-1.000 \mathrm{E}+00 & 2.669 \mathrm{E}-12 & 7.068 \mathrm{E}-13\end{array}$ $2.223 \mathrm{E}-15 \quad 5.890 \mathrm{E}-13 \quad 1.010 \mathrm{E}-12 \quad 3.773 \mathrm{E}-17-1.000 \mathrm{E}+00 \quad 2.300 \mathrm{E}-12 \quad 3.183 \mathrm{E}-13$ 
OVARIES $\quad 1.516 E-15 \quad 4.021 E-13 \quad 6.581 E-13 \quad 2.581 E-17-1.000 E+00 \quad 2.896 E-12 \quad 3.757 E-12$ EDEWBODY 2.569E-15 1.848E-12 2.653E-12 $1.192 \mathrm{E}-16-1.000 \mathrm{E}+00 \quad 1.529 \mathrm{E}-10 \quad 3.750 \mathrm{E}-10$ $\begin{array}{llllllll}\text { THYROIDH } & 2.099 \mathrm{E}-15 & 5.594 \mathrm{E}-13 & 9.643 \mathrm{E}-13 & 3.583 \mathrm{E}-17 & 2.195 \mathrm{E}-12 & 2.447 \mathrm{E}-12 & 5.284 \mathrm{E}-14\end{array}$ LA - 142

STOMACH $1.123 \mathrm{E}-13 \quad 1.396 \mathrm{E}-11 \quad 1.440 \mathrm{E}-11 \quad 1.744 \mathrm{E}-15 \quad 4.911 \mathrm{E}-11 \quad 4.911 \mathrm{E}-11 \quad 8.530 \mathrm{E}-10$ SMALL IN $1.053 \mathrm{E}-13 \quad 1.307 \mathrm{E}-11 \quad 1.349 \mathrm{E}-11 \quad 1.633 \mathrm{E}-15 \quad 4.066 \mathrm{E}-11 \quad 4.066 \mathrm{E}-11 \quad 7.750 \mathrm{E}-10$ LUNGS RED MARR THYROID LOWER LI $\begin{array}{lllllll}1.195 \mathrm{E}-13 & 1.490 \mathrm{E}-11 & 1.537 \mathrm{E}-11 & 1.861 \mathrm{E}-15 & 3.508 \mathrm{E}-10 & 3.508 \mathrm{E}-10 & 8.370 \mathrm{E}-12\end{array}$ $\begin{array}{lllllll}1.221 E-13 & 1.521 E-11 & 1.569 E-11 & 1.899 E-15 & 6.799 E-12 & 6.799 E-12 & 1.930 E-11\end{array}$ $1.113 \mathrm{E}-13 \quad 1.384 \mathrm{E}-11 \quad 1.427 \mathrm{E}-11 \quad 1.728 \mathrm{E}-15 \quad 1.143 \mathrm{E}-11 \quad 1.144 \mathrm{E}-11 \quad 1.901 \mathrm{E}-10$ BONE SUR 1.262E-13 $1.579 \mathrm{E}-11 \quad 1.629 \mathrm{E}-11 \quad 1.972 \mathrm{E}-15-1.000 \mathrm{E}+00 \quad 5.372 \mathrm{E}-12 \quad 7.400 \mathrm{E}-12$ BREAST TESTES $\begin{array}{llllll}1.427 \mathrm{E}-13 & 1.780 \mathrm{E}-11 & 1.836 \mathrm{E}-11 & 2.223 \mathrm{E}-15-1.000 \mathrm{E}+00 & 6.257 \mathrm{E}-12 & 1.540 \mathrm{E}-11\end{array}$ OVARIES EDEWBODY THYROIDH CE-141 STOMACH SMALL IN LUNGS $\begin{array}{llllll}1.560 \mathrm{E}-13 & 1.945 \mathrm{E}-11 & 2.006 \mathrm{E}-11 & 2.429 \mathrm{E}-15-1.000 \mathrm{E}+00 & 2.103 \mathrm{E}-12 & 5.290 \mathrm{E}-12\end{array}$ $1.037 \mathrm{E}-13 \quad 1.297 \mathrm{E}-11 \quad 1.338 \mathrm{E}-11 \quad 1.620 \mathrm{E}-15-1.000 \mathrm{E}+00 \quad 5.887 \mathrm{E}-12 \quad 6.970 \mathrm{E}-11$ $\begin{array}{llllll}1.366 \mathrm{E}-13 & 1.761 \mathrm{E}-11 & 1.816 \mathrm{E}-11 & 2.199 \mathrm{E}-15-1.000 \mathrm{E}+00 & 5.587 \mathrm{E}-11 & 1.790 \mathrm{E}-10\end{array}$

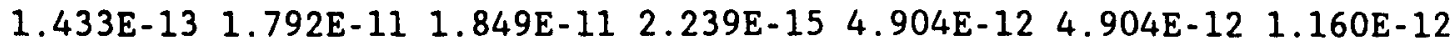

$\begin{array}{lllllll}2.388 E-15 & 1.547 E-12 & 3.029 E-11 & 5.391 E-17 & 9.040 E-11 & 1.603 E-10 & 2.240 E-10\end{array}$

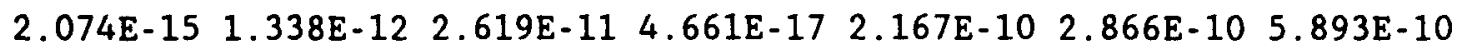
$\begin{array}{lllllll}2.664 \mathrm{E}-15 & 1.729 \mathrm{E}-12 & 3.385 \mathrm{E}-11 & 6.025 \mathrm{E}-17 & 1.192 \mathrm{E}-09 & 1.669 \mathrm{E}-08 & 1.429 \mathrm{E}-12\end{array}$ RED MARR THYROID LOWER LI BONE SUR BREAST TESTES OVARIES EDEWBODY THYROIDH CE - 143 STOMACH SMALL IN LUNGS RED MARR THYROID LOWER LI BONE SUR BREAST TESTES OVARIES EDEWBODY THYROIDH CE-144 STOMACH $2.419 \mathrm{E}-15 \quad 1.556 \mathrm{E}-12 \quad 3.047 \mathrm{E}-11 \quad 5.422 \mathrm{E}-17 \quad 2.434 \mathrm{E}-11 \quad 8.891 \mathrm{E}-11 \quad 3.396 \mathrm{E}-11$ $3.678 \mathrm{E}-15 \quad 2.412 \mathrm{E}-12 \quad 4.721 \mathrm{E}-11 \quad 8.403 \mathrm{E}-17-1.000 \mathrm{E}+00 \quad 2.543 \mathrm{E}-11 \quad 1.800 \mathrm{E}-13$ 2.328E-15 $1.502 E-12 \quad 2.940 E-11 \quad 5.232 E-17 \quad 1.972 E-09 \quad 4.100 E-09 \quad 8.641 E-09$ 4.154E-15 2.703E-12 5.292E-11 9.418E-17-1.000E+00 2.657E-10 2.375E-11 4.249E-15 2.821E-12 5.523E-11 9.830E-17-1.000E+00 4.456E-11 $1.105 \mathrm{E}-11$ 3.773E-15 2.484E-12 4.864E-11 8.657E-17-1.000E+00 5.369E-12 7.580E-12 $2.093 \mathrm{E}-15 \quad 1.356 \mathrm{E}-12 \quad 2.655 \mathrm{E}-11 \quad 4.725 \mathrm{E}-17-1.000 \mathrm{E}+00 \quad 5.544 \mathrm{E}-11 \quad 1.076 \mathrm{E}-10$ $3.426 \mathrm{E}-15$ 2.229E-12 4.364E-11 7.766E-17-1.000E+00 2.436E-09 7.850E-10 $3.678 \mathrm{E}-15 \quad 2.412 \mathrm{E}-12 \quad 4.721 \mathrm{E}-11 \quad 8.403 \mathrm{E}-17 \quad 3.186 \mathrm{E}-12 \quad 2.543 \mathrm{E}-11 \quad 1.800 \mathrm{E}-13$ 8.720E-15 4.926E-12 $3.092 \mathrm{E}-11 \quad 1.858 \mathrm{E}-16 \quad 1.854 \mathrm{E}-10 \quad 2.121 \mathrm{E}-10 \quad 5.608 \mathrm{E}-10$

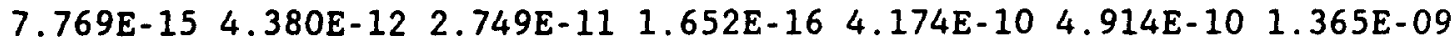
$\begin{array}{lllllll}9.545 E-15 & 5.422 \mathrm{E}-12 & 3.403 \mathrm{E}-11 & 2.045 \mathrm{E}-16 & 1.429 \mathrm{E}-09 & 3.884 \mathrm{E}-09 & 3.829 \mathrm{E}-12\end{array}$ $\begin{array}{lllllll}9.545 E-15 & 5.330 E-12 & 3.345 E-11 & 2.010 E-16 & 2.039 E-11 & 2.953 E-11 & 5.074 E-11\end{array}$ 1.234E-14 7.087E-12 4.448E-11 2.673E-16-1.000E+00 6.239E-12 4.351E-13 8.498E-15 4.792E-12 3.007E-11 $1.807 \mathrm{E}-16 \quad 2.311 \mathrm{E}-09 \quad 4.281 \mathrm{E}-09 \quad 1.167 \mathrm{E}-08$ $1.195 \mathrm{E}-14 \quad 6.893 \mathrm{E}-12 \quad 4.326 \mathrm{E}-11 \quad 2.600 \mathrm{E}-16-1.000 \mathrm{E}+00 \quad 1.647 \mathrm{E}-11 \quad 1.610 \mathrm{E}-11$ 1. 303E-14 7.675E-12 4.817E-11 2.895E-16-1.000E+00 $1.661 \mathrm{E}-112.327 \mathrm{E}-11$ $1.319 \mathrm{E}-14 \quad 7.616 \mathrm{E}-12 \quad 4.780 \mathrm{E}-11 \quad 2.873 \mathrm{E}-16-1.000 \mathrm{E}+00 \quad 5.816 \mathrm{E}-12 \quad 1.531 \mathrm{E}-11$ 8.245E-15 4.657E-12 2.923E-11 $1.757 \mathrm{E}-16-1.000 \mathrm{E}+00 \quad 7.556 \mathrm{E}-112.131 \mathrm{E}-10$

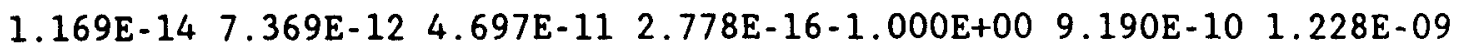

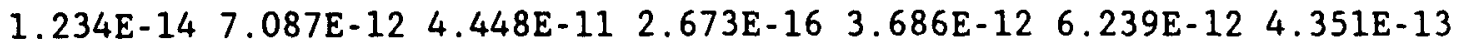

SMALL IN LUNGS RED MARR THYROID LOWER LI BONE SUR BREAST TESTES

$\begin{array}{lllllll}1.793 E-15 & 9.314 \mathrm{E}-13 & 2.002 \mathrm{E}-11 & 3.343 \mathrm{E}-17 & 6.003 \mathrm{E}-10 & 1.185 \mathrm{E}-09 & 1.110 \mathrm{E}-09\end{array}$ $\begin{array}{lllllll}1.636 \mathrm{E}-15 & 8.416 \mathrm{E}-13 & 1.811 \mathrm{E}-11 & 3.024 \mathrm{E}-17 & 1.461 \mathrm{E}-09 & 2.166 \mathrm{E}-09 & 3.711 \mathrm{E}-09\end{array}$ $\begin{array}{lllllll}1.960 \mathrm{E}-15 & 1.025 \mathrm{E}-12 & 2.201 \mathrm{E}-11 & 3.675 \mathrm{E}-17 & 1.811 \mathrm{E}-08 & 7.896 \mathrm{E}-07 & 6.514 \mathrm{E}-12\end{array}$ $\begin{array}{lllllll}1.882 E-15 & 9.613 E-13 & 2.070 E-11 & 3.457 E-17 & 4.025 E-11 & 2.786 E-09 & 8.660 E-11\end{array}$ $2.505 \mathrm{E}-15 \quad 1.344 \mathrm{E}-12 \quad 2.880 \mathrm{E}-11 \quad 4.810 \mathrm{E}-17-1.000 \mathrm{E}+00 \quad 2.908 \mathrm{E}-10 \quad 5.137 \mathrm{E}-12$ $\begin{array}{lllllll}1.761 E-15 & 9.113 \mathrm{E}-13 & 1.960 \mathrm{E}-11 & 3.272 \mathrm{E}-17 & 1.526 \mathrm{E}-08 & 3.410 \mathrm{E}-08 & 6.638 \mathrm{E}-08\end{array}$ $2.426 \mathrm{E}-15 \quad 1.328 \mathrm{E}-12 \quad 2.838 \mathrm{E}-11 \quad 4.738 \mathrm{E}-17-1.000 \mathrm{E}+00 \quad 4.819 \mathrm{E}-09 \quad 1.304 \mathrm{E}-10$ $2.649 \mathrm{E}-15 \quad 1.467 \mathrm{E}-12 \quad 3.136 \mathrm{E}-11 \quad 5.235 \mathrm{E}-17-1.000 \mathrm{E}+00 \quad 3.478 \mathrm{E}-10 \quad 1.215 \mathrm{E}-11$ $2.660 \mathrm{E}-15 \quad 1.426 \mathrm{E}-12 \quad 3.057 \mathrm{E}-11 \quad 5.105 \mathrm{E}-17-1.000 \mathrm{E}+00 \quad 1.922 \mathrm{E}-10 \quad 1.013 \mathrm{E}-11$ 
OVARIES

EDEWBODY

THYROIDH

PR - 143

STOMACH

SMALL IN

LUNGS

RED MARR

THYROID

LOWER LI

BONE SUR

BREAST

TESTES

OVARIES

EDEWBODY

THYROIDH

ND - 147

STOMACH

SMALL IN

LUNGS

RED MARR

THYROID

LOWER LI

BONE SUR

BREAST

TESTES

OVARIES

EDEWBODY

THYROIDH

NP-239

STOMACH

SMALL IN

LUNGS

RED MARR

THYROID

LOWER LI

BONE SUR

BREAST

TESTES

OVARIES

EDEWBODY

THYROIDH

PU- 238

STOMACH

SMALL IN

LUNGS

RED MARR

THYROID

LOWER LI

BONE SUR

BREAST

TESTES $\begin{array}{llllll}1.651 \mathrm{E}-15 & 8.577 \mathrm{E}-13 & 1.845 \mathrm{E}-11 & 3.081 \mathrm{E}-17-1.000 \mathrm{E}+00 & 2.383 \mathrm{E}-10 & 6.961 \mathrm{E}-11\end{array}$ 8.079E-16 5.398E-13 $1.124 \mathrm{E}-11 \quad 1.875 \mathrm{E}-17-1.000 \mathrm{E}+00 \quad 1.012 \mathrm{E}-07 \quad 5.710 \mathrm{E}-09$ $2.505 \mathrm{E}-15$ 1.344E-12 2.880E-11 4.810E-17 $3.907 \mathrm{E}-12 \quad 2.908 \mathrm{E}-10 \quad 5.137 \mathrm{E}-12$

$\begin{array}{lllllll}3.234 E-22 & 1.802 E-19 & 3.209 E-18 & 6.310 E-24 & 1.398 E-10 & 1.661 E-10 & 3.620 E-10\end{array}$ $\begin{array}{lllllll}2.917 \mathrm{E}-22 & 1.630 \mathrm{E}-19 & 2.902 \mathrm{E}-18 & 5.708 \mathrm{E}-24 & 3.371 \mathrm{E}-10 & 4.126 \mathrm{E}-10 & 8.939 \mathrm{E}-10\end{array}$ $3.488 \mathrm{E}-22 \quad 1.947 \mathrm{E}-19 \quad 3.467 \mathrm{E}-18 \quad 6.818 \mathrm{E}-24 \quad 1.631 \mathrm{E}-09 \quad 1.332 \mathrm{E}-08 \quad 6.518 \mathrm{E}-15$ $3.552 \mathrm{E}-22 \quad 1.983 \mathrm{E}-19 \quad 3.531 \mathrm{E}-18 \quad 6.944 \mathrm{E}-24 \quad 4.864 \mathrm{E}-12 \quad 1.497 \mathrm{E}-11 \quad 1.039 \mathrm{E}-12$ $4.376 \mathrm{E}-22 \quad 2.454 \mathrm{E}-19 \quad 4.370 \mathrm{E}-18 \quad 8.593 \mathrm{E}-24-1.000 \mathrm{E}+00 \quad 9.391 \mathrm{E}-14 \quad 6.518 \mathrm{E}-15$ $\begin{array}{llllllll}3.158 E-22 & 1.766 E-19 & 3.144 E-18 & 6.183 E-24 & 3.304 E-09 & 6.763 E-09 & 1.466 E-08\end{array}$ $3.900 \mathrm{E}-22 \quad 2.173 \mathrm{E}-19 \quad 3.870 \mathrm{E}-18$ 7.610E-24-1.000E $+00 \quad 1.495 \mathrm{E}-11 \quad 1.037 \mathrm{E}-12$ $4.186 \mathrm{E}-22 \quad 2.345 \mathrm{E}-19 \quad 4.176 \mathrm{E}-18$ 8.213E-24-1.000E $+00 \quad 9.391 \mathrm{E}-14 \quad 6.519 \mathrm{E}-15$ 4.630E-22 2.590E-19 4.611E-18 9.069E-24-1.000E+00 9.390E-14 6.519E-15 $3.168 \mathrm{E}-22 \quad 1.775 \mathrm{E}-19 \quad 3.160 \mathrm{E}-18 \quad 6.215 \mathrm{E}-24-1.000 \mathrm{E}+00 \quad 9.390 \mathrm{E}-14 \quad 6.523 \mathrm{E}-15$ $\begin{array}{lllllll}1.771 E-16 & 5.553 \mathrm{E}-13 & 9.887 \mathrm{E}-12 & 1.944 \mathrm{E}-17-1.000 \mathrm{E}+00 & 2.190 \mathrm{E}-09 & 1.264 \mathrm{E}-09\end{array}$ $4.376 E-22 \quad 2.454 E-19 \quad 4.370 E-18$ 8.593E-24 8.229E-14 9.391E-14 6.518E-15 $\begin{array}{lllllll}4.313 E-15 & 2.675 E-12 & 4.589 E-11 & 9.386 E-17 & 1.419 E-10 & 1.977 E-10 & 3.571 E-10\end{array}$ $3.805 \mathrm{E}-15 \quad 2.359 \mathrm{E}-12 \quad 4.046 \mathrm{E}-11 \quad 8.276 \mathrm{E}-17 \quad 3.388 \mathrm{E}-10 \quad 4.346 \mathrm{E}-10 \quad 9.403 \mathrm{E}-10$

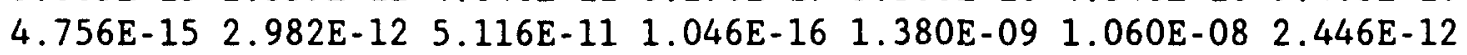
$\begin{array}{llllllll}4.471 E-15 & 2.729 \mathrm{E}-12 & 4.682 \mathrm{E}-11 & 9.576 \mathrm{E}-17 & 3.426 \mathrm{E}-11 & 9.219 \mathrm{E}-11 & 5.042 \mathrm{E}-11\end{array}$ $6.279 \mathrm{E}-15 \quad 4.003 \mathrm{E}-12 \quad 6.868 \mathrm{E}-11 \quad 1.405 \mathrm{E}-16-1.000 \mathrm{E}+00 \quad 1.825 \mathrm{E}-112.699 \mathrm{E}-13$ $\begin{array}{lllllll}4.154 \mathrm{E}-15 & 2.567 \mathrm{E}-12 & 4.403 \mathrm{E}-11 & 9.006 \mathrm{E}-17 & 2.868 \mathrm{E}-09 & 5.843 \mathrm{E}-09 & 1.279 \mathrm{E}-08\end{array}$ $6.247 \mathrm{E}-15 \quad 4.003 \mathrm{E}-12 \quad 6.868 \mathrm{E}-11 \quad 1.405 \mathrm{E}-16-1.000 \mathrm{E}+00 \quad 3.258 \mathrm{E}-10 \quad 2.219 \mathrm{E}-11$ $6.818 \mathrm{E}-15 \quad 4.473 \mathrm{E}-12 \quad 7.674 \mathrm{E}-11 \quad 1.570 \mathrm{E}-16-1.000 \mathrm{E}+00 \quad 3.465 \mathrm{E}-11 \quad 1.872 \mathrm{E}-11$ $6.723 E-15 \quad 4.302 E-12 \quad 7.379 E-11 \quad 1.509 E-16-1.000 E+00 \quad 6.749 E-12 \quad 1.364 E-11$ 4.091E-15 2.539E-12 4.356E-11 8.911E-17-1.000E+00 8.412E-11 1.790E-10 $5.891 \mathrm{E}-15 \quad 3.991 \mathrm{E}-12 \quad 6.847 \mathrm{E}-11 \quad 1.400 \mathrm{E}-16-1.000 \mathrm{E}+00 \quad 1.849 \mathrm{E}-09 \quad 1.184 \mathrm{E}-09$ $6.279 \mathrm{E}-15$ 4.003E-12 6.868E-11 $1.405 \mathrm{E}-16 \quad 4.598 \mathrm{E}-12 \quad 1.825 \mathrm{E}-11 \quad 2.699 \mathrm{E}-13$

$\begin{array}{lllllll}5.296 \mathrm{E}-15 & 3.236 \mathrm{E}-12 & 3.022 \mathrm{E}-11 & 1.180 \mathrm{E}-16 & 1.059 \mathrm{E}-10 & 1.291 \mathrm{E}-10 & 3.457 \mathrm{E}-10\end{array}$ $\begin{array}{lllllll}4.630 \mathrm{E}-15 & 2.818 \mathrm{E}-12 & 2.632 \mathrm{E}-11 & 1.027 \mathrm{E}-16 & 2.413 \mathrm{E}-10 & 2.988 \mathrm{E}-10 & 8.735 \mathrm{E}-10\end{array}$ $\begin{array}{lllllll}5.8 .98 E-15 & 3.610 \mathrm{E}-12 & 3.371 \mathrm{E}-11 & 1.316 \mathrm{E}-16 & 9.014 \mathrm{E}-10 & 2.348 \mathrm{E}-09 & 2.402 \mathrm{E}-12\end{array}$ $5.454 \mathrm{E}-15$ 3.314E-12 3.095E-11 $1.208 \mathrm{E}-16 \quad 7.943 \mathrm{E}-11 \quad 2.075 \mathrm{E}-10 \quad 4.660 \mathrm{E}-11$ $7.991 \mathrm{E}-15 \quad 4.915 \mathrm{E}-12 \quad 4.590 \mathrm{E}-11 \quad 1.792 \mathrm{E}-16-1.000 \mathrm{E}+00 \quad 7.599 \mathrm{E}-12 \quad 2.063 \mathrm{E}-13$ $5.137 \mathrm{E}-15 \quad 3.149 \mathrm{E}-12 \quad 2.941 \mathrm{E}-11 \quad 1.148 \mathrm{E}-16 \quad 1.509 \mathrm{E}-09 \quad 2.916 \mathrm{E}-09 \quad 8.684 \mathrm{E}-09$ 8.815E-15 5.437E-12 5.077E-11 $1.982 \mathrm{E}-16-1.000 \mathrm{E}+00 \quad 2.027 \mathrm{E}-09 \quad 3.609 \mathrm{E}-11$ $9.037 \mathrm{E}-15 \quad 6.028 \mathrm{E}-12 \quad 5.629 \mathrm{E}-11 \quad 2.198 \mathrm{E}-16-1.000 \mathrm{E}+00 \quad 1.623 \mathrm{E}-11 \quad 1.714 \mathrm{E}-11$ 8.245E-15 5.141E-12 4.801E-11 $1.874 \mathrm{E}-16-1.000 \mathrm{E}+00 \quad 2.296 \mathrm{E}-11 \quad 1.144 \mathrm{E}-11$ $\begin{array}{llllll}4.725 \mathrm{E}-15 & 2.897 \mathrm{E}-12 & 2.705 \mathrm{E}-11 & 1.056 \mathrm{E}-16-1.000 \mathrm{E}+00 & 7.449 \mathrm{E}-11 & 1.621 \mathrm{E}-10\end{array}$

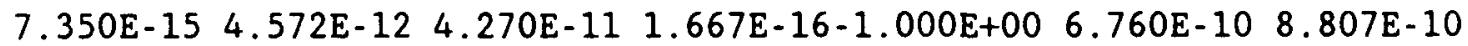
$7.991 \mathrm{E}-15$ 4.915E-12 4.590E-11 1.792E-16 4.130E-12 7.599E-12 2.063E-13

$\begin{array}{llllllll}6.723 E-19 & 1.396 E-15 & 2.935 E-14 & 4.852 E-20 & 2.725 E-10 & 1.134 E-09 & 1.277 E-09\end{array}$ $4.566 \mathrm{E}-19 \quad 4.249 \mathrm{E}-16 \quad 8.939 \mathrm{E}-15 \quad 1.478 \mathrm{E}-20 \quad 6.381 \mathrm{E}-10 \quad 2.222 \mathrm{E}-09 \quad 3.186 \mathrm{E}-09$ $1.008 \mathrm{E}-18 \quad 3.021 \mathrm{E}-15 \quad 6.348 \mathrm{E}-14 \quad 1.050 \mathrm{E}-19 \quad 9.682 \mathrm{E}-07 \quad 3.186 \mathrm{E}-04 \quad 8.643 \mathrm{E}-14$ $4.535 \mathrm{E}-19 \quad 1.113 \mathrm{E}-15 \quad 2.340 \mathrm{E}-14 \quad 3.869 \mathrm{E}-20 \quad 2.552 \mathrm{E}-09 \quad 5.785 \mathrm{E}-05 \quad 1.266 \mathrm{E}-08$ $1.465 \mathrm{E}-18 \quad 3.724 \mathrm{E}-15 \quad 7.825 \mathrm{E}-14 \quad 1.294 \mathrm{E}-19-1.000 \mathrm{E}+00 \quad 3.852 \mathrm{E}-10 \quad 7.993 \mathrm{E}-14$ $6.659 \mathrm{E}-19 \quad 1.780 \mathrm{E}-15 \quad 3.740 \mathrm{E}-14 \quad 6.183 \mathrm{E}-20 \quad 6.605 \mathrm{E}-09 \quad 3.294 \mathrm{E}-08 \quad 5.656 \mathrm{E}-08$ $1.792 \mathrm{E}-18 \quad 5.057 \mathrm{E}-15 \quad 1.063 \mathrm{E}-13 \quad 1.757 \mathrm{E}-19-1.000 \mathrm{E}+00 \quad 7.211 \mathrm{E}-04 \quad 1.576 \mathrm{E}-07$ $\begin{array}{llllll}1.547 \mathrm{E}-17 & 1.004 \mathrm{E}-13 & 2.110 \mathrm{E}-12 & 3.488 \mathrm{E}-18-1.000 \mathrm{E}+00 & 4.394 \mathrm{E}-10 & 1.804 \mathrm{E}-13\end{array}$

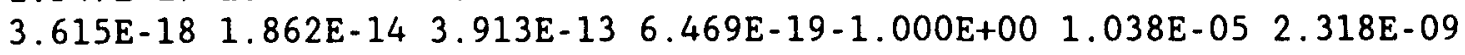


OVARIES $\quad 6.659 \mathrm{E}-19 \quad 1.716 \mathrm{E}-15 \quad 3.606 \mathrm{E}-14 \quad 5.961 \mathrm{E}-20-1.000 \mathrm{E}+00 \quad 1.041 \mathrm{E}-05 \quad 2.325 \mathrm{E}-09$ EDEWBODY 4.028E-18 2.290E-14 4.809E-13 7.951E-19-1.000E+00 7.759E-05 1.337E-08 THYROIDH PU -239

STOMACH

SMALL IN LUNGS

RED MARR THYROID

LOWER LI

BONE SUR

BREAST

TESTES

OVARIES

EDEWBODY

THYROIDH

PU -240

STOMACH

SMALL IN

LUNGS

RED MARR

THYROID

LOWER LI

BONE SUR

BREAST

TESTES

OVARIES

EDEWBODY

THYROIDH

PU - 241

STOMACH

SMALL IN

LUNGS

RED MARR

THYROID

LOWER LI

BONE SUR

BREAST

TESTES

OVARIES

EDEWBODY

THYROIDH

AM- 241

STOMACH

SMALL IN

LUNGS

RED MARR

THYROID

LOWER LI

BONE SUR

BREAST

TESTES
$1.465 \mathrm{E}-18 \quad 3.724 \mathrm{E}-15 \quad 7.825 \mathrm{E}-14 \quad 1.294 \mathrm{E}-19 \quad 1.480 \mathrm{E}-11 \quad 3.852 \mathrm{E}-10 \quad 7.993 \mathrm{E}-14$

$\begin{array}{lllllll}1.776 \mathrm{E}-18 & 1.489 \mathrm{E}-15 & 2.909 \mathrm{E}-14 & 5.169 \mathrm{E}-20 & 2.533 \mathrm{E}-10 & 1.069 \mathrm{E}-09 & 1.196 \mathrm{E}-09\end{array}$ $\begin{array}{lllllll}1.481 E-18 & 9.863 E-16 & 1.881 E-14 & 3.425 E-20 & 5.952 E-10 & 2.097 E-09 & 2.991 E-09\end{array}$ $2.074 \mathrm{E}-18 \quad 2.21 .0 \mathrm{E}-15 \quad 4.401 \mathrm{E}-14 \quad 7.674 \mathrm{E}-20 \quad 9.116 \mathrm{E}-07 \quad 3.213 \mathrm{E}-04 \quad 7.881 \mathrm{E}-14$ $\begin{array}{llllllll}1.671 E-18 & 1.379 E-15 & 2.665 E-14 & 4.788 E-20 & 2.400 E-09 & 6.568 E-05 & 1.405 E-08\end{array}$ $2.901 E-18 \quad 2.877 E-15 \quad 5.717 E-14 \quad 9.989 E-20-1.000 E+00 \quad 3.757 E-10 \quad 7.495 E-14$ $\begin{array}{lllllll}1.735 \mathrm{E}-18 & 1.616 \mathrm{E}-15 & 3.182 \mathrm{E}-14 & 5.613 \mathrm{E}-20 & 6.175 \mathrm{E}-09 & 3.099 \mathrm{E}-08 & 5.316 \mathrm{E}-08\end{array}$ $3.425 \mathrm{E}-18 \quad 3.635 \mathrm{E}-15 \quad 7.276 \mathrm{E}-14 \quad 1.262 \mathrm{E}-19-1.000 \mathrm{E}+00 \quad 8.167 \mathrm{E}-04 \quad 1.748 \mathrm{E}-07$ 8.562E-18 3.991E-14 8.344E-13 $1.386 \mathrm{E}-18-1.000 \mathrm{E}+00 \quad 3.990 \mathrm{E}-10 \quad 1.207 \mathrm{E}-13$ $\begin{array}{llllll}3.710 \mathrm{E}-18 & 8.548 \mathrm{E}-15 & 1.762 \mathrm{E}-13 & 2.968 \mathrm{E}-19-1.000 \mathrm{E}+00 & 1.197 \mathrm{E}-05 & 2.631 \mathrm{E}-09\end{array}$ $\begin{array}{llllll}1.573 E-18 & 1.479 \mathrm{E}-15 & 2.915 \mathrm{E}-14 & 5.137 \mathrm{E}-20-1.000 \mathrm{E}+00 & 1.194 \mathrm{E}-05 & 2.624 \mathrm{E}-09\end{array}$

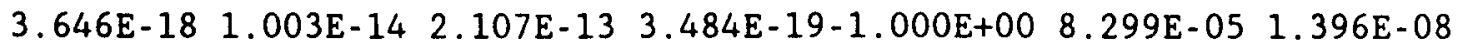
$2.901 \mathrm{E}-18 \quad 2.877 \mathrm{E}-15 \quad 5.717 \mathrm{E}-14 \quad 9.989 \mathrm{E}-20 \quad 1.381 \mathrm{E}-11 \quad 3.757 \mathrm{E}-10 \quad 7.495 \mathrm{E}-14$

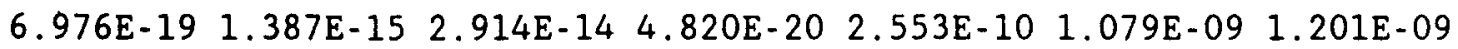
$\begin{array}{llllllll}4.820 \mathrm{E}-19 & 4.516 \mathrm{E}-16 & 9.487 \mathrm{E}-15 & 1.570 \mathrm{E}-20 & 6.007 \mathrm{E}-10 & 2.108 \mathrm{E}-09 & 3.005 \mathrm{E}-09\end{array}$ $\begin{array}{llllllll}1.034 \mathrm{E}-18 & 2.949 \mathrm{E}-15 & 6.193 \mathrm{E}-14 & 1.024 \mathrm{E}-19 & 9.117 \mathrm{E}-07 & 3.213 \mathrm{E}-04 & 8.206 \mathrm{E}-14\end{array}$ $\begin{array}{lllllll}4.661 E-19 & 1.095 E-15 & 2.301 E-14 & 3.805 E-20 & 2.400 E-09 & 6.562 E-05 & 1.405 E-08\end{array}$

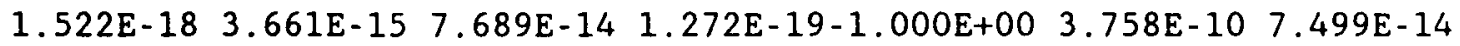
6.849E-19 $1.744 \mathrm{E}-15 \quad 3.662 \mathrm{E}-14 \quad 6.057 \mathrm{E}-20 \quad 6.224 \mathrm{E}-09 \quad 3.112 \mathrm{E}-08 \quad 5.337 \mathrm{E}-08$ $\begin{array}{lllllll}1.842 \mathrm{E}-18 & 4.948 \mathrm{E}-15 & 1.039 \mathrm{E}-13 & 1.719 \mathrm{E}-19-1.000 \mathrm{E}+00 & 8.145 \mathrm{E}-04 & 1.746 \mathrm{E}-07\end{array}$

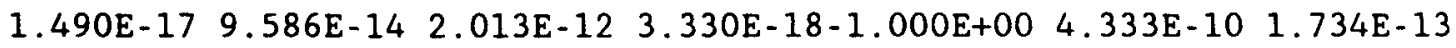
$3.583 E-18 \quad 1.789 E-14 \quad 3.758 E-13 \quad 6.215 E-19-1.000 E+00 \quad 1.197 E-05 \quad 2.631 E-09$ 6.881E-19 1.689E-15 3.547E-14 5.866E-20-1.000E+00 1.197E-05 2.632E-09 $3.958 \mathrm{E}-18 \quad 2.190 \mathrm{E}-14 \quad 4.599 \mathrm{E}-13 \quad 7.604 \mathrm{E}-19-1.000 \mathrm{E}+00 \quad 8.291 \mathrm{E}-05 \quad 1.397 \mathrm{E}-08$ $1.522 E-18 \quad 3.661 E-15 \quad 7.689 E-14 \quad 1.272 E-19 \quad 1.381 E-11 \quad 3.758 E-10 \quad 7.499 E-14$

$\begin{array}{lllllll}0.000 E+00 & 8.927 E-19 & 3.289 E-16 & 0.000 E+00 & 2.415 E-12 & 2.455 E-11 & 6.080 E-12\end{array}$ $\begin{array}{llllllll}0.000 E+00 & 7.733 E-19 & 2.849 E-16 & 0.000 E+00 & 5.838 E-12 & 2.436 E-11 & 1.513 E-11\end{array}$ $0.000 \mathrm{E}+00 \quad 1.015 \mathrm{E}-18 \quad 3.739 \mathrm{E}-16 \quad 0.000 \mathrm{E}+00 \quad 5.369 \mathrm{E}-10 \quad 3.172 \mathrm{E}-06 \quad 4.468 \mathrm{E}-15$ $\begin{array}{lllllll}0.000 E+00 & 8.539 E-19 & 3.146 E-16 & 0.000 E+00 & 4.411 E-13 & 1.426 E-06 & 2.780 E-10\end{array}$ $\begin{array}{lllllll}0.000 \mathrm{E}+00 & 1.430 \mathrm{E}-18 & 5.268 \mathrm{E}-16 & 0.000 \mathrm{E}+00-1.000 \mathrm{E}+00 & 9.130 \mathrm{E}-12 & 1.011 \mathrm{E}-15\end{array}$ $\begin{array}{lllllll}0.000 E+00 & 8.569 E-19 & 3.157 E-16 & 0.000 E+00 & 6.199 E-11 & 1.805 E-10 & 2.694 E-10\end{array}$ $0.000 \mathrm{E}+00 \quad 1.615 \mathrm{E}-18 \quad 5.950 \mathrm{E}-16 \quad 0.000 \mathrm{E}+00-1.000 \mathrm{E}+00 \quad 1.776 \mathrm{E}-05 \quad 3.467 \mathrm{E}-09$ $0.000 \mathrm{E}+00 \quad 1.860 \mathrm{E}-18 \quad 6.852 \mathrm{E}-16 \quad 0.000 \mathrm{E}+00-1.000 \mathrm{E}+00 \quad 2.141 \mathrm{E}-11 \quad 2.780 \mathrm{E}-15$ $0.000 \mathrm{E}+00 \quad 1.496 \mathrm{E}-18 \quad 5.510 \mathrm{E}-16 \quad 0.000 \mathrm{E}+00-1.000 \mathrm{E}+00 \quad 2.753 \mathrm{E}-07 \quad 5.636 \mathrm{E}-11$ $0.000 \mathrm{E}+00 \quad 7.912 \mathrm{E}-192.915 \mathrm{E}-16 \quad 0.000 \mathrm{E}+00-1.000 \mathrm{E}+00 \quad 2.755 \mathrm{E}-07 \quad 5.650 \mathrm{E}-11$ $\begin{array}{lllllll}7.153 \mathrm{E}-20 & 6.100 \mathrm{E}-17 & 1.281 \mathrm{E}-15 & 2.118 \mathrm{E}-21-1.000 \mathrm{E}+00 & 1.334 \mathrm{E}-06 & 2.069 \mathrm{E}-10\end{array}$ $0.000 \mathrm{E}+00 \quad 1.430 \mathrm{E}-18 \quad 5.268 \mathrm{E}-16 \quad 0.000 \mathrm{E}+00 \quad 1.872 \mathrm{E}-15 \quad 9.130 \mathrm{E}-12 \quad 1.011 \mathrm{E}-15$ $\begin{array}{lllllll}4.883 E-16 & 4.036 \mathrm{E}-13 & 8.475 \mathrm{E}-12 & 1.402 \mathrm{E}-17 & 5.091 \mathrm{E}-10 & 3.251 \mathrm{E}-09 & 1.345 \mathrm{E}-09\end{array}$ $\begin{array}{llllllll}4.122 E-16 & 3.378 E-13 & 7.095 E-12 & 1.173 E-17 & 8.464 E-10 & 4.191 E-09 & 3.330 E-09\end{array}$ $\begin{array}{llllllll}5.930 \mathrm{E}-16 & 4.958 \mathrm{E}-13 & 1.041 \mathrm{E}-11 & 1.722 \mathrm{E}-17 & 4.703 \mathrm{E}-07 & 1.837 \mathrm{E}-05 & 3.364 \mathrm{E}-11\end{array}$ $\begin{array}{lllllll}3.203 E-16 & 2.657 \mathrm{E}-13 & 5.580 \mathrm{E}-12 & 9.228 \mathrm{E}-18 & 4.847 \mathrm{E}-08 & 1.738 \mathrm{E}-04 & 1.448 \mathrm{E}-06\end{array}$ $9.418 \mathrm{E}-16 \quad 7.834 \mathrm{E}-13 \quad 1.645 \mathrm{E}-11 \quad 2.721 \mathrm{E}-17-1.000 \mathrm{E}+00 \quad 1.596 \mathrm{E}-09 \quad 1.321 \mathrm{E}-11$ 4.313E-16 3.588E-13 $7.536 \mathrm{E}-12 \quad 1.246 \mathrm{E}-17 \quad 6.275 \mathrm{E}-09 \quad 3.161 \mathrm{E}-08 \quad 5.810 \mathrm{E}-08$ $\begin{array}{lllllll}1.091 E-15 & 9.112 \mathrm{E}-13 & 1.914 \mathrm{E}-11 & 3.165 \mathrm{E}-17-1.000 \mathrm{E}+00 & 2.166 \mathrm{E}-03 & 1.802 \mathrm{E}-05\end{array}$ $\begin{array}{llllll}1.259 \mathrm{E}-15 & 1.370 \mathrm{E}-12 & 2.876 \mathrm{E}-11 & 4.756 \mathrm{E}-17-1.000 \mathrm{E}+00 & 2.666 \mathrm{E}-09 & 2.625 \mathrm{E}-11\end{array}$ $9.862 \mathrm{E}-16 \quad 8.738 \mathrm{E}-13 \quad 1.835 \mathrm{E}-11 \quad 3.035 \mathrm{E}-17-1.000 \mathrm{E}+00 \quad 3.243 \mathrm{E}-05 \quad 2.693 \mathrm{E}-07$ 
OVARIES $\quad 4.344 \mathrm{E}-16 \quad 3.606 \mathrm{E}-13 \quad 7.574 \mathrm{E}-12 \quad 1.253 \mathrm{E}-17-1.000 \mathrm{E}+00 \quad 3.251 \mathrm{E}-05 \quad 2.706 \mathrm{E}-07$ EDEWBODY 8.264E-16 7.533E-13 1.582E-11 2.616E-17-1.000E+00 1.197E-04 9.819E-07 $\begin{array}{llllllll}\text { THYROIDH } \quad 9.418 \mathrm{E}-16 & 7.834 \mathrm{E}-13 & 1.645 \mathrm{E}-11 & 2.721 \mathrm{E}-17 & 2.761 \mathrm{E}-10 & 1.596 \mathrm{E}-09 & 1.321 \mathrm{E}-11\end{array}$ CM- 242

STOMACH $\quad 7.008 \mathrm{E}-19 \quad 1.652 \mathrm{E}-15 \quad 3.421 \mathrm{E}-14 \quad 5.740 \mathrm{E}-20 \quad 5.453 \mathrm{E}-10 \quad 1.627 \mathrm{E}-09 \quad 1.423 \mathrm{E}-09$ SMALL IN $4.439 \mathrm{E}-19 \quad 4.317 \mathrm{E}-16 \quad 8.939 \mathrm{E}-15 \quad 1.500 \mathrm{E}-20 \quad 8.934 \mathrm{E}-10 \quad 2.656 \mathrm{E}-09 \quad 3.545 \mathrm{E}-09$ LUNGS

RED MARR THYROID $1.081 \mathrm{E}-18 \quad 3.541 \mathrm{E}-15 \quad 7.333 \mathrm{E}-14 \quad 1.230 \mathrm{E}-19 \quad 4.630 \mathrm{E}-07 \quad 1.546 \mathrm{E}-05 \quad 8.833 \mathrm{E}-12$ 4.915E-19 1.296E-15 2.684E-14 4.503E-20 5.125E-08 3.908E-06 3.581E-08 LOWER LI BONE SUR BREAST TESTES OVARIES EDEWBODY THYROIDH CM- 244 STOMACH $1.560 \mathrm{E}-18 \quad 4.581 \mathrm{E}-15 \quad 9.487 \mathrm{E}-14 \quad 1.592 \mathrm{E}-19-1.000 \mathrm{E}+00 \quad 9.384 \mathrm{E}-10 \quad 8.805 \mathrm{E}-12$ $\begin{array}{lllllll}7.135 \mathrm{E}-19 & 2.090 \mathrm{E}-15 & 4.328 \mathrm{E}-14 & 7.262 \mathrm{E}-20 & 6.546 \mathrm{E}-09 & 3.118 \mathrm{E}-08 & 6.234 \mathrm{E}-08\end{array}$ $1.899 \mathrm{E}-18 \quad 5.886 \mathrm{E}-15 \quad 1.219 \mathrm{E}-13 \quad 2.045 \mathrm{E}-19-1.000 \mathrm{E}+00 \quad 4.862 \mathrm{E}-05 \quad 4.463 \mathrm{E}-07$

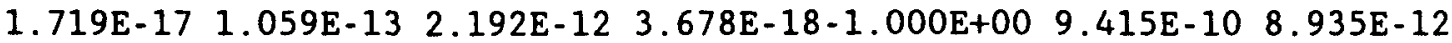
4.186E-18 2.172E-14 4.498E-13 7.547E-19-1.000E+00 5.695E-07 5.190E-09 7.040E-19 2.017E-15 4.177E-14 7.008E-20-1.000E+00 5.699E-07 5.198E-09

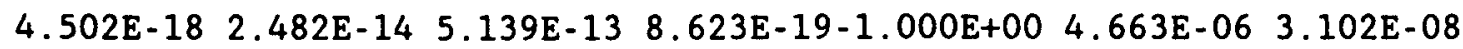

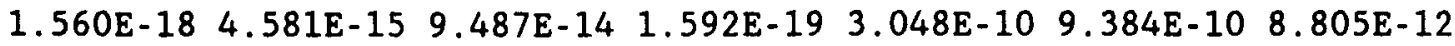

SMALL IN LUNGS

RED MARR THYROID LOWER II BONE SUR BREAST TESTES $\begin{array}{lllllll}5.105 E-19 & 1.389 \mathrm{E}-15 & 2.914 \mathrm{E}-14 & 4.820 \mathrm{E}-20 & 5.191 \mathrm{E}-10 & 1.712 \mathrm{E}-09 & 1.356 \mathrm{E}-09\end{array}$ $\begin{array}{lllllll}3.000 \mathrm{E}-19 & 3.090 \mathrm{E}-16 & 6.480 \mathrm{E}-15 & 1.072 \mathrm{E}-20 & 8.501 \mathrm{E}-10 & 2.737 \mathrm{E}-09 & 3.362 \mathrm{E}-09\end{array}$ 8.308E-19 3.052E-15 $6.404 \mathrm{E}-14$ 1.059E-19 $4.950 \mathrm{E}-07 \quad 1.925 \mathrm{E}-05 \quad 8.846 \mathrm{E}-12$ $\begin{array}{lllllll}3.583 E-19 & 1.097 E-15 & 2.301 E-14 & 3.805 E-20 & 5.102 E-08 & 9.330 E-05 & 7.766 E-07\end{array}$

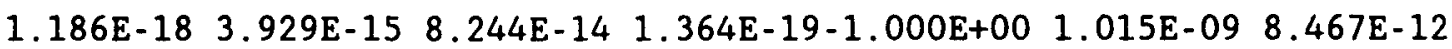
$\begin{array}{lllllll}5.359 E-19 & 1.791 E-15 & 3.758 E-14 & 6.215 E-20 & 6.289 E-09 & 3.147 E-08 & 5.967 E-08\end{array}$ $\begin{array}{llllll}1.446 \mathrm{E}-18 & 5.072 \mathrm{E}-15 & 1.064 \mathrm{E}-13 & 1.760 \mathrm{E}-19-1.000 \mathrm{E}+00 & 1.171 \mathrm{E}-03 & 9.748 \mathrm{E}-06\end{array}$ $1.509 \mathrm{E}-17$ 9.504E-14 1.994E-12 3.298E-18-1.000E+00 1.052E-09 8.920E-12 OVARIES $\quad 5.296 \mathrm{E}-19 \quad 1.727 \mathrm{E}-15 \quad 3.624 \mathrm{E}-14 \quad 5.993 \mathrm{E}-20-1.000 \mathrm{E}+00 \quad 1.587 \mathrm{E}-05 \quad 1.324 \mathrm{E}-07$ EDEWBODY $3.831 \mathrm{E}-18 \quad 2.208 \mathrm{E}-14 \quad 4.633 \mathrm{E}-13 \quad 7.662 \mathrm{E}-19-1.000 \mathrm{E}+00 \quad 6.687 \mathrm{E}-05 \quad 5.429 \mathrm{E}-07$ THYROIDH $1.186 \mathrm{E}-18 \quad 3.929 \mathrm{E}-15 \quad 8.244 \mathrm{E}-14 \quad 1.364 \mathrm{E}-19 \quad 2.909 \mathrm{E}-10 \quad 1.015 \mathrm{E}-09 \quad 8.467 \mathrm{E}-12$ 


\section{D.2 Site Data File For the Sample Problems - SURSIT}

MACCS SITE DATA FILE FOR SURRY (JLS, 11/10/88)

SECPOP POP DISTRIBUTION FROM 1980 CENSUS DATA ALTERED USING $0-10$ MI NRC DATA

26 SPATIAL INTERVALS

16 WIND DIRECTIONS

7 CROP CATEGORIES

4 WATER PATHWAY ISOTOPES

2 WATERSHEDS

59 ECONOMIC REGIONS

SPATIAL DISTANCES

$\begin{array}{rrrrrrrr}0.16 & 0.52 & 1.21 & 1.61 & 2.13 & 3.22 & 4.02 & 4.83 \\ 5.63 & 8.05 & 11.27 & 16.09 & 20.92 & 25.75 & 32.19 & 40.23 \\ 48.28 & 64.37 & 80.47 & 112.65 & 160.93 & 241.14 & 321.87 & 563.27 \\ 804.67 & 1609.34 & & & & & & \end{array}$

POPULATION

$\begin{array}{rrrrrrrr}0 . & 0 . & 0 . & 0 . & 0 . & 0 . & 4 . & 5 .\end{array}$

6. 25. 3341. 7107. 2173. 0.474.

2252. 2945. 5403. 20169. 112004. 3431358. 1355700. 2742710.

2487346. 104331 .

$\begin{array}{rrrrrrrr}0 . & 0 . & 0 . & 0 . & 1 . & 2 . & 9 . & 13 .\end{array}$

15. 63. 1667. 3550. 1330. 1072. 3198. 2425.

515. 9469. 5317. 7120. 13586. 198785. 1058744. 20508438.

3290082 . 830354 .

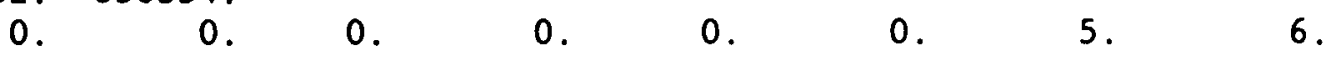

8. 31. 822. 1752. 4543. 1713. 1597. 2296.

6535. 1775. 0. 8555. 48596. 119411. 233382. 3003954 .

7620063. 1169436

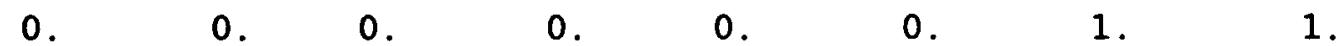

2. 11. 543. 1157. 3820. 1621. 3364. 0.

0. 129. 6679. 11858. 0.0 .0000$.

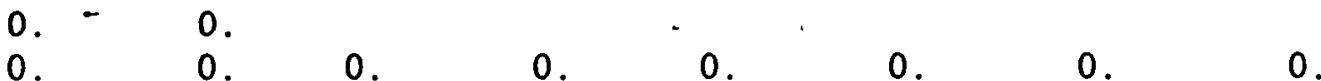

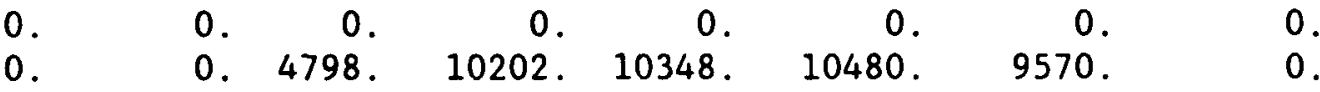

0. 2317. 1756. 0.00 .0000$.

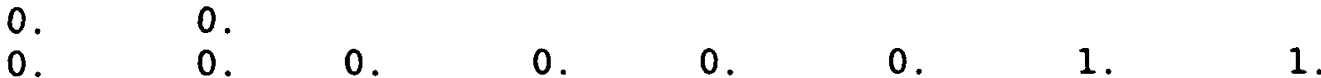

1. 7. 8316. 17684. 16340. 30419. 39474. 74998.

24195. 80412. 57477. 0.00000.

$\begin{array}{lllllll}0 . & 0 . & & & & 0 . & 0 .\end{array}$

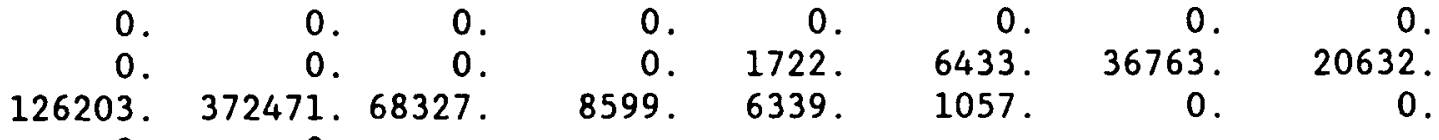

$\begin{array}{rrrrrrrr}0 . & 0 . & & & & & \\ 0 . & 0 . & 0 . & 0 . & 0 . & 0 . & 2 .\end{array}$

3. $13.2127 . \quad 273.21649 . \quad 4571 . \quad 3441 . \quad 7838$.

11747. 19019. 3360. 36387. 10447. 12402. 0.

$\begin{array}{rrrrrrrr}0 . & 0 . & & & & & \\ 0 . & 0 . & 5 . & 4 . & 8 . & 23 . & 14 . & 20 . \\ 23 . & 93 . & 301 . & 650 . & 0 . & 0 . & 1264 . & 4065 . \\ 1106 . & 14665 . & 4071 . & 18006 . & 37417 . & 89072 . & 81626 . & 0 .\end{array}$ 


\begin{tabular}{|c|c|c|c|c|c|c|c|}
\hline 0. & 0 . & & & & & & \\
\hline $\begin{array}{r}0 . \\
29 \\
1223 \\
280809\end{array}$ & $\begin{array}{r}0 . \\
117 . \\
17636 \\
8801784\end{array}$ & $\begin{array}{r}0 . \\
45 \\
4926\end{array}$ & $\begin{array}{r}0 . \\
105 . \\
30765 \text {. }\end{array}$ & $\begin{array}{r}0 \\
0 . \\
53265 .\end{array}$ & $\begin{array}{r}0 . \\
510 \\
289674 .\end{array}$ & $\begin{array}{r}19 \\
951 \\
216165 \text {. }\end{array}$ & $\begin{array}{r}25 \\
1521 \\
479431\end{array}$ \\
\hline $\begin{array}{r}0 \\
23 \\
2765 \\
526840\end{array}$ & $\begin{array}{r}0 . \\
93 \\
154 . \\
3099458\end{array}$ & $\begin{array}{r}0 \\
155 . \\
5296 .\end{array}$ & $\begin{array}{r}0 . \\
338 . \\
21409 .\end{array}$ & $\begin{array}{r}1 \\
125 \\
62228\end{array}$ & $\begin{array}{r}2 . \\
1079 \\
523803\end{array}$ & $\begin{array}{r}14 . \\
0 . \\
479588 .\end{array}$ & $\begin{array}{r}20 . \\
1355 . \\
1538059 .\end{array}$ \\
\hline $\begin{array}{r}0 . \\
23 . \\
915 . \\
3957581 .\end{array}$ & $\begin{array}{r}0 . \\
93 . \\
3153 . \\
0560254 .\end{array}$ & $\begin{array}{r}0 . \\
110 . \\
4132 .\end{array}$ & $\begin{array}{r}0 . \\
240 . \\
16295 .\end{array}$ & $\begin{array}{r}1 . \\
1056 \\
35596\end{array}$ & $\begin{array}{r}2 . \\
0 . \\
239712 \text {. }\end{array}$ & $\begin{array}{r}14 . \\
50 \\
709522\end{array}$ & $\begin{array}{r}20 \\
1396 \\
2845970\end{array}$ \\
\hline $\begin{array}{r}0 \\
38 \\
155 \\
2250273\end{array}$ & $\begin{array}{r}0 \\
154 \\
66531 \\
2145932\end{array}$ & $\begin{array}{r}0 . \\
30 \\
40902 .\end{array}$ & $\begin{array}{r}0 . \\
70 . \\
9557 .\end{array}$ & $\begin{array}{r}0 \\
450 \\
44818\end{array}$ & $\begin{array}{r}0 . \\
0 . \\
194801\end{array}$ & $\begin{array}{r}25 \\
980 \\
376828\end{array}$ & $\begin{array}{r}33 . \\
517 \\
1492286 .\end{array}$ \\
\hline $\begin{array}{r}0 . \\
12 \\
1986 \\
5086913\end{array}$ & $\begin{array}{r}0 . \\
47 \\
32459 \\
9537940\end{array}$ & $\begin{array}{r}0 . \\
31 \\
183133 .\end{array}$ & $\begin{array}{r}0 \\
69 \\
69 \\
193630\end{array}$ & $\begin{array}{r}0 \\
0 \\
30369\end{array}$ & $\begin{array}{r}0 . \\
380 \\
203275\end{array}$ & $\begin{array}{r}7 . \\
281 . \\
94113 .\end{array}$ & $\begin{array}{r}9 \\
445 \\
4328987 .\end{array}$ \\
\hline $\begin{array}{r}0 . \\
0 . \\
2794 . \\
7535605\end{array}$ & $\begin{array}{r}0 . \\
0 \\
6593 \\
9667977\end{array}$ & $\begin{array}{r}0 . \\
223 . \\
96857 .\end{array}$ & $\begin{array}{r}0 \\
477 \\
107328\end{array}$ & $\begin{array}{r}0 \\
0 \\
47585\end{array}$ & $\begin{array}{r}0 \\
1026 \\
156826\end{array}$ & $\begin{array}{r}0 . \\
609 . \\
101785 .\end{array}$ & $\begin{array}{r}0 . \\
2575 \\
4175263\end{array}$ \\
\hline $\begin{array}{r}0 . \\
23 \\
3965\end{array}$ & $\begin{array}{r}0 \\
92 \\
2084\end{array}$ & $\begin{array}{r}0 \\
2503 \\
6270\end{array}$ & $\begin{array}{r}0 . \\
5326 \\
10765\end{array}$ & $\begin{array}{r}0 . \\
3508 \\
103787\end{array}$ & $\begin{array}{r}0 \\
1826 \\
970659\end{array}$ & $\begin{array}{r}15 \\
1884 \\
472558\end{array}$ & $\begin{array}{r}20 . \\
275 \\
1396088 .\end{array}$ \\
\hline
\end{tabular}

1969210. 73968 .

LAND FRACTION

$\begin{array}{lllllllllllllllllll}1.00 & 1.00 & 0.00 & 0.00 & 0.00 & 0.00 & 0.00 & 0.00 & 0.00 & 0.80 & 1.00 & 1.00 & 0.95 & 0.75 & 0.70 & 0.85\end{array}$ $\begin{array}{llllllllllll}1.00 & 0.85 & 0.70 & 0.75 & 0.55 & 0.70 & 0.60 & 1.00 & 1.00 & 0.95\end{array}$

$\begin{array}{lllllllllllllllllll}1.00 & 1.00 & 1.00 & 1.00 & 0.90 & 0.70 & 0.40 & 0.00 & 0.00 & 0.45 & 1.00 & 0.95 & 0.40 & 0.60 & 1.00 & 1.00\end{array}$ $\begin{array}{lllllllllllll}0.90 & 0.45 & 0.60 & 0.20 & 0.50 & 0.50 & 0.30 & 0.25 & 0.50 & 0.60\end{array}$

$\begin{array}{lllllllllllllllll}1.00 & 1.00 & 1.00 & 1.00 & 1.00 & 1.00 & 0.00 & 0.00 & 0.00 & 0.05 & 1.00 & 1.00 & 0.20 & 1.00 & 0.70 & 0.30\end{array}$ $\begin{array}{lllllllllllllll}0.85 & 0.20 & 0.00 & 0.20 & 0.00 & 0.00 & 0.00 & 0.00 & 0.00 & 0.00\end{array}$

$\begin{array}{lllllllllllllllllll}1.00 & 1.00 & 1.00 & 1.00 & 1.00 & 0.80 & 0.10 & 0.00 & 0.00 & 0.00 & 1.00 & 1.00 & 0.75 & 0.30 & 0.40 & 0.00\end{array}$ $\begin{array}{llllllllllllll}0.15 & 0.00 & 0.45 & 0.30 & 0.00 & 0.00 & 0.00 & 0.00 & 0.00 & 0.00\end{array}$

$\begin{array}{llllllllllllllllll}1.00 & 1.00 & 1.00 & 1.00 & 1.00 & 0.60 & 0.00 & 0.00 & 0.00 & 0.00 & 0.95 & 1.00 & 1.00 & 0.70 & 0.40 & 0.10\end{array}$ $\begin{array}{llllllllllllllll}0.00 & 0.50 & 0.00 & 0.00 & 0.00 & 0.00 & 0.00 & 0.00 & 0.00 & 0.00\end{array}$

$\begin{array}{llllllllllllllllllll}1.00 & 1.00 & 1.00 & 1.00 & 1.00 & 0.70 & 0.00 & 0.00 & 0.00 & 0.80 & 0.90 & 0.75 & 0.85 & 1.00 & 1.00 & 0.70\end{array}$ $\begin{array}{llllllllllllll}0.15 & 0.25 & 0.25 & 0.00 & 0.00 & 0.00 & 0.00 & 0.00 & 0.00 & 0.00\end{array}$

$\begin{array}{lllllllllllllllllll}1.00 & 1.00 & 1.00 & 1.00 & 1.00 & 1.00 & 0.55 & 0.50 & 0.25 & 0.15 & 0.00 & 0.10 & 0.00 & 0.10 & 0.50 & 0.60\end{array}$ $\begin{array}{llllllllllll}0.85 & 1.00 & 1.00 & 0.40 & 0.40 & 0.05 & 0.00 & 0.00 & 0.00 & 0.00\end{array}$

$\begin{array}{llllllllllllllllll}1.00 & 1.00 & 1.00 & 1.00 & 1.00 & 1.00 & 1.00 & 1.00 & 1.00 & 0.90 & 0.35 & 0.40 & 0.80 & 1.00 & 1.00 & 1.00\end{array}$ $\begin{array}{llllllllll}1.00 & 1.00 & 1.00 & 1.00 & 0.95 & 0.20 & 0.00 & 0.00 & 0.00 & 0.00\end{array}$

$\begin{array}{llllllllllllllll}1.00 & 1.00 & 1.00 & 1.00 & 1.00 & 1.00 & 1.00 & 1.00 & 1.00 & 1.00 & 1.00 & 1.00 & 1.00 & 1.00 & 1.00 & 1.00\end{array}$ $\begin{array}{llllllllll}1.00 & 1.00 & 1.00 & 1.00 & 1.00 & 0.80 & 0.40 & 0.00 & 0.00 & 0.20\end{array}$

$\begin{array}{llllllllllllllll}1.00 & 1.00 & 1.00 & 1.00 & 1.00 & 1.00 & 1.00 & 1.00 & 1.00 & 1.00 & 1.00 & 1.00 & 1.00 & 1.00 & 1.00 & 1.00\end{array}$

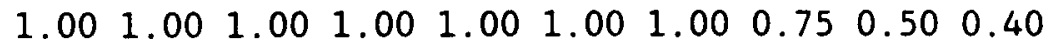

$\begin{array}{lllllllllllllllllll}1.00 & 1.00 & 0.70 & 0.40 & 0.20 & 0.75 & 0.95 & 1.00 & 1.00 & 1.00 & 1.00 & 1.00 & 1.00 & 1.00 & 1.00 & 1.00\end{array}$ $1.001 .001 .001 .00 \quad 0.951 .001 .001 .001 .00 \quad 0.80$ 
$\begin{array}{llllllllllllllllllllllllll}1 & 1 & 2 & 2 & 2 & 2 & 2 & 2 & 2 & 1 & 1 & 1 & 1 & 1 & 1 & 1 & 1 & 1 & 1 & 1 & 1 & 1 & 1 & 1 & 1 & 1\end{array}$ $\begin{array}{llllllllllllllllllllllllll}1 & 1 & 1 & 1 & 1 & 1 & 2 & 2 & 2 & 2 & 1 & 1 & 2 & 1 & 1 & 1 & 1 & 1 & 1 & 2 & 2 & 2 & 2 & 2 & 2 & 1\end{array}$ $\begin{array}{llllllllllllllllllllllllll}1 & 1 & 1 & 1 & 1 & 1 & 2 & 2 & 2 & 2 & 1 & 1 & 2 & 1 & 1 & 2 & 1 & 2 & 2 & 2 & 2 & 2 & 2 & 2 & 2 & 2\end{array}$ $\begin{array}{llllllllllllllllllllllllll}1 & 1 & 1 & 1 & 1 & 1 & 2 & 2 & 2 & 2 & 1 & 1 & 1 & 2 & 2 & 2 & 2 & 2 & 2 & 2 & 2 & 2 & 2 & 2 & 2 & 2\end{array}$ $\begin{array}{llllllllllllllllllllllllll}1 & 1 & 1 & 1 & 1 & 1 & 2 & 2 & 2 & 2 & 1 & 1 & 1 & 1 & 2 & 2 & 2 & 2 & 2 & 2 & 2 & 2 & 2 & 2 & 2 & 2\end{array}$ $\begin{array}{llllllllllllllllllllllllll}1 & 1 & 1 & 1 & 1 & 1 & 2 & 2 & 2 & 1 & 1 & 1 & 1 & 1 & 1 & 1 & 2 & 2 & 2 & 2 & 2 & 2 & 2 & 2 & 2 & 2\end{array}$ $\begin{array}{llllllllllllllllllllllllll}1 & 1 & 1 & 1 & 1 & 1 & 1 & 2 & 2 & 2 & 2 & 2 & 2 & 2 & 2 & 1 & 1 & 1 & 1 & 2 & 2 & 2 & 2 & 2 & 2 & 2\end{array}$ $\begin{array}{llllllllllllllllllllllllll}1 & 1 & 1 & 1 & 1 & 1 & 1 & 1 & 1 & 1 & 2 & 2 & 1 & 1 & 1 & 1 & 1 & 1 & 1 & 1 & 1 & 2 & 2 & 2 & 2 & 2\end{array}$ $\begin{array}{llllllllllllllllllllllllll}1 & 1 & 1 & 1 & 1 & 1 & 1 & 1 & 1 & 1 & 1 & 1 & 1 & 1 & 1 & 1 & 1 & 1 & 1 & 1 & 1 & 1 & 2 & 2 & 2 & 2\end{array}$ $\begin{array}{llllllllllllllllllllllllll}1 & 1 & 1 & 1 & 1 & 1 & 1 & 1 & 1 & 1 & 1 & 1 & 1 & 1 & 1 & 1 & 1 & 1 & 1 & 1 & 1 & 1 & 1 & 1 & 2 & 2\end{array}$ $\begin{array}{llllllllllllllllllllllllll}1 & 1 & 1 & 2 & 2 & 1 & 1 & 1 & 1 & 1 & 1 & 1 & 1 & 1 & 1 & 1 & 1 & 1 & 1 & 1 & 1 & 1 & 1 & 1 & 1 & 1\end{array}$ $\begin{array}{llllllllllllllllllllllllll}1 & 1 & 2 & 2 & 2 & 2 & 2 & 1 & 1 & 1 & 1 & 1 & 1 & 1 & 1 & 1 & 1 & 1 & 1 & 1 & 1 & 1 & 1 & 1 & 1 & 1\end{array}$ $\begin{array}{llllllllllllllllllllllllll}1 & 1 & 2 & 2 & 2 & 2 & 2 & 2 & 1 & 1 & 1 & 1 & 1 & 1 & 1 & 1 & 1 & 1 & 1 & 1 & 1 & 1 & 1 & 1 & 1 & 1\end{array}$ $\begin{array}{llllllllllllllllllllllllll}1 & 1 & 2 & 2 & 2 & 2 & 2 & 2 & 2 & 2 & 1 & 1 & 1 & 1 & 1 & 1 & 1 & 1 & 1 & 1 & 1 & 1 & 1 & 1 & 1 & 1\end{array}$ $\begin{array}{llllllllllllllllllllllllll}1 & 1 & 2 & 2 & 2 & 2 & 2 & 2 & 1 & 1 & 1 & 1 & 1 & 1 & 1 & 1 & 1 & 1 & 1 & 1 & 1 & 1 & 1 & 1 & 1 & 1\end{array}$ $\begin{array}{llllllllllllllllllllllllll}1 & 1 & 2 & 2 & 2 & 2 & 2 & 2 & 2 & 1 & 1 & 1 & 1 & 1 & 1 & 1 & 1 & 1 & 1 & 1 & 1 & 1 & 1 & 1 & 1 & 1\end{array}$ CROP SEASON AND SHARE

\begin{tabular}{|c|c|c|}
\hline 1 PASTURE & 90.270. & 0.41 \\
\hline STORED FORAGE & 150. 240 & 0.13 \\
\hline GRAINS & 150. 240. & 0.21 \\
\hline GRN LEAFY VEGETABLES & 150. & 0.002 \\
\hline IHER FOOD CROPS & 150. & 0.004 \\
\hline GUMES AND SEEDS & 150. & 0.15 \\
\hline TS AND TUBERS & 150 & 0.003 \\
\hline
\end{tabular}




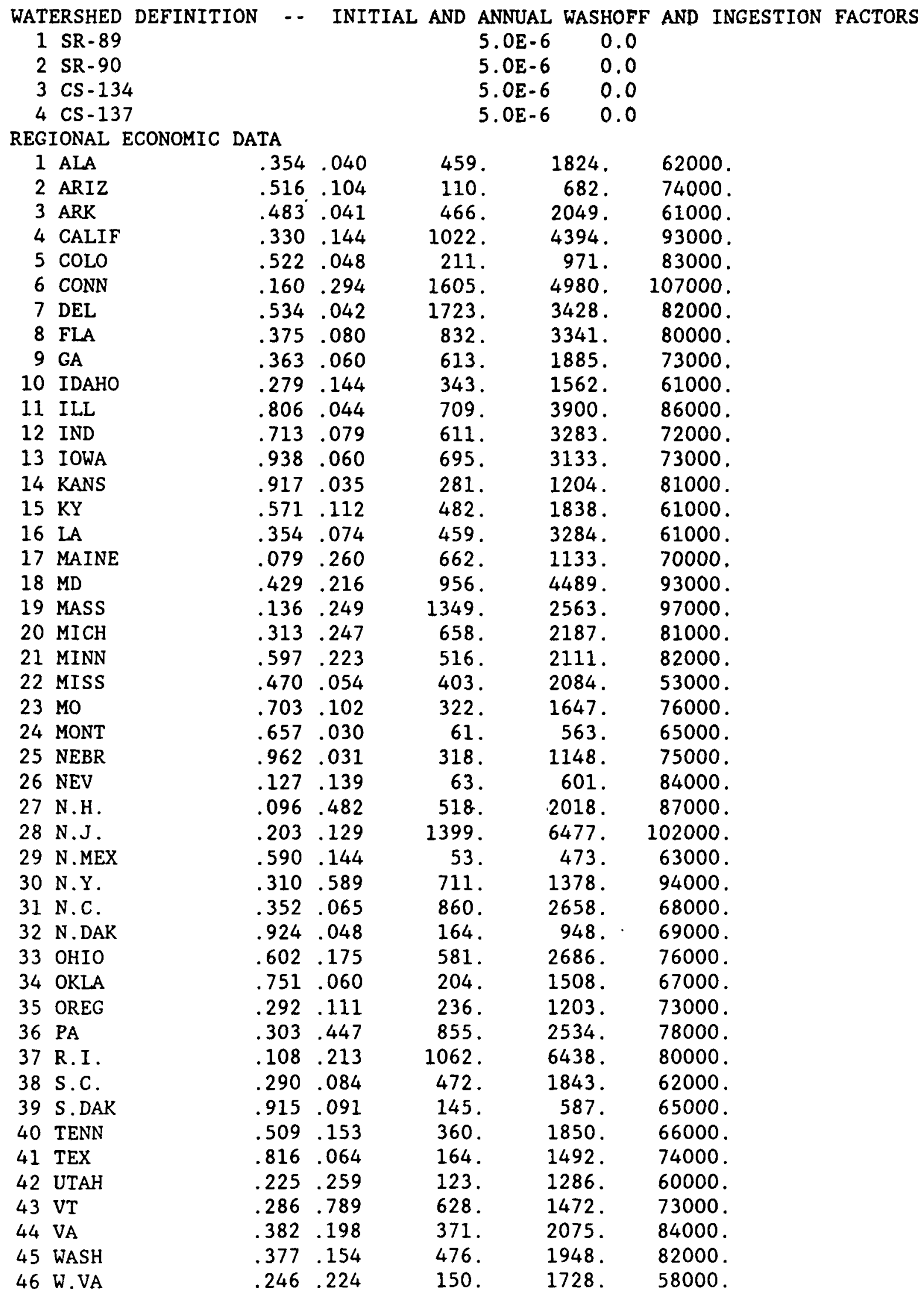




$\begin{array}{lrrrrr}47 \text { WIS } & .517 & .591 & 723 . & 1751 . & 76000 . \\ 48 \text { WYO } & .561 & .028 & 43 . & 380 . & 70000 . \\ 49 \text { BRIT COL } & .377 & .154 & 476 . & 1948 . & 60000 . \\ 50 \text { OCEAN } & .000 & .000 & 0 . & 0 . & 0 . \\ 51 \text { SASKAT } & .657 & .030 & 61 . & 563 . & 60000 . \\ 52 \text { MANITOBA } & .924 & .048 & 164 . & 948 . & 60000 . \\ 53 \text { ONTARIO } & .597 & .223 & 516 . & 2111 . & 60000 . \\ 54 \text { QUEBEC } & .310 & .589 & 711 . & 1378 . & 60000 . \\ 55 \text { NOVA SCOT } & .079 & .260 & 662 . & 1133 . & 60000 . \\ 56 \text { BAJA CAL } & .330 & .144 & 1022 . & 4394 . & 10000 . \\ 57 \text { SONORA } & .516 & .104 & 110 . & 682 . & 10000 . \\ 58 \text { CHIHUAHUA } & .590 & .144 & 53 . & 473 . & 10000 . \\ 59 \text { COAHUILA } & .816 . .064 & 164 . & 1492 . & 10000 .\end{array}$




\section{3 List Output File for Sample Problem A}

MACCS11, ATMINP=IN1A, EARINP=IN2A, CHRINP IN3A, METFIL=METSUR, SITDAT $=$ SURSIT, LISOUT-LISTA

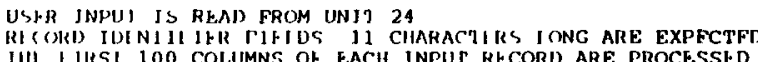

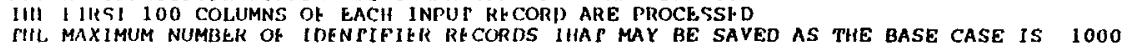

RHCORD
NUMHCR RECORD

IBING THIS "ATMOS" INPUT

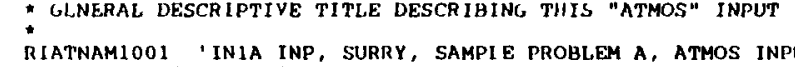

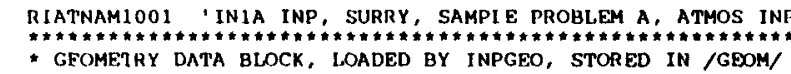

: numbr of radial spatial, elements

2 GE NUMREADOOL 26

SURRY

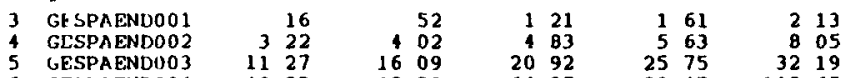

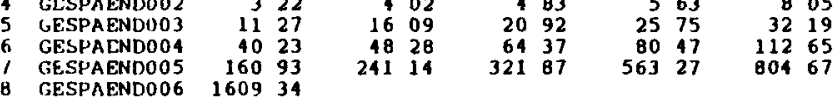

等

- number of nuclides

9 isNumiso001 60

* NUMBer of nUCLide GROUPS

10 ISMAXGRPOOI 9

" wet and dRY deposition flags for each nuclide group

WETDEP DRYDEP

11 ISDEPFLAOOI

ISDEPFLAOO2

ISDCPFLAO004

I SDEPTLAOO

ISDEPFLAOOB

DEEPFLAOO

FALSE FALSE

TRUE

TRUE

TRUE

TRUE
TRUE
TRUE

ISOTPGRPOOI

DATA FOR

ISOTPGRPO02

ISOTPGRPO0

ISOTPGRPO0

ISOTPGRPO07
ISOTPGRPO08

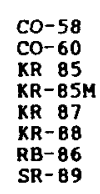

PARENT IGROUP

$\begin{array}{llll}\text { NONE } & 6 & 6 & 160 E+06 \\ \text { NONE } & 6 & 1 & 660 E+08 \\ \text { NONE } & 1 & 3 & 386 E+08 \\ \text { NONE } & 1 & 1 & 613 E+04 \\ \text { NONE } & 1 & 4560+03 \\ \text { NONE } & 1 & 1 & 008 E+04 \\ \text { NONE } & 3 & 1 & 11 E+06 \\ \text { NONE } & 5 & 193 E+06\end{array}$

HAFLIF 


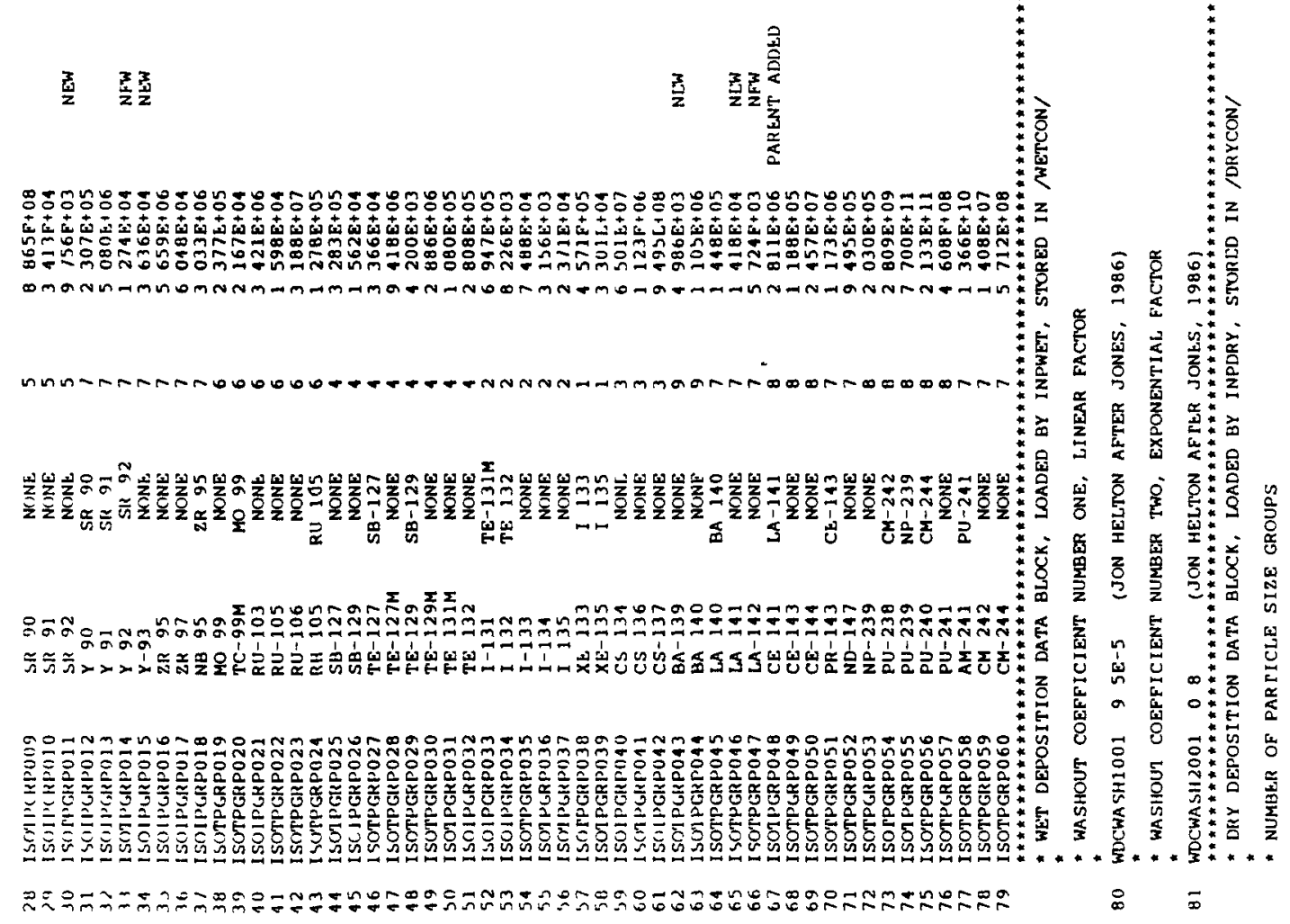




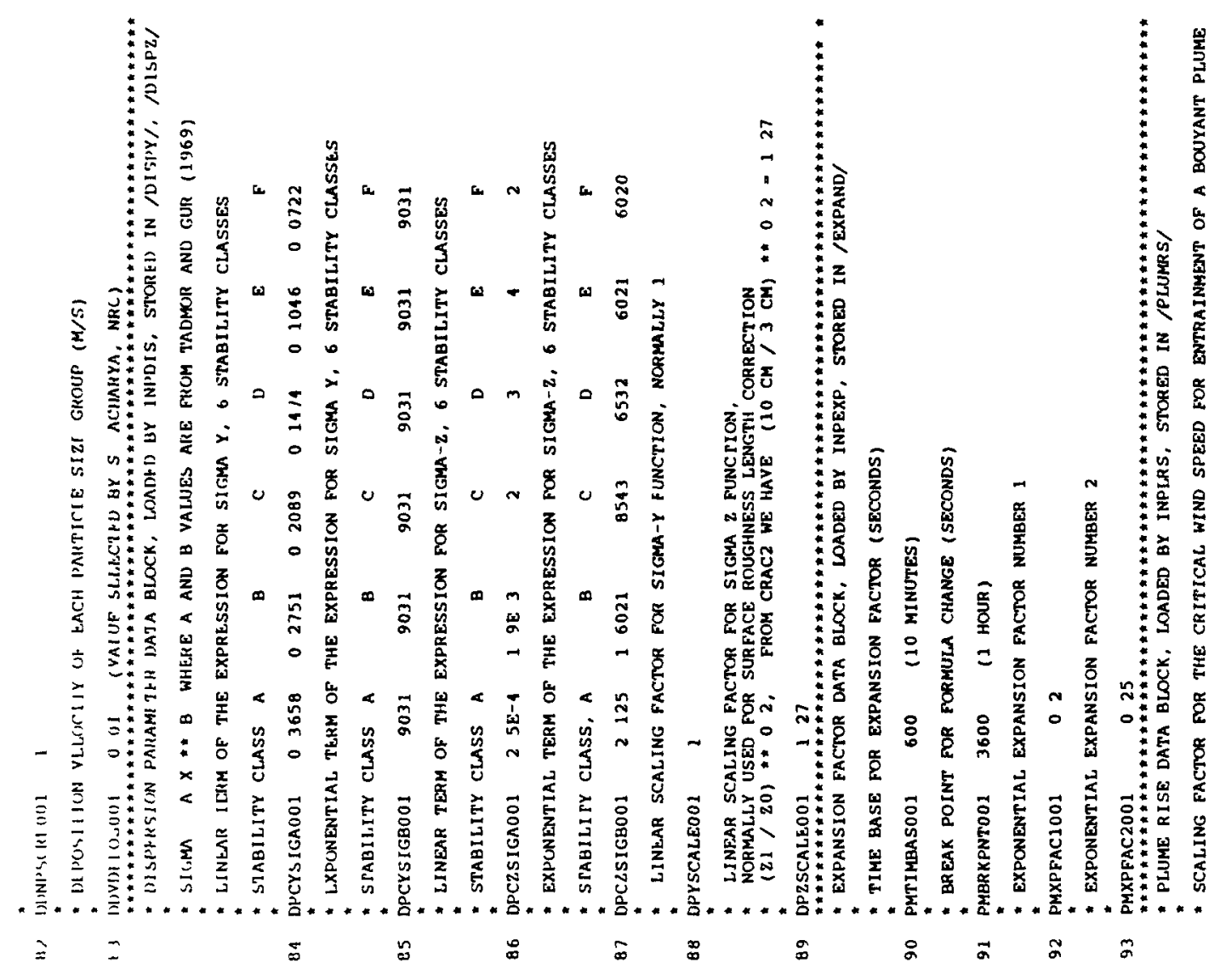


- (USED BY FUNCTION CAUGHT)

91 PRSCLCRWOO1 1

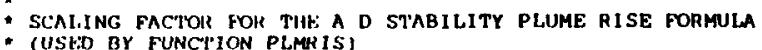

: (USLE BY FUNCI'ION PLMHIS)

9') PRSCLADPOOI 1 .

: SCALING FACTOR FOR THE E-F STABILITY PLUME RISE Formula
(USED BY FUNCTION PLMRIS)

96 PRSCLEFPOO1 1.

- WAKE EFFECTS DATA BLOCK, LOADED BY INPWAK, STORED IN /BILHAK/

- BUILOING HIDTH (METERS)

97 HEBUI LDWOO1 10. - SURRY

" butloing hetght (METERS)

98 WEBUILDHOU1 50. SURRY

" RELEASE DATA BLOCK, LOADED BY INPREL, STORED IN /ATMAM2/, MULREL/

99 RDATNAM2001 'SECOND DRAFT 1150, WORST CASE SOURCE TERM POR EARLY FATALITIES'

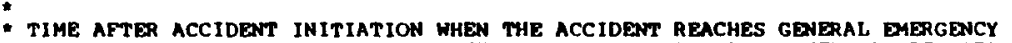
- CONDITIONS (AS DEEINED IN NUREG-0654), OR WHEN PLANT PERSONNEL CAN RELIABLY

100 RDOALARMOD 1300

" number of plume segments that are released

101 RDNUMRELO01 2

" selection of risk dominant plume

102 RDMAXRISO0

* reference time for dispersion and radioactive decay

103 RDREFTIMO01 0.00

0.50

- HEAT CONTENT OF THE RELEASE SECMENTS (W)

104 RDPLHEAT001 $3.7 E+6 \quad 1.7 E 5$

- height of THE PLUME segments at Release (M)

- A VALUE SPECIFIED For EACH OF THE RELEASE SEGMENTS

105 RDPLHITE0O

- DURATION OF THE PLUME SEGMENTS (S)

106 RDPLUDUROOI 1800. 22000 .

* TIME OF RELEASE FOR EACH PLUME (S AFTER SCRAM)

107 RDPDELAYO01 3700. 10000. 
: PARTICLE SIZE DISTRIBUTION OF EACH NUCLIDE GROUP

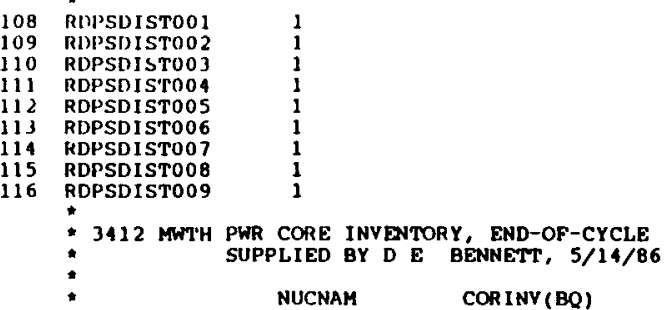

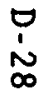
NUCNAM COR INY (BQ)
$\begin{array}{lll}\text { CO-5B } & 3 & 223 E+16 \\ \text { CO } 60 & 2 & 465 E+16\end{array}$
$K R-85$
$K R-85 M$
$\mathrm{KR}-88$
$\mathrm{RB}-86$
$\mathrm{SR}-89$
SR -90
SR-91
SR -92
$\begin{array}{ll}Y-90 & 2079 E+17 \\ Y-91 & 1374+18 \\ Y-92 & 1821 E+18 \\ Y-93 & 5\end{array}$
$\begin{array}{ll}2 R-95 & 5526 E+18 \\ 2 R-97 & 5759 E+18\end{array}$
$\begin{array}{lll}N B-95 & 5224 E+18 \\ M O-99 & 6 & 098 E+18\end{array}$
RU-103 $1542 \mathrm{E}+18$
RU-106 1. $032 \mathrm{E}+18$
$S \mathrm{~B}-127$
$\mathrm{SB}$
$\begin{array}{lll}S 8-129 & 9872 \mathrm{E}+1\end{array}$
$\begin{array}{ll}T E-127 M & 3564 E+16 \\ T E-129 & 9265 E+17\end{array}$
TE-129M $2443 \mathrm{E}+17$
TE-132
$1-131$
$1-132$
$1-133$
4
6
$6779 \mathrm{E}+18$
778
1 $782 \mathrm{E}+18$
$\mathrm{CS}$
$\mathrm{CS}-136$
$B A-139$
$B A-140$
$\begin{array}{ll}6 & 216 \mathrm{E}+1 \\ \mathrm{LA}-140 & 6\end{array}$
$L A-141$
$L A-142$
$C E-141$

RDCORINYOO1

RDCOR INVO0
RDCOR INYO0

RDCOR INVO0S

RDCOR INVO07

RDCOR INV009

RDCOR INV010

RDCOR INV012
RDCOR INV013

RDCOR INVO1

RDCORINY016

RDCOR INVO17
RDCORINVO19

RDCOR INV02

RDCOR INVO21

RDCOR INY023

RDCOR INV025

DDCORINVO

RDCOR INV029
RDCOR I INV030

RDCOR INVO 31

RDCOR INVO33

RDCOR INV03

RDCOR INV03

RDCOR INVOJ

RDCOR INV0 4

RDCORINVA

RDCORINYO4

RDCORINVO4

RDCOR INV04 


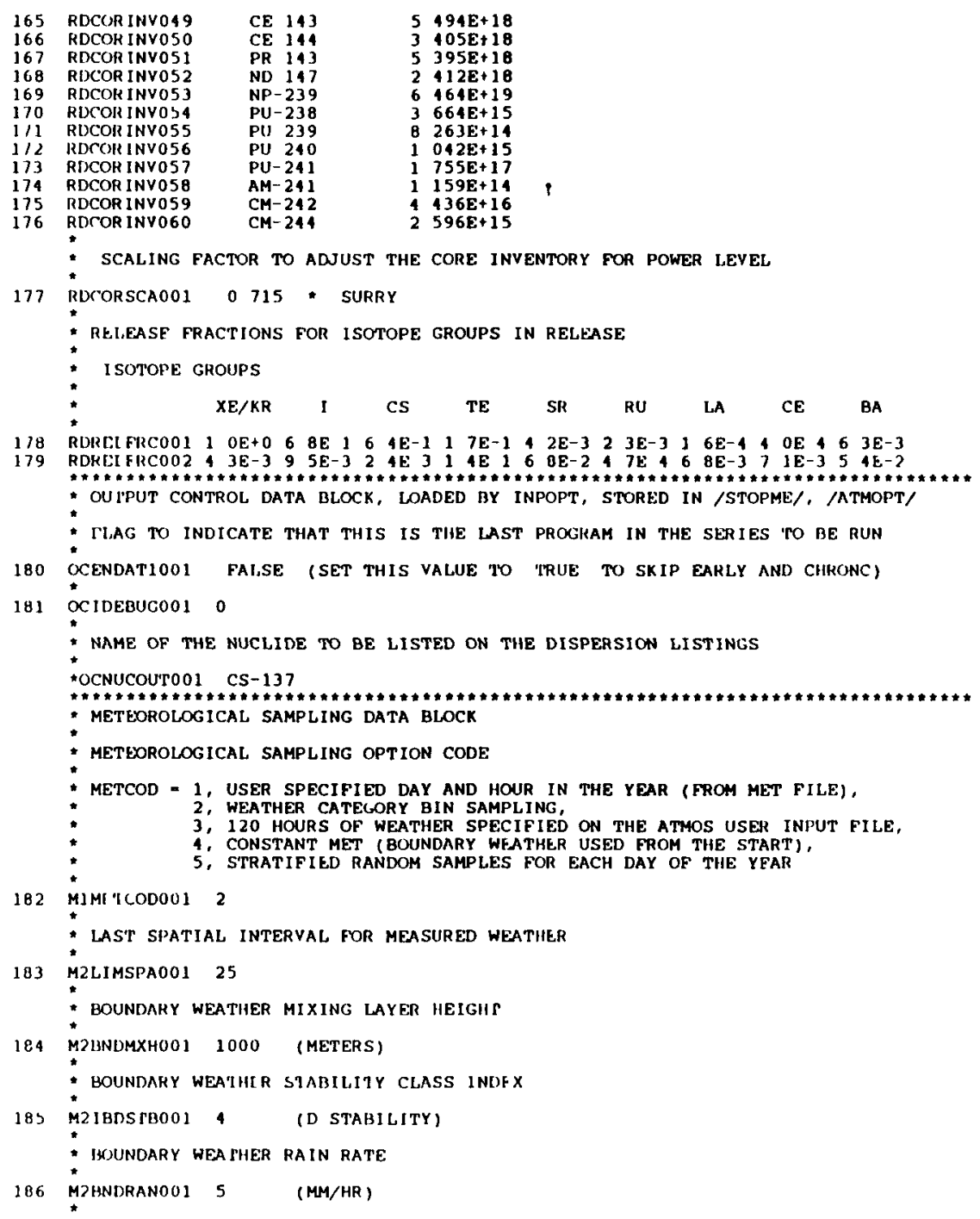


18) M2BNIWNISUOL 5 (M/S)

* numbir of rain distance intlivals for binning

la minitintous 5

- LNIPO)INTS OF THE RAIN DISTANCE inTERVALS (KILOMETtRS)

" NOTE TIESE MIJST BE CHOSEN TO MATCH THE SPATIAL ENDPOINT DISTANCES

189 MARNDSTSO01 $3222553 \quad 11 \quad 27 \quad 2092 \quad 32 \quad 19$

- Number of rain INTENSITIY breakPoINTS

190 M4NRINTNOO1 3

: RAIN INTENSITY BREAKPOINTS FOR WEATHER BINNING (MILLIMETERS PER HOUR)

191 M4RNRATEU01 246

* number of samples per bin

192 MANSMPLSOO1 \& (THIS NUMBER SHOULD BE SET TO 4 FOR RISK ASSESSMENT)

: initial seed for random number generator

193 MA IRSEED001 79

........ TERMINATOR RECORD ENCOUNTEHED -- END OF BASE CASE USER INPUT .......*

ISER INPUT PROCESSING SUMMARY - BASE CASE

NUMBFK OF RTCORDS READ

NUMHER OF BLANR OR COMMENT RECORDS FEEAD

NUMBER OF RECOHDS PHOCESSED
NUMBER OF PROCESSED RECORDS DUPLICATED
NIMMER OF PROCESSED RECORDS SORTED

416
$-\quad 222$ 


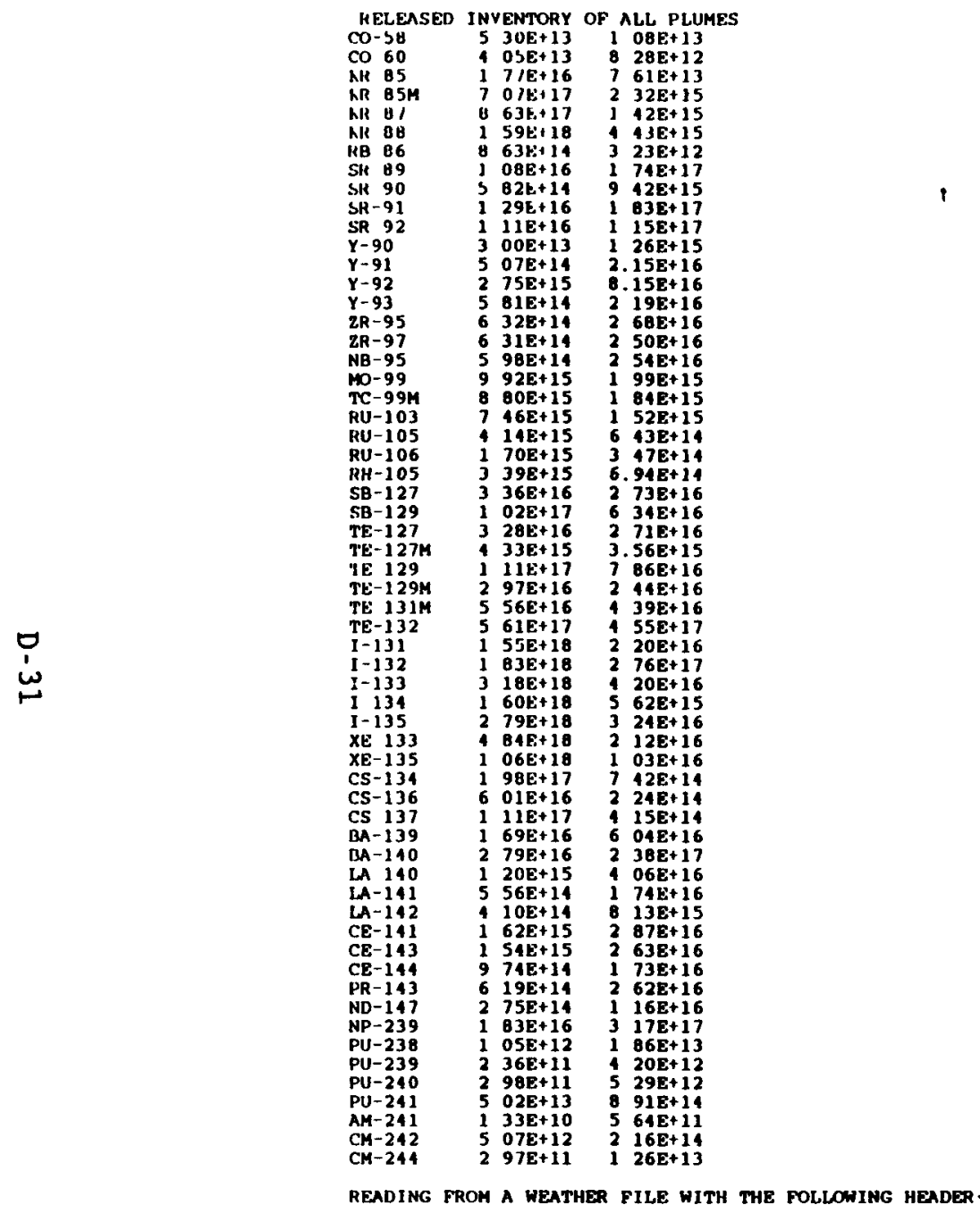

READING FROH A HEATHER FILE HITH THE FOLLOHING HEADER.

SURRY MET, NRC-12/12/88, CREATED 12/22/8B
MACCS PORMAT-NUREG-1150
METEOROOLGICAL DATA FILE CONTAINS 451 HOURS OF OBSERVED RAIN DATA 


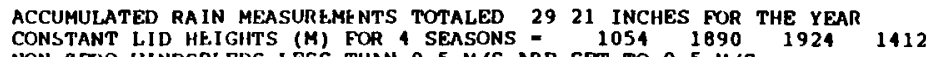

NON ZERO WINDSPEEDS LESS THAN O 5 M/S ARE SET TO 05 M/S

HIN PRIORIIIFS

* met gorological bin sumary $\ldots$.

RI XX RAIN INFENSITY I WITHIN THE INTERVAL, ENDING AT $X X$

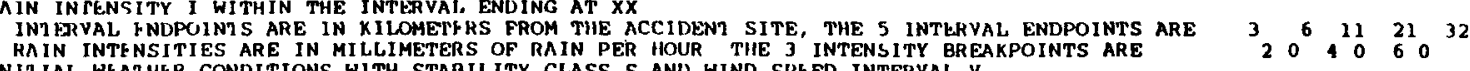

s $v$

WIN STABLITY CLASS $S$ ANU HIND SPEED INTERYAI $Y$

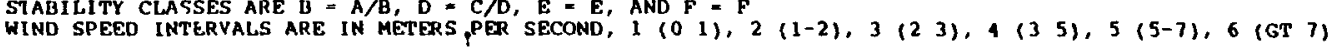

Me TBIN 1 ${ }_{7}^{\text {HIND DIRECTION }}$

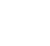
12 $14 \quad 15$ 16 TOTAL PER CENT

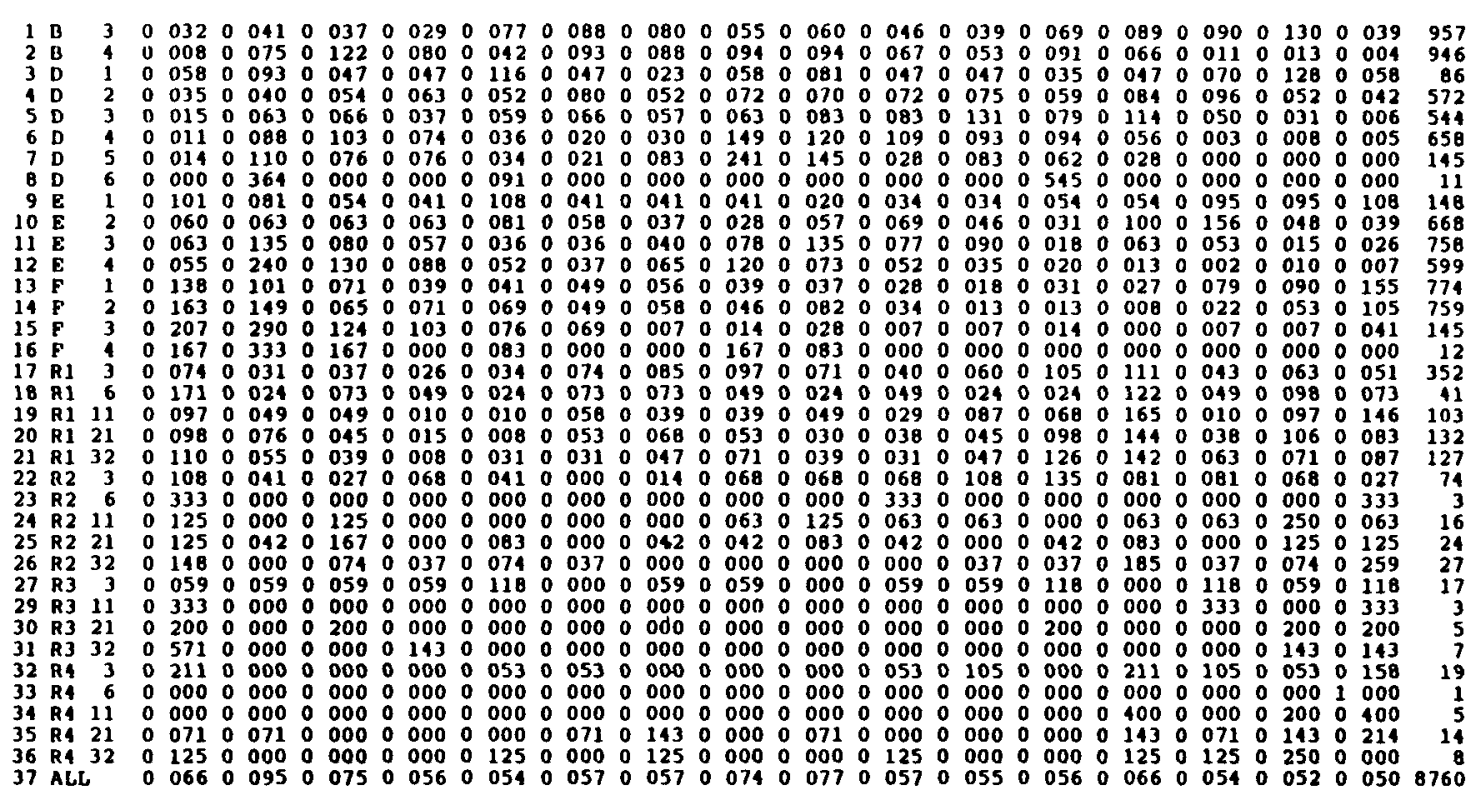

$\begin{array}{rl}10 & 9247 \\ 10 & 7991 \\ 0 & 9817 \\ 6 & 5297 \\ 6 & 2100 \\ 7 & 5114 \\ 1 & 6553 \\ 0 & 1256 \\ 1 & 6895 \\ 7 & 6256 \\ 0 & 6530 \\ 6 & 8379 \\ 8 & 0356 \\ 8 & 6644 \\ 1 & 6553 \\ 0 & 1370 \\ 1 & 0183 \\ 0 & 4680 \\ 1 & 1758 \\ 1 & 5068 \\ 1 & 9198 \\ 0 & 8447 \\ 0 & 0342 \\ 0 & 1826 \\ 0 & 2740 \\ 0 & 3082 \\ 0 & 1941 \\ 0 & 0342 \\ 0 & 0571 \\ 0 & 0799 \\ 0 & 2169 \\ 0 & 0114 \\ 0 & 0571 \\ 0 & 1598 \\ 0 & 0913\end{array}$ 


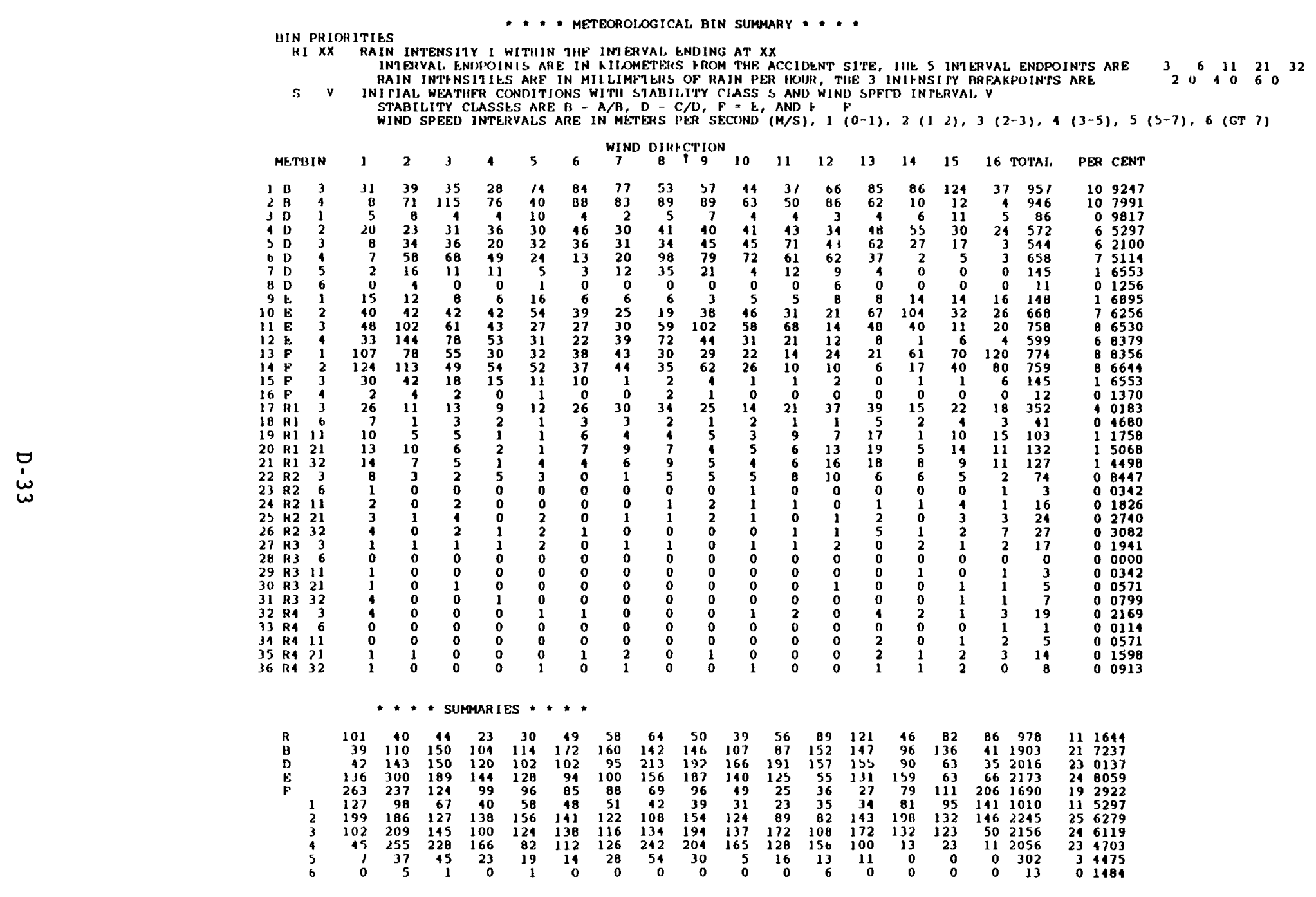




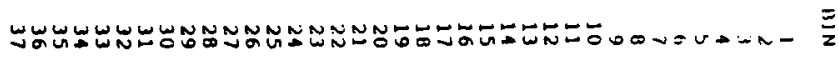
$000000000000000000000000000000000=000$

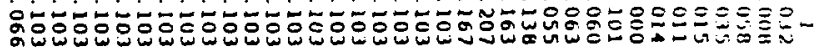

$000000000000000000000000000000000=0=0$

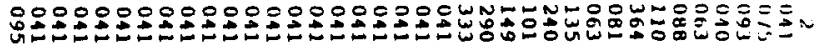
$00000000000000000000000000000000000=$

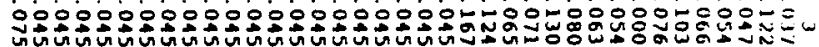

0000000000000000000000000000000000000

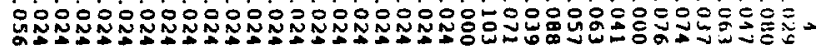

0000000000000000000000000000000000000

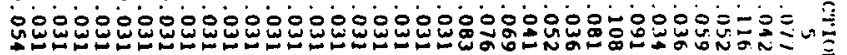

$000000000000000000000000000000000000=$

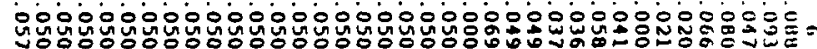
0000000000000000000000000000000000000

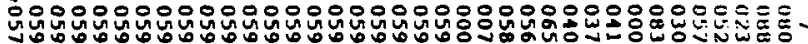
$000000000000000000000000000000000000=$

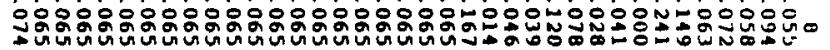

0000000000000000000000000000000000000

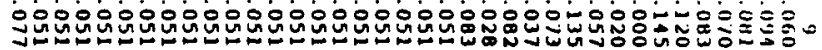
0000000000000000000000000000000000000

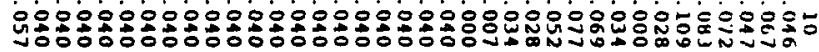
0000000000000000000000000000000000000

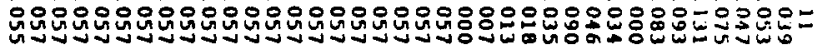

0000000000000000000000000000000000000

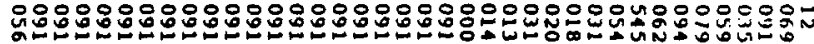

0000000000000000000000000000000000000

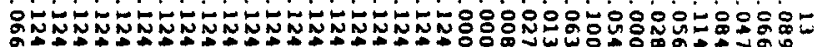

0000000000000000000000000000000000000

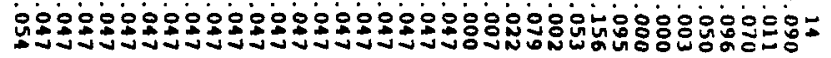
0000000000000000000000000000000000000

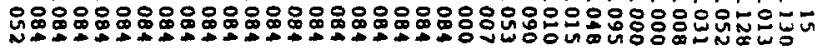
0000000000000000000000000000000000000

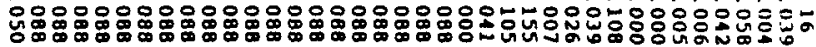




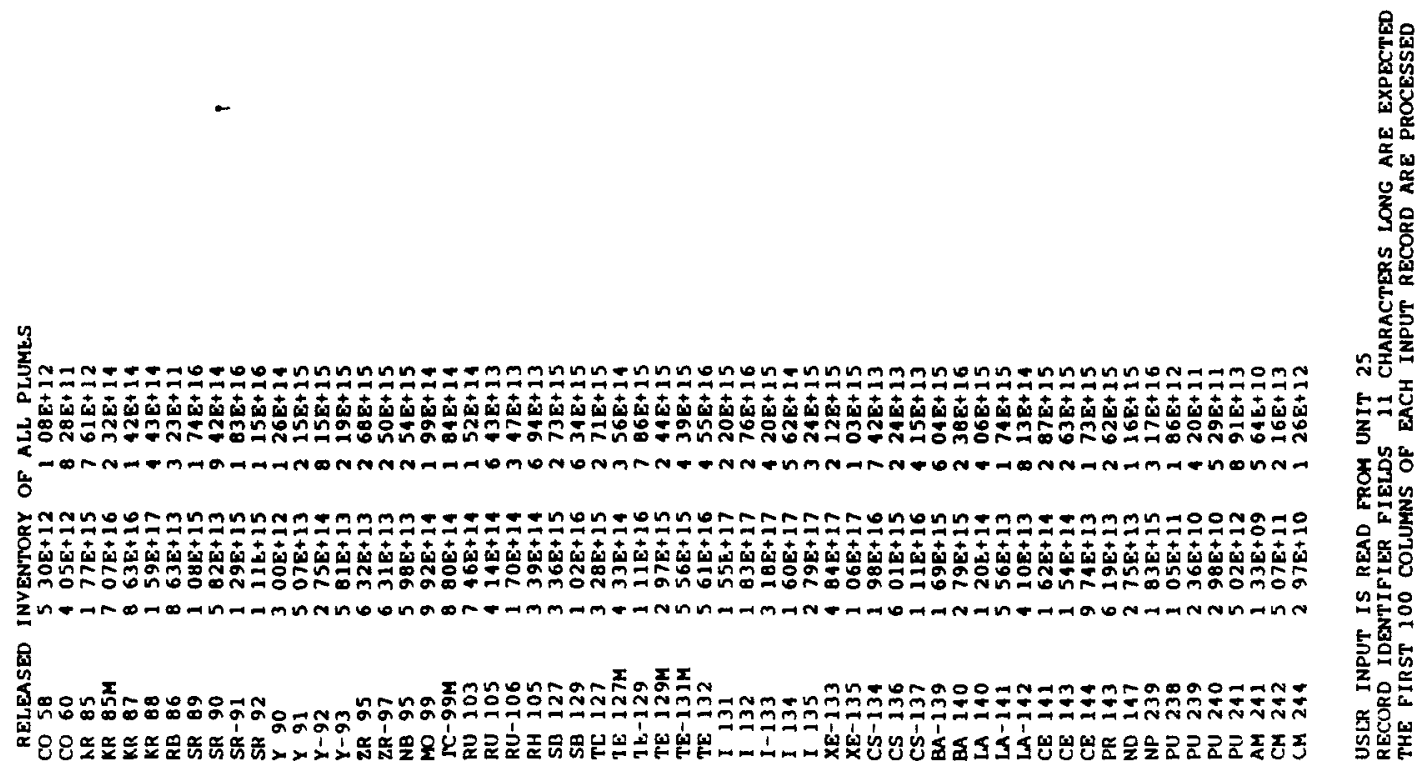


THE MAXIMUM NUMBER OF IDENTIFIER RECORdS THAT MAY BE SAVED AS THE bASE CASE IS 1000 ,

RECORD)
NUMABER

RECORD

* Gremeral, descriplitive tittie vescribing this "early" input file

1 MIFANAMIO01 'IN2A. INP, SURRY, SAMPLE Prol3LEM A, EARLY INPU'T"

* Hlag to indicate that this is the last program in the series to be run

2 mi ENdatroo1 false. (SET this value to .true. to skip chronc)

" DISPEHSION MODEL OPTION CODE: $\frac{1}{2}$ * STRAIGHT LINE

- WIND-SHIFT WITH ROTATION

3 MitPLUMEOOI 2

- NUMBER OF FINE GRID SUBDIVISIONS USED BY THE MODEL

4 minumpinod 7 (3, 5 OR 7 ALlowed)

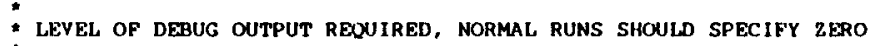

5 mi IPRINTO01 0

: LOGICAL FLAG SIGNIFY IMG THAT THE BREAKDOWN OF RISK BY WEATHER CATEGORY

: risein

6 Miriscat001 false

- Flag indicating if wind-roses from atmos are to be overridden

7 MIOVRRIDO01 FALSE. (USE THE WIND ROSE CALCULATED FOR EACH WEATIER BIN

POPULATION Distribution DATA BLOCK, LOADED BY INPOPU, STORED IN/POPDAT/

a PUPOPflgoor FILE

PPPPOPFIGO01 UNIFORM
PDIBEgINO01 1 (SPATIAL INTERVAL AT WHICH POPULATION BEgINS)

-PDPOPUEN001 50. (POPULATION DENSITY (PEOPLE PLR SQUARE KILOMETER))

: ORGAN DEFINITION DATA BLOCK, LOADED BY INORGA, STORED IN / EARDIM/ AND /ORGNAM/

" number of organs defined for health efFects

9 ODNUMORGOO1 9

NAMEs of the organs defined for health efFects

10 ODORGMAMOO1 'SKIN' ' 'EDEWBODY ', 'LUNGS', 'RED MARR', 'LOWER LI', 'STOMACH',

- SHIELDING AND EXPOSURE FACTORS, LOADED DY INDFAC, STORED IN /EADFAC/

: THREE VAlUes OF EACH PROTECTION Factor aRe supplied,

: ONE ROR EAch Trp

- ACTIVITY TYPE:

1 - EVACUEES WHILE MOVING
2 - NORMAL ACTIVITY IN SHELTER ING AND EVACUATION ZONE 
: cloud shielding factoro

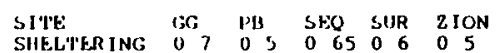

$$
\begin{aligned}
& \text { EVACUtes hommal SHelter }
\end{aligned}
$$

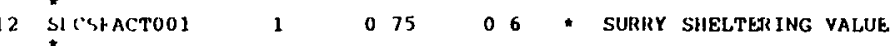

18 SFRESHAFOOI $1.82 E 5$ (2) 11 DAYS)

- Evacuarion zone data block, LOADED dy guneriw, smore

* Specific description of thie emergency response scenar 10 being used

19 Ezeanam2001 'evacuation hithin 10 miles, Relocation models apply eisewhere' : THE TYPE OF WEIGHTING TO BE APPLIED TO THE EMERGENCY RESPONSE SCENARIOS

EZW'NAME001 'PEOPLE

" weighting fraction applicable to this scenario

21 EZWTFRACOO1 0.95

: LAST Ring in the movement zone

22 EZLASMOVO01 15 (EVACUEes DisAPPEAR AFTER TRAYELING TO 20 MILES)

" girst spatial interval in the evacuation zone 
 - 012 (SINGlik EVAcuatrion zone out to 10 miles)

* EVACUATION DELAY TIMES FOR THE 3 EVACUATION ZONES
"THIS IS THE DEIAY TIME FROM OALARM (ATMOS) TO WHEN PEOPLE START MOVING

2b E\%EDEIAYOOI O. O. 7200. (SURRY) i

* havial, evacuátion SPEed (M/S)

26 EZFSPEPDO01 1.8 (SURRY)

: Silelter and relocation zone data block, loaded gy inPEgr,

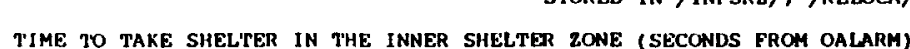

27 SRTYOSH1001 O. O. (THERE IS NO INNER SHELTER ZONE)

" sheltet deration in the inner shelter zone (SEconds from taking shelter)

28 SRSHELT1001 O. O. (THERE IS NO INNER SHELTER ZONE)

- LAST Ring of THE OUTER SHelter zONE

29 SRLASHE2001 0 (THERE IS NO OUTER SHELTER ZONE)

- Time to take shelter in the outer shelter zone (SEconos hrom oAlarm)

30 SRTTOSH2001 O. O. (THERE IS NO OU'TER SHELTER ZONE)

- SHELTER DURATION IN THE OUTER SHELTER zONE (SECONDS FRom taking SHELTER)

31 SRSHELT2001 0 0. (THERE IS NO OUTER SHELTER ZONE)

- Duration of the emergency phase (SEcondos from plume arrival)

32 SRENDEMPOO1 604800. (ONE HEEK)

- critical organ for relocation decisions

33 SRCH TORGOO1 'EDEWBODY.

: hot SPOT RELOCATION TIME (SECONDS from PLUme ARR IVAL)

34 SRTIMHOTO01 43200. (ONE-HALF DAY)

- mormal relocation time (SEcondos from plume arrival)

35 SRTIMNRMOO1 86400. (ONE DAY)

HOT SPOT RELOCATION DOSE CRITERION THRESHOLD (SIEVERTS)

36 SRDOSHOTOO) 0.5 (50 REM DOSE TO WHOLE BODY IN 1 WEEK TRIGGERS RELOCATION) " normal relocation dose criterion threshold (SIEverts)

37 SRDOSNRMOO1 0.25 (25 REM DOSE TO HHOLE BODY IN 1 WEEK TR IGGERS RELOCATION) - early fatality model parameters, loaded by inefat, Stored in /efataly : Number of early fatality efFects 
38 FFNUMEFAOD 3

\begin{tabular}{|c|c|c|c|}
\hline 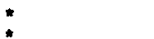 & ORGNAM & EFFACA & EFFACB \\
\hline $\begin{array}{l}\text { ETATACBRPOOLI } \\
\text { EFATAGRPOOL }\end{array}$ & $\begin{array}{l}\text { 'REI) MARR " } \\
\text { 'LUNGS' }\end{array}$ & $\begin{array}{r}3.8 \\
10.0\end{array}$ & $\begin{array}{l}5.0 \\
7.0\end{array}$ \\
\hline
\end{tabular}

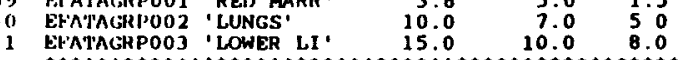

EARLY INJURY MODEL PARAMETERS, LOADED BY INEINJ, STORED

* number of garly inuury effects

12 Einumeinool 7
- Einame
ORGNAM EISUSC EITHRE EIFACA EIFACB

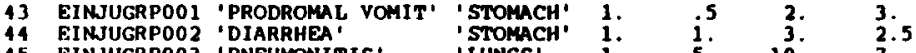

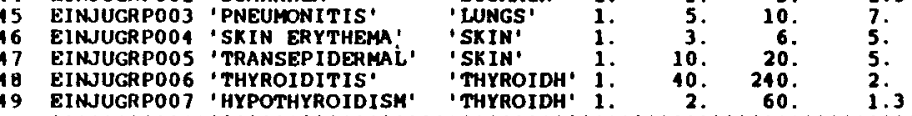

ACUTE EXPOSURE CANCER PARAMETERS, LOADED BY INACAN STORED IN /ACANCR/

* number of acute exposure cancer efpects

50 LCNUMACAOOI 7

DOSE THREShold for linear dose Response (sV)

51 LCACTHREOO1 1.5

\begin{tabular}{|c|c|c|c|c|c|c|c|}
\hline & ACNAME & ORGNAM & Acsusc & DOSEFA & DOSEFB & CFRISK & CIRISK \\
\hline 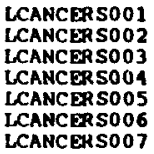 & $\begin{array}{l}\text { 'LEUKEPIA' } \\
\text { 'BONE' ' } \\
\text { 'BREAST' } \\
\text { 'LUNG' } \\
\text { 'THYROID' } \\
\text { 'GI' } \\
\text { 'OTHER' }\end{array}$ & $\begin{array}{l}\text { 'RED MARR' } \\
\text { 'BONE SUR' } \\
\text { 'BREAST' } \\
\text { 'LUNGS' } \\
\text { 'THYRODH' } \\
\text { 'LOWER LI' } \\
\text { ' LOWBR LI' }\end{array}$ & $\begin{array}{l}1 . \\
1: \\
1: \\
1: \\
1 .\end{array}$ & $\begin{array}{l}.39 \\
.39 \\
.19 \\
.39 \\
.39 \\
.39\end{array}$ & $\begin{array}{l}.61 \\
.61 \\
0 . \\
.61 \\
.00 \\
.61 \\
.61\end{array}$ & $\begin{array}{l}3.7 E-3 \\
1.5 E-4 \\
6.0 E-3 \\
5.1 E-3 \\
7.2 E-4 \\
1.5 E-2 \\
7.5 E-3\end{array}$ & $\begin{array}{l}3.7 E-3 \\
1.5 E-4 \\
1.7 E-2 \\
5.7 E-3 \\
7.2 E-3 \\
2.5 E-2 \\
1.3 E-2\end{array}$ \\
\hline
\end{tabular}

: RESULT I OPTIONS BLOCK, LADED BY INOUT1 C'STORED IN / INOUTH'

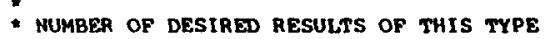

\section{TYPEINUMBER 27}

60 TYPEIOUTOO1 'ERL FAT/TOTAL'

'ERE FAT/TOTAL'
'ERL INJ/PRODROMAL VOMIT'
IERL INJ/DIARREA'

62 TYPE1OUTO03

TYYPEIOUTOOS

65 TYPEIOUTO06

67 TYPEIOUTO08

69 TYPE1OUT010

TYPE1OUT011

TYPE1OUTO13

4 TYPE1OUT015

'ERL INJJPNEUMONITIS'

'ERL INJ/THYROIDITIS'

'ERL. INJ/SKIN ERYTHEMA'

'ERL INJ/TRANSEP IDERMAL

'CAN FAT/TOTAL'

CAN FAT/THYROID

'CAN FAT/BREAST

'CAN FAT/LEUEMAIA'

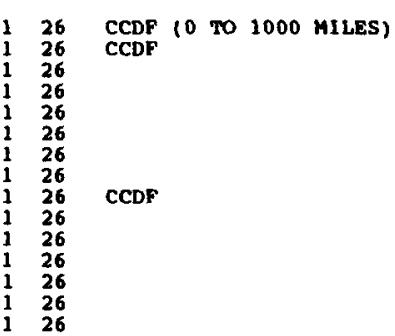




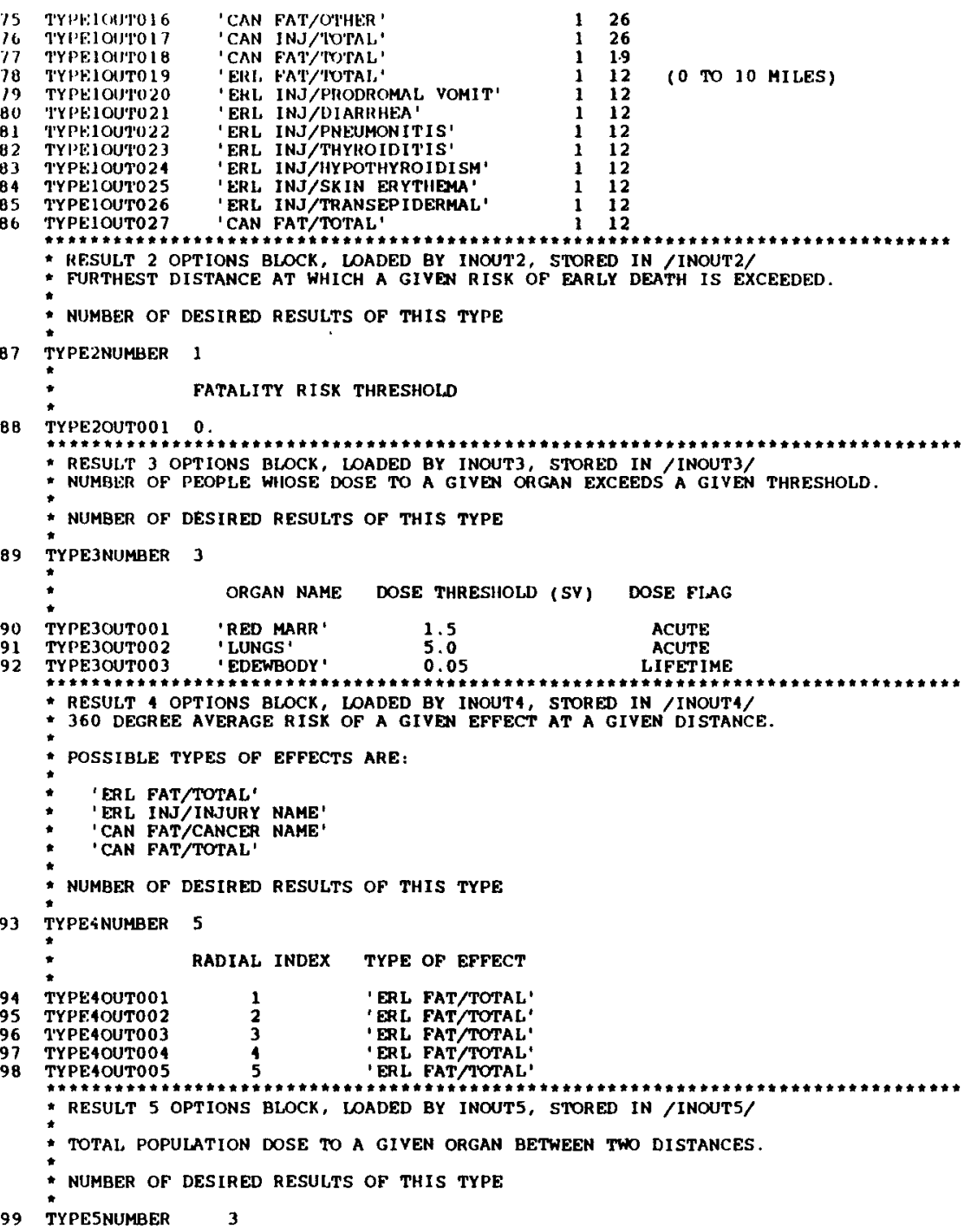




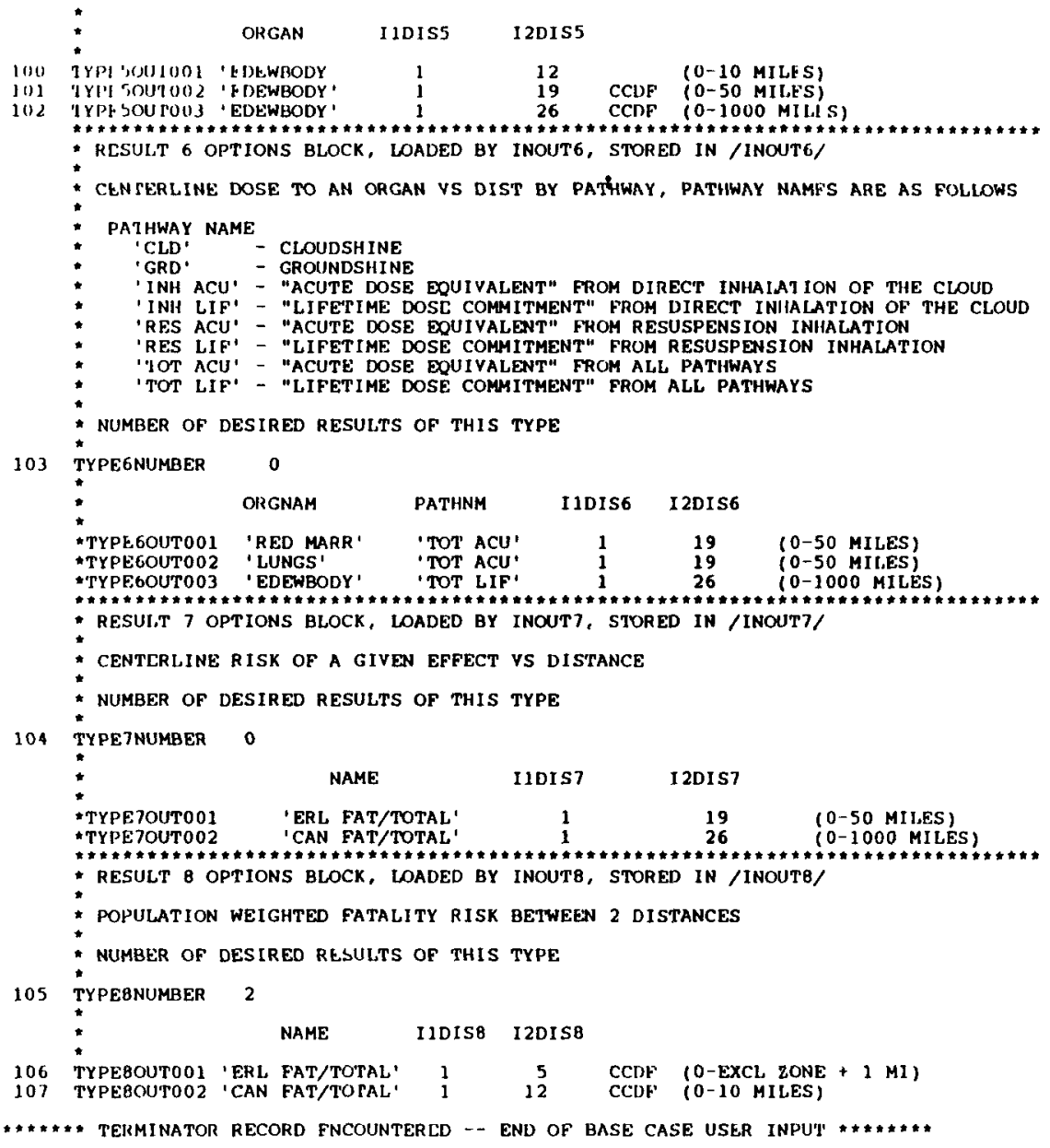

USIR INPUT PROCESSTNG SUMMARY - BASE CASE

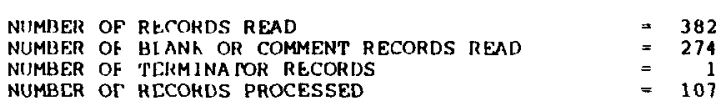




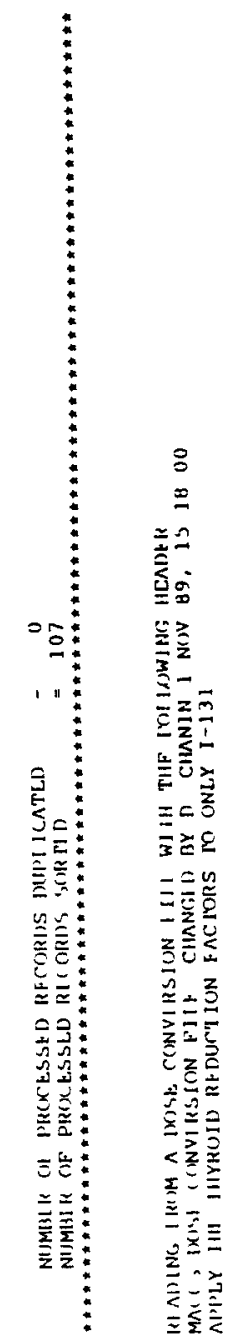

D-42 


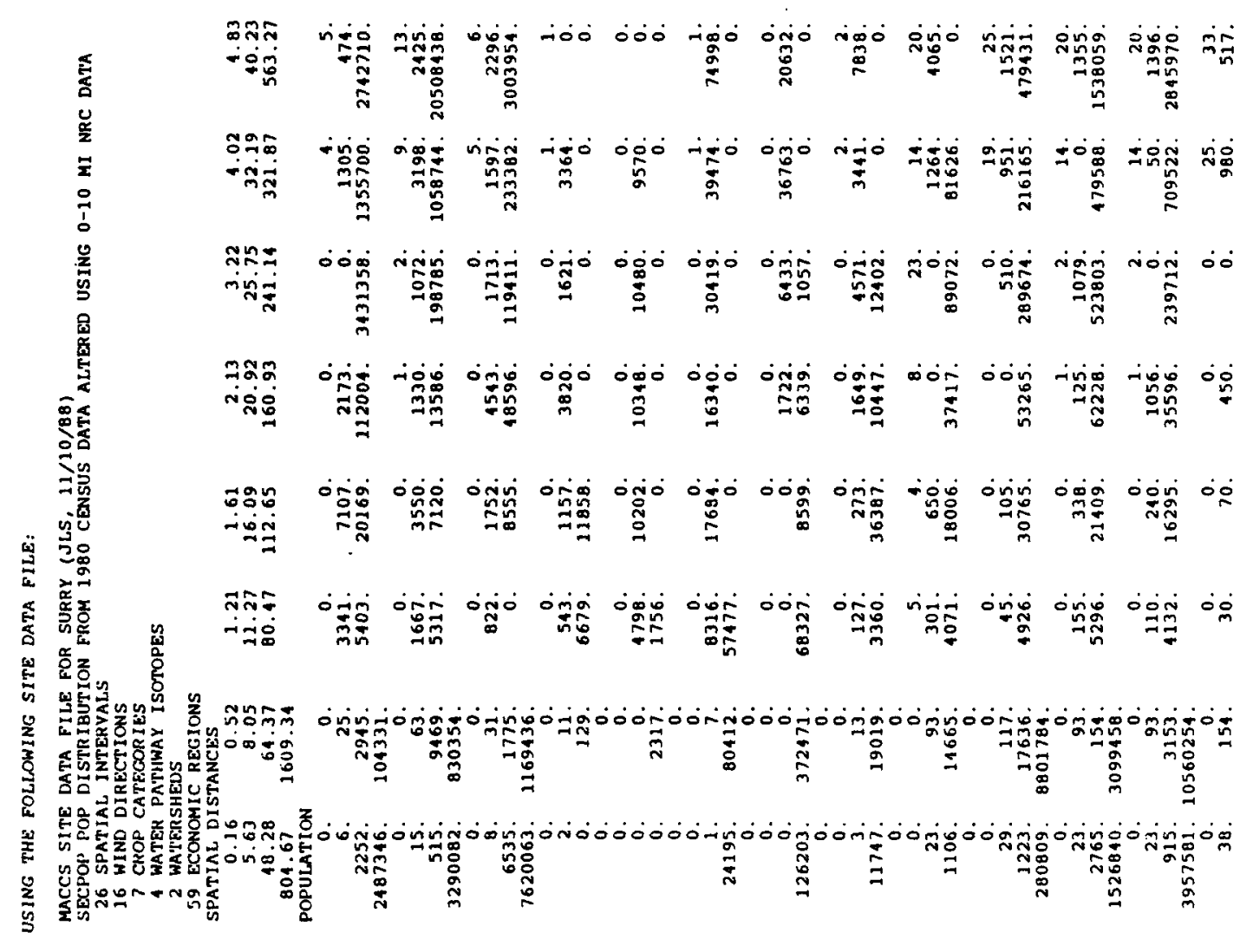




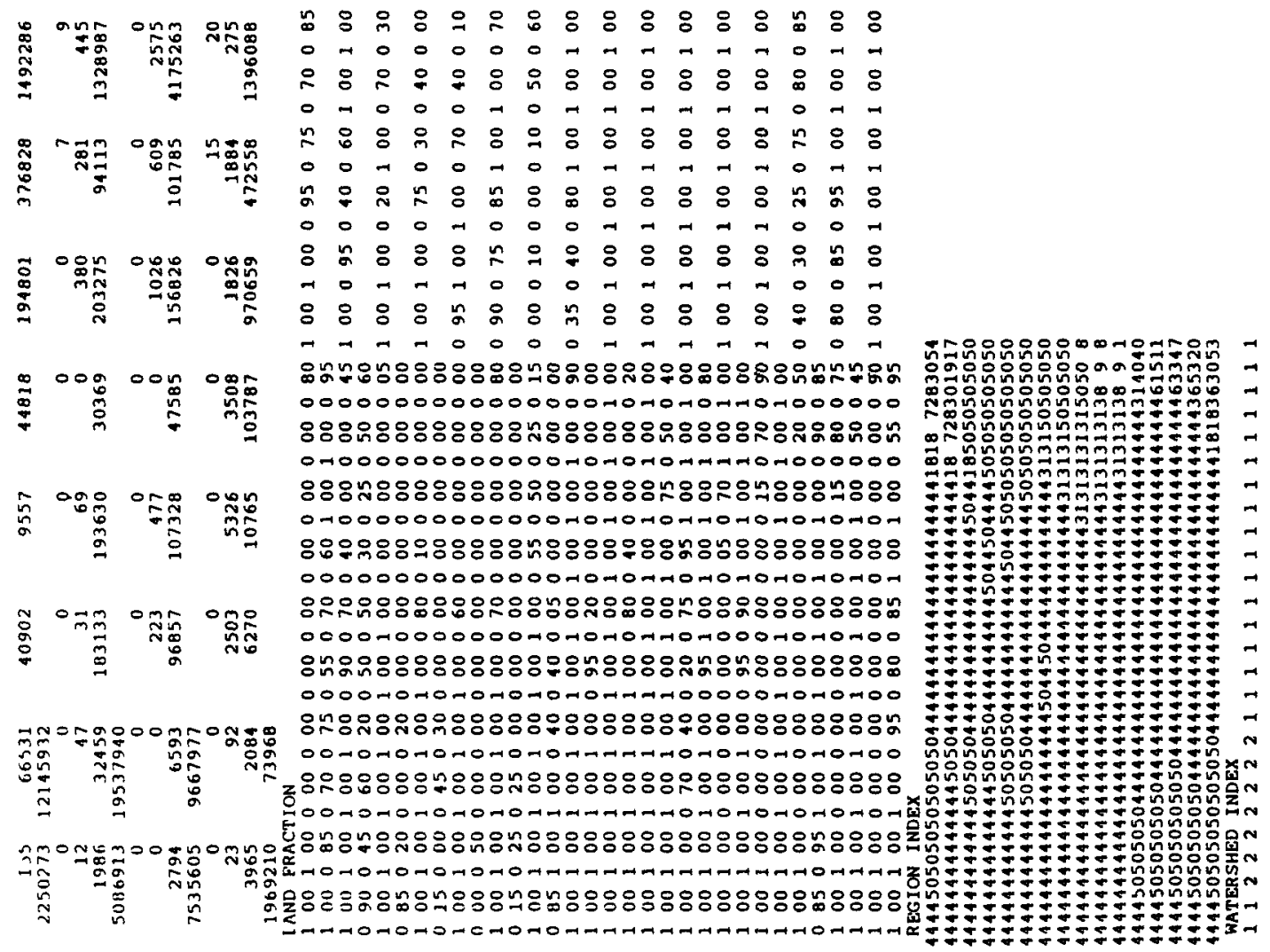



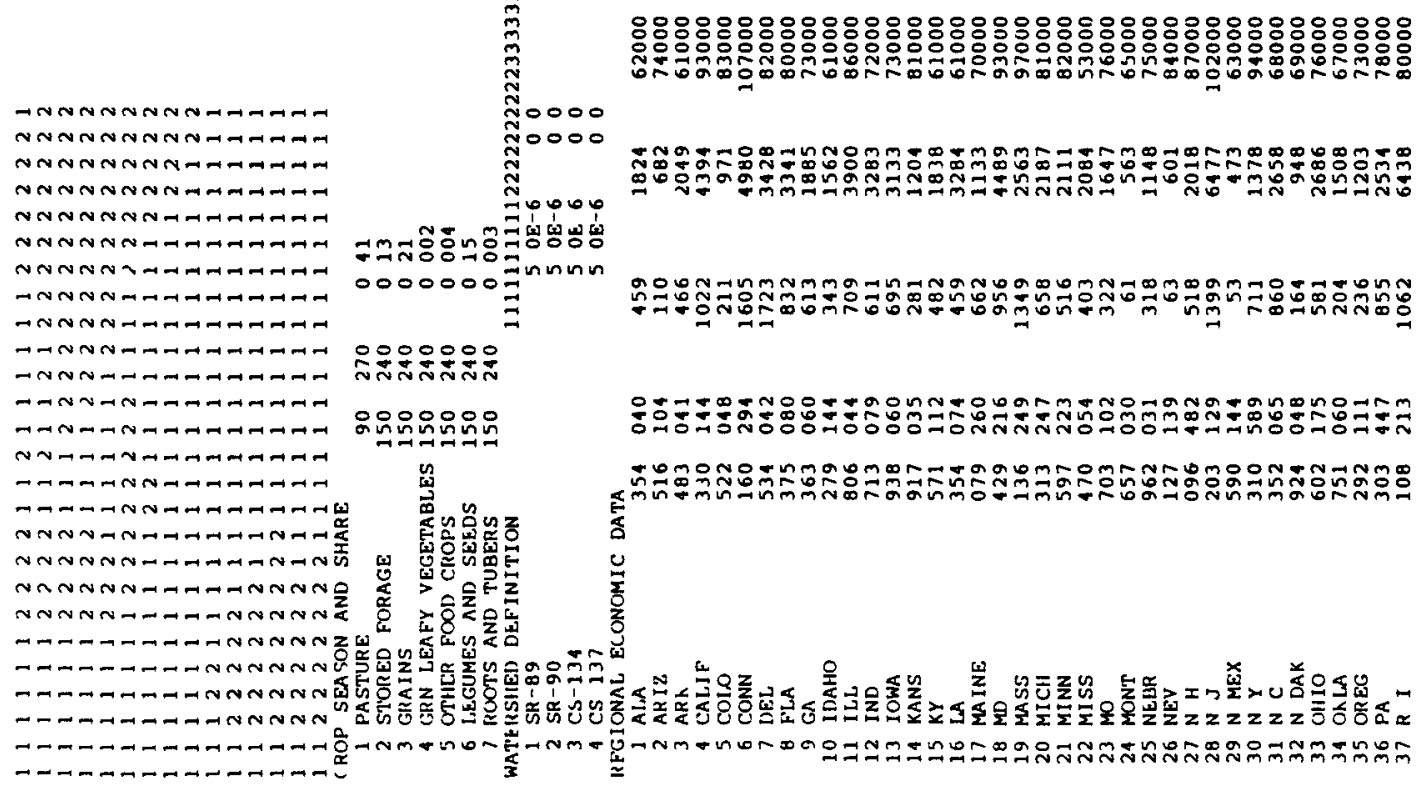

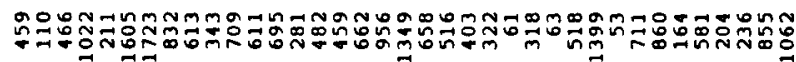

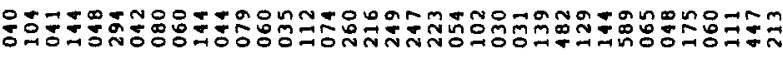

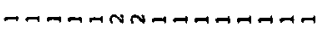

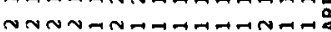

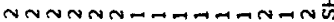

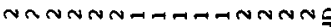

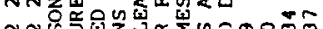
- - -

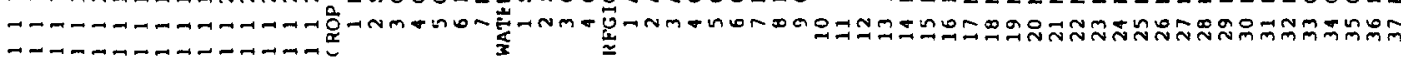




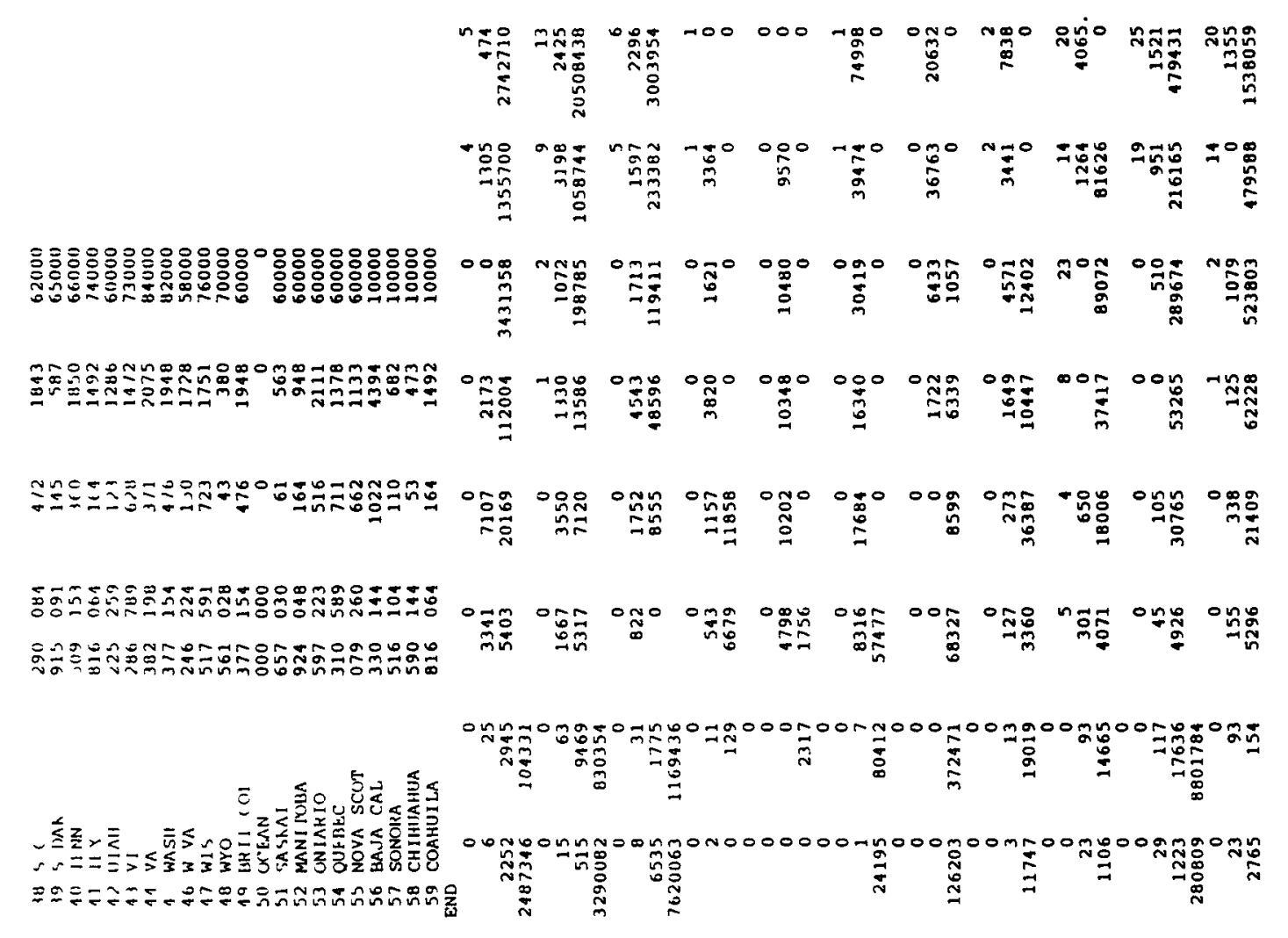




\begin{tabular}{|c|c|c|c|c|c|c|c|}
\hline 13268 & $\begin{array}{r}3099458 \\
90 \\
93 \\
1153\end{array}$ & $\begin{array}{r}110 \\
4132 \\
4132\end{array}$ & $\begin{array}{r}240^{\circ} \\
16295\end{array}$ & $\begin{array}{r}1056 \\
35596\end{array}$ & $\begin{array}{r}2 \\
0 \\
239712\end{array}$ & $\begin{array}{r}14 \\
50 \\
709522\end{array}$ & $\begin{array}{r}20 \\
2066 \\
2845970\end{array}$ \\
\hline & & $\begin{array}{r}0 \\
30 \\
40902\end{array}$ & $\begin{array}{r}70 \\
9557\end{array}$ & $\begin{array}{r}450 \\
41818\end{array}$ & $\begin{array}{r}0 \\
0 \\
194801\end{array}$ & $\begin{array}{r}25 \\
900 \\
376828\end{array}$ & \\
\hline & & $\begin{array}{r}30 \\
31 \\
183133\end{array}$ & $\begin{array}{r}09 \\
193630\end{array}$ & $\begin{array}{r}0 \\
30369\end{array}$ & $\begin{array}{r}380 \\
203275\end{array}$ & $\begin{array}{r}287 \\
9413\end{array}$ & \\
\hline & & $\begin{array}{r}223 \\
96857\end{array}$ & $\begin{array}{r}47 \\
107328\end{array}$ & $\begin{array}{r}0 \\
47585 \\
4\end{array}$ & $\begin{array}{r}1026 \\
156826\end{array}$ & $\begin{array}{r}609 \\
101785\end{array}$ & $\begin{array}{r}2575 \\
4175263\end{array}$ \\
\hline $\begin{array}{r}32 \\
1969 \\
196921\end{array}$ & $\begin{array}{r}92 \\
2084 \\
73968\end{array}$ & $\begin{array}{r}2500 \\
6270 \\
6270\end{array}$ & $\begin{array}{r}5326 \\
10765\end{array}$ & $\begin{array}{r}3508 \\
103787\end{array}$ & $\begin{array}{r}1826 \\
970659\end{array}$ & $\begin{array}{r}185 \\
1884 \\
472558\end{array}$ & $\begin{array}{r}20 \\
275 \\
1396008\end{array}$ \\
\hline
\end{tabular}

......... gEgINNING OF CHANGE CASE 1 USER INPUT ..........

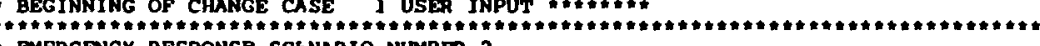

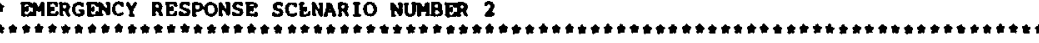

EVACUATION ZONE DATA BLCKK, LOADED BY EVNETW, STORED IN /NETWOR/, /DOPTIO/

SPECIFIC DESCRIPTION OF THE EHERGEHCY RESPONSE SCENARIO BEING USED

108 EZEANAM2001 'NO EVACUATION, RELOCATION MOOELS APPLYY EVERYWHERE'

: WEIGHTING FRACTION APPLicable to this SCENARIO

109 EZWTFRACO01 005 OS

LAST RING IN THE Movement zone

...110..... EZLASMOVOO 1 RECORD NUMBER (A 2ERO TURNS OFF THE EVACUATION MODEL)....

**.**** terminator Record encoUntered -- END OF CHANGe CASE 1 USER INPUT ....*...**

USER INPUT PROCESSING SUMMARY - CHANGE CASE 1

NUMBER OF RECORDS CHANGED $=3$

\section{no EVACUATION REQUESTED}

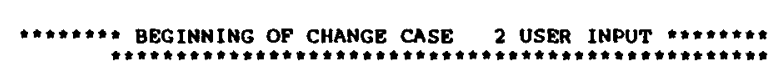

- EMERGENCY RESPONSE SCENARIO NUMBER 3

EVACUATION ZONE DATA BLOCK, LOADED BY EVEETW, STORED IN /NETWOR/, /BOPTIO/

- SPECIFIC DESCRIPTION OF THE EMERGENCY RESPONSE SCENARIO BEING USED

111. EZEAMAM2001
........ SHELTER ING WITHTN 10 MILES, RELOCATION MODELS APPLY ELSEWHERE' 
- weighting fraction applicable to this scenario

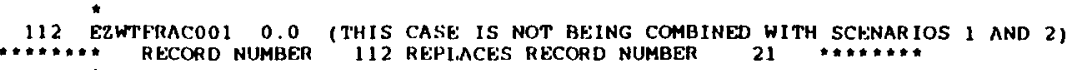

* time to take shelipel in the outer sheltem zone (SEcondos from oalarim)

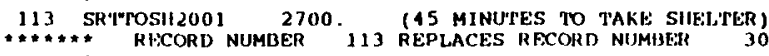

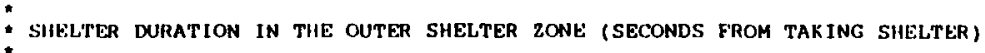

114 SRSIIELI'2001 43200 i1 (12 HOUR SHELTER DURATION)
RECORD NUMBER

: LAS'T uing of the outer shelter zone

115 SRLASHE2001 12 (OUTER SHELTER ZONE EXTENDS FROM O TO 10 MILES)

........ terminator ReCORD ENCOUNTERED -- END OF CHANGE CASE 2 USER INPUT .........

USER INPUT PROCESSING SUMATAYY - CHANGE CASE 2

NUMBER OF RECORDS ADDED

NO EVACUATION REQUESTED

USER INPUT IS READ FROM UNIT 26
RECORD IOENTIIER FIELOS II CHARACTERS LONG ARE EXPECTED.

THE FIRST 100 COLUNANS OF EACH INPUT RECORD ARE PROCESSED.

RECORD

\section{RECORD}

- general descriptive title describing this "Chronc" inPut file

1 CHCHNAMEOO1 ' IN3A. INP, SURRY, SAMPLE PROBLLM A, CHRONC'

- gaergeancy Response cost data block

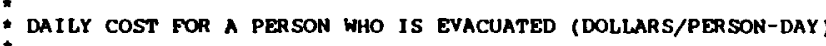

2 ChEVACSTO01 27.00 (INCLUDES FOOD AND hOUSING COSTS BUT NOT LOST INCOME)

DAILY COST FOR A PERSON HHO IS RELOCATED (DOLLARS/PERSON-DAY)

3 CHRELCSTO01 27.00 (INCLUDES FOOD AND HOUSING COSTS BUT NOT LOST INCOME)

* LONG TERH PROTECTIVE ACtION DATA BLOCK

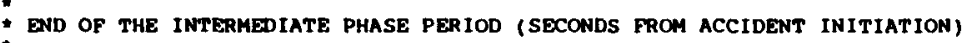

4 CHTMIPNDOO1 604800

(7 DAYs, NO INTERMEDIATE PHASE)

- LONG-TERM PHASE DOSE PROJECTION PERIOD, THE DURATION OF THE EXPOSURE

5 CHTMPactool $1.58 E 6$ (5 YeArs)

DOSE CRITERION FOR INTERmediate phase RELOCATION (sv) 
6 CHDSCRTIO01 1 OES (NO INTERMEDIATE PHASE RELOCATION)

" dose chiterion for long-tekm phase relocation (SV)

7 CHDSCRLtool 0 04

" critical organ name for long-term actions

B CHCRTOCROOI 'EDEWBODY'

- DEconpraminarion plan oata bloce

* number of LEvels of decontamination

9 CHLVLdeCO01 2

DEContamination times corresponding to the lvLdec levels of Decontamination

: (SECONDS)

10 CHTIMUEC001 5 184E6 1 0368E 7 (60, 120 DAYS $)$

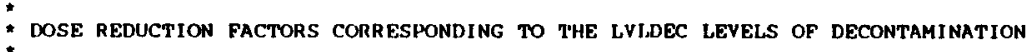

11 CHDSRFCTO01 $3 \quad 15$

cost of Farm decontamination per farmland unit aRea (DOLlars/hectake)

* for the various levels of Decontamination

12 CHCDFRMOOON $5625 \quad 1250$

* COST OF nONFARM DEContamination PER RES IDENT PERSON (DOLlars/PERSON)

13 CHCDNFRMO01 $3000 \quad 8000$

- fraction of farmland decontamination cost due to labor

: FRACTION OF FARMLAND DECONTAMINATION COS

14. Cherfoloo01 3

35

* FRACTION OF NON-Farm DEcontamination COST DUE to labor
FOR THE VAR IOUS DECONTAMINATION LEVELS

15 CHFRnFDLO01 $7 \quad 5$

- fraction of tTime workers in farm areas spend in contaminated areas

- For trHe various decontamination levels

16 CHTI'WKFO001 10

: Fraction of time worhers in non farm areas SPEnd in contamina fed areas

17 CHTFWhNF001 $33 \quad 33$

* average cost of decontamination labor (Dollars/man-year)

18 CHDLBCSTOO1 35000

- interdicítion cost Data block

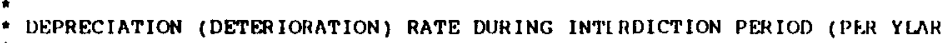

19 Cidprate001 20 (VALUe Obtained from wash 1400, appendix 6)

* INYESTMENT INCOME RETURN (DISCOUNT RATE) DURING INTERDICTION PERIOD (PER YEAR ) 
* 'Illis value should be drrivid as a real, return rate adjusted for inflation

20

CHISGKAlloU1 12 (VALUE UBTAINED FROM WASH 1400, APPFNDIX 6)

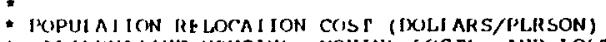

* AL 1H HNAIIVE HOUSING, MOVING COSTS, ANI) IOST INCOME FOR PROPIE IN

21 CIIX)PCS1001 5000

" GKOINDSHINE WEATHERING DEFINITION DATA BLOCK

* number of terms in the groundsiline weathering relationship (E1THer 1 or 2)

22 CHNGWTRMOOI 2

* Groundshine weathering coefricients

23 CIIGWCOEFOO1 O 5 O 5

(JON HELTON)

* lali lives corresponding to the groundshine weather ing coeffictents (s)

24 CHTGHHLFOO1 1 6E7 $2869 \quad$ (JON HELTON)

"RESUSPENSION WEATHER ING DEFINITION DATA BLOCK

- number of terms in the resuspension heathering relationship

25 CHNRWTRMOO1 3

: RESUSPENSION CONCENTRATION COEFFICIENTS (/ MEIER)

26 CHRWCOEF 001 1 OE 5 1 OE-7 1 OE-9 (VALUES HERE SELECTED HY JON HEI TON)

Half-lives corresponding to the Resuspension Concenthation coefficients (s)

27 CHTRHHLFO01 1687 16E8 1 6E9 16 MONTHS, 5 YEARS, 50 YEARS

* SITE Region Description data block

- HRaction of arga that is Land in the region

28 CHFracloool 095 (ROUGH GUess Value, Site file overrtoes this value)

- Fraction of land devớted to farming in the region

29 Chfrcfrmoo1 0382 (VIrginia state value, Site file overrides this value)

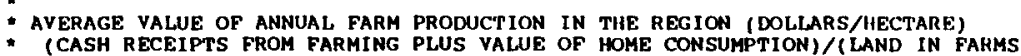

30 ChFrmprdo01 3710 (VIrginia state value, site file overkides this value)

: FRACTION OF FARM PRODUCTION RESULTING FROM DAIRY PRODUC'ION IN THE RLGION
(VALUE OF MILK PRODUCED)/(CASH RECEIPTS FROM FARHIHG PLUS HOME CONSUMPTION)

31 CHDPFRCT001 0198 (VIRGINIA STATE VALUE, SITE FILE OVERRIDES THIS VALUE)

: Value of Farm wealqu (Dollars Hectare)

PUE

32 CHYALWF0001 2613 * SURRY

Fraction of farm WEALTH IN IMPRovements for the REgion 


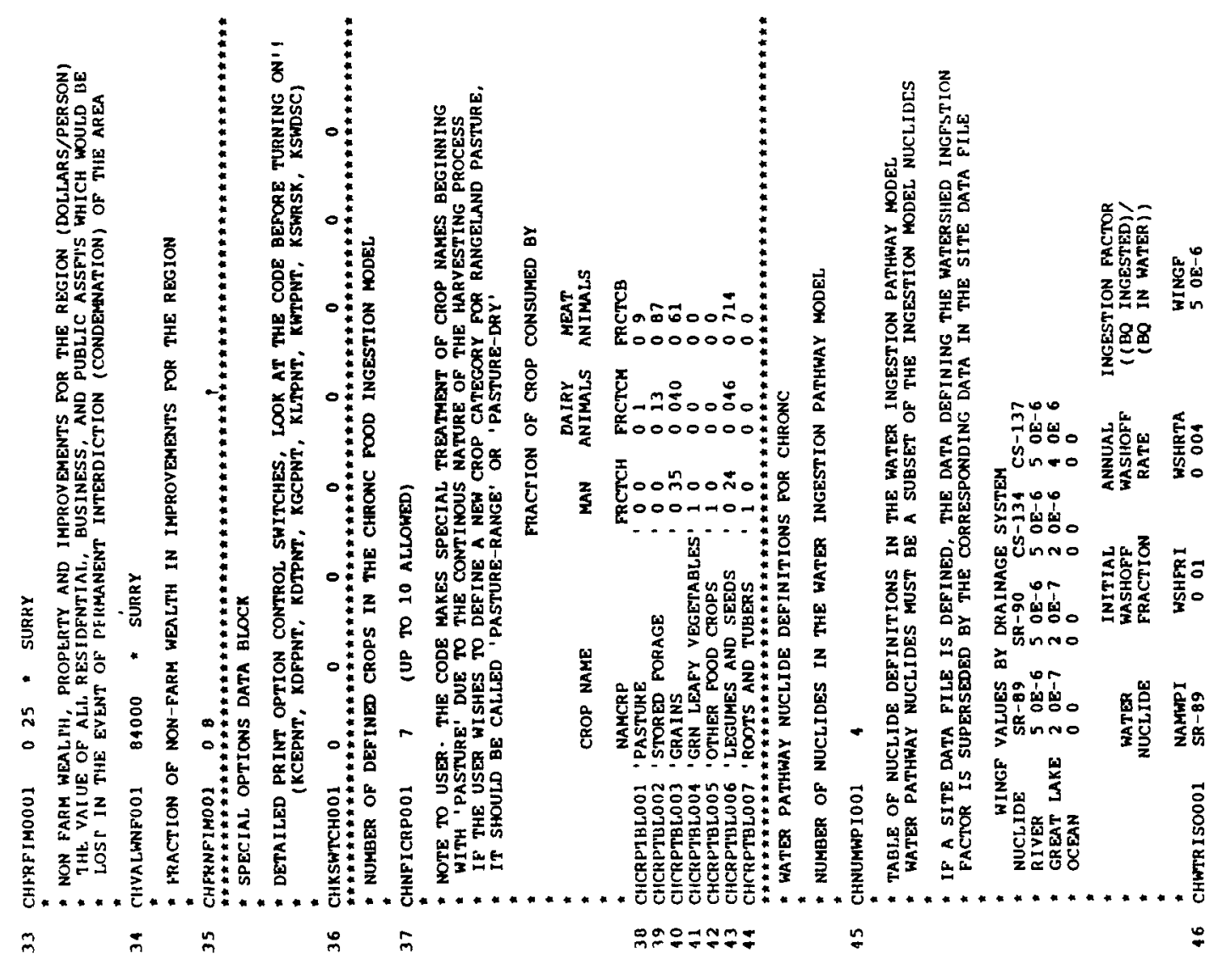




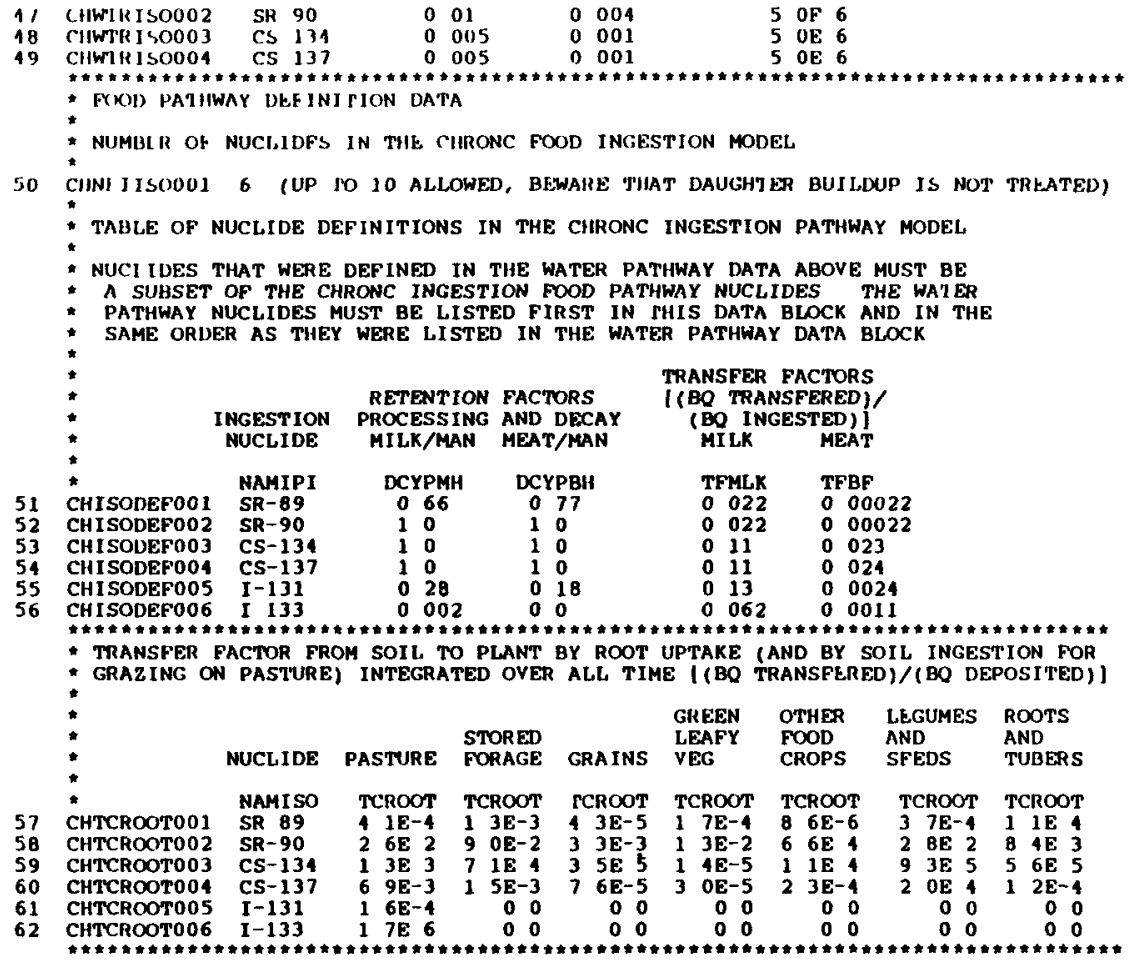

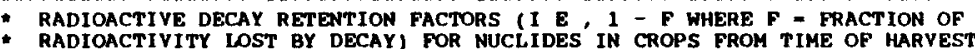
- RADIOACTIVITY LOST BY DECAY) FOR NUCLIDES' IN CROPS FFOM
TO TIME OF CONSUMPTION BY HUMANS (FRACTION RETAINED)

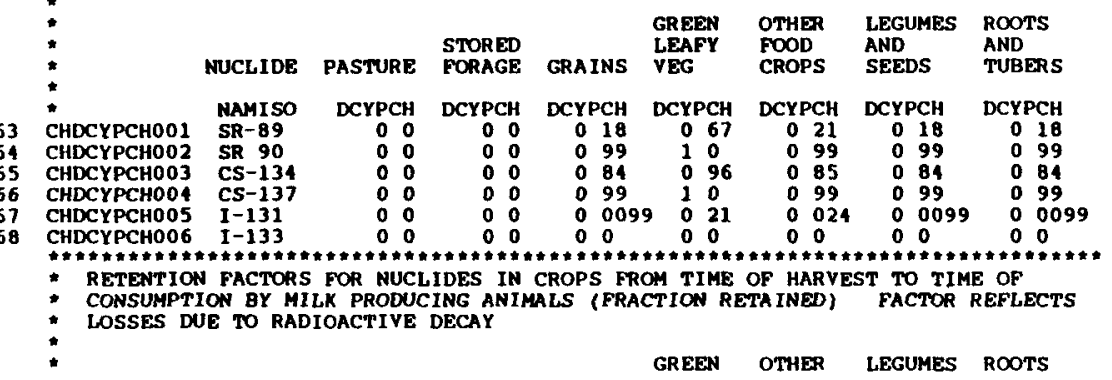




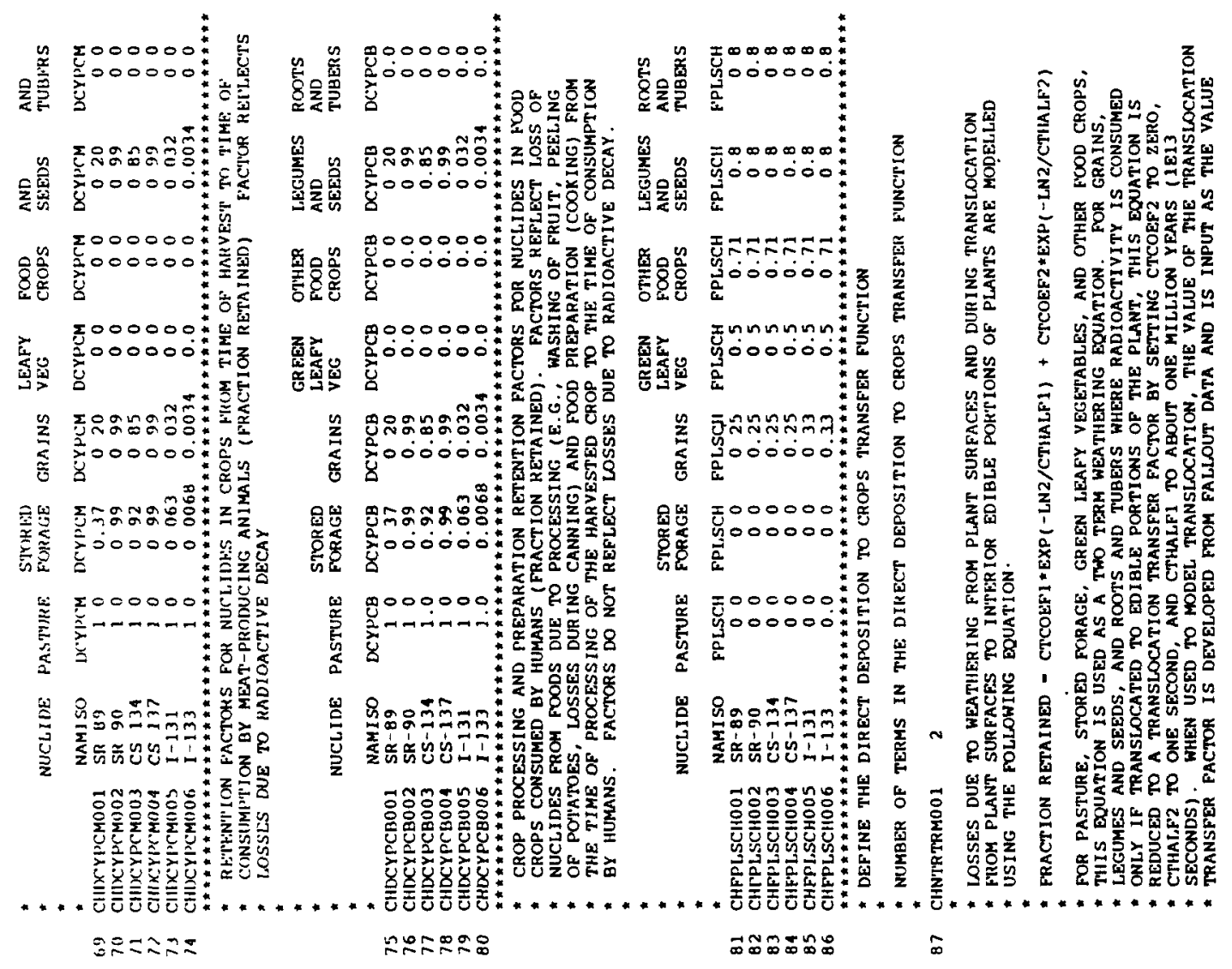


(1) CICOEF1.

'WWO TIME PER IODS ARE ISSED FOR WEATHERING, THE FIRST IS 14 DAYS LONG (1.21E6
SECONDS) AND THE SECOND IS 50 DAYS LONG (4.32E6 SECONDS).

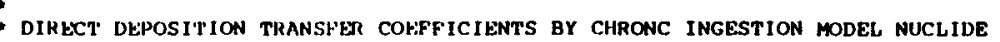

- ((DQ TRANSFERED)/(BQ DEPOSITED))

- term 1 nuclide pasture STOReo

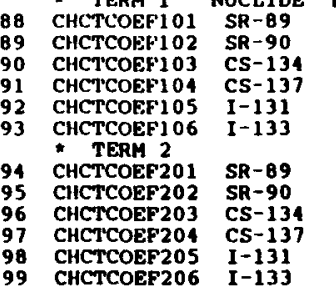

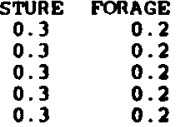

$\begin{array}{lr}\text { OGE } & \text { GRAINS } \\ 0.2 & 0.01 \\ 0.2 & 0.01 \\ 0.2 & 0.05 \\ 0.2 & 0.05 \\ 0.2 & 0.0\end{array}$

GREEN OTHER LEGUMES ROOTS

$\begin{array}{lll}0.2 & \text { SEEDS } & \text { TUBERS } \\ 0.2 & 0.005 & 0.0006 \\ 0.2 & 0.005 & 0.0006\end{array}$

0.3

0.076
0.076
0.076

0.076
0.076
0.076
0.076

0.05
0.05
0.05
0.05
0.05
0.05

$\begin{array}{ll}0.0 & 0.24 \\ 0.24\end{array}$

$\begin{array}{lll}0.2 & 0.01 & 0.025 \\ 0.2 & 0.01 & 0.025\end{array}$

$\begin{array}{llll}0.076 & 0.05 & 0.0 & 0.06 \\ 0.05 & 0.0 & 0.06\end{array}$

$\begin{array}{lll}0.2 & 0.0 & 0.0 \\ 0.2 & 0.0 & 0.0\end{array}$

$0.05 \quad 0.0 \quad 0.0$

:

CROP TRANSFEr hALF-LIVES BY CHRONC INGESTION MODEL NUCLIDE (SECONDS)

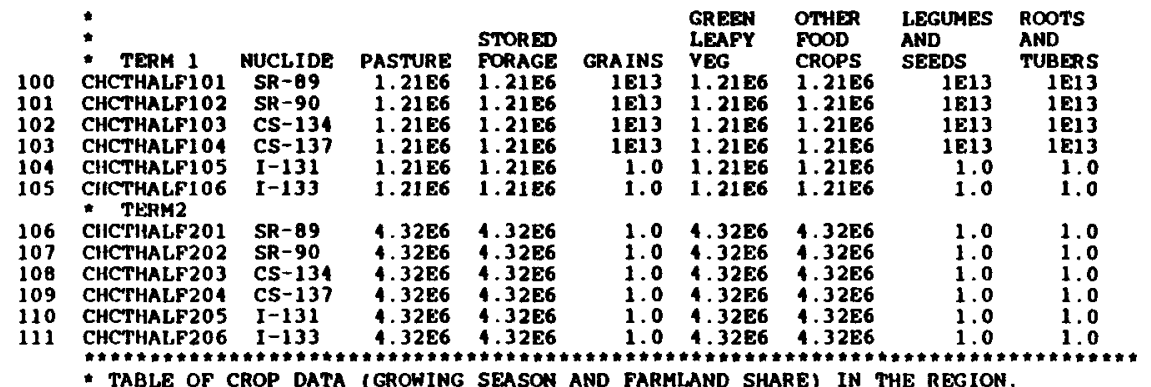

- TABLE OF CROP DATa (GROHING SEASON AND FARMLAND SHARE) IN THE REGion.

* if a site data file is being used (as specified on the early user inPut file), : THEN DATA FROM THE STTE FILE (AND NOT THE DATA BELOW) IS USED FOR THE belon aRE not uTilized in THE calculations.

: if a site data file is not being used, the data below is used in its stread.

- in all cases, the user must supply valid values for the parameters below.

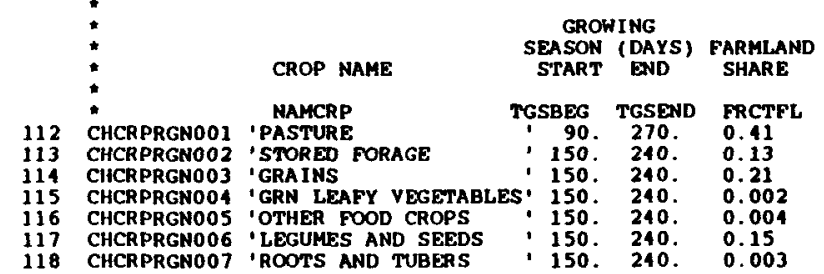


119 CHCOUPLDO01 .FALSE. (GRONING-SEASON ACTIONS INDEPENDENT OF LONG-TERM ACTIONS) * PUOTRCTIVE ACt ION GUIDES EOR THE GRONING-SEASON PATHWAY FOR BOTH MILK DEFINED SEPARATELYY AS PSCHLX AHD PSCOTH.

* THese values are calculated outside of the code by assuming that the - ARE THE GROUND CONCENTRATION OF EACH NUCLIDE (ASSUMING A SINGLE NUCLIDE * RELEASE) WIIICH WOULD RESULT IN A MAXIMALLY EXPOSED INDIVIDUAL RECEIVING * A DOSE NOT EXCEEDING O.O5 SIEVERT EFFECTIVE-WHOLE-BODY-DOSE-EOUIVALENT VALENT

:

$$
\text { NUCLIDE }
$$$$
\begin{aligned}
& \text { MILK AND OTHER CROPS } \\
& \text { PRODUCTS AND PRODUCTS }
\end{aligned}
$$

NAMIPI

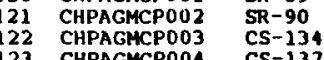

124 CHPAGHCP005 $1-131$

$$
\text { PSCMLK PSCOTH }
$$

$\begin{array}{ll}\text { PSCMLK } & \text { PSCOTH } \\ 2.2 \mathrm{EO} 07 & \mathbf{2 . 2 E 0 7}\end{array}$

2.4E05

2.7805

2.2807
2.4805
2.7805

- PROTECTIVE ACTION GUIDES FOR LONG-TERM AGRICULTURAL PRODUCTION, GCMAXR, AND
THE RATE AT WHICH EACH RADIONUCLIDE BECOMES UNAVAILABLE FOR UPTAKE, OROOT.

* gcmaxr values are calculated outside of the code by assuming that the accident - concentration of each nuclide (Assuming a single nuClide RELEase) which * WOULD RESULT IN A MAXIMALLY EXPOSED INDIVIDUAL RECEIVING A DOSE COMMI TMENT FROM T=0 TO T-INFINITY. THE DECISION TO CALCULATE GCMAXR VALUES THE UNITS ARE (BQ/SOUARE-MERER).

OROOT VAlUes aRe DER IVED to take ACCOUNT OF THE COMBINED EFFECT OF * PERCOLATION, CHEMICAL BINDING AND RADIOACTIVE DECAY IN REDUCING THE AMOUNT
OF MATER IAL AVAILABLE FOR LONG-TERM UPTAKE INTO FOOD IN UNITS OF (PER YEAR). :

126
127
128
129
130
131

SR-B9
SR-90

$\begin{array}{ll}\text { CHPAGLTSOOOS } & \mathrm{I}-131 \\ \mathrm{I}-133\end{array}$

GCMAXR
1.8EQ
$3.7 E 4$

1. 1.820

OROOT

- AN ALTERNATIVE METHOD FOR DERIVING YALUES FOR GCMAXR IS TO BASE THEM

- IF THE USER WOULD LIKE TO ADOPT THIS APPROACH, A SET OF APPROPRIATE - CORRESPOND TO A MAXIMALLY EXPOSED INDIVIDUAL RECEIVING A DOSE COMMITMENT - NO GREATER THAN O. 005 SIEVERT EFFECTIVE-HIOLL-BODY-DOSE-EOUIVALENT OR

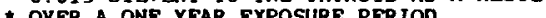

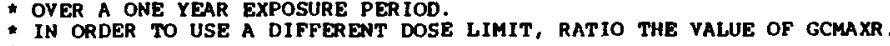

*

*CHPAGLTSOO

*CHPAGLTSO03

$\underset{S R \rightarrow-89}{\text { NAMIPI }}$

SR -89
$\mathrm{SR}-90$
$\mathrm{CS}-134$
$\mathrm{CS}-137$

GCMAXR
$1.8 E 8$
$5.9 E 5$
$9.2 E 6$
$7.4 E 6$

OROOT
4.9
0.065
0.59 


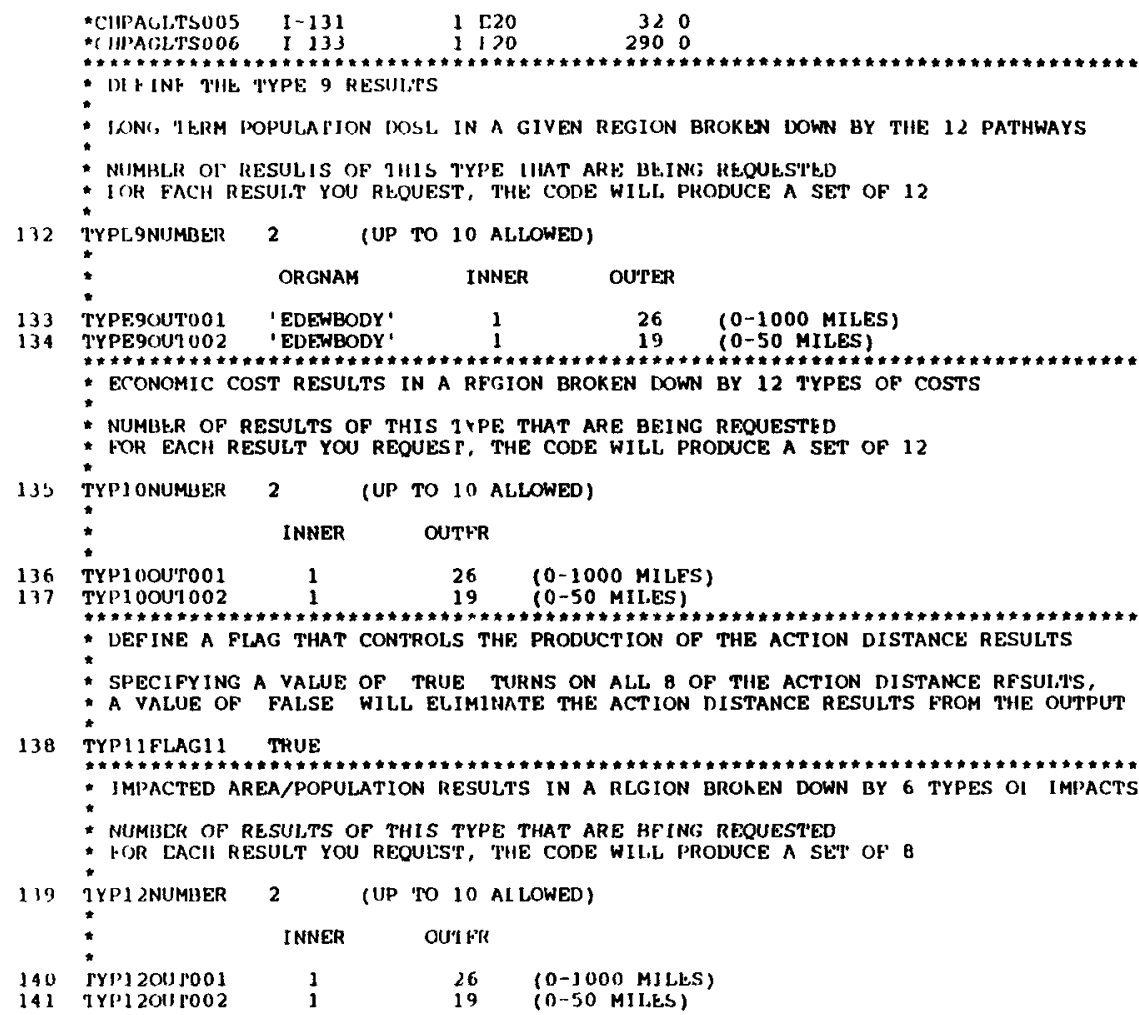

* *.**** 1ERMTHATOR RECORD ENCOUN FERED - - END OF BASF CASE USER INPUI * ******

USLK INPIT PROCESSING SUMMARY BASE CASE

NUIMIBER OF RFCORIS READ

NUMBLR OF BI.ANK OR COMTNT RECORDS READ

NIMHER OF TFRMINATOR RECORUS
NIMBESA OF KLCORUS PROCESSED

NUMIJER OF PRUCESSED RECORIS DUPLICATED

$=531$
$=395$
$=1$
$=141$
$=0$

A SITE DATA FILE IS BEING USED FOR BOTH "EARLY" AND "CHRONC"

9 ORGANS ARE DEFINED IN THE "EARLY" MODEL 


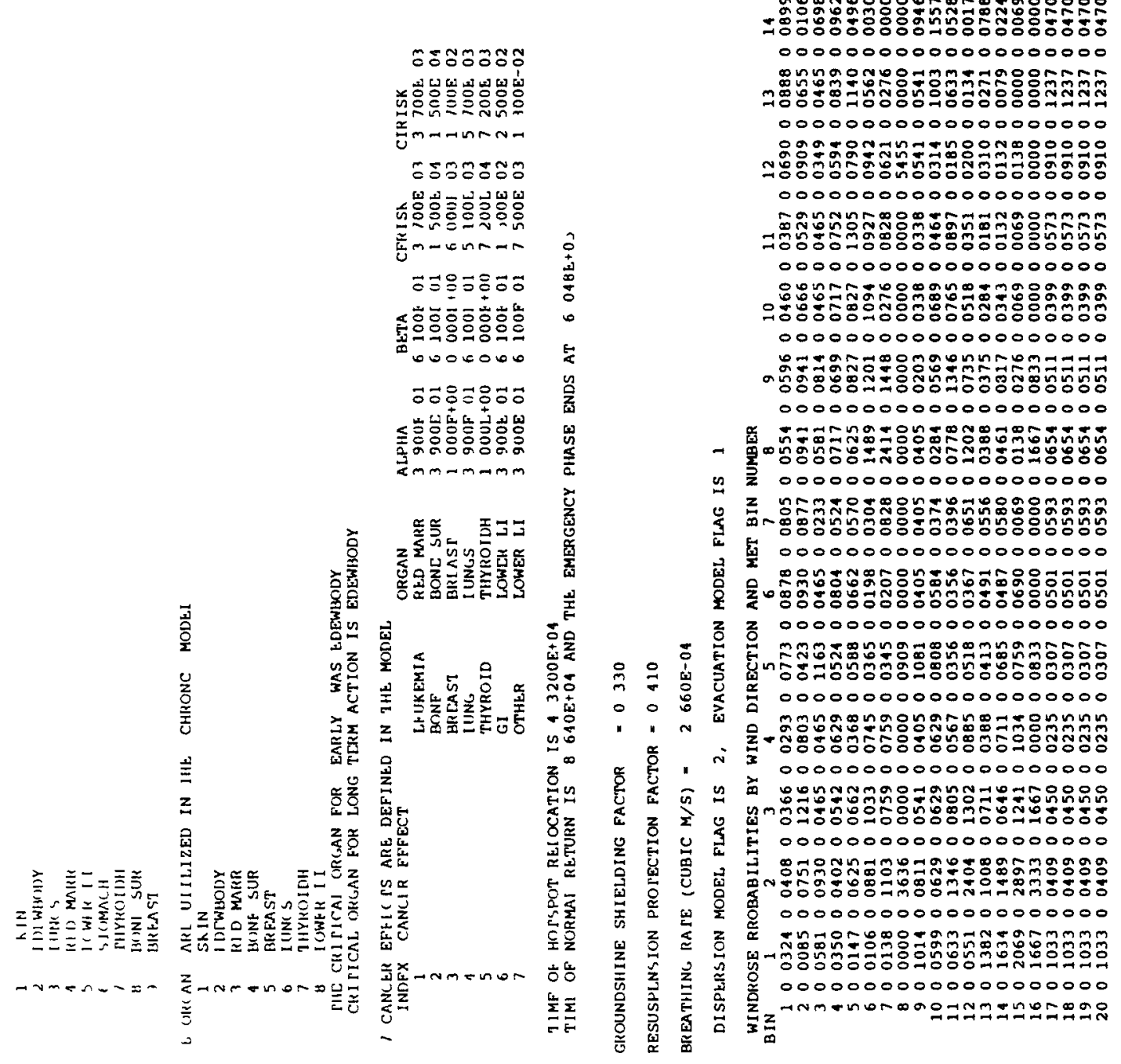




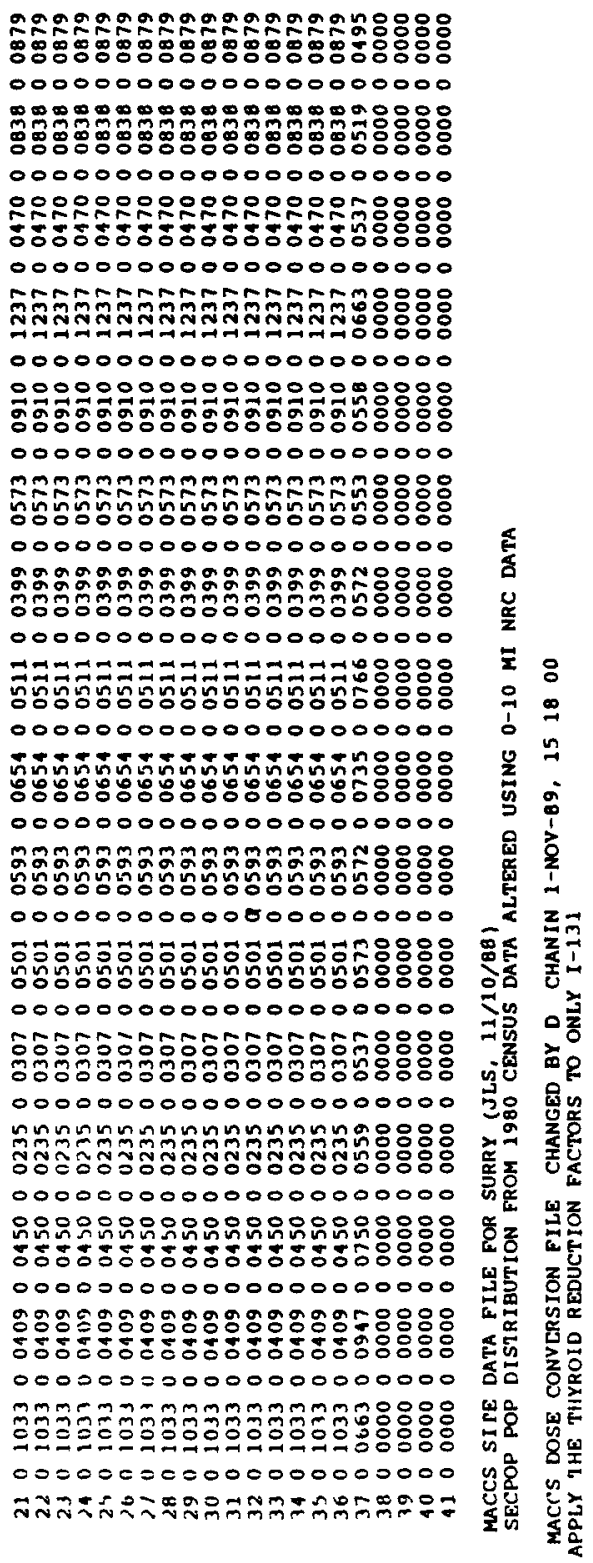




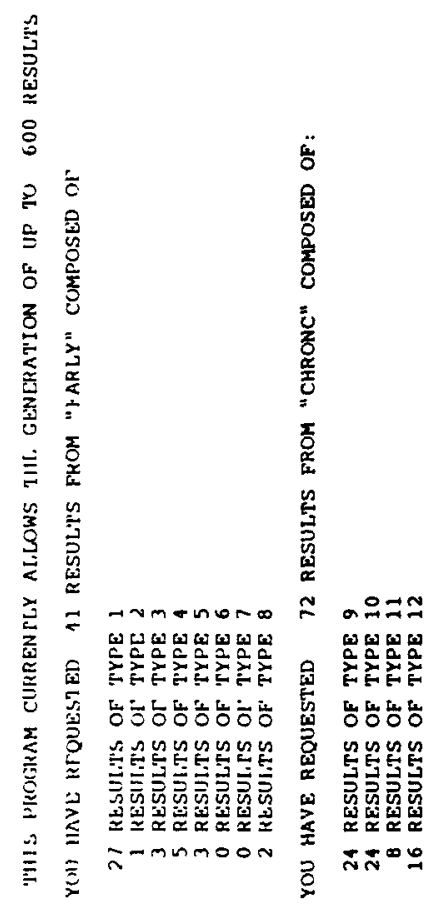

D-59 


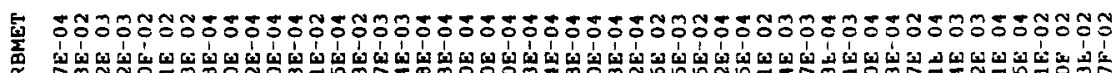

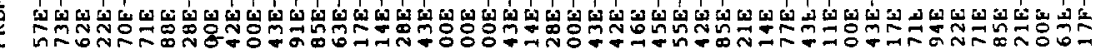

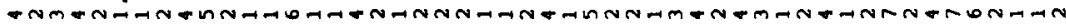

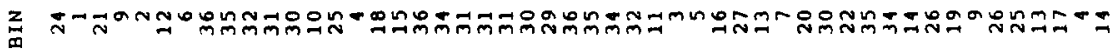

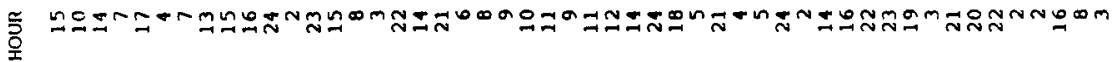

亡ั.

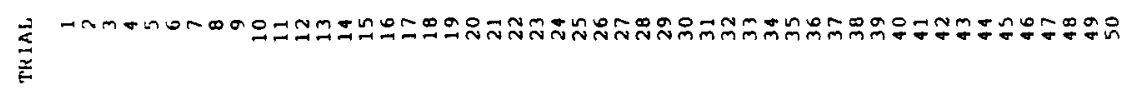

D- 60 


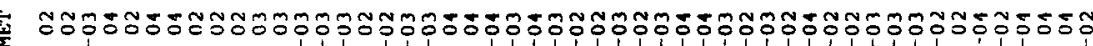

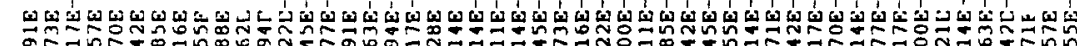

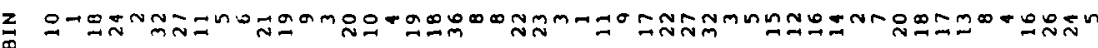

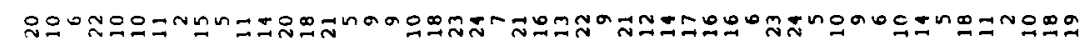
○

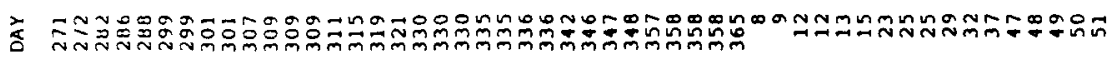

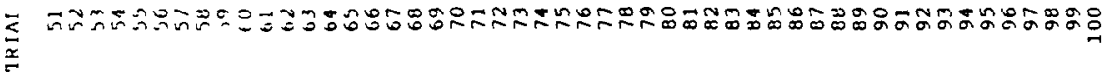




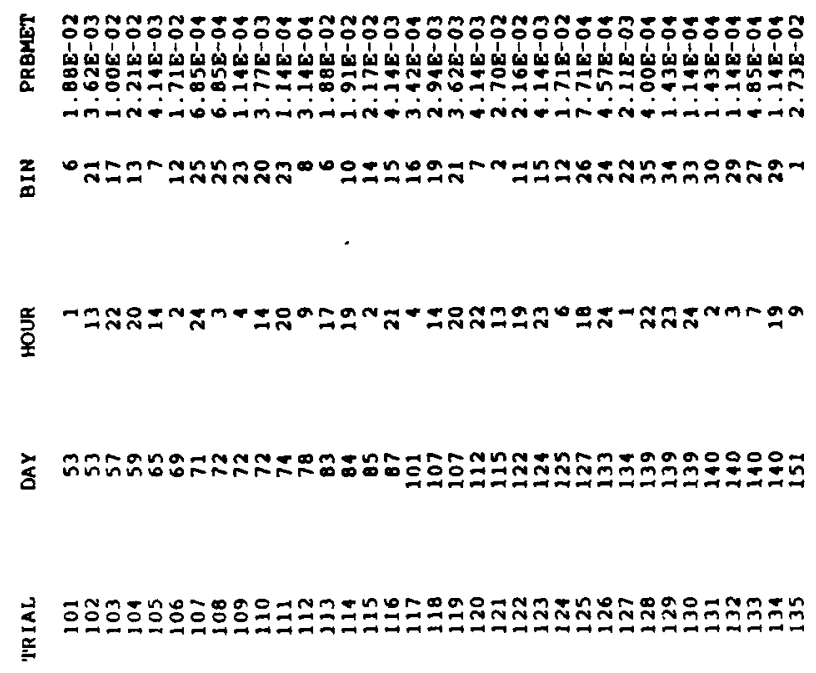

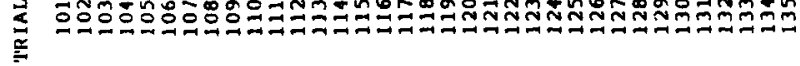




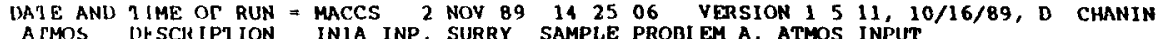

ATRIY DI SCRIPTION = INIA INP, SURRY SAMPLE PROBI EMA A, ATMOS INPUT

SAMPLE PROBLEM $A$, CHRONC

SOUIRCE PHKM 1 OH 2
SLCONU DHAF"1 1150 WOHST CASE SOURCE TERM FOR EARLY FATALITIES

OVERAIL RESULIS OBTAINED BY COMBINING 3 EMERGENCY RESPONSE COHORTS FROM "EARIY" WITH THE WEIGHTING FRACTIONS BELOW APPLIED TO THEM

COHORT 1 = EVACUATION WITHIN 10 MILES, RELOCATIÓN MODELS APPLY ELSEWHERE

FACTION OF THE PEOPLE

0950

COHORT 2 = NO EVACUATION, RELOCATION MODELS APPLY EVERYHHERE

0.050

COHORI 3 - SHELTERING WITHIN 10 MILES, RELOCATION MODELS APPLY ELSEWHERE

0000

AND THEN MERGING THE 3 RESULTS ABOVE WITH THE SINGLE SET OF RESULTS FROM "CHRONC" DESCRIBED BELOH

COHORT 4 = IN3A INP, SURRY, SAMPLE PROBLEM A, CHRONC

RESULTS WHICH ARE PRODUCED ONLY BY "EARLY" OR ONLY BY "CHRONC" ARE PRESENTED IN LATER SECTIONS

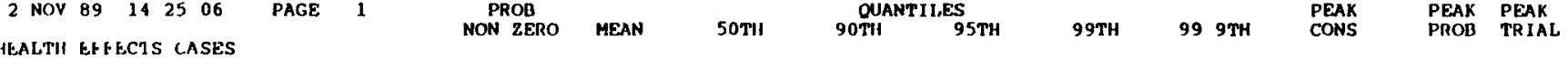

Hethe

FRL INJ/PRODROMAL, VOMIT

ERL INJ/DIARRIIEA

ERL INJ/PNEUMONI IIS

ERL INJ/HYPOTHYROIDISM

ERL INJ/SKIN ERYTHEMA

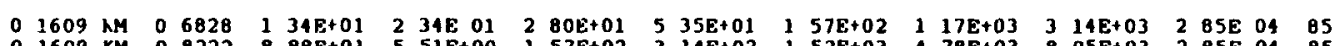

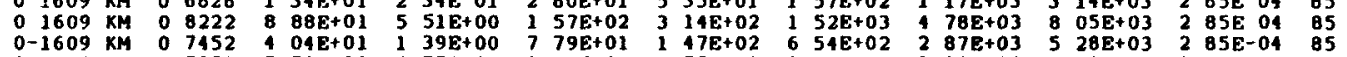

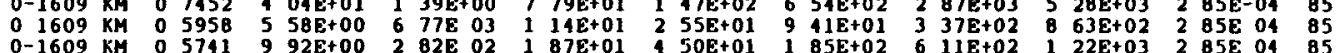

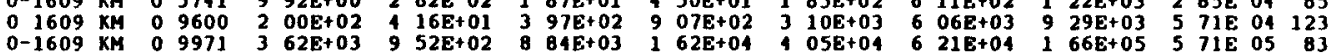

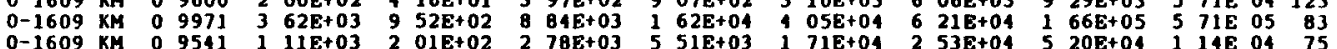

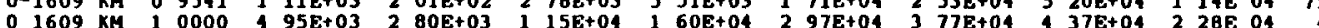

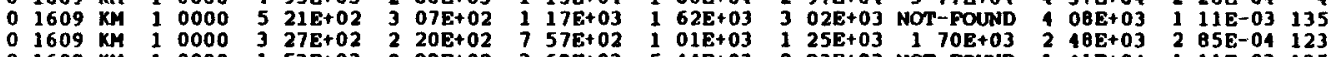

CAN FAT/LUNG

CAN FAT/BREAS

CAN FAT/LEUKEMIA

CAN INJ/TOTAL

CAN FAT/TOTAL

ERL FAT/TOTAL

ERL INJ/DIARRHEA

ERL INJ/PNEUMONITIS

THJ/HYPOTHYROIDISH

ERL INJ/TRANSEPIDEPMA

CAN FAT/TOTAL

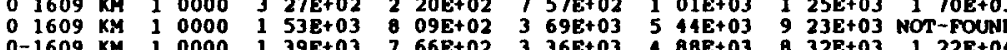

$01609 \mathrm{KM}$

0

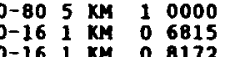

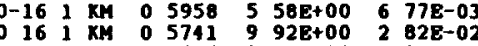

$0161 \mathrm{kM} 09929167 \mathrm{E}+03 \quad 3 \quad 01 \mathrm{E}+02$

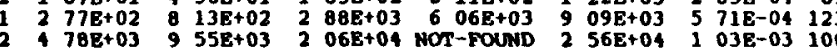

EARLY FATALITY DISTANCE (XM)

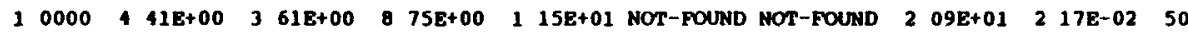




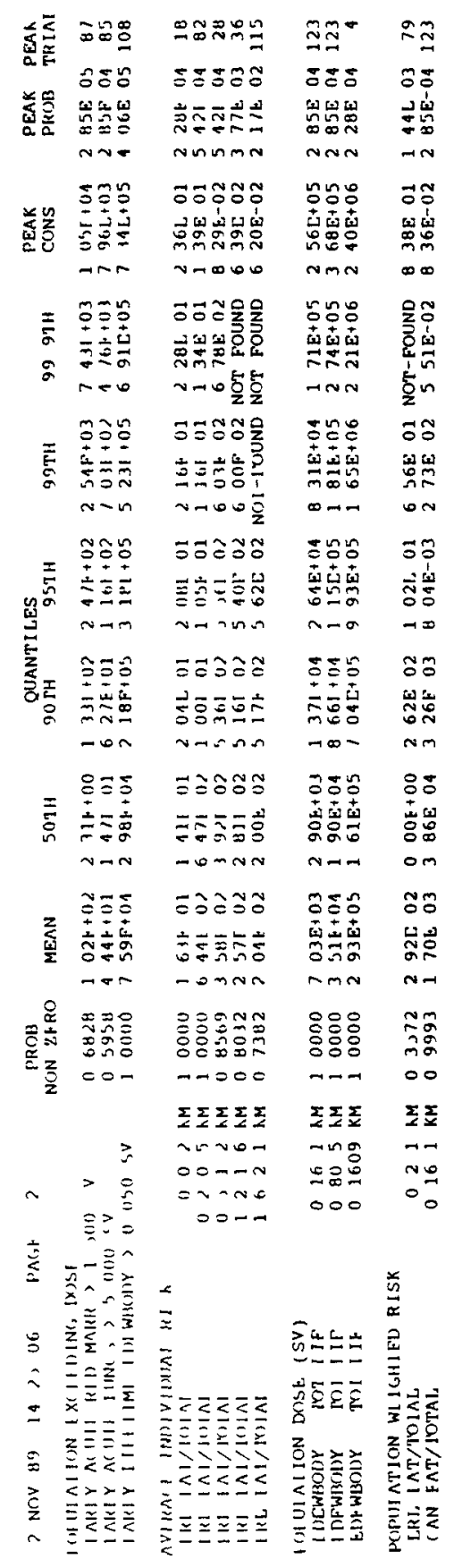




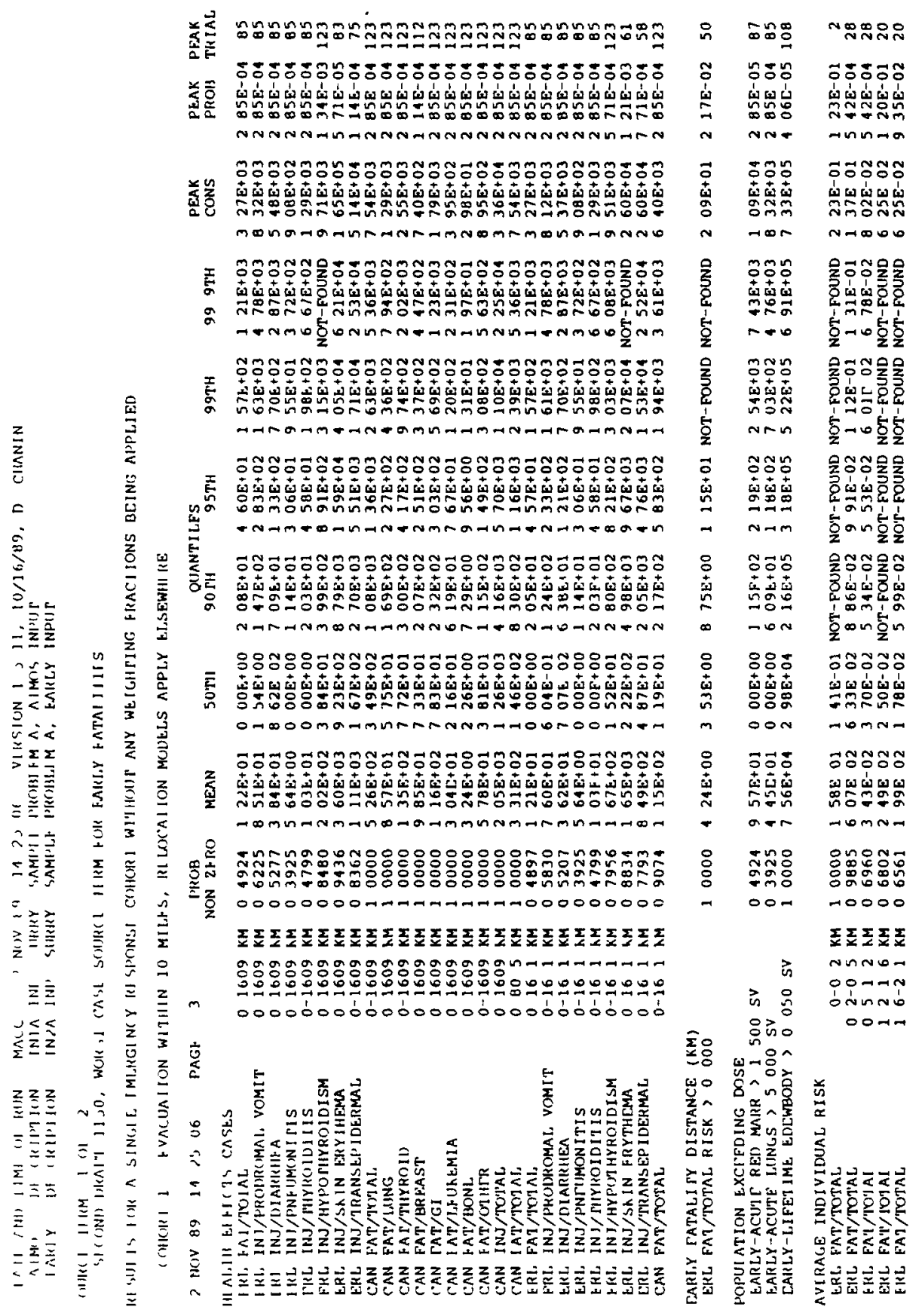




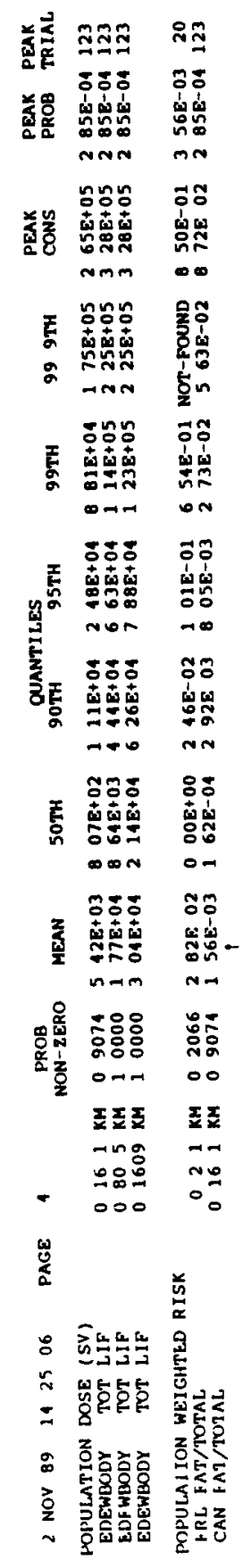


DATC AND TIMF OF RUN - MACCS 2-NOV 891425 O6 VERSION $15511,10 / 16 / 89$, D CHANIN

"ATMOS" DI SCR IPTION : INIA INP, SURRY, SAMPLE PROBI,EM A, ATHOS INPUT

SOURCE TERM 1 OF 2
SECOND DRAFT 1150 , WORST CASE SOURCE TERM FOR EARLY FATALITIES

RFSUITS FOK A SINGLE FMERGENCY RESPONSE COHORT WITHOUT ANY WEIGHTING PRACTIONS BEING APPLIED COHORT 2 - NO EVACUATION, RELOCATION MODELS APPLY EVERYHHEHE

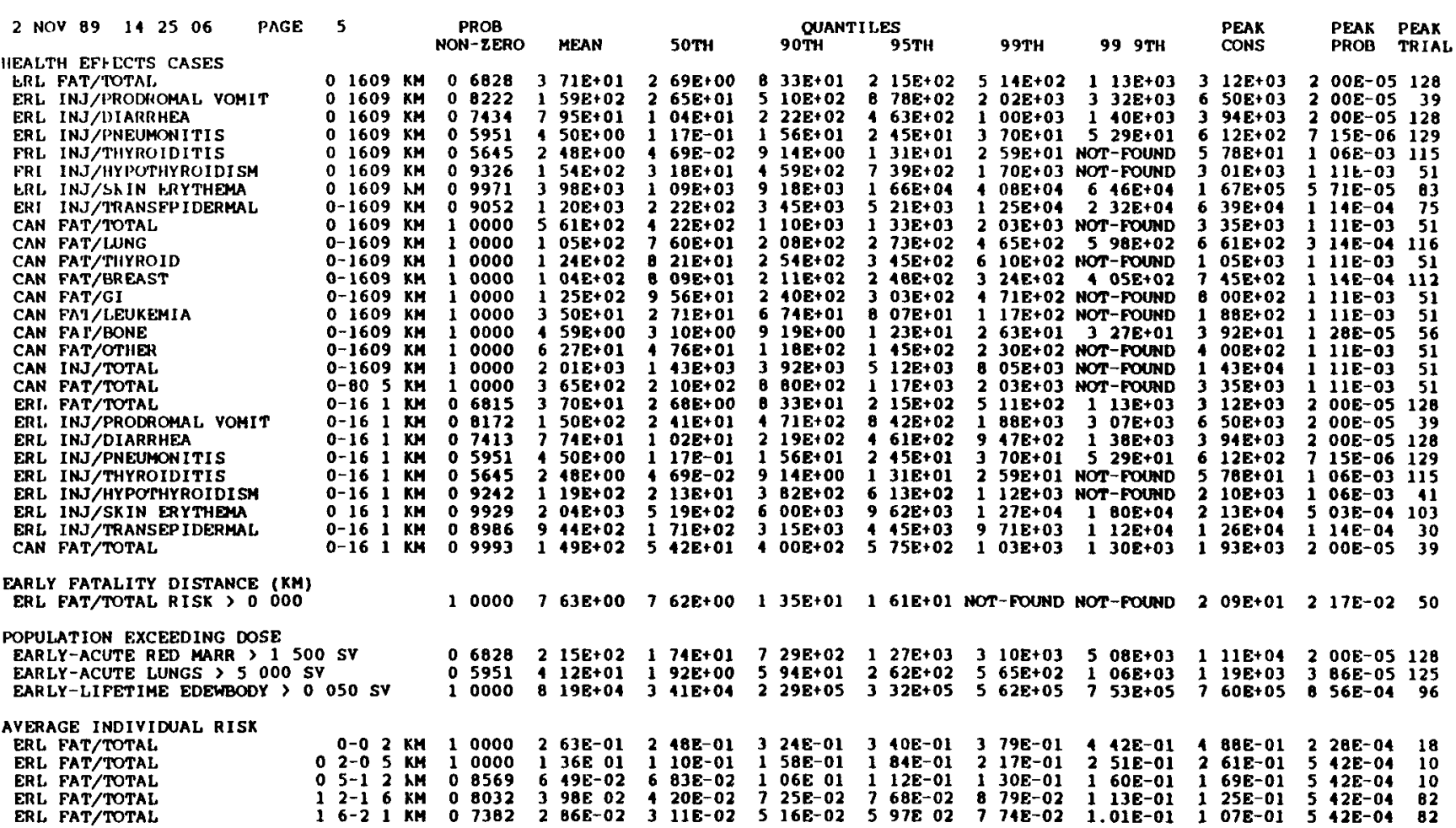




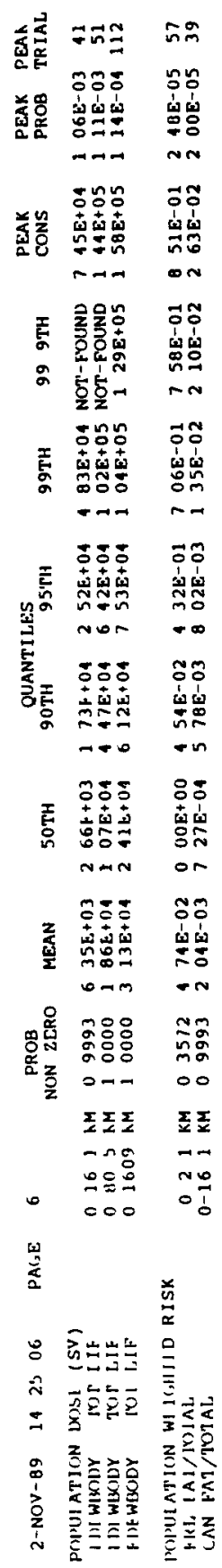

D- 68 


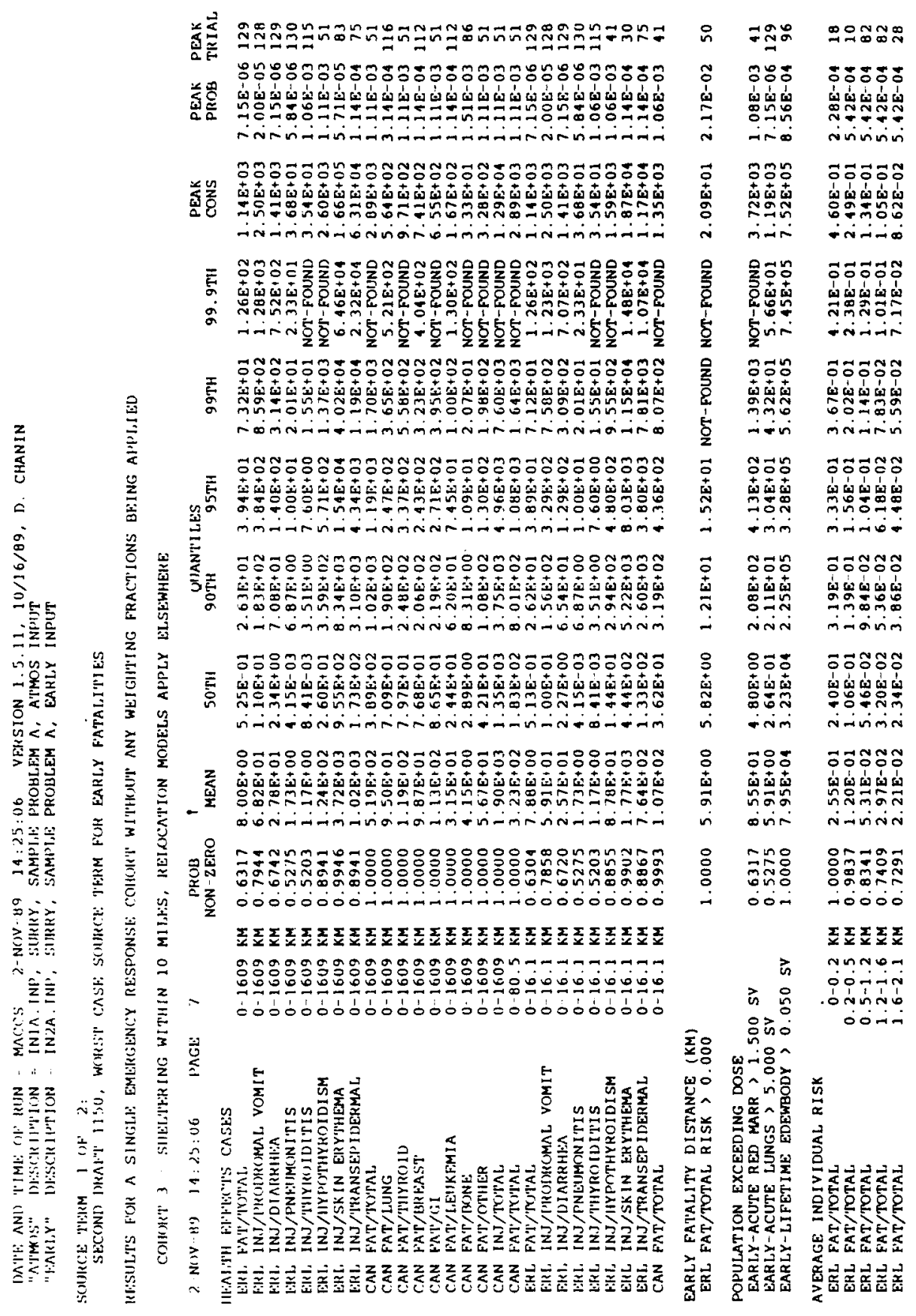




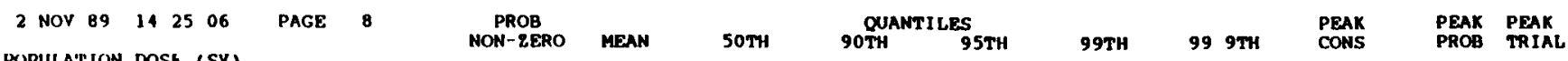

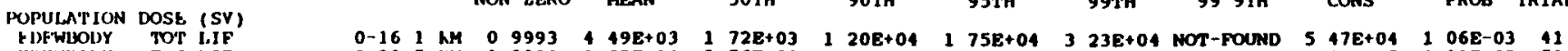

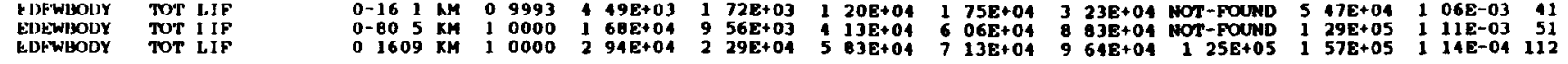

PMPUIATION WEIGHTED RISK

TRI, HATYIOTAL

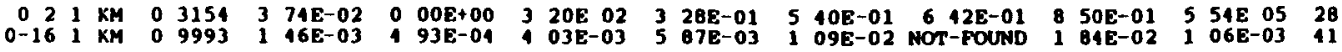


DATE: AND TIME OF RUN = MACCS 2-NOV-89 14:25:06 VERSION 1.5.11, 10/16/89, D. CHANIN

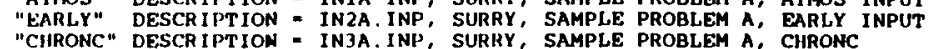

SOURCE TRRM 1 OF

WORT CASE SOURCE TERM FOR EARLY FATALITIES

ESULTS FROM THE "CHRONC" MODULE ALONE

COHOR'T 4 = IN3A. INP, SURRY, SAMPLE PROBLEM A, CHRONC

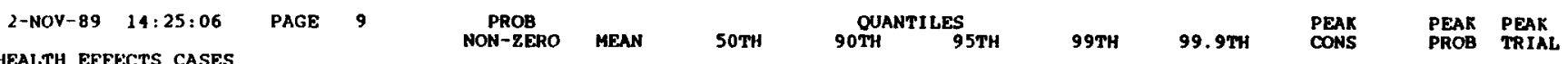

MEALTH EFFECTS CASES

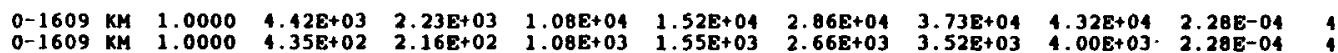
CAN FAT/LUNG

CAN FAT/THYROID

CAN FAT/BREAST

CAN FAT/LEUKEMIA

CAN FAT/BONE

CAN INJ/TOTA

CAN FAT/TOTAL

POPULATION DOSE (SV)

$\begin{array}{ll}\text { EDEWBODY } & \text { TOT LIF } \\ \text { EDEWBODY } & \text { TOT LIF } \\ \text { RDEWBODY } & \text { TOT LIF }\end{array}$

POPULATION WEIGHTED RISK

EDEWBODY POP. DOSE (SV)

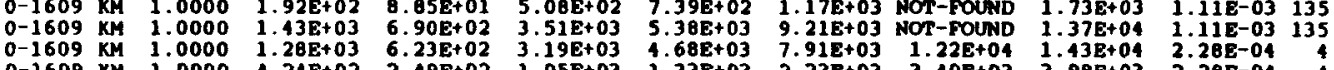

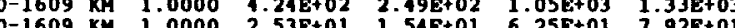

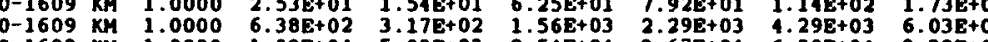

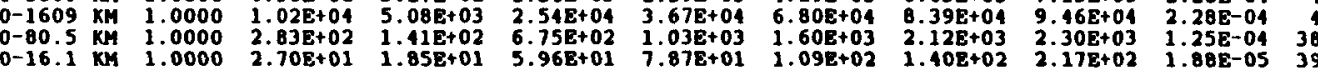

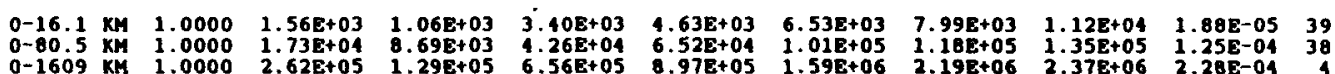

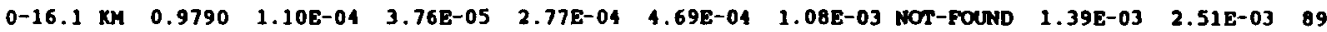
$0-1609 \mathrm{KM}$

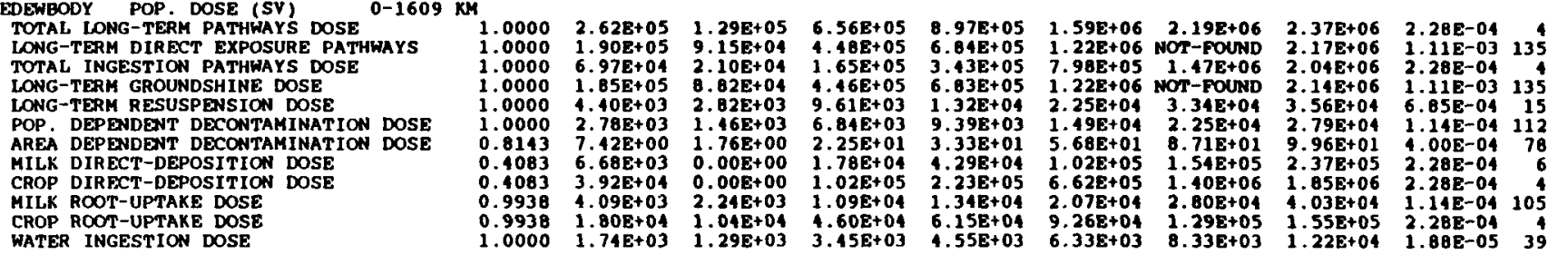

EDEWBODY POP. DOSE (SV) $0-80.5 \mathrm{kM}$ TOTAL LONG-TERM PATHWAYS DOSE TOTAL INGESTION PATHWAYS DOS

LONG-TERM GROUNDSHINE DOSE
LONG-TERM RESUSPENSION DOSE

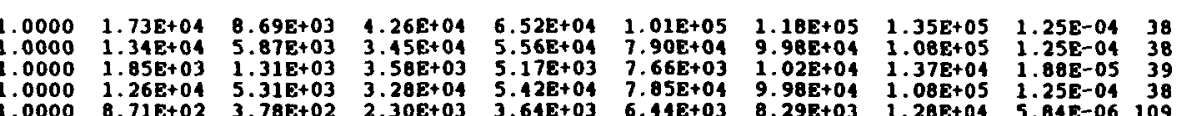




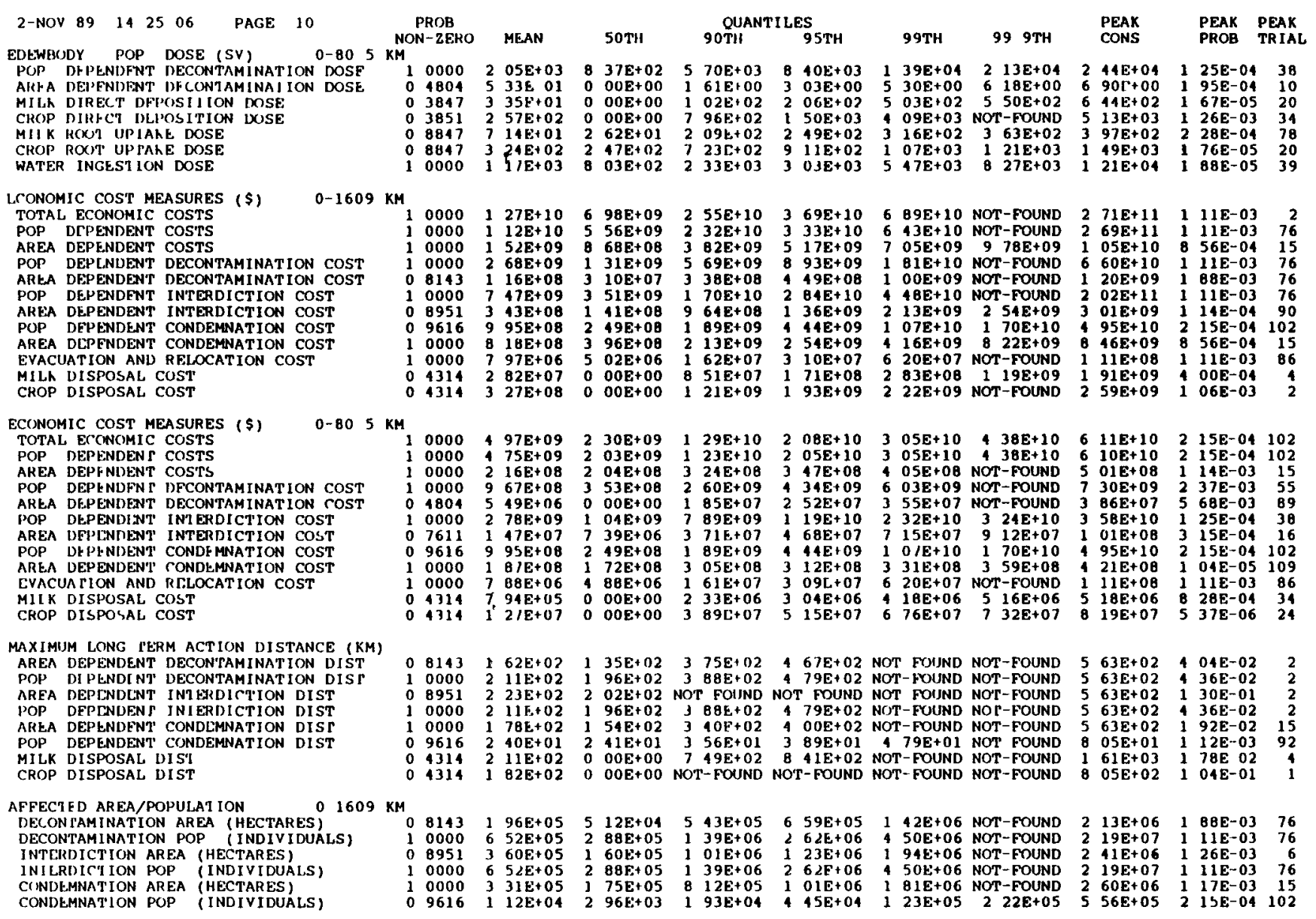




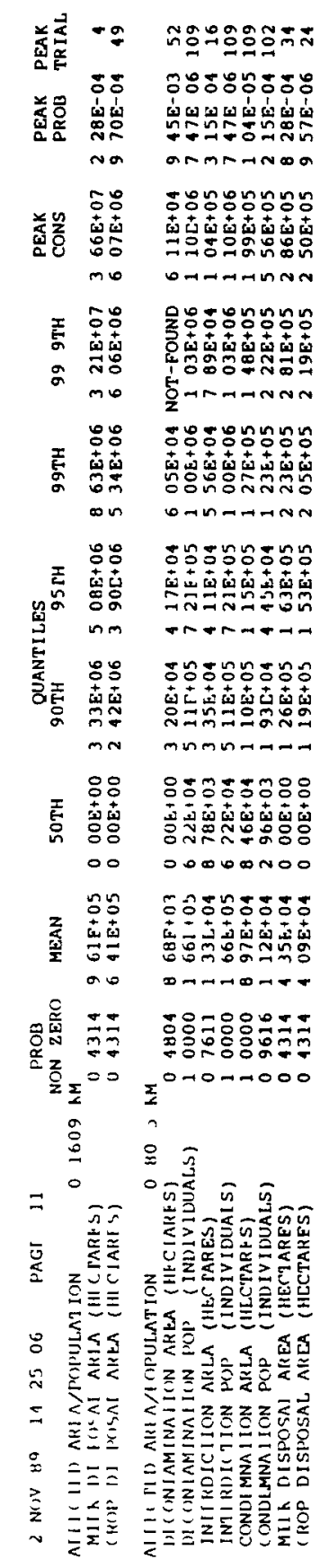


ร

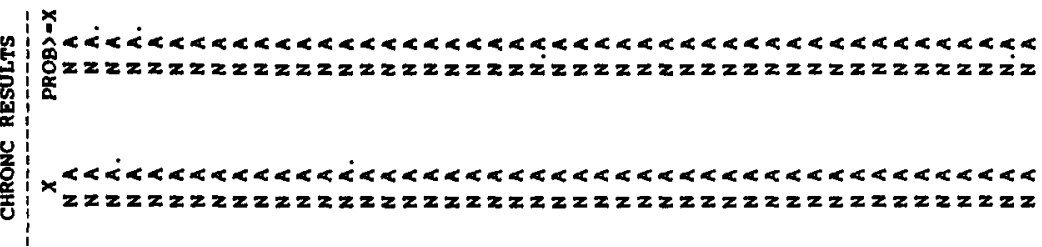

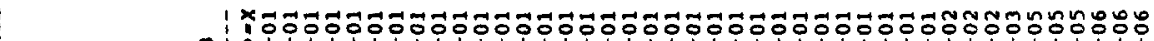

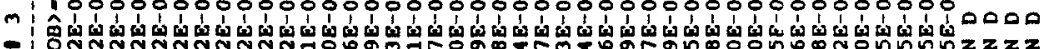
:

:

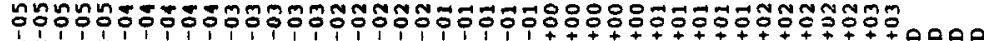

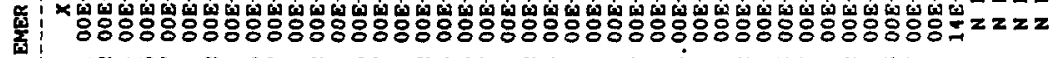

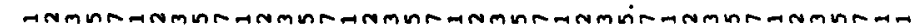

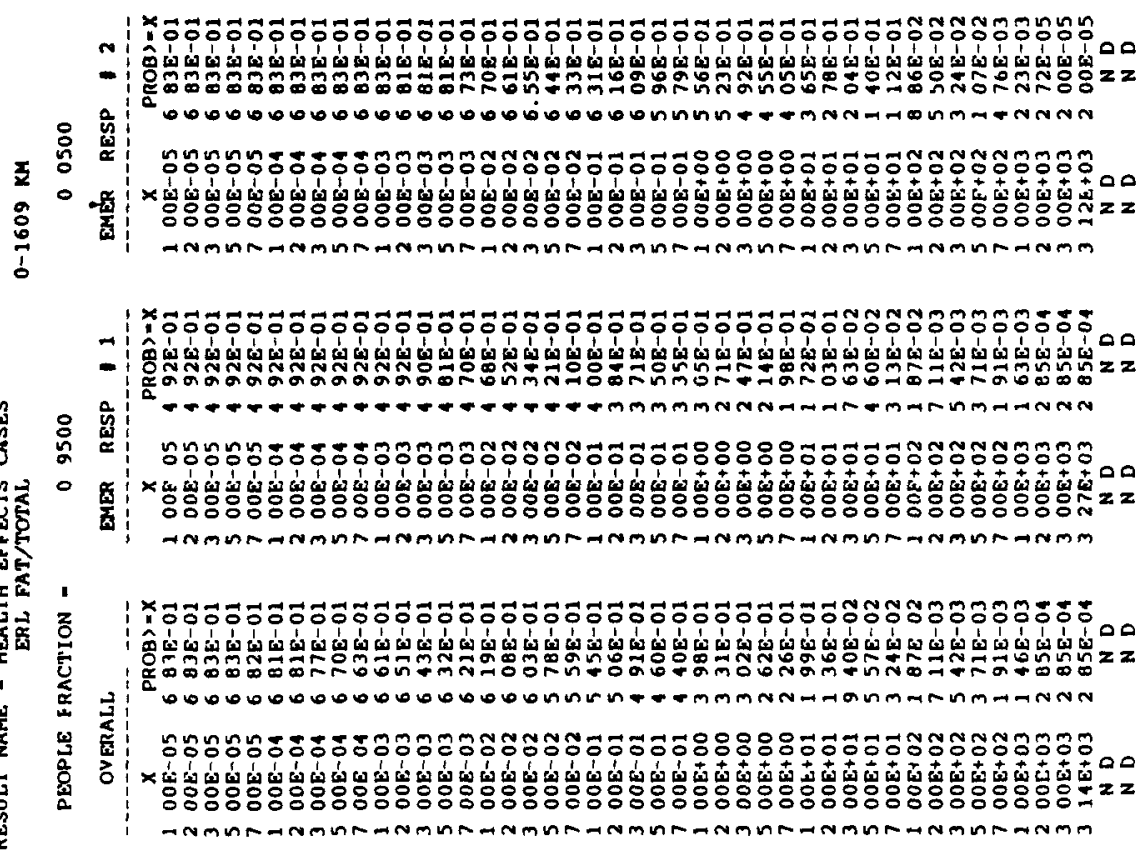




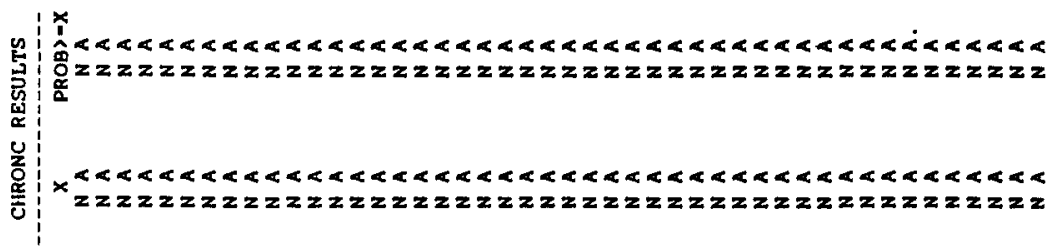

$m$ 的

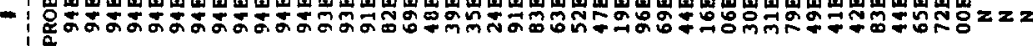

容

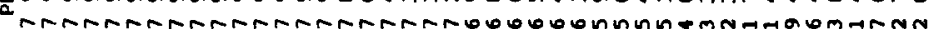

$\circ$

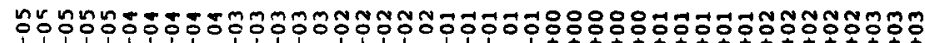

产

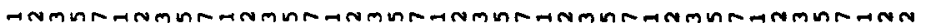

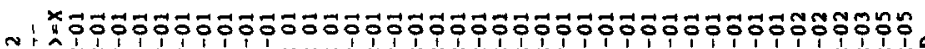

⿵人一

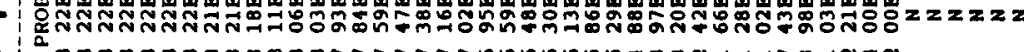
की

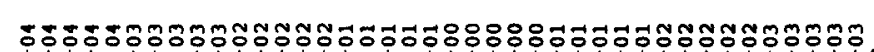

$\times 1 \times$ 군

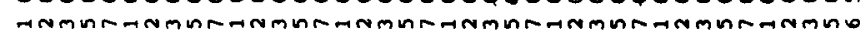

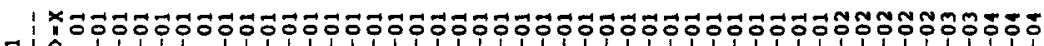

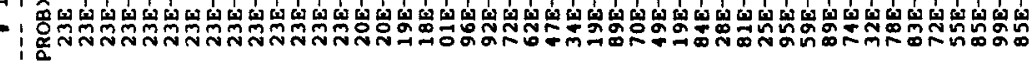

8 空

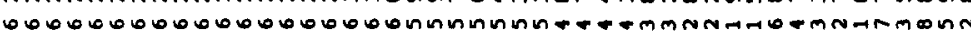

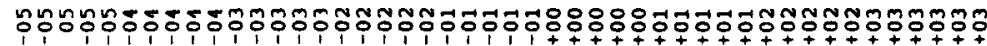

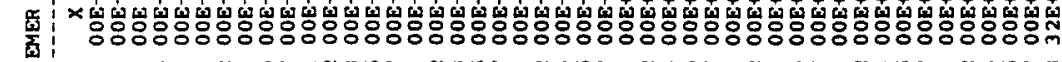
-nmunanmunanmunanmunmnmummmunammunmmmnnmmun

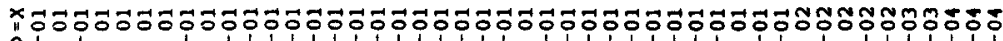

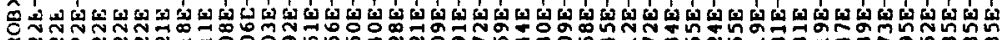
1

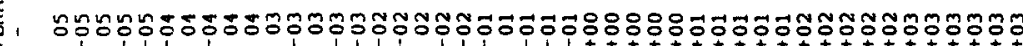

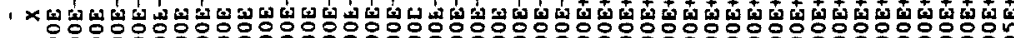

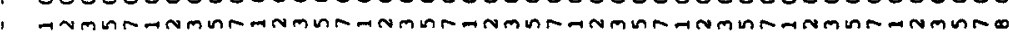




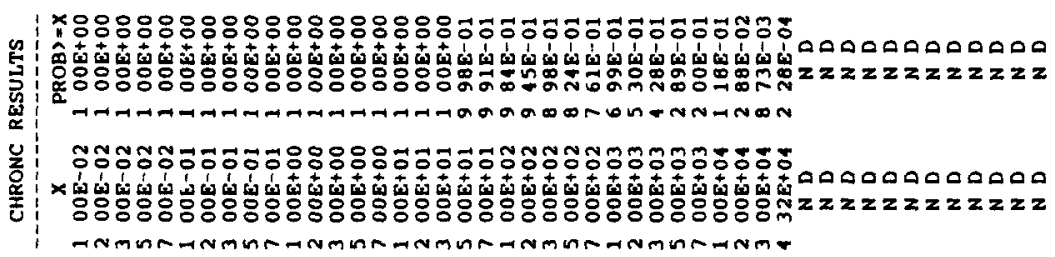

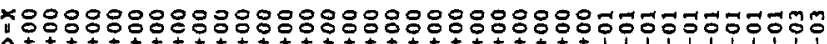

M -

言

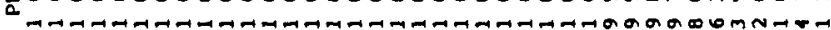

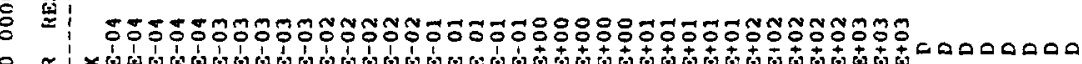
㪯

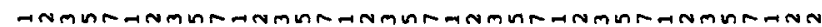

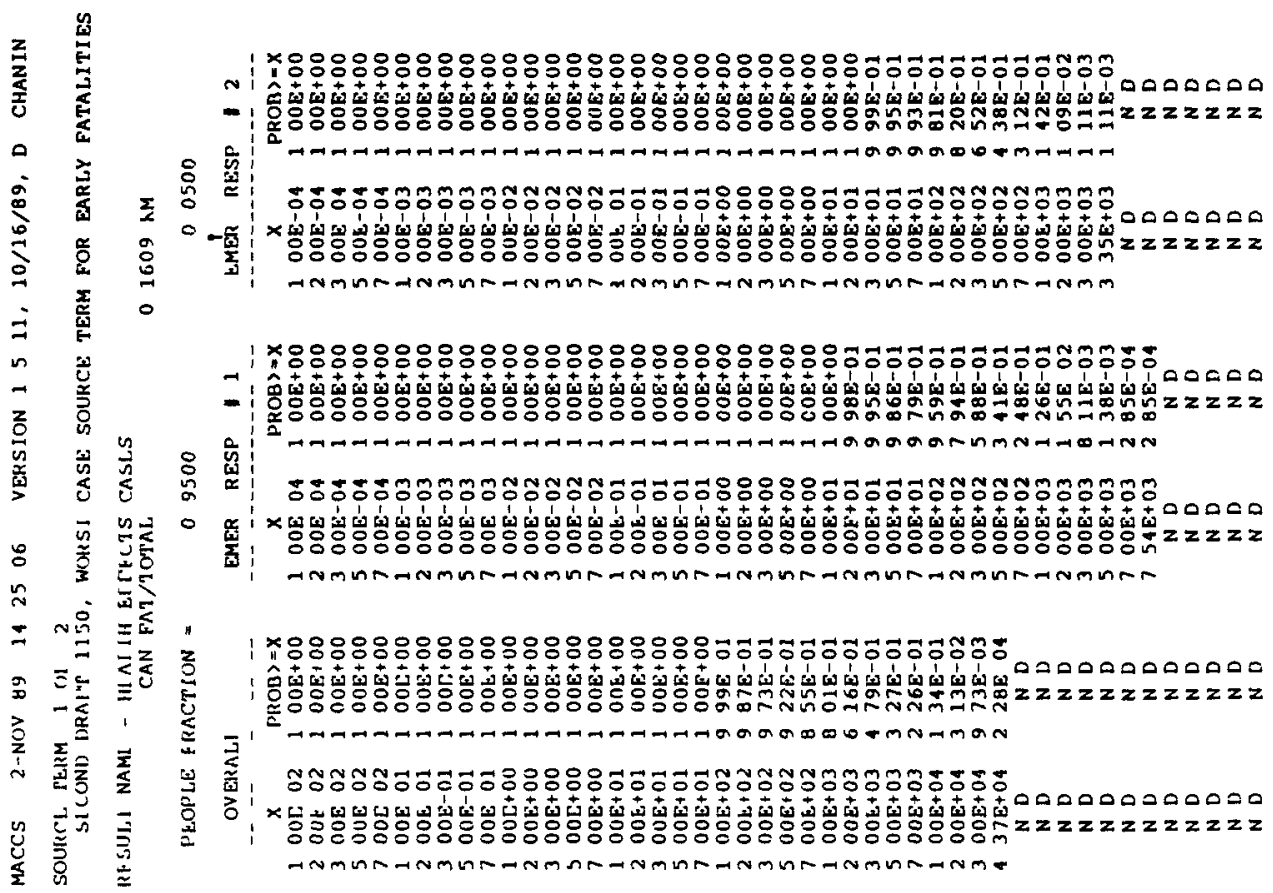




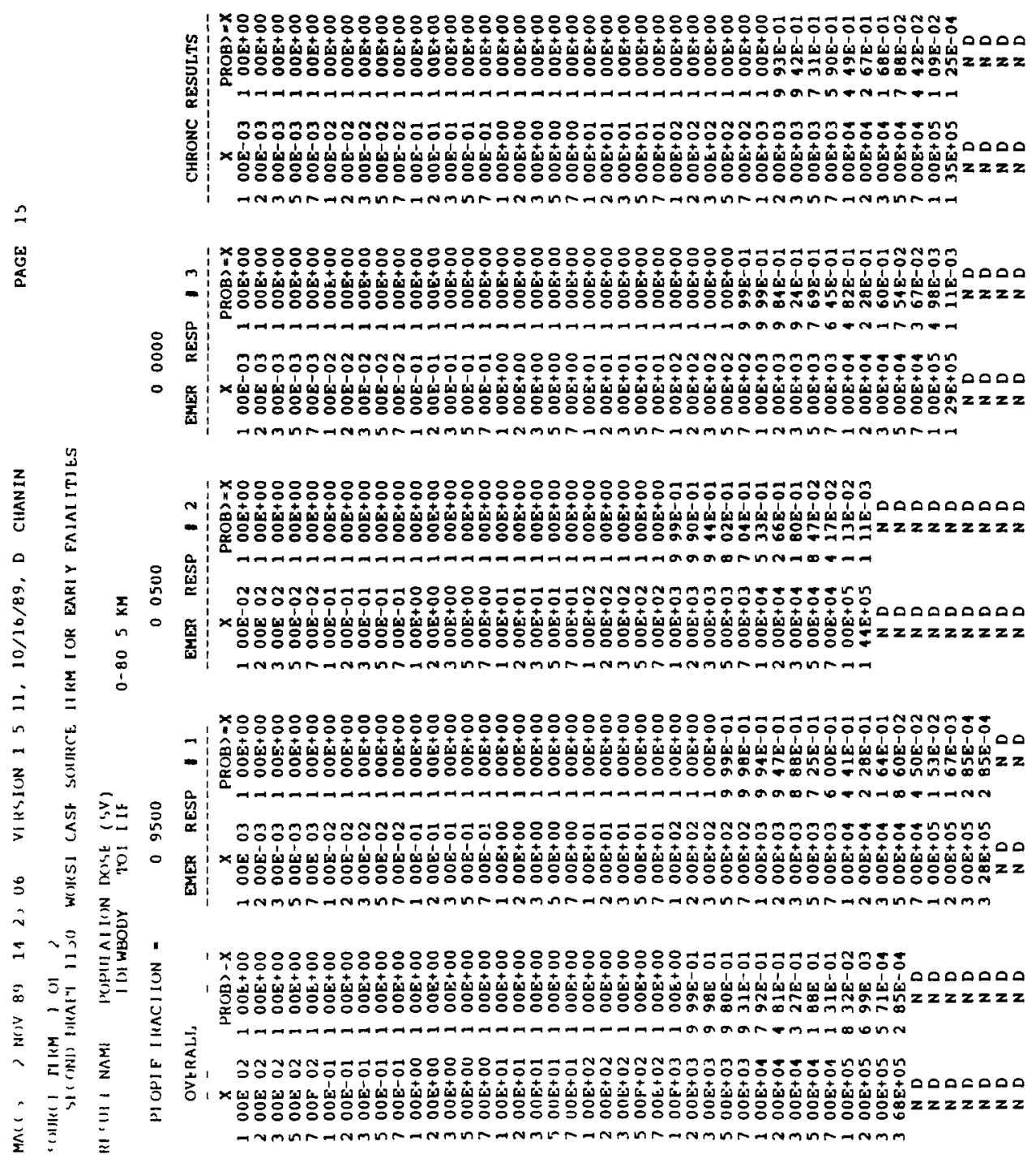




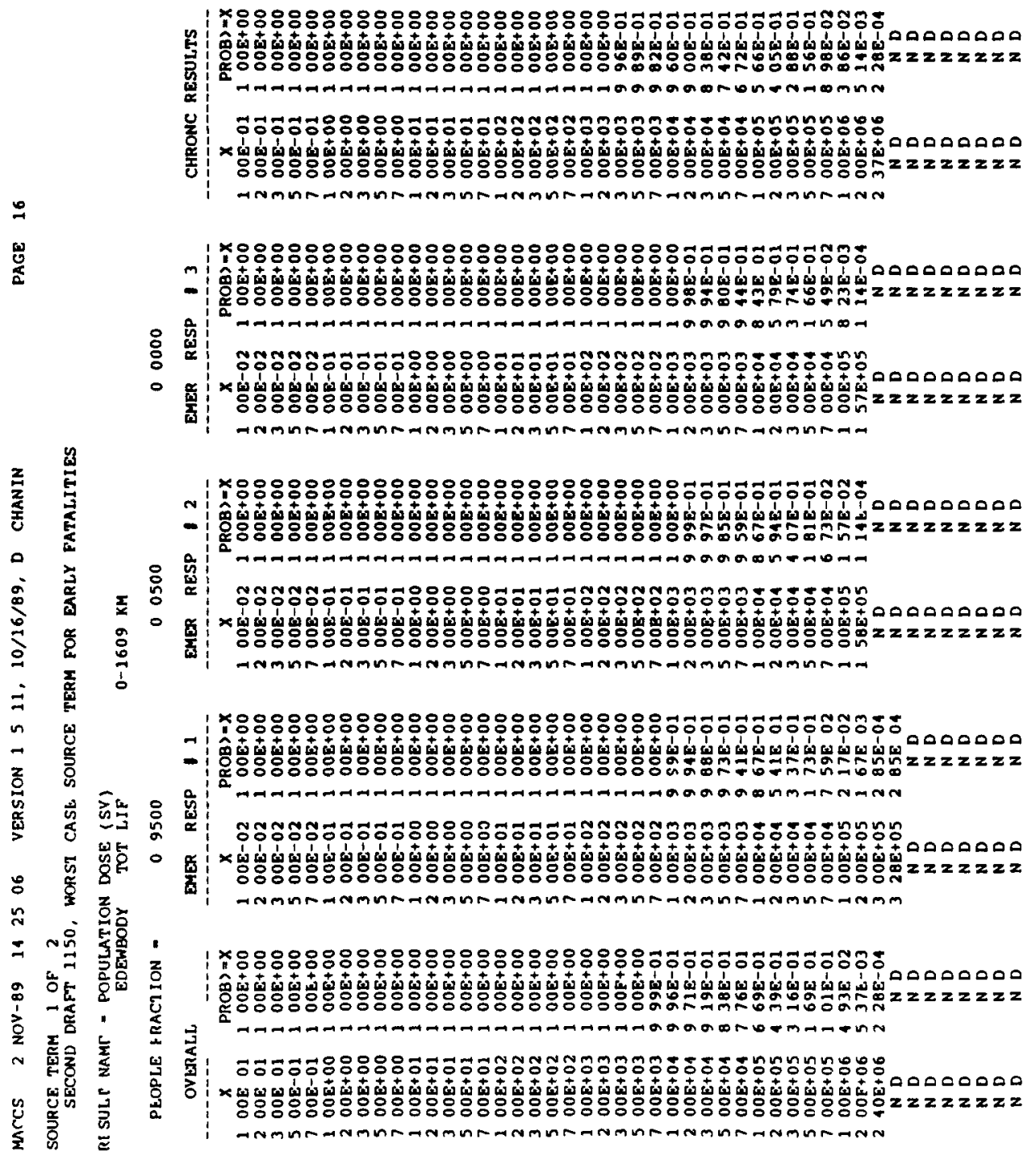




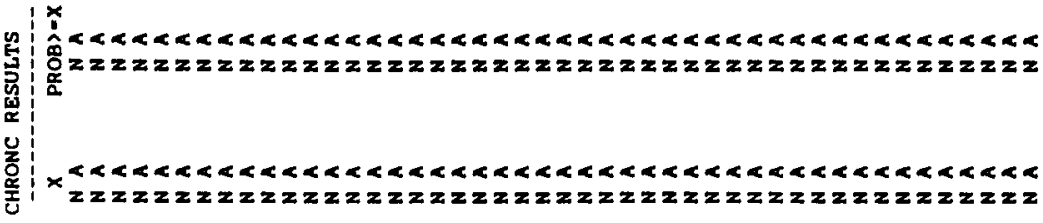

^

娄

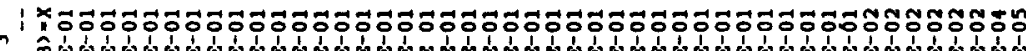

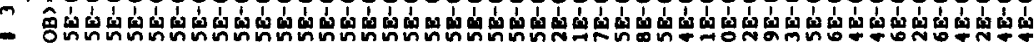

8

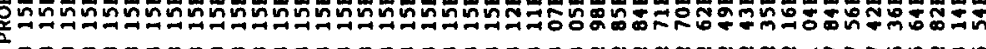

:

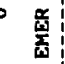

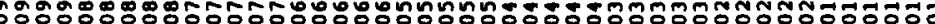
× mnmn-nmnn

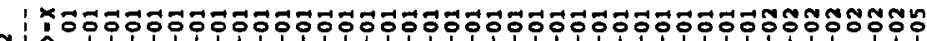

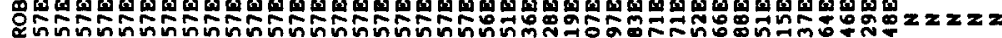
(n)

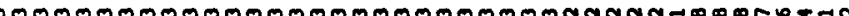

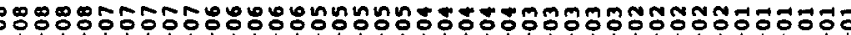

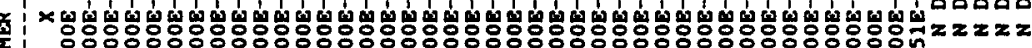

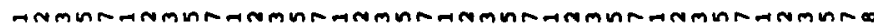

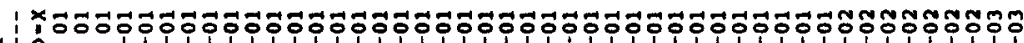

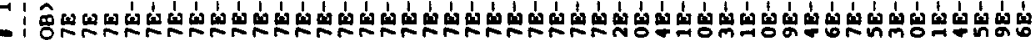

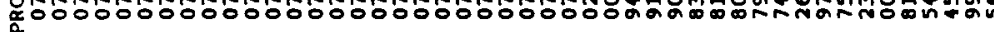

象

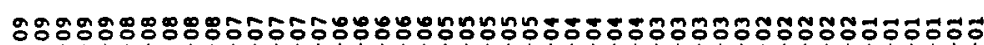

신

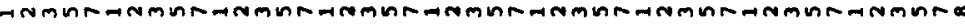

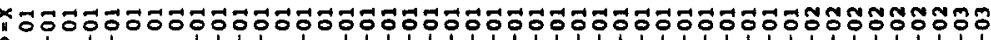

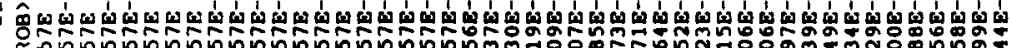

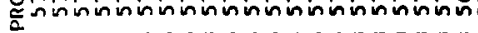

ב

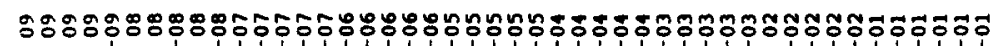

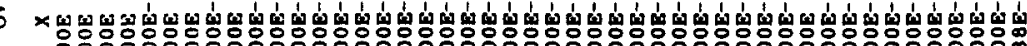

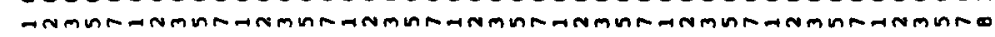



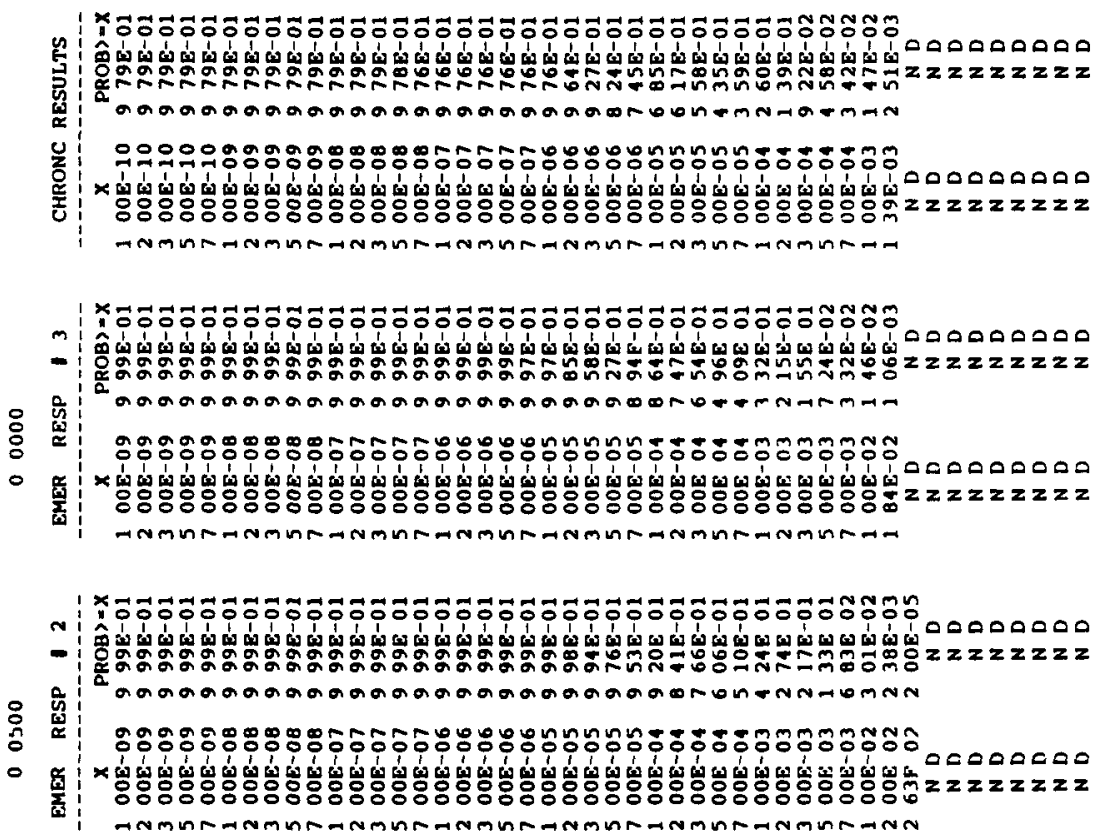


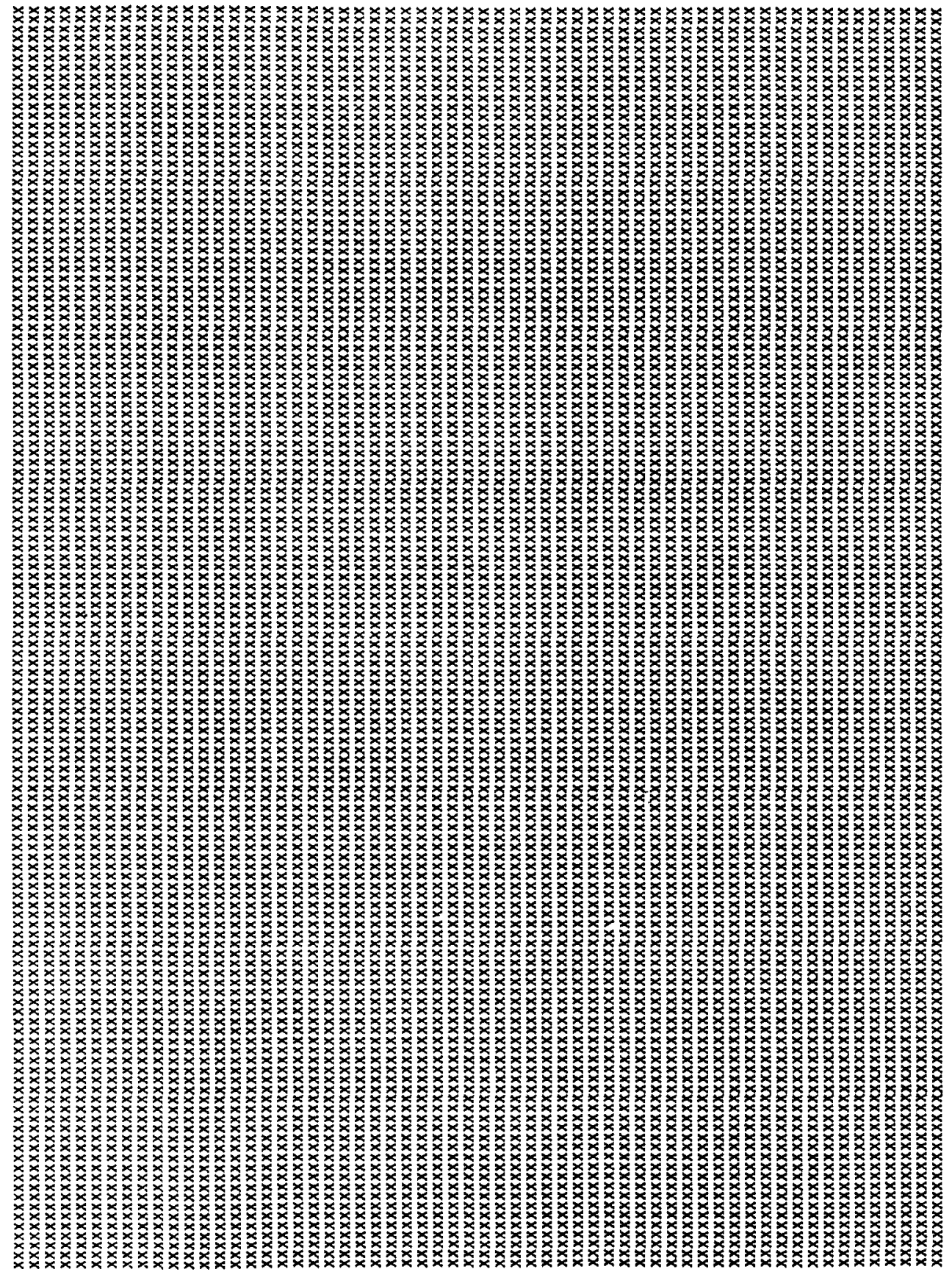


DATP AND TIME OF RUN - MACCS 2-NOV-89 1425 O6 VERSION $1511,10 / 16 / 89$, D CHANIN "EARY" DESCRIPTION = IN1A INP, SURRY, SAMPLE PROBLEM A,' ATMOS INPUT

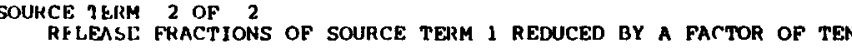

OY ERALL RESULTS OBTAINED BY COMBINING 3 EMERGENCY RESPONSE COHORTS FROM "EARLY" WITH THE WEIGHTING FRACTIONS BELOW APPLICD TO THEM

COHOR'T 1 = EVACUATION WITHIN 10 MILES, RELOCATION MODELS APPLY ELSEHHERE

COHORT 2 - NO EVACUATION, RELOCATION MODELS APPLY EVERYWHERE

FRACTION OF THE PEOPLE

T. SHELTERING WITHIN 10 MILES, RELOCATION MODELS APPLY EDSBHHERE

AND THEN MERGING THE 3 RESULTS ABOVE WITH THE SINGLE SET OF RESULTS FROM "CHRONC" DESCRIBED BELOW

COHORT 4 - IN3A INP, SURRY, SAMPLE PROBLEM A, CHRONC

RESUlts hHICH ARE PRODUCEd ONLY BY "EARLY" OR ONLY BY "CHRONC" ARE PRESENTED IN LATER SECTIONS

2-NOY-89 $14 \quad 2506 \quad$ PAGE

PROB
MON-2ERO MEA SUANTILES

HEALTH EFFECTS CASES

ERL FAT/MOTAL

ERL INJ/PRODROMA

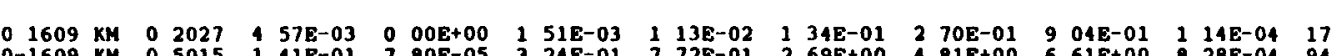

ERL INJ/PNEUMONITIS

ERL INJ/HYPOTIYYOIDISM

ERL INJ/SKIN ERYTHEMA

CAN FAT/TOTAL

CAN FAT/LUNG

CAN FAT/THYROID

CAN FAT/GI

CAN FAT/LEUKEM

CAN FAY'OTHER

CAN IN.JTTOTAL

ERL. INJJPRODROMAL VOHIT

ERL INJJPNEUMONIT

ERL INJ/THYROIDITIS

ERL INJ/HYPOTHYROIDISM

ERL INJ/SKIN ERYTHEMA

EARLY FATALITY DISTANCE (KM)
ERL FAT/TOTAL RISK $>0$ OOO

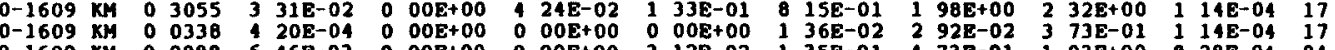

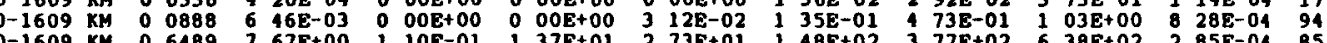

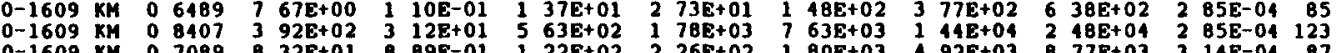

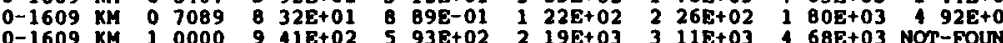

(1609 KM 10000 9 41E+0

$0-1609 \mathrm{KM} 10000 \quad 548 \mathrm{E}+01$

$01609 \mathrm{KM}$

2
1

0-1609 KM

01609
$0-1609$
KM

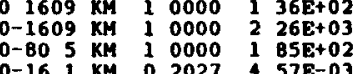

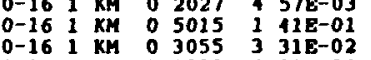

$\begin{array}{lllllll}0-16 & 1 & \mathrm{KM} & 0 & 088 \mathrm{~B} & 6 & 60 \mathrm{E}-0 \\ 0-16 & \mathrm{KM} & 0 & 6476 & 7 & 58 \mathrm{E}-0\end{array}$

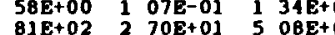

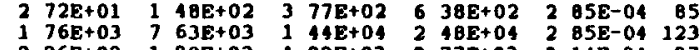

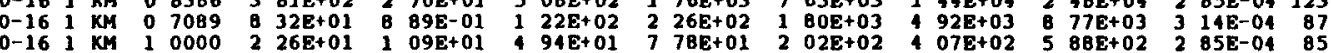

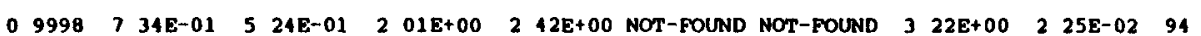




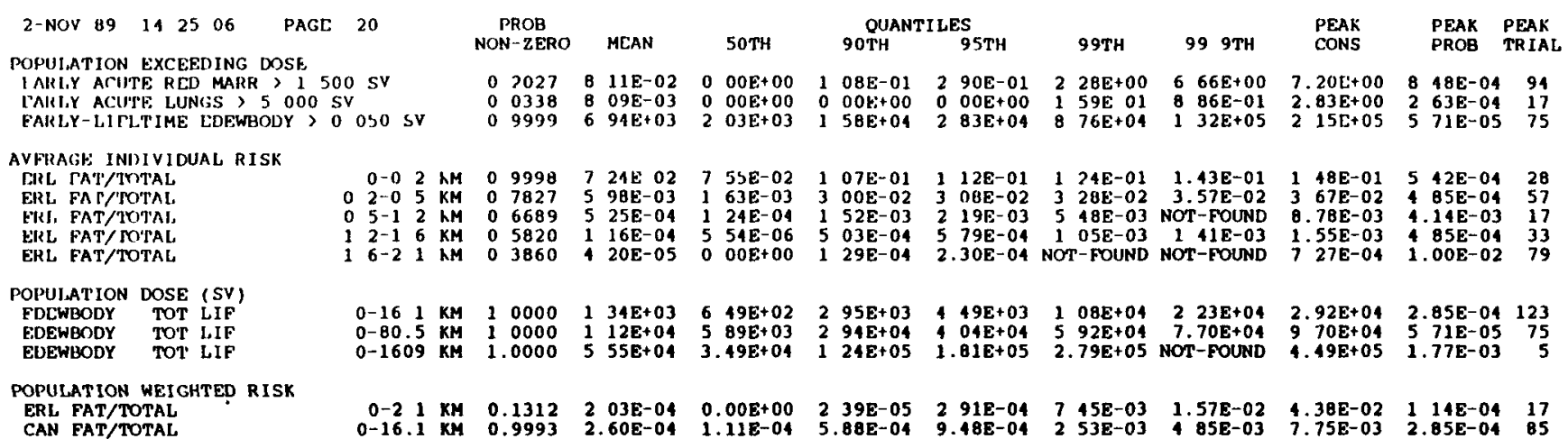


DATE AND TIME OF RUN = MACCS 2 NOV 891425 O6 VERSION $1511,10 / 16 / 89$, D CHANIN

"EARLY" DESCR IPTION = IN1A INP, SURRY, SAMPLE PROBLEM $A$, ATMOS INPUT

SOUKCE TERM 2 OF 2
RELEASE FRACTIONS OF SOUACE TERM 2 REDUCED UY A FACTOR OF TEN

RESULTS FOR A SINGLE EMERGENCY RESPONSE COHORT WITHOUT ANY WEIGHTING ERACTIONS BEING APPLIED

COHOR' 1 = EVACUATION WITHIN 10 MILES, RELOCATION MODELS APPLY ELSEWHERE

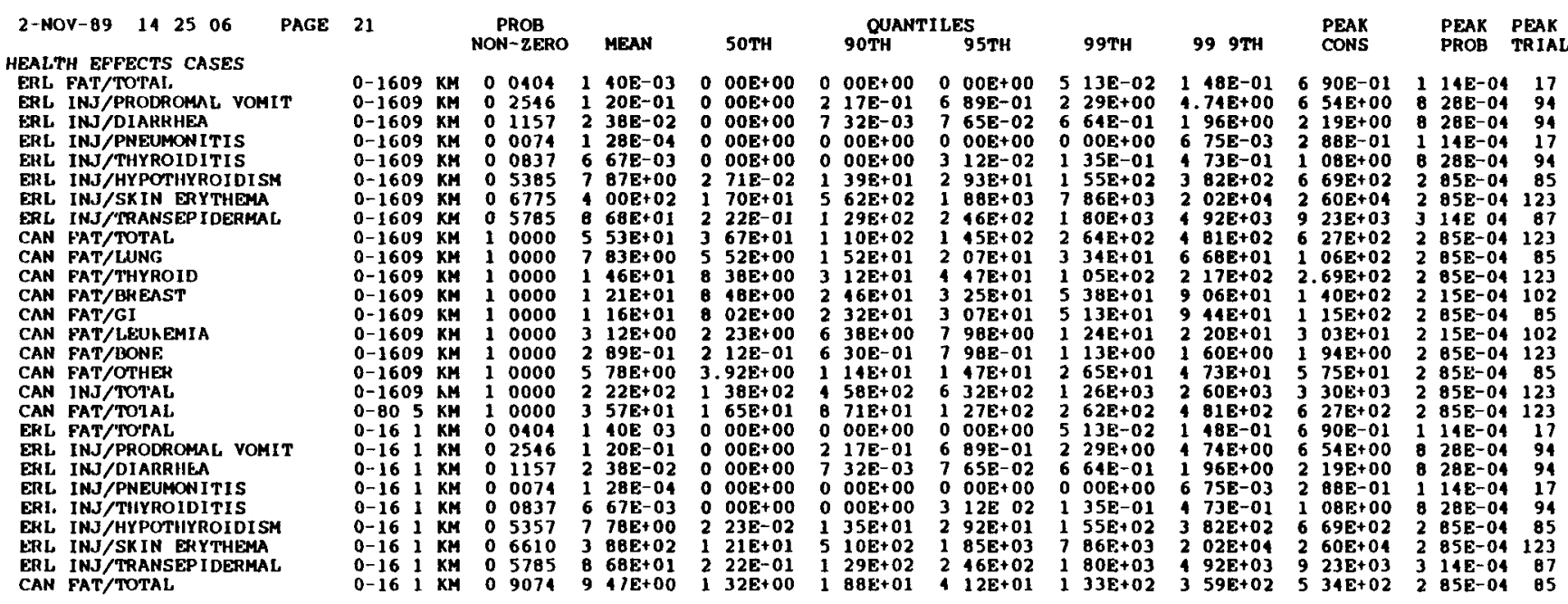

EARLY FATALITY DISTANCE (KM)
ERL FAT/TOTAL RISK $>0$ OOO

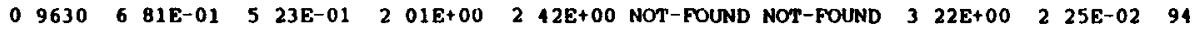

POPULATION EXCEEDING DOSE

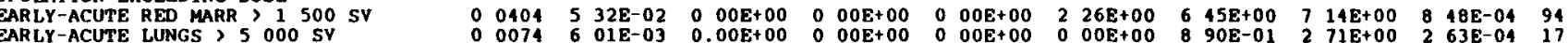

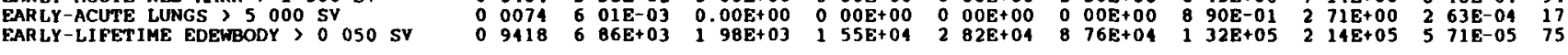

AYTRAGE INDIYIDUAL RISK

ERI. FAT/TOTAL

ERL FAT/TYTPAL

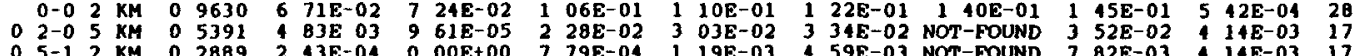

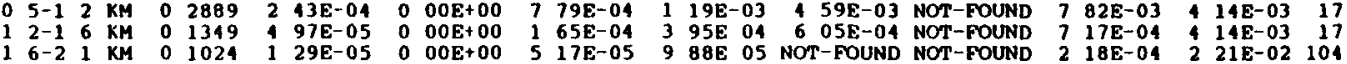




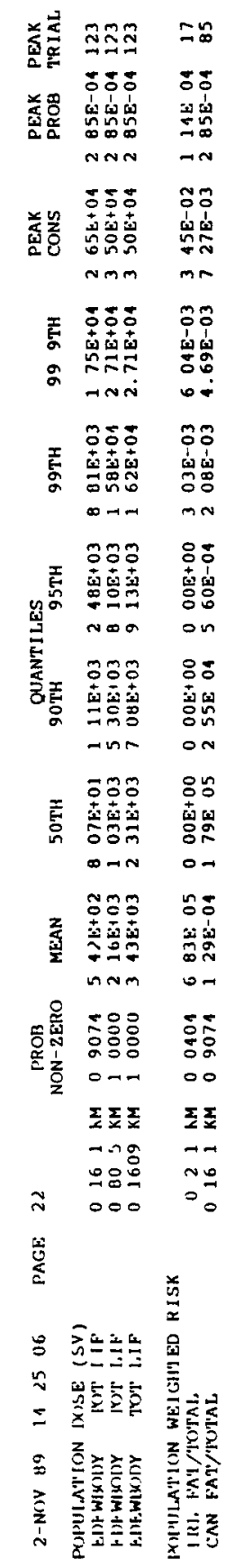

D- 85 
DAIE AND TIME OF RUN - MACCS 2 NOV 89142506 VERSION $1511,10 / 16 / 89$, D CHANIN

"ATMOS" DESCR IPTION = INIA INP, SURRY, SAMPLE PROBLEMA A,' ATMOS INPUT

SOURCE IERM 2 OF 2
RELEASE T'KACIIONS OF SUURCF PETM 1 REDUCED BY A FACTOR OF TEN

HESULTS HOR A SINGLE EMERGENCY RESPONSE COHORT WITHOUT ANY WEIGHTING FRACTIONS BEING APPLIED

COHORT 2 = NO EVACIJATION, RELOCATION MODELS APPLY EVERYWHERE

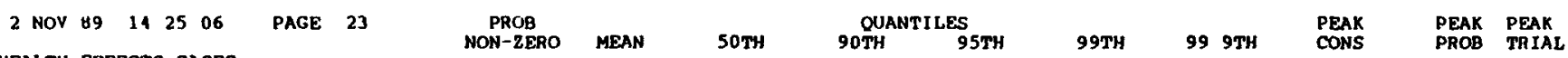
HEALTH EFFECTS CASES

ERL FAT/TOTAL ERL INJ/PRODROMAL, VOMIT

ERL, INJ/DIAROA

ERT. INJ/THYYOID ITTS

ERL INJ/HYPOTHYROIDISM

ERL INJ/TRANSEP IDERMA

CAN FAT/TOTAL

CAN FAT/THYROID

CAN FAT/GI

CAN FAT JOTHER

CAN INJ/TOTAL

GRL GACTOTAL

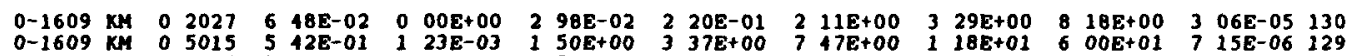

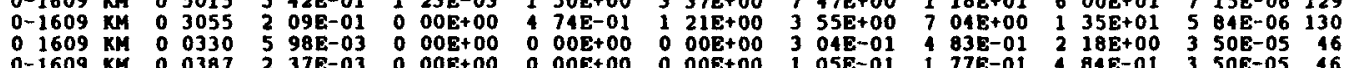

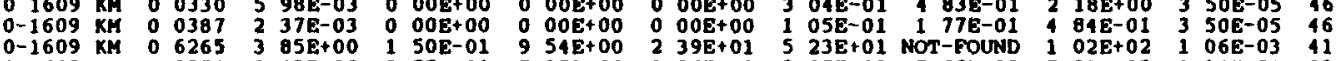

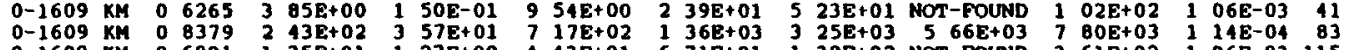

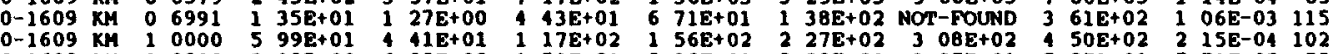

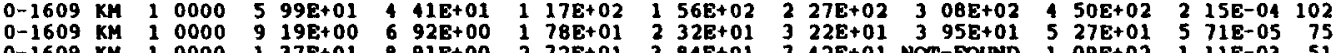

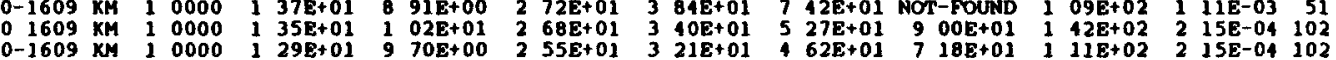

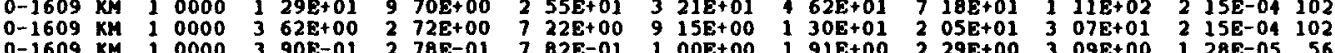

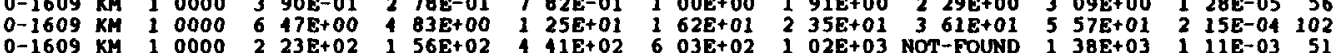

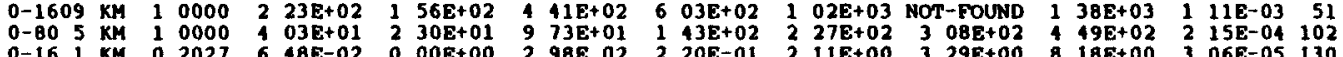

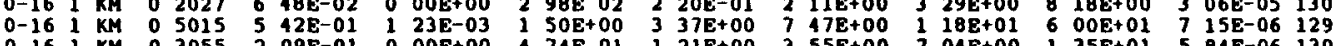

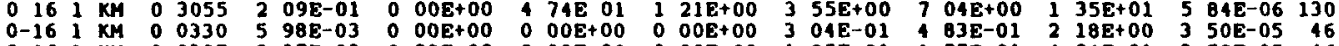

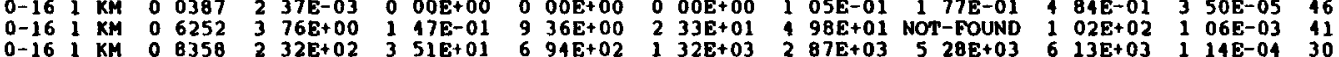

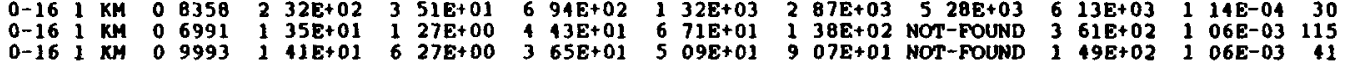
GR. INJ/THYROIDITIS FRL INJ/HYPOI'HYROIDISA ERL INJ/TRANSEPIDERMAL EARLY FATALITY DISTANCE (KM)
ERL FAT/TOTAL RISK 20 OOO

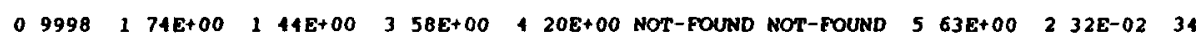
POPULATION EXCEEDING DOSE EARLY-ACUTE RED MARR 3 I 500 ST 作

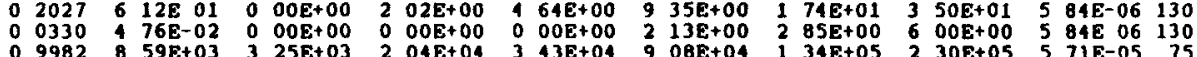
AVERAGE INDIVIDUAL RISK

ERL FAT/TOTAL

ERL FAT/TOTAL

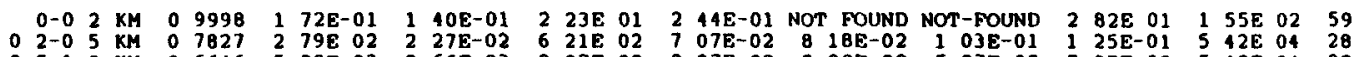

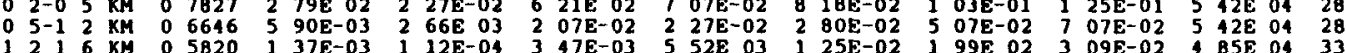

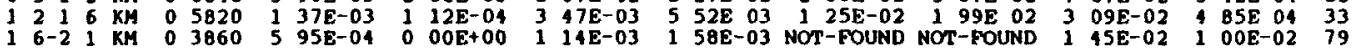




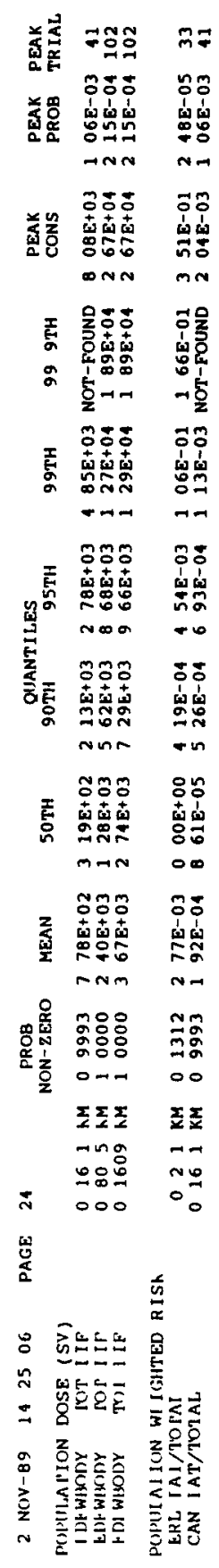


DATE AND TIME OF RUN = MACCS 2-NOV-89 1425 O6 VERSION $1511,10 / 16 / 89$, D CHANIN "ATMOS" DESCR IPTION = IN1A INP, SURRY, SAMPLE PROBLEM A, ATMOS INPUT
"EARLY" DESCH IPTION = IN2A INP," SURPY, SAMPLE PROBLEM A,. EARLY INPUT

SOURCE RERM 2 OF 2
RELEASE FRACTIONS OF SOURCE TERM 1 REDUCED BY A FACTOR OF TEN

RESULTS FOR A SINGLE EMERGENCY RESPONSE COHORT WITHOUT ANY WEIGHTING FRACTIONS BEING APPLIED

COHORT 3 = SHELTERING WITHIN 10 MILES, RELOCATTON MODELS APPLY ELSEWHERE

HIRALTH LEFEC TS CASES

NON-ZERO MEAN

$50 \mathrm{TH}$

QUTANTI LES

$99 \mathrm{TH}$

99 9TH

PEAK

PEAR PEAK
PROB TRIAL

ERL INJ/PRODROMAL VOMIT

FRI INJ/DIARRIIEA

$\begin{array}{llllllllllllllllllllll}01609 & K M & 0 & 0987 & 1 & 62 E-02 & 0 & 00 E+00 & 0 & 00 E+00 & 1 & 32 E-02 & 7 & 51 E-01 & 1 & 59 E+00 & 3 & 89 E+00 & 2 & 48 E-05 & 33\end{array}$

INJ/PNEUMONITIS

ERI INJ/IYYPOTHYROIDISM

ERL INJ/SKIN EHYTHEMA

CAN FAT/TOTAL

CAN FAT/LUNG

CAN FAT/BREAST

CAN FAT/GI

CAN FAT/BONE

CAN INJTTOTAL

CAN FAT/TOTAL.

ERL FATJ/POTAL

ERL INJJDIARRHEA

ERL INJ/PNEUMONITIS

ERL INJ SKIN

ERL INJ/TRANSEPIDERMAL

CAN FAT/TOTAL

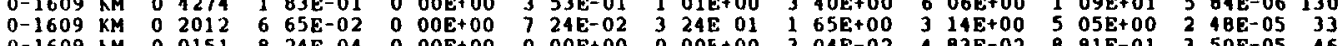

$\begin{array}{llll} & \end{array}$

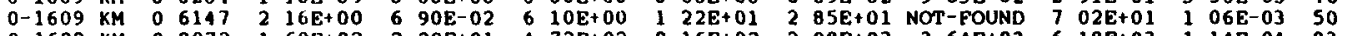

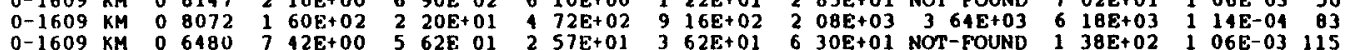

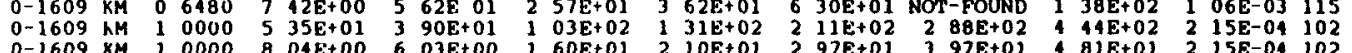

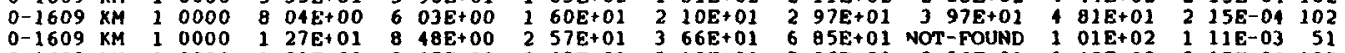

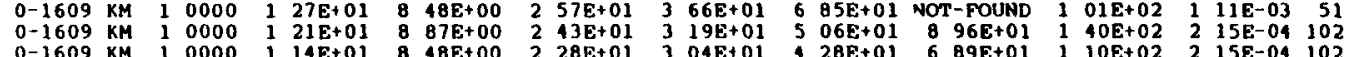

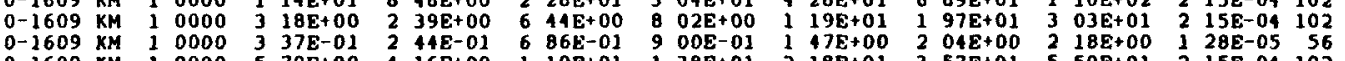

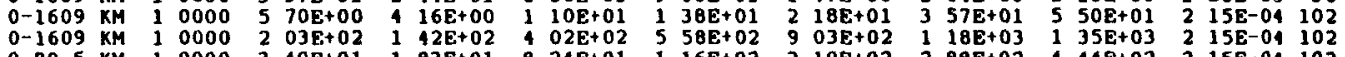

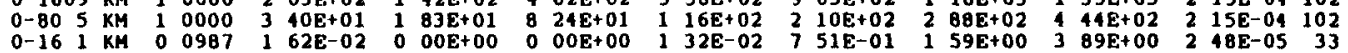

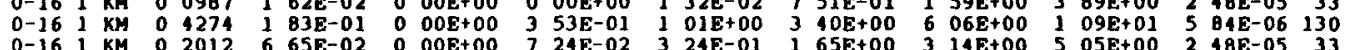

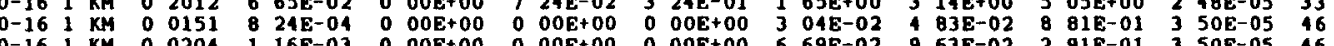

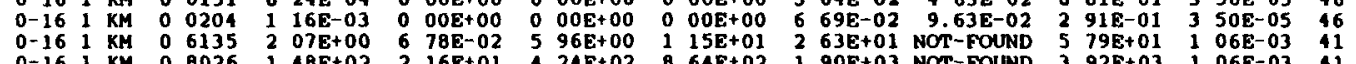

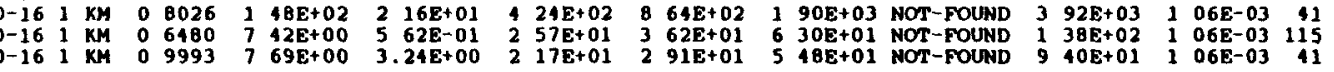

EARLY FATALITY DISTANCE (KM)
ERL FAT/TOTAL RISK $>0$ 000

$09998115 \mathrm{E}+00 \quad 109 \mathrm{E}+00 \quad 277 \mathrm{E}+00 \quad 310 \mathrm{E}+00 \quad 348 \mathrm{E}+00 \quad 412 \mathrm{E}+00 \quad \& \quad 83 \mathrm{E}+00 \quad 114 \mathrm{E}-04130$

POPULATION EXCEEDING DOSE

EARLY-ACUTE LUNGS $>5$ O00 SV
EARLY LIFETIME FDEWBODY $>050$ SV

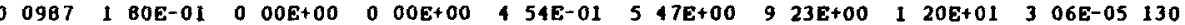

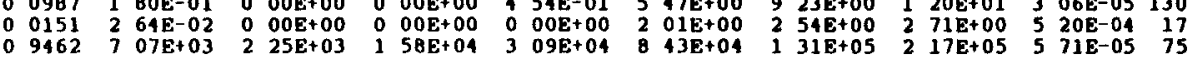
AVERAGE INDIVIDUAL RISK

ERL FAT/TOTAL
ERL FA1/ /O1AL

ERL FAT/TOTAL

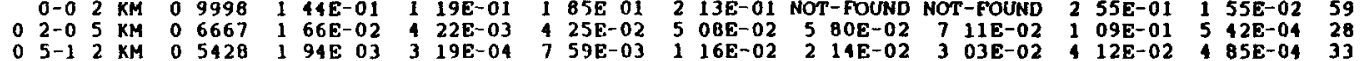

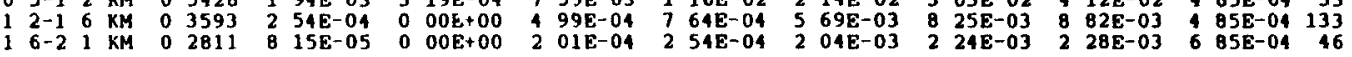




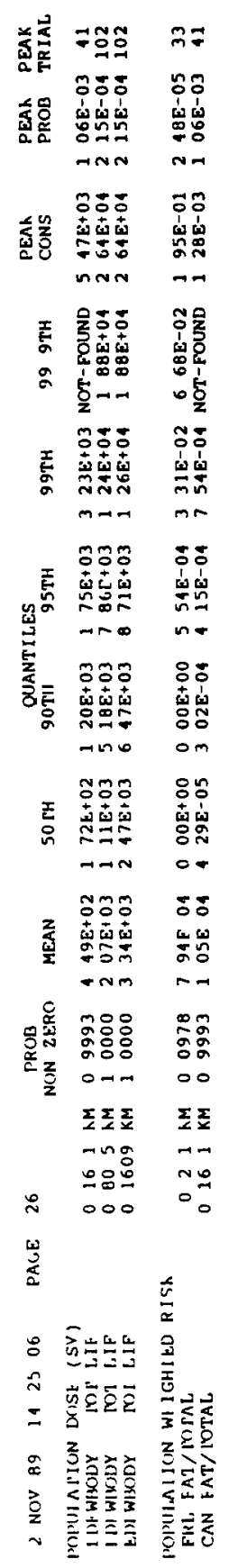


DATE AND PIME OF RUN = MACCS 2 NOV-89 1425 O6 VERSION $1511,10 / 16 / 89$, D CHANIN

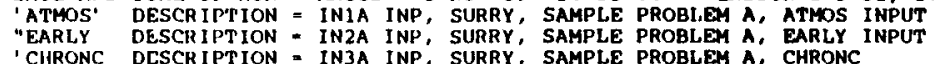

SOURCE TERM 2 OF 2

SOURCE TERM I REDUCED BY A FACTOR OF TEN

RESULIS PROM THE 'CHRONC" MODULE ALONE

COHORT 4 - IN3A INP, SURRY, SAMPLE PROBLEM A, CHRONC

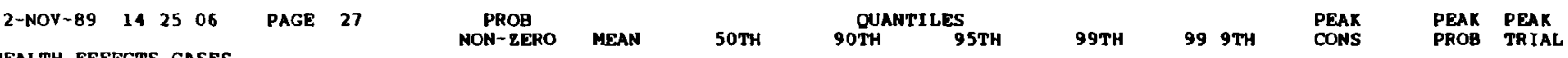
HEALTH EFFECTS CASES

CAN FAT/TOTAL

CAN FAT/THYROID

CAN FAT/GI

CAN FAT/LEUKEMIA

CAN FAT/OTHE

CAN INJ/TOTAL

CAN FAT/TOTAL

POPULATION DOSE (SV)

EDEWBODY TOT LIF
EDEWBODY TOT LIF

POPULATION WEIGHIED RISK

CAN FAT/ DOTAL

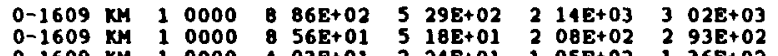

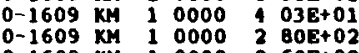

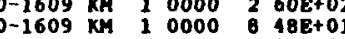

-1609 $\mathrm{km}$

$0-1609 \mathrm{kM} 1000020 \mathrm{OB}+02$

$1198+03 \quad 512 E+03 \quad 717 E+03$

$19 E+02>42 E+02$

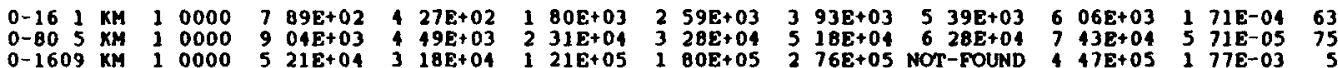

EDEWBODY POP DOSE (SV) $0-1609 \mathrm{KM}$

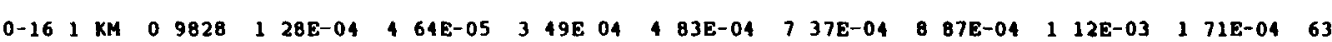

LONG TERM DIRECT EXPOSURE PATHWAYS

LONG-TESM GHOUNDSHINE DOSE

LONG-TERM RESUSPENSION DOSE

POP DEPENDENT DECONTAMINATION DOSE

MILK DIRECT-DEPOSI'PION DOSE
CROP DIRECT-DEPOSITION DOSE

MLK ROOT-UPTAKE DOSE
CROP ROO-UPTAKE DOSE

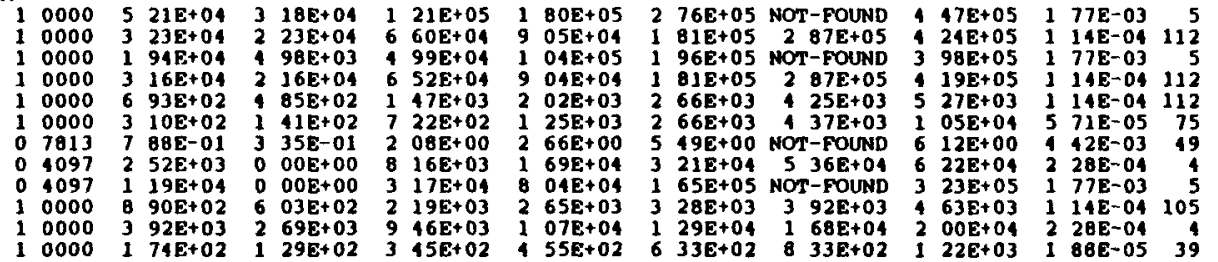

EDEWBODY POP DOSE (SV) 0-80 $5 \mathrm{kM}$

TONA-TERM DIRECT EXPOSURE PATHWAYS

TOTAL INGESTION PATHWAYS DOSE

LONG-TERM GROUNDSHNE DOSE

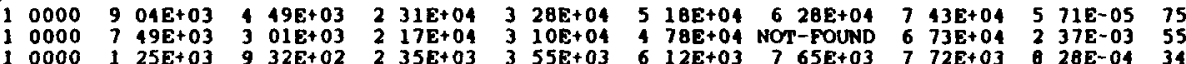

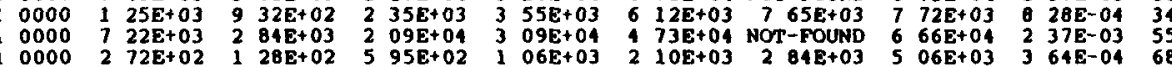




\begin{tabular}{|c|c|c|c|c|c|c|c|c|c|}
\hline $\begin{array}{llllll}\text { NOV 89 } & 14 & 25 & 06 & \text { PAGE } & 28\end{array}$ & $\begin{array}{l}\text { PROB } \\
\text { NON ZERO } \\
\text { KM }\end{array}$ & MEAN & 50TH & $90 \mathrm{OPHAN}$ & $\begin{array}{l}\mathrm{ESS}_{95 \mathrm{TH}} \\
\end{array}$ & 99TH & 99 9TH & $\begin{array}{l}\text { PEAK } \\
\text { CONS }\end{array}$ & \\
\hline 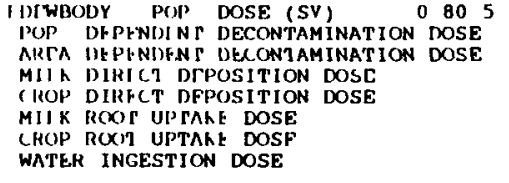 & $\begin{array}{rl}\text { KM } & 9999 \\
0 & 9999 \\
0 & 7619 \\
0 & 4096 \\
0 & 1096 \\
0 & 1090 \\
1 & 0000 \\
1 & 0000 \\
1 & 0000\end{array}$ & 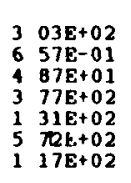 & $\begin{array}{ll}1 & 31 E+02 \\
2 & 71 E+02 \\
0 & 00 E+00 \\
0 & 00 E+00 \\
1 & 008+02 \\
1 & 08 E+02 \\
5 & 23 E+02 \\
8 & 03 E+01\end{array}$ & $\begin{array}{ll}7 & 20 E+02 \\
1 & 55 E+00 \\
1 & 34 E+02 \\
1 & 31 E+03 \\
2 & 49 E+02 \\
9 & 79 E+02 \\
2 & 33 E+02\end{array}$ & 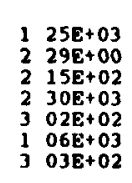 & 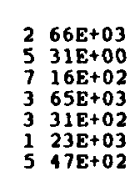 & 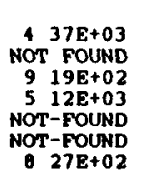 & & \\
\hline 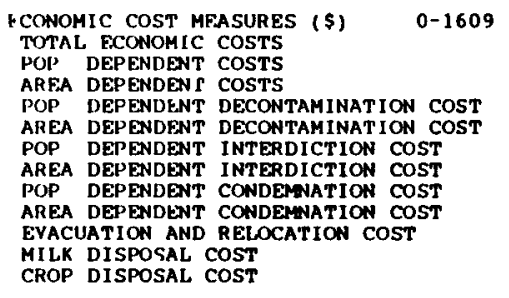 & $\begin{array}{ll}K M & \\
1 & 0000 \\
1 & 0000 \\
1 & 0000 \\
1 & 0000 \\
0 & 0000 \\
1 & 7813 \\
0 & 0000 \\
0 & 9698 \\
0 & 6839 \\
1 & 0000 \\
1 & 0000 \\
0 & 9314 \\
0 & 4314\end{array}$ & 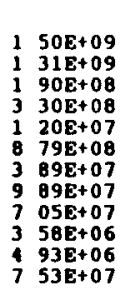 & 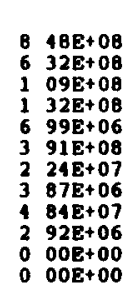 & 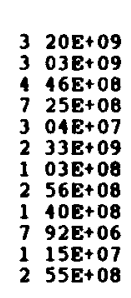 & 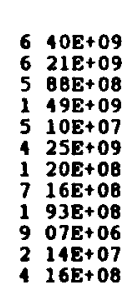 & 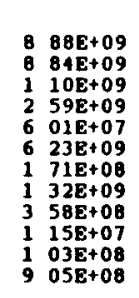 & 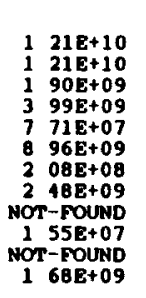 & & \\
\hline 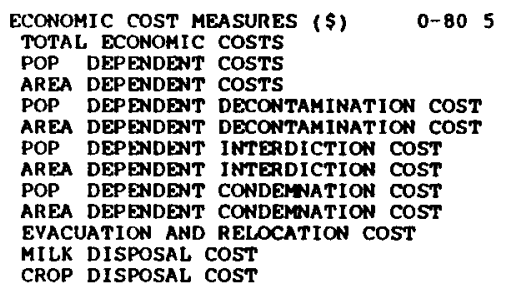 & $\begin{array}{ll}\mathrm{KM} & \\
1 & 0000 \\
1 & 0000 \\
1 & 0000 \\
1 & 0000 \\
0 & 9999 \\
0 & 7619 \\
0 & 9999 \\
0 & 9953 \\
0 & 9553 \\
0 & 6839 \\
1 & 0000 \\
1 & 0000 \\
0 & 0014 \\
0 & 4314 \\
0 & 4314\end{array}$ & 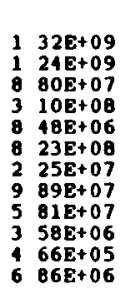 & 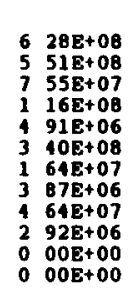 & 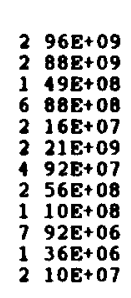 & 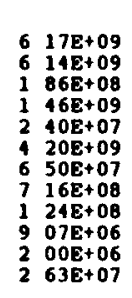 & & $\begin{array}{rl}1 & 2 \\
1 & 2 \\
2 & \\
3 & \\
\text { NOT- } \\
8 \\
\text { NOT- } \\
2 \\
2 \\
2 \\
1 \\
\text { NOT }\end{array}$ & & \\
\hline 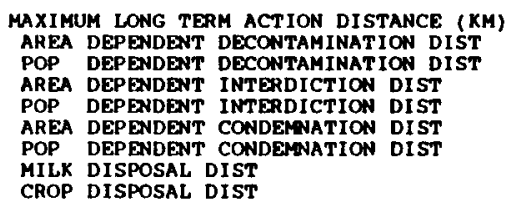 & $\begin{array}{ll}0 & 7813 \\
1 & 0000 \\
0 & 9698 \\
1 & 0000 \\
1 & 0000 \\
0 & 0000 \\
0 & 6839 \\
0 & 4314 \\
0 & 4314\end{array}$ & 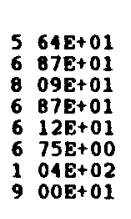 & $\begin{array}{ll}5 & 43 E+1 \\
6 & 14 E+01 \\
7 & 140 E+01 \\
6 & 14 E+01 \\
5 & 10 E+01 \\
7 & 64 E+00 \\
0 & 00 E+00 \\
0 & 00 E+00\end{array}$ & $\begin{array}{ll}1 & 14 E+02 \\
1 & 13 E+02 \\
1 & 14 E+02 \\
9 & 78 E+01 \\
1 & 20 E+01 \\
3 & 61 E+02 \\
3 & 09 E+02\end{array}$ & 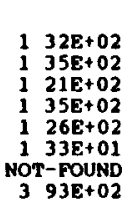 & & & & \\
\hline 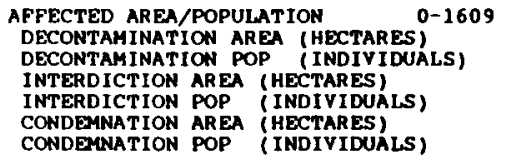 & $\begin{array}{lll} & 7 & 7 \\
1 & 0 \\
0 & 9 \\
1 & 0 & 0\end{array}$ & $\begin{array}{l}204 \\
748 \\
746 \\
746 \\
74\end{array}$ & $\begin{array}{ll}1 & 05 \\
2 & 73 \\
2 & 72 \\
2 & 73 \\
2 & 28\end{array}$ & & $\begin{array}{l}3678 \\
11080 \\
3078 \\
9 \\
9\end{array}$ & & $\begin{array}{l}05 \\
05 \\
05 \\
05 \\
05 \\
\text { ND }\end{array}$ & & \\
\hline
\end{tabular}




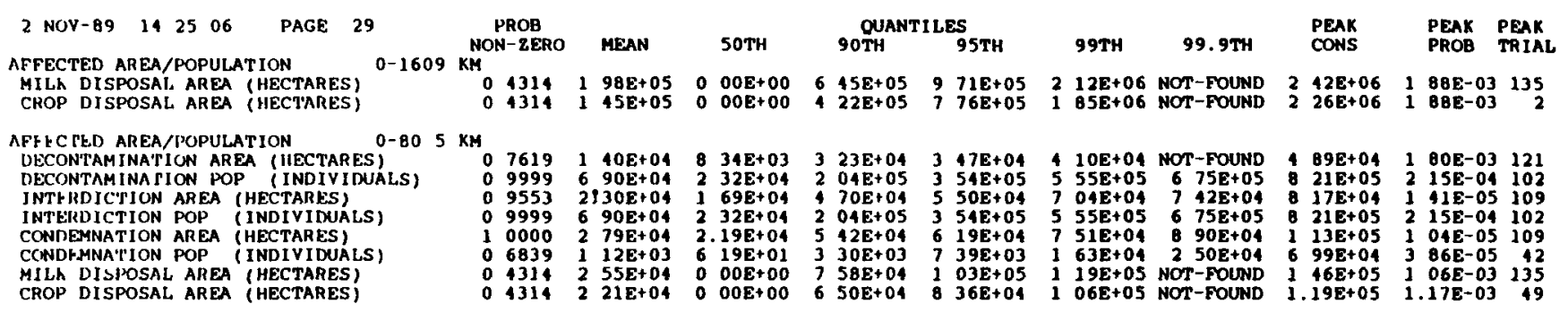



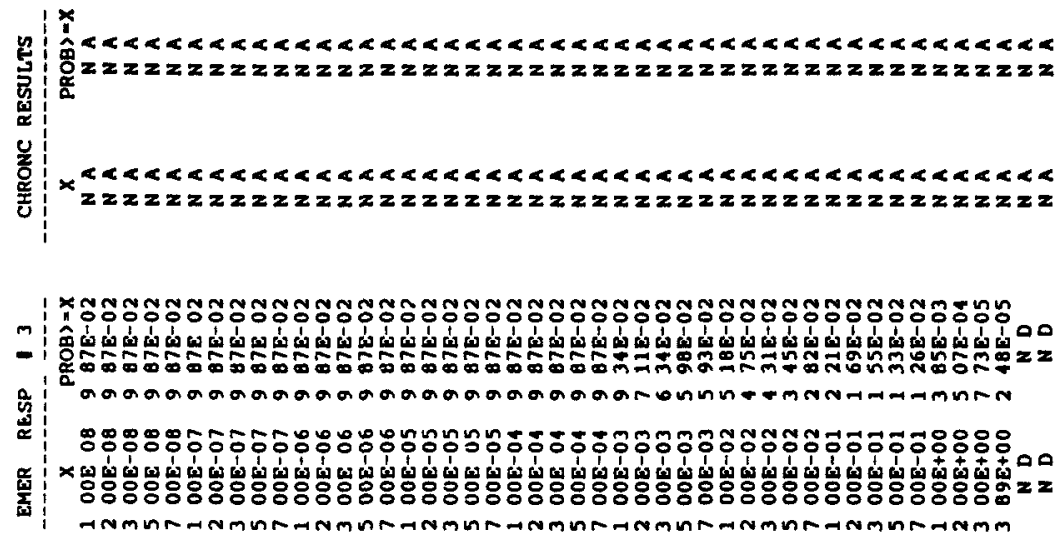
-Nmnn-NmnN-Nmnn-NmnN-Nmnn-NmnNaNmnn-Nmnn-Nmm

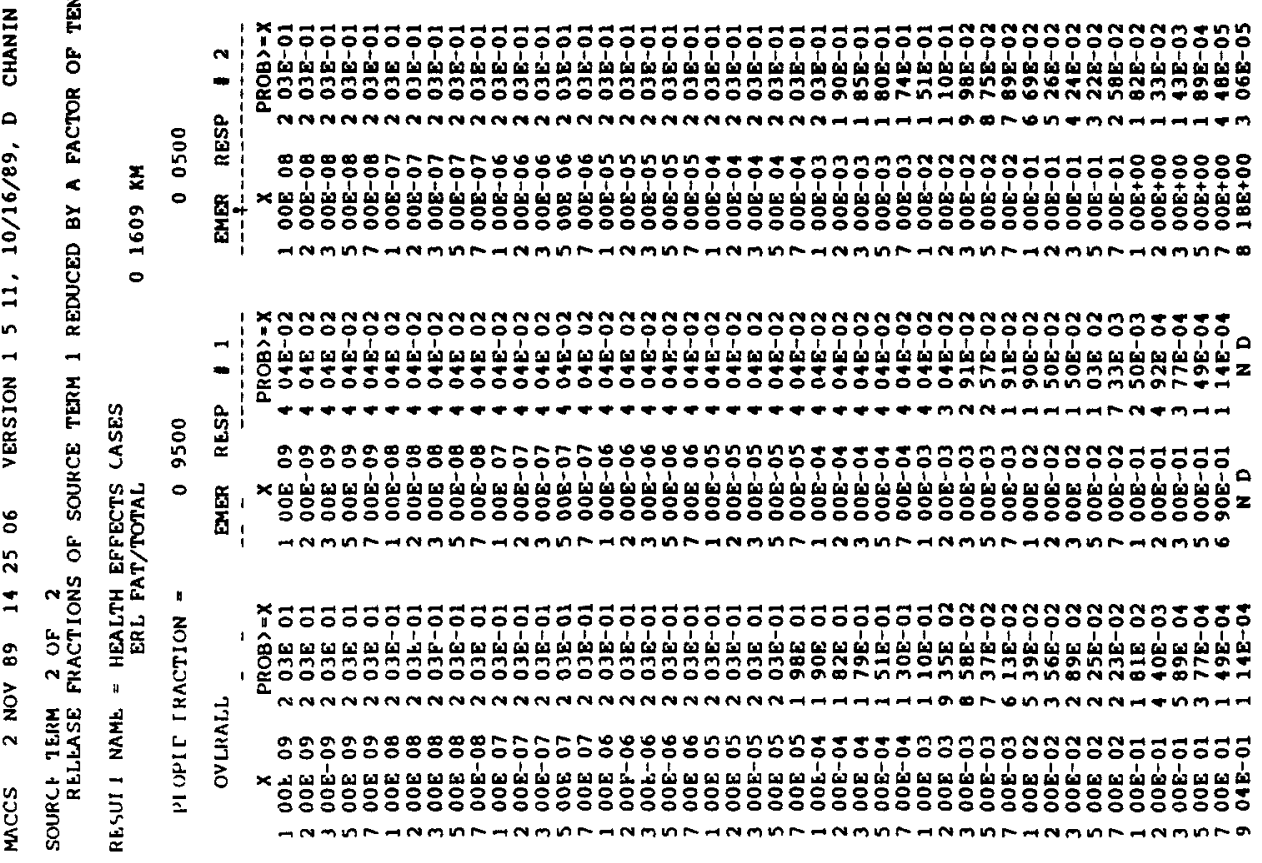


m

苋

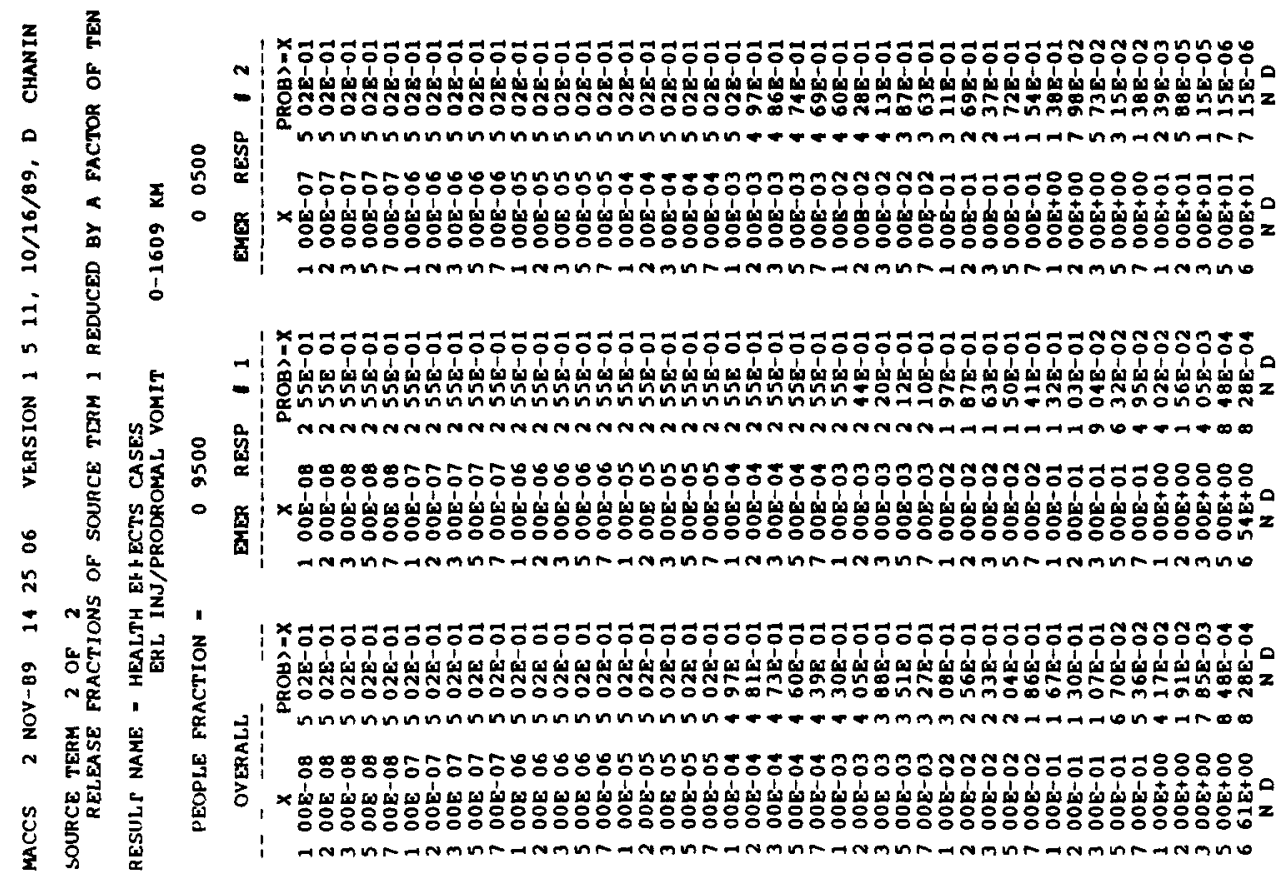

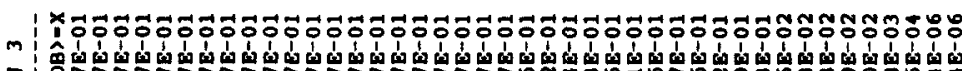

丹

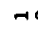

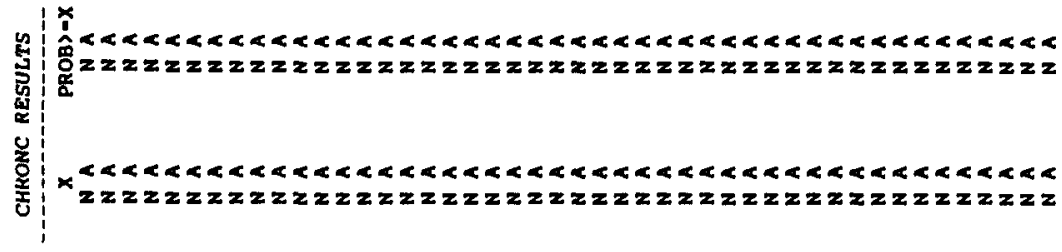
茴

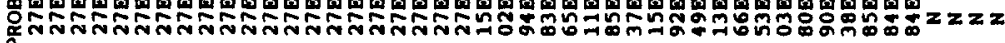

落

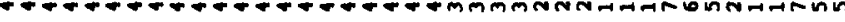

×

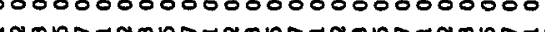




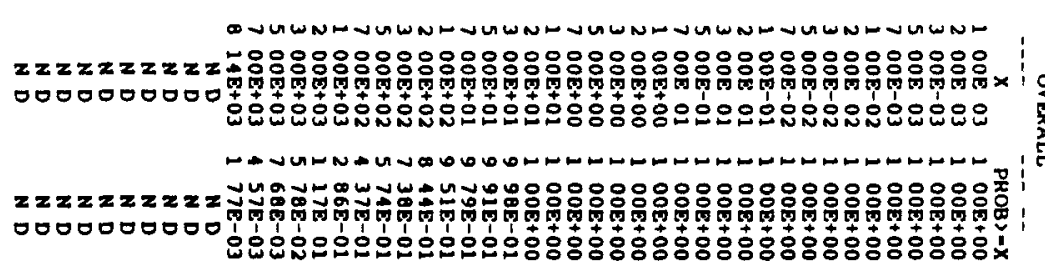

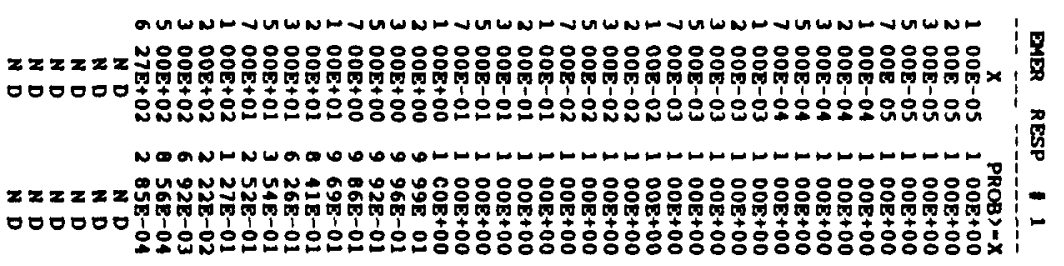

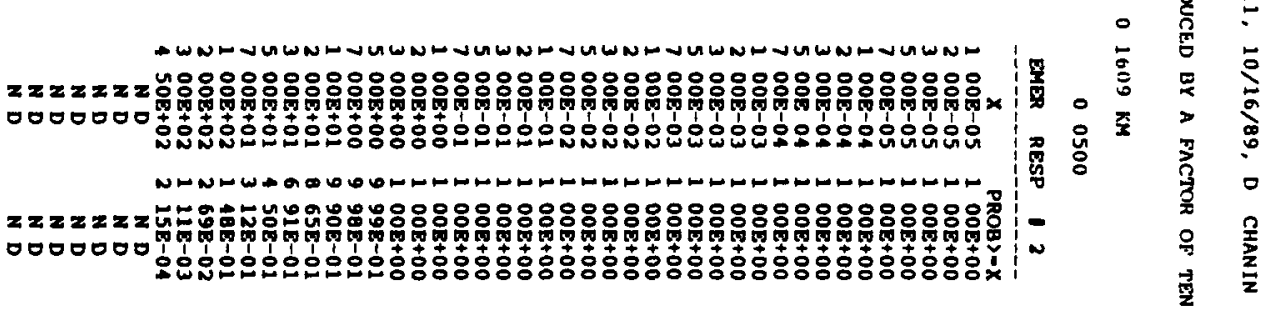
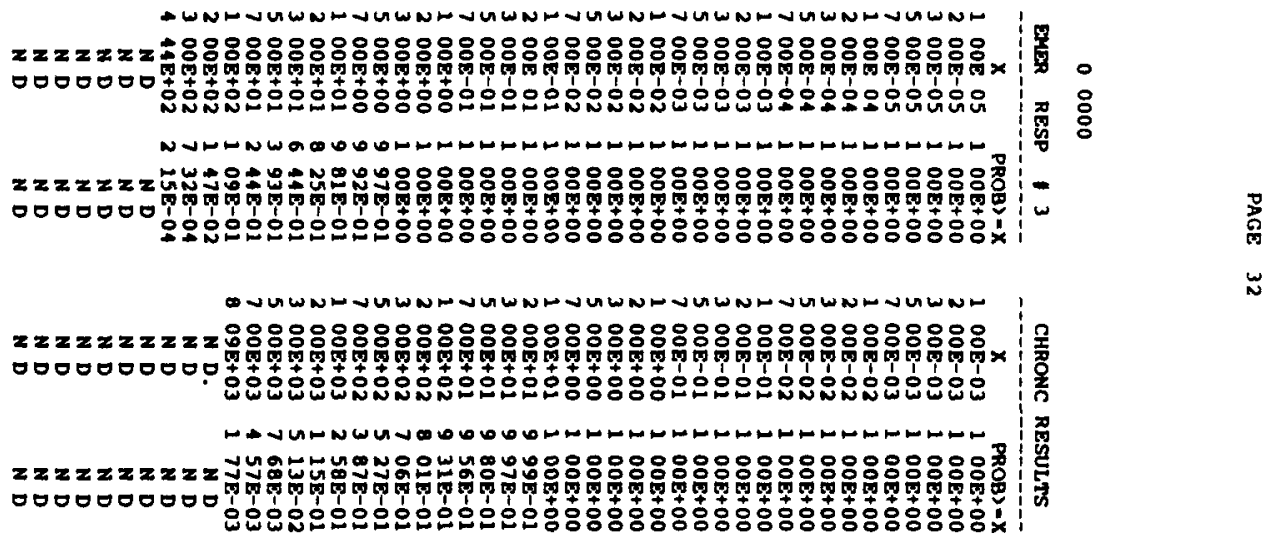


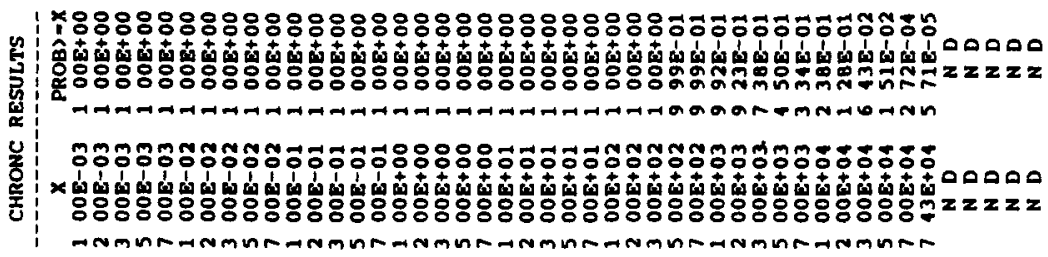

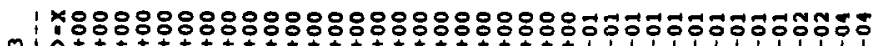

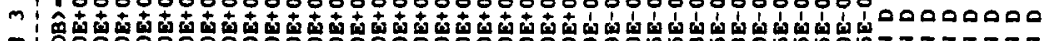

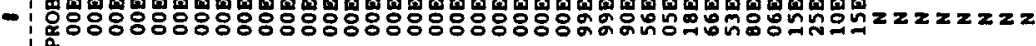

芒商

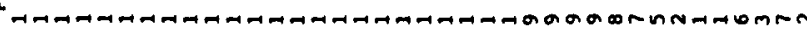

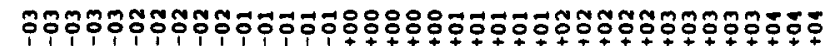

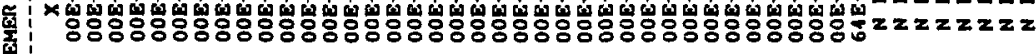

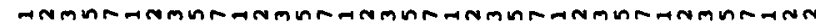

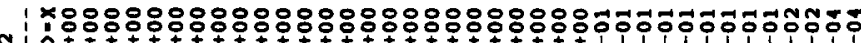

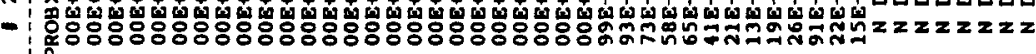
・

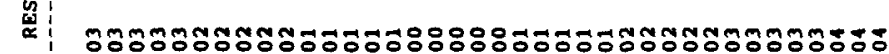

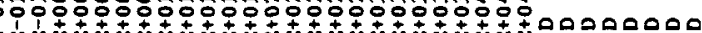
|

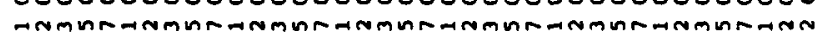

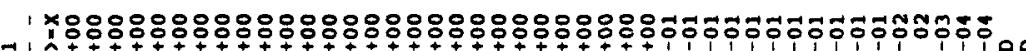

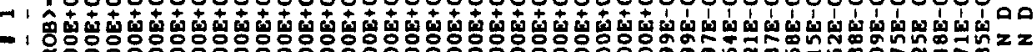

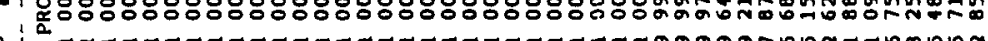

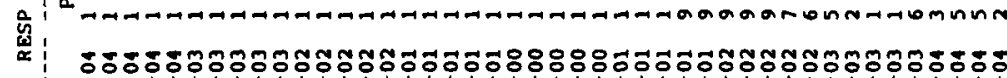

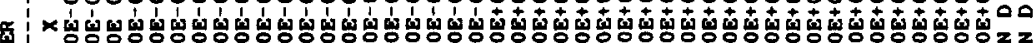
质

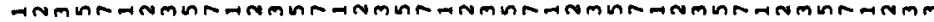

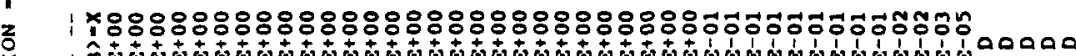

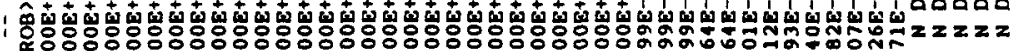

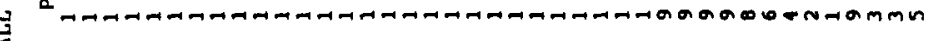

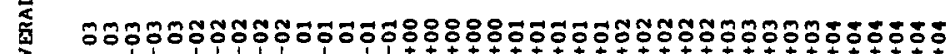

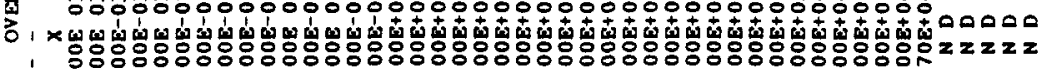

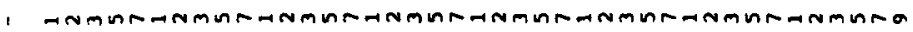



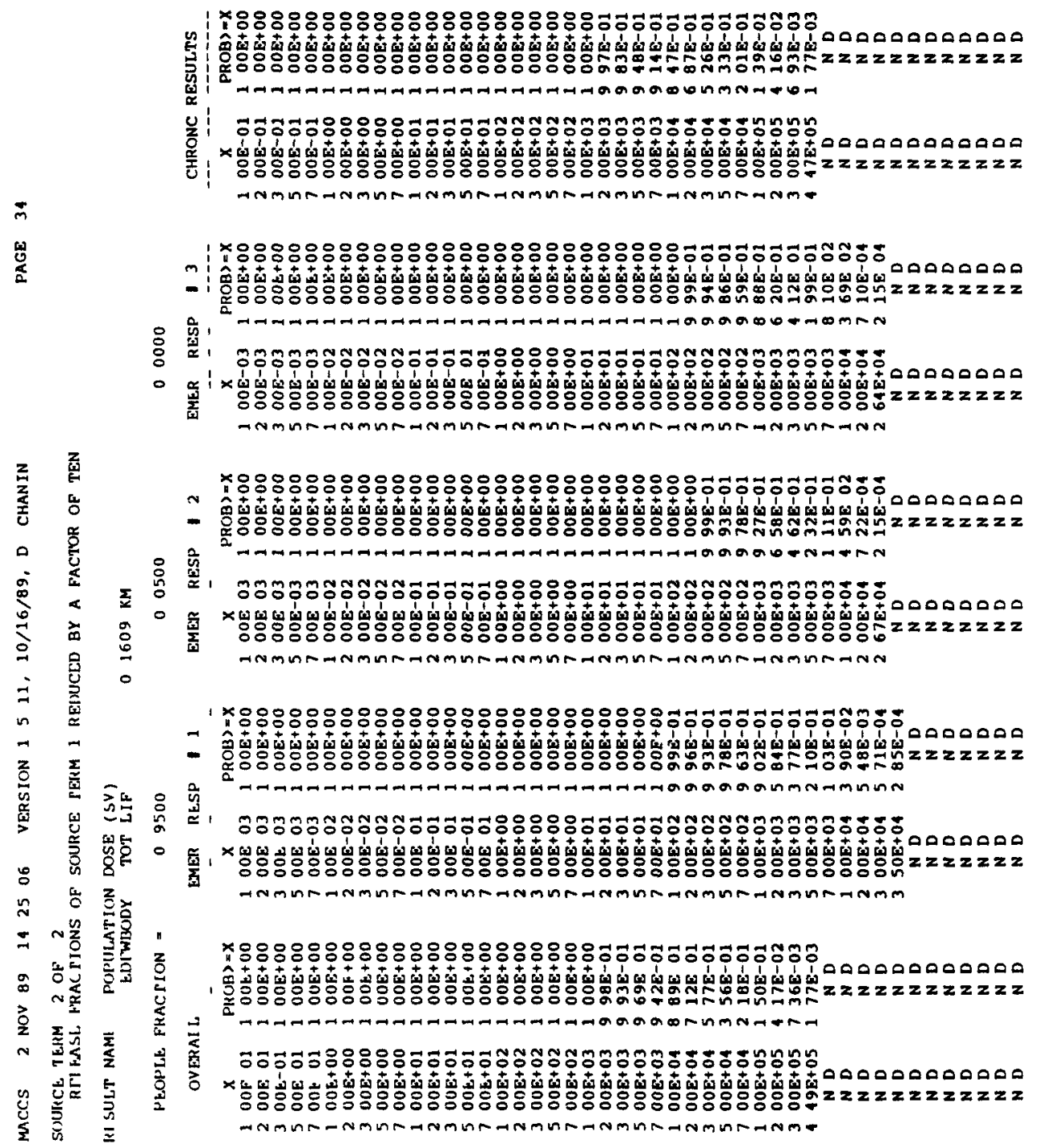


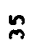

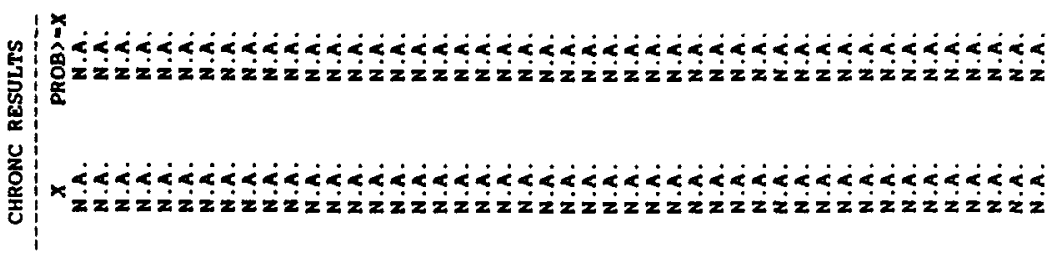

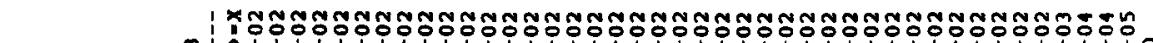

$m$ holo - Oำ

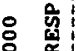

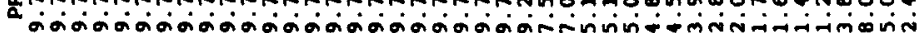

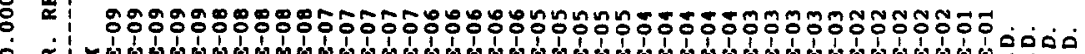

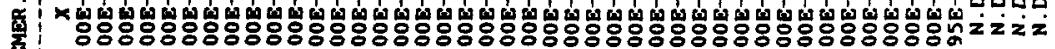

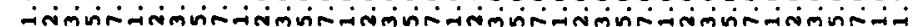

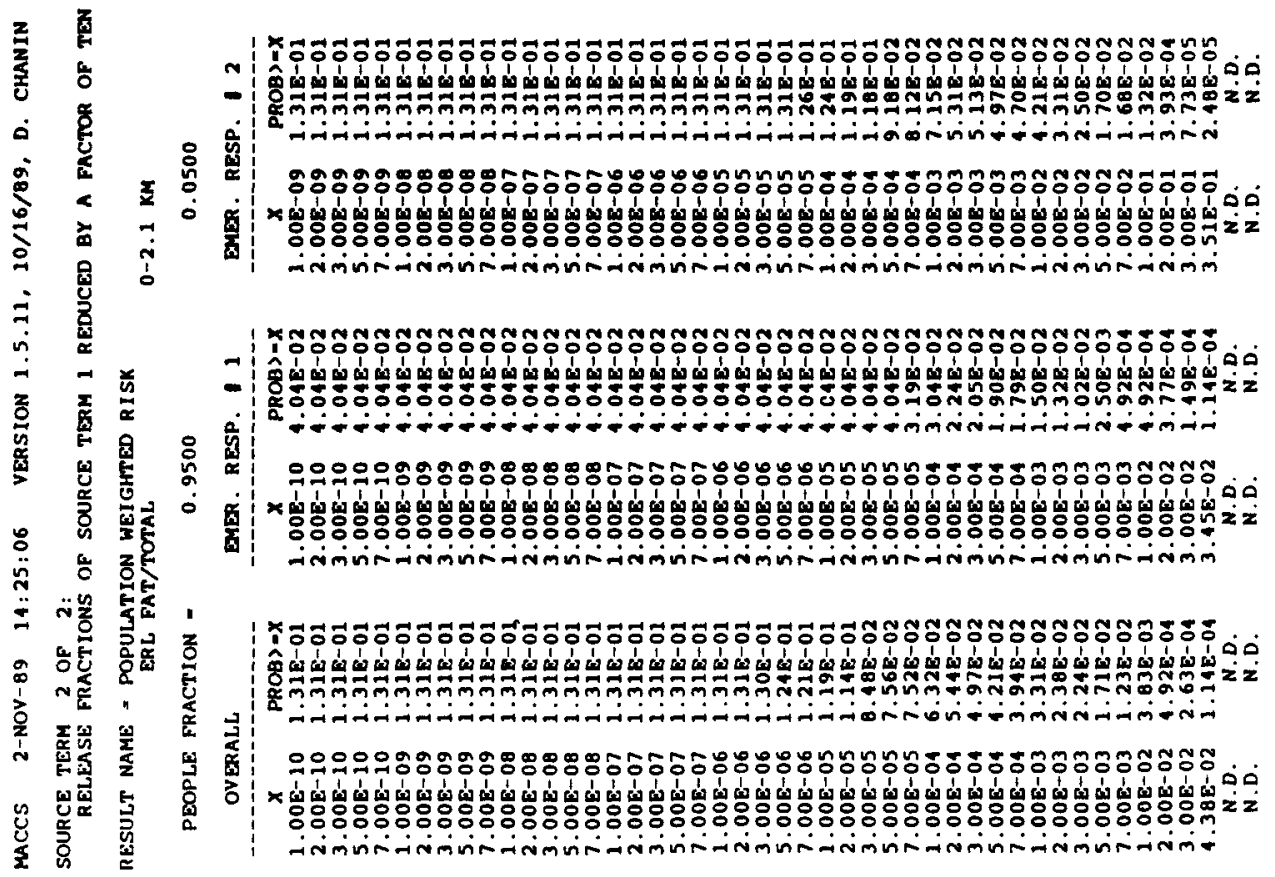




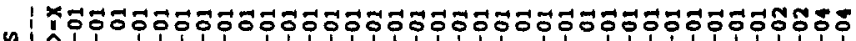

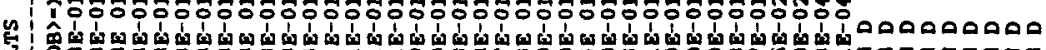

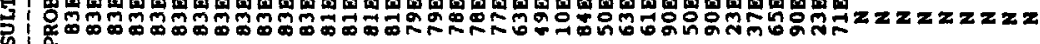

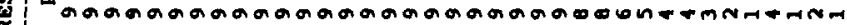

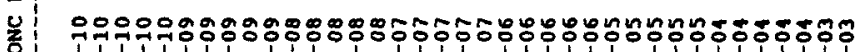

× ×

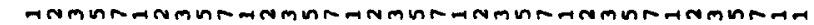

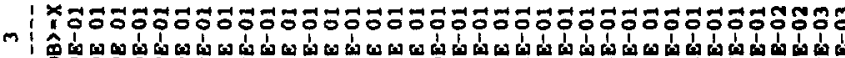

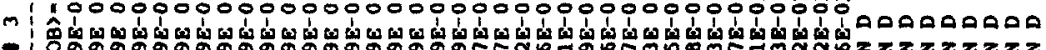

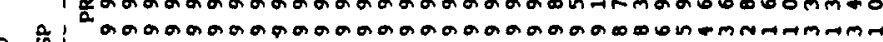

: 舟

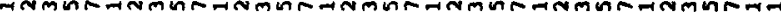

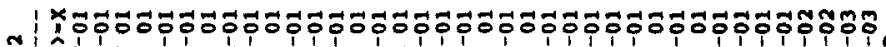
๑ ॠूँ 总

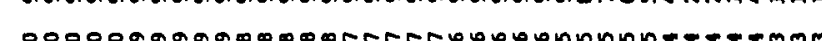

1×1

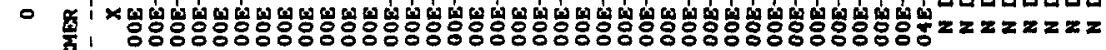

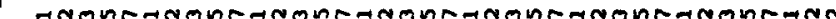

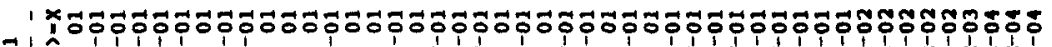
- - 鲑

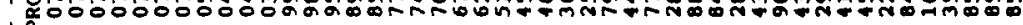

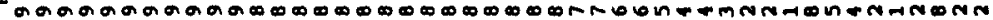

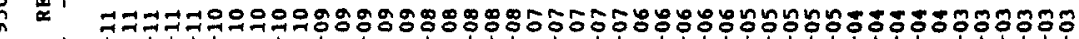

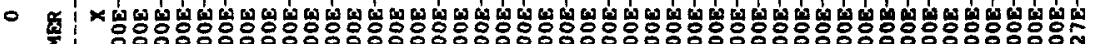

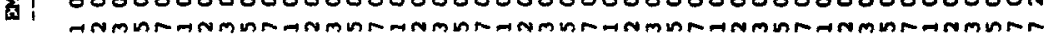

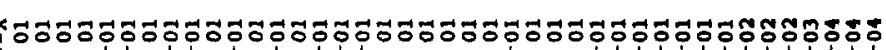

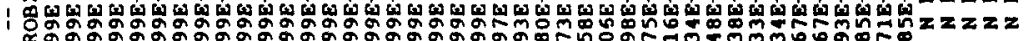

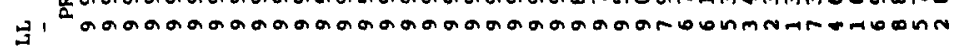
(a)

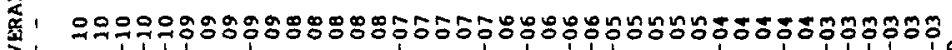

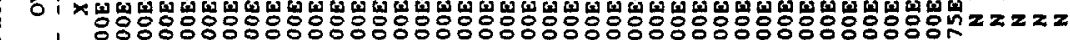

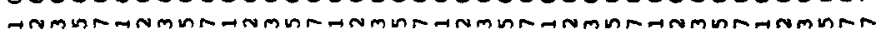


U, S, NRC

Denwood Ross, RES, MS - NLOO7

Themis Speis, RES, MS-NLOO7

Brian Sheron, RES/DSR, MS-NLOO7

Joseph Murphy, RES/DSR, MS-NL007

Mark Cunningham, RES/PRAB, MS-NLS 372

Mat Taylor, NRC/EDO, MS-17G21

R Wayne Houston, RES, MS-NLO07

Bill Morris, RES/DRA, MS-NLO07

Zoltan Rosztoczy, RES/DRA, MS-NLOO7

Donald Cool, RES/RPHEB, MS-NLS139

Warren Minners, RES/DSIR, MS-NLS 360

Thomas King, RES/DSIR, MS-NLS360

William Beckner, RES/SAIB, MS-NLS 324

Frank Congel, NRR/DREP, MS - 10E4

Charles Willis, NRR/DREP, MS-1OE4

Richard Barrett, NRR/PRAB, MS-10A2

Lemoine Cunningham, NRR/PRAB, MS-11D23

Ashok Thadani, NRR/DST, MS-8E2

William Russell, RI

Stewart Ebneter, RII

A Bert Davis, RIII

Robert Martin, RIV

John Martin, RV

James Glynn, RES/PRAB, MS-NLS 372

Harold VanderMolen, RES/PRAB, MS - NLS 372

Sarbes Acharya, RES/PRAB, MS-NLS372 (10)

James Johnson, RES/PRAB, MS-NLS 372

Les Lancaster, RES/PRAB, MS-NLS 372

Pradyot Niyogi, RES/PRAB, MS-NLS 372

Chris Ryder, RES/PRAB, MS - NLS 372

Michael Jamgochian, RES/SAIB, MS-NLS 324

Jocelyn Mitchell, RES/SAIB, MS-NLS324

Leonard Soffer, RES/SAIB, MS-NLS 324

John Ridgely, RES/SAiB, MS-NLS 324

Harold Peterson, RES/RPHEB, MS-NLS139

Shlomo Yaniv, RES/RPHEB, MS-NLS139

Robert Kornasiewicz, RES/WMB, MS-NLS 260

Tim Margulies, RES/WMB, MS-NLS260

Joe Levine, NRR/PRPB, MS-11D23

Jim Martin, NRR/PRPB, MS-11D23

Frank Skopec, NRR/PRPB, MS-11D23

Edward Podolak, NRR/PEPB, MS-10D4

Robert Palla, NRR/PRAB, MS-10A2

Tom McKenna, AEOD/IRB, MS-3206 
Natl. Energy Software Center (20) Argonne National Laboratory Attn: Mr. Larry Eyberger 9700 S. Cass Avenue Argonne, IL 60439

Argonne National Laboratory (3)

Attn: Mr. S. Y. Chen

Mr. Kou-John Hong

Mr. Brad Micklich

9700 S. Cass Avenue

Argonne, IL 60439

Brookhaven National Laboratory (3)

Attn: Mr. Arthur Tingle

Mr. Eric Cazzoli

Ms. Carrie Grimshaw

Building 130

Upton, NY 11973

EG\&G Idaho, Inc. (4)

Attn: Mr. Jack Dallman

Mr. Chuck Dobbe

Mr. John Tolli

Ms. Sandra Brereton MS 3523

P.O. Box 1625

Idaho Falls, ID 83415

Knolls Atomic Power

Laboratory (2)

Attn: Mr. Ken McDonough

Mr. Dominic Sciaudone

Box 1072

Schenectady, NY 12301-1072

Mr. Dennis Strenge

Pacific Northwest Laboratory

RTO /125

P.O. Box 999

Richland, WA 99352

Mr. Fred Mann

Westinghouse Hanford Co.

W/A. 53

P.O. Box 1970

Richland, WA 99352

Savannah River Laboratory (2)

Attn: Mr. Dave Sharp

Mr. Kevin 0'Kula

Aiken, SC 29808

omimoroflin

IIIS PRGE
Oak Ridge National Laboratory (2)

Attn: Mr. Keith F. Eckerman

Mr. Robert W. Roussin

P. O. Box 2008

Oak Ridge, TN 37831

Los Alamos National Laboratory (2)

Analysis and Assessment Division

Attn: Ms. Mary Meyer

Ms. Jane Booker

Los Alamos, NM 87545

Lawrence Livermore National

Laboratory (3)

Attn: Mr. George Greenly

Mr. Marvin Dickerson

Mr. Rolf Lange

Livermore, CA 94550

Mr. Terry Foppe

Safety Analysis Engineering

Rocky Flats Plant

Energy Systems Group

Rockwell International Corp.

P.0. Box 464

Golden, CO 80401

U.S. Environmental Protection Agency (2)

Office of Radiation Programs

Environmental Analysis Division

Attn: Mr. Allen Richardson

Mr. Joe Logsdon

Washington, D.C. 20460

U.S. Department of Energy (2)

Attn: Mr. Ken Murphy (EH351)

Mr. Ed Branagan (EH332)

Washington, D.C. 20545

Mr. Robert Ostmeyer

U.S. Dept. of Energy

Rocky Flats Area Office

P.0. Box 928

Golden, CO 80402-0928 
Mr. Bruce Burnett

CDRH (HFZ - 60)

U.S. Department of Health and Human Services

Food and Drug Administration

5600 Fishers Lane

Rockville, MD 20857

Mr. Scott Bigelow

$S$ - CUBED

2501 Yale SE, Suite 300

Albuquerque, NM 87106

Mr. David Black

American Electric Power

1 Riverside Plaza

Columbus, $\mathrm{OH} 43215$

Mr. Gerald Davidson

Fauske and Associates, Inc. $16 \mathrm{~W} 070$ West 83rd Street

Burr Ridge, IL 60521

Mr. Keith Woodard

Pickard, Lowe, and Garrick

Suite 730

1615 M. Street

Washington, DC 20036

Mr. Jim Mayberry

Ebasco Services

160 Chubb Ave.

Lyndhurst, NJ 07071

Ms. Christine Miller

F- 30

Koshland Way

Santa Cruz, CA 95064

Mr. Mike Cheok

NUS

910 Clopper Road

Gaithersburg, MD 20878

Mr. Ken $0^{\prime} B r i e n$

University of Wisconsin

Nuclear Engineering Dept.

153 Engineering Research Bldg.

Madison, WI 53706
Mr. Harold Careway

General Electric Co., M/C 754

175 Curtner Ave.

San Jose, CA 95129

Ms. Judy Rollstin

GRAM, Inc.

1709 Moon NE

Albuquerque, NM 87112

Ms. Zen Mendoza

SAIC

5150 E1 Camino Real

Suite C31

Los Altos, CA 94022

SAIC (2)

Attn: Mr. Chris Amos

Mr. Paul Mattingly

2109 Air Park Rd. SE

Albuquerque, NM 87106

SAIC (3)

Attn: Mr. Roger Blond

Mr. Dave Aldrich

Mr. Geoff Kaiser

Mail Stop 2-5-1

1710 Goodridge Drive

McLean, VA 22102

Mr. John Luke

Florida Power \& Light

P.O. Box 14000

Juno Beach, FL 33408

Prof. F. Eric Haskin

Dept. of Nuclear Engineering

University of New Mexico

Albuquerque, NM 87131

Duke Power Co. (2)

Design Engineering

Attn: Mr. Duncan Brewer

Mr. Steve Deskevich

422 South Church Street

Charlotte, NC 28242 
Professor Jon Helton

Mathematics Dept.

Arizona State University

Tempe, AZ 85287

Mr. Griff Holmes

Westinghouse Electric Co.

Energy Center East

B1dg. 371

P.0. Box 355

Pittsburgh, Pa 15230

Mr. Edward Warman

Stone \& Webster Engineering Corp.

P. 0. Box 2325

Boston, MA 02107

Mr. William Hopkins

Bechtel Power Corporation

15740 Shady Grove Road

Gaithersburg, MD 20877-1454

Mr. R. Toossi

Physical Research, Inc.

25500 Hawthorne Blvd.

Torrance, CA 90505-6828

Technadyne Engineering

Consultants, Inc. (3)

Attn: Mr. Burt Newmark

Mr. David Chanin

Mr. Mel Piepho

P.0. Box 13928

Albuquerque, NM 87192

Mr. Bill Eakin

Northeast Utilities

Box 270

Hartford, CT 06141-0270

Mr. Ian Wall

Electric Power Reseach Institute 3412 Hillview Avenue

Palo Alto, CA 94304

Mr. Jim Meyer

Scientech

11821 Parklawn Dr.

Suite 100

Rockville, MD 20852
Mr. Ray Ng

NUMARC

1776 Eye St, NW

Suite 300

Washington, DC 20006-2496

Mr. Robert Gobel

Clark University

Center for Technology,

Environment and Development

950 Main St.

Worcester, MA 01610-1477

Mr. Ken Keith

TVA

W 10 D 201

400 West Summit Hill

Knoxville, TN 37902

Mr. Shengdar Lee

Yankee Atomic Electric Company 580 Main St.

Bolton, MA 01740

Mr. Paul Govaerts

Studiecentrum voor Kernenergie (SCK/CEN)

Boeretang, 200

B-2400 Mol

Belgium

Mr. S. Daggupaty

Environment Canada

4905 Dufferin Street

Downsview

Ontario, M3H 5T4

Canada

Mr. Soren Thykier-Nielsen

Riso National Laboratory

Postbox 49

DK-4000 Roskilde

Denmark 
Mr. Seppo Vuori

Technical Research Centre of

Finland (VTT)

Nuclear Engineering Laboratory

(YDI)

Lonnrotinkatu 37

P.O. Box 169

SF-00181 Helsinki 18

Finland

Mr. Daniel Manesse

IPSN

Boite Postale 6

F-92265 Fontenay-aux-Roses CEDEX

France

Mr. Joachim Ehrhardt

Institut fur Neutronenphysik und Reaktortechnik (INR)

Kernforschungszentrum Karlsruhe

GmbH

Postfach 3640

D-7500 Karlsruhe 1

Federal Republic of Germany

Mr. John G. Kollas

Institute of Nuclear Technology and

Radiation Protection

N.R.C.P.S. "Demokritos"

P.O. Box 60228

GR-153 10 Aghia Paraskevi

Attiki

Greece

ENEA/DISP

Attn: Mr. Alvaro Valeri

Mr. Alfredo Bottino

Via Vitaliano Brancati, 48

00144 Roma EUR

Italy

Mr. Hideo Matsuzuru

Tokai Research Establishment

Tokai-mura

Maka-gun

Ibaraki-ken, 319-11

Japan
Mr. Jan Van der Steen

KEMA Laboratories

Utrechtseweg, 310

Postbus 9035

NL-6800 ET Arnhem

Netherlands

Mr. D. Eugenio Gil Lopez

Consejo de Seguridad Nuclear

Calle Justo Dorado, 11

E-28040 Madrid

Spain

Mr. Lennart Devell

Studsvik Nuclear

Studsvik Energiteknik AB

S-611 82 Nykoping

Sweden

Mr. Hanspeter Isaak

Abteilung Strahlenschutz

Hauptabteilung fur die Sicherheit der Kernanlagen (HSK)

$\mathrm{CH}-5303$ Wurenlingen

Switzerland

Ms. Marion Hill

National Radiological Protection Board

Chilton

Didcot

Oxon. OXII ORQ

United Kingdom

Mr. William Nixon

$\mathrm{AEA} / \mathrm{SRD}$

Wigshaw Lane

Culcheth

Warrington

Cheshire WA3 4NE

United Kingdom.

Mr. G. Neale Kelly

Nuclear Safety Research

Commission of the European

Communities

Rue de la Loi, 200

B-1049 Bruxelles

Belgium 
Mr. Ephraim Asculai

Division of Nuclear Safety

Wagramestrasse, 5

P.0. Box 100

A-1400 Wien

Austria

Mr. Ulf Tveten, Head

Environmental Physics Section

Institutt for Energiteknikk

Postboks 40

$\mathrm{N}-2007$ Kjeller

Norway

Mr. M. K. Yeung

University of Hong Kong

Mechanical Engineering Dept.

Pokfulam

Hong Kong

Mr. Leonel Canelas

New University of Lisbon

Quinta de Torre

2825 Monte da Caparica

Portugal

Mr. Stephen Boult

Electrowatt Engineering Services

(UK) Ltd.

Grandford House

16 Carfax, Horsham

West. Sussex RH12 IUP

England

Ms. Nadia Soido Falcao Martins

Comissao Nacional de Energia Nuclear

R General Severiano 90 S/408-A

Rio de Janeiro

Brazil

Mr. Eli Stern

Israel AEC Licensing Div.

P.0. Box 7061

Tel-Aviv 61070

Israel
Mr. Der-Yu Hsia

Atomic Energy Council

67, Lane 144

Keelung Road, Section 4

Taipei, Taiwan 10772

Taiwan

Mr. Shankaran Nair

Central Electricity Generating Board

Berkeley Nuclear Laboratories

Berkeley

Gloucestershire GL13 9PB

United Kingdom

Mr. Paul Kayser

Division de la Radioprotection

1 , Avenue des Archiducs

L-1135 Luxembourg-Belair

Luxembourg

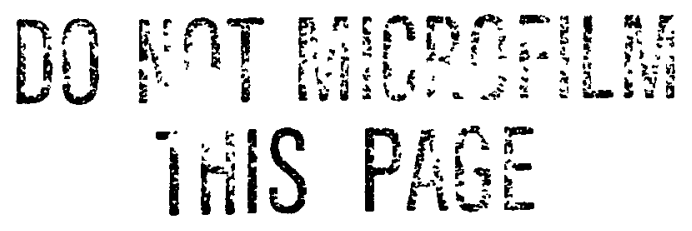


Sandia National Laboratories, Albuquerque, NM, 87185

3141 S. A. Landenberger (5)

3151 W. I. Klein

3212 H. N. Jow (10)

6216 D. J. Alpert

6216 J. I. Sprung (3)

6400 D. J. McCloskey

6410 D. A. Dahlgren

6412 A. L. Camp

6412 D. M. Kunsman

6412 A. C. Payne

6413 K. G. Adams

6413 R. J. Breeding

6413 T. D. Brown

6413 J. J. Gregory

6413 F. T. Harper

6413 S. J. Higgins

6415 R. M. Cranwell (10)

6415 B. L. O'Neal

6416 E. J. Bonano

6418 J. E. Kelly

6422 D. A. Powers

6429 K. D. Bergeron

6429 D. C. Williams

6453 L. F. Restrepo

7254 L. T. Ritchie

8524 J. A. Wackerly

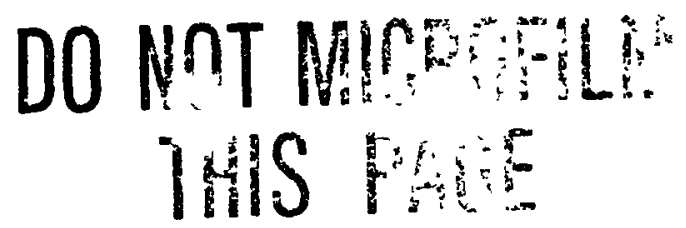

* U.S. GOVERNMENT PRINTING OFFICE: 1990--258-467/49754 


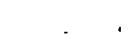


MELCOR Accident Consequence Code System (MACCS)

Volume 1: User's Guide

SAND86-1562

Volume 1

5 AUTHORISI

David 1. Chanin, Jeremy L. Sprung, Lynn T. Ritchie, Hong-Nian Jow

3 DATE REPORIT PUBLISHED

February 1990

4 FIN OR GAANT NUMBER

A1853

6 TYPE OF REPORT

Technical

7. PERIOD COVERED Inclusure Dores

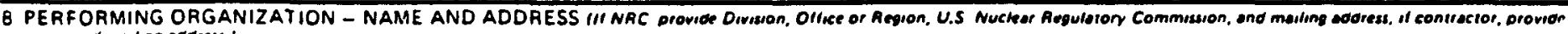
name and mailing oddress.

Sandia National Laboratories

Division 6415

P.O. Box 5800

Albuquerque, NM $\mathbf{8 7 1 8 5}$

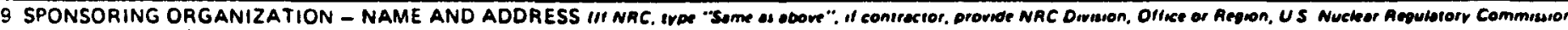
end moiling odoress.)

Division of Systems Research

Ofice of Nuclear Regulatory Research

U.S. Nuclear Regulatory Commission

Washington, DC 20555

10. SUPPLEMENTARY NOTES

11. ABSTRACT 1200 words or kas)

This report describes the MACCS computer code. The purpose of this code is to simulate the impact of severe accidents at nuclear power plants on the surrounding environment. Maccs has been developed for the U.S. Nuclesr Regulatory Commission to replace the previous CRAC2 code and it incorporates many improvements in modeling flexibllity in comparison to CRAC2.

The principal phenomena considered in MACCS are atmospheric transport. mitigative actions based on dose projection, dose accumulation by a number of pathways including food and water lngestion, early and latent health effects, and economic costs.

The MACCS code can be used for variety of applications. These include (1) probebilistic risk essessment (PRA) of nuclear power plants and other nuclear facilities, (2) sensitivity studies to gain a better understanding of the parameters important to PRA, and (3) cost-benefit analysis.

This report is composed of three volumes. Volume I, the User's Gulde, descrlbes the Input data requlrements of the MACCS code and provides directions for 1ts use es 1llustrated by three sample problems. Volume II. the Model Description, describes the underlying models that are implemented in the code, and Volume III, the Programer's Reference Manual, descrlbes the code's structure and database management.

12 KEY WORDSIDESCR!PTORS (List woros or phreses thal will essus mesereners in localung ine moort.)

Accidents, Atmospheric Dispersion, Dosimetry, Economic Costs, Emergency Response, Evacuation, Health Effects, MACCS Computer Code, Mitigative Actions, Nuclear, Offsite Consequences, Radiation, Radioactive Releases, Reactor Accidents, Relocation, Sheltering, Weather Sampling.

13 AVAILABILITY STATEMENT

Unlimited 14 SECURIT Y CLASSIFICATION (Thes Pape)

Unclassified TThes Reporti

Unclassified 15 NUMBER OF PAGES

16 PRICE 